\title{
De Novo Asymmetric Syntheses of Bioactive Natural Products and Carbohydrate Motifs
}

Yalan Xing

West Virginia University

Follow this and additional works at: https://researchrepository.wvu.edu/etd

\section{Recommended Citation}

Xing, Yalan, "De Novo Asymmetric Syntheses of Bioactive Natural Products and Carbohydrate Motifs" (2011). Graduate Theses, Dissertations, and Problem Reports. 4819.

https://researchrepository.wvu.edu/etd/4819

This Dissertation is protected by copyright and/or related rights. It has been brought to you by the The Research Repository @ WVU with permission from the rights-holder(s). You are free to use this Dissertation in any way that is permitted by the copyright and related rights legislation that applies to your use. For other uses you must obtain permission from the rights-holder(s) directly, unless additional rights are indicated by a Creative Commons license in the record and/ or on the work itself. This Dissertation has been accepted for inclusion in WVU Graduate Theses, Dissertations, and Problem Reports collection by an authorized administrator of The Research Repository @ WVU.

For more information, please contact researchrepository@mail.wvu.edu. 


\title{
De Novo Asymmetric Syntheses of Bioactive Natural Products and Carbohydrate Motifs
}

\author{
Yalan Xing \\ Dissertation submitted to the \\ Eberly College of Arts and Sciences \\ at West Virginia University \\ in partial fulfillment of the requirements \\ for the degree of
Doctor of Philosophy
in
Organic Chemistry \\ John H. Penn, Ph.D., Chair \\ George A. O'Doherty, Ph.D. \\ Patrick Callery, Ph.D. \\ Alan Stolzenberg, Ph.D. \\ Kung K. Wang, Ph.D. \\ C. Eugene Bennett Department of Chemistry
}

Morgantown, West Virginia

2011

Keywords: Glycosylation, Daumone, Deoxysugars, Cladospolides, Aspergillides Copyright 2011 Yalan Xing 


\title{
ABSTRACT \\ De Novo Asymmetric Syntheses of Bioactive Natural Products and Carbohydrate Motifs
}

\author{
Yalan Xing
}

De novo asymmetric syntheses of daumone I-1 and the fluorescent analog have been described. This route took advantage of the Noyori asymmetric hydrogenation of acetyl furan and alkynyl ketone, the Pd-catalyzed glycosylation and various postglycosylation transformations to finish the total synthesis of Daumone I-1, which provided materials for mechanism of action studies.

In an effort to mimics natures use of rare sugars in the synthesis of biologically important natural products (e.g., the carbohydrate portion of Daumone I-1), the O'Doherty group has been interested in developing new methods for practical and stereoselective synthesis. As part of these efforts, a concise synthesis of benzyl $\alpha-6$ deoxy-altro-pyranoside and benzyl-ascarylo-pyranoside has been developed. The synthesis relied upon a Pd-catalyzed glycosylation reaction, a highly diastereoselecitve epoxidation and ring opening.

An enantioselective synthesis of cladospolide B, C and (ent)-cladospolide D has been achieved. This synthesis not only provided access to these antimicrobial natural products but also corrected the structural assignment of cladospolide $\mathrm{D}$ by means of a de novo synthesis and kinetic/thermodynamic alkene isomerization. The synthesis coupled the Noyori reduction of alkynyl ketone and the alkyl zipper reaction with the conversion to and asymmetric oxidation of dienoates to enable the successful synthesis of cladospolides B-D.

A de novo asymmetric total synthesis of aspergilliges has been investigated. The challenge of our route is using a novel palladium-catalyzed addition of a vinyl group to install the key trans-pyran ring system. To date, an intermediate which is five steps away from a formal synthesis of aspergillide $\mathrm{C}$ has been prepared by Pd-catalyzed $C$ glycosylation and Julia olefination. Noyori reductions of furyl and propargyl ketone, alkyne zipper reaction and Au-catalyzed alkyne hydration have been utilized. 


\section{DEDICATED TO}

My husband, Wuming Yan, my daughter, Emma J. Yan

And my parents, Xinhui Zhang, Yifeng Xing 


\section{ACKNOWLEDGEMENTS}

I would like to express my deepest gratitude to my research advisor, Dr. George A. O'Doherty, I have been so fortunate to have an advisor who gave me the freedom to explore on my own, and at the same time the guidance to recover when I failed. George taught me how to become a better organic chemist, his patience and support helped me overcome many crisis situations and finish this dissertation.

I am deeply grateful to the chair of my committee Dr. John Penn for the valuable assistance and helpful suggestions. I also would like to give my sincere appreciation to other members of my committee, Dr. Patrick Callery, Dr. Wang and Dr. Alan Stolzenberg.

Though only my name appears on the cover of this dissertation, a great many people have contributed to its production. I would like to express my heart-felt gratitude to my family. My parents, my husband and my daughter have been a constant source of love, concern, support and strength all these years. I have to give a special mention for the support and love given by my parents-in-law. Without their understanding, encouragement and support, I could not have finished this thesis.

I would like to acknowledge the members of the O'Doherty group who I've had the pleasure of working with, Dr. Haibing Guo, Dr. Mingde Shan, Dr. Maoquan Zhou, Dr. Miaosheng Li, Dr. Dong Gao, Dr. Sanjeeva Rao Guppi, Dr. Matt Mortensen, Dr. SangWoo Kang, Dr. Qian Chen, Dr. Rajender Vemula, Dr. Hongyan Li, Dr. Mingzong Li, Phillip, Harsh, Xiaomei Yu, Bulan Wu, Leo Wang, Melvin Rajaratnam, Ehesan Sharif, 
Qi Zhang, Mike Cuccarese, Sumit Bajaj, Yashan Zhong, Pei Shi, Yangping Wang for all of the assistance they have given me in the last five years. I would also like to thank Dr. Novruz Akhmedov for his kind and friendly help during NMR experiments.

Financial supports from Dissertation fellowship WVU, the C. Eugene Bennett department of chemistry at West Virginia University, the National Institutes of Health and the National Science Foundation are also gratefully acknowledged. 


\section{TABLE OF CONTENTS}

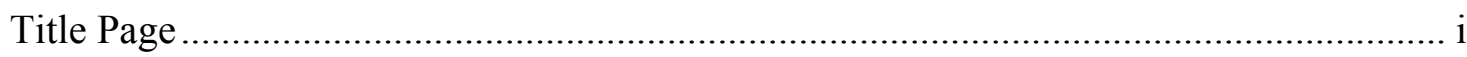

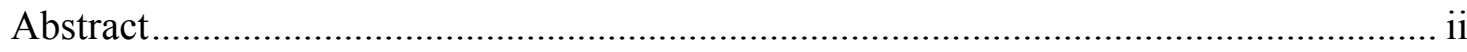

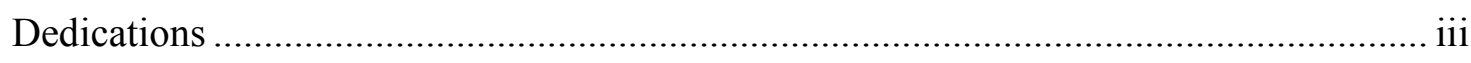

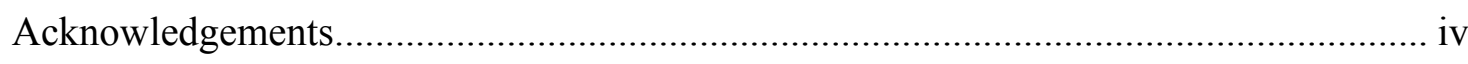

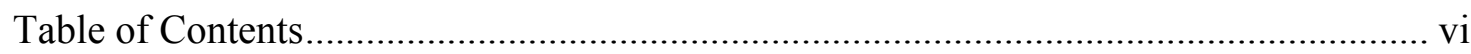

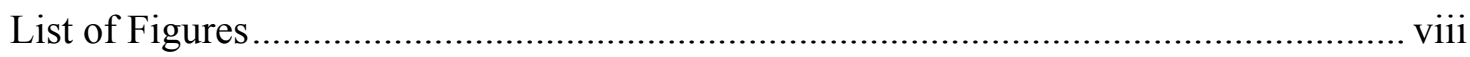

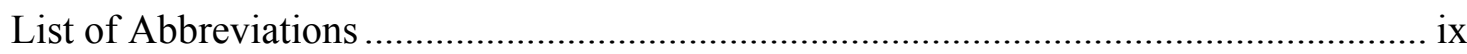

Chapter 1. De novo asymmetric synthesis of pheromone daumone I-1 ...........................

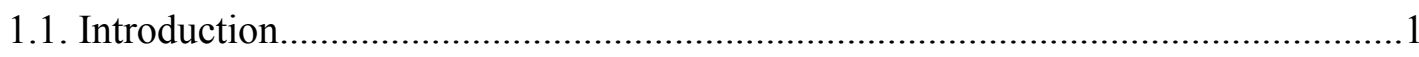

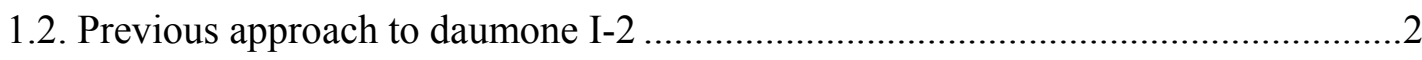

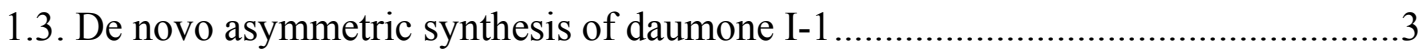

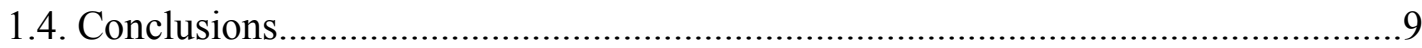

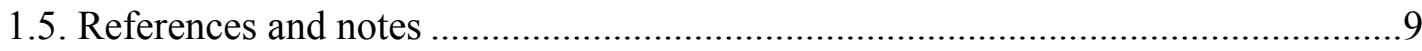

Chapter 2. De novo asymmetric synthesis of $\alpha$-6-deoxy-altro-pyranoside and $\alpha-3,6-$

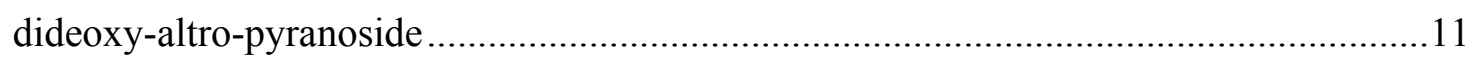

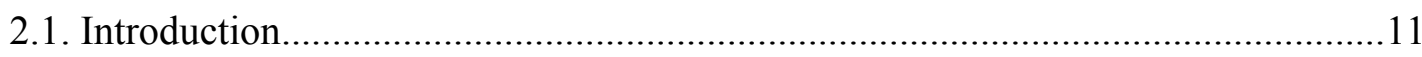

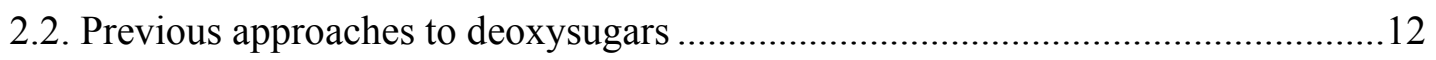

2.3. De Novo Synthesis of $\alpha$-6-deoxy-altro-pyranoside and $\alpha$-3, 6-dideoxy-altro-

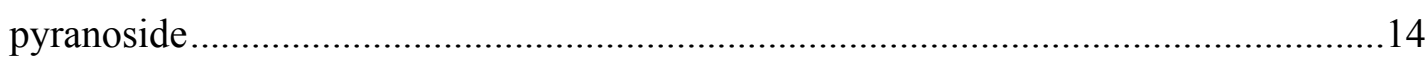

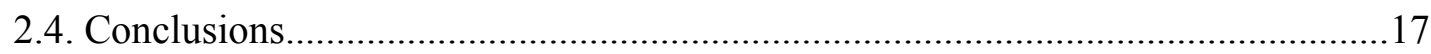

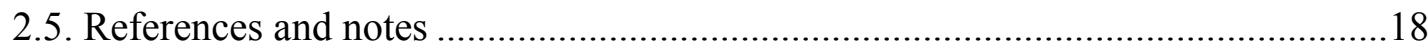

Chapter 3. Structure investigations of ent-cladospolide D by de novo synthesis and kinetic and thermodynamic isomerization. 


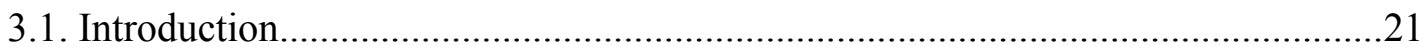

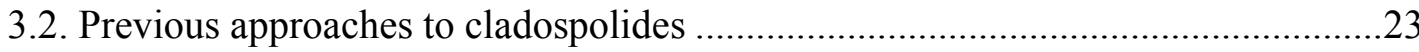

3.3. De Novo approach to natural product natural products cladospolides ...................25

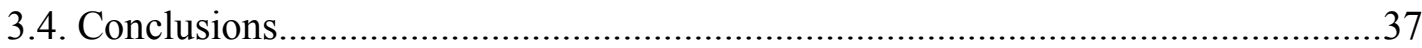

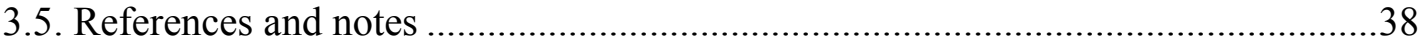

Chapter 4. De novo asymmetric total synthesis of aspergillids .......................................41

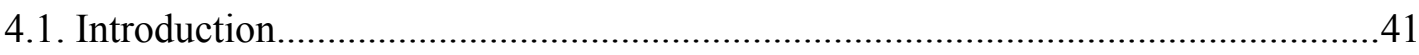

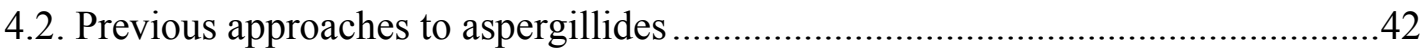

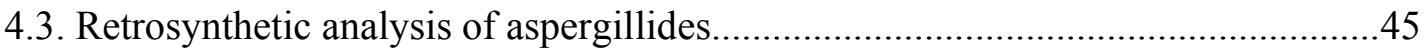

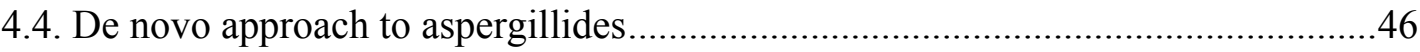

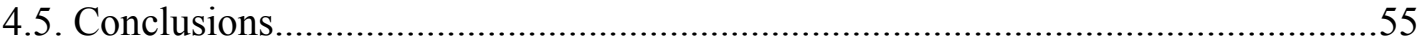

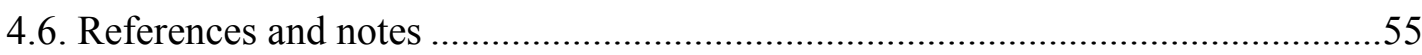

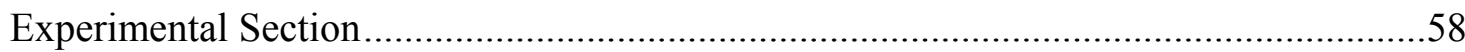




\section{List of Figures}

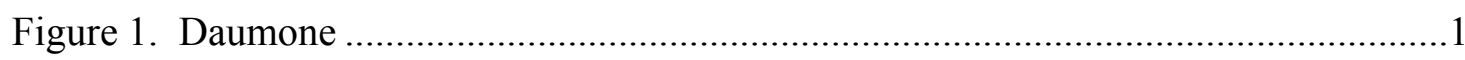

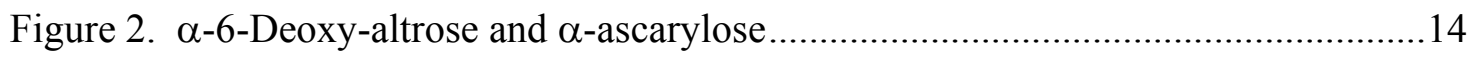

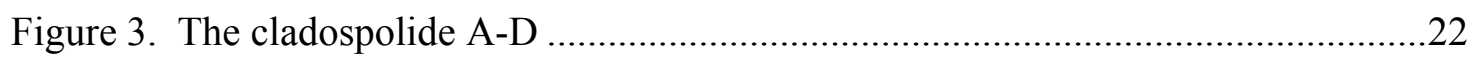

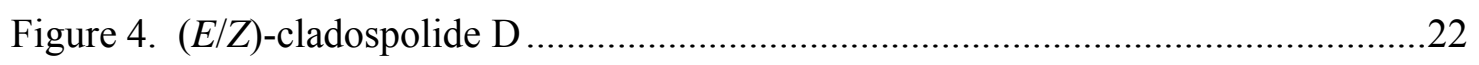

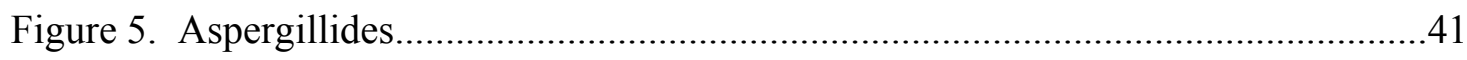




\section{List of Abbreviations}

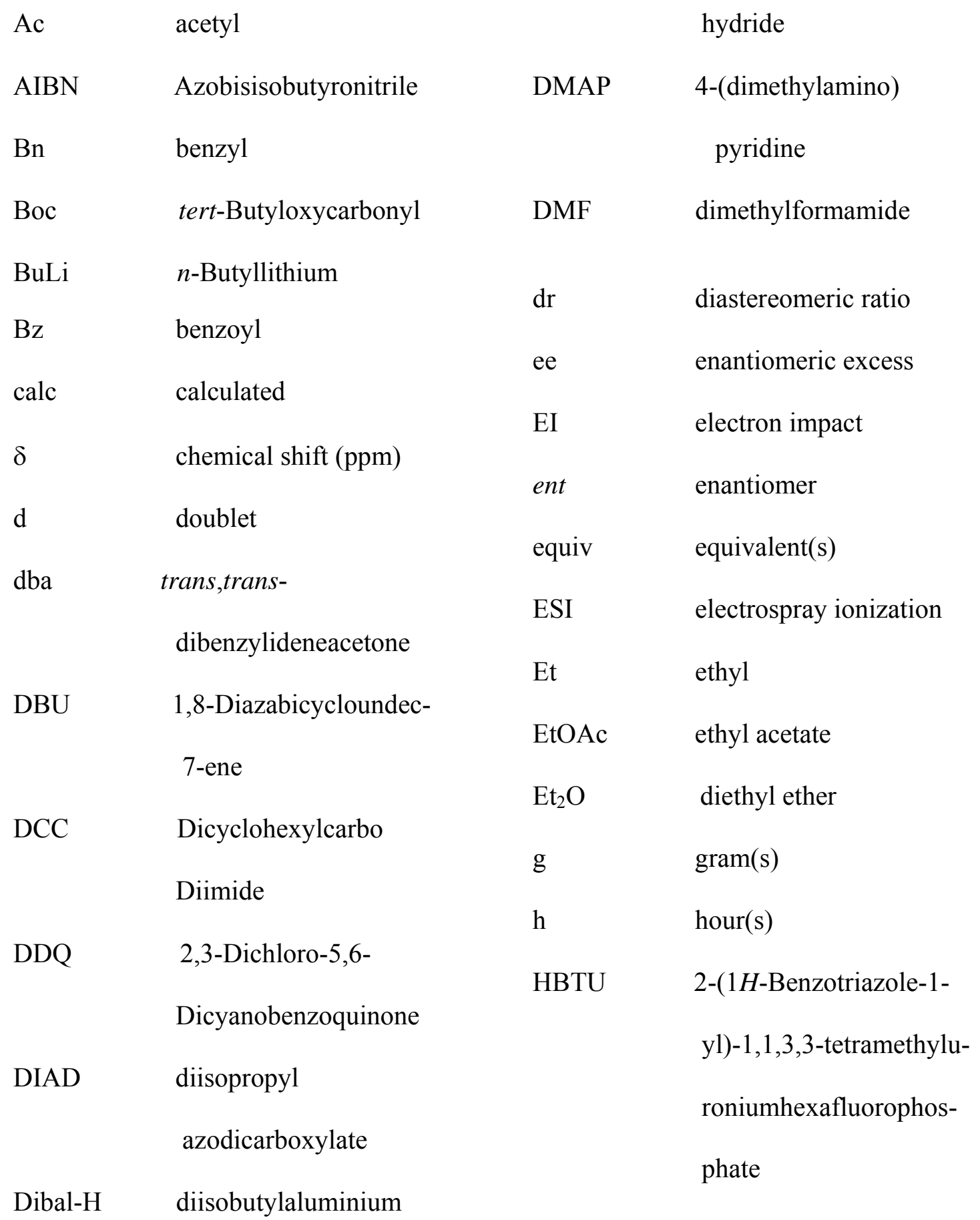




\begin{tabular}{|c|c|c|c|}
\hline Hex & hexanes & Piv & Pivalate \\
\hline \multirow[t]{2}{*}{ HPLC } & high pressure liquid & PMB & para-methoxybenzyl \\
\hline & Chromatography & ppm & parts per million \\
\hline \multirow[t]{2}{*}{ HRMS } & High resolution mass & py & pyridine \\
\hline & Spectrum & q & quartet \\
\hline $\mathrm{Hz}$ & hertz (cycles per second) & $\mathrm{R}_{f}$ & ratio to front \\
\hline IR & infrared & $\mathrm{rt}$ & room temperature \\
\hline \multirow[t]{2}{*}{$J$} & spin-spin coupling & $\mathrm{t}$ & triplet \\
\hline & Constant & TBAF & tetrabutylammonium \\
\hline \multirow[t]{2}{*}{ KAPA } & potassium 3- & & Fluoride \\
\hline & aminopropylamide & TBDPS & t-Butyldiphenylsilyl \\
\hline $\mathrm{Me}$ & methyl & TBS & t-butyldimethylsilyl \\
\hline $\mathrm{MHz}$ & megahertz & TEMPO & 2,2,6,6-tetramethyl- \\
\hline $\min$ & minute(s) & & piperidin-1-oxyl \\
\hline mol & mole(s) & THF & tetrahydrofuran \\
\hline mmol & millimole(s) & TLC & thin layer \\
\hline $\mathrm{mp}$ & melting point & & chromatography \\
\hline NBS & $N$-Bromosuccinimide & TMS & trimethylsilyl \\
\hline \multirow[t]{2}{*}{ NMR } & nuclear magnetic & Tf & trifluoromethanesulfonyl \\
\hline & resonance & $p$-TsOH & para-toluenesulfonic \\
\hline $\mathrm{Ph}$ & phenyl & & acid \\
\hline
\end{tabular}




\section{Chapter 1}

\section{De Novo Asymmetric Synthesis of Pheromone Daumone I-1}

\subsection{Introduction}

Daumone, a family of small molecule pheromones, can control multiple behaviors in the nematode in Caenorhabditis elegans. ${ }^{1}$ Developmental arrest at the dauer stage and male mating behavior are the two basic C. elegans traits which are controlled by daumone pheromones. Under harsh growth condition, daumone could signal C. elegans to enter the dauer stage, an enduring and non-ageing stage of the nematode life cycle with distinctive adaptive features and extended life. ${ }^{2}$ At low concentrations, a synergistic mixture of daumone produced by hermaphrodites causes male-specific attraction.

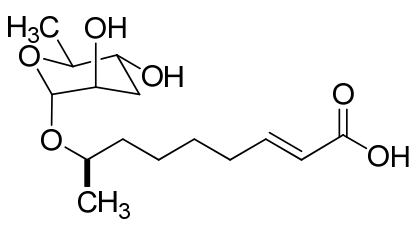

Daumone I-1

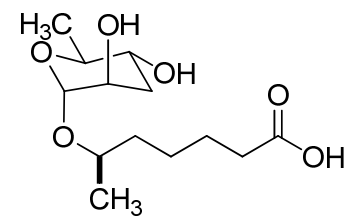

Daumone I-2

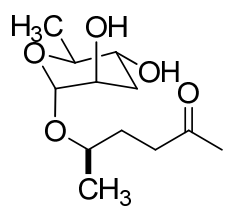

Daumone I-3

Figure 1. Daumone

In 2005, Jeong et al. have first isolated, characterized and completed a total synthesis of daumone $\mathbf{I}-2,{ }^{3}$ recently, another two daumone pheromones (I-1 and $\left.\mathbf{I}-\mathbf{3}\right)$ were isolated and characterized by the Clardy’s group. ${ }^{2}$ Because of the fascinating biological activity, we desired to access synthetic samples of daumone.

Herein, I wish to present a de novo route of preparing daumone using our recently developed palladium catalyzed glycosylation reaction. ${ }^{4,5}$ This de novo route starts from 
the non-chiral materials and uses asymmetric catalysis such as Noyori reduction to install the stereochemistry of daumone. Our strategies allowed us to not only access the natural products but also synthesize many daumone analogs which could be studied for the biological activities.

\subsection{Previous Approach to Daumone I-2}

In our group, former student Haibing Guo has developed a de novo asymmetric route ${ }^{5}$ for daumone I-2 and provided the synthetic sample of $\mathbf{I}-\mathbf{2}$ for the mechanism of action study in the Noel labs at The Salk Institute. ${ }^{6}$ After the biological activity study, daumone I-2 was not as active as people thought before. Thus, based on Haibing's synthesis, I finished the total synthesis of daumone $\mathbf{I - 1}$ as well as its fluorescent analog. The mechanism of action study showed that daumone $\mathbf{I}-\mathbf{1}$ is the most active daumone component in terms of the dauer activity.

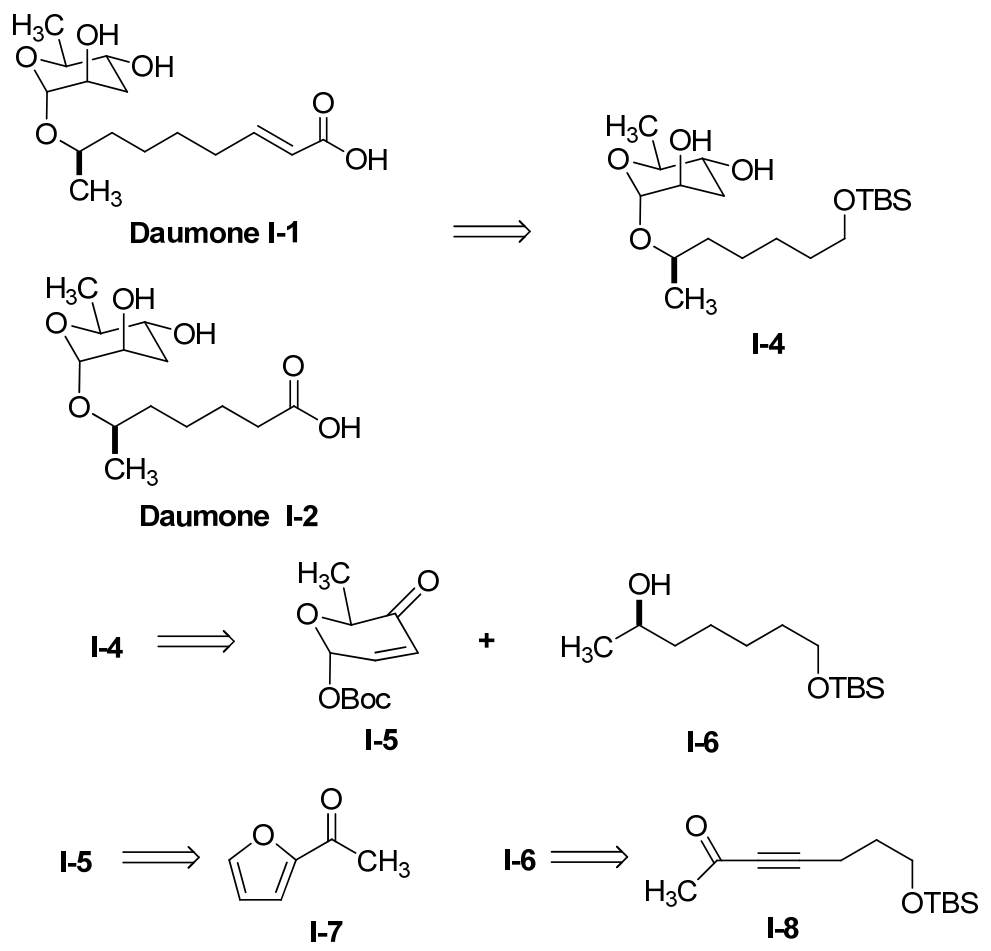


Scheme 1. Retrosynthetic analysis of Daumone I-1 and I-2

As we can see from the retrosynthetic analysis (Scheme 1), both daumone I-1 and daumone I-2 could be established from diol I-4, which could be stereoselectively derived by a palladium-catalyzed glycosylation of the pyranone I-5 and alcohol I-6 and several post-glycosylation transformations. The Noyori reductions of acylfuran I-7 and propargyl ketone $\mathbf{I - 8}$ respectively could install the absolute stereochemistry of both $\mathbf{I}-\mathbf{5}$ and $\mathbf{I}-\mathbf{6}$. $^{7}$ For the synthesis of daumone $\mathbf{I}-\mathbf{2}$, the TBS protecting group of common intermediate $\mathbf{I}-\mathbf{4}$ can be removed by TBAF, the resulting primary alcohol I-9 was oxidized by TEMPO condition to provide Daumone $\mathbf{I}-2$.

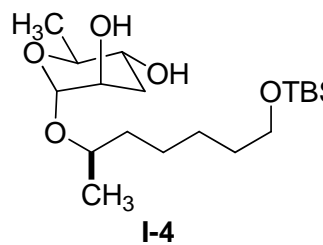<smiles>CC(CCCCCO)OC(OC(C)(C)C)C(O)(O)C(C)(O)C(C)(C)C(C)(C)C</smiles><smiles>CC(CCCCCO)OC(O)C(C)(C)O</smiles>
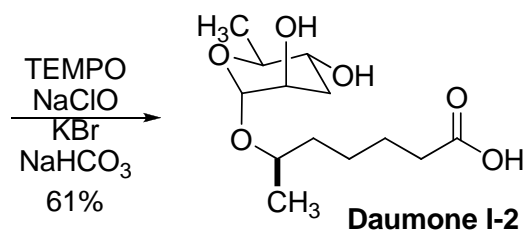

Scheme 2. Synthesis of Daumone I-2

\subsection{De Novo Asymmetric Synthesis of Daumone I-1}

As a de novo route, we wish to introduce both of the absolute stereochemistry of furan alcohol I-10 and propargyl alcohol I-12 by Noyori reduction respectively (Scheme 3). ${ }^{8}$ Furan alcohol I-10 was obtained from acylfuran I-7 using Noyori $(S, S)$ catalyst in a very high enantiomeric excess (93\% yield and > 96\% ee). Noyori reduction of propargyl 
ketone I-8 provided propargyl alcohol I-12 with high yield (81\%) and enantiomeric purity (> 96\% ee). Propargyl ketone I-8 was established from 4-pentynol I-11 by metalation of the TBS ether with $n$-BuLi and then quenching with acetaldehyde followed by oxidation with $\mathrm{MnO}_{2}(98 \%)$.

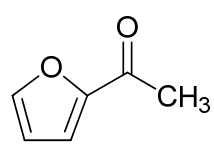

I-7

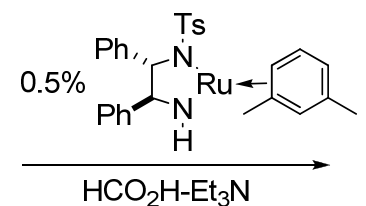

$(1: 1)$<smiles>CC(O)c1ccco1</smiles>

$\mathrm{I}-10$

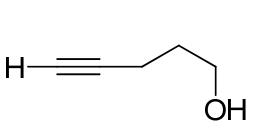

I-11

$93 \%$

1) $\mathrm{TBSCl}$, imid. $99 \%$

2) n-BuLi

3) $\mathrm{MnO}_{2} \quad 98 \%$

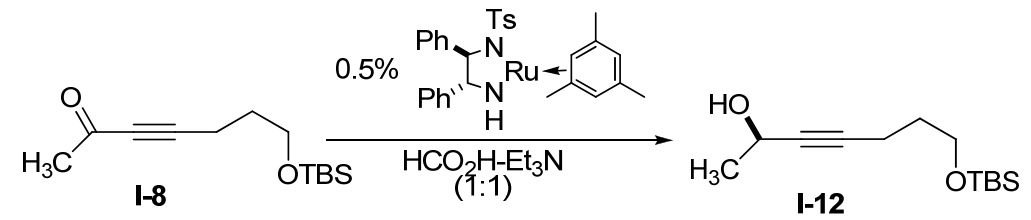

Scheme 3. Noyori reduction to install stereochemistry

With successful installation of the stereochemistry of $\mathbf{I - 1 0}$ and $\mathbf{I - 1 2}$, we next proceeded to couple I-5 and I-6 (Scheme 4) using palladium-catalyzed glycosylation reaction. Achmatowicz reaction (NBS in $\mathrm{THF} / \mathrm{H}_{2} \mathrm{O}$ ) of $\mathbf{I - 1 0}$ gave the ring-expanded pyranone product I-13 in good yield (91\%). ${ }^{9}$ The mechanism of Achmatowicz reaction is shown in scheme 5. The hemiacetal of $\mathbf{I}-\mathbf{1 3}$ was then protected with (Boc) $)_{2} \mathrm{O}$ to obtain Bocprotected pyranone $\mathbf{I}-5$ in good yield (78\%) . 


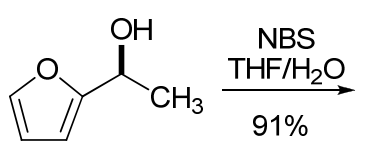

I-10

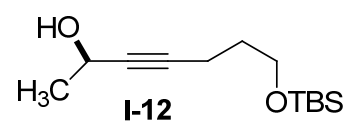

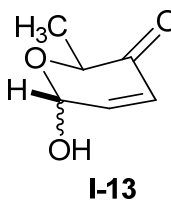

I-13

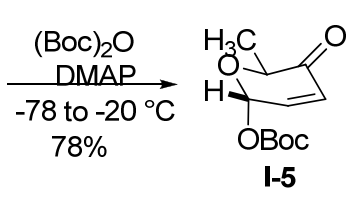

$\mathrm{H}_{2}, 10 \% \mathrm{Pd} / \mathrm{C}$

$\underset{82 \%}{\stackrel{5 \mathrm{~min} .}{\longrightarrow}}$<smiles>CC(O)CCCCCO[SbH2]</smiles>
OTBS

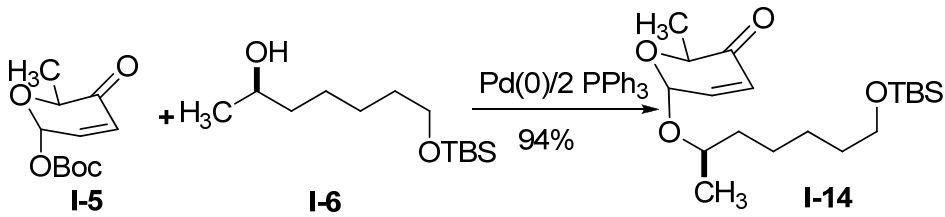

Scheme 4. Palladium-catalyzed glycosylation

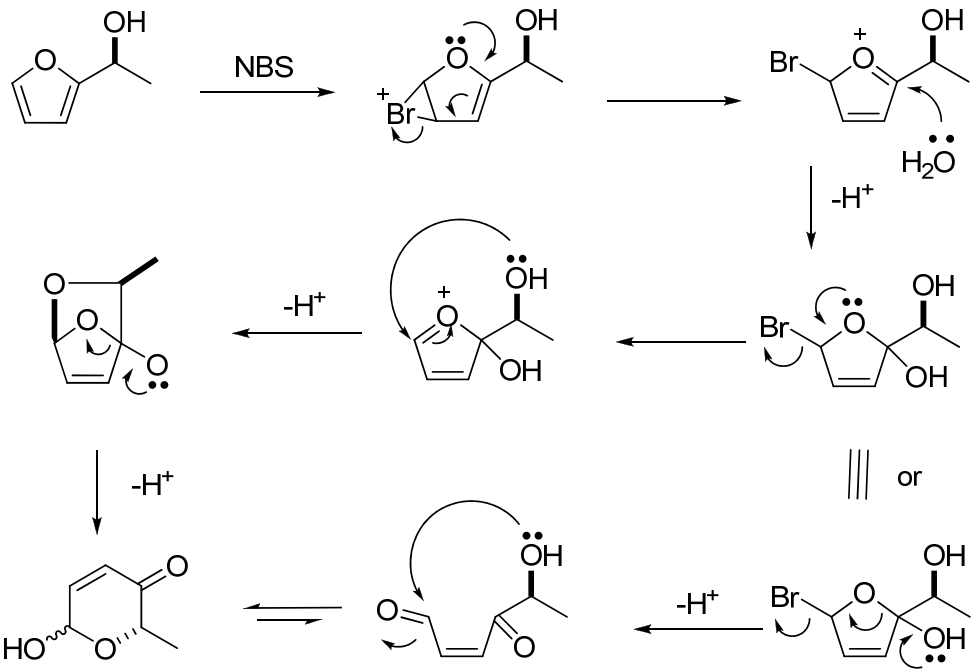

Scheme 5. Achmatowicz reaction

Propargyl alcohol I-12 was reduced $\left(\mathrm{H}_{2}, 10 \% \mathrm{Pd} / \mathrm{C}\right)$ to generate the coupling partner alcohol I-6 (82\%). The palladium-catalyzed glycosylation of pyranone I-5 and alcohol I6 by using 5\% palladium (0) and 10\% triphenylphosphine provided enone I-14 as a single diastereomer (94\%). ${ }^{10}$ The mechanism of the glycosylation reaction is shown in scheme 6 . First, the Pd- $\pi$-allyl specie was formed from the opposite face of the Boc group, and then 
the alcohol nucleophile comes to attack the anomeric position from the top face to give the glycosylation product with the retention of stereochemistry.
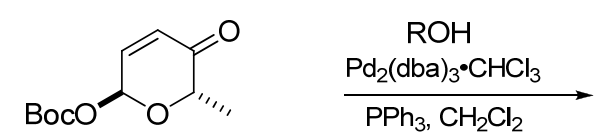<smiles>[R20]C1C=CC(=O)[C@H](C)O1</smiles>
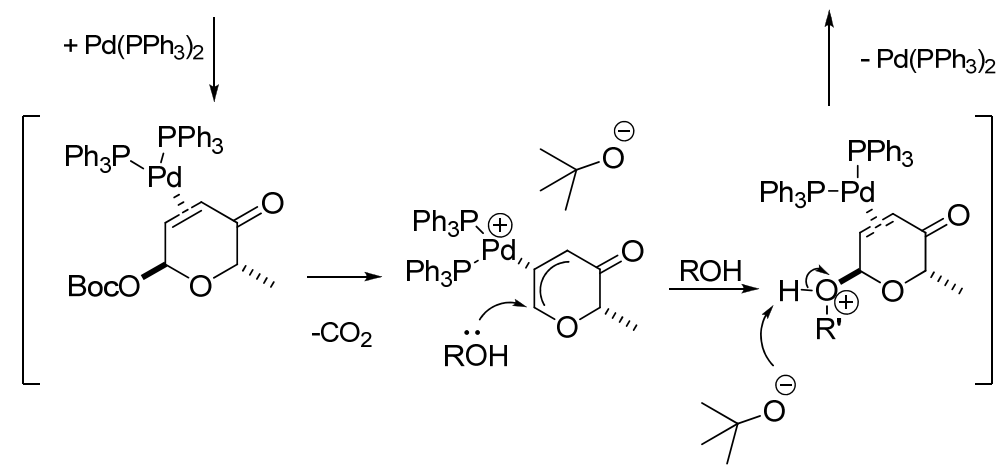

Scheme 6. Palladium-catalyzed glycosylation

As shown in scheme 7, enone I-14 was diastereoselectively epoxidized to give epoxyenone I-15 (81\%) using hydrogen peroxide in the presence of a catalytic amount of base $(10 \mathrm{~mol} \% \mathrm{NaOH}) .{ }^{11}$ Then we turned our efforts to the diastereoselectivly opening of the expoxide ring. We decided to use reductive hydration strategy. Epoxy-enone I-15 was diatereoselectviely reduced to provide the equatorial alcohol I-16 (93\%) with $\mathrm{NaBH}_{4}(-78$ to $-20{ }^{\circ} \mathrm{C}$ ). The epoxide $\mathbf{I}-16$ was regioselectively and diastereoselectively opened with $\mathrm{LiAlH}_{4}$ to generate diol $\mathbf{I}-\mathbf{4}$ (88\%) in a trans-diaxial way. ${ }^{12}$ Diol I-4 can also be achieved in a one-step reaction by treating $\mathbf{I - 1 5}$ with $\mathrm{LiAlH}_{4}$ at $-78{ }^{\circ} \mathrm{C}$ followed by warming to room temperature (86\%). 

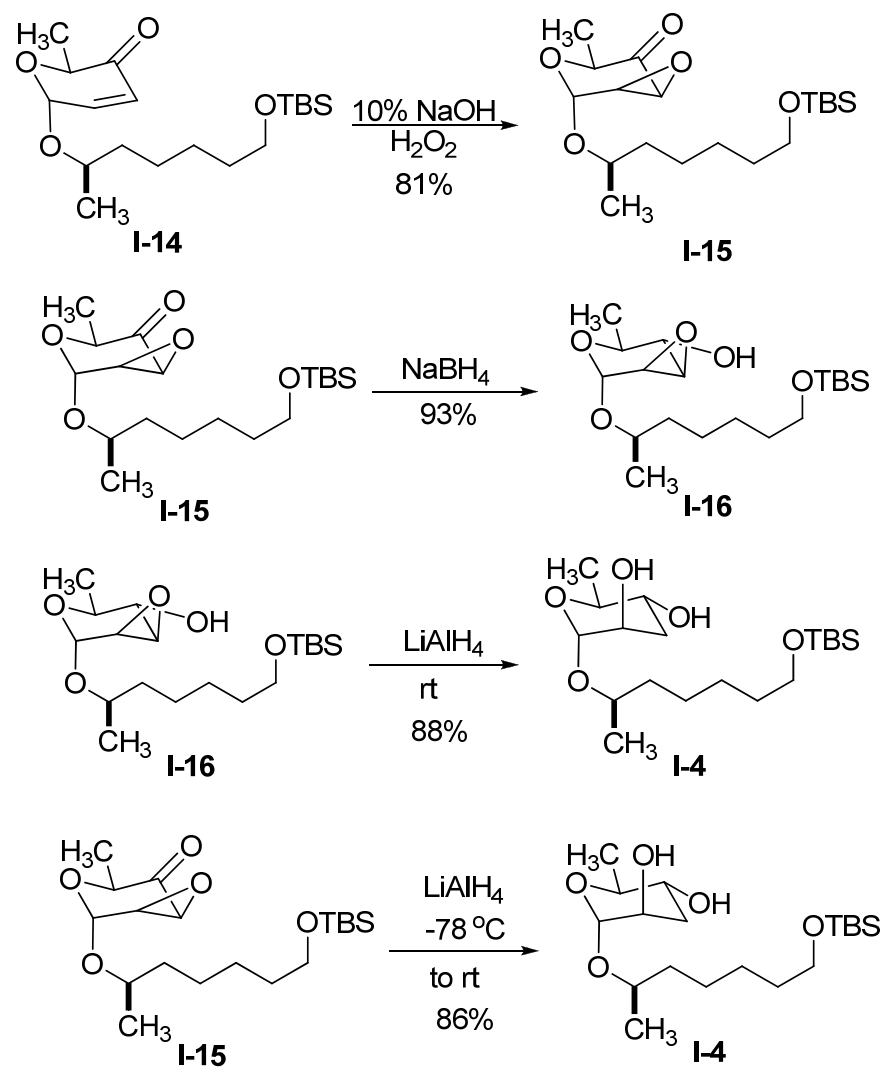

Scheme 7. Reductive hydration

With diol I-4 in hand, we next protected it with acetic anhydride to provide the acetate I-17 (85\%), which was treated with aq. HCl to remove TBS group to give alcohol I-18 (97\%). The aldehyde I-19 was obtained from oxidation of alcohol I-18 with TEMPO in 88\% yield. Wittig reaction of corresponding aldehyde I-19 installed ethyl ester I-20 in 97\% yield. Treatment of $\mathbf{I}-\mathbf{2 0}$ with $\mathrm{LiOH}$ in $\mathrm{MeOH}$ provided the acetyl deprotected methyl ester I-21 in 99\% yield. Finally, hydrolysis of methyl ester I-21 with $\mathrm{LiOH}$ in THF/ $\mathrm{H}_{2} \mathrm{O}$ generated daumone $\mathbf{I}-\mathbf{1}$ in 99\% yield (Scheme 8). 

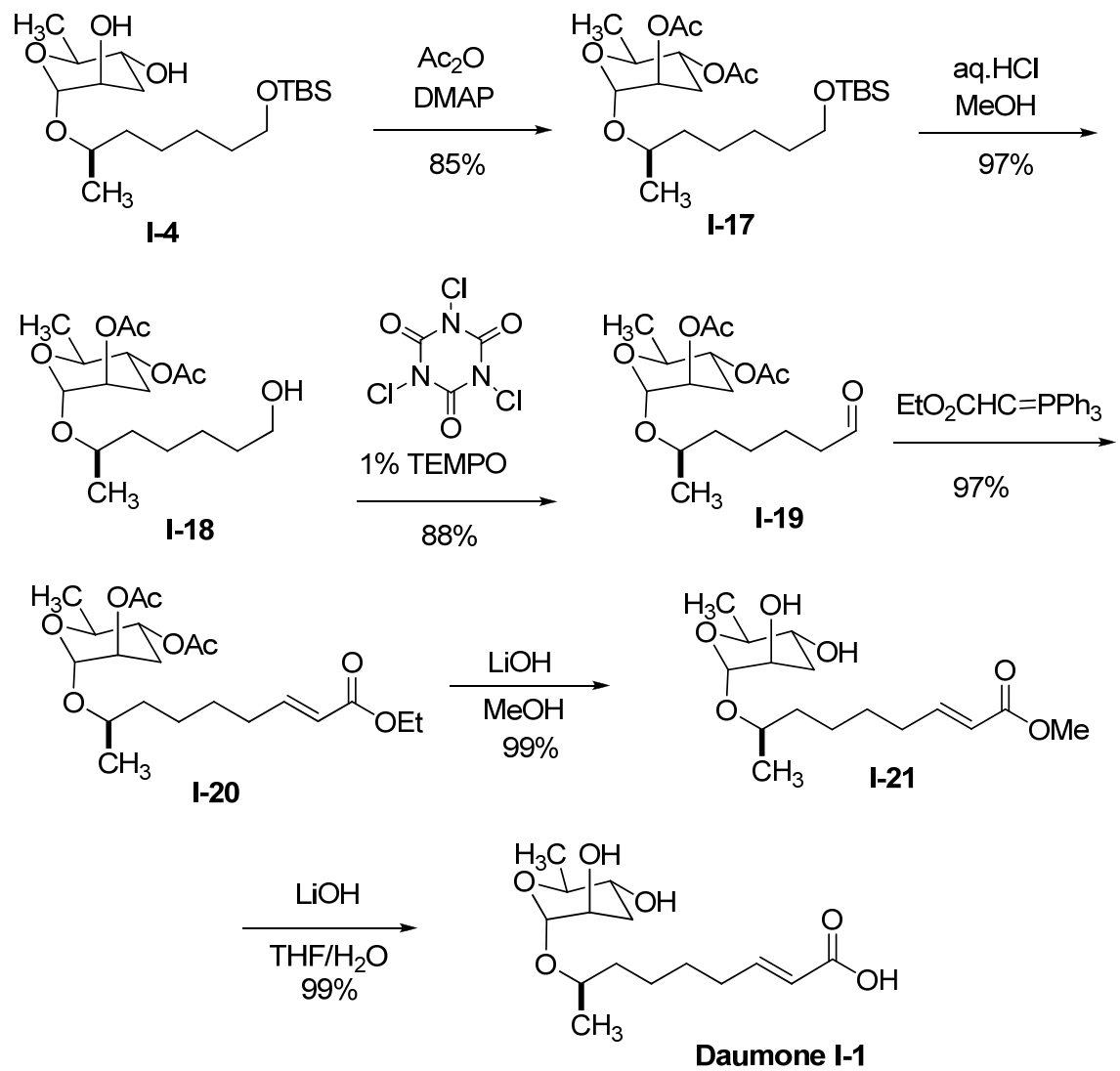

Scheme 8. Synthesis of Daumone I-1

As an effort to study the dauer activity of $C$. elegant, we also made the fluorescent daumone analog I-23. Simple peptide coupling linked fluorescent probe $\mathbf{I}-22$ and daumone I-1 to provide fluorescent daumone analog I-23 which has been used for the mechanism of action study in The Salk Institute. 


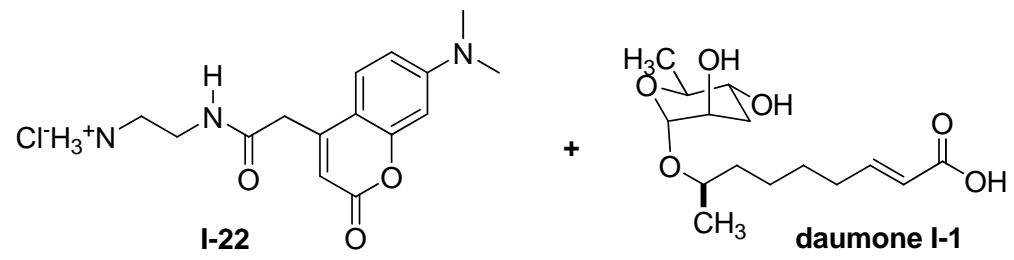

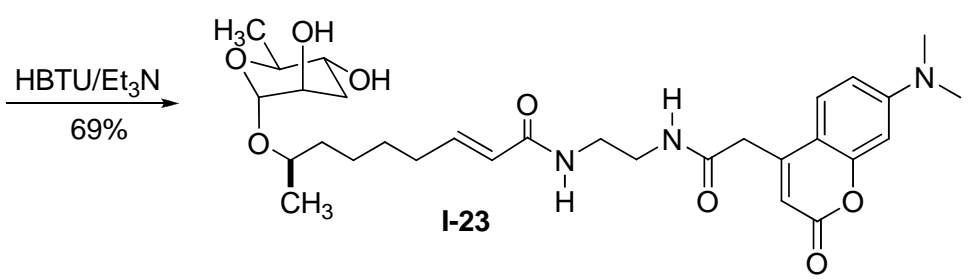

Scheme 9. Synthesis of fluorescent Daumone.

\subsection{Conclusion:}

In conclusion, the de novo enantioselective total syntheses of daumone $\mathbf{I - 1}$ and its fluorescent analog have been accomplished. This approach takes advantage of our highy enantio- and diatereocontrolled palladium-catalyzed glycosylation reaction and Noyori reduction of two ketones. Daumone $\mathbf{I - 1}, \mathbf{I}-2$ and the fluorescent analogs we accessed were utilized in the biological study of the dauer stage of $C$. elegans in The Salk Institute along with antihelmycin studies at USDA. These compounds have been used as probes to track the uptake and localization of dauer stage of $C$. elegans and the fluorescent probes have been shown to have activity in dauer formation. ${ }^{6}$

\section{Reference:}

\footnotetext{
${ }^{1}$ Edison, A.S. Neurobiology. 2009, 19, 378-388.
}

${ }^{2}$ (a) Butcher, R. A.; Fujita, M.; Schroeder, F. C.; Clardy, J. Nature Chem. Bio. 2007, 3, 
420-422. (b) Boucher, Y. \& Doolittle, W. F. Nature. 2002, 417, 27-28. (c) Gordon, J. W.; Riddle Dev. Biol. 1984, 102, 368-378. (d) Cassada, R. C.; Russell, R. L. Dev. Biol. 1975, 46, 326-342.

${ }^{3}$ Jeong, P.-Y.; Jung, M.; Yim, Y.-H.; Kim, H.; Kim, K.; Paik, Y.-K. Nature 2005, 433, 541-545.

${ }^{4}$ (a) Babu, R. S.; O'Doherty, G. A. J. Am. Chem. Soc. 2003, 125, 12406-12407. (b) Babu, R. S.; M. Zhou; O'Doherty, G. A. J. Am. Chem. Soc. 2004, 126, 3428-3429. (c) Babu, R. S.; O'Doherty, G. A. J. Carb. Chem. 2005, 24, 169-177.

${ }^{5}$ H. Guo; O’Doherty, G.A. Org. Lett. 2005; 7(18); 3921-3924.

${ }^{6}$ Baiga, T. J.; Guo, H.; Xing, Y.; O'Doherty, G. A.; Dillin, A.; Austin, M. B.; Noel, J. P.; La Clair, J. J. ACS Chem. Bio. 2008, 3, 294-304

${ }^{7}$ (a) Fujii, A.; Hashiguchi, S.; Uematsu, N.; Ikariya, T.; Noyori, R. J. Am. Chem. Soc. 1996, 118, 2521-2522. (b) Noyori, R.; Ohkuma, T. Angew. Chem. Int. Ed. 2001, 40, 4073.

${ }^{8}$ Li, M.; Scott, J. G.; O'Doherty, G. A. Tetrahedron Lett. 2004, 45, 1005-1009.

${ }^{9}$ (a) Achmatowicz, O.; Bielski, R. Carbohydr. Res. 1977, 55, 165-176. (b) Balachari, D.; O’Doherty, G. A. Org. Lett. 2000, 2, 863-866. (c) Balachari, D.; O’Doherty, G. A. Org. Lett. 2000, 2, 4033-4036.

${ }^{10}$ Tsuji, J. Palladium Reagents and Catalysts. Innovations in Organic Synthesis; John Wiley \& Sons: New York, 1995; pp 290-422.

${ }^{11}$ Jung, M. E.; Pontillo, J. J. Org. Chem. 2002, 67, 6848-6851.

${ }^{12}$ Mastihubova, M; Biely, P. Tetrahedron Lett. 2001, 42, 9065-9067. 


\section{Chapter 2}

\section{De Novo Asymmetric Synthesis of $\alpha$-6-deoxy-altro-pyranoside and $\alpha-3,6$-dideoxy-altro-pyranoside ${ }^{\dagger}$}

\subsection{Introduction}

Deoxysugars are a common structural motif in biologically active natural products. ${ }^{1}$ The removal of the hydroxyl groups in sugars along with the use of unusual stereochemistry imparts resistance to most glycosidases and thus conveys improved metabolic stability. ${ }^{1}$ Examples of nature's reliance of these structural motifs are seen in the use of $\alpha$-6-deoxy-altrose as the main constituent of bacterial lipopolysaccharides, ${ }^{2}$ as well as, the use of $\alpha-2,6-$ and $\alpha$-3,6-dideoxy-altrose (i.e., $\alpha$-digitoxose and $\alpha$-ascarylose) in biologically active natural products, such as jadomycin $\mathrm{B}^{3}$ and daumone, ${ }^{4}$ respectively. ${ }^{5}$
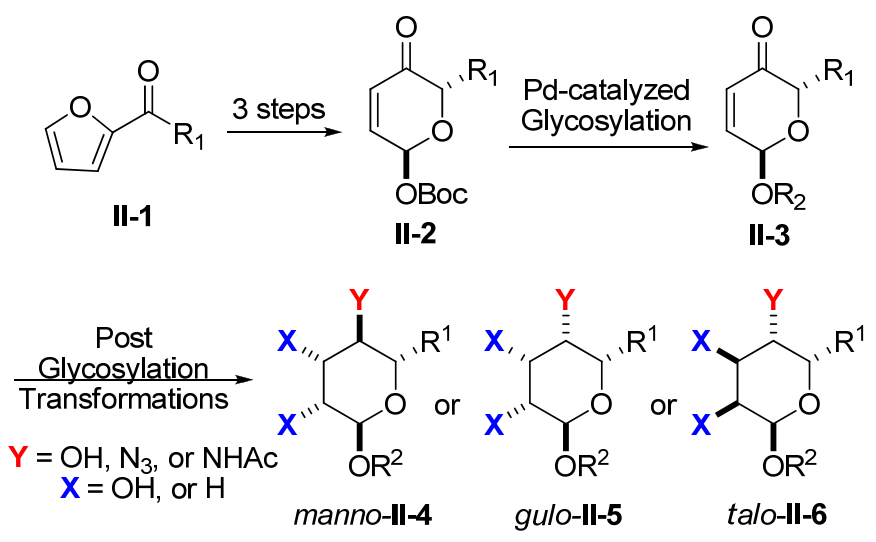

Scheme 1. De novo approach to carbohydrate

\footnotetext{
$\dagger$ Reproduced with permission from J. Org. Chem. 2009, 74, 5961-5966. Copyright 2009, with permission from American Chemical Society
} 
Over the last 10 years, there has been considerable effort made toward the synthesis of monosaccharides from achiral starting materials. ${ }^{6}$ In this regard, our group has developed a de novo asymmetric synthesis of carbohydrates using achiral acylfurans II-1 (Scheme 1). ${ }^{7}$ Our approach used a Noyori reduction, an Achmatowicz reaction and a $\operatorname{Pd}(0)-$ catalyzed glycosylation (II-2 to II-3) to prepare pyranone intermediate $\mathbf{I I - 3},{ }^{7,8,9}$ which via post-glycosylation transformations were converted into corresponding carbohydrates (e.g., II-3 to II-4, II-5 and II-6). ${ }^{7,9}$ This method has allowed for the stereoselective synthesis of a variety of sugars, including rare sugars, aminosugars and even unnatural sugars. ${ }^{10}$

These approaches primarily rely on the osmium catalyzed dihydroxylation reaction for the syn-addition of two hydroxyl groups across the C-2/C-3 pyran double bond, which has been used to prepare D- or L-sugars with manno-, gulo- and talo-stereochemistry. ${ }^{9,10}$ A related dihydroxylation on the $\mathrm{C}-3 / \mathrm{C}-4$ pyran double bond provided the sugars with galacto- and allo-stereochemistry. ${ }^{10,}{ }^{11}$ By combining this approach with protection/inversion reactions, this route has also been used to access to the glucostereoisomers. ${ }^{12}$

\subsection{Previous approaches to deoxysugars}

In 2009, Lowary and co-workers reported the use of 2, 3-anhydrosugars as glycosylating agents for the preparation of 2-deoxypyranosides. ${ }^{5 a}$ Glycosylation of a panel of alcohols with one of two 6-deoxy-2,3-anhydrosugar thioglycosides (II-7 and II8) afforded 2,6-dideoxy-2-thiotolyl glycoside products II-9 and II-11 in excellent yields with an exclusively syn relationship between the aglycon and the C-3 hydroxyl group. 
Removal of the 2-thiotolyl group can be achieved via reaction with tri-n-butyltin hydride and AIBN to give the corresponding 2, 6-dideoxy pyranosides II-10 and II-12 (Scheme 2).

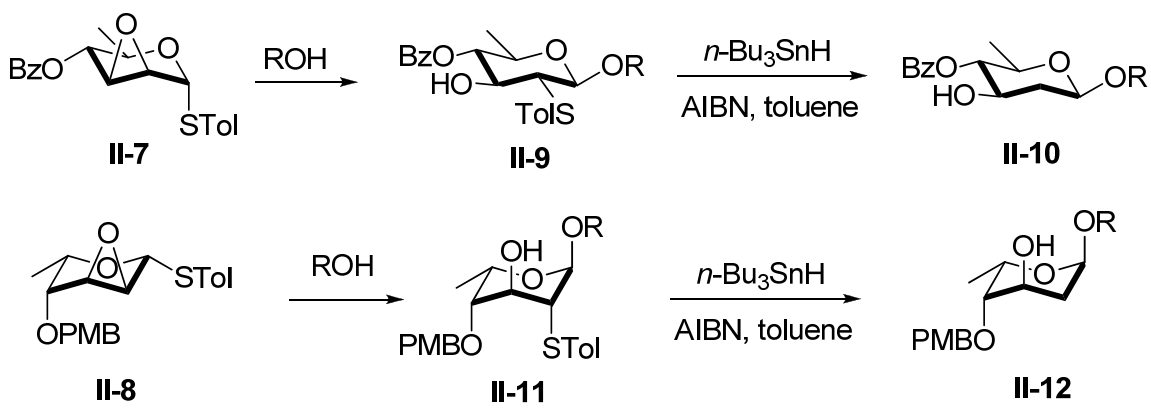

Scheme 2. Lowary's approach to 2, 6-dideoxysugars.

The synthesis of glycosyl donors II-7 and II-8 is outline in Scheme 3. The preparation of II-7 started with the known benzylidene acetal protected 6-deoxy-D-mannopyranoside II-13. The Hanessian-Huller reaction to II-13 led to the formation of II-14 in 75\% yield. After five steps transformations, II-14 was converted to glycosyl donor II-7. The preparation of II-8 proved more challenging than II-7. They first synthesized thioglycoside II-15, which underwent five steps transformations to provide II-16. PMB protection followed by the removal of allyl group and reaction with sodium hydride led the formation of glycosyl donor II-8.
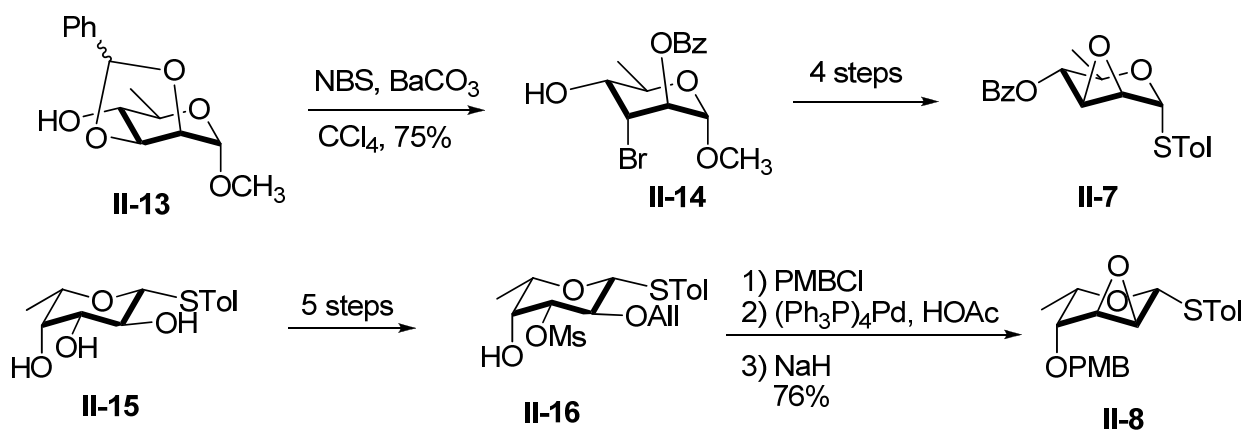

Scheme 3. Lowary's synthesis of glycosyl donors II-7 and II-8. 


\subsection{De Novo Asymmetric Synthesis of $\alpha$-6-deoxy-altro- pyranoside and $\alpha-3,6$-dideoxy-altro-pyranoside}

As part of our efforts to expand upon this methodology, we have been investigating epoxidation/nucleophilic ring-opening reactions for the net anti-addition of hydroxyl groups across the C-2/C-3 pyran double bond. Herein I would like to introduce our successful application of this new post-glycosylation transformation for the de novo synthesis of two deoxysugar targets (i.e., benzyl pyranosides with $\alpha$-altrostereochemistry and its 3-deoxy ( $\alpha$-ascarylose) congeners) (Figure 2).
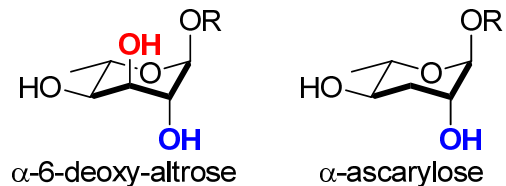

Figure 2. $\alpha$-6-Deoxy-altrose and $\alpha$-ascarylose

\subsubsection{Retrosynthetic Analysis}

Retrosynthetically, we envisioned both $\alpha$-6-deoxy-altro-pyranoside and $\alpha-3$, 6dideoxy-altro-pyranoside II-17 as being derived from the trans-diaxial opening of epoxide II-18 with either a formal hydroxy or hydride nucleophile, respectively (Scheme 4). Epoxide II-18 could be prepared by epoxidation of pyran II-19, which in turn could be prepared by a ketone reduction of pyranone II-20. Because our route began with the Noyori reduction of acylfuran, it is amenable to the synthesis of either D- or Lenantiomers of these sugars. ${ }^{13}$ 


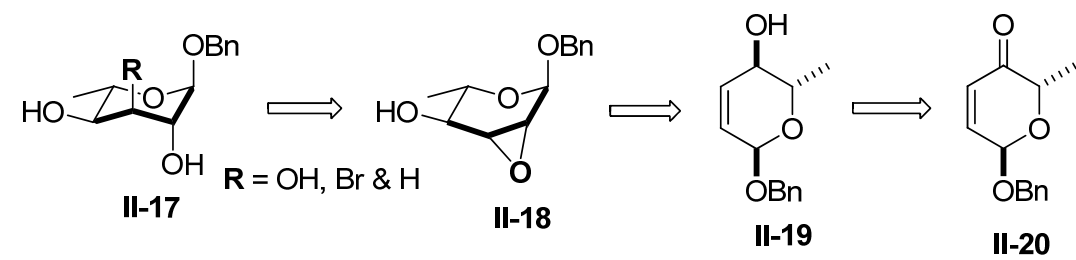

Scheme 4. Retrosynthetic analysis

\subsubsection{De Novo Synthesis of $\alpha$-Boc-pyranone}

As we have previously reported, ${ }^{13 b}$ the Noyori reduction of acylfuran II-21, with formic acid/triethylamine as the hydride source, produced either enantiomer of furfuryl alcohol II-22 in 96\% yield with high enantiomeric excess (>96\% ee). ${ }^{13}$ An Achmatowicz rearrangement transformed the furfuryl alcohol to pyranone in $91 \%$ yield. The hemiacetal was then protected as t-butyl carbonate $\mathbf{I I - 2 3}$ at low temperature in $78 \%$ yield with 3:1 ratio of $\alpha$ - and $\beta$-diastereomers (Scheme 5).

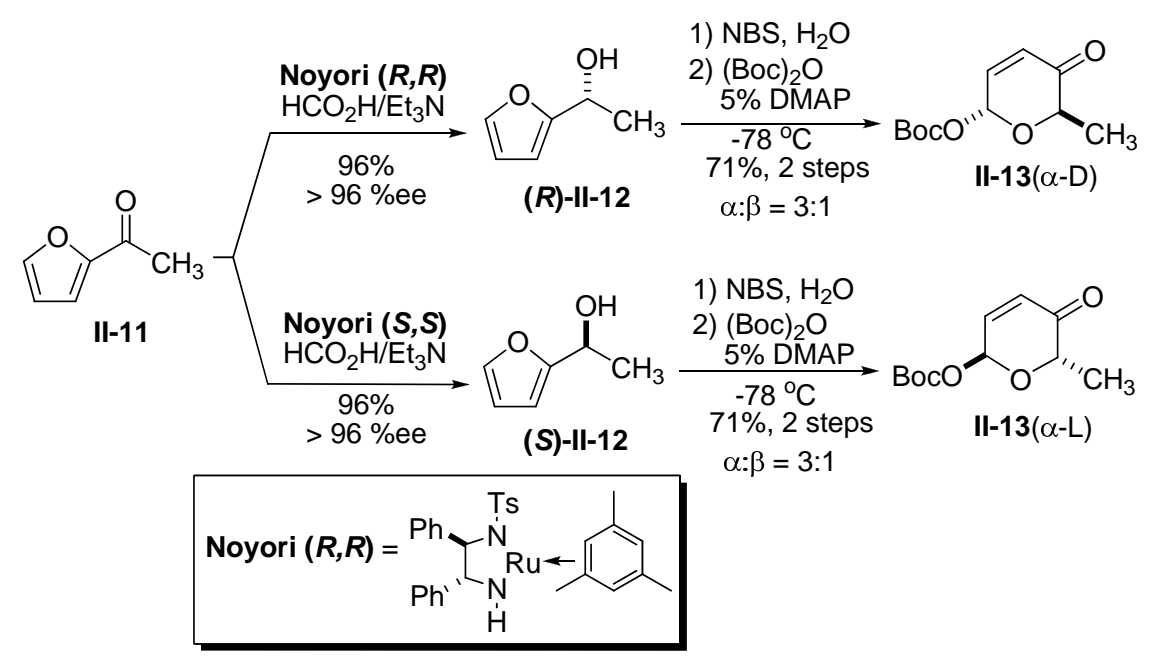

Scheme 5. De Novo synthesis of $\alpha$-Boc-pyranone

\subsubsection{Synthesis of $\alpha$-6-deoxy-altro-pyranoside}

Our proposed synthesis of $\alpha$-6-deoxy-altro-pyranoside started with the attempted 
epoxidation of pyran II-19. Unfortunately, our efforts to epoxidize allylic alcohol II-19 were not satisfactory. As a result, we decided to reverse the reduction/oxidation sequence. These efforts began with our $\mathrm{Pd}(0)$-catalyzed glycosylation reaction to diastereoselectively install the anomeric benzyloxy group with complete $\alpha$-selectivity (Scheme 6). Similarly, treating pyranone II-20 with hydrogen peroxide in the presence of catalytic amount of base $\left(10 \mathrm{~mol} \% \mathrm{NaOH} / \mathrm{H}_{2} \mathrm{O}_{2}\right)$ diastereoselectively installed the epoxide II-24 in $88 \%$ yield. $^{4 \mathrm{~b}}$ In an equally diastereoselective fashion, the ketone II-24 was reduced with $\mathrm{NaBH}_{4}\left(-78\right.$ to $\left.-20{ }^{\circ} \mathrm{C}\right)$ to form the equatorial alcohol $\mathbf{I I - 1 8}$ in $85 \%$ yield.

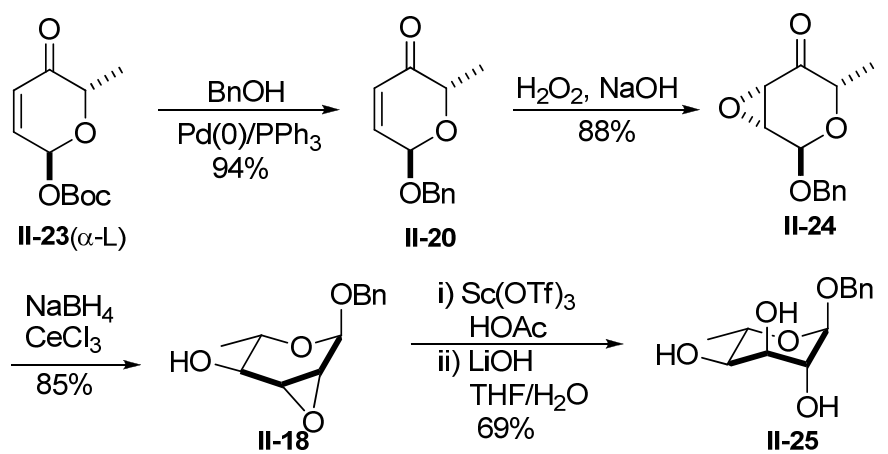

Scheme 6. Synthesis of $\alpha$-6-deoxy-altro-pyranoside

With the alcohol II-18 in hand, we turned our attention to the epoxide ring opening with acetic acid. After investigating the use of a variety of Lewis acids, we found $\mathrm{Sc}(\mathrm{OTf})_{3}$ gave the best results. In practice, treatment of epoxide $\mathbf{I I - 1 8}$ with $\mathrm{Sc}(\mathrm{OTf})_{3}$ in HOAc afforded an inseparable mixture of acetates which presumably resulted from acid catalyzed ester migration of the initial epoxide ring opening product. This mixture was directly hydrolyzed with $\mathrm{LiOH}$ in aqueous THF producing $\alpha$-6-deoxy-altrose II-25 in $69 \%$ yield. The stereochemistry of the ring opening was confirmed by analysis of the 
corresponding proton/proton coupling constants at C-2, C-3 and C-4 positions of II-25.

\subsubsection{Synthesis of $\alpha$-ascarylo-pyranoside}

As we previously reported, ${ }^{4 \mathrm{~b}}$ epoxide II-18 was converted into the $\alpha$-ascarylose sugar II-26 in 85\% yield using $\mathrm{LiAlH}_{4}$ (Scheme 7). This same regioselective Lewis acid promoted ring opening reaction can also be used for 3-deoxy-sugars, which provides a less basic alternative to our previously reported $\mathrm{LiAlH}_{4}$ method. ${ }^{4 \mathrm{~b}}$ Simply switching the Lewis acid to $\mathrm{MgBr}_{2} \bullet \mathrm{Et}_{2} \mathrm{O}$ promoted a regioselective opening to form bromide II-27. The axial bromide in II-27 was cleanly removed to give II-26 in 76\% yield, using an AIBN promoted tris(trimethylsilyl)silane (TTMSS) radical reduction.

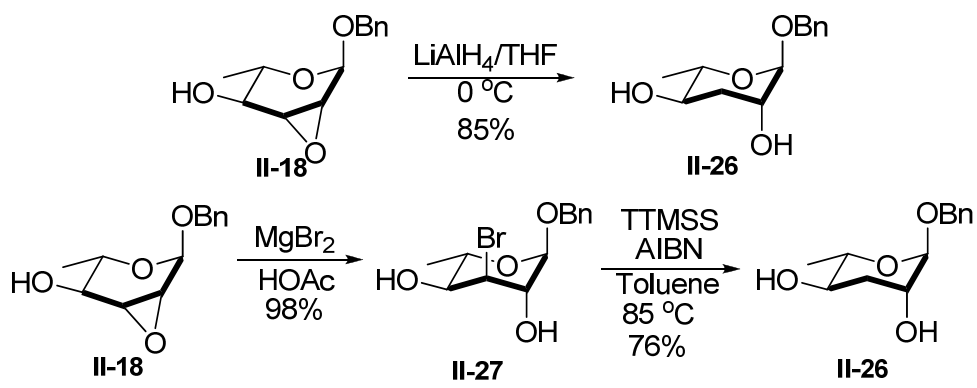

Scheme 7. Synthesis of $\alpha$-ascarylo-pyranoside

\subsection{Conclusions}

In summary, a highly enantio- and diastereoselective procedure for the preparation of C-3 and C-6 deoxypyranosides has been developed. Our approach to these sugars provides rapid and practical access to these rare and naturally occurring sugars, which should be of use for further natural product synthesis. Critical to the success of this synthesis was the use of the $\operatorname{Pd}(0)$-catalyzed glycosylation and corresponding postglycosylation transformations. It is also worth noting that our method is structurally 
divergent in that it produces four different sugar congeners from the same advanced intermediate. We believe that this approach will be particularly useful for medicinal chemistry. Finally, by selecting either enantiomeric form of the Noyori catalyst $(R, R)$ or $(S, S)$, our strategy is also amenable to the synthesis of both D- or L-forms of these sugars.

\section{Reference:}

${ }^{1}$ (a) Johnson, D. A.; Liu, H.-w. Comprehensive Natural Products Chemistry; Elsevier: Amsterdam, New York, 1999; Vol. 3, pp 311-365. (b) Thibodeaux, C.; Melancüon, C. E.,

III; Liu, H.-w. Nature 2007, 446, 1008-1016. (c) Kren, V.; Martinkova, L. Curr. Med. Chem. 2001, 8, 1303-1328.

${ }^{2}$ Gorshkova, R. P.; Kalmykova, E. N.; Isakov, V. V.; Ovodov, Y. S. Eur. J. Biochem. $1985,150,527-531$.

${ }^{3}$ (a) Doull, J. L.; Singh, A. K.; Hoare, M.; Ayer, S. W. J. Ind. Microbiol. 1994, 13, 120 125. (b) Jakeman, D. L.; Borissow, C. N.; Graham, C. L.; Timmons, S. C.; Reid, T. R.; Syvitski, R. T. Chem. Commun. 2006, 3738-3740.

${ }^{4}$ (a) Jeong, P.-Y.; Jung, M.; Yim, Y.-H.; Kim, H.; Kim, K.; Paik, Y.-K. Nature 2005, 433, 541-545. (b) Guo, H.; O’Doherty, G. A. Org. Lett. 2005, 7, 3921-3924. (c) Baiga, T. J.; Guo, H.; Xing, Y. O'Doherty, G. A.; Parrish, A.; Dillin, A.; Austin, M. B.; Noel, J. P.; La Clair, J. J. ACS Chem. Biol. 2008, 3, 294-304. 
${ }^{5}$ For a recent approach to 2,6-dideoxy-sugars, see: (a) Hou, D.; Lowary, T. L. J. Org. Chem. 2009, 74, 2278-2289. For approaches to 3,6-dideoxy-sugars, see: (b) Weigel, T. M.; Liu, H.-w. Tetrahedron Lett. 1988, 29, 4221-4224 and ref. 4b.

${ }^{6}$ (a) Zamoiski, A., Banaszek, A.; Grynkiewicz, G. Advances in Carbohydrate Chemistry and Biochemistry 1982, 40, 1. (b) Ernst, B.; Hart, G. W.; Sinay, P., Eds.; Carbohydrates in Chemistry and Biology; Wiley-VCH: New York, 2000. (c) Ko, S. Y.; Lee, A. W. M.; Masamune, S.; Reed, L. A.; Sharpless, K. B. Science 1983, 220, 949-951. (d) Northrup, A. B.; MacMillan, D. W. C. Science 2004, 305, 1752-1755.

${ }^{7}$ (a) Harris, J. M.; Keranen, M. D.; O’Doherty, G. A. J. Org. Chem. 1999, 64, 29822983. (b) Harris, J. M.; Keranen, M. D.; Nguyen, H.; Young, V. G.; O’Doherty, G. A. Carbohydr. Res. 2000, 328, 17-36. For its use in oligosaccharide synthesis see: (c) Babu, R. S.; Zhou, M.; O’Doherty, G. A. J. Am. Chem. Soc. 2004, 126, 3428-3429. (d) Ahmed, Md. M.; O'Doherty, G. A. Tetrahedron Lett. 2005, 46, 3015-3019. (e) Ahmed, Md. M.; Berry, B. P. ; Hunter, T. J. ; Tomcik, D. J.; O'Doherty, G. A. Org. Lett. 2005, 7, 745-748. (f) Guo, H.; O’Doherty, G. A. Angew. Chem. Int. Ed. 2007, 46, 5206-5208. (g) Zhou, M.; O’Doherty, G. A. Org. Lett. 2008, 10, 2283-2286.

${ }^{8}$ Jones, R. A.; Krische, M. J. Org. Lett. 2009, 11, 1849-1851.

${ }^{9}$ (a) Babu, R. S.; Guppi, S. R.; O’Doherty, G. A. Org. Lett., 2006, 8, 1605-1608. (b) Shan, M.; O’Doherty, G. A. Org. Lett. 2006, 8, 5149-5152. (c) Guppi, S. R.; O'Doherty, G. A. J. Org. Chem., 2007, 72, 4966-4969. 
${ }^{10}$ (a) Haukaas, M. H.; O’Doherty, G. A. Org. Lett. 2001, 3, 3899-3992. (b) Haukaas, M. H.; O’Doherty, G. A. Org. Lett. 2002, 4, 1771-1774. (c) Ahmed, Md. M.; O'Doherty, G. A. Tetrahedron Lett. 2005, 46, 4151-4155.

${ }^{11}$ (a) Zhou, M.; O’Doherty, G. A. Org. Lett. 2006, 8, 4339-4342. (b) Zhou, M.; O’Doherty, G. A. J. Org. Chem. 2007, 72, 2485-2493.

${ }^{12}$ Balachari, D.; O'Doherty, G. A. Org. Lett. 2000, 2, 4033-4036.

${ }^{13}$ (a) Fujii, A.; Hashiguchi, S.; Uematsu, N.; Ikariya, T.; Noyori, R. J. Am. Chem. Soc. 1996, 118, 2521-2522. (b) Li, M.; O'Doherty, G. A. Tetrahedron Lett. 2004, 45, 64076411. 


\section{Chapter 3}

\section{Structure Investigations of ent-Cladospolide D by De Novo Synthesis and Kinetic and Thermodynamic Isomerization}

\subsection{Introduction}

In an interesting synergistic relationship between parasite and host, the Cladosporium species produce secondary metabolites that can regulate the host plant's growth. ${ }^{1} \quad$ This growth regulation is believed to occur via the inhibition of gibberellin biosynthesis. ${ }^{1 c}$ Structurally these fugal products/plant pheromones are made up of a group of diastereoisomeric 12-membered macrolactone diols, which differ in stereochemistry at the C-4/C-5 diol and C-2/C-3 double bond stereochemistry (Figure 3). ${ }^{1}$ These stereochemical differences affect the biological activity. For instance, cladospolides A-C inhibit the shoot elongation in rice seedlings, ${ }^{1 \mathrm{C}}$ whereas, cladospolides $\mathrm{A}$ and $\mathrm{B}$ have the opposite effect on the root growth of lettuce seedlings. ${ }^{1 \mathrm{~b}}$

${ }^{\dagger}$ Reproduced with permission from Synthesis. 2009, 17, 2847-2854. Copyright 2009, with permission from Thieme Stuttgart. Reproduced with permission from Org. Lett. . 2009, 11, 1107-1110. Copyright 2009, with permission from American Chemical Society. 
<smiles>CC1CCCCC[C@@H](O)[C@@H](O)/C=C/C(=O)O1</smiles>

cladospolide A (III-1)<smiles>C[C@@H]1CCCCC[C@H](O)[C@@H](O)/C=C\C(=O)O1</smiles>

cladospolide B (III-2)<smiles>CC1CCCCCC(O)[C@@H](O)/C=C/C(=O)O1</smiles>

cladospolide C (III-3)<smiles>CC1CCCCCC(O)C(=O)/C=C\C(=O)O1</smiles>

cladospolide D (III-4)

Figure 3: The cladospolide A-D

Because these three natural products, cladospolides A-C, ${ }^{2,3,4}$ are produced by the same and related organisms, it was initially suggested that they should share the $C-11$ carbinol stereochemistry, which biosynthetically would be formed earliest in the fatty acid biosynthetic pathway. The relative stereochemistry for the cladospolides A-C have been confirmed by several total syntheses, although it should be noted that questions remain around cladospolide $\mathrm{B}$, where the different signs of rotation were found for the same absolute stereoisomer for synthetic cladospolide B by Banwell and us. $^{2,3,4}$<smiles>CC1CCCCCC(O)C(=O)/C=C/C(=O)O1</smiles>

purported cladospolide D (III-5)<smiles>C[C@H]1CCCCC[C@@H](O)C(=O)/C=C/C(=O)O1</smiles>

E-cladospolide D (III-6)<smiles>CC1CCCCCC(O)C(=O)/C=C\C(=O)O1</smiles>

natural

Z-cladospolide D (III-4)

Figure $4:(E / Z)$-cladospolide D

In contrast to the plant pheromone activity of cladospolide A-C, cladospolide D possesses antimicrobial activity with $\mathrm{IC}_{50}$ values of 0.1 and $29 \mathrm{~g} / \mathrm{mL}$ against $M$. racemosus and $P$. oryzae, respectively. ${ }^{1 \mathrm{~d}}$ Until our work, the absolute and relative stereochemistry of cladospolide D had not been correctly determined. ${ }^{5}$ At issue was 
the stereochemical assignment of the C2/C3 double bond, which was solely based on a $13.5 \mathrm{~Hz}$ vicinal coupling constant for the purported trans-enoate carbon/carbon double bond. ${ }^{1 \mathrm{~d}}$

Using asymmetric synthesis of all the possible diastereomers and the comparison of optical rotation data, we assigned the absolute and relative stereochemistry for cladospolide D (Figure 4). ${ }^{3 \mathrm{c}}$ This work resulted in a correction of the initially assigned $C$-2/C-3 double bond stereochemistry, which the first synthesis of cladospolide $\mathrm{D}$, by Hou, initially confirmed the structure as E-isomer III-6. ${ }^{5}$ Unfortunately, this synthetic study missed a facile E/Z-double bond isomerization and thus they actually prepared the enantiomer of the Z-isomer, (ent)-cladospolide D III-4. Herein, I describe the full account of our synthetic studies toward the successful synthesis of cladospolide B/C and the (ent)-cladospolide $\mathrm{D}$, which includes our study of the E/Z-double bond isomerization of cladospolides and their diastereomers.

\subsection{Previous Approaches to Cladospolides}

In Hou's synthesis of cladospolide D, they started from chiral starting material diol III-7 which was converted to allylic alcohol III-8 after four steps (Scheme 1). The cross-metathesis between the allylic alcohol III-8 and both enantiomerically pure $(R)$ and (S)-2-(6-heptenyl) acetates (III-9a and III-9b, respectively) provided III-10a and III-10b. The allylic alcohol of III-10 was protected as its TBDPS ether followed by Michael addition to give III-11a and III-11b respectively. Acid III-12 was achieved by five steps transformations from III-11. 


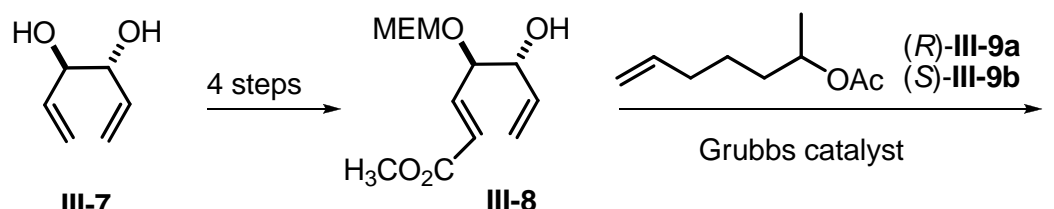

III-7

III-8

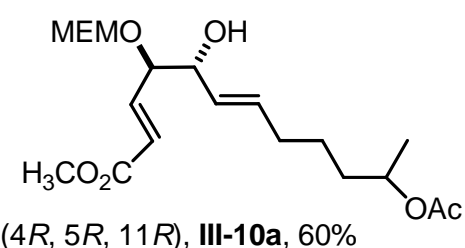

(4R, 5R, 11R), III-10a, 60\% (4R, 5R, 11S), III-10b, 60\%

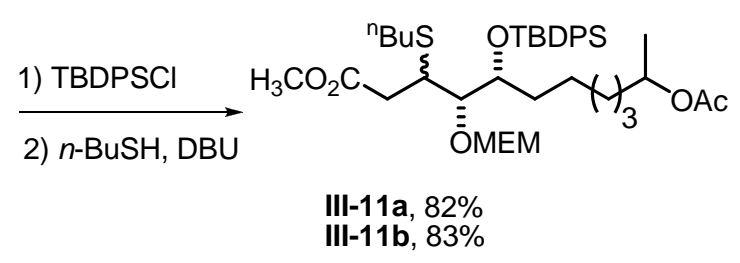

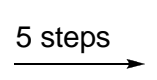

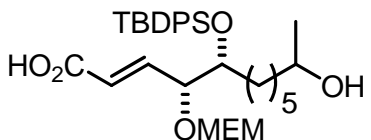

III-12a, $75 \%$ III-12b, $70 \%$

Scheme 1. Total synthesis of cladospolide D by Hou

With III-12 in hand, they next preceded the Yamaguchi lactonization to generate lactone III-13 (Scheme 2). After deprotection of MEM group and oxidation with DMP, III-15 was prepared in good yield. Diastereomers III-16a and III-16b were established after removing the tert-butyldiphenyl silyl (TBDPS) groups by tetrabutylammonium fluoride (TBAF) or hydrogen fluoride/pyridine. They noticed that the diastereomer III-16b was only generated by HF-Py (46\%).

After comparing the spectra data with the isolation paper, Hou claimed that the structure III-16b was the natural product cladospolide D. However, in their last deprotection of TBDPS step using HF-Py, they missed a facile double bond isomerization. Therefore, they actually prepared the natural product cladospolide D, but they mis-assigned the structure as $E$ isomer III-16b. 


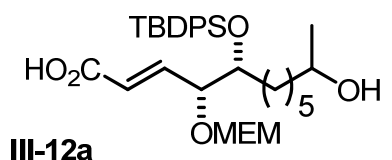
III-12b

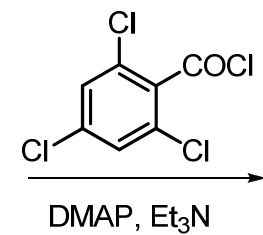

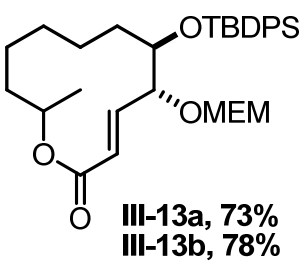<smiles>Br[Bi]1Oc2ccccc2O1</smiles>

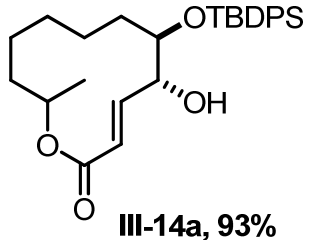
III-14b, $98 \%$

Fluoride<smiles>CC1CCCCCC(O)C(=O)/C=C/C(=O)O1</smiles>

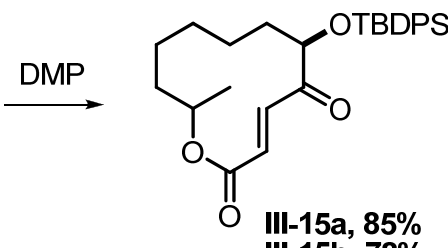

(5R, 11R), III-16a, 42\%, TBAF (5R, 11S), III-16b, 46\%, HF-Py

Scheme 2. Lactonization to form cladospolide D by Hou

\subsection{De Novo Approach to Natural Products Cladospolides}

Our de novo synthesis linked the use of Noyori asymmetric hydrogenation of propargyl ketone, alkyne zipper reaction and Sharpless dihydroxylation together to construct natural products cladospolides B, C and (ent)-cladospolide D as well as all the possible isomers of cladospolide D.

\subsubsection{Retrosynthetic Analysis}

At the outset of this project, we had reservations regarding the assignment of double bond stereochemistry for purported cladospolide D (III-5). Thus our retrosynthetic analysis envisioned that Z-cladospolide D (III-4) could be prepared from cladospolide B (III-2) or its bis-epimer III-17. Similarly, E-cladospolide D (III-6) could be 
prepared from cladospolide C (III-3) or its bis-epimer III-18. In turn, cladospolides B, C (III-2, III-3) and their bis-epimers III-17 and III-18 could come from the lactonization reactions of diols III-19 and III-20. Diols III-19 and III-20 could come from the diastereoselective and regioselective dihydroxylation ${ }^{6}$ and diene conjugation of ynone III-21, ${ }^{7}$ which could be prepared from commercially available 1-nonyne III-22 employing the Noyori asymmetric reduction/alkyne zipper isomerization (Scheme 3).

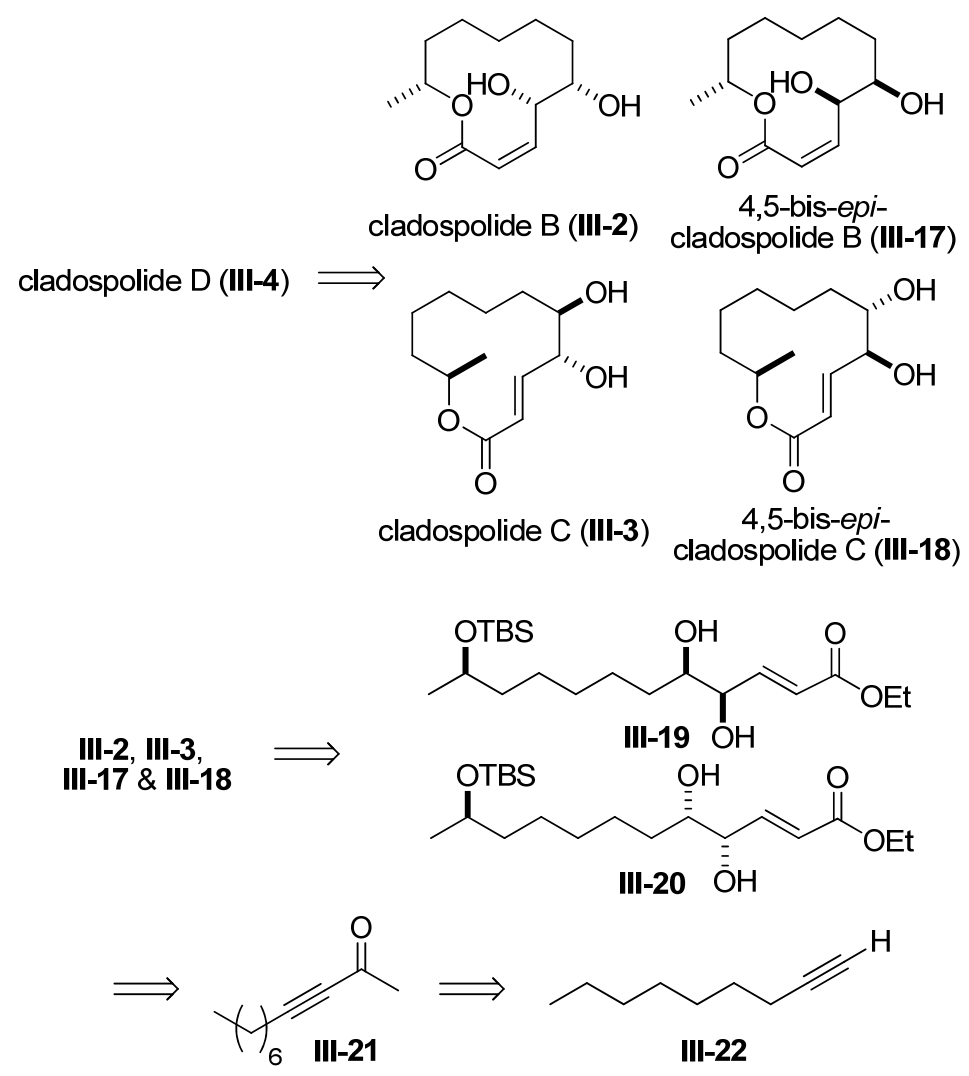

Scheme 3. Retrosynthetic analysis

\subsubsection{Synthesis of diols}

Lithiation of commercially available 1-nonyne III-22 and alkylation with acetylaldehyde gave racemic propargylic alcohol (rac)- III-23 (69\%, two steps) (Scheme 4). Propargylic oxidation of (rac)-III-23 with $\mathrm{MnO}_{2}$ gave the ynone III-21. 
Exposure of ynone III-21 to our modified Noyori conditions provided an excellent yield (85\%) of propargyl alcohol III-23 with high enantiomeric purity (> 96\% ee). Exposure of III-23 to the KAPA reagent readily isomerized it to the terminal undecynol, which was protected as a TBS-ether (TBSCl/imid. in DMF) in good overall yield (82\%) and with no loss of enantiomeric purity (> 96\% ee). ${ }^{8}$

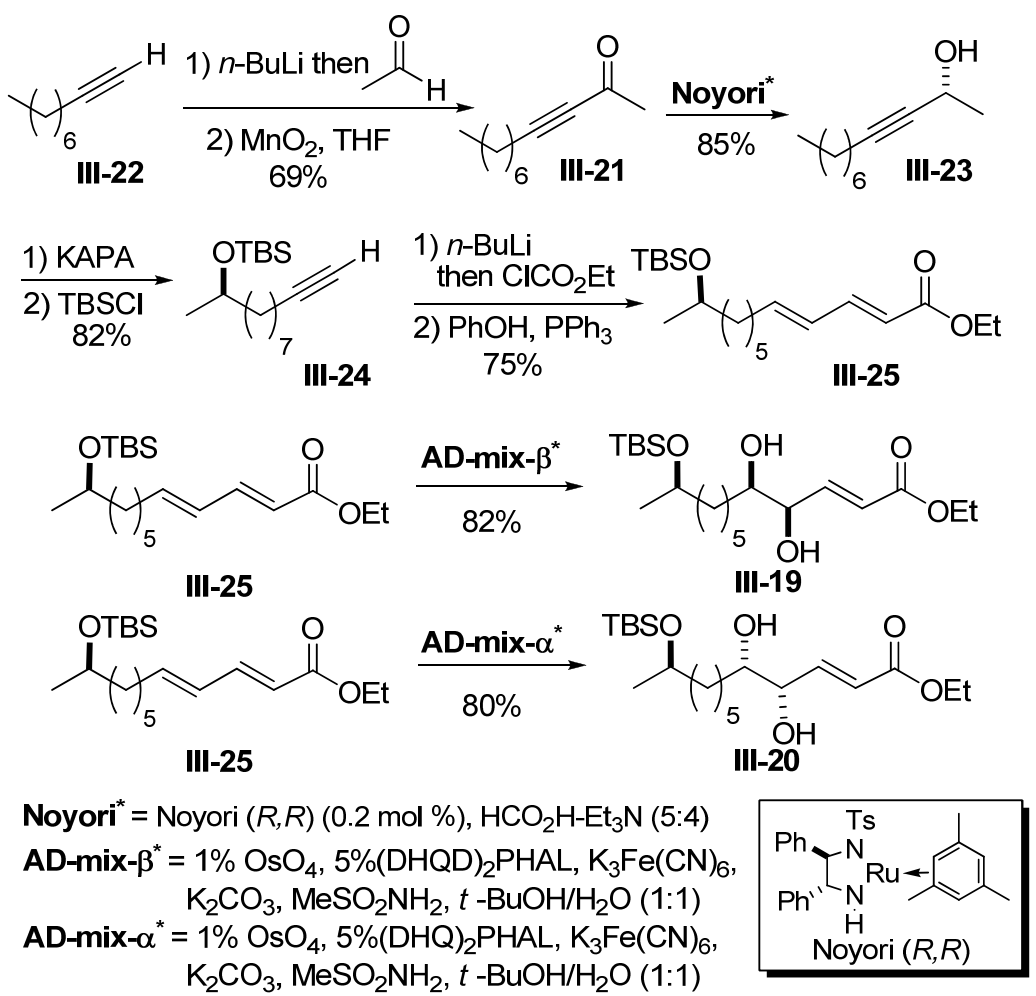

Scheme 4. Synthesis of diol

The mechanism of alkyne zipper reaction is shown in Scheme $5 .{ }^{9}$ KAPA reagent which refers to potassium 3-aminopropylamide is a readily prepared difunctional superbase. KAPA is prepared by quantitative reaction of $\mathrm{KH}$ with excess 3-aminopropylamine. In this KAPA catalyzed alkyne zipper reaction, the triple bond from the middle of the carbon chain migrates to the end of the carbon chain because of the formation of stable acetylide ion. 


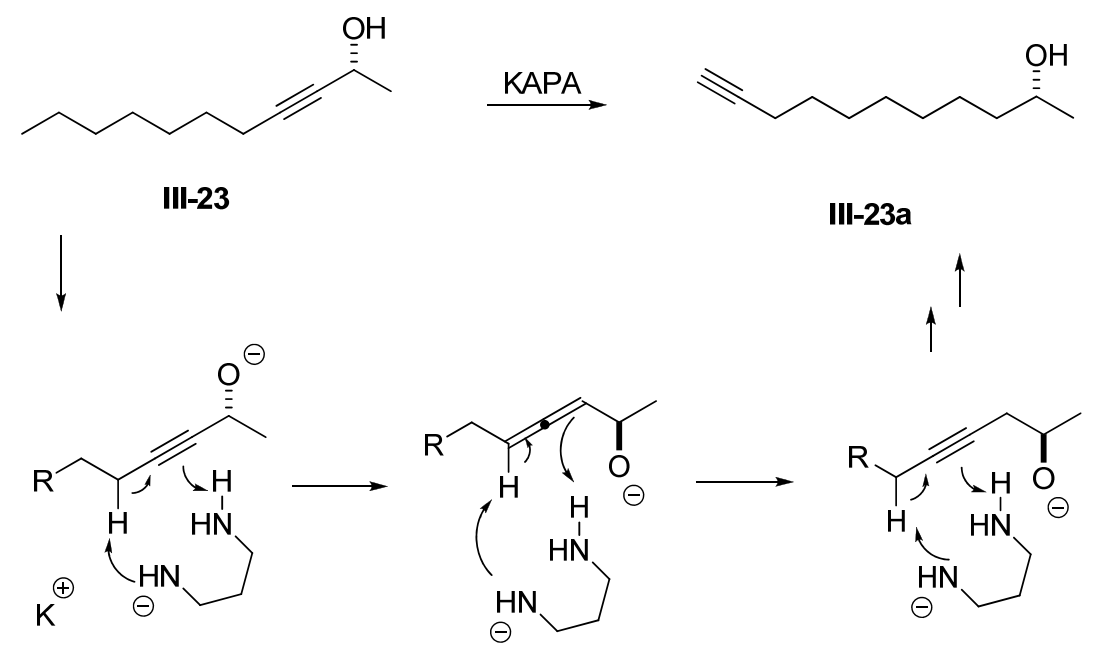

Scheme 5. Mechanism of alkyne zipper reaction

The terminal alkyne III-24 was carboxylated ( $n$-BuLi/ClCO ${ }_{2} \mathrm{Et}, 83 \%$ ) to give an ynoate, which by means of the Rychnovsky variant of the Trost ynoate to dienoate isomerization $\left(\mathrm{Ph}_{3} \mathrm{P} / \mathrm{PhOH}\right)$ was cleanly converted into $E$,E-dienoate III-25 in good yields (90\%) and stereoselectivity. ${ }^{7}$ Dienoate III-25 was subjected to Sharpless asymmetric dihydroxylation conditions (2\% $\mathrm{OsO}_{4}$ and $4 \%$ (DHQD) ${ }_{2} \mathrm{PHAL}$ ) to give diol III-19 in approximately $80 \%$ yield and as a single diastereomer. ${ }^{6}$ Similarly, dienoate III-25 was dihydroxylated with the pseudo-enantiomeric reagent (2\% $\mathrm{OsO}_{4}$ and 4\% (DHQ) $)_{2}$ PHAL) to give diol III-20 in approximately $80 \%$ yield and as a single diastereomer. The mechanism of Rychnovsky variant of the Trost ynoate to dienoate isomerization is outlined in scheme 6. 

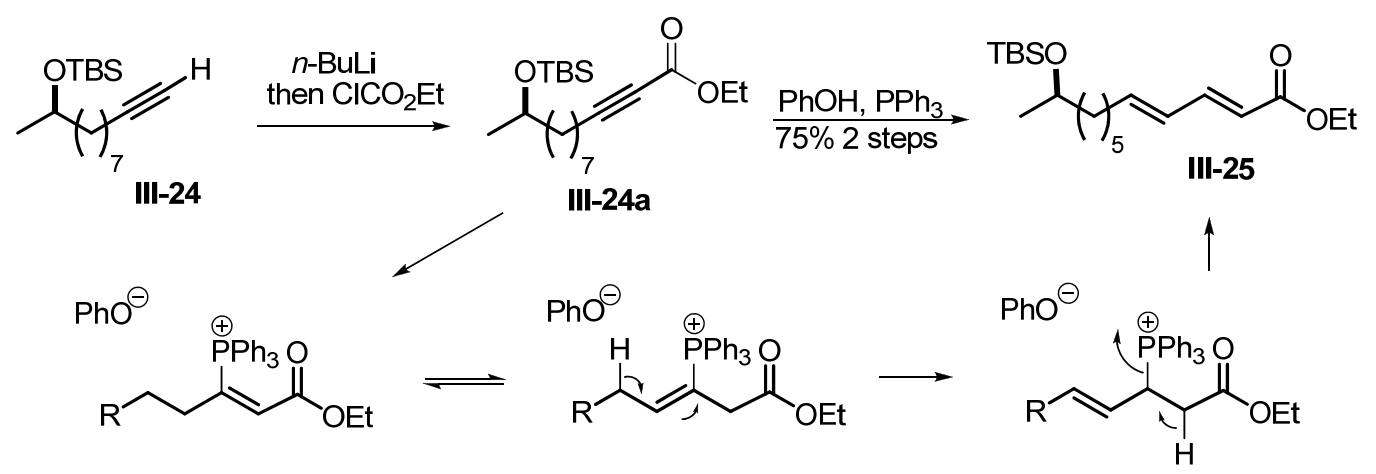

Scheme 6. Mechanism of ynoate to dienoate isomerization

\subsubsection{Synthesis of cladospolide $B, C$ and isomers}

With all the $\mathrm{sp}^{3}$ stereocenters established for the cladospolides, we turned to the assembly of the macrocycle (Scheme 7). To our delight this occurred with a small amount of double bond isomerization. Thus exposing diol III-19 to acid catalyzed acetonide formation/TBS deprotection conditions gave the desired seco-acid, which when exposed to the Yamaguchi lactonization conditions ${ }^{10}$ gave a 6:1 mixture of cladospolide C (III-3) (36\%) and its double bond isomer III-17 (6\%), after TFA promoted acetonide deprotection. Following a virtually identical macrocyclization protocol, diol III-20 was converted into a similar mixture of cladospolide B (III-2) (6\%) and its double bond isomer III-18 (34\%). 


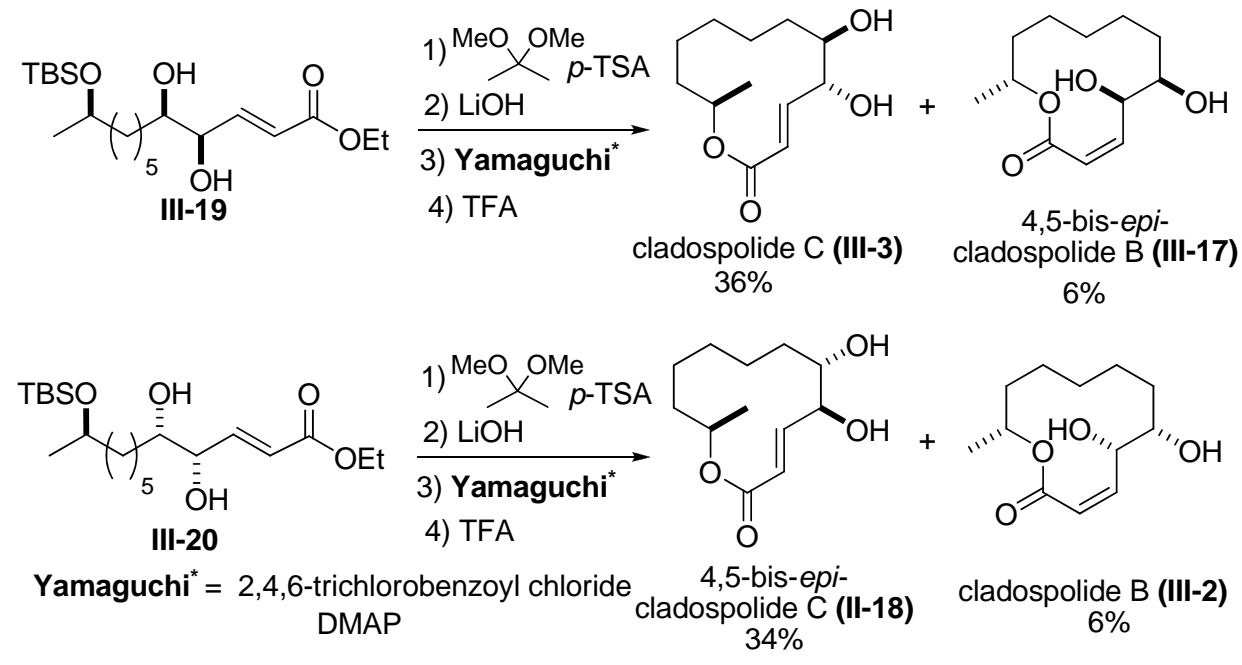

Scheme 7. Synthesis of cladospolide B, C and isomers

\subsubsection{Synthesis of cladospolide $D$ and diastereomers}

With the construction of the required four stereoisomeric macrolactone diols, we turned our attention to the synthesis and structural proof of cladospolide D. This began with the investigation of the selective oxidation of the allylic alcohol in cladospolide $\mathrm{C}$ (III-3) (Scheme 8). Unfortunately, several oxidants (e.g., $\mathrm{MnO}_{2}$ and Dess-Martin Periodinane) led to oxidative diol cleavage giving a dialdehyde product. In our grahamimycin A synthesis, we had previously found that the enone functionality could be installed by a stochiometric TEMPO oxidation of the allylic alcohol in colletodiol, without the need for selective protection. ${ }^{6 \mathbf{c}}$ Unfortunately, when we applied these same conditions to the cladospolides, only products of double oxidation were obtained. Thus, when we exposed cladospolide C (III-3) and its C-4,5-bis-epimer III-18 to the catalytic TEMPO oxidation conditions (1 mol\% 2,2,6,6-tetramethylpiperidinooxy/1 equiv trichloroisocyanuric acid), ${ }^{11}$ only diketone III-26 was observed. When only one equiv 
of co-oxidant was used (1 mol\% 2,2,6,6-tetramethylpiperidinooxy/0.33 equiv trichloroisocyanuric acid), only low yields of III-26 and starting material were observed. Similarly, cladospolide B (III-2) and its C-4,5-bis-epimer III-17 reacted under the TEMPO oxidation conditions to give diketone III-27. Thus, we turned to a selective protection/oxidation/deprotection sequence.<smiles></smiles>

cladospolide C (III-3)<smiles>C[C@H]1CCCCC[C@@H](O)[C@H](O)/C=C\C(=O)O1</smiles>

4,5-bis-epicladospolide B (III-17)<smiles></smiles>

III-26<smiles>CC1CCCCC[C@H](O)C(O)/C=C/C(=O)O1</smiles>

4,5-bis-epicladospolide C (III-18)

EMPO $=1 \%$ 2,2,6,6-tetramethylpiperidinooxy, trichloroisocynanuric acid

Scheme 8. Diol per-oxidation

Because the absolute stereochemistry of cladospolide D was not known and we had our doubts about the assigned olefin geometry, we decided to perform the protection/oxidation/deprotection sequence on both cladospolide B (III-2) and C (III-3) as well as their 4,5-bis-epimers III-17 and III-18. Because of the exploratory nature of these studies, no effort was made to maximize the regioselectivity in the protection-step. Rather a regio-unselective TBS-protection was chosen for simplicity's sake (i.e., no bis-protection was observed and the minor regioisomer could be recycled). This in turn helped with the assignment of the regioisomers. 

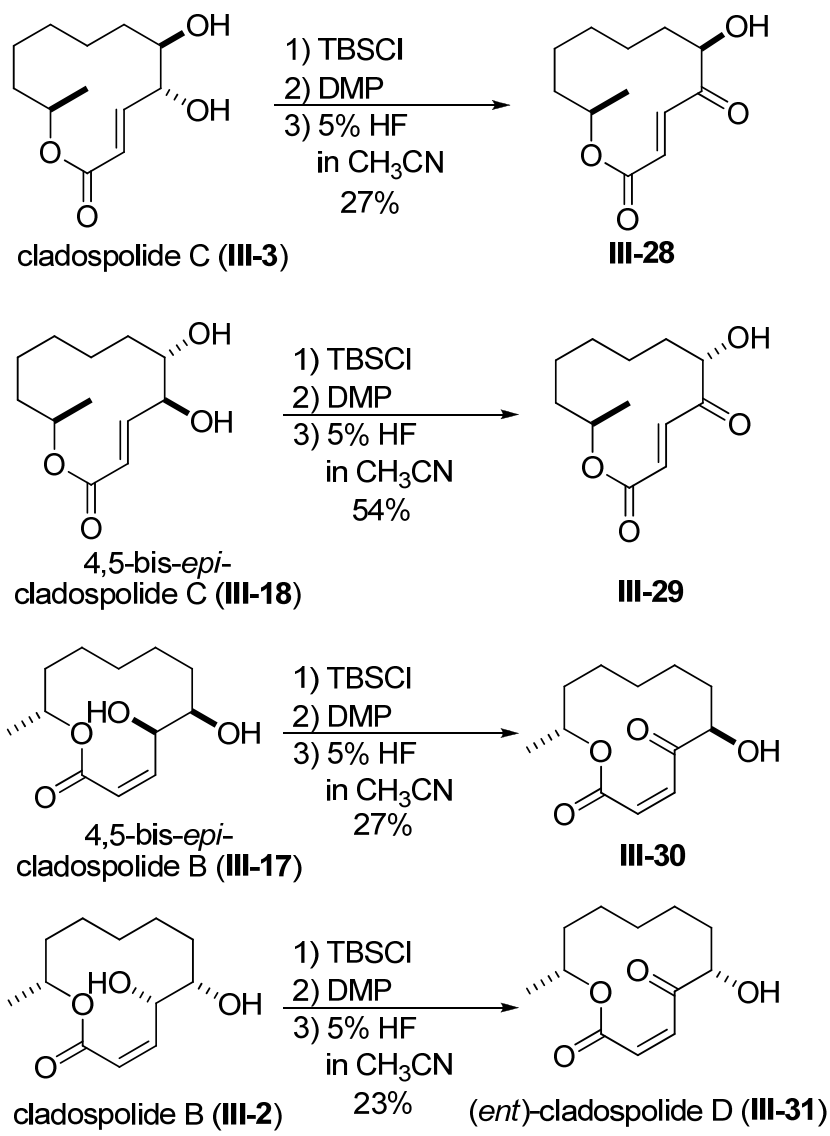

Scheme 9. Synthesis of cladospolide D and diastereomers

Thus exposure of cladospolide $\mathrm{C}$ (III-3) to the three-step TBS mono-protection/Dess-Martin oxidation/HF deprotection gave a 27\% yield of enone III-28 (Scheme 9). Cladospolide B (III-2) and its 4,5-bis-epimer III-17 gave the corresponding enones III-31 and III-30, respectively, with similar yields for this three-step sequence. The low yield for these sequences was due to the poor regioselectivity (1:2 against) in the TBS-protection step. In contrast, when the 4,5-bis-epimer III-18 was exposed to the same three-step sequence a higher yield of enone III-29 was obtained due to improved regioselectivity in the TBS-protection (2:1). Of the enones produced only III-31 had spectral data that matched cladospolide $\mathrm{D},{ }^{1 \mathrm{~d}}$ although the rotation was opposite in sign. The spectral data also matched the data 
reported by Hou for (ent)-cladospolide D, who reported its structure to be the $E$-isomer III-29. Therefore, we concluded that a hidden alkene isomerization reaction must have occurred during the Hou synthesis.

\subsubsection{Photo Isomerization of Double Bond}

Thus, we decided to investigate the isomerization of the olefin III-29. When enone III-29 was irradiated with UV-light (300 nm) with and without iodine, a 1:1 ratio of III-29 and (ent)-cladospolide D (III-31) was found along with a significant amount of photo-degradation products. While this isomerization explained the problem with Hou's assignment of cladospolide $\mathrm{D}$, the photochemical isomerization reaction was not clean enough to be consistent with his reported yield. Similarly byproducts were also observed, when we explored the photoisomerization of III-28 and III-30. However, much cleaner isomerization reactions were observed for the TBS-protected enones, III-32, III-33, III-34 and III-35 (Scheme 10).

Thus when we irradiated $\mathrm{D}_{6}$-benzene solutions of pure III-32 and III-33 for $24 \mathrm{~h}$ with $300 \mathrm{~nm}$ light through a Pyrex filter, a photo-equilibrium (1:1.3, E/Z) was established with the Z-isomer III-33 being slightly favored. When we irradiated $\mathrm{D}_{6}$-benzene solutions of III-34 and III-35 under identical conditions a photo-equilibrium (1:2, E/Z) was established, which slightly favored the Z-isomer III-35 (Scheme 10). 


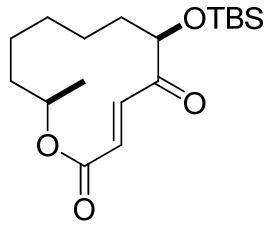

III-32

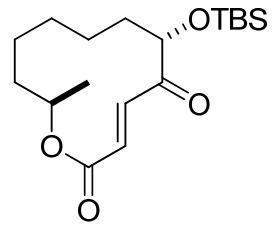

III-34

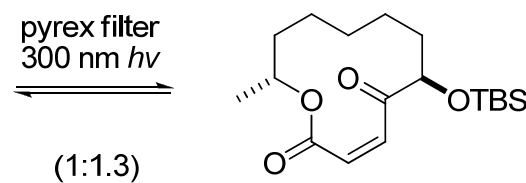

III-33

pyrex filter

$\stackrel{300 \mathrm{~nm} \mathrm{hv}}{\rightleftharpoons}$

$(1: 2)$

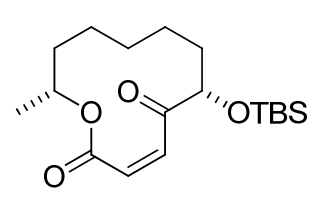

III-35

Scheme 10. Photo equilibration

Because the photo-isomerization give photo-equilibriums and not true $\mathrm{K}_{\mathrm{eq}}$, we decided to isomerize the enones under iodine radical conditions. Thus we exposed pure $\mathrm{D}_{6}$-benzene solutions of III-32 and III-33 to a catalytic amount of $\mathrm{I}_{2}$ under thermal and photochemical conditions (Scheme 11). Under these conditions, both solutions rapidly $(<1 \mathrm{~h})$ reached equilibrium $\left(\mathrm{K}_{\mathrm{eq}}=1: 4, E / Z\right)$ with the $Z$-isomer being more stable. When we turned to the E/Z-isomers of the natural cladospolide D diastereomer, we found a much greater difference in stability. When the same isomerization was preformed with pure solutions of III-34 and III-35 the equilibrium was reached much more quickly and the equilibrium achieved was in even greater preference for the Z-isomer III-35 ( $\mathrm{K}_{\mathrm{eq}}>$ 20, Z/E). Thus, we can conclude that the driving force and barrier to isomerization of the cladospolide D diastereomer III-35 is quite strong. 
<smiles></smiles>

III-32<smiles>CC1CCCCC[C@H](OC(=O)/C=C/C(=O)O[SnH3])C1</smiles>

III-34

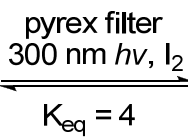<smiles>C[C@@H]1CCCCCC(O[SnH3])C(=O)/C=C\C(=O)O1</smiles>

III-33
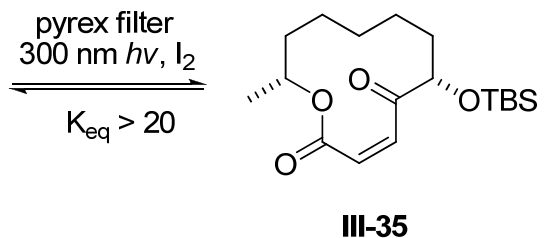

Scheme 11. Photochemical equilibrium

For comparison, we decided to study the double bond isomerization between cladospolide C (III-3) and its 4,5-bis-epimer III-18 using the same equilibration condition (Scheme 12). This study showed that no strong thermodynamic preference for the Z-isomers could be found. For example, when cladospolide C (III-3) and its double bond isomer III-17 were isomerized under radical iodine conditions (24 h irradiation with $\mathrm{I}_{2}$ ), we found equal isomers of III-3 and III-17 $\left(\mathrm{K}_{\mathrm{eq}}=1\right)$. In contrast, we were not able to reach to full equilibirum for the isomerization of 4,5-bis-epi-cladospolide C (III-18) to cladospolide B (III-2), but we could bracket the equilibrium constant to be between 0.3 and 6 (Scheme 12). Thus, we can conclude that the C-4 carbonyl in cladospolide D has a strong effect on the E/Z-stability. 

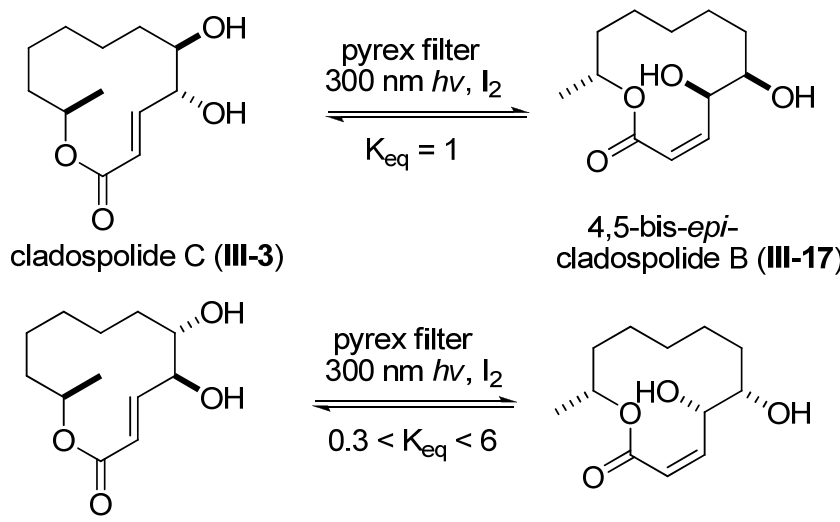

cladospolide C (III-18)

cladospolide B (III-2)

Scheme 12. Photochemical equilibrium

\subsubsection{Improved approaches to cladospolide D}

With the knowledge of the structure of the desired target molecule, we next sought an improved synthesis (Scheme 13). These efforts lead to the discovery of the double bond isomerization found in the Hou's synthesis. Thus we returned to the trans-diol III-18, which could be regioselectively protected to give III-36 in good yield. Dess-Martin oxidation of III-36 cleanly gave enone III-34. At this stage a photo-chemical isomerization of III-34 cleanly gave the previous prepared cis-isomer III-35 in near quantitative yield (> 95\%). Interestingly, when III-34 was treated with the conditions used by Hou (HF/Py), instead of our preferred conditions (5 \% $\mathrm{HF} / \mathrm{CH}_{3} \mathrm{CN}$ ), a clean double bond isomerization occurred to give III-35. As before, TBS-deprotection with $5 \% \mathrm{HF} / \mathrm{CH}_{3} \mathrm{CN}$ occurred to give $\gamma$-keto-enoate III-31. Synthetic III-31 material was spectroscopically $\left({ }^{1} \mathrm{H}\right.$ NMR, ${ }^{13} \mathrm{C}$ NMR, IR and MS) identical with natural cladospolide $\mathrm{D}$, although the optical rotation was opposite in sign $\left([\alpha]_{\mathrm{D}}-58\right.$ vs +56 in $\left.\mathrm{MeOH}\right)$. Thus structure III-31 must be the enantiomer of 
cladospolide D and the structure should be revised to structure III-4 as shown in Figure 1.

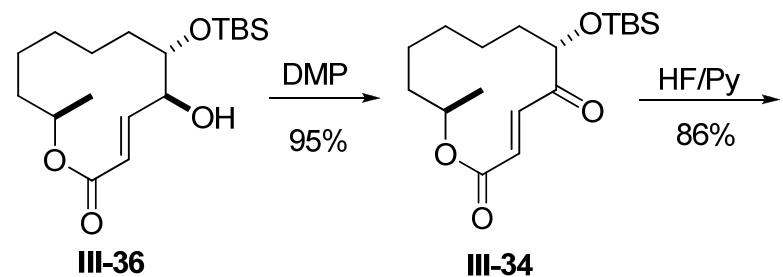

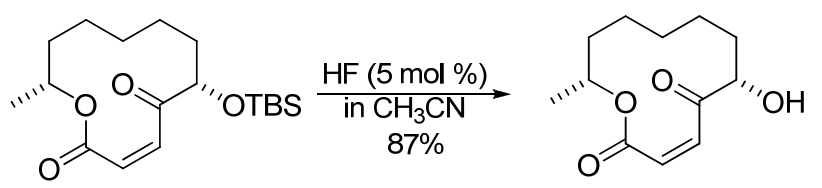

III-35 (ent)-cladospolide D (III-31)

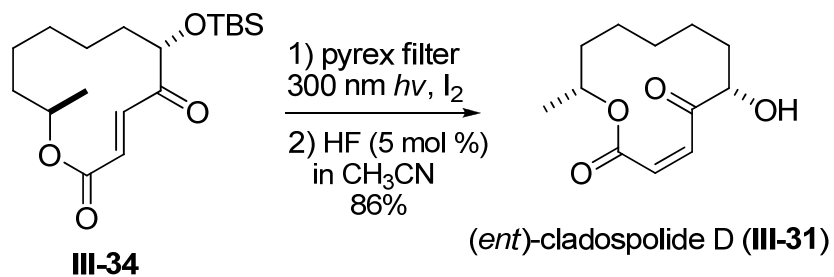

Scheme 13. Improved approaches to cladospolide D

\subsection{Conclusions}

In conclusion, a short and enantioselective synthesis of (ent)-cladospolide D has been developed, which clears up any ambiguities with its structural assignment. This structural proof involved the synthesis of all the possible stereoisomers of cladospolide D as well as to other members of this family of natural products, cladospolides B and C. This de novo asymmetric approach used two remote catalytic asymmetric reactions (Noyori reduction and Sharpless asymmetric dihydroxylation) to establish its asymmetry, therefore, this synthesis is also a formal synthesis of the natural stereoisomer of cladospolide $\mathrm{D}$. This de novo route to the cladospolides compares favorably (i.e., less steps and greater overall efficiency) to the previous 
routes from chiral starting materials. In addition, this route enabled the study of the C-2/3 alkene isomerization that was of central importance to the structural proof of cladospolide D.

\section{Reference:}

${ }^{1}$ (a) Hirota, A.; Isogai, A.; Sakai, H. Agric. Biol. Chem. 1981, 45, 799. (b) Hirota, A.; Sakai, H.; Isogai, A. Agric. Biol. Chem. 1985, 49, 731. (c) Fujii, Y.; Fukuda, A.; Hamasaki, T.; Ichimoto I.; Nakajima, H. Phytochemistry. 1995, 40, 1443. (d) Zhang, H.; Tomoda, H.; Tabata, N.; Miura, H.; Namikoshi, M.; Yamaguchi, Y.; Masuma R.; Omura, S, J. Antibiot. 2001, 54, 635. (e) Hirota, A.; Sakai, H.; Isogai, A.; Kitano, Y.; Ashida, T.; Hirota H.; Takahashi, T. Agric. Biol. Chem. 1985, 49, 903. (f) Hirota, H.; Hirota, A.; Sakai, H.; Isogai, A.; Takahashi, T. Bull. Chem. Soc. Jpn. 1985, 58, 2147. (g) Smith, C. J.; Abbanat, D.; Bernan, V. S.; Maiese, W. M.; Greenstein, M.; Jompa, J.; Tahir A.; Ireland, C. M. J. Nat. Prod. 2000, 63, 142.

${ }^{2}$ For the synthesis of cladospolide A, see: (a) Banwell, M. G.; Loong, D. T. J. Org. Biomol. Chem. 2004, 2, 2050. (b) Banwell, M. G.; Jolliffe, K. A.; Loong, D. T. J.; McRae, K. J.; Vounatsos, F. J. Chem. Soc., Perkin Trans. 1 2002, 22. (c) Solladie, G.; Almario, A. Tetrahedron: Asymmetry 1995, 6, 559. (d) Solladie, G.; Almario, A. Pure Appl. Chem. 1994, 66, 2159. (e) Ichimoto, I.; Sato, M.; Kirihata, M.; Ueda, H. Chem. 
Express. 1987, 2, 495. (f) Maemoto, S.; Mori, K. Chem. Lett. 1987, 109. (g) Mori, K.; Maemoto, S. Liebigs Ann. Chem. 1987, 863.

${ }^{3}$ For the synthesis of cladospolide B, see: (a) Pandey, S. K.; Kumar, P. Tetrahedron Lett. 2005, 46, 6625. (b) Austin, K. A. B.; Banwell, M. G.; Loong, D. T. J.; Rae, A. D.; Willis, A. C. Org. Biomol. Chem. 2005, 3, 1081. (c) Xing, Y.; O’Doherty, G. A. Org. Lett. 2009, 11, 1107-1110.

${ }^{4}$ For the synthesis of cladospolide C, see: ref. 3c and (a) Banwell, M. G.; Loong, D. T. J.; Willis, A. C. Aust. J. Chem. 2005, 58, 511. (b) Chou, C.-Y. Hou, D.-R. J. Org. Chem. 2006, 71, 9887. (c) Sharma, G. V. M.; Reddy, K. L.; Reddy, J. J. Tetrahedron Lett. 2006, 47, 6537. (d) Reddy, C. R.; Rao, N. N. Tetrahedron Lett. 2009, 50, 2478-2480.

${ }^{5}$ For the synthesis of cladospolide D, see: ref. 3c and Lu, K.-J.; Chen, C.-H.; Hou, D.-R. Tetrahedron 2009, 65, 225.

${ }^{6}$ The regioselectivity of the asymmetric dihydroxylation of dienoates has been studied by Sharpless and our group, see: (a) Berker, H.; Soler, M. A.; Sharpless, K. B. Tetrahedron 1995, 51, 1345. (b) Zhang, Y.; O'Doherty, G. A. Tetrahedron 2005, 61, 6337-6351. For synthetic application of this, see: (c) Hunter, T. J.; O’Doherty, G. A. Org. Lett. 2002, 4, 4447-4450. (d) Guo, H.; Mortensen, M. S.; O’Doherty, G. A. Org. Lett. 2008, 10, 3149-3152. (e) Ahmed, Md., M. Mortensen, M. S.; O’Doherty, G. A. J. Org. Chem. 2006, 71, 7741-7746. (f) Ahmed, Md. M.; O’Doherty, G. A. Tetrahedron 
Lett. 2005, 46, 3015-3019. (g) Ahmed, Md. M.; Berry, B. P. ; Hunter, T. J. ; Tomcik, D. J.; O’Doherty, G. A. Org. Lett. 2005, 7, 745-748.

${ }^{7}$ (a) Rychnovsky, S. D.; Kim, J. J. Org. Chem. 1994, 59, 2659-2660. (b) Trost, B.; Kazmaier, U. J. Am. Chem. Soc. 1992, 114, 7933-7935. For synthetic application of this, see: (c) Li, M.; O’Doherty, G. A. Org. Lett. 2006, 8, 3987-3990.

${ }^{8}$ Trost, B. M.; Horne, D. B.; Woltering M. J. Chem. Eur. J. 2006, 12, 6607.

${ }^{9}$ Brown, C. A.; Yamashita, A. J. Am. Chem. Soc. 1975, 97, 891-892

${ }^{10}$ Inanaga, J.; Hirata, K.; Saeki, H.; Katsuki, T.; Yamaguchi, M. Bull. Chem. Soc. Jpn. 1979, 52, 1989-1993.

11 (a) Luca, L. D.; Giacomelli, G.; Porcheddu, A. Org. Lett. 2001, 3, 3041-3043. (b)

Md. M. Ahmed, H. Cui and G. A. O’Doherty, J. Org. Chem. 2006, 71, 6686-6689. 


\section{Chapter 4}

\section{De Novo Asymmetric Total Synthesis of Aspergillides}

\subsection{Introduction}

In 2007, Kusumi and coworkers had isolated three new 14-membered macrolides, aspergillides A (IV-1), B (IV-2), and C (IV-3) from marine-derived fungus Aspergillus ostianus strain $01 \mathrm{~F} 313$ (Figure 5). ${ }^{1}$ The aspergillides contain interesting tri-substituted tetrahydro and dihydropyran rings. These compounds were found to exhibit significant cytotoxicity against mouse lymphocytic leukemica cells (L1210) with $\mathrm{LD}_{50}$ values of 2.1, 71.0, and $2.0 \mu \mathrm{g} / \mathrm{Ml}$ for aspergillide A (IV-1), B (IV-2), and C (IV-3), respectively. The structures and absolute configurations of aspergillides were determined by analyses of NMR spectra and modified Mosher’s method.

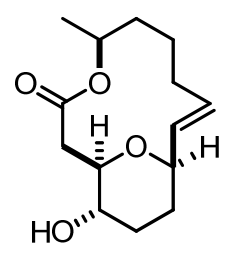

Aspergillide A (IV-1)

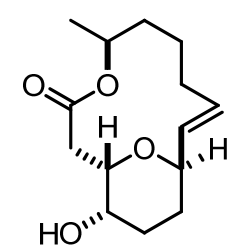

Aspergillide B (IV-2)

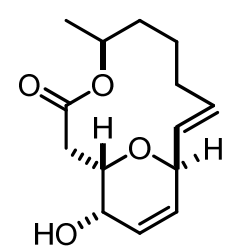

Aspergillide C (IV-3)

Figure 5. Aspergillides

Based on the unique molecular architectures and important biological features of these natural products, they have attracted interest from synthetic community. To date, five total syntheses of $\mathbf{I V - 1},{ }^{2}$ seven of $\mathbf{I V}-\mathbf{2}^{3,2 \mathrm{e}}$ and two of $\mathbf{I V - \mathbf { 3 } ^ { 4 }}$ have been reported. 
Herein I would like to describe our efforts towards total synthesis of aspergillides using our de novo methodology.

\subsection{Previous Approaches to Aspergillides}

Most recently, Shishido and coworkers published a total synthesis of aspergillide A (IV-1) and B (IV-2) based on transannular oxy-Michael reaction in Angewandte Cheme. ${ }^{2 \mathrm{e}}$ Their retrosynthetic analysis showed that the trisubstituted pyran moiety could be formed through a base-mediated transannular oxy-Michael reaction from the 14-membered macrolactone IV-4 (Scheme 1). The macrolactone IV-4 could be established by using a sequential cross-metathesis and intramolecular Horner-Wadsworth-Emmons reaction of IV-5 and IV-6. Compound IV-5 could be prepared from the chiral building block IV-7 and compound IV-6 was known in the literature (Scheme 1).

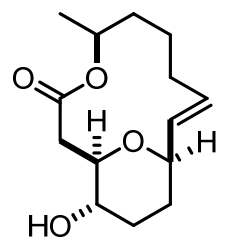

Aspergillide A (IV-1) and<smiles>CC1CCC/C=C\[C@H]2CC[C@@H](O)[C@H](CC(=O)O1)O2</smiles>

Aspergillide B (IV-2)

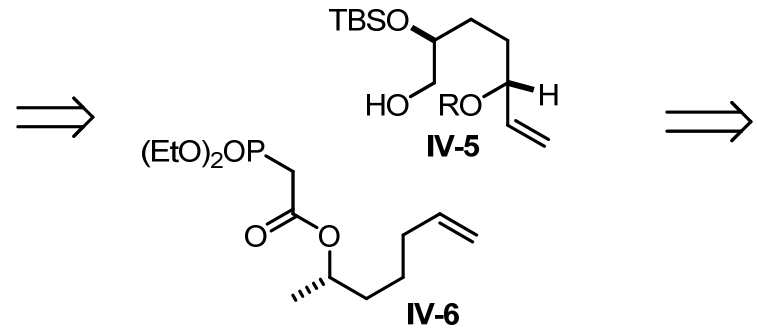

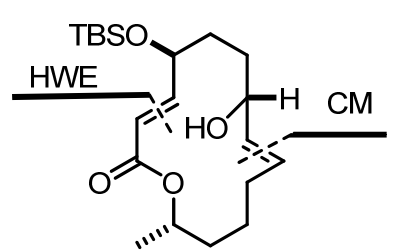

IV-4

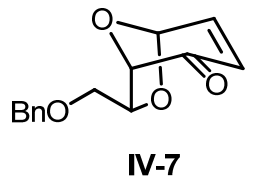

Scheme 1. Retrosynthetic analysis of Shishido’s synthesis

Chiral building block IV-7 was first converted to alcohol IV-8, which then underwent five transformations to give hemiacetal IV-9 (Scheme 2). Reduction of IV-9 with $\mathrm{LiBH}_{4}$ 
provided resulting diol, the secondary alcohol was protected as a TES ether to generate alcohol IV-10. Cross-metathesis of IV-10 with the phosphonoacetate IV-6 in the presence of the second-generation Grubbs catalyst provided the coupled product as the $E$ alkene IV-11 (>20:1) in 98\% yield. Oxidation of IV-11 with Dess-Martin periodinane and a subsequent intramolecular Horner-Wadsworth-Emmons reaction gave the macrolactone. Selective removal of the TES ether provided intermediate IV-4.
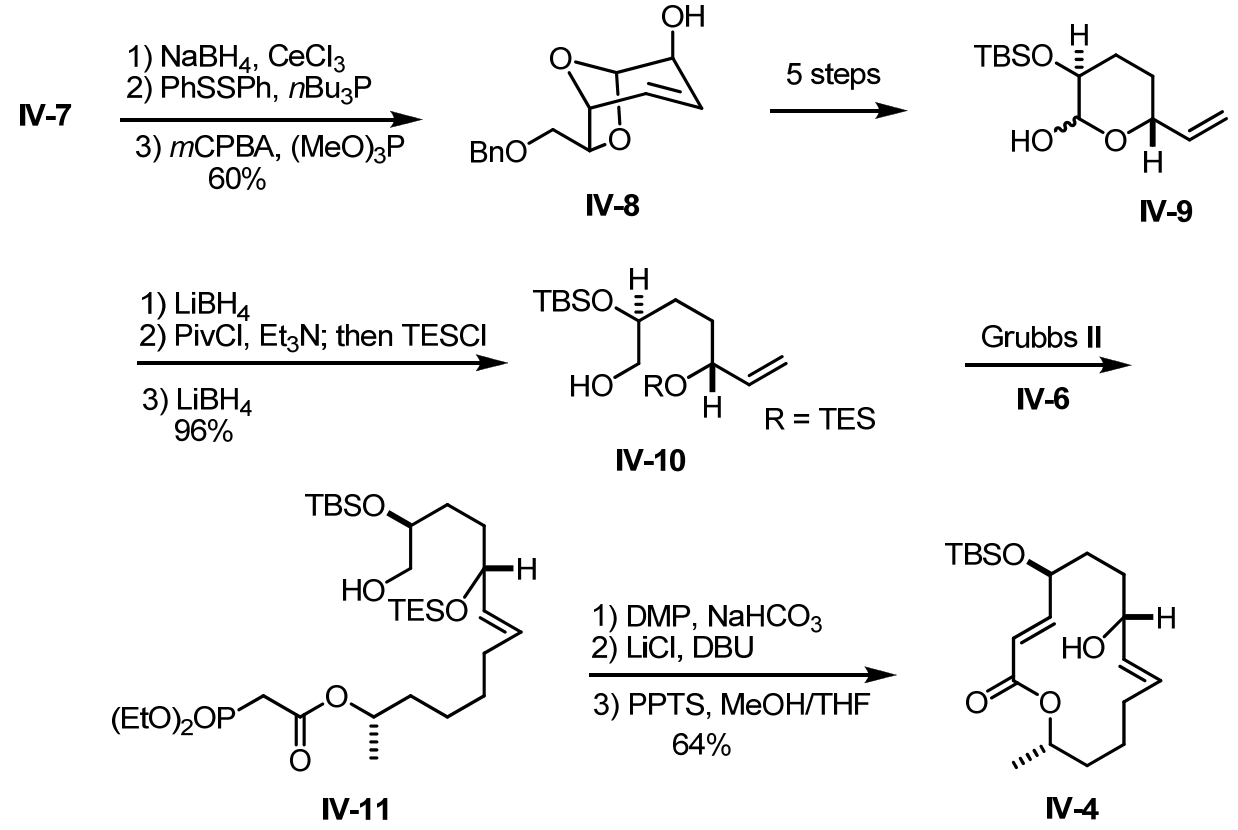

Scheme 2. Total synthesis of aspergillide A and B by Shishido

With the precursor IV-4 in hand, Shishido next investigated the transannular oxy-Michael reaction (Scheme 3). They found that the best result was for the treatment of IV-4 with $\mathrm{DBU}$ and $\mathrm{LiCl}$ in acetonitrile at room temperature for 1.5 hours which provided a quantitative amount of the syn-adduct $\mathbf{I V - 1 2}$ as a single product (entry 1). The best condition for quantitative formation of $\mathbf{I V - 1 3}$ was achieved by using $\mathrm{KH}$ and 
[18]crown-6 at $0{ }^{\circ} \mathrm{C}$ for 0.5 hours. Removal of the TBS group of IV-12 and IV-13 generated aspergillide A (IV-1) and aspergillide B (IV-2), respectively.

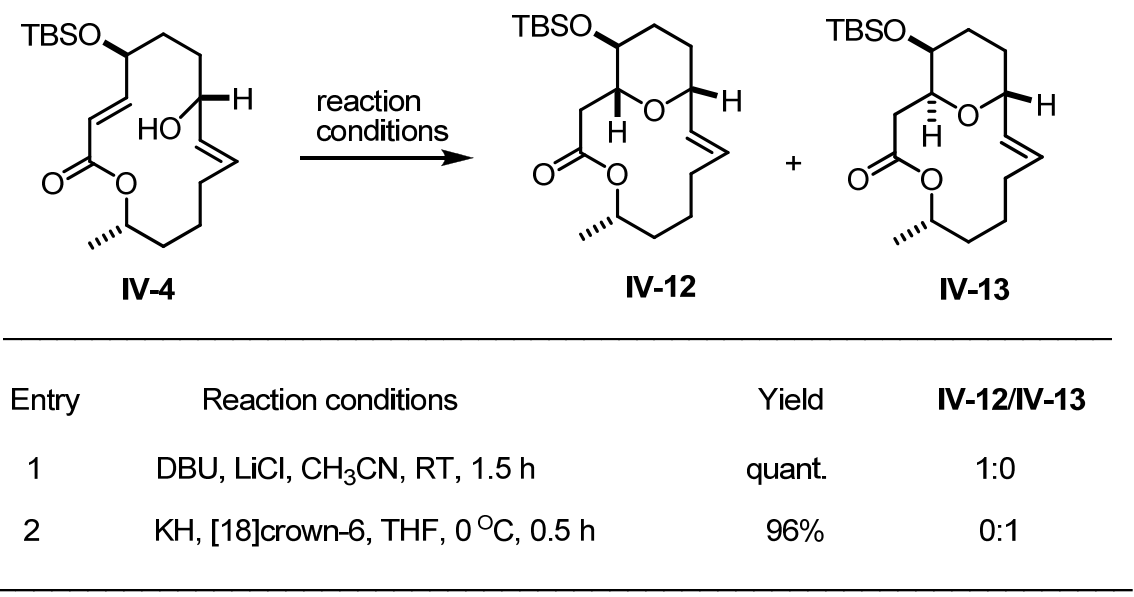

Scheme 3. Transannular oxy-Michael reaction

In 2007, Kuwahara reported the first total synthesis of aspergillide C. ${ }^{4 a}$ They envisioned that aspergillide C (IV-3) could be established from a macrolactonization of seco acid IV-14, which can be synthesized from compound IV-15. To construct IV-15, they took advantage of a Ferrier-type reaction of acetal IV-16, which could be formed by Julia olefination of aldehyde IV-18 and sulfone IV-17. The aldehyde IV-18 could be prepared via epoxide opening of commercially available epoxide IV-19 and alkyne IV-20 (Scheme 4).

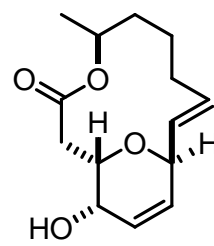

Aspergillide C (IV-3)<smiles></smiles><smiles>[C+]#CCOCCO</smiles>

IV-20

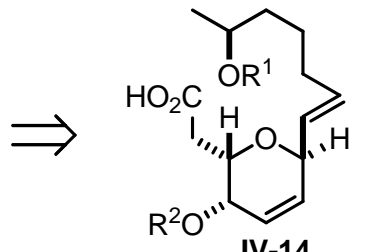

IV-14

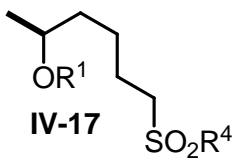<smiles>CCO[C@H]1C=CC[C@@H](C=O)O1</smiles><smiles>[R6][C@H]1C=CC[C@@H](/C=C\CCCC(C)O)O1</smiles>

IV-15

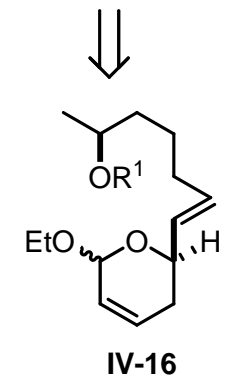

Scheme 4. Retrosynthetic analysis of Kuwahara's synthesis 
E-isomer IV-16 was established within 10 steps from starting materials and then converted to desired trans-substituted ester IV-21 by Ferrier-type reaction using silyl ketene acetal (Scheme 5). Treating IV-21 with aqueous $\mathrm{NaOH}$ solution followed by $\mathrm{KI}_{3} / \mathrm{NaHCO}_{3}$ provided iodolactone which was exposed to DBU in THF to give IV-22. With lactone intermediate $\mathbf{I V - 2 2}$ in hand, they proceeded the final construction of aspergillide C. After a hydrolysis, protection/deprotection and macrolactonization sequence, asptergillide C (IV-3) was finally established.<smiles>CCOC(C)CCC/C=C\C1CC=CC(OCC)O1</smiles>

IV-16

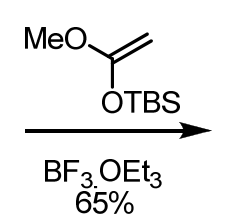

$65 \%$<smiles>COC(C)CCC/C=C\[C@H]1CC=CC(CC(C)=O)O1</smiles>

IV-21<smiles></smiles>

IV-22

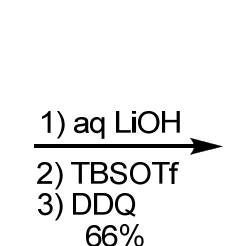

$66 \%$

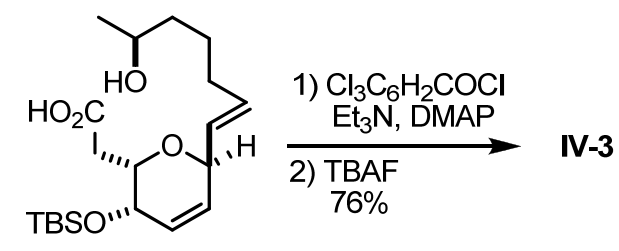

IV-23

Scheme 5. Total synthesis of aspergillide C by Kuwahara

\subsection{Retrosynthetic Analysis of Aspergillides}

Our strategy proposed to link the Noyori reductions of furyl ketone and alkynyl ketone, the alkyne zipper reaction and palladium-catalyzed glycosylation together to construct aspergillides. The challenge of our route is using a novel palladium-catalyzed addition of a vinyl group to install the key trans-pyran ring system. 


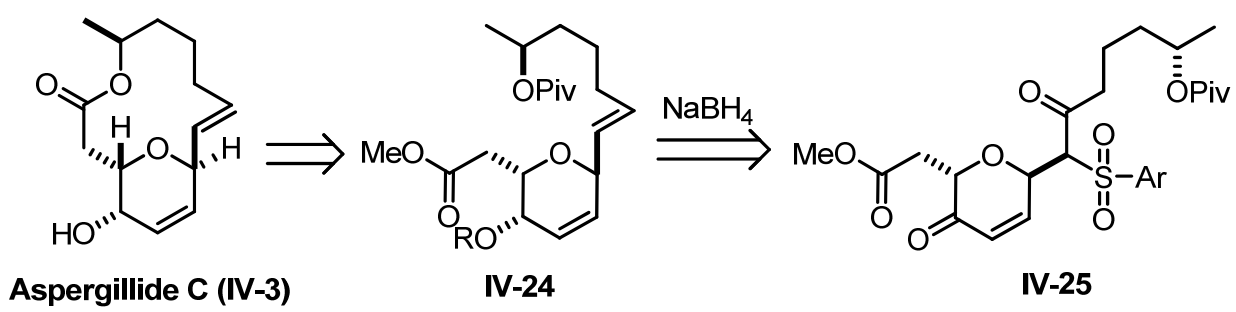

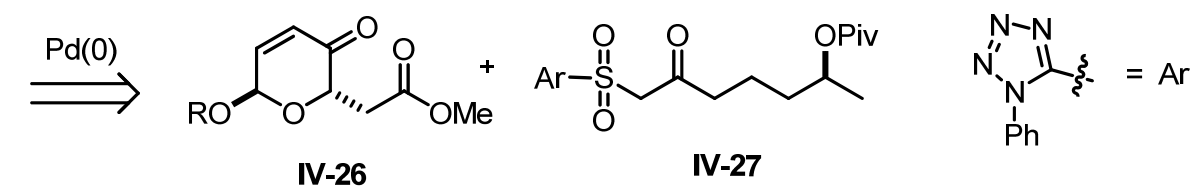

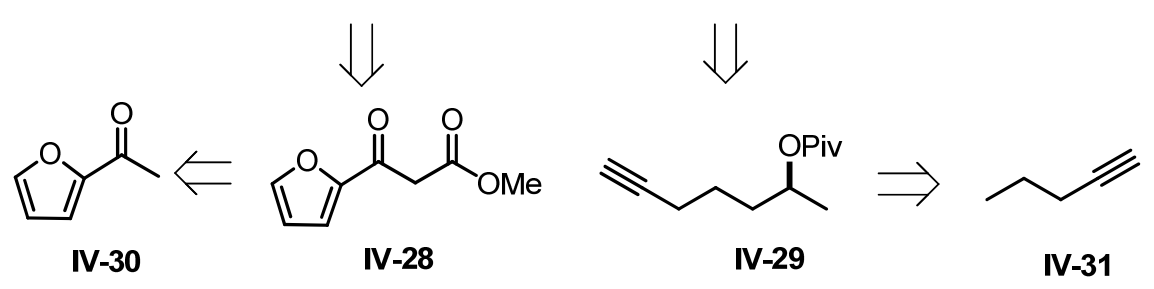

Scheme 6. Retrosynthetic analysis

Retrosynthetically, we envisioned that aspergillide C (IV-3) could be prepared from the macrolactonization of compound IV-24, which could be synthesized from Julie-Kocienski olefination of sulfone precursor $\mathbf{I V - 2 5 .}{ }^{5}$ The key transformation Pd-catalyzed C-glycosylation should allow the coupling of pyranone $\mathbf{I V - 2 6}$ and sulfone IV-27. Pyranone IV-26 could be established from acetyl-furan by three steps. The other coupling partner sulfone IV-27 could be prepared from pivalate protected alkynol IV-29 by Au-catalyzed alkyne hydration ${ }^{6}$ and the following transformations. Alkynol IV-29 could be derived by Noyori reduction and alkyne zipper reaction from non-chiral starting material pentyne IV-31 (Scheme 6).

\subsection{De Novo Approach to Aspergillides}



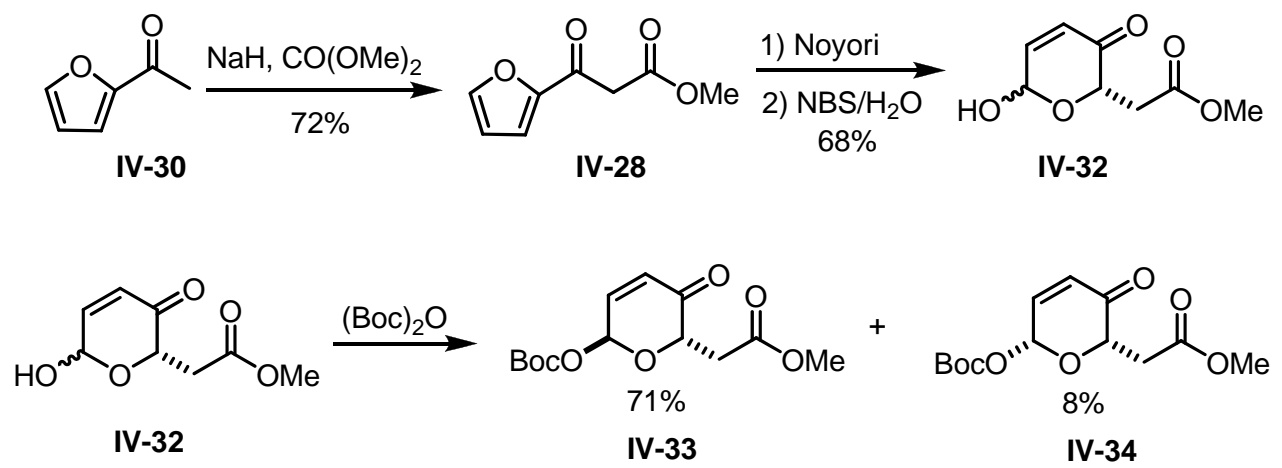

Scheme 7. Synthesis of Boc-pyranone

Taking advantage of our classic three-step Noyori reduction/Achmatowicz reaction/Boc-protection sequence, ${ }^{7}$ we successfully installed $\alpha$-Boc pyranone in $48 \%$ yield (3 steps) from furan IV-28, which was derived from acetyl furan IV-30 by alkylation with dimethyl carbonate (Scheme 7). ${ }^{8}$
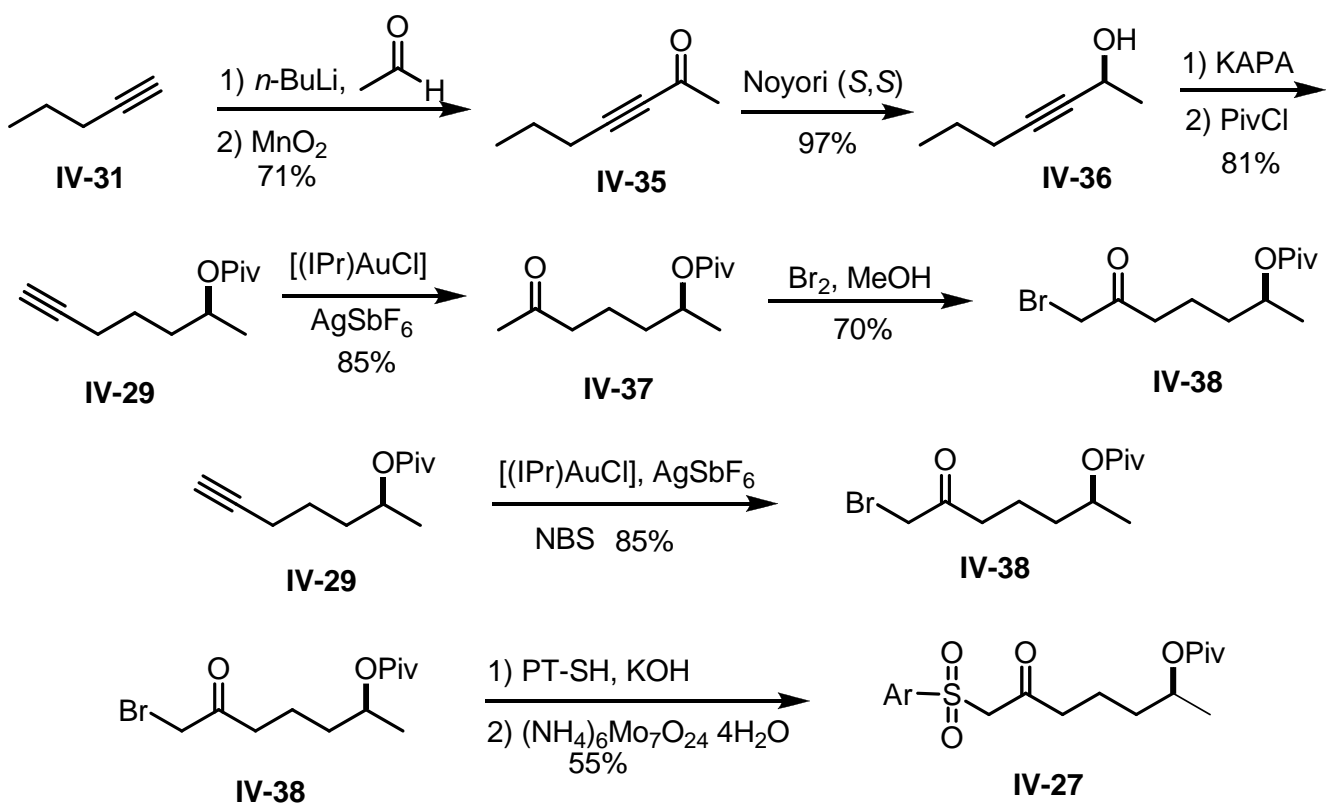<smiles>[Y10][Na]</smiles>

Scheme 8. Synthesis of sulfone precursor

With the pyranone building block IV-33 in hand, we next proceeded to establish the other coupling partner sulfone intermediate IV-27 (Scheme 8). Treating commercially 
available non-chiral starting material 1-pentyne with $n$-BuLi followed by quenching with acetyl aldehyde generated racemic propargyl alcohol. The corresponding propargyl alcohol was oxidized with manganese dioxide to provide propargyl ketone IV-35. Exposure of the propargyl ketone IV-36 with Noyori $(S, S)$ catalyst generated enantioenriched propargyl alcohol IV-36. The propargyl alcohol IV-36 was treated with KAPA reagent to give the terminal alkyne, the secondary alcohol was protected as its pivalate ether IV-29. With the alkynol IV-29 in hand, we next converted the triple bond functional group to a $\alpha$-bromo-ketone. Au-catalyzed alkyne hydration ${ }^{6}$ of pivalate ether IV-29 provided ketone IV-37 in 85\% yield. Treatment of ketone IV-37 with bromine in methanol generated less hindered $\alpha$-bromo-ketone IV-38 as the major product (70\% yield). ${ }^{9}$ Alternatively, one pot reaction of Au-catalyzed hydration and bromination with NBS gave $\alpha$-bromo-ketone $\mathbf{I V}$-38 in $85 \%$ yield. The $\alpha$-bromo-ketone IV-38 was converted to a sulfide by $\mathrm{S}_{\mathrm{N}} 2$ substitution, the resulting sulfide was oxidized by Mo catalyst to provide sulfone intermediate IV-27. 


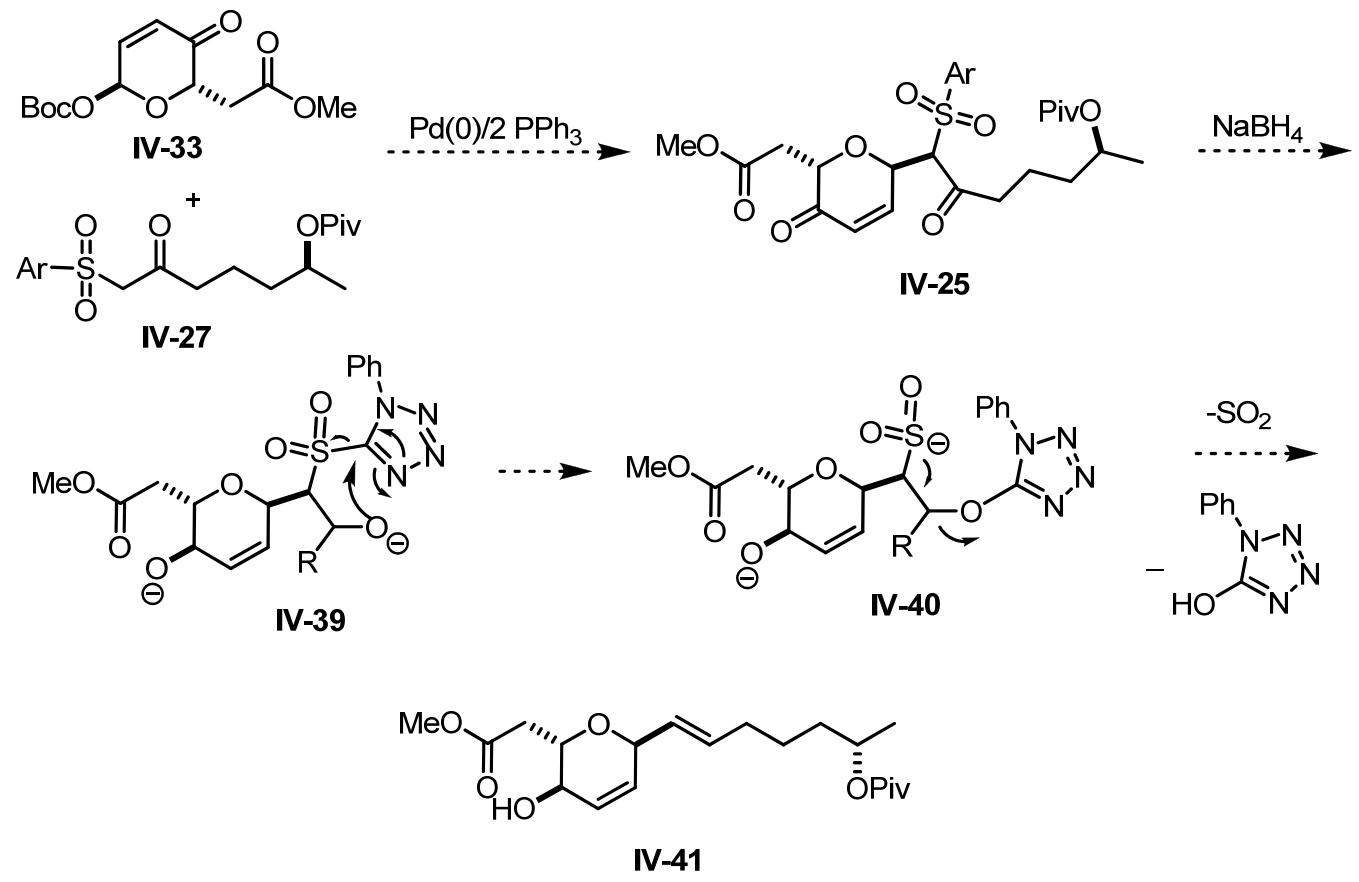

Scheme 9. Proposed Pd-catalyzed C-glycosylation and Julia olefination

With two coupling partners IV-33 and IV-27 prepared, we next investigated Pd-catalyzed glycosylation to link the two precursors. As we desired, treatment of IV-33 and IV-27 with $\mathrm{Pd}_{2}(\mathrm{dba})_{3} \cdot \mathrm{CHCl}_{3}$ and $\mathrm{PPh}_{3}$ could give us the $C$-glycosylation product IV-25, which is a mixture of the two diastereomers. $\mathrm{NaBH}_{4}$ reduction of $\mathbf{I V - 2 5}$ could reduce two ketones and the resulting alcohol next to sufone would eliminate to give E-olefin IV-41 as the major product (Scheme 9). ${ }^{5}$ 
<smiles>COC(=O)CC1OC(OC(C)(C)C)C=CC1=O</smiles><smiles>COC(=O)C[C@H]1O[C@@H](O)C=CC1=O</smiles>

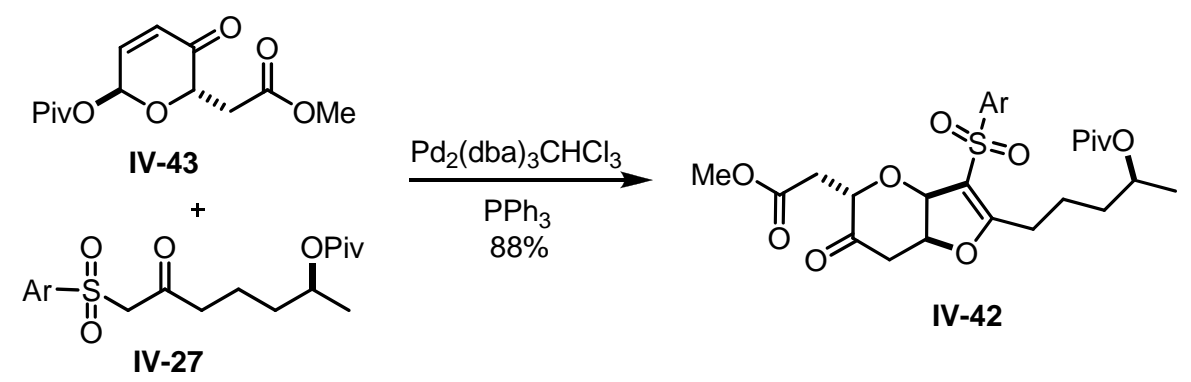

Scheme 10. Double addition

In practice, when we treated $\alpha$-Boc-pyranone IV-33 and sulfone IV-27 with $\mathrm{Pd}_{2}(\mathrm{dba})_{3} \cdot \mathrm{CHCl}_{3}$ and $\mathrm{PPh}_{3}$, it actually gave us the Michael addition product IV-42 (Scheme 10). We thought maybe Boc protecting group provided too much basic condition in the glycosylation reaction, therefore the double addition occured. Thus, we made $\alpha$-Piv-pyranone IV-43 to undergo the glycosylation reaction. However, similar result was observed from the coupling of $\alpha$-Piv-pyranone IV-43 and sulfone IV-27, we still received the double addition product IV-42. The mechanism of this double addition is shown in scheme 11. After the coupling of pyranone IV-26 and sulfone IV-27, a further 1, 4 addition took place to generate the double addition product IV-42. 


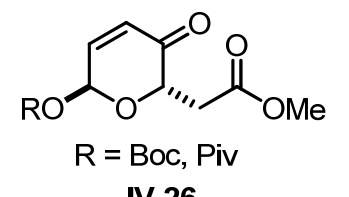<smiles>CC(CCCC(=[OH+])CS(=O)(=O)[AlH2])O[Na]</smiles><smiles>COC(=O)C[C@H]1OC2C(S(=O)(=O)[Ge])=C(CCCC(C)OP)O[C@H]2CC1=O</smiles><smiles>[Te]C1CC1</smiles>

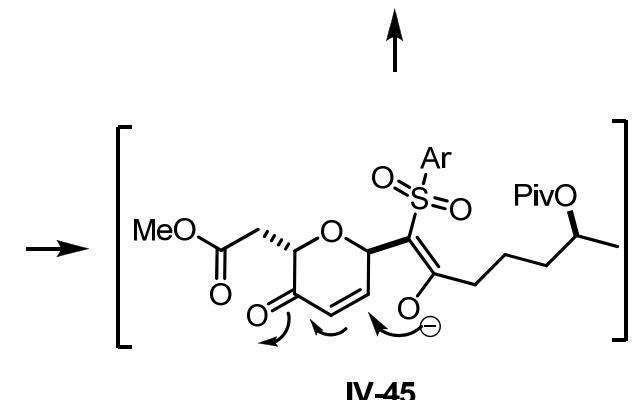

IV-25

IV-45

Scheme 11. Mechanism of double addition

With the double addition product IV-42 in hand, we thought if we treat it with some base, the glycosylation product $\mathbf{I V - 2 5}$ would be reformed. However, we could not find a suitable base to do this transformation. In addition, we planned to reduce the double bond of the double addition product IV-42 to provide compound IV-46 which could be converted to $E$ olefine IV-47 when treated with some base. Unfortunately, many attempts of the hydrogenation of IV-42 failed (Scheme 12). 


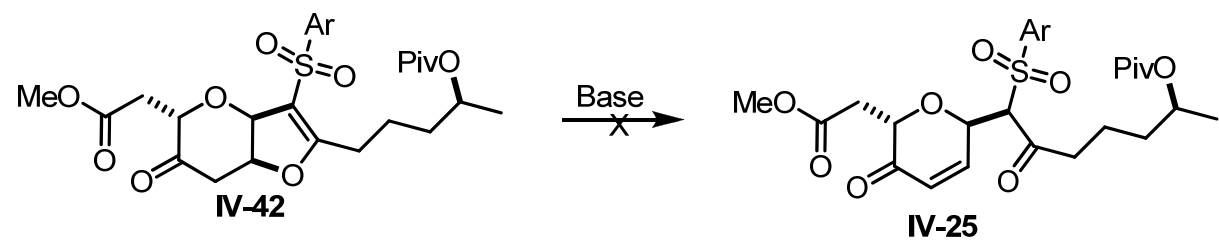

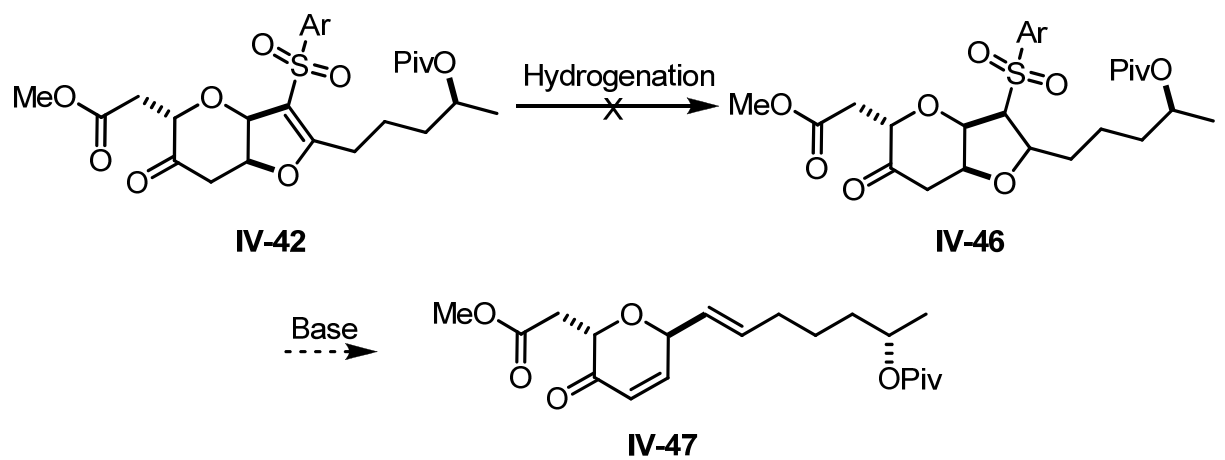

Scheme 12. Attempt to correct double addition product

Consequently, we decided to avoid the double addition by diastereoselectivly reducing the $\alpha, \beta$ unsaturated ketone $\mathbf{I V}-\mathbf{3 3}$ to an equatorial allylic alcohol $\mathbf{I V - 4 8}$ using $\mathrm{NaBH}_{4}$. When we utilized our Pd-catalyzed glycosylation reaction followed by one-pot reduction with $\mathrm{NaBH}_{4}$ without isolation of the coupling product, we accessed the mixture of trans/cis double bond isomers of $\mathbf{I V - 4 1}$ and IV-49. After careful chromatography separation, E-olefine IV-41 was collected in 26\% yield (Scheme 13).

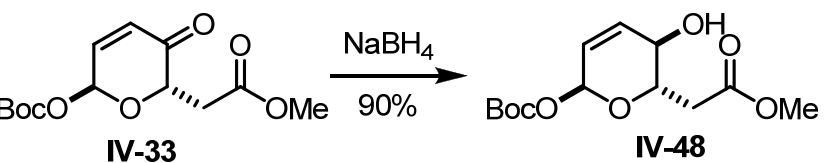

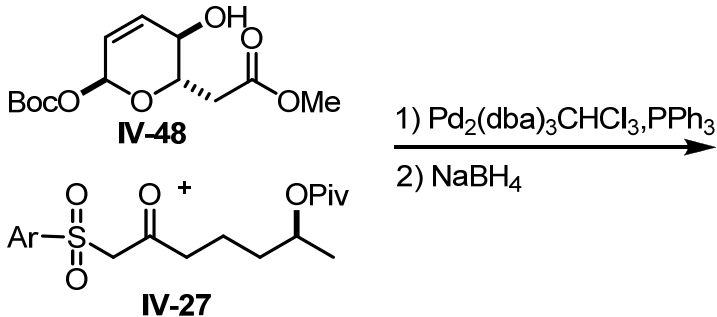

IV-27

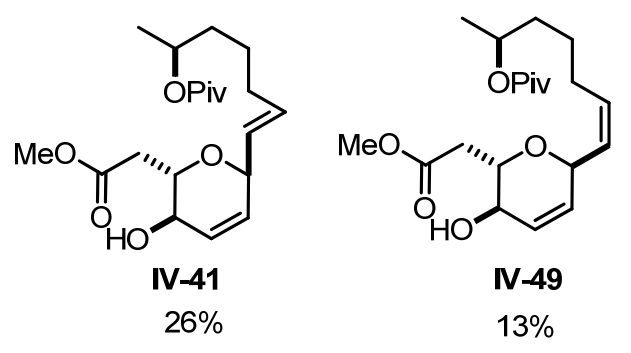

$13 \%$

Scheme 13. Glycosylation of equatorial allylic alcohol with sulfone 
We were delighted that the E-olefine IV-41 had been established by our glycosylation reaction, but both the yield and selectivity needed to be improved. Thus, we tried many different substitutes for the glycosylation reactions. Firstly, we protected the allylic alcohol IV-48 as a TBS ether IV-50, however, the Pd-catalyzed glycosylation of the TBS ether IV-50 and the sulfone IV-27 failed (Scheme 14), and we could only isolate the unknown side products.

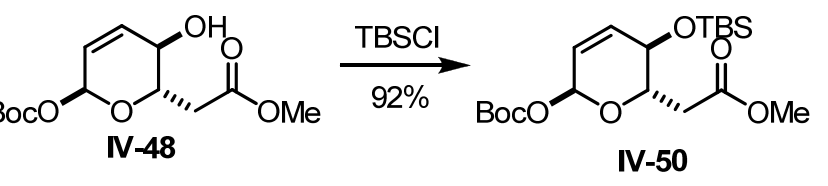<smiles>COC(=O)CC1OC(O[18O])C=CC1[O-]</smiles>

Scheme 14. Glycosylation of TBS ether of the equatorial allylic alcohol with sulfone
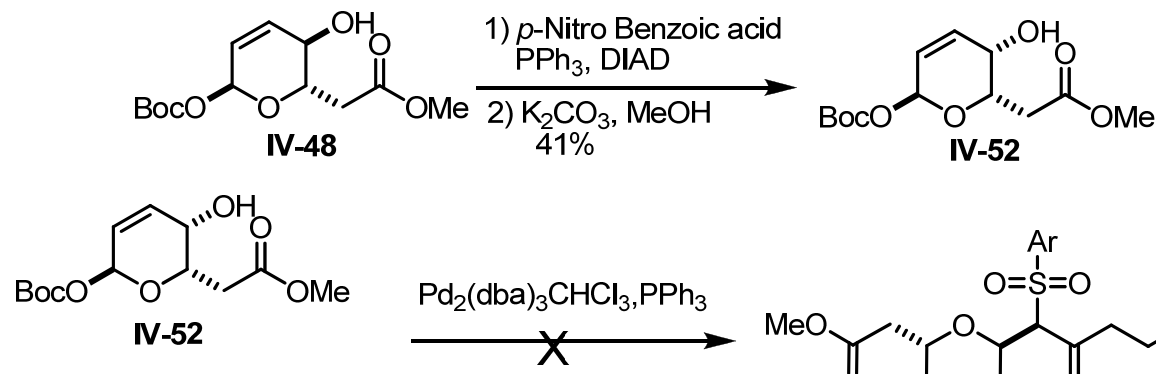<smiles></smiles><smiles>COC(=O)C[C@H]1O[C@H](C(=O)CCCC(C)O[Na])C=C[C@@H]1O</smiles>

Scheme 15. Glycosylation of axial allylic alcohol with sulfone 

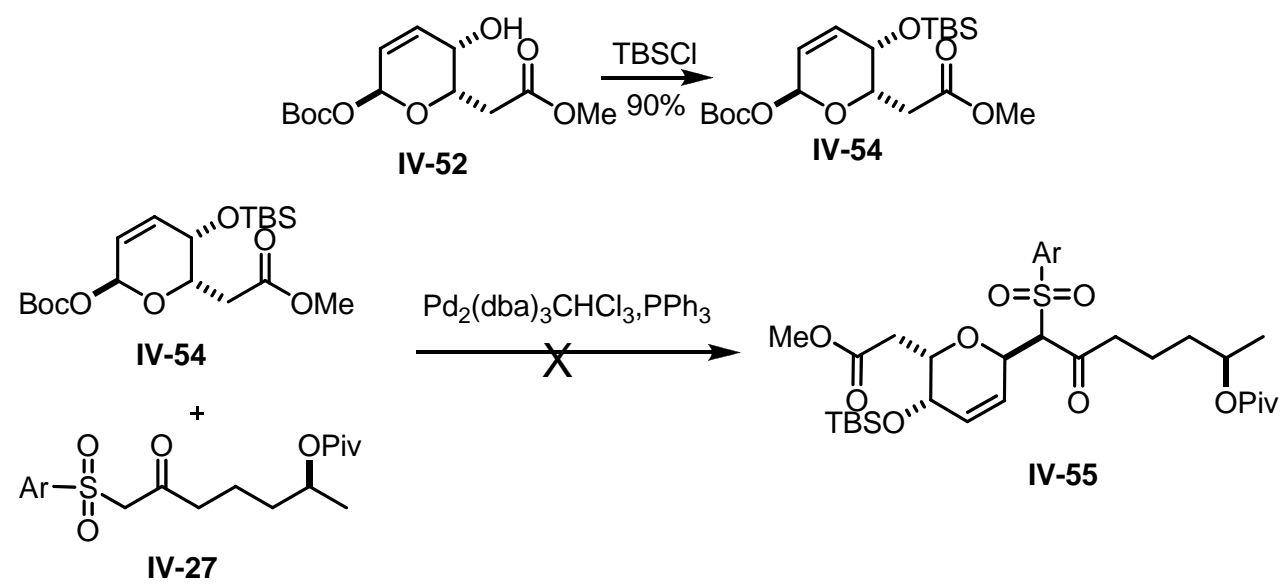

Scheme 16. Glycosylation of TBS ether of the axial allylic alcohol with sulfone

Secondly, because the stereochemistry of the allylic alcohol IV-48 was opposite with the natural product aspergillides, we reversed the stereochemistry by Mitsunobu reaction to generate axial allylic alcohol IV-52 (Scheme 15). Unfortunately, no product can be isolated from the glycosylation reaction to couple IV-52 and IV-27. Consequently, we tried the glycosylation reaction on the TBS protected allylic alcohol IV-54, however, no coupling reaction occurred (Scheme 16).
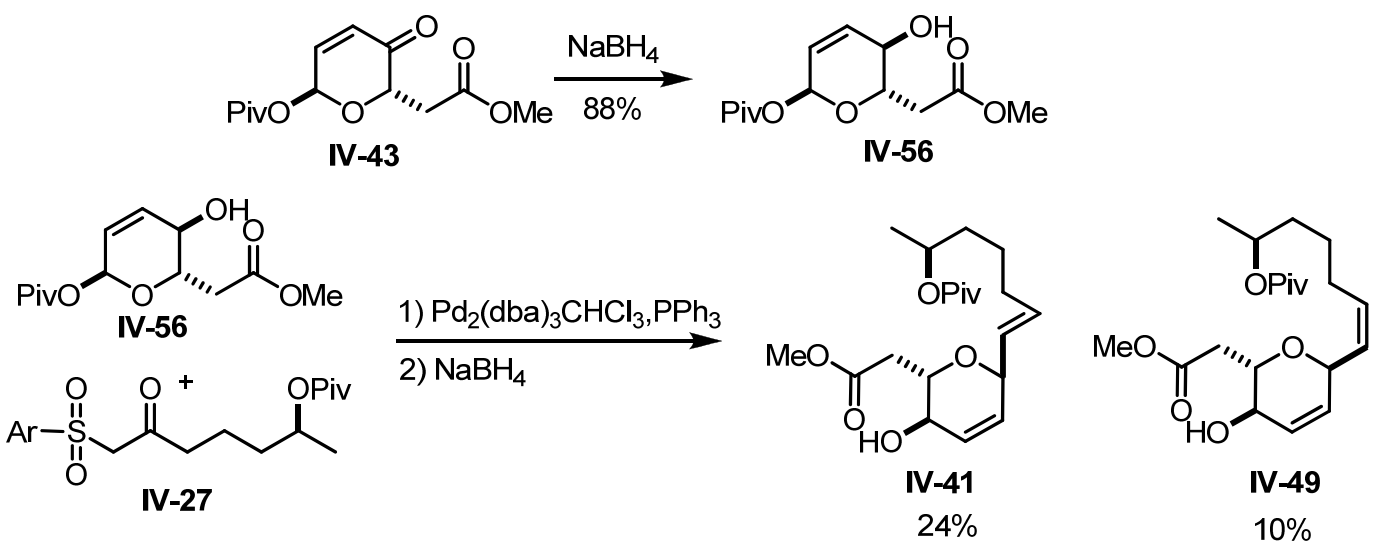

Scheme 17. Glycosylation of pivalate equatorial allylic alcohol with sulfone 

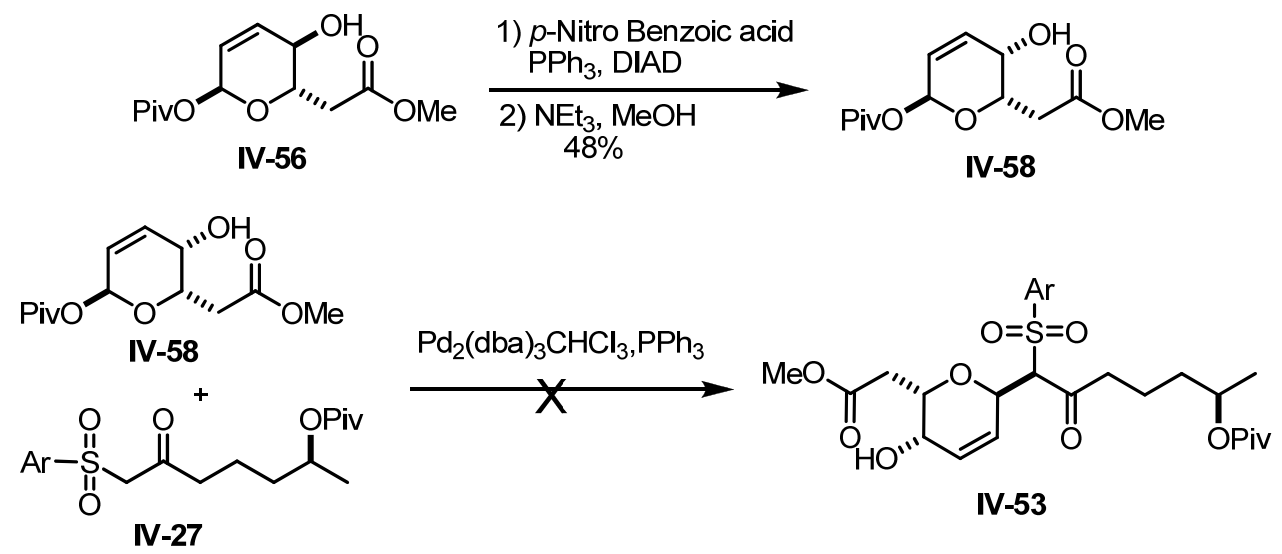

Scheme 18. Glycosylation of pivalate axial allylic alcohol with sulfone

Finally, we prepared pivalate protected allylic alchol IV-56 and IV-58, when we treated these two diastereomers with Pd-catalyzed glycosylation condition, IV-56 gave us the similar result as $\mathbf{I V - 4 8}$, however, we still could not could IV-58 with the sulfone under this condition, only starting materials were recovered.

\subsection{Conclusions}

In summary, a de novo asymmetric total synthesis of aspergilliges has been investigated. To date, intermediate IV-41 which is five steps away from a formal synthesis $^{4 a}$ (compound IV-23) of aspergillide C (IV-3) has been prepared by Pd-catalyzed C-glycosylation and the following Julia olefination. Noyori reductions of furyl and propargyl ketone, alkyne zipper reaction and Au-catalyzed alkyne hydration have been utilized.

\section{Reference:}


${ }^{1}$ K. Kito, R. Ookura, S. Yoshida, M. Namikoshi, T. Ooi, T. Kusumi, Org. Lett. 2008, 10, 225-228.

${ }^{2}$ Syntheses of aspergillide A: a) T. Nagasawa, S. Kuwahara,Tetrahedron Lett. 2010, 51, 875-877; b) S. Diaz-Oltra, C. A. Angulo-Pachon, J. Murga, M. Carda, J. A. Marco, J. Org. Chem. 2010, 75, 1775-1778; c) H. Fuwa, H. Yamaguchi, M. Sasaki, Org. Lett. 2010, 12, 1848-1851; H. Fuwa, H. Yamaguchi, M. Sasaki, Tetrahedron 2010, 66, 7492-7503; d) G. Sabitha, D. V. Reddy, A. S. Rao, J. S. Yadav, Tetrahedron Lett. 2010, 51, 4195-4198; e) M. Kanematsu, M. Yoshida, K, Shishido, Angew. Chem. Int. Ed. 2011, 50, 1-4.

${ }^{3}$ Syntheses of aspergillide B: a) S. M. Hande, J. Uenishi, Tetrahedron Lett. 2009, 50, 189-192; b) S. Diaz-Oltra, C. A. Angulo-Pachon, M. N. Kneeteman, J. Murga, M. Carda, J. A. Marco, Tetrahedron Lett. 2009, 50, 3783-3785; c) T. Nagasawa, S. Kuwahara, Biosci. Biotechnol. Biochem. 2009, 73, 1893-1894; d) J. Liu, K. Xu, J. He, L. Zhang, X. Pan, X. She, J. Org. Chem. 2009, 74, 5063-5066; e) A. J. Mueller, M. P. Jennings, Tetrahedron Lett. 2010, 51, 4260-4262.

${ }^{4}$ Syntheses of aspergillide C: a) T. Nagasawa, S. Kuwahara, Org. Lett. 2009, 11, 761-764; b) J. D. Panarese, S. P.Waters, Org. Lett. 2009, 11, 5086-5088.

${ }^{5}$ M. Nielsen, C. B. Jacobsen, M.W. Paixao, N. Holub, K. Jorgensen, J. Am. Chem. Soc. 2009, 131, 10581-10586.

${ }^{6}$ a) N. Marion, R. S. Ramon, S. P. Nolan, J. Am, Chem. Soc. 2009, 131, 448-449. b) Fremont, N. M. Scott, E. D. Stevens, S. P. Nolan, Organometallics, 2005, 24, 2411-2418. 
7 a) Fujii, A.; Hashiguchi, S.; Uematsu, N.; Ikariya, T.; Noyori, R. J. Am. Chem. Soc. 1996, 118, 2521-2522. b) Noyori, R.; Ohkuma, T. Angew. Chem. Int. Ed. 2001, 40, 40-73. C) Li, M.; Scott, J. G.; O'Doherty, G. A. Tetrahedron Lett. 2004, 45, 1005-1009. d) Achmatowicz, O.; Bielski, R. Carbohydr. Res. 1977, 55, 165-176. e) Balachari, D.; O’Doherty, G. A. Org. Lett. 2000, 2, 863-866. f) Balachari, D.; O’Doherty, G. A. Org. Lett. 2000, 2, 4033-4036.

${ }^{8}$ Organic Synthesis, Coll. Vol. 5, p. 198(1973)

${ }^{9}$ H. Ha, S. Lee, Y. Ha. J. Park, Synthetic Communications, 1994, 24, 2557-2562 


\section{Experimental Section}

\section{Section A: General Information}

General Methods and materials: ${ }^{1} \mathrm{H}$ and ${ }^{13} \mathrm{C}$ spectra were recorded on 270 and 600 spectrometers. Chemical shifts were reported relative to internal tetramethylsilane $(\delta 0.00$ ppm) or $\mathrm{CDCl}_{3}(\delta 7.26 \mathrm{ppm})$ or $\mathrm{CD}_{3} \mathrm{OD}(\delta 4.89 \mathrm{ppm})$ for ${ }^{1} \mathrm{H}$ and $\mathrm{CDCl}_{3}(\delta 77.1 \mathrm{ppm})$ or $\mathrm{CD}_{3} \mathrm{OD}(\delta 49.15 \mathrm{ppm})$ for ${ }^{13} \mathrm{C}$. Optical rotations were measured with a digital polarimeter in the solvent specified. Infrared (IR) spectra were obtained on a FT-IR spectrometer. Flash column chromatography was performed on ICN reagent 60 (60-200 mesh) silica gel. Analytical thin-layer chromatography was performed with precoated glass-backed plates (K6F $\left.60 \AA, F_{254}\right)$ and visualized by quenching of fluorescence and by charring after treatment with $p$-anisaldehyde or phosphomolybdic acid or potassium permanganate stain. $R_{f}$ values were obtained by elution in the stated solvent ratios $(\mathrm{v} / \mathrm{v})$. Ether, THF, methylene chloride and triethylamine were dried by passing through activated alumina ( 8 x 14 mesh) column with nitrogen gas pressure. Commercial reagents were used without purification unless otherwise noted. Air and/or moisture-sensitive reactions were carried out under an atmosphere of argon/nitrogen using oven/flamed-dried glassware and standard syringe/septa techniques. 


\section{Section B: Experimental Procedures}

\section{(S)-1-(furan-2-yl)ethanol (I-10) ${ }^{1}$}<smiles>CC(O)c1ccco1</smiles>

To a solution of acylfuran I-7 $(15 \mathrm{~g}, 136.4 \mathrm{mmol})$ in $\mathrm{CH}_{2} \mathrm{Cl}_{2}(20 \mathrm{~mL})$ was added a prepared solution of formic acid/triethylamine $(40 \mathrm{~mL}, 2: 1(\mathrm{~mol} / \mathrm{mol}))$ and Noyori asymmetric transfer hydrogenation catalyst $(R)-\mathrm{Ru}\left(\eta^{6}\right.$-mesitylene $)-(S, S)-\mathrm{TsDPEN}(0.2 \mathrm{~g}$, $0.25 \mathrm{~mol} \%$ ). The resulting solution was stirred at room temperature for $24 \mathrm{~h}$. Then it was diluted with water $(90 \mathrm{~mL})$ and extracted with $\mathrm{Et}_{2} \mathrm{O}(200 \mathrm{~mL} \times 3)$. The combined organic layer was washed with saturated aqueous $\mathrm{NaHCO}_{3}(50 \mathrm{~mL})$ saturated brine $(50 \mathrm{~mL})$, dried over $\mathrm{Na}_{2} \mathrm{SO}_{4}$ and then concentrated under reduced pressure to give a residue. Flash chromatography on silica gel eluting with hexane/ $\mathrm{Et}_{2} \mathrm{O}(1: 1, \mathrm{v} / \mathrm{v})$ gave furfuryl alcohol I$10(14 \mathrm{~g}, 92 \%)$ as colorless oil: $R f 0.45(7: 3(\mathrm{v} / \mathrm{v})$ hexane/EtOAc $) ;[\alpha]^{25}{ }_{\mathrm{D}}=-20.1(\mathrm{c} 1.0$, $\mathrm{CH} 2 \mathrm{Cl} 2) ;{ }^{1} \mathrm{H} \mathrm{NMR}\left(600 \mathrm{MHz} \mathrm{CDCl}_{3}\right) \delta 7.30(\mathrm{~d}, J=1.8,1 \mathrm{H}), 6.26(\mathrm{dd}, J=3.0,1.8 \mathrm{~Hz}$, $1 \mathrm{H}), 6.15(\mathrm{~d}, J=3.0,1 \mathrm{H}), 4.78(\mathrm{dq}, J=6.6,6.6 \mathrm{~Hz}, 1 \mathrm{H}), 3.11(\mathrm{~s}, 1 \mathrm{H}), 1.46(\mathrm{~d}, J=6.6 \mathrm{~Hz}$, $3 \mathrm{H}) ;{ }^{13} \mathrm{C}$ NMR $\left(150 \mathrm{MHz}, \mathrm{CDCl}_{3}\right) \delta 157.7,141.6,109.9,104.9,63.3,21.1$.

\footnotetext{
${ }^{1} 1$ (a) Shan, M., O’Doherty, G. A. Org. Lett. 2006, 8, 5149-5152. (b) Guo, H.; O’Doherty, G. A. Org. Lett. 2005, 7, 3921-3924. (c) Li, M.; Scott, J. G.; O’Doherty, G. A. Tetrahedron Lett. 2004, 45, 1005 1009.
} 
(2S, 6R)-6-hydroxy-2-methyl-2H-pyran-3(6H)-one (I-13) ${ }^{1}$<smiles>C[C@H]1OC(O)C=CC1=O</smiles>

To a solution of furfuryl alcohol I-10 (14 g, $125 \mathrm{mmol})$ in THF/ $\mathrm{H}_{2} \mathrm{O}(416 \mathrm{~mL}, 3: 1(\mathrm{v} / \mathrm{v}))$ was added $\mathrm{NaHCO}_{3}(21 \mathrm{~g}, 250 \mathrm{mmol}), \mathrm{NaOAc} \cdot 3 \mathrm{H}_{2} \mathrm{O}(17 \mathrm{~g}, 125 \mathrm{mmol})$, and NBS (22.3 g, $125 \mathrm{mmol}$ ) at $0{ }^{\circ} \mathrm{C}$. The reaction mixture was kept stirring at this temperature for 1 hour, then at $0{ }^{\circ} \mathrm{C}$ saturated aqueous $\mathrm{NaHCO}_{3}(200 \mathrm{~mL})$ was added to quench the reaction. The reaction mixture was directly extracted with $\mathrm{Et}_{2} \mathrm{O}(300 \mathrm{~mL} \times 3)$ and the combined organic layer was washed by saturated brine $(100 \mathrm{~mL})$, dried over $\mathrm{Na}_{2} \mathrm{SO}_{4}$ and then concentrated under reduced pressure to give a residue, which was rapidly subjected to flash chromatography on silica gel. Elution with hexane/EtOAc $(1: 1, \mathrm{v} / \mathrm{v})$ afforded diastereomeric mixture of pyranone alcohol I-13 (14.4 g, 90\%, $\alpha: \beta=2.8: 1)$ : White solid; $R f 0.25\left(7: 3(\mathrm{v} / \mathrm{v})\right.$ hexane/EtOAc); ${ }^{1} \mathrm{H}$ NMR $(600 \mathrm{MHz}, \mathrm{CDCl} 3, \alpha$ isomer $) \delta 6.82(\mathrm{dd}, J=$ 10.2, $3.0 \mathrm{~Hz}, 1 \mathrm{H}), 5.96(\mathrm{~d}, J=10.2,1 \mathrm{H}), 5.48(\mathrm{~d}, J=3.0 \mathrm{~Hz}, 1 \mathrm{H}), 3.99(\mathrm{q}, J=7.2 \mathrm{~Hz}$, 1H), $1.23(\mathrm{~d}, J=7.2 \mathrm{~Hz}, 3 \mathrm{H}) ;{ }^{13} \mathrm{C} \mathrm{NMR}(150 \mathrm{MHz}, \mathrm{CDCl} 3, \alpha$ isomer $) \delta 197.6,145.3$, $126.6,87.2,74.8,15.1$.

tert-Butyl (2S,6S)-6-methyl-5-oxo-5,6-dihydro-2H-pyran-2-yl carbonate (I-5)) ${ }^{1}$<smiles>CC(=O)OC1C=CC(=O)[C@@H](C)O1</smiles> 
To a solution of pyranone alcohol $\mathbf{I}-13(7.4 \mathrm{~g}, 57.8 \mathrm{mmol})$ in $\mathrm{CH}_{2} \mathrm{Cl}_{2}(80 \mathrm{~mL})$ was added DMAP (528 mg, $4.33 \mathrm{mmol})$ at $-78{ }^{\circ} \mathrm{C}$. A pre-cooled solution of $(\mathrm{Boc})_{2} \mathrm{O}(25.2 \mathrm{~g}, 115.6$ mmol) in $\mathrm{CH}_{2} \mathrm{Cl}_{2}(30 \mathrm{~mL})$ was added dropwise to the reaction mixture via a cannula. The reaction mixture was stirred at $-78{ }^{\circ} \mathrm{C}$ for 12 hours. The reaction was quenched by saturated aqueous $\mathrm{NaHCO}_{3}(100 \mathrm{~mL})$ and then extracted with $\mathrm{Et}_{2} \mathrm{O}(300 \mathrm{~mL} \times 3)$. The combined organic layers was washed by saturated brine $(70 \mathrm{~mL})$, dried over $\mathrm{Na}_{2} \mathrm{SO}_{4}$ and concentrated under reduced pressure to give a residue. Flash chromatography on silica gel eluting with hexane/Et ${ }_{2} \mathrm{O}(100: 7, \mathrm{v} / \mathrm{v})$ gave $\alpha$-Boc-pyranone I-5 (7.8 g, 60\%). Elution with hexane/Et ${ }_{2} \mathrm{O}(10: 1, \mathrm{v} / \mathrm{v})$ gave $\beta$-Boc-pyranone (2.5g, 19\%). $\alpha$-Boc-pyranone 15( $\alpha$-L): $R f=0.60\left(7: 3(\mathrm{v} / \mathrm{v})\right.$ hexane/EtOAc); $[\alpha]_{\mathrm{D}}^{25}=+98(c 1.0, \mathrm{CH} 2 \mathrm{Cl} 2) ; \mathrm{IR}($ thin film, $\mathrm{cm}-1) v$ 2986, 1752, 1703, 1633, 1278, 1258, 1159, 1090, 1058, 1029, 944. ${ }^{1} \mathrm{H}$ NMR (600 MHz, CDCl3) $\delta 6.78(\mathrm{dd}, J=10.2,3.6 \mathrm{~Hz}, 1 \mathrm{H}), 6.22(\mathrm{~d}, J=3.6 \mathrm{~Hz}, 1 \mathrm{H}), 6.09(\mathrm{~d}, J=10.2 \mathrm{~Hz}$

$1 \mathrm{H}), 4.53(\mathrm{q}, J=6.6 \mathrm{~Hz}, 1 \mathrm{H}), 1.40(\mathrm{~s}, 9 \mathrm{H}), 1.28(\mathrm{~d}, J=6.6 \mathrm{~Hz}, 3 \mathrm{H}) ;{ }^{13} \mathrm{C}$ NMR $(150.8$ $\mathrm{MHz}, \mathrm{CDCl} 3) \delta 195.5,151.7,140.9,128.2,89.1,83.3,72.0,27.5,15.1$; HRMS (CI): Calculated for $\left[\mathrm{C}_{11} \mathrm{H}_{16} \mathrm{O}_{5}+\mathrm{Na}\right]^{+}$251.0890, Found 251.0883.

1-(tert-butyldimethylsilyloxy)-pen-4-yne (I-11a): ${ }^{2}$

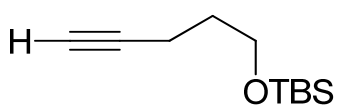

${ }^{2}$ H. Guo; O’Doherty, G.A. Org. Lett. 2005; 7(18); 3921-3924. 
To a magnetically stirred solution of 4-pentynol I-11 (5.0 g, $59.4 \mathrm{mmol})$ and imidazole (10.1 g, $148.6 \mathrm{mmol})$ in dry DMF $(100 \mathrm{~mL})$, was added dropwise tert-butyldimethylsilyl chloride $(9.4 \mathrm{~g}, 62.4 \mathrm{mmol})$ in $\mathrm{DMF}(50 \mathrm{~mL})$ over $10 \mathrm{~min}$ at $0{ }^{\circ} \mathrm{C}$ under an atmosphere of nitrogen. The reaction mixture was warmed to room temperature and stirred overnight. Water $(300 \mathrm{~mL})$ was added and then the reaction mixture was extracted with $\mathrm{Et}_{2} \mathrm{O}(3 \mathrm{x}$ $300 \mathrm{~mL})$, dried $\left(\mathrm{Na}_{2} \mathrm{SO}_{4}\right)$, and evaporated under reduced pressure to leave a light yellow oil. The crude product was purified using silica gel flash chromatography eluting with $5 \%$ EtOAc/hexane to give TBS-ether I-11a $(11.8 \mathrm{~g}, 147.1,99 \%)$ as a colorless oil; $R_{f}=0.89$ (30\% EtOAc/hexane); IR (thin film, $\mathrm{cm}^{-1}$ ) 3314, 2954, 2930, 2858, 1473, 1256, 1106, 835, 776; ${ }^{1} \mathrm{H}$ NMR $\left(600 \mathrm{MHz}, \mathrm{CDCl}_{3}\right) \delta 3.68(\mathrm{t}, J=6.6 \mathrm{~Hz}, 2 \mathrm{H}), 2.26(\mathrm{td}, J=7.2,2.4$ $\mathrm{Hz}, 2 \mathrm{H}), 1.90(\mathrm{t}, J=2.4,1 \mathrm{H}), 1.71(\mathrm{tt}, J=7.2,6.6 \mathrm{~Hz}, 2 \mathrm{H}), 0.89(\mathrm{~s}, 9 \mathrm{H}), 0.05(\mathrm{~s}, 6 \mathrm{H}) ;{ }^{13} \mathrm{C}$ $\operatorname{NMR}\left(150 \mathrm{MHz}, \mathrm{CDCl}_{3}\right) \delta 84.3,68.3,61.5,31.6,26.0,18.4,14.9,-5.3$.

\section{(+/-)-7-(tert-butyldimethylsilyloxy)-hept-3-yne-2-ol (I-11b): ${ }^{2}$}

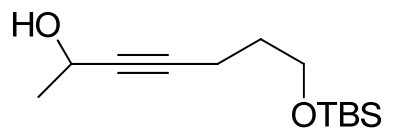

A solution of $\mathrm{BuLi}$ in hexane $(24.71 \mathrm{~mL}, 59.3 \mathrm{mmol})$ was added dropwise to the magnetically stirred solution of TBS-ether I-11a $(11.8 \mathrm{~g}, 59.3 \mathrm{mmol})$ in dry THF (59.3 $\mathrm{mL})$ at $-78{ }^{\circ} \mathrm{C}$ under the atmosphere of argon. After $30 \mathrm{~min}$, acetaldehyde $(1.54 \mathrm{~g}, 35.1$ mmol) was added and stirred for $10 \mathrm{~min}$ at $0{ }^{\circ} \mathrm{C}$ and then the resulting solution was warmed to room temperature for $50 \mathrm{~min}$. $100 \mathrm{~mL}$ of $\mathrm{CH}_{2} \mathrm{Cl}_{2}$ was added to the reaction 
mixture followed by addition of $100 \mathrm{~mL}$ of $\mathrm{pH}=7.0$ phosphate buffer. The aqueous phase was separated and extracted with $\mathrm{CH}_{2} \mathrm{Cl}_{2}(3 \times 100 \mathrm{~mL})$. The combined organic phase was dried $\left(\mathrm{Na}_{2} \mathrm{SO}_{4}\right)$ and concentrated under reduced pressure. The crude product was purified using silica gel flash chromatography eluting with $6 \%$ EtOAc/hexane to give propargyl alcohol I-11b $(8.4 \mathrm{~g}, 34.6 \mathrm{mmol}, 98 \%)$ : light yellow oil; $R_{f}=0.42(20 \%$ EtOAc/hexane); IR (thin film, $\mathrm{cm}^{-1}$ ), 3364, 2953, 2930, 2858, 1740, 1472, 1254, 1103, $1071,835,775 ;{ }^{1} \mathrm{H}$ NMR $\left(600 \mathrm{MHz} \mathrm{CDCl}_{3}\right) \delta 4.65(\mathrm{qd}, J=6.6,1.8 \mathrm{~Hz}, 1 \mathrm{H}), 3.64(\mathrm{t}, J=$ $6.0 \mathrm{~Hz}, 2 \mathrm{H}), 2.24(\mathrm{td}, J=7.2,1.8 \mathrm{~Hz}, 2 \mathrm{H}), 1.66(\mathrm{tt}, J=7.2,6.6 \mathrm{~Hz}, 2 \mathrm{H}), 1.38(\mathrm{~d}, J=6.6$ $\mathrm{Hz}, 3 \mathrm{H}), 0.86$ (s, 9H), $0.02(\mathrm{~s}, 6 \mathrm{H}) ;{ }^{13} \mathrm{C} \mathrm{NMR}\left(150 \mathrm{MHz}, \mathrm{CDCl}_{3}\right) \delta 84.0,82.5,61.6,58.4$, 31.7, 25.9, 24.7, 18.3, 15.1, -5.0; CIHRMS: Calculated for $\left[\mathrm{C}_{13} \mathrm{H}_{26} \mathrm{O}_{2} \mathrm{SiNa}^{+}\right]$: 265.1594, found: 265.1630 .

\section{7-(tert-butyldimethylsilyloxy)-hept-3-yne-2-one (I-8): ${ }^{2}$}

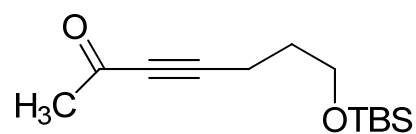

To a solution of alcohol I-11b ( $2 \mathrm{~g}, 8.23 \mathrm{mmol})$ in THF $(82.3 \mathrm{~mL})$, was added activated $\mathrm{MnO}_{2}(10 \mathrm{~g})$ and left to stir for $30 \mathrm{~h}$, the reaction mixture was filtered and concentrated under reduced pressure. The crude product was purified by silica gel flash chromatography eluting with 5\% EtOAc/hexane to yield propargyl ketone I-8 (1.96 g, $8.13 \mathrm{~mol}, 98 \%): R_{f}=0.85\left(30 \%\right.$ EtOAc/hexane); IR (thin film, $\left.\mathrm{cm}^{-1}\right) 2955,2930,2858$, $2210,1678,1472,1226,1103,966,8345,775 ;{ }^{1} \mathrm{H}$ NMR $\left(600 \mathrm{MHz}, \mathrm{CDCl}_{3}\right) \delta 3.64(\mathrm{t}, J=$ $6.0 \mathrm{~Hz}, 2 \mathrm{H}), 2.40(\mathrm{t}, J=7.2 \mathrm{~Hz}, 2 \mathrm{H}), 2.25(\mathrm{~s}, 3 \mathrm{H}), 1.71(\mathrm{tt}, J=7.2,6.0 \mathrm{~Hz}, 2 \mathrm{H}), 0.84(\mathrm{~s}$, 
9H), $0.02(\mathrm{~s}, J=0.05 \mathrm{~Hz}, 6 \mathrm{H}) ;{ }^{13} \mathrm{C} \mathrm{NMR}\left(150 \mathrm{MHz}, \mathrm{CDCl}_{3}\right) \delta 184.5,93.5,81.4,61.1$, $32.6,30.7,25.8,18.2,15.3,-5.4$.

(R)-7-(tert-butyldimethylsilyloxy)-hept-3-yne-2-ol (I-12): ${ }^{2}$

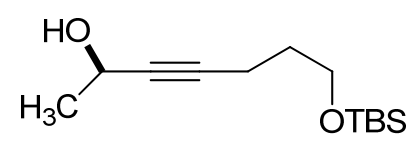

To a $20 \mathrm{~mL}$ flask was added propargyl ketone I-8 (704 mg, $2.92 \mathrm{mmol}), \mathrm{CH}_{2} \mathrm{Cl}_{2}(1.48$ $\mathrm{mL}$ ), formic acid/triethylamine $(1: 1,5.38 \mathrm{~mL})$ and Noyori asymmetric transfer hydrogenation catalyst $(S)-\mathrm{Ru}\left(\eta^{6}\right.$-mesitylene)-(R,R)-TsDPEN (8.9 mg, $\left.0.5 \mathrm{~mol} \%\right)$. The resulting solution was stirred at room temperature for $24 \mathrm{~h}$. Water $(10 \mathrm{~mL})$ was added to dilute and extracted with EtOAc $(3 \times 20 \mathrm{~mL})$. The combined organic layers were washed with saturated $\mathrm{NaHCO}_{3}$, dried over $\mathrm{Na}_{2} \mathrm{SO}_{4}$, and concentrated under reduced pressure to afford crude oil. The crude product was purified by silica gel flash chromatography eluting with 6\% EtOAc/hexane to give propargyl alcohol I-12 (605 mg, 2.49 mmol, 86\%): colorless oil; $R_{f}=0.42\left(20 \% \mathrm{Et}_{2} \mathrm{O} /\right.$ hexane $) ;[\alpha]^{25}=+16\left(c=1.1, \mathrm{CH}_{2} \mathrm{Cl}_{2}\right)$; IR (thin film, $\mathrm{cm}^{-1}$ ) 3346, 2954, 2930, 2858, 1739, 1472, 1253,1103,1070,835, 775; ${ }^{1} \mathrm{H}$ NMR (600 MHz CDCl $) \delta 4.43(\mathrm{qd}, J=6.0,1.8 \mathrm{~Hz}, 1 \mathrm{H}), 3.64(\mathrm{t}, J=6.0 \mathrm{~Hz}, 2 \mathrm{H}), 2.71$ (s, 1H), $2.22(\mathrm{td}, J=7.2,1.8 \mathrm{~Hz}, 2 \mathrm{H}), 1.63(\mathrm{tt}, J=7.2,6.0 \mathrm{~Hz}, 2 \mathrm{H}), 1.34(\mathrm{~d}, J=6.0 \mathrm{~Hz}$, $3 \mathrm{H}), 0.86(\mathrm{~s}, 9 \mathrm{H}), 0.02(\mathrm{~s}, 6 \mathrm{H}) ;{ }^{13} \mathrm{C} \mathrm{NMR}\left(150 \mathrm{MHz}, \mathrm{CDCl}_{3}\right) \delta 83.8,82.5,61.5,58.2$, 31.6, 25.9, 24.7, 18.3, 15.0, -5.3; CI HRMS: Calculated for $\left[\mathrm{C}_{13} \mathrm{H}_{26} \mathrm{O}_{2} \mathrm{SiNa}^{+}\right]$: 265.1594, found: 265.1630 . 
(R)-7-(tert-butyldimethylsilyloxy) -heptan-2-ol (I-6): ${ }^{2}$<smiles>CC(O)CCCCC[SeH3]</smiles>

To a solution of propargyl alcohol $\mathbf{I - 1 2}(2 \mathrm{~g}, 8.23 \mathrm{mmol})$ in dry EtOAc $(33 \mathrm{~mL})$, was carefully added $\mathrm{Pd} / \mathrm{C}(20 \mathrm{mg})$ and the resulting suspension was stirred under $\mathrm{H}_{2}(1 \mathrm{~atm})$ for $5 \mathrm{~min}$ at room temperature. The catalyst was filtered off through a short pad of celite, concentrated under reduced pressure to afford colorless oil. The crude product was purified by silica gel flash chromatography eluting with $6 \%$ EtOAc/hexane to give alcohol I-6 (1.73 g, $6.75 \mathrm{mmol}, 82 \%)$ : colorless oil; $R_{f}=0.40\left(20 \% \mathrm{Et}_{2} \mathrm{O} / \mathrm{hexane}\right) ;[\alpha]^{25}$ $=+8\left(c=1.0, \mathrm{CH}_{2} \mathrm{Cl}_{2}\right)$; IR (thin film, $\left.\mathrm{cm}^{-1}\right) 3363,2929,2857,1472,1254,1096,835$, 774; ${ }^{1} \mathrm{H}$ NMR $\left(600 \mathrm{MHz}, \mathrm{CDCl}_{3}\right) \delta 3.74(\mathrm{ddq}, J=6.0,6.0,6.0,1 \mathrm{H}), 3.56(\mathrm{t}, J=6.6 \mathrm{~Hz}$, 2H), $1.85(\mathrm{~s}, 1 \mathrm{H}), 1.49(\mathrm{~m}, 2 \mathrm{H}), 1.43(\mathrm{~m}, 2 \mathrm{H}), 1.39(\mathrm{~m}, 2 \mathrm{H}), 1.31(\mathrm{~m}, 2 \mathrm{H}), 1.14(\mathrm{~d}, J=6.0$ $\mathrm{Hz}, 3 \mathrm{H}), 0.86$ (s, 9H), $0.02(\mathrm{~s}, 6 \mathrm{H}) ;{ }^{13} \mathrm{C} \mathrm{NMR}\left(150 \mathrm{MHz}, \mathrm{CDCl}_{3}\right) \delta$ 68.0, 63.2, 39.4, 32.8, 26.0, 25.9, 25.6, 23.5, 18.4, -5.3; CIHRMS: Calculated for $\left[\mathrm{C}_{13} \mathrm{H}_{30} \mathrm{O}_{2} \mathrm{Si}+\mathrm{Na}\right]^{+}:$269.1907, found: 269.1903 .

(2S, 6R)-6-((R)-7-(tert-butyldimethylsilyloxyl)heptan-2-yloxy)-2-methyl-2H-pyran3(6H)-one (I-14): ${ }^{2}$ 


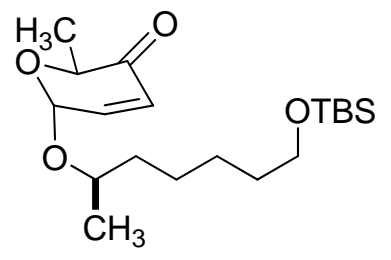

To a solution of pyranone I-5 (473 mg, $2.07 \mathrm{mmol})$ and alcohol I-6 (430 mg, $1.74 \mathrm{mmol})$ in dry $\mathrm{CH}_{2} \mathrm{Cl}_{2}(1 \mathrm{~mL})$, was added a $\mathrm{CH}_{2} \mathrm{Cl}_{2}(1 \mathrm{~mL})$ solution of $\mathrm{Pd}_{2}(\mathrm{DBA})_{3} \cdot \mathrm{CHCl}_{3}(43.9$ mg, $2.5 \mathrm{~mol} \%)$ and $\mathrm{PPh}_{3}(45.6 \mathrm{mg}, 10 \mathrm{~mol} \%)$ at $0{ }^{\circ} \mathrm{C}$ under argon atmosphere. After stirring for $3 \mathrm{~h}$ at $0{ }^{\circ} \mathrm{C}$ to room temperature, the reaction mixture was quenched with 15 $\mathrm{mL}$ of saturated $\mathrm{NaHCO}_{3}$, extracted $(3 \times 25 \mathrm{~mL})$ with $\mathrm{Et}_{2} \mathrm{O}$, dried $\left(\mathrm{Na}_{2} \mathrm{SO}_{4}\right)$, and concentrated under reduced pressure. The crude product was purified using silica gel flash chromatography eluting with 4\% EtOAc/hexane to give pyranone I-14 (656 mg, $1.64 \mathrm{mmol}, 94 \%): R_{f}=0.64(20 \% \mathrm{EtOAc} /$ hexane $) ;[\alpha]_{\mathrm{D}}^{25}=+13\left(c=1.0, \mathrm{CH}_{2} \mathrm{Cl}_{2}\right) ; \mathrm{IR}$ (thin film, $\mathrm{cm}^{-1}$ ) 2930, 2857, 1701, 1463, 1375, 1254, 1097, 1023, 836; ${ }^{1} \mathrm{H}$ NMR (600 $\left.\mathrm{MHz}, \mathrm{CDCl}_{3}\right) \delta 6.77(\mathrm{dd}, J=10.2,3.6 \mathrm{~Hz}, 1 \mathrm{H}), 6.04(\mathrm{~d}, J=10.2 \mathrm{~Hz}, 1 \mathrm{H}), 5.25(\mathrm{~d}, J=3.6$ $\mathrm{Hz}, 1 \mathrm{H}), 4.56(\mathrm{q}, J=6.6 \mathrm{~Hz}, 1 \mathrm{H}), 3.89(\mathrm{ddq}, J=6.0,6.0,6.0 \mathrm{~Hz}, 1 \mathrm{H}), 3.58(\mathrm{t}, J=6.6 \mathrm{~Hz}$, 2H), $1.58(\mathrm{~m}, 2 \mathrm{H}) 1.49(\mathrm{~m}, 2 \mathrm{H}), 1.47(\mathrm{~m}, 2 \mathrm{H}), 1.41(\mathrm{~m}, 2 \mathrm{H}), 1.35(\mathrm{~d}, J=6.6 \mathrm{~Hz}, 3 \mathrm{H})$, $1.17(\mathrm{~d}, J=6.6 \mathrm{~Hz}, 3 \mathrm{H}), 0.87(\mathrm{~s}, 9 \mathrm{H}), 0.02(\mathrm{~s}, 6 \mathrm{H}) ;{ }^{13} \mathrm{C} \mathrm{NMR}\left(150 \mathrm{MHz}, \mathrm{CDCl}_{3}\right) \delta 197.2$, $144.2,127.1,90.8,73.8,70.4,63.2,37.3,32.8,26.0,25.8,25.6,19.5,18.3,15.2,-5.3$; CIHRMS: Calculated for $\left[\mathrm{C}_{19} \mathrm{H}_{36} \mathrm{O}_{4} \mathrm{SiNa}^{+}\right]$: 379.2275 , found: 379.2277 .

(1S, 3S, 5R, 6R)-5-((R)-7-(tert-butyldimethylsilyloxyl)heptan-2-yloxy)-3-methyl-4,7dioxa-bicyclo[4.1.0]heptan-2-one (I-15): ${ }^{2}$ 


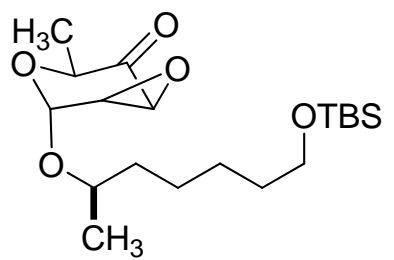

To the solution of pyranone $\mathbf{I - 1 4}(440 \mathrm{mg}, 1.18 \mathrm{mmol})$ in methanol $(3.93 \mathrm{~mL})$ at $0{ }^{\circ} \mathrm{C}$, $30 \%$ aqueous hydrogen peroxide $(0.553 \mathrm{~mL}, 4.7 \mathrm{mmol})$ was added dropwise, followed by aqueous sodium hydroxide $(0.236 \mathrm{~mL}, 0.5 \mathrm{M})$. The reaction mixture was warmed to room temperature. After stirring for $5 \mathrm{~h}$, the reaction mixture was quenched using saturated $\mathrm{NaHCO}_{3}(25 \mathrm{~mL})$, extracted with diethyl ether $(5 \times 20 \mathrm{~mL})$, dried $\left(\mathrm{Na}_{2} \mathrm{SO}_{4}\right)$, and concentrated under reduced pressure. The crude product was purified using silica gel flash chromatography eluting with 4\% EtOAc/hexane to give $656 \mathrm{mg}(1.64 \mathrm{mmol}, 94 \%)$ of epoxy-enone I-15: $R_{f}=0.62(20 \%$ EtOAc/hexane $) ;[\alpha]_{\mathrm{D}}^{25}=-103\left(c=1.0, \mathrm{CH}_{2} \mathrm{Cl}_{2}\right)$; IR (thin film, $\mathrm{cm}^{-1}$ ) 2930, 2858, 1726, 1463, 1380, 1254, 1143, 1094, 1057, 833; ${ }^{1} \mathrm{H}$ NMR $\left(600 \mathrm{MHz}, \mathrm{CDCl}_{3}\right) \delta 5.28(\mathrm{~d}, J=0.6 \mathrm{~Hz}, 1 \mathrm{H}), 4.19(\mathrm{q}, J=7.0 \mathrm{~Hz}, 1 \mathrm{H}), 3.86$ (ddq, $J$ $=6.0,6.0,6.0 \mathrm{~Hz}, 1 \mathrm{H}), 3.60(\mathrm{t}, J=6.6 \mathrm{~Hz}, 2 \mathrm{H}), 3.48(\mathrm{dd}, J=4.2,0.6 \mathrm{~Hz}, 1 \mathrm{H}), 3.42(\mathrm{~d}, J$ $=4.2 \mathrm{~Hz}, 1 \mathrm{H}), 1.54(\mathrm{~m}, 2 \mathrm{H}), 1.51(\mathrm{~m}, 2 \mathrm{H}), 1.37(\mathrm{~d}, J=6.6 \mathrm{~Hz}, 3 \mathrm{H}), 1.32(\mathrm{~m}, 2 \mathrm{H}), 1.24$ $(\mathrm{m}, 2 \mathrm{H}),, 1.20(\mathrm{~d}, J=7 \mathrm{~Hz}, 3 \mathrm{H}), 0.89(\mathrm{~s}, 9 \mathrm{H}), 0.04(\mathrm{~s}, 6 \mathrm{H}) ;{ }^{13} \mathrm{C} \mathrm{NMR}\left(150 \mathrm{MHz}, \mathrm{CDCl}_{3}\right)$ $\delta 203.3,91.3,73.7,71.9,63.1,54.3,53.0,37.1,32.8,26.0,25.8,25.5,19.2,18.4,16.8,-$ 5.2; CIHRMS: Calculated for $\left[\mathrm{C}_{19} \mathrm{H}_{36} \mathrm{O}_{5} \mathrm{SiNa}^{+}\right]$: 395.2224, found: 379.2228 .

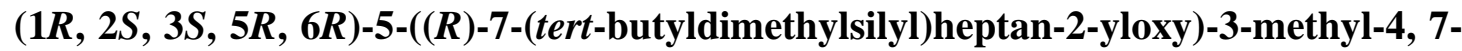
dioxa-bicyclo[4.1.0]heptan-2-ol (I-16): ${ }^{2}$ 


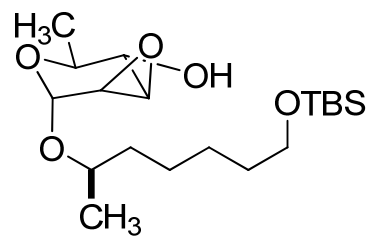

A $\mathrm{CH}_{2} \mathrm{Cl}_{2}(0.46 \mathrm{~mL})$ solution of epoxy enone $\mathbf{I}-15(181 \mathrm{mg}, 0.46 \mathrm{mmol})$ and $\mathrm{CeCl}_{3} / \mathrm{MeOH}(0.3 \mathrm{~mL}, 0.4 \mathrm{M})$ was cooled to $-78{ }^{\circ} \mathrm{C} . \mathrm{NaBH}_{4}(38.4 \mathrm{mg}, 10.2 \mathrm{mmol})$ was added and the reaction mixture was stirred at -78 to $-20{ }^{\circ} \mathrm{C}$ for $4 \mathrm{~h}$. The reaction mixture was diluted with ether $(5 \mathrm{~mL})$ and quenched with $2.5 \mathrm{~mL}$ of saturated $\mathrm{NaHCO}_{3}$, extracted (3 x $5 \mathrm{~mL})$ with $\mathrm{Et}_{2} \mathrm{O}$, dried $\left(\mathrm{Na}_{2} \mathrm{SO}_{4}\right)$, and concentrated under reduced pressure. The crude product was purified using silica gel flash chromatography eluting with $20 \%$ EtOAc/hexane to give $170 \mathrm{mg}(0.43 \mathrm{mmol}, 93 \%)$ of epoxy alcohol I-16: $R_{f}=0.34(40 \%$ EtOAc/hexane); $[\alpha]^{25}=-54\left(c=1.0, \mathrm{CH}_{2} \mathrm{Cl}_{2}\right)$; IR (thin film, $\left.\mathrm{cm}^{-1}\right) 3441,2930,2857$, 1463, 1387, 1254, 1097, 1055, 834; ${ }^{1} \mathrm{H}$ NMR $\left(600 \mathrm{MHz}, \mathrm{CDCl}_{3}\right) \delta 5.02(\mathrm{~s}, 1 \mathrm{H}), 3.84$ (ddq, $J=6.0,6.0,6.0 \mathrm{~Hz} 1 \mathrm{H}), 3.60(\mathrm{t}, J=6.6 \mathrm{~Hz}, 2 \mathrm{H}), 3.59-3.55(\mathrm{~m}, 1 \mathrm{H}), 3.50(\mathrm{~m}, 1 \mathrm{H})$, $3.26(\mathrm{~d}, J=3.6 \mathrm{~Hz}, 1 \mathrm{H}), 3.05(\mathrm{~d}, J=3.6 \mathrm{~Hz}, 1 \mathrm{H}), 1.61-1.53(\mathrm{~m}, 2 \mathrm{H}) 1.52(\mathrm{~m}, 2 \mathrm{H}), 1.49-$ $1.40(\mathrm{~m}, 2 \mathrm{H}), 1.38-1.31(\mathrm{~m}, 2 \mathrm{H}), 1.23(\mathrm{~d}, J=6.6 \mathrm{~Hz}, 3 \mathrm{H}), 1.16(\mathrm{~d}, J=6.6 \mathrm{~Hz}, 3 \mathrm{H}), 0.89$ (s, 9H), $0.04(\mathrm{~s}, 6 \mathrm{H}) ;{ }^{13} \mathrm{C} \mathrm{NMR}\left(150 \mathrm{MHz}, \mathrm{CDCl}_{3}\right) \delta$ 92.5, 73.1, 68.0, 65.6, 63.3, 56.0, 50.6, 37.3, 32.8, 26.0, 25.8, 25.5, 19.4, 18.4, 18.3, -5.2; CIHRMS: Calculated for $\left[\mathrm{C}_{19} \mathrm{H}_{38} \mathrm{O}_{5} \mathrm{SiNa}^{+}\right]$: 397.2380, found: 397.2386 .

(2R, 3R, 5R, 6S)-2-((R)-7-(tert-butyldimethylsilyloxyl)heptan-2-yloxy)-tetrahydro-6methyl-2H-pyran-3,5-diol (I-4): ${ }^{2}$ 


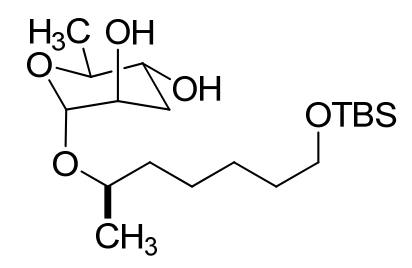

To a solution of epoxy ketone $\mathbf{I}-16(35 \mathrm{mg}, 0.09 \mathrm{mmol})$ in dry THF $(0.15 \mathrm{~mL})$ at $-78{ }^{\circ} \mathrm{C}$, was added dropwise the suspension solution of $\mathrm{LiAlH}_{4}(6.8 \mathrm{mg}, 0.18 \mathrm{mmol})$ in dry THF $(0.15 \mathrm{~mL})$ under argon atmosphere. After stirring $2 \mathrm{~h}$, the resulting solution was warmed to the room temperature and $\mathrm{LiAlH}_{4}(6.8 \mathrm{mg}, 0.18 \mathrm{mmol})$ in dry THF $(0.15 \mathrm{~mL})$ was added dropwise again, keeping reaction overnight. Water $(1 \mathrm{~mL})$ was added dropwise with cooling. Then the mixture was extracted with EtOAc $(3 \times 3 \mathrm{~mL})$, dried $\left(\mathrm{Na}_{2} \mathrm{SO}_{4}\right)$, and concentrated under reduced pressure. The crude product was purified using silica gel flash chromatography eluting with $50 \%$ EtOAc/hexane to give $30.5 \mathrm{mg}(0.078 \mathrm{mmol}$, $86 \%)$ of diol I-4: $R_{f}=0.21(50 \%$ EtOAc/hexane $) ;[\alpha]^{25}{ }_{\mathrm{D}}=-67\left(c=1.0, \mathrm{CH}_{2} \mathrm{Cl}_{2}\right) ; \mathrm{IR}$ (thin film, $\mathrm{cm}^{-1}$ ) 3360, 2929, 2857, 1463, 1378, 1255, 1098, 1028, 984, 835; ${ }^{1} \mathrm{H}$ NMR (600 MHz, CD $\left.\mathrm{CD}_{3} \mathrm{OD}\right) \delta 4.69(\mathrm{~s}, 1 \mathrm{H}), 3.83(\mathrm{ddq}, J=6.0,6.0,6.0 \mathrm{~Hz}, 1 \mathrm{H}), 3.76(\mathrm{~m}, 1 \mathrm{H})$, $3.69(\mathrm{t}, J=6.6 \mathrm{~Hz}, 2 \mathrm{H}), 3.66(\mathrm{~m}, 1 \mathrm{H}), 3.56(\mathrm{ddd}, J=11,11,4.2 \mathrm{~Hz}, 1 \mathrm{H}), 2.00(\mathrm{ddd}, J$ $=13.2,3.6,3.6 \mathrm{~Hz}, 1 \mathrm{H}), 1.82(\mathrm{ddd}, J=13.2,11.4,3 \mathrm{~Hz}, 1 \mathrm{H}), 1.58(\mathrm{~m}, 2 \mathrm{H}) 1.51(\mathrm{~m}, 2 \mathrm{H})$, $1.43(\mathrm{~m}, 2 \mathrm{H}), 1.34(\mathrm{~m}, 2 \mathrm{H}), 1.26(\mathrm{~d}, J=6.6 \mathrm{~Hz}, 3 \mathrm{H}), 1.17(\mathrm{~d}, J=6.0 \mathrm{~Hz}, 3 \mathrm{H}), 0.95(\mathrm{~s}$, 9H), $0.10(\mathrm{~s}, 6 \mathrm{H}) ;{ }^{13} \mathrm{C} \mathrm{NMR}\left(150 \mathrm{MHz}, \mathrm{CD}_{3} \mathrm{OD}\right) \delta 96.3,72.5,71.2,70.0,68.8,63.1$, 38.6, 36.1, 34.0, 27.1, 26.7, 26.6, 19.5, 19.3, 18.3, -4.9; CIHRMS: Calculated for $\left[\mathrm{C}_{19} \mathrm{H}_{40} \mathrm{O}_{5} \mathrm{SiNa}^{+}\right]$: 399.2537, found: 399.2539. 


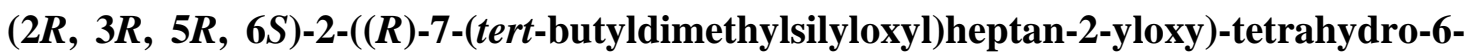
methyl-2H-pyran-3,5-diol (I-17): ${ }^{2}$

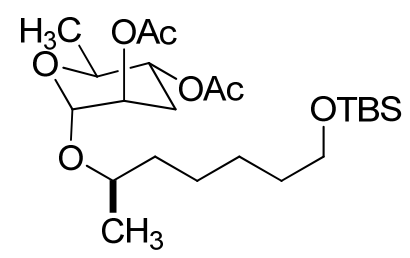

To a solution of diol I-4 $(752 \mathrm{mg}, 2 \mathrm{mmol})$ in pyridine $(2 \mathrm{~mL})$, was added $\mathrm{Ac}_{2} \mathrm{O}(1 \mathrm{~mL})$ and DMAP (35 mg). The reaction mixture was stirred for $12 \mathrm{~h}$. Water was added to destroy the excess acetic anhydride, extracted $(3 \times 50 \mathrm{~mL})$ with $\mathrm{Et}_{2} \mathrm{O}$ washed with $40 \mathrm{~mL}$ of saturated $\mathrm{CuSO}_{4}$ solution for three times, dried $\left(\mathrm{Na}_{2} \mathrm{SO}_{4}\right)$, and concentrated under reduced pressure. The crude product was purified using silica gel flash chromatography eluting with 5\% ether/hexane to give acetate I-17 (912 mg, $1.98 \mathrm{mmol}, 99 \%$ ); a clean oil; $R_{f}=0.49(10 \%$ EtOAc/hexane $) ;[\alpha]^{25}=-66\left(c=1.1, \mathrm{CH}_{2} \mathrm{Cl}_{2}\right) ; \mathrm{IR}\left(\right.$ thin film, $\left.\mathrm{cm}^{-1}\right) 2934$, 2958, 1743, 1371, 12309, 1037, 835; ${ }^{1} \mathrm{H}$ NMR (600 MHz, $\left.\mathrm{CDCl}_{3}\right) \delta 4.74(\mathrm{~m}, 1 \mathrm{H}), 4.70$ (ddd, $J=11.4,10.2,4.2 \mathrm{~Hz}, 1 \mathrm{H}), 4.65(\mathrm{~s}, 1 \mathrm{H}), 3.85(\mathrm{dq}, J=9.6,6.6 \mathrm{~Hz}, 1 \mathrm{H}), 3.76(\mathrm{~m}$, 1H), $3.60(\mathrm{t}, J=6.6 \mathrm{~Hz}, 2 \mathrm{H}), 2.11(\mathrm{ddd}, J=13.2,3.6,3.6 \mathrm{~Hz}, 1 \mathrm{H}), 2.10(\mathrm{~s}, 3 \mathrm{H}), 2.05(\mathrm{~s}$, 3H), $1.93(\mathrm{ddd}, J=13.2,11.4,3 \mathrm{~Hz}, 1 \mathrm{H}), 1.58(\mathrm{~m}, 2 \mathrm{H}), 1.54(\mathrm{~m}, 2 \mathrm{H}), 1.43(\mathrm{~m}, 2 \mathrm{H}), 1.34$ $(\mathrm{m}, 2 \mathrm{H}), 1.17(\mathrm{~d}, J=6.6 \mathrm{~Hz}, 3 \mathrm{H}), 1.12(\mathrm{~d}, J=6.0 \mathrm{~Hz}, 3 \mathrm{H}), 0.89(\mathrm{~s}, 9 \mathrm{H}), 0.04(\mathrm{~s}, 6 \mathrm{H}) ;{ }^{13} \mathrm{C}$ NMR $\left(150 \mathrm{MHz}, \mathrm{CDCl}_{3}\right) \delta 170.4,170.1,93.7,72.4,70.7,70.0,68.8,63.3,37.2,32.9$, 29.5, 26.1, 26.0, 25.9, 25.6, 21.3, 21.2, 19.1, 18.4, 17.7, -5.2; CIHRMS: Calculated for $\left[\mathrm{C}_{23} \mathrm{H}_{44} \mathrm{O}_{7} \mathrm{SiNa}^{+}\right]:$483.2754, found: 483.2755 . 
2-((R)-7-hydroxyheptan-2-yloxy)-tetrahydro-6-methyl-2H-pyran-3，5-diacetate (I18): ${ }^{2}$

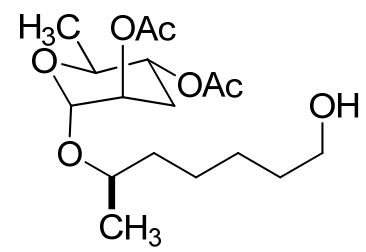

To a solution of TBS-ether I-17 (460.6 mg, $1 \mathrm{mmol})$ in dry $\mathrm{MeOH}(2 \mathrm{~mL}), 6 \mathrm{~N} \mathrm{HCl}(2$ $\mathrm{mL}$ ) was added at room temperature under the argon atmosphere. After $4 \mathrm{~h}$, the reaction mixture was diluted with ether $(20 \mathrm{~mL})$ and quenched with saturated $\mathrm{NaHCO}_{3}$, extracted ( 3 x $5 \mathrm{~mL}$ ) with $\mathrm{Et}_{2} \mathrm{O}$, dried $\left(\mathrm{Na}_{2} \mathrm{SO}_{4}\right)$, and concentrated under reduced pressure. The crude product was purified by passing a silica gel pad eluting with pure ether to give alcohol I-18 (350 mg, $0.97 \mathrm{mmol}, 97 \%)$; a colorless oil, $[\alpha]^{25}=-86\left(c=1.12, \mathrm{CH}_{2} \mathrm{Cl}_{2}\right)$; IR (thin film, $\left.\mathrm{cm}^{-1}\right)$ 2975, 2935, 2861, 1739, 1230, 1105, 1032, 983; ${ }^{1} \mathrm{H}$ NMR (600 MHz, $\left.\mathrm{CDCl}_{3}\right) \delta 4.81(\mathrm{~m}, 1 \mathrm{H}), 4.77(\mathrm{ddd}, J=11.4,10.2,4.2 \mathrm{~Hz}, 1 \mathrm{H}), 4.72(\mathrm{~s}, 1 \mathrm{H}), 3.84(\mathrm{dq}, J=$ 9.6, $6.6 \mathrm{~Hz}, 1 \mathrm{H}), 3.74(\mathrm{~m}, 1 \mathrm{H}), 3.62(\mathrm{t}, J=6.6 \mathrm{~Hz}, 2 \mathrm{H}), 2.10$ (ddd, $J=13.2,3.6,3.6 \mathrm{~Hz}$, 1H), $2.09(\mathrm{~s}, 3 \mathrm{H}), 2.03(\mathrm{~s}, 3 \mathrm{H}), 1.92(\mathrm{ddd}, J=13.2,11.4,3 \mathrm{~Hz}, 1 \mathrm{H}), 1.57(\mathrm{~m}, 2 \mathrm{H}), 1.54$ $(\mathrm{m}, 2 \mathrm{H}), 1.43(\mathrm{~m}, 2 \mathrm{H}), 1.36(\mathrm{~m}, 2 \mathrm{H}), 1.15(\mathrm{~d}, J=6.6 \mathrm{~Hz}, 3 \mathrm{H}), 1.11(\mathrm{~d}, J=6.0 \mathrm{~Hz}, 3 \mathrm{H})$ ${ }^{13} \mathrm{C} \mathrm{NMR}\left(150 \mathrm{MHz}, \mathrm{CDCl}_{3}\right) \delta 170.4,170.1,93.7,72.3,70.7,70.0,66.8,62.7,37.1,32.7$, 29.4, 25.8, 25.5, 21.2, 21.1, 19.1, 17.7; CIHRMS: Calculated for $\left[\mathrm{C}_{17} \mathrm{H}_{30} \mathrm{O}_{7} \mathrm{Na}^{+}\right]$: 369.1889, found: 369.1891 .

2-methyl-6-((R)-7-oxoheptan-2-yloxy)-tetrahydro-2H-pyran-3, 5-yl diacetate (I-19): 


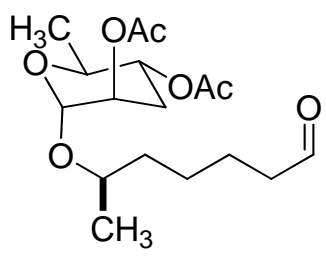

A solution of alcohol I-18 $(240 \mathrm{mg}, 0.693 \mathrm{mmol})$ and trichloroisocyanuric acid (171.2 $\mathrm{mg}, 1.386 \mathrm{mmol})$ in ethyl ether $(30 \mathrm{ml})$ was stirred at $-30{ }^{\circ} \mathrm{C}$, and then $2,2,6,6-$ tetramethylpeperidinooxy was added in one portion. The mixture was stirred for $20 \mathrm{~min}$ and then quenched with $30 \mathrm{ml}$ saturated sodium carbonate. The organic layer was separated and the aqueous layer was extracted with ether. Combined the organic layer and washed with brine, dried $\left(\mathrm{Na}_{2} \mathrm{SO}_{4}\right)$, filtered and concentrated. Chromatography on silica gel (20\% EtOAc/Hexane) gave aldehyde I-19 (210 mg, $0.61 \mathrm{mmol}, 88 \%)$ as colorless oil; $R f(40 \%$ EtOAc/Hexane $)=0.66 ;[\alpha]^{25}=-41(c=1.0, \mathrm{MeOH}) ;$ IR (thin film, $\left.\mathrm{cm}^{-1}\right), 2929,1741,1234,1040 ;{ }^{1} \mathrm{H}$ NMR $\left(600 \mathrm{MHz}, \mathrm{CD}_{3} \mathrm{Cl}\right) \delta 9.76(\mathrm{t}, J=1.8 \mathrm{~Hz}$, $1 \mathrm{H}), 4.82(\mathrm{~m}, 1 \mathrm{H}), 4.78(\mathrm{ddd}, J=11.4,10.2,4.8 \mathrm{~Hz}, 1 \mathrm{H}), 4.72(\mathrm{~s}, 1 \mathrm{H}), 3.82(\mathrm{dq}, J=12.6$, $6 \mathrm{~Hz}, 1 \mathrm{H}), 3.76(\mathrm{ddq}, J=6.0,6.0,6.0 \mathrm{~Hz}, 1 \mathrm{H}), 2.44(\mathrm{dt}, J=7.2,1.8 \mathrm{~Hz}, 1 \mathrm{H}), 2.09(\mathrm{~m}$, 3H), $2.04(\mathrm{~m}, 3 \mathrm{H}), 1.91(\mathrm{ddd}, J=13.8,11.4,3 \mathrm{~Hz}, 1 \mathrm{H}), 1.68-1.46(\mathrm{~m}, 8 \mathrm{H}), 1.33(\mathrm{~d}, J=$

$6.6 \mathrm{~Hz}, 6 \mathrm{H}) ;{ }^{13} \mathrm{C}$ NMR $\left(150 \mathrm{MHz}, \mathrm{CD}_{3} \mathrm{Cl}\right) \delta 202.6,170.6,170.3,93.8,72.3,70.8,70.0$, 67.0, 44.003, 37.01, 29.6, 25.4, 22.3, 21.4, 21.3, 19.19, 17.9. CIHRMS: Calculated for $\left[\mathrm{C}_{17} \mathrm{H}_{28} \mathrm{O}_{7} \mathrm{Na}^{+}\right]:$367.1227, Found: 367.1733 .

(8R,E)-ethyl 8-(3,5-diacetoxy-6-methyl-tetrahydro-2H-pyran-2-yloxy)non-2-enoate (I-20): 


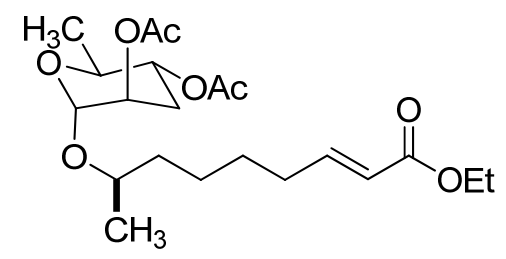

Ylide (396.7 mg, $1.14 \mathrm{mmol}$ ) was dissolved in $0.6 \mathrm{ml}$ of $\mathrm{CH}_{2} \mathrm{Cl}_{2}$, into which aldehyde $\mathbf{I}$ 19 (190 mg, $0.55 \mathrm{mmol})$ was added. The reaction was stirred vigorously at room temperature for $12 \mathrm{hrs}$ before solvent was removed in vacuum. Chromatography on silica gel (30\% EtOAc/Hexane) gave ethyl ester I-20 (220 mg, $0.53 \mathrm{mmol}, 96.5 \%)$ as colorless oil. $R f(40 \%$ EtOAc/Hexane $)=0.57 ;[\alpha]^{25}=-22(c=1.0, \mathrm{MeOH}) ;$ IR (thin film, $\left.\mathrm{cm}^{-1}\right)$, 2936, 1743, 1233, 1038; ${ }^{1} \mathrm{H}$ NMR $\left(600 \mathrm{MHz}, \mathrm{CD}_{3} \mathrm{Cl}\right) \delta 6.96(\mathrm{dt}, J=13.8,7.2 \mathrm{~Hz}, 1 \mathrm{H})$, $5.82(\mathrm{dt}, J=15.6,1.8 \mathrm{~Hz}, 1 \mathrm{H}), 4.83(\mathrm{~m}, 1 \mathrm{H}), 4.79$ (ddd, $J=10.8,9.6,4.2 \mathrm{~Hz}, 1 \mathrm{H}), 4.73$ $(\mathrm{s}, 1 \mathrm{H}), 4.18(\mathrm{q}, J=7.2 \mathrm{~Hz}, 2 \mathrm{H}), 3.84(\mathrm{dq}, J=12,6 \mathrm{~Hz}, 1 \mathrm{H}), 3.76(\mathrm{ddq}, J=6.0,6.0,6.0$ $\mathrm{Hz}, 1 \mathrm{H}), 2.22(\mathrm{dt}, J=7.2,1.2 \mathrm{~Hz}, 1 \mathrm{H}), 2.11(\mathrm{~m}, 3 \mathrm{H}), 2.06(\mathrm{~m}, 3 \mathrm{H}), 1.93(\mathrm{ddd}, J=13.8$, 11.4, $3 \mathrm{~Hz}, 1 \mathrm{H}), 1.60-1.35(\mathrm{~m}, 8 \mathrm{H}), 1.28(\mathrm{t}, J=7.2 \mathrm{~Hz}, 3 \mathrm{H}) ; 1.17(\mathrm{~d}, J=6.6 \mathrm{~Hz}, 3 \mathrm{H})$, $1.13(\mathrm{~d}, J=6 \mathrm{~Hz}, 3 \mathrm{H}) ;{ }^{13} \mathrm{C} \mathrm{NMR}\left(150 \mathrm{MHz}, \mathrm{CD}_{3} \mathrm{Cl}\right) \delta 170.3,170.1,166.7,148.9,121.5$, $93.6,72.2,70.6,69.8,66.8,60.4,60.2,36.8,32.2,29.3,27.9,25.3,21.2,19.0,17.6,14.3$; CIHRMS: Calculated for $\left[\mathrm{C}_{21} \mathrm{H}_{34} \mathrm{O}_{8} \mathrm{Na}^{+}\right]$: 437.2146, Found: 437.2151.

(8R,E)-methyl 8-(3,5-dihydroxy-6-methyl-tetrahydro-2H-pyran-2-yloxy)non-2enoate (I-21):<smiles>COC(=O)/C=C/CCCCC(C)OC1C2COC(O)C(C)(O)C1C2(C)C</smiles> 
To a $20 \mathrm{ml}$ flask was added ethyl ester I-20 (136 mg, $0.328 \mathrm{mmol})$, MeOH (3 ml), Lithium hydroxide (30 mg, $1.25 \mathrm{mmol}$ ). The resulting solution was stirred at room temperature for $24 \mathrm{hrs}$. Aqueous $\mathrm{HCl}(0.5 \mathrm{M}, 3 \mathrm{ml})$ was added before solvent was removed in vacuum. Chromatography on silica gel (80\% EtOAc/Hexane) gave ethyl ester I-21 (103 mg, $0.309 \mathrm{mmol}, 99 \%)$ as colorless oil. $R f($ EtOAc $)=0.58 ;[\alpha]^{25}=-81(c=$ 1.0, MeOH); IR (thin film, $\mathrm{cm}^{-1}$ ), 3404, 2934, 2180, 1991, 1723, 1655, 1438, 1273, $1203,1101,1128,1029,984,854 ;{ }^{1} \mathrm{H}$ NMR $\left(600 \mathrm{MHz}, \mathrm{CD}_{3} \mathrm{Cl}\right) \delta 6.96(\mathrm{dt}, J=15.6,6.6$ Hz, 1H), $5.83(\mathrm{dt}, J=15.6,1.2 \mathrm{~Hz}, 1 \mathrm{H}), 4.69(\mathrm{~s}, 1 \mathrm{H}), 3.80-3.77(\mathrm{~m}, 1 \mathrm{H}), 3.72(\mathrm{~s}, 3 \mathrm{H})$, $3.65(\mathrm{dt}, J=6.6,3.0 \mathrm{~Hz}, 1 \mathrm{H}), 3.59-3.57(\mathrm{~m}, 1 \mathrm{H}), 2.22(\mathrm{ddd}, J=9.0,7.2,1.8 \mathrm{~Hz}, 1 \mathrm{H})$, $1.88-1.72(\mathrm{~m}, 2 \mathrm{H}), 1.61(\mathrm{~s}, 1 \mathrm{H}), 1.55-1.35(\mathrm{~m}, 6 \mathrm{H}), 1.27(\mathrm{~d}, J=6 . \mathrm{Hz}, 3 \mathrm{H}), 1.22(\mathrm{~d}, J=$ $6 \mathrm{~Hz}, 3 \mathrm{H}) ;{ }^{13} \mathrm{C} \mathrm{NMR}\left(150 \mathrm{MHz}, \mathrm{CD}_{3} \mathrm{Cl}\right) \delta 167.3,149.5,121.7,96.1,71.3,70.0,69.3$, $69.1, \quad 51.5,36.9,35.2,32.0,27.8,25.0,20.0,17.7$; CIHRMS: Calculated for $\left[\mathrm{C}_{16} \mathrm{H}_{28} \mathrm{O}_{6} \mathrm{Na}^{+}\right]: 339.1778$, Found: 339.1784 .

(8R,E)-8-(3,5-dihydroxy-6-methyl-tetrahydro-2H-pyran-2-yloxy)non-2-enoic acid (I-1)

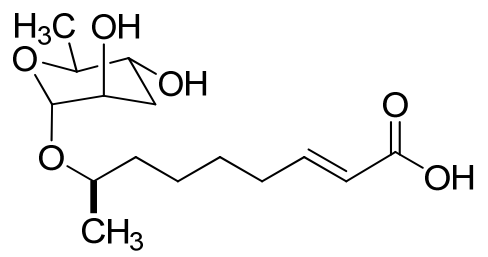

To a $20 \mathrm{ml}$ flask was added methyl ester $\mathbf{I}-21$ (13 mg, $0.04 \mathrm{mmol}), \mathrm{THF} / \mathrm{H}_{2} \mathrm{O}(4: 1,0.4$ $\mathrm{ml})$, Lithium hydroxide $(1.44 \mathrm{mg}, 0.06 \mathrm{mmol})$. The resulting solution was stirred at room temperature for $24 \mathrm{hrs}$. Aqueous $\mathrm{HCl}(0.5 \mathrm{M}, 0.4 \mathrm{ml})$ was added to make the $\mathrm{pH}$ equal to 7 , and then the solvent was removed in vacuum. Chromatography on silica gel $(5 \%$ 
$\mathrm{MeOH} / \mathrm{EtOAc})$ gave acid I-1 $(12 \mathrm{mg}, 0.0397 \mathrm{mmol}, 99 \%)$ as colorless oil. $R f($ EtOAc $)=$ $0.28 ;[\alpha]^{25}=-62(c=1.0, \mathrm{MeOH}) ;$ IR (thin film, $\left.\mathrm{cm}^{-1}\right), 3703,3364,2930,2357,2164$, 2084, 2010, 1954, 1696, 1272, 1127, 981, 667; ${ }^{1} \mathrm{H}$ NMR $\left(600 \mathrm{MHz}, \mathrm{CD}_{3} \mathrm{Cl}\right) \delta 6.93(\mathrm{dt}, J$ $=15.6,7.2 \mathrm{~Hz}, 1 \mathrm{H}), 5.79(\mathrm{dt}, J=15.6,1.2 \mathrm{~Hz}, 1 \mathrm{H}), 4.62(\mathrm{~s}, 1 \mathrm{H}), 3.78-3.75(\mathrm{~m}, 2 \mathrm{H})$, $3.69(\mathrm{~s}, 1 \mathrm{H}), 3.63-3.58(\mathrm{~m}, 1 \mathrm{H}), 3.49(\mathrm{ddd}, J=10.8,9.6,4.8 \mathrm{~Hz}, 1 \mathrm{H}), 2.23-2.21(\mathrm{~m}, 2 \mathrm{H})$, 2.05-1.91 (m, 2H), $1.74(\mathrm{ddd}, J=12.6,11.4,2.4 \mathrm{~Hz}, 1 \mathrm{H}), 1.58-1.23(\mathrm{~m}, 8 \mathrm{H}), 1.19(\mathrm{~d}, J$ $=6.6 \mathrm{~Hz}, 3 \mathrm{H}), 1.11(\mathrm{~d}, J=6 \mathrm{~Hz}, 3 \mathrm{H}) ;{ }^{13} \mathrm{C} \mathrm{NMR}\left(150 \mathrm{MHz}, \mathrm{CD}_{3} \mathrm{OD}\right) \delta 169.9,148.8$, 122.4, 96.4, 71.3, 70.0, 68.8, 67.2, 36.9, 34.8, 31.9, 28.0, 25.2, 18.2, 16.9; CIHRMS: Calculated for $\left[\mathrm{C}_{15} \mathrm{H}_{26} \mathrm{O}_{6} \mathrm{Na}^{+}\right]$: 325.1622 , Found: 325.1627 .

(8R,E)-8-(3,5-dihydroxy-6-methyl-tetrahydro-2H-pyran-2-yloxy)-N-(2-(2-(7(dimethylamino)-2-oxo-2H-chromen-4-yl)acetamido)ethyl)non-2-enamide (I-23)<smiles>CC(CCCC/C=C/C(=O)NCCNC(=O)Cc1cc(=O)oc2cc(N(C)C)ccc12)OC1CC2CC(O)C(O)C1C2(C)C</smiles>

To a solution of amine $(6 \mathrm{mg}, 0.015 \mathrm{mmol})$ in THF $(0.125 \mathrm{ml})$, was added $\mathrm{Et}_{3} \mathrm{~N}(3 \mu \mathrm{L})$, HBTU (4.7 mg, $0.0125 \mathrm{mmol})$ and acid I-1 $(5 \mathrm{mg}, 0.0125 \mathrm{mmol})$. The reaction mixture was stirred overnight, concentrated under reduced pressure, purified using silica gel flash chromatography eluting with $10 \% \mathrm{MeOH} / \mathrm{EtOAc}$ to give amide I-23 (5 mg, 0.00872 mmol, 69\%); Rf (10\% MeOH/EtOAc) $=0.55 ;[\alpha]^{25}=-30(c=0.5, \mathrm{MeOH}) ; \quad$ IR (thin 
film, $\left.\mathrm{cm}^{-1}\right), 3781,3358,2933,2252,2149,1997,1699,1615,1531,1453,1403,1252$, 1128, 1030, 983, 834; ${ }^{1} \mathrm{H}$ NMR $\left(600 \mathrm{MHz}, \mathrm{CD}_{3} \mathrm{Cl}\right) \delta 7.32(\mathrm{~d}, J=9 \mathrm{~Hz}, 1 \mathrm{H}), 6.92(\mathrm{dt}, J$ $=15.6,7.2 \mathrm{~Hz}, 1 \mathrm{H}), 6.77-6.71(\mathrm{~m}, 1 \mathrm{H}), 6.54(\mathrm{~d}, J=3 \mathrm{~Hz}, 1 \mathrm{H}), 6.03(\mathrm{~s}, 1 \mathrm{H}), 5.81(\mathrm{dt}, J=$ 15.6, $1.2 \mathrm{~Hz}, 1 \mathrm{H}), 4.63(\mathrm{~s}, 1 \mathrm{H}), 3.79-3.78(\mathrm{~m}, 1 \mathrm{H}), 3.70(\mathrm{~s}, 1 \mathrm{H}), 3.67-3.60(\mathrm{~m}, 2 \mathrm{H})$, $3.41(\mathrm{t}, J=6.0 \mathrm{~Hz}, 2 \mathrm{H}), 3.34(\mathrm{t}, \mathrm{J}=6.0 \mathrm{~Hz}, 2 \mathrm{H}), 3.06(\mathrm{~s}, 6 \mathrm{H}), 2.26-2.22(\mathrm{~m}, 1 \mathrm{H}), 1.97-$ $1.88(\mathrm{~m}, 2 \mathrm{H}), 1.78-1.73(\mathrm{~m}, 1 \mathrm{H}), 1.58-1.54(\mathrm{~m}, 1 \mathrm{H}), 1.50-1.45(\mathrm{~m}, 2 \mathrm{H}), 1.43-1.38(\mathrm{~m}=$ 1H), $1.28(\mathrm{~m}, 2 \mathrm{H}), 1.20(\mathrm{~d}, J=6.6 \mathrm{~Hz}, 3 \mathrm{H}), 1.11(\mathrm{~d}, J=6 \mathrm{~Hz}, 3 \mathrm{H}) ;{ }^{13} \mathrm{C}$ NMR $(150$ $\left.\mathrm{MHz}, \mathrm{CD}_{3} \mathrm{OD}\right) \delta 170.3,167.9,163.1,156.0,153.6,151.4,149.2,144.6,125.7,123.5$, $121.9,109.5,108.7,97.6,96.4,71.2,70.0,68.8,67.2,39.3,39.0,38.7,36.9,34.8,31.8$, 28.1, 28.0, 25.2, 18.1, 16.9; CIHRMS: Calculated for $\left[\mathrm{C}_{30} \mathrm{H}_{43} \mathrm{O}_{8} \mathrm{~N}_{3} \mathrm{Na}^{+}\right]$: 596.2942, Found: 596.2948.

(2S, 6R)-6-(benzyloxy)-2-methyl-2H-pyran-3(6H)-one (II-20) ${ }^{3}$<smiles>C[C@H]1OC(OCc2ccccc2)C=CC1=O</smiles>

To a solution of Boc-protected pyranone II-23 (22.8 g, $100 \mathrm{mmol})$ and benzyl alcohol $(21.6 \mathrm{~g}, 200.0 \mathrm{mmol})$ in dry $\mathrm{CH}_{2} \mathrm{Cl}_{2}(100 \mathrm{~mL})$ was added $\mathrm{Pd}_{2}(\mathrm{DBA})_{3} \cdot \mathrm{CHCl}_{3}(257 \mathrm{mg}$, $0.25 \mathrm{~mol} \% \mathrm{Pd})$ and $\mathrm{PPh}_{3}(262 \mathrm{mg}, 0.5 \mathrm{~mol} \%)$ at $0{ }^{\circ} \mathrm{C}$ under argon atmosphere. After stirring for $2 \mathrm{~h}$ from $0{ }^{\circ} \mathrm{C}$ to room temperature, the reaction mixture was quenched with of

\footnotetext{
${ }^{3}$ Guo, H.; O’Doherty, G. A. Angew. Chem. Int. Ed. 2007, 46, 5206-5208.
} 
saturated aqueous $\mathrm{NaHCO}^{3}(300 \mathrm{~mL})$, extracted with $\mathrm{Et}_{2} \mathrm{O}(300 \mathrm{~mL} \times 3)$, dried over $\mathrm{Na}_{2} \mathrm{SO}_{4}$, and concentrated under reduced pressure. The crude product was purified using silica gel flash chromatography eluting with 4\% EtOAc/hexane to give Bn-ether II-20 (19.6 g, 90 mmol, 90\%); colorless oil; $R f=0.71(30 \%$ EtOAc/hexane $) ;[\alpha]^{25}{ }_{\mathrm{D}}=+46(c=$ 1.11, $\mathrm{CH}_{2} \mathrm{Cl}_{2}$ ); IR (thin film, cm-1) 2985, 2939, 2873, 1697, 1231, 1022, 953; ${ }^{1} \mathrm{H}$ NMR $\left(270 \mathrm{MHz}, \mathrm{CDCl}_{3}\right) \delta$ 7.32-7.38 (m, 5H), $6.85(\mathrm{dd}, J=10.2,3.6 \mathrm{~Hz}, 1 \mathrm{H}), 6.11(\mathrm{~d}, J=10.2$ $\mathrm{Hz}, 1 \mathrm{H}), 5.28(\mathrm{~d}, J=3.6 \mathrm{~Hz}, 1 \mathrm{H}), 4.86(\mathrm{~d}, J=11.4 \mathrm{~Hz}, 1 \mathrm{H}), 4.70(\mathrm{~d}, J=11.4 \mathrm{~Hz}, 1 \mathrm{H})$, $4.57(\mathrm{q}, J=6.7 \mathrm{~Hz}, 1 \mathrm{H}), 1.36(\mathrm{~d}, J=6.7 \mathrm{~Hz}, 3 \mathrm{H}) ;{ }^{13} \mathrm{C} \mathrm{NMR}\left(67.5 \mathrm{MHz}, \mathrm{CDCl}_{3}\right) \delta 197.0$, 143.5, 137.2, 126.6, 128.2, 128.1, 127.5, 92.4, 70.8, 70.5, 15.3; HRMS (CI): Calculated for $\left[\mathrm{C}_{13} \mathrm{H}_{14} \mathrm{O}_{3}+\mathrm{Na}\right]^{+}$: 241.0835, found: 241.0843 .

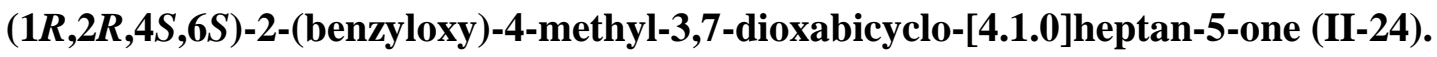<smiles>C[C@H]1OC(OCc2ccccc2)[C@H]2O[C@H]2C1=O</smiles>

To the solution of pyranone $\mathbf{I I - 2 0}(220 \mathrm{mg}, 1.0 \mathrm{mmol})$ in methanol $(3 \mathrm{ml})$ at $0{ }^{\circ} \mathrm{C}, 30 \%$ aqueous hydrogen peroxide $(0.26 \mathrm{ml}, 2.5 \mathrm{mmol})$ was added dropwise, followed by aqueous sodium hydroxide $(0.1 \mathrm{ml}, 0.5 \mathrm{M})$. The reaction mixture was stirred at RT for 4 $\mathrm{h}$ and then the reaction mixture was extracted with diethyl ether. The extracts were washed with saturated aqueous $\mathrm{NaHCO}_{3}$, dried over $\mathrm{Na}_{2} \mathrm{SO}_{4}$ and concentrated under reduced pressure. The crude product was purified using silica gel flash chromatography 
to give epoxide II-24 (207 mg, $0.88 \mathrm{mmol}, 88 \%)$ as colorless oil: $R_{f}(20 \%$ EtOAc/hexane) $0.47 ;[\alpha]^{20}{ }_{\mathrm{D}}=-59.3\left(c\right.$ 1.0, MeOH); IR (thin film, $\mathrm{cm}^{-1}$ ) v 3034, 2937, $1726,1455,1369,1256,1146,1060,996,859,699 ;{ }^{1} \mathrm{H} \mathrm{NMR}\left(270 \mathrm{MHz}, \mathrm{CDCl}_{3}\right) \delta 7.36$ (m, 5H), $5.30(\mathrm{~d}, J=1.2 \mathrm{~Hz}, 1 \mathrm{H}), 4.80(\mathrm{~d}, J=11.6 \mathrm{~Hz}, 1 \mathrm{H}), 4.64(\mathrm{~d}, J=11.6 \mathrm{~Hz}, 1 \mathrm{H})$, $4.17(\mathrm{q}, J=6.9 \mathrm{~Hz}, 1 \mathrm{H}), 3.59(\mathrm{dd}, J=3.9,1.2 \mathrm{~Hz}, 1 \mathrm{H}), 3.46(\mathrm{~d}, J=4.0 \mathrm{~Hz}, 1 \mathrm{H}), 1.38(\mathrm{~d}$, $J=6.9 \mathrm{~Hz}, 3 \mathrm{H}) ;{ }^{13} \mathrm{C} \mathrm{NMR}\left(67.5 \mathrm{MHz}, \mathrm{CDCl}_{3}\right) \delta 203.1,136.6,128.7,128.4,128.2,93.3$, 93.2, 72.0, 70.8, 53.8, 53.1; HRMS: Calculated for $\left[\mathrm{C}_{13} \mathrm{H}_{14} \mathrm{O}_{4}+\mathrm{Na}\right]^{+}:$257.0784, Found: 257.0784 .

(1R,2R,4S,5S,6R)-2-(benzyloxy)-4-methyl-3,7-dioxabicyclo[4.1.0]heptan-5-ol (II-18).

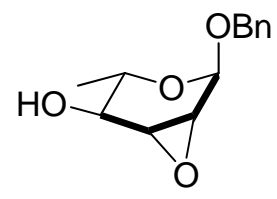

To a solution of epoxide II-20 $(672 \mathrm{mg}, 2.87 \mathrm{mmol})$ in $\mathrm{CH}_{2} \mathrm{Cl}_{2}(3 \mathrm{~mL})$ was added a solution of $\mathrm{CeCl}_{3} / \mathrm{MeOH}(3 \mathrm{~mL}, 0.4 \mathrm{M})$. The mixture was cooled to $-78{ }^{\circ} \mathrm{C}$. $\mathrm{NaBH}_{4}(130$ $\mathrm{mg}, 3.4 \mathrm{mmol}$ ) was added and the reaction mixture was stirred at -78 to $-20{ }^{\circ} \mathrm{C}$ for $4 \mathrm{~h}$. The reaction was quenched with saturated aqueous $\mathrm{NaHCO}_{3}$. The mixture was extracted with $\mathrm{Et}_{2} \mathrm{O}$, and the organic layer was washed with saturated brine, dried over $\mathrm{Na}_{2} \mathrm{SO}_{4}$ and concentrated under reduced pressure. The crude product was purified using silica gel flash chromatography to give epoxide II-18 (576 mg, $2.44 \mathrm{mmol}, 85 \%)$ as colorless oil: $R_{f}\left(80 \%\right.$ EtOAc/hexane) $0.47 ;[\alpha]^{20}{ }_{\mathrm{D}}-62.8$ (c 1.0, MeOH); IR (thin film, $\left.\mathrm{cm}^{-1}\right) v 3430$, 2911, 1454, 1370, 1241, 1114, 1054, 980, 848, 805, 738, 698; ${ }^{1} \mathrm{H}$ NMR (600 MHz, 
$\left.\mathrm{CDCl}_{3}\right) \delta 7.34(\mathrm{~m}, 5 \mathrm{H}), 5.03(\mathrm{~s}, 1 \mathrm{H}), 4.75(\mathrm{~d}, J=11.4 \mathrm{~Hz}, 1 \mathrm{H}), 4.57(\mathrm{~d}, J=11.4 \mathrm{~Hz}, 1 \mathrm{H})$, $3.58(\mathrm{dq}, J=9.0,6.0 \mathrm{~Hz}, 1 \mathrm{H}), 3.45(\mathrm{~d}, J=9.0 \mathrm{~Hz}, 1 \mathrm{H}), 3.23(\mathrm{~d}, J=3.6 \mathrm{~Hz}, 1 \mathrm{H}), 3.12(\mathrm{~d}$, $J=3.6 \mathrm{~Hz}, 1 \mathrm{H}), 1.21(\mathrm{~d}, J=6.0 \mathrm{~Hz}, 3 \mathrm{H}) ;{ }^{13} \mathrm{C} \mathrm{NMR}\left(150 \mathrm{MHz}, \mathrm{CDCl}_{3}\right) \delta 137.5,128.8$, $128.4,128.3,94.5,70.3,68.0,65.8,56.1,50.5,18.5$; HRMS: Calculated for $\left[\mathrm{C}_{13} \mathrm{H}_{16} \mathrm{O}_{4}+\mathrm{H}\right]^{+}:$237.1121, Found: 237.1121.

(2R,3R,4S,5R,6S)-2-(benzyloxy)-6-methyltetrahydro-2H-pyran-3,4,5-triol (II-25).

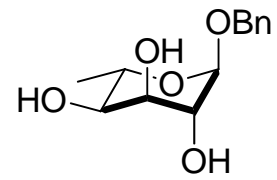

To a round bottom flask was added epoxide II-18 (13 mg, $0.055 \mathrm{mmol})$, HOAc (0.5 ml) and $\mathrm{Sc}(\mathrm{OTf})_{3}(5 \mathrm{mg}, 0.01 \mathrm{mmol})$. The reaction solution was stirred for $1 \mathrm{~h}$ at $\mathrm{RT}$. The reaction mixture was diluted with EtOAc and saturated aqueous $\mathrm{NaHCO}_{3}$ was added at 0 ${ }^{\circ} \mathrm{C}$. The mixture was extracted with EtOAc, and the EtOAc layer was washed with saturated brine, dried over $\mathrm{Na}_{2} \mathrm{SO}_{4}$ and concentrated under reduced pressure. The crude product was dissolved in $\mathrm{THF} / \mathrm{H}_{2} \mathrm{O}(0.4 \mathrm{~mL}, 3: 1)$ at $\mathrm{RT}$, and lithium hydroxide monohydrate $(5 \mathrm{mg}, 0.13 \mathrm{mmol})$ was added to the solution. The reaction mixture was stirred at RT for $5 \mathrm{~h}$. Then the reaction mixture was directly loaded onto silica gel column to give $\alpha$-6-deoxy-altrose II-25 (9.6 $\mathrm{mg}, 0.038 \mathrm{mmol}, 69 \%)$ as colorless oil: $R_{f}($ EtOAc) $0.49 ;[\alpha]^{20}{ }_{\mathrm{D}}-73.3\left(c\right.$ 1.0, MeOH); IR (thin film, $\mathrm{cm}^{-1}$ ) $v 3384,2930,1455,1375,1259$, 1127, 1061, 1014, 970, 852, 737, 698; ${ }^{1} \mathrm{H}$ NMR (600 MHz, $\left.\mathrm{CDCl}_{3}\right) \delta 7.35$ (m, 5H), 4.81 (s, 1H), $4.75(\mathrm{~d}, J=12 \mathrm{~Hz}, 1 \mathrm{H}), 4.55(\mathrm{~d}, J=12 \mathrm{~Hz}, 1 \mathrm{H}), 3.98(\mathrm{dd}, J=3.6,1.8 \mathrm{~Hz}, 1 \mathrm{H})$, 
$3.89(\mathrm{dd}, J=3.6,3.6 \mathrm{~Hz}, 1 \mathrm{H}), 3.79(\mathrm{dq}, J=9.0,6.0 \mathrm{~Hz}, 1 \mathrm{H}), 3.51(\mathrm{dd}, J=9.6,3.6 \mathrm{~Hz}$, 1H), $1.21(\mathrm{~d}, J=6.0 \mathrm{~Hz}, 3 \mathrm{H}) ;{ }^{13} \mathrm{C} \mathrm{NMR}\left(150 \mathrm{MHz}, \mathrm{CDCl}_{3}\right) \delta 136.5,128.9,128.6,128.4$, 99.0, 70.9, 70.1, 69.8, 69.6, 65.8, 17.8; HRMS: Calculated for $\left[\mathrm{C}_{13} \mathrm{H}_{18} \mathrm{O}_{5}+\mathrm{Na}\right]^{+}$: 277.1046, Found: 277.1047.

(2R,3S,4S,5S,6S)-2-(benzyloxy)-4-bromo-6-methyltetrahydro-2H-pyran-3,5-diol (II27).

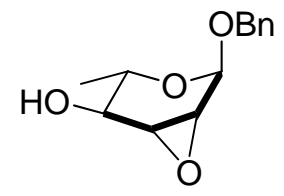

To a round bottom flask was added epoxide II-18 $(96 \mathrm{mg}, 0.41 \mathrm{mmol})$, HOAc $(0.5 \mathrm{~mL})$ and $\mathrm{MgBr}_{2} \bullet \mathrm{Et}_{2} \mathrm{O}(106 \mathrm{mg}, 0.41 \mathrm{mmol})$. The reaction mixture was stirred at RT for $1 \mathrm{~h}$. The reaction mixture was diluted with EtOAc and saturated aqueous $\mathrm{NaHCO}_{3}$ was added at $0{ }^{\circ} \mathrm{C}$. The mixture was extracted with EtOAc, and the organic layer was washed with saturated brine, dried over $\mathrm{Na}_{2} \mathrm{SO}_{4}$ and concentrated under reduced pressure. The crude product was purified using silica gel flash chromatography to give bromide II-27 (137 mg, 0.4 mmol, 98\%) as colorless oil: $R_{f}\left(50 \%\right.$ EtOAc/hexane) $0.28 ;[\alpha]^{25}-57.2$ (c 1.0, $\mathrm{MeOH}$ ); IR (thin film, $\mathrm{cm}^{-1}$ ) $v$ 3402, 2919, 1718, 1454, 1245, 1055, 994, 739; ${ }^{1} \mathrm{H}$ NMR $\left(600 \mathrm{MHz}, \mathrm{CDCl}_{3}\right) \delta 7.34(\mathrm{~m}, 5 \mathrm{H}), 4.80(\mathrm{~d}, J=12.0 \mathrm{~Hz}, 1 \mathrm{H}), 4.72(\mathrm{~d}, J=3.6 \mathrm{~Hz}, 1 \mathrm{H})$, $4.54(\mathrm{~d}, J=12.0 \mathrm{~Hz}, 1 \mathrm{H}), 4.35(\mathrm{dd}, J=6.0,3.6 \mathrm{~Hz}, 1 \mathrm{H}), 4.12(\mathrm{dd}, J=6.0,3.6 \mathrm{~Hz}, 1 \mathrm{H})$, $4.08(\mathrm{qd}, J=6.6,6.6 \mathrm{~Hz}, 1 \mathrm{H}), 3.67(\mathrm{dd}, J=6.0,3.6 \mathrm{~Hz}, 1 \mathrm{H}), 1.27(\mathrm{~d}, J=6.6 \mathrm{~Hz}, 3 \mathrm{H})$; 
${ }^{13} \mathrm{C} \mathrm{NMR}\left(150 \mathrm{MHz}, \mathrm{CDCl}_{3}\right) \delta 137.2,128.4,127.8,127.7,99.0,71.8,70.4,69.8,68.5$, 56.0, 16.9; HRMS: Calculated for $\left[\mathrm{C}_{13} \mathrm{H}_{17} \mathrm{BrO}_{4}+\mathrm{Na}\right]^{+}: 339.0202$, Found: 339.0204 .

(2R,3R,5R,6S)-2-(benzyloxy)-6-methyltetrahydro-2H-py-ran-3,5-diol (II-26).

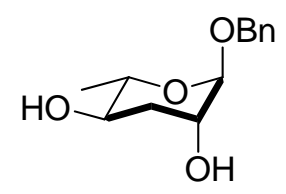

To a solution of bromide II-27 $(17 \mathrm{mg}, 0.05 \mathrm{mmol})$ in dry toluene $(0.5 \mathrm{ml})$, tris(trimethylsilyl)silane (TTMSS) $(0.033 \mathrm{ml}, 0.1 \mathrm{mmol})$ and AIBN (4 mg, $0.025 \mathrm{mmol})$ was added at $0{ }^{\circ} \mathrm{C}$. At this stage reaction mixture was degassed using freeze-pump-thaw technique three times. Then the reaction mixture was stirred at $80{ }^{\circ} \mathrm{C}$ for $4 \mathrm{~h}$ under Ar. The mixture was directly loaded onto silica gel column for purification to give $\alpha$ ascarylose II-28 (10 mg, $0.038 \mathrm{mmol}, 76 \%)$ as colorless oil.

Alternative procedure for (II-26): To a round bottom flask was added epoxide 18 (23 $\mathrm{mg}, 0.094 \mathrm{mmol})$, THF $(0.5 \mathrm{ml})$ and $\mathrm{LiAlH}_{4}(7.1 \mathrm{mg}, 0.19 \mathrm{mmol})$ at $0{ }^{\circ} \mathrm{C}$. The reaction solution was stirred for $30 \mathrm{~min}$ at $0{ }^{\circ} \mathrm{C}$. Water was added dropwise to quench the reaction, then the mixture was extracted with EtOAc. The organic layer was washed with saturated brine, dried over $\mathrm{Na}_{2} \mathrm{SO}_{4}$ and concentrated under reduced pressure. The crude product was purified using silica gel flash chromatography to give $\alpha$-ascarylose 26 (19 $\mathrm{mg}, 0.08 \mathrm{mmol}, 85 \%)$ as colorless oil: $R_{f}\left(60 \%\right.$ EtOAc/hexane) $0.17 ;[\alpha]^{20}{ }_{\mathrm{D}}-90.8$ (c 1.0, $\mathrm{MeOH}$ ); IR (thin film, $\mathrm{cm}^{-1}$ ) v 3405, 2935, 1718, 1686, 1256, 1123, 1080, 995; ${ }^{1} \mathrm{H}$ NMR $\left(600 \mathrm{MHz}, \mathrm{CDCl}_{3}\right) \delta 7.34(\mathrm{~m}, 5 \mathrm{H}), 4.74(\mathrm{~d}, J=12.0 \mathrm{~Hz}, 1 \mathrm{H}), 4.68(\mathrm{~s}, 1 \mathrm{H}), 4.53(\mathrm{~d}, J=$ 
$12.0 \mathrm{~Hz}, 1 \mathrm{H}), 3.91(\mathrm{dd}, J=4.8,3.0 \mathrm{~Hz}, 1 \mathrm{H}), 3.70(\mathrm{dq}, J=9.6,6.0 \mathrm{~Hz}, 1 \mathrm{H}), 3.61(\mathrm{ddd}, J=$ $10.8,9.0,4.2 \mathrm{~Hz}, 1 \mathrm{H}), 2.09(\mathrm{ddd}, J=13.2,3.6,3.6 \mathrm{~Hz}, 1 \mathrm{H}), 1.89(\mathrm{ddd}, J=13.2,11.4,3.0$ $\mathrm{Hz}, 1 \mathrm{H}), 1.30(\mathrm{~d}, J=6.6 \mathrm{~Hz}, 3 \mathrm{H}) ;{ }^{13} \mathrm{C} \mathrm{NMR}\left(150 \mathrm{MHz}, \mathrm{CDCl}_{3}\right) \delta 137.4,128.5,127.9$, 127.8, 98.2, 69.8, 69.0, 68.8, 68.1, 35.2, 17.7; HRMS: Calculated for $\left[\mathrm{C}_{13} \mathrm{H}_{18} \mathrm{O}_{4}+\mathrm{H}\right]^{+}$: 239.0202, Found: 239.0204.

\section{(+/-) undec-3-yn-2-ol (III-22a)}

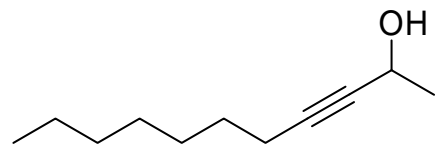

A solution of $n$-BuLi in hexane $(48 \mathrm{~mL}, 121 \mathrm{mmol})$ was added dropwise to the solution of 1-nonyne III-22 $(20 \mathrm{~mL}, 121 \mathrm{mmol})$ in dry THF $(120 \mathrm{~mL})$ at $-78{ }^{\circ} \mathrm{C}$ under argon. After $30 \mathrm{~min}$, acetaldehyde $(6.7 \mathrm{~mL}, 120 \mathrm{mmol})$ was added. The reaction was stirred for 10 min at $0{ }^{\circ} \mathrm{C}$ and then the resulting solution was warmed to room temperature for 50 min. Additional $\mathrm{CH}_{2} \mathrm{Cl}_{2}(80 \mathrm{ml})$ was added to the reaction mixture followed by addition of $80 \mathrm{ml}$ saturated aqueous $\mathrm{NH}_{4} \mathrm{Cl}(80 \mathrm{ml})$. The aqueous phase was separated and extracted with $\mathrm{CH}_{2} \mathrm{Cl}_{2}$. The combined organic layers were washed with brine, dried $\left(\mathrm{Na}_{2} \mathrm{SO}_{4}\right)$, filtered and concentrated. Chromatography on silica gel $(10 \%$ EtOAc/hexane $)$ gave alcohol III-22a $(16.8 \mathrm{~g}, 96.8 \mathrm{mmol}, 80 \%)^{4}$ as a colorless oil; $R f(20 \%$ EtOAc/hexane $)=0.32 ;$ IR (thin film, $\left.\mathrm{cm}^{-1}\right), 3345,2930,2857,2227,1467,1156,1078$; ${ }^{1} \mathrm{H}$ NMR $\left(600 \mathrm{MHz}, \mathrm{CDCl}_{3}\right) \delta 4.48(\mathrm{qdt}, J=6.6,4.8,1.8 \mathrm{~Hz} 1 \mathrm{H}), 4.15(\mathrm{td}, J=7.2,1.8$

\footnotetext{
${ }^{4}$ James, S. A.; Edward, R. D.; Antonio, Z. J. Org. Chem. 1989.54, 5854-5
} 
$\mathrm{Hz}, 2 \mathrm{H}), 1.93(\mathrm{~d}, J=4.8 \mathrm{~Hz}, 1 \mathrm{H}), 1.46$ (tt, $J=7.2,7.2 \mathrm{~Hz}, 2 \mathrm{H}), 1.38(\mathrm{~d}, J=6.6 \mathrm{~Hz}, 3 \mathrm{H})$, 1.35-1.25 (m, 8H), $0.85(\mathrm{t}, J=7.2 \mathrm{~Hz}, 3 \mathrm{H}) ;{ }^{13} \mathrm{C} \mathrm{NMR}\left(150 \mathrm{MHz}, \mathrm{CD}_{3} \mathrm{Cl}\right) \delta 84.9,82.5$, $58.8,31.9,29.0,28.9,28.8,25.0,22.8,18.8,14.2$.

undec-3-yn-2-one (III-21)

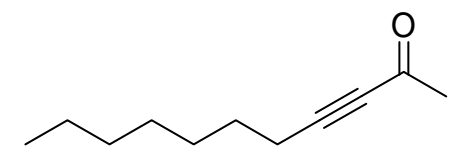

To a solution of alcohol III-22a (22 g, $131 \mathrm{mmol})$ in THF $(100 \mathrm{~mL})$ at room temperature was added activated $\mathrm{MnO}_{2}(65 \mathrm{~g})$. The reaction mixture was stirred for $24 \mathrm{~h}$ and then filtered through celite and concentrated under pressure to give ketone III-21 (18.7 g, 116 mmol, $86 \%)^{5}$ as a colorless oil. $R f(20 \%$ EtOAc/hexane $)=0.70 ;$ IR (thin film, $\left.\mathrm{cm}^{-1}\right)$, 2926, 2213, 1750, 1358, 1228; ${ }^{1} \mathrm{H}$ NMR $\left(600 \mathrm{MHz}, \mathrm{CDCl}_{3}\right) \delta 2.35(\mathrm{t}, J=7.2 \mathrm{~Hz}, 1 \mathrm{H})$, $2.32(\mathrm{~s}, 3 \mathrm{H}), 1.57(\mathrm{tt}, J=7.2,7.2 \mathrm{~Hz}, 2 \mathrm{H}), 1.41-1.26(\mathrm{~m}, 8 \mathrm{H}), 0.89(\mathrm{t}, J=7.2,3 \mathrm{H}) ;{ }^{13} \mathrm{C}$ NMR $\left(150 \mathrm{MHz}, \mathrm{CD}_{3} \mathrm{Cl}\right) \delta 184.9,94.2,81.4,32.7,31.6,28.8,28.7,27.7,22.6,18.9$, 14.0.

(R)-undec-3-yn-2-ol (III-23)

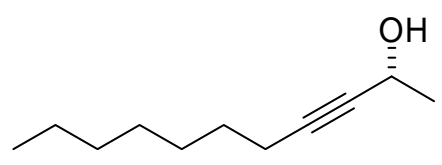

${ }^{5}$ Marshall, J. A.; Robinson, E. D.; Antonio, Z. J. Org. Chem. 1989.54, 5854-5 
To a flask was added ketone III-21 $(0.136 \mathrm{~g}, 0.82 \mathrm{mmol}), \mathrm{CH}_{2} \mathrm{Cl}_{2}(0.42 \mathrm{~mL})$, formic $\operatorname{acid} / \mathrm{Et}_{3} \mathrm{~N}(5: 4,1.5 \mathrm{~mL})$ and Noyori asymmetric transfer hydrogenation catalyst $(S)$ $\operatorname{Ru}\left(\eta^{6}\right.$-mesitylene)-(R,R)-TsDPEN (2.5 mg, $\left.0.0016 \mathrm{mmol}, 0.2 \mathrm{~mol} \%\right)$. The resulting solution was stirred at room temperature for $24 \mathrm{~h}$, water was added to dilute and the reaction mixture was extracted with saturated $\mathrm{NaHCO}_{3}$, dried over $\mathrm{Na}_{2} \mathrm{SO}_{4}$ and concentrated under reduced pressure to afford a crude oil. The crude product was purified by silica gel flash chromatography eluting with $10 \%$ EtOAc/hexane to give alcohol III-23 $(0.118 \mathrm{~g}, 0.71 \mathrm{mmol}, 86 \%)^{6}$ as a colorless oil. $R f\left(20 \% \mathrm{Et}_{2} \mathrm{O} / \mathrm{hexane}\right)=$ $0.32 ;[\alpha]^{25}=+18(\mathrm{MeOH}) ;$ IR (thin film, $\left.\mathrm{cm}^{-1}\right), 3303,2926,2856,2253,1458,1376$, 1076, 889; ${ }^{1} \mathrm{H}$ NMR $\left(600 \mathrm{MHz}, \mathrm{CDCl}_{3}\right) \delta 4.48(\mathrm{qdt}, J=6.6,4.8,1.8 \mathrm{~Hz} 1 \mathrm{H}), 4.15(\mathrm{td}, J$ $=7.2,1.8 \mathrm{~Hz}, 2 \mathrm{H}), 1.93(\mathrm{~d}, J=4.8 \mathrm{~Hz}, 1 \mathrm{H}), 1.46(\mathrm{tt}, J=7.2,7.2 \mathrm{~Hz}, 2 \mathrm{H}), 1.38(\mathrm{~d}, J=$ $6.6 \mathrm{~Hz}, 3 \mathrm{H}), 1.35-1.25(\mathrm{~m}, 8 \mathrm{H}), 0.85(\mathrm{t}, J=7.2 \mathrm{~Hz}, 3 \mathrm{H}) ;{ }^{13} \mathrm{C} \mathrm{NMR}\left(150 \mathrm{MHz}, \mathrm{CD}_{3} \mathrm{Cl}\right) \delta$ $84.9,82.5,58.8,31.9,29.0,28.9,28.8,25.0,22.8,18.8,14.2$.

\section{(R)-undec-10-yn-2-ol (III-23a)}

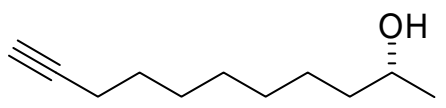

A $30 \%$ suspension of $\mathrm{KH}$ in mineral oil $(171 \mathrm{mg}, 4.28 \mathrm{mmol})$ was washed three times under Argon with dry ether and then the excess solvent was removed under vacuum, Dry 1,3-diaminopropane $(1.14 \mathrm{~mL})$ was added with cooling $\left(15^{\circ} \mathrm{C}\right)$. After the mixture was

${ }^{6}$ Marshall, J. A.; Wang, X.; J. Org. Chem. 1990.55, 2995-6 
stirred for $1 \mathrm{~h}$, the alkynol III-23 (172 $\mathrm{mg}, 1.02 \mathrm{mmol})$ was added dropwise (10 min) at $\left(10-15^{\circ} \mathrm{C}\right)$ to the mixture and the resulting slurry was stirred further under argon for 2 hrs. With cooling $\left(0{ }^{\circ} \mathrm{C}\right)$, water $(11 \mathrm{~mL})$ was added dropwise and the mixture was extracted with ether, the combined extracts were washed with $1 \mathrm{~N} \mathrm{HCl}$, saturated aqueous sodium bicarbonate and brine, and then dried with anhydrous sodium sulfate. Flash chromatography gave $(R)$-undec-10-yn-2-ol III-23a (143 mg, 85\%) as a colorless oil: $[\alpha]^{25}{ }_{\mathrm{D}}=-4\left(\mathrm{CH}_{2} \mathrm{Cl}_{2}\right) ; \operatorname{Rf}(30 \%$ EtOAc/Hexane $)=0.32 ;$ IR (thin film, $\left.\mathrm{cm}^{-1}\right) 3296,2931$, $2856,2116,1611,1512,1244,1034,821 ;{ }^{1} \mathrm{H}$ NMR $\left(600 \mathrm{MHz}, \mathrm{CDCl}_{3}\right) \delta 3.79(\mathrm{t}, J=5.4$ $\mathrm{Hz}, 1 \mathrm{H}), 2.18(\mathrm{td}, J=7.2,2.4 \mathrm{~Hz}, 2 \mathrm{H}), 1.93(\mathrm{t}, J=2.4 \mathrm{~Hz}, 1 \mathrm{H}), 1.57 \sim 1.25(\mathrm{~m}, 12 \mathrm{H}), 1.18$ $(\mathrm{d}, J=6.0 \mathrm{~Hz}, 3 \mathrm{H}) ;{ }^{13} \mathrm{C} \mathrm{NMR}\left(150 \mathrm{MHz}, \mathrm{CDCl}_{3}\right) \delta 84.7,68.2,68.1,39.3,29.5,29.0$, 28.7, 28.4, 25.7, 23.5, 18.4; ESI HRMS Calcd for $\left[\mathrm{C}_{11} \mathrm{H}_{20} \mathrm{O}+\mathrm{H}\right]^{+}: 169.1587$, Found: 169.1586.

\section{(R)-tert-butyldimethyl(undec-10-yn-2-yloxy)silane (III-24)}

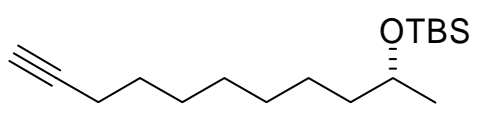

To a magnetically stirred solution of alcohol III-23a (300 mg, $1.79 \mathrm{mmol})$ and imidazole (304 mg, $4.48 \mathrm{mmol})$ in dry $\mathrm{CH}_{2} \mathrm{Cl}_{2}(3 \mathrm{~mL})$ was added dropwise $\mathrm{TBSCl}(281 \mathrm{mg}, 1.87$ mmol) at $0{ }^{\circ} \mathrm{C}$. The reaction mixture was warmed to room temperature and stirred overnight. Water was added and then the reaction mixture was extracted with $\mathrm{Et}_{2} \mathrm{O}$, dried with $\mathrm{Na}_{2} \mathrm{SO}_{4}$ and evaporated under reduced pressure to leave the crude product which was then purified using silica gel flash chromatography to give alkyne III-24 (490 mg, 
$1.74 \mathrm{mmol}, 97 \%)$ as a colorless oil. $R f\left(5 \% \mathrm{Et}_{2} \mathrm{O} /\right.$ hexane $)=0.68 ;[\alpha]^{25}=-13\left(\mathrm{CH}_{2} \mathrm{Cl}_{2}\right)$; IR (thin film, $\mathrm{cm}^{-1}$ ), 3314, 2929, 2857, 2253, 1463, 1374, 1254, 1134, 1069, 1005, 834, 807, 773; ${ }^{1} \mathrm{H}$ NMR $\left(600 \mathrm{MHz}, \mathrm{CDCl}_{3}\right) \delta 3.76(\mathrm{qdd}, J=6.0,6.0,6.0 \mathrm{~Hz}, 1 \mathrm{H}), 2.16(\mathrm{td}, J=$ 6.6, $2.4 \mathrm{~Hz}, 2 \mathrm{H}), 1.92(\mathrm{t}, J=2.4 \mathrm{~Hz}, 1 \mathrm{H}), 1.58-1.26(\mathrm{~m}, 12 \mathrm{H}), 1.11(\mathrm{~d}, J=6.0 \mathrm{~Hz}, 3 \mathrm{H})$, $0.88(\mathrm{~s}, 9 \mathrm{H}), 0.04(\mathrm{~s}, 6 \mathrm{H}) ;{ }^{13} \mathrm{C} \mathrm{NMR}\left(150 \mathrm{MHz}, \mathrm{CD}_{3} \mathrm{Cl}\right) \delta 84.8,68.6,68.0,39.7,29.5$, 29.2, 29.0, 28.7, 28.5, 26.0, 25.7, 23.8, 18.4, 18.2, - 4.37, - 4.69 ; ESI HRMS Calcd for $\left[\mathrm{C}_{17} \mathrm{H}_{34} \mathrm{OSi}+\mathrm{H}\right]^{+}:$283.2452, Found: 283.2453.

\section{(R)-ethyl 11-(tert-butyldimethylsilyloxy)dodec-2-ynoate (III-24a)}

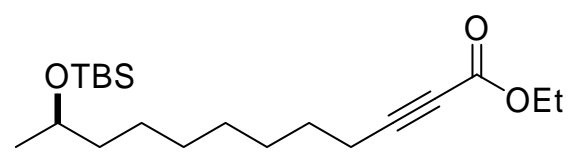

To a solution of alkyne III-24 $(358 \mathrm{mg}, 1.27 \mathrm{mmol})$ in THF $(2.5 \mathrm{~mL})$ at $-78{ }^{\circ} \mathrm{C}$ was added $n$-BuLi $(0.5 \mathrm{~mL}, 1.4 \mathrm{mmol})$ dropwise and the reaction mixture was stirred for $1 \mathrm{~h}$. Ethylchloroformate $(0.15 \mathrm{~mL}, 1.55 \mathrm{mmol})$ was added at $-78{ }^{\circ} \mathrm{C}$ and the reaction mixture was stirred for another $1 \mathrm{~h}$. The reaction mixture was then warmed to $0{ }^{\circ} \mathrm{C}$ and stirred for $15 \mathrm{~min}$. The reaction was quenched with saturated aqueous $\mathrm{NH}_{4} \mathrm{Cl}(10 \mathrm{~mL})$ and then extracted with $\mathrm{Et}_{2} \mathrm{O}$, washed with brine and dried with $\mathrm{Na}_{2} \mathrm{SO}_{4}$. Evaporated under reduced pressure gave the crude product which was then purified using silica gel flash chromatography to give ester III-24a $(373 \mathrm{mg}, 1.05 \mathrm{mmol}, 83 \%)$ as a colorless oil. $R f$ $\left(10 \% \mathrm{Et}_{2} \mathrm{O} /\right.$ hexane $)=0.65 ;[\alpha]^{25}{ }_{\mathrm{D}}=-7\left(\mathrm{CH}_{2} \mathrm{Cl}_{2}\right) ;$ IR (thin film, $\left.\mathrm{cm}^{-1}\right), 2930,2857,2235$, $1713,1366,1246,1134,1070,1005,834,807,773,752 ;{ }^{1} \mathrm{H}$ NMR $\left(600 \mathrm{MHz}, \mathrm{CDCl}_{3}\right) \delta$ 
$4.20(\mathrm{q}, J=7.2 \mathrm{~Hz}, 2 \mathrm{H}), 3.75(\mathrm{qdd}, J=6.0,6.0,6.0 \mathrm{~Hz}, 1 \mathrm{H}), 2.31(\mathrm{t}, J=7.2 \mathrm{~Hz}, 2 \mathrm{H})$, $1.57(\mathrm{tt}, J=7.2,7.2 \mathrm{~Hz}, 2 \mathrm{H}), 1.44-1.22(\mathrm{~m}, 10 \mathrm{H}), 1.30(\mathrm{t}, J=7.2 \mathrm{~Hz}, 3 \mathrm{H}), 1.10(\mathrm{~d}, J=$ $6.0 \mathrm{~Hz}, 3 \mathrm{H}), 0.88(\mathrm{~s}, 9 \mathrm{H}), 0.04(\mathrm{~s}, 3 \mathrm{H}), 0.03(\mathrm{~s}, 3 \mathrm{H}) ;{ }^{13} \mathrm{C} \mathrm{NMR}\left(150 \mathrm{MHz}, \mathrm{CD}_{3} \mathrm{Cl}\right) \delta$ $153.9,89.4,73.2,68.6,61.7,39.7,29.4,29.0,28.8,27.5,25.9,25.7,23.8,18.6,18.1$, 14.0, - 4.20, - 4.51; ESI HRMS Calcd for $\left[\mathrm{C}_{20} \mathrm{H}_{38} \mathrm{O}_{3} \mathrm{Si}+\mathrm{H}\right]^{+}: 355.2663$, Found: 355.2665.

\section{(R, 2E, 4E)-ethyl 11-(tert-butyldimethylsilyloxy)dodeca-2, 4-dienoate (III-25)}<smiles>CCOC(=O)/C=C/C=C/CCCCCC(C)[OH2+]</smiles>

In to a round bottom flask were added ester III-24a (276 mg, $0.78 \mathrm{mmol}), \mathrm{PPh}_{3}(204 \mathrm{mg}$, $0.78 \mathrm{mmol})$, phenol $(73 \mathrm{mg}, 0.78 \mathrm{mmol})$ and benzene $(1.5 \mathrm{~mL})$. The mixture was stirred at $50{ }^{\circ} \mathrm{C}$ for $12 \mathrm{~h}$. The solution was diluted with ether $(1 \mathrm{~mL})$ and $1 \mathrm{~N} \mathrm{NaOH}(1 \mathrm{~mL})$. The layer was separated and the aqueous layer was extracted with ether. The combined organic layers were washed (water, brine), dried $\left(\mathrm{Na}_{2} \mathrm{SO}_{4}\right)$ and concentrated. The residue was dissolved in ether $(3 \mathrm{~mL})$ and MeI $(0.06 \mathrm{~mL})$ was added to the solution. The reaction mixture was refluxed for $12 \mathrm{hrs}$. Then the solution was filtered, concentrated and purified by flash chromatography on silica gel to give diene III-25 (249 $\mathrm{mg}, 0.70 \mathrm{mmol})$ as a light yellow oil. $R f\left(10 \% \mathrm{Et}_{2} \mathrm{O} /\right.$ hexane $)=0.64 ;[\alpha]^{25}{ }_{\mathrm{D}}=-14\left(\mathrm{CH}_{2} \mathrm{Cl}_{2}\right)$; IR (thin film, $\left.\mathrm{cm}^{-1}\right)$, $2930,2857,2200,1717,1643,1368,1255,1138,1047,1001,836,774 ;{ }^{1} \mathrm{H}$ NMR $(600$ $\left.\mathrm{MHz}, \mathrm{CDCl}_{3}\right) \delta 7.25(\mathrm{dd}, J=15.6,10.2 \mathrm{~Hz}, 1 \mathrm{H}), 6.16(\mathrm{dd}, J=15.0,10.2 \mathrm{~Hz}, 1 \mathrm{H}), 6.11$ $(\mathrm{dt}, J=15.0,6.6 \mathrm{~Hz}, 1 \mathrm{H}), 5.77(\mathrm{~d}, J=15.6 \mathrm{~Hz}, 1 \mathrm{H}), 4.19$ (q, $J=7.2 \mathrm{~Hz}, 2 \mathrm{H}), 3.76(\mathrm{qdd}, J$ $=6.0,6.0,6.0 \mathrm{~Hz}, 1 \mathrm{H}), 2.16(\mathrm{dt}, J=6.6,6.6 \mathrm{~Hz}, 2 \mathrm{H}), 1.44-1.26(\mathrm{~m}, 8 \mathrm{H}), 1.29(\mathrm{t}, J=7.2$ 
$\mathrm{Hz}, 3 \mathrm{H}), 1.10(\mathrm{~d}, J=6.0 \mathrm{~Hz}, 3 \mathrm{H}), 0.88(\mathrm{~s}, 9 \mathrm{H}), 0.04(\mathrm{~s}, 6 \mathrm{H}) ;{ }^{13} \mathrm{C} \mathrm{NMR}(150 \mathrm{MHz}$, $\left.\mathrm{CD}_{3} \mathrm{Cl}\right) \delta 167.3,145.1,144.6,128.4,119.2,68.6,60.1,39.6,32.9,29.2,28.7,26.0,23.8$, 18.2, 14.3, - 4.40, - 4.69; ESI HRMS Calcd for $\left[\mathrm{C}_{20} \mathrm{H}_{38} \mathrm{O}_{3} \mathrm{Si}+\mathrm{H}\right]^{+}:$355.2663, Found: 355.2664 .

(4R,5R,11R,E)-ethyl11-(tert-butyldimethylsilyloxy)-4,5-dihydroxydodec-2-enoate (III-19)<smiles>CCOC(=O)/C=C/C(O)C(O)CCCCCC(C)O[Sb](C)(=O)c1ccccc1</smiles>

In a round bottom flask were added $t$ - $\mathrm{BuOH}(40 \mathrm{~mL})$, water $(40 \mathrm{~mL}), \mathrm{K}_{3} \mathrm{Fe}(\mathrm{CN})_{6}(8.29 \mathrm{~g}$, $25.31 \mathrm{mmol}), \mathrm{K}_{2} \mathrm{CO}_{3}(3.49 \mathrm{~g}, 35.31 \mathrm{mmol}), \mathrm{MeSO}_{2} \mathrm{NH}_{2}(806 \mathrm{mg}, 8.48 \mathrm{mmol})$, (DHQD) ${ }_{2}-$ PHAL (130 mg), and $\mathrm{O}_{\mathrm{S}} \mathrm{O}_{4}(3 \mathrm{mg}$.). The reaction mixture was stirred at room temperature for about $15 \mathrm{~min}$. and then cooled to $0{ }^{\circ} \mathrm{C}$. To this solution was added diene III-25 (3.00 $\mathrm{g}, 8.48 \mathrm{mmol}$ ) and the reaction was stirred at $0{ }^{\circ} \mathrm{C}$ overnight. The reaction was quenched with saturated aqueous sodium sulfite $(30 \mathrm{~mL})$ at room temperature and then extracted with ethyl acetate, washed with $2 \mathrm{~N} \mathrm{NaOH}$ and brine to remove methanesulfonamide. After removal of solvents in vacuum, flash chromatography on silica gel gave diol III-19 $(2.70 \mathrm{~g}, 6.95 \mathrm{mmol}, 82 \%)$ as a colorless oil. $R f\left(30 \% \mathrm{Et}_{2} \mathrm{O} / \mathrm{hexane}\right)=0.40 ;[\alpha]_{\mathrm{D}}^{25}=-14$ $\left(\mathrm{CH}_{2} \mathrm{Cl}_{2}\right)$; IR (thin film, $\mathrm{cm}^{-1}$ ), 3406, 2932, 2859, 1720, 1463, 1370, 1256, 1177, 1047, 836, 774; ${ }^{1} \mathrm{H}$ NMR $\left(600 \mathrm{MHz}, \mathrm{CDCl}_{3}\right) \delta 6.93(\mathrm{dd}, J=15.6,5.4 \mathrm{~Hz}, 1 \mathrm{H}), 6.14(\mathrm{dd}, J=$ 15.6, $1.8 \mathrm{~Hz}, 1 \mathrm{H}), 4.21(\mathrm{q}, J=7.2 \mathrm{~Hz}, 2 \mathrm{H}), 4.12$ (ddd, $J=5.4,5.4,1.8 \mathrm{~Hz}, 1 \mathrm{H}), 3.76$ 
(qdd, $J=6.0,6.0,6.0 \mathrm{~Hz}, 1 \mathrm{H}), 3.55(\mathrm{ddd}, J=5.4,4.2,3.6 \mathrm{~Hz}, 1 \mathrm{H}), 1.54-1.25(\mathrm{~m}, 10 \mathrm{H})$, $1.30(\mathrm{t}, J=7.2 \mathrm{~Hz}, 3 \mathrm{H}), 1.11(\mathrm{~d}, J=6.0 \mathrm{~Hz}, 3 \mathrm{H}), 0.88(\mathrm{~s}, 9 \mathrm{H}), 0.04(\mathrm{~s}, 6 \mathrm{H}) ;{ }^{13} \mathrm{C} \mathrm{NMR}$ $\left(150 \mathrm{MHz}, \mathrm{CD}_{3} \mathrm{Cl}\right) \delta 166.2,146.6,122.6,74.1,74.0,68.9,60.6,39.6,33.1,29.6,25.9$, 25.7, 25.6, 23.8, 18.1, 14.2, - 4.42, - 4.75; ESI HRMS Calcd for $\left[\mathrm{C}_{20} \mathrm{H}_{40} \mathrm{O} 5 \mathrm{~S}^{\mathrm{Si}+\mathrm{H}}\right]^{+}$: 389.2718, Found: 389.2719.

$(4 S, 5 S, 11 R, E)$-ethyl

11-(tert-butyldimethylsilyloxy)-4,5-dihydroxydodec-2-enoate (III-20)<smiles>CCOC(=O)/C=C/[C@@H](O)[C@H](O)CCCCCC(C)O[Sb]</smiles>

In a round bottom flask were added $t$ - $\mathrm{BuOH}(2 \mathrm{~mL})$, water $(2 \mathrm{~mL}), \mathrm{K}_{3} \mathrm{Fe}(\mathrm{CN})_{6}(649 \mathrm{mg}$, $1.97 \mathrm{mmol}), \mathrm{K}_{2} \mathrm{CO}_{3}(272 \mathrm{mg}, 1.97 \mathrm{mmol}), \mathrm{MeSO}_{2} \mathrm{NH}_{2}(71.8 \mathrm{mg}, 0.65 \mathrm{mmol}),(\mathrm{DHQ})_{2}-$ PHAL (10 mg), and $\mathrm{O}_{\mathrm{S}} \mathrm{O}_{4}(4 \mathrm{mg}$.). The reaction mixture was stirred at room temperature for about $15 \mathrm{~min}$. and then cooled to $0{ }^{\circ} \mathrm{C}$. To this solution was added diene III-25 (233 $\mathrm{mg}, 0.65 \mathrm{mmol}$ ) and the reaction was stirred at $0{ }^{\circ} \mathrm{C}$ overnight. The reaction was quenched with saturated aqueous sodium sulfite $(3 \mathrm{~mL})$ at room temperature and then extracted with ethyl acetate, washed with $2 \mathrm{~N} \mathrm{NaOH}$, and brine to remove methanesulfonamide. After removal of solvents in vacuum, flash chromatography on silica gel gave diol III-20 (202 $\mathrm{mg}, 0.52 \mathrm{mmol}, 80 \%)$ as a colorless oil. $R f(30 \%$ $\mathrm{Et}_{2} \mathrm{O} /$ hexane $)=0.40 ;[\alpha]^{25}=-31\left(\mathrm{CH}_{2} \mathrm{Cl}_{2}\right) ; \mathrm{IR}$ (thin film, $\left.\mathrm{cm}^{-1}\right), 3402,2930,2857$, $1721,1658,1370,1254,1133,1040,835,774 ;{ }^{1} \mathrm{H}$ NMR $\left(600 \mathrm{MHz}, \mathrm{CDCl}_{3}\right) \delta 6.91(\mathrm{dd}, J$ 
$=15.6,4.8 \mathrm{~Hz}, 1 \mathrm{H}), 6.14(\mathrm{dd}, J=15.6,1.2 \mathrm{~Hz}, 1 \mathrm{H}), 4.17(\mathrm{q}, J=7.2 \mathrm{~Hz}, 2 \mathrm{H}), 4.11(\mathrm{ddd}, J$ $=5.4,5.4,1.2 \mathrm{~Hz}, 1 \mathrm{H}), 3.76(\mathrm{qdd}, J=6.0,6.0,6.0 \mathrm{~Hz}, 1 \mathrm{H}), 3.53(\mathrm{ddd}, J=4.8,4.8,4.8$ $\mathrm{Hz}, 1 \mathrm{H}), 1.54-1.25(\mathrm{~m}, 10 \mathrm{H}), 1.28(\mathrm{t}, J=7.2 \mathrm{~Hz}, 3 \mathrm{H}), 1.11(\mathrm{~d}, J=6.0 \mathrm{~Hz}, 3 \mathrm{H}), 0.87(\mathrm{~s}$, 9H), 0.03 (s, 3H), $0.02(\mathrm{~s}, 3 \mathrm{H}) ;{ }^{13} \mathrm{C} \mathrm{NMR}\left(150 \mathrm{MHz}, \mathrm{CD}_{3} \mathrm{Cl}\right) \delta 166.4,146.9,122.4,74.1$, $74.0,68.6,60.6,39.6,33.1,29.6,25.9,25.7,25.6,23.8,18.1,14.2,-4.43,-4.74$; ESI HRMS Calcd for $\left[\mathrm{C}_{20} \mathrm{H}_{40} \mathrm{O}_{5} \mathrm{Si}+\mathrm{H}\right]^{+}: 389.2718$, Found: 389.2720 .

\section{(E)-ethyl-3-((4R,5R)-5-((R)-6-hydroxyheptyl)-2,2-dimethyl-1,3-dioxolan-4-yl)-} acrylate (III-19a)

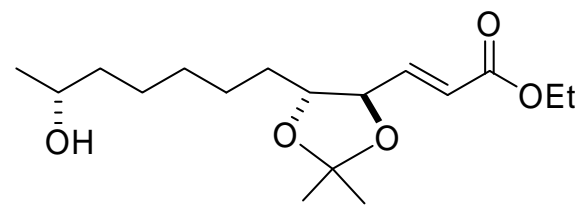

The monohydrate of $p$-toluenesulfonic acid $(100 \mathrm{mg}, 5.27 \mathrm{mmol})$ was added to a solution of diol III-19 (2.05 g, $5.27 \mathrm{mmol})$, 2,2-dimethyoxypropane (1.5 mL, $10.54 \mathrm{mmol})$ and $\mathrm{CH}_{2} \mathrm{CH}_{2}(4 \mathrm{~mL})$. The solution was stirred for $5 \mathrm{~h}$. The reaction mixture was diluted with $\mathrm{CH}_{2} \mathrm{CH}_{2}$ and washed with $1 \mathrm{~N} \mathrm{NaOH}$. The organic layer was dried over $\mathrm{Na}_{2} \mathrm{SO}_{4}$, filtered and concentrated to give the crude product. The crude product was further purified by column chromatography to give alcohol III-19a (1.33 g, $4.25 \mathrm{mmol}, 81 \%)$ as a colorless oil. $R f\left(30 \% \mathrm{Et}_{2} \mathrm{O} /\right.$ hexane $)=0.40 ;[\alpha]_{\mathrm{D}}^{25}=+24\left(\mathrm{CH}_{2} \mathrm{Cl}_{2}\right) ;$ IR (thin film, $\left.\mathrm{cm}^{-1}\right), 3473$, $2934,2861,1722,1661,1371,1259,1174,1036,979 ;{ }^{1} \mathrm{H}$ NMR $\left(600 \mathrm{MHz}, \mathrm{CDCl}_{3}\right) \delta$ $6.85(\mathrm{dd}, J=15.6,6.0 \mathrm{~Hz}, 1 \mathrm{H}), 6.10(\mathrm{dd}, J=15.6,1.2 \mathrm{~Hz}, 1 \mathrm{H}), 4.20(\mathrm{q}, J=7.2 \mathrm{~Hz}, 2 \mathrm{H})$, $4.11(\mathrm{ddd}, J=6.6,6.0,1.2 \mathrm{~Hz}, 1 \mathrm{H}), 3.78(\mathrm{qdd}, J=6.0,6.0,6.0 \mathrm{~Hz}, 1 \mathrm{H}), 3.72(\mathrm{ddd}, J=$ 
6.6, 5.4, 3.0 Hz, 1H), 1.61-1.24 (m, 10H), $1.43(\mathrm{~s}, 3 \mathrm{H}), 1.40(\mathrm{~s}, 3 \mathrm{H}), 1.29$ (t, J= 7.2 Hz, 3H), $1.17(\mathrm{~d}, J=6.0 \mathrm{~Hz}, 3 \mathrm{H}) ;{ }^{13} \mathrm{C} \mathrm{NMR}\left(150 \mathrm{MHz}, \mathrm{CD}_{3} \mathrm{Cl}\right) \delta 166.0,144.1,122.8,109.4$, $80.6,80.2,68.0,60.6,39.2,31.9,29.5,27.2,26.7,25.9,25.5,23.5,14.2$; ESI HRMS Calcd for $\left[\mathrm{C}_{17} \mathrm{H}_{30} \mathrm{O}_{5}+\mathrm{Na}\right]^{+}:$337.1986, Found: 337.1987 .

(E)-ethyl-3-((4S,5S)-5-((R)-6-hydroxyheptyl)-2,2-dimethyl-1,3-dioxolan-4-yl)acrylate (III-20a)

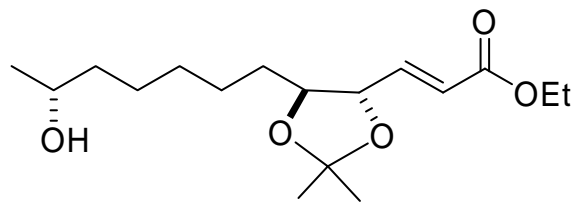

The monohydrate of $p$-toluenesulfonic acid $(2.3 \mathrm{mg}, 0.012 \mathrm{mmol})$ was added to a solution of diol III-20 (95.5 mg, $0.246 \mathrm{mmol}), 2$,2-dimethyoxypropane (0.06 mL, $0.492 \mathrm{mmol})$ and $\mathrm{CH}_{2} \mathrm{CH}_{2}(0.2 \mathrm{~mL})$. The solution was stirred for $5 \mathrm{~h}$. The reaction mixture was diluted with $\mathrm{CH}_{2} \mathrm{CH}_{2}$ and washed with $1 \mathrm{~N} \mathrm{NaOH}$. The organic layer was dried over $\mathrm{Na}_{2} \mathrm{SO}_{4}$, filtered and concentrated to give the crude product. The crude product was further purified by column chromatography to give alcohol III-20a (60 $\mathrm{mg}, 0.19 \mathrm{mmol}, 78 \%$ ) as a colorless oil. $R f\left(30 \% \mathrm{Et}_{2} \mathrm{O} /\right.$ hexane $)=0.40 ;[\alpha]^{25}{ }_{\mathrm{D}}=-20\left(\mathrm{CH}_{2} \mathrm{Cl}_{2}\right) ; \mathrm{IR}$ (thin film, $\left.\mathrm{cm}^{-1}\right)$, $3443,2934,2860,1722,1662,1460,1371,1261,1173,1036,979,877 ;{ }^{1} \mathrm{H}$ NMR $(600$ $\left.\mathrm{MHz}, \mathrm{CDCl}_{3}\right) \delta 6.84(\mathrm{dd}, J=15.6,6.0 \mathrm{~Hz}, 1 \mathrm{H}), 6.10(\mathrm{dd}, J=15.6,1.2 \mathrm{~Hz}, 1 \mathrm{H}), 4.20(\mathrm{q}, J$ $=7.2 \mathrm{~Hz}, 2 \mathrm{H}), 4.13(\mathrm{ddd}, J=8.4,6.0,1.2 \mathrm{~Hz}, 1 \mathrm{H}), 3.78(\mathrm{qdd}, J=6.0,6.0,6.0 \mathrm{~Hz}, 1 \mathrm{H})$, $3.72(\mathrm{ddd}, J=8.4,6.0,6.0 \mathrm{~Hz}, 1 \mathrm{H}), 1.61-1.27(\mathrm{~m}, 10 \mathrm{H}), 1.42(\mathrm{~s}, 3 \mathrm{H}), 1.40(\mathrm{~s}, 3 \mathrm{H}), 1.29$ $(\mathrm{t}, J=7.2 \mathrm{~Hz}, 3 \mathrm{H}), 1.17(\mathrm{~d}, J=6.0 \mathrm{~Hz}, 3 \mathrm{H}) ;{ }^{13} \mathrm{C} \mathrm{NMR}\left(150 \mathrm{MHz}, \mathrm{CD}_{3} \mathrm{Cl}\right) \delta 166.0,144.1$, 
$122.7,109.3,80.5,80.2,68.0,60.6,39.1,31.9,29.5,27.2,26.6,25.9,25.5,23.5,14.2$;

ESI HRMS Calcd for $\left[\mathrm{C}_{17} \mathrm{H}_{30} \mathrm{O}_{5}+\mathrm{Na}\right]^{+}: 337.1986$, Found: 337.1987.

(E)-3-((4R,5R)-5-((R)-6-hydroxyheptyl)-2,2-dimethyl-1,3-dioxolan-4-yl)acrylic acid (III-19b)

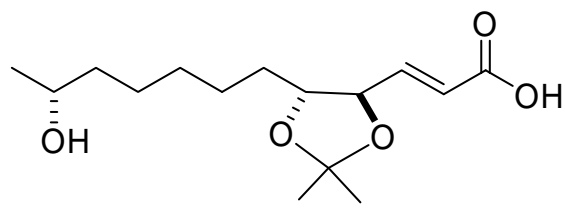

LiOH (452 mg, $10.78 \mathrm{mmol})$ was added to a solution of alcohol III-19a (1.35 g, 4.31 mmol), THF (4 mL) and $\mathrm{H}_{2} \mathrm{O}(1 \mathrm{~mL})$. The reaction mixture was stirred for $5 \mathrm{~h}$, then diluted with $\mathrm{CH}_{2} \mathrm{CH}_{2}$ and washed with $1 \mathrm{~N} \mathrm{HCl}$ and brine. The organic layer was dried over $\mathrm{Na}_{2} \mathrm{SO}_{4}$, filtered and concentrated to give the crude product. The crude product was further purified by column chromatography to give acid III-19b $(1.12 \mathrm{~g}, 3.92 \mathrm{mmol}$, 91\%) as a colorless oil. $R f\left(50 \% \mathrm{Et}_{2} \mathrm{O} /\right.$ hexane $)=0.26 ;[\alpha]^{25}=+22\left(\mathrm{CH}_{2} \mathrm{Cl}_{2}\right)$; IR (thin film, $\left.\mathrm{cm}^{-1}\right), 3450,2933,2862,1701,1380,1261,1238,1111,1054,980 ;{ }^{1} \mathrm{H}$ NMR $(600$ $\left.\mathrm{MHz}, \mathrm{CDCl}_{3}\right) \delta 6.96(\mathrm{dd}, J=15.6,5.4 \mathrm{~Hz}, 1 \mathrm{H}), 6.13(\mathrm{dd}, J=15.6,1.2 \mathrm{~Hz}, 1 \mathrm{H}), 4.17$ (ddd, $J=8.4,5.4,1.2 \mathrm{~Hz}, 1 \mathrm{H}), 3.80$ (qdd, $J=6.0,6.0,6.0 \mathrm{~Hz}, 1 \mathrm{H}), 3.72$ (ddd, $J=8.4$, 6.0, $6.0 \mathrm{~Hz}, 1 \mathrm{H}), 1.63-1.32(\mathrm{~m}, 10 \mathrm{H}), 1.44(\mathrm{~s}, 3 \mathrm{H}), 1.41(\mathrm{~s}, 3 \mathrm{H}), 1.18(\mathrm{~d}, J=6.0 \mathrm{~Hz}, 3 \mathrm{H})$; ${ }^{13} \mathrm{C}$ NMR $\left(150 \mathrm{MHz}, \mathrm{CD}_{3} \mathrm{Cl}\right) \delta 170.3,146.6,121.8,109.5,80.5,80.1,68.2,39.0,31.9$, 29.5, 27.2, 26.6, 25.9, 25.5, 23.4; ESI HRMS Calcd for $\left[\mathrm{C}_{15} \mathrm{H}_{25} \mathrm{O}_{5}+\mathrm{H}\right]^{+}:$287.1853, Found: 287.1854. 
(E)-3-((4S,5S)-5-((R)-6-hydroxyheptyl)-2,2-dimethyl-1,3-dioxolan-4-yl)acrylic acid (III-20b)

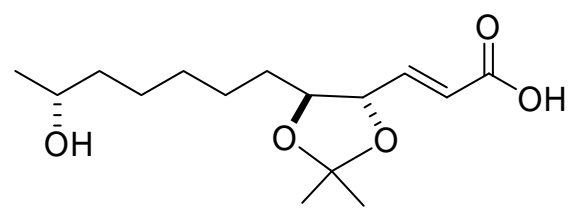

LiOH (19.4 mg, $0.463 \mathrm{mmol})$ was added to a solution of alcohol III-20a (58 mg, 0.185 mmol), THF $(1.7 \mathrm{~mL})$ and $\mathrm{H}_{2} \mathrm{O}(0.3 \mathrm{~mL})$. The reaction mixture was stirred for $5 \mathrm{~h}$, then diluted with $\mathrm{CH}_{2} \mathrm{CH}_{2}$ and washed with $1 \mathrm{~N} \mathrm{HCl}$ and brine. The organic layer was dried over $\mathrm{Na}_{2} \mathrm{SO}_{4}$, filtered and concentrated to give the crude product. The crude product was further purified by column chromatography to give acid III-20b (48 mg, $0.167 \mathrm{mmol}$, $90 \%)$ as a colorless oil. $R f\left(50 \% \mathrm{Et}_{2} \mathrm{O} /\right.$ hexane $)=0.26 ;[\alpha]^{25}=-22\left(\mathrm{CH}_{2} \mathrm{Cl}_{2}\right)$; IR (thin film, $\left.\mathrm{cm}^{-1}\right), 3311,2934,2860,1702,1373,1240,1169,1052,980,863 ;{ }^{1} \mathrm{H}$ NMR (600 $\left.\mathrm{MHz}, \mathrm{CDCl}_{3}\right) \delta 6.95(\mathrm{dd}, J=15.6,5.4 \mathrm{~Hz}, 1 \mathrm{H}), 6.13(\mathrm{dd}, J=15.6,1.2 \mathrm{~Hz}, 1 \mathrm{H}), 4.17$ $(\mathrm{ddd}, J=8.4,5.4,1.2 \mathrm{~Hz}, 1 \mathrm{H}), 3.80(\mathrm{qdd}, J=6.0,6.0,6.0 \mathrm{~Hz}, 1 \mathrm{H}), 3.73(\mathrm{ddd}, J=8.4$, 6.0, $6.0 \mathrm{~Hz}, 1 \mathrm{H}), 1.63-1.32(\mathrm{~m}, 10 \mathrm{H}), 1.44(\mathrm{~s}, 3 \mathrm{H}), 1.41(\mathrm{~s}, 3 \mathrm{H}), 1.18(\mathrm{~d}, J=6.0 \mathrm{~Hz}, 3 \mathrm{H})$; ${ }^{13} \mathrm{C}$ NMR $\left(150 \mathrm{MHz}, \mathrm{CD}_{3} \mathrm{Cl}\right) \delta 170.3,146.6,121.8,109.5,80.5,80.1,68.2,39.0,32.0$, 29.5, 27.2, 26.6, 25.9, 25.5, 23.4; ESI HRMS Calcd for $\left[\mathrm{C}_{15} \mathrm{H}_{26} \mathrm{O}_{5}+\mathrm{H}\right]^{+}: 287.1853$, Found: 287.1854.

(3aR,8R,13aR,E)-2,2,8-trimethyl-9,10,11,12,13,13a-hexahydro-3aH-[1,3]dioxolo[4,5e][1] loxacyclododecin-6(8H)-one (III-19c) 


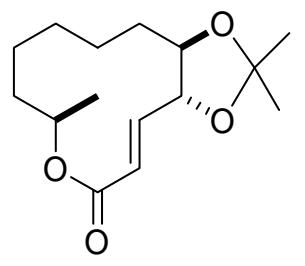

(3aR,8R,13aR,Z)-2,2,8-trimethyl-9,10,11,12,13,13a-hexahydro-3aH-[1,3]dioxolo[4,5-

\section{e][1]oxacyclododecin-6(8H)-one (III-19d)}

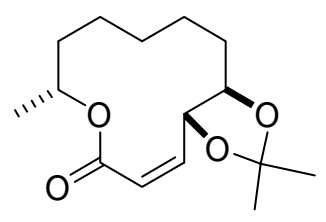

To a solution of acid III-19b $(47.5 \mathrm{mg}, 0.17 \mathrm{mmol})$ and $\mathrm{Et}_{3} \mathrm{~N}(0.028 \mathrm{~mL}, 0.2 \mathrm{mmol})$ in THF $(0.83 \mathrm{~mL})$ was added 2,4,6-trichlorobenzoyl chloride $(0.029 \mathrm{~mL}, 0.18 \mathrm{mmol})$ dropwise. The reaction mixture was stirred at room temperature for $2 \mathrm{~h}$, followed by dilution with dry toluene $(20 \mathrm{~mL})$ and added dropwise to a refluxing solution of DMAP (646 mg, $5.3 \mathrm{mmol})$ in dry toluene $(50 \mathrm{~mL})$. After addition, the mixture was refluxed for $10 \mathrm{~h}$ and then concentrated in vacuum. The residue was dissolved in EtOAc $(30 \mathrm{~mL})$ and washed with $1 \mathrm{~N} \mathrm{HCl}(20 \mathrm{~mL})$, saturated $\mathrm{NaHCO}_{3}$, brine, dried over $\mathrm{Na}_{2} \mathrm{SO}$, filtered and evaporated. The residue was purified by flash chromatography on silica gel to give the macrolactone III-19c $(28.5 \mathrm{mg}, 0.10 \mathrm{mmol}, 60 \%)^{7}$ and macrolactone III-19d $(4.74 \mathrm{mg}$, $0.017 \mathrm{mmol}, 10 \%)$.

\footnotetext{
${ }^{7}$ These known compounds were characterized based on the comparison of ${ }_{1} \mathrm{H}$ and ${ }_{13} \mathrm{C} \mathrm{NMR}$ data with the published ones: Chou, C.-Y. Hou, D.-R. J. Org. Chem. 2006, 71, 9887.
} 
III-19c: colorless oil; $R f\left(10 \% \mathrm{Et}_{2} \mathrm{O} /\right.$ hexane $)=0.33 ;[\alpha]_{\mathrm{D}}^{25}=+6(\mathrm{MeOH}) ; \mathrm{IR}$ (thin film, $\left.\mathrm{cm}^{-1}\right), 2982,2934,2866,1718,1463,1371,1252,1163,1050,988,857 ;{ }^{1} \mathrm{H}$ NMR (600 $\left.\mathrm{MHz}, \mathrm{CDCl}_{3}\right) \delta 6.77(\mathrm{dd}, J=15.6,9.6 \mathrm{~Hz}, 1 \mathrm{H}), 6.22(\mathrm{~d}, J=15.6 \mathrm{~Hz}, 1 \mathrm{H}), 4.98(\mathrm{dqd}, J=$ 9.6, 6.0, $4.2 \mathrm{~Hz}, 1 \mathrm{H}), 4.04(\mathrm{dd}, J=9.6,8.4 \mathrm{~Hz}, 1 \mathrm{H}), 3.91(\mathrm{ddd}, J=11.4,8.4,4.2 \mathrm{~Hz}, 1 \mathrm{H})$, $1.96(\mathrm{ddt}, J=13.8,13.8,4.2 \mathrm{~Hz}, 1 \mathrm{H}), 1.68$ (dddd, $J=15.6,9.0,4.2,1.8 \mathrm{~Hz}, 1 \mathrm{H}), 1.61-$ $1.35(\mathrm{~m}, 8 \mathrm{H}), 1.44(\mathrm{~s}, 3 \mathrm{H}), 1.42(\mathrm{~s}, 3 \mathrm{H}), 1.30(\mathrm{~d}, J=6.0 \mathrm{~Hz}, 3 \mathrm{H}) ;{ }^{13} \mathrm{C}$ NMR $(150 \mathrm{MHz}$, $\left.\mathrm{CD}_{3} \mathrm{Cl}\right) \delta 166.6,143.8,125.9,109.3,80.4,80.1,75.4,35.4,29.5,27.8,27.1,26.8,25.1$, 24.9, 20.6; ESI HRMS Calcd for $\left[\mathrm{C}_{15} \mathrm{H}_{24} \mathrm{O}_{4}+\mathrm{Na}\right]^{+}:$291.1567, Found: 291.1568.

III-19d: colorless oil; $R f\left(10 \% \mathrm{Et}_{2} \mathrm{O} /\right.$ hexane $)=0.41 ;[\alpha]_{\mathrm{D}}^{25}=+53(\mathrm{MeOH}) ;$ IR (thin film, $\left.\mathrm{cm}^{-1}\right), 2982,2939,2866,1718,1380,1206,1047,861 ;{ }^{1} \mathrm{H} \mathrm{NMR}\left(600 \mathrm{MHz}, \mathrm{CDCl}_{3}\right) \delta$ $5.97(\mathrm{~d}, J=12.0 \mathrm{~Hz}, 1 \mathrm{H}), 6.22(\mathrm{dd}, J=12.0,8.4 \mathrm{~Hz}, 1 \mathrm{H}), 5.08(\mathrm{qdd}, J=6.0,4.2,3.0 \mathrm{~Hz}$, 1H), $4.87(\mathrm{dd}, J=9.0,9.0 \mathrm{~Hz}, 1 \mathrm{H}), 3.87(\mathrm{ddd}, J=9.0,5.4,1.8 \mathrm{~Hz}, 1 \mathrm{H}), 1.85-1.41(\mathrm{~m}$, $8 \mathrm{H}), 1.42(\mathrm{~s}, 3 \mathrm{H}), 1.41(\mathrm{~s}, 3 \mathrm{H}), 1.25(\mathrm{~d}, J=6.6 \mathrm{~Hz}, 3 \mathrm{H}) ;{ }^{13} \mathrm{C} \mathrm{NMR}\left(150 \mathrm{MHz}, \mathrm{CD}_{3} \mathrm{Cl}\right) \delta$ $166.3,139.1,125.3,109.3,79.9,75.1,72.9,30.8,27.4,27.2,26.4,25.4,18.3,18.2,17.9$; ESI HRMS Calcd for $\left[\mathrm{C}_{15} \mathrm{H}_{24} \mathrm{O}_{4}+\mathrm{H}\right]^{+}:$: 269.1747, Found: 269.1748.

(3aS,8R,13aS,E)-2,2,8-trimethyl-9,10,11,12,13,13a-hexahydro-3aH-[1,3]dioxolo[4,5e][1] loxacyclododecin-6(8H)-one (III-20c)<smiles>CC1CCCCCC(/C=C/C(=O)OC2CCCC2)O1</smiles> 
(3aS,8R,13aS,Z)-2,2,8-trimethyl-9,10,11,12,13,13a-hexahydro-3aH-[1,3]dioxolo[4,5-

\section{e][1]oxacyclododecin-6(8H)-one (III-20d)}

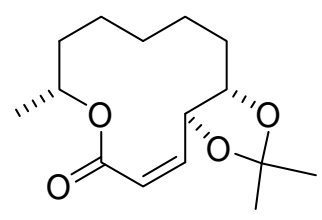

To a solution of acid III-20b $(180 \mathrm{mg}, 0.63 \mathrm{mmol})$ and $\mathrm{Et}_{3} \mathrm{~N}(0.1 \mathrm{~mL})$ in THF $(3.1 \mathrm{~mL})$ was added 2,4,6-trichlorobenzoyl chloride $(0.11 \mathrm{~mL}, 0.65 \mathrm{mmol})$ dropwise. The reaction mixture was stirred at room temperature for $2 \mathrm{~h}$, followed by dilution with dry toluene ( 76 $\mathrm{mL})$ and added dropwise to a refluxing solution of DMAP (169 mg, $20.16 \mathrm{mmol})$ in dry toluene $(190 \mathrm{~mL})$. After addition, the mixture was refluxed for $10 \mathrm{~h}$ and then concentrated in vacuum. The residue was dissolved in EtOAc $(100 \mathrm{~mL})$ and washed with $1 \mathrm{~N} \mathrm{HCl}$, saturated $\mathrm{NaHCO}_{3}$, brine, dried over $\mathrm{Na}_{2} \mathrm{SO}_{4}$, filtered and evaporated. The residue was purified by flash chromatography on silica gel to give the macrolactone III20c (108 mg, $0.39 \mathrm{mmol}, 62 \%)$ and macrolactone III-20d (17 mg, $0.06 \mathrm{mmol}, 10 \%)^{8}$ III-20c: colorless oil. $R f\left(10 \% \mathrm{Et}_{2} \mathrm{O} / \mathrm{hexane}\right)=0.56 ;[\alpha]_{\mathrm{D}}^{25}=+30(\mathrm{MeOH}) ; \mathrm{IR}$ (thin film, $\left.\mathrm{cm}^{-1}\right), 2983,2937,2870,1717,1465,1230,1163,1057,993,848 ;{ }^{1} \mathrm{H}$ NMR (600 MHz, $\left.\mathrm{CDCl}_{3}\right) \delta 6.86(\mathrm{dd}, J=16.2,6.6 \mathrm{~Hz}, 1 \mathrm{H}), 6.20(\mathrm{dd}, J=16.2,1.2 \mathrm{~Hz}, 1 \mathrm{H}), 5.02(\mathrm{qdd}, J=$ 6.0, 5.4, $5.4 \mathrm{~Hz}, 1 \mathrm{H}), 4.11$ (ddd, $J=7.8,7.2,1.2 \mathrm{~Hz}, 1 \mathrm{H}), 3.63(\mathrm{ddd}, J=10.8,8.4,3.6$ $\mathrm{Hz}, 1 \mathrm{H}), 1.99-1.44(\mathrm{~m}, 8 \mathrm{H}), 1.44(\mathrm{~s}, 3 \mathrm{H}), 1.43(\mathrm{~s}, 3 \mathrm{H}), 1.28(\mathrm{~d}, J=6.0 \mathrm{~Hz}, 3 \mathrm{H}) ;{ }^{13} \mathrm{C} \mathrm{NMR}$ $\left(150 \mathrm{MHz}, \mathrm{CD}_{3} \mathrm{Cl}\right) \delta 166.8,143.9,123.7,109.5,83.0,81.2,73.1,33.3,29.0,27.4,27.3$,

\footnotetext{
${ }^{8}$ Sharma, G. V. M.; Reddy, J. J.; Reddy, K. L. Tetrahedron Lett. 2006, 47, 6531-6535.
} 
26.9, 25.3, 24.2, 20.2; ESI HRMS Calcd for $\left[\mathrm{C}_{15} \mathrm{H}_{24} \mathrm{O}_{4}+\mathrm{Na}\right]^{+}:$291.1567, Found: 291.1568.

III-20d: colorless oil. $R f\left(10 \% \mathrm{Et}_{2} \mathrm{O} /\right.$ hexane $)=0.38 ;[\alpha]_{\mathrm{D}}^{25}=-50(\mathrm{MeOH})$; IR (thin film, $\left.\mathrm{cm}^{-1}\right), 2941,2937,1710,1460,1379,1298,1211,1074,1045,993,833 ;{ }^{1} \mathrm{H}$ NMR (600 $\left.\mathrm{MHz}, \mathrm{CDCl}_{3}\right) \delta 6.14(\mathrm{dd}, J=12.0,8.4 \mathrm{~Hz}, 1 \mathrm{H}), 6.22(\mathrm{dd}, J=12.0,1.2 \mathrm{~Hz}, 1 \mathrm{H}), 5.31$ (ddd, $J=9.0,8.4,1.2 \mathrm{~Hz}, 1 \mathrm{H}), 4.89$ (dqd, $J=12.0,6.0,3.6 . \mathrm{Hz}, 1 \mathrm{H}), 3.87$ (dd, $J=8.4$, $6.0, \mathrm{~Hz}, 1 \mathrm{H}), 1.75-1.31(\mathrm{~m}, 16 \mathrm{H}), 1.45(\mathrm{~s}, 3 \mathrm{H}), 1.42(\mathrm{~s}, 3 \mathrm{H}), 1.27(\mathrm{~d}, J=6.0 \mathrm{~Hz}, 3 \mathrm{H}) ;{ }^{13} \mathrm{C}$ NMR $\left(150 \mathrm{MHz}, \mathrm{CD}_{3} \mathrm{Cl}\right) \delta 165.8,144.7,123.7,109.3,79.5,74.2,74.0,32.5,27.3,27.0$, 26.5, 24.4, 20.8, 20.1, 17.1; ESI HRMS Calcd for $\left[\mathrm{C}_{15} \mathrm{H}_{24} \mathrm{O}_{4}+\mathrm{Na}\right]^{+}: 291.1567$, Found: 291.1568.

(5R,6R,12R,E)-5,6-dihydroxy-12-methyloxacyclododec-3-en-2-one (Cladospolide C)<smiles>CC1CCCCC(O)[C@@H](O)/C=C/C(=O)O1</smiles>

To a solution of lactone III-19c (140 mg, $0.50 \mathrm{mmol})$ and acetonitrile/water $(2: 1, \mathrm{v} / \mathrm{v}, 5$ $\mathrm{mL})$ was added trifluoroacetic acid $(3.75 \mathrm{~mL})$ at $0{ }^{\circ} \mathrm{C}$. Then the reaction was stirred at room temperature for another $2 \mathrm{~h}$, diluted with ether acetate, washed with sat. $\mathrm{NaHCO}_{3}(\mathrm{aq})$, dried over $\mathrm{Na}_{2} \mathrm{SO}_{4}$, filtered, and concentrated. The residue was purified by flash chromatography on silica gel to give the Cladospolide C (91 mg, $0.40 \mathrm{mmol}, 80 \%$ ) 
as a colorless solid. ${ }^{9} R f\left(50 \% \mathrm{Et}_{2} \mathrm{O} /\right.$ hexane $)=0.27 ; \mathrm{Mp} 91-92{ }^{\circ} \mathrm{C} ;[\alpha]^{25}{ }_{\mathrm{D}}=-90(\mathrm{MeOH})$; IR (thin film, $\left.\mathrm{cm}^{-1}\right), 3415,2940,2864,1705,1650,1460,1258,1035 ;{ }^{1} \mathrm{H}$ NMR (600 $\left.\mathrm{MHz}, \mathrm{CDCl}_{3}\right) \delta 6.81(\mathrm{dd}, J=15.6,9.0 \mathrm{~Hz}, 1 \mathrm{H}), 6.05(\mathrm{~d}, J=15.6 \mathrm{~Hz}, 1 \mathrm{H}), 4.98(\mathrm{dqd}, J=$ 8.4, 6.0, $1.8 \mathrm{~Hz}, 1 \mathrm{H}), 3.98(\mathrm{dd}, J=8.4,7.8 \mathrm{~Hz}, 1 \mathrm{H}), 3.57$ (ddd, $J=7.8,7.8,1.8 \mathrm{~Hz}, 1 \mathrm{H})$, $1.30(\mathrm{~d}, J=6.0 \mathrm{~Hz}, 3 \mathrm{H}) 1.69-1.11(\mathrm{~m}, 10 \mathrm{H}) ;{ }^{13} \mathrm{C} \mathrm{NMR}\left(150 \mathrm{MHz}, \mathrm{CD}_{3} \mathrm{Cl}\right) \delta 166.7$, 145.3, 124.5, 77.5, 76.5, 74.3, 33.9, 32.1, 27.3, 24.5, 24.1, 20.8; ESI HRMS Calcd for $\left[\mathrm{C}_{12} \mathrm{H}_{20} \mathrm{O}_{4}+\mathrm{H}\right]^{+}:$229.1434, Found: 229.1435.

(5R, 6R, 12R, Z)-5,6-dihydroxy-12-methyloxacyclododec-3-en-2-one (III-17)<smiles>C[C@@H]1CCCCC[C@@H](O)[C@@H](O)/C=C\C(=O)O1</smiles>

To a solution of lactone III-19d (58 mg, $0.21 \mathrm{mmol})$ and acetonitrile/water $(2: 1, \mathrm{v} / \mathrm{v}, 2$ $\mathrm{mL})$ was added trifluoroacetic acid $(1.58 \mathrm{~mL})$ at $0{ }^{\circ} \mathrm{C}$. Then the reaction was stirred at room temperature for another $2 \mathrm{~h}$, diluted with ether acetate, washed with sat. $\mathrm{NaHCO}_{3}(\mathrm{aq})$, dried over $\mathrm{Na}_{2} \mathrm{SO}_{4}$, filtered, and concentrated. The residue was purified by flash chromatography on silica gel to give the lactone III-17 (48 $\mathrm{mg}, 0.17 \mathrm{mmol}, 81 \%$ ) as a colorless oil; $R f\left(50 \% \mathrm{Et}_{2} \mathrm{O} /\right.$ hexane $)=0.33 ;[\alpha]_{\mathrm{D}}^{25}=+1(\mathrm{MeOH}) ; \mathrm{IR}\left(\right.$ thin film, $\left.\mathrm{cm}^{-1}\right)$, 3272, 2943, 2862, 1710, 1461, 1278, 1050; ${ }^{1} \mathrm{H}$ NMR $\left(600 \mathrm{MHz}, \mathrm{CDCl}_{3}\right) \delta 6.12(\mathrm{dd}, J=$ 12.0, $8.4 \mathrm{~Hz}, 1 \mathrm{H}), 6.05(\mathrm{dd}, J=12.0,1.2 \mathrm{~Hz}, 1 \mathrm{H}), 5.10$ (qdd, $J=6.0,5.4,3.0 \mathrm{~Hz}, 1 \mathrm{H})$,

\footnotetext{
${ }^{9}$ These known compounds were characterized based on the comparison of ${ }_{1} \mathrm{H}$ and ${ }_{13} \mathrm{C}$ NMR data with the published ones: Chou, C.-Y. Hou, D.-R. J. Org. Chem. 2006, 71, 9887.
} 
4.97 (ddd, $J=8.4,4.8,1.2 \mathrm{~Hz}, 1 \mathrm{H}), 3.87(\mathrm{ddd}, J=9.0,4.8,3.0 \mathrm{~Hz}, 1 \mathrm{H}), 2.04-1.35(\mathrm{~m}$, $10 \mathrm{H}), 1.25(\mathrm{~d}, J=6.0 \mathrm{~Hz}, 3 \mathrm{H}) ;{ }^{13} \mathrm{C} \mathrm{NMR}\left(150 \mathrm{MHz}, \mathrm{CD}_{3} \mathrm{Cl}\right) \delta 166.7,145.3,124.5,77.5$, 76.5, 74.3, 33.9, 32.1, 27.3, 24.5, 24.1, 20.8; ESI HRMS Calcd for $\left[\mathrm{C}_{12} \mathrm{H}_{20} \mathrm{O}_{4}+\mathrm{H}\right]^{+}$: 229.1434, Found: 229.1435.

(5S, 6S, 12R, E)-5, 6-dihydroxy-12-methyloxacyclododec-3-en-2-one (III-18)<smiles>CC1CCCCC[C@@H](O)C(O)/C=C/C(=O)O1</smiles>

To a solution of lactone III-20c $(68 \mathrm{mg}, 0.24 \mathrm{mmol})$ and acetonitrile/water $(2: 1, \mathrm{v} / \mathrm{v}, 2.5$ $\mathrm{mL})$ was added trifluoroacetic acid $(1.83 \mathrm{~mL})$ at $0{ }^{\circ} \mathrm{C}$. Then the reaction was stirred at room temperature for another $2 \mathrm{~h}$, diluted with ether acetate, washed with sat. $\mathrm{NaHCO}_{3}(\mathrm{aq})$, dried over $\mathrm{Na}_{2} \mathrm{SO}_{4}$, filtered, and concentrated. The residue was purified by flash chromatography on silica gel to give the lactone III-18 (45 mg, $0.20 \mathrm{mmol}, 83 \%)$ as a colorless oil; $R f\left(50 \% \mathrm{Et}_{2} \mathrm{O} /\right.$ hexane $)=0.41 ;[\alpha]_{\mathrm{D}}^{25}=-10(\mathrm{MeOH}) ; \mathrm{IR}\left(\right.$ thin film, $\left.\mathrm{cm}^{-1}\right)$, 3396, 2937, 2870, 1711, 1464, 1247, 1028; ${ }^{1} \mathrm{H}$ NMR (600 MHz, $\left.\mathrm{CDCl}_{3}\right) \delta 6.94(\mathrm{dd}, J=$ 15.6, $6.0 \mathrm{~Hz}, 1 \mathrm{H}), 6.12(\mathrm{dd}, J=15.6,1.2 \mathrm{~Hz}, 1 \mathrm{H}), 4.96(\mathrm{dqd}, J=6.6,6.0,3.0 \mathrm{~Hz}, 1 \mathrm{H})$, $4.12(\mathrm{ddd}, J=7.2,6.0,1.2 \mathrm{~Hz}, 1 \mathrm{H}), 3.32(\mathrm{ddd}, J=9.6,7.8,1.8 \mathrm{~Hz}, 1 \mathrm{H}), 1.74-1.20(\mathrm{~m}$, $10 \mathrm{H}), 1.28(\mathrm{~d}, J=6.0 \mathrm{~Hz}, 3 \mathrm{H}) ;{ }^{13} \mathrm{C} \mathrm{NMR}\left(150 \mathrm{MHz}, \mathrm{CD}_{3} \mathrm{Cl}\right) \delta 167.2,146.9,122.5,78.0$, 77.8, 73.1, 33.2, 32.6, 28.3, 25.0, 22.8, 19.0; ESI HRMS Calcd for $\left[\mathrm{C}_{12} \mathrm{H}_{20} \mathrm{O}_{4}+\mathrm{Na}\right]^{+}$: 251.1254, Found: 251.1254. 
(5S,6S,12R,Z)-5,6-dihydroxy-12-methyloxacyclododec-3-en-2-one (Cladospolide B)<smiles>C[C@@H]1CCCCC[C@H](O)[C@@H](O)/C=C\C(=O)O1</smiles>

To a solution of lactone III-20d (34 mg, $0.12 \mathrm{mmol})$ and acetonitrile/water (2:1, v/v, 1.2 $\mathrm{mL})$ was added trifluoroacetic acid $(0.90 \mathrm{~mL})$ at $0{ }^{\circ} \mathrm{C}$. Then the reaction was stirred at room temperature for another $2 \mathrm{~h}$, diluted with ether acetate, washed with sat. $\mathrm{NaHCO}_{3}$ (aq), dried over $\mathrm{Na}_{2} \mathrm{SO}_{4}$, filtered, and concentrated. The residue was purified by flash chromatography on silica gel to give the Cladospolide B (27 mg, $0.10 \mathrm{mmol}, 81 \%$ ) as a white solid; ${ }^{10} R f\left(50 \% \mathrm{Et}_{2} \mathrm{O} /\right.$ hexane $)=0.57 ; \mathrm{Mp} 108-109{ }^{\circ} \mathrm{C} ;[\alpha]^{25}=+5(\mathrm{MeOH})$; IR (thin film, $\mathrm{cm}^{-1}$ ), 3345, 2934, 2865, 1707, 1461, 1280, 1046 ; ${ }^{1} \mathrm{H}$ NMR (600 MHz, $\left.\mathrm{CDCl}_{3}\right) \delta 6.23(\mathrm{dd}, J=12.0,8.4 \mathrm{~Hz}, 1 \mathrm{H}), 5.77(\mathrm{dd}, J=12.0,1.2 \mathrm{~Hz}, 1 \mathrm{H}), 5.26(\mathrm{ddd}, J$ $=8.4,4.2,1.2 \mathrm{~Hz}, 1 \mathrm{H}), 4.88(\mathrm{dqd}, J=10.2,6.0,1.8 \mathrm{~Hz}, 1 \mathrm{H}), 3.77(\mathrm{ddd}, J=9.0,4.2,2.4$ $\mathrm{Hz}, 1 \mathrm{H}), 1.84-1.35(\mathrm{~m}, 10 \mathrm{H}), 1.28(\mathrm{~d}, J=6.0 \mathrm{~Hz}, 3 \mathrm{H}) ;{ }^{13} \mathrm{C} \mathrm{NMR}\left(150 \mathrm{MHz}, \mathrm{CD}_{3} \mathrm{Cl}\right)$ $\delta 165.8,148.5,121.9,74.4,73.9,67.5,32.0,30.6,25.7,24.1,21.3,19.7$; ESI HRMS Calcd for $\left[\mathrm{C}_{12} \mathrm{H}_{20} \mathrm{O}_{4}+\mathrm{H}\right]^{+}:$229.1434, Found: 229.1435 .

\footnotetext{
${ }^{10}$ These known compounds were characterized based on the comparison of $1 \mathrm{H}$ and ${ }_{13} \mathrm{C}$ NMR data with the published ones: Austin, K. A. B.; Banwell, M. G.; Loong, D. T. J.; Rae, A. D.; Willis, A. C. Org. Biomol. Chem. 2005, 3, 1081.
} 
(5R,6R,12R,E)-6-(tert-butyldimethylsilyloxy)-5-hydroxy-12-methyloxacyclododec-3en-2-one (III-3a)<smiles>CCCCCC(O[Sb])[C@H](O)/C=C/C(=O)OC(C)CCCC</smiles>

(5R,6R,12R,E)-5-(tert-butyldimethylsilyloxy)-6-hydroxy-12-methyloxacyclododec-3en-2-one (III-3a1)

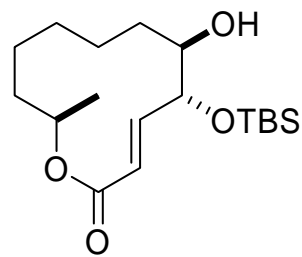

To a magnetically stirred solution of Cladospolide C (10 $\mathrm{mg}, 0.044 \mathrm{mmol})$ and imidazole $(7.5 \mathrm{mg}, 0.11 \mathrm{mmol})$ in dry $\mathrm{CH}_{2} \mathrm{Cl}_{2}(0.5 \mathrm{~mL})$ was added dropwise $\mathrm{TBSCl}(19$ $\mathrm{mg}, 0.13 \mathrm{mmol}$ ) at $0{ }^{\circ} \mathrm{C}$. The reaction mixture was warmed to room temperature and stirred overnight. Water was added and then the reaction mixture was extracted with $\mathrm{Et}_{2} \mathrm{O}$, dried with $\mathrm{Na}_{2} \mathrm{SO}_{4}$ and evaporated under reduced pressure to leave the crude product which was then purified using silica gel flash chromatography to give alcohol III-3a (4.5 mg, $0.013 \mathrm{mmol}, 30 \%$ ) and alcohol III-3a1 (9 mg, $0.026 \mathrm{mmol}, 60 \%)$.

III-3a: colorless oil; $R f\left(10 \% \mathrm{Et}_{2} \mathrm{O} /\right.$ hexane $)=0.20 ;[\alpha]_{\mathrm{D}}^{25}=50\left(\mathrm{CH}_{2} \mathrm{Cl}_{2}\right)$; IR (thin film, $\left.\mathrm{cm}^{-1}\right), 3457,2930,2858,1722,1463,1255,1048,998,776 \mathrm{~cm}-1 ;{ }^{1} \mathrm{H}$ NMR $(600 \mathrm{MHz}$, $\left.\mathrm{CDCl}_{3}\right) \delta 6.84(\mathrm{dd}, J=15.6,9.0 \mathrm{~Hz}, 1 \mathrm{H}), 6.07(\mathrm{~d}, J=15.6 \mathrm{~Hz}, 1 \mathrm{H}), 5.02(\mathrm{dqd}, J=9.0$, 
6.0, 3.0 Hz, 1H), $4.04(\mathrm{dd}, J=9.0,7.2 \mathrm{~Hz}, 1 \mathrm{H}), 3.64(\mathrm{ddd}, J=8.4,7.2,1.2 \mathrm{~Hz}, 1 \mathrm{H}), 1.70$ $1.10(\mathrm{~m}, 10 \mathrm{H}), 1.28(\mathrm{~d}, J=6.0 \mathrm{~Hz}, 3 \mathrm{H}), 0.90(\mathrm{~s}, 9 \mathrm{H}), 0.11(\mathrm{~s}, 3 \mathrm{H}), 0.09(\mathrm{~s}, 3 \mathrm{H}) ;{ }^{13} \mathrm{C} \mathrm{NMR}$ $\left(150 \mathrm{MHz}, \mathrm{CD}_{3} \mathrm{Cl}\right) \delta 167.2,145.4,123.7,77.2,76.8,73.7,33.5,32.1,27.6,25.8,24.4$, 23.4, 20.3, 18.0, - 4.49, - 4.78; ESI HRMS Calcd for $\left[\mathrm{C}_{18} \mathrm{H}_{34} \mathrm{O}_{4} \mathrm{Si}+\mathrm{H}\right]^{+}: 343.2299$, Found: 343.2300 .

III-3a1: colorless oil; $R f\left(10 \% \mathrm{Et}_{2} \mathrm{O} / \mathrm{h}\right.$ exane $)=0.08 ;[\alpha]_{\mathrm{D}}^{25}=+34\left(\mathrm{CH}_{2} \mathrm{Cl}_{2}\right)$; IR (thin film, $\left.\mathrm{cm}^{-1}\right), 3482,2931,2859,1721,1463,1254,1039,997,778 ;{ }^{1} \mathrm{H}$ NMR (600 MHz, $\left.\mathrm{CDCl}_{3}\right) \delta 6.77(\mathrm{dd}, J=16.2,9.0 \mathrm{~Hz}, 1 \mathrm{H}), 6.01(\mathrm{dd}, J=16.2,0.6 \mathrm{~Hz}, 1 \mathrm{H}), 4.99(\mathrm{dqd}, J=$ 10.2, 6.0, $2.4 \mathrm{~Hz}, 1 \mathrm{H}), 3.93(\mathrm{ddd}, J=9.0,7.2,0.6 \mathrm{~Hz}, 1 \mathrm{H}), 3.54(\mathrm{ddd}, J=7.2,7.2,2.4$ $\mathrm{Hz}, 1 \mathrm{H}), 1.70-1.11(\mathrm{~m}, 10 \mathrm{H}), 1.30(\mathrm{~d}, J=6.0 \mathrm{~Hz}, 3 \mathrm{H}), 0.91(\mathrm{~s}, 9 \mathrm{H}), 0.10(\mathrm{~s}, 3 \mathrm{H}), 0.06(\mathrm{~s}$, $3 \mathrm{H}) ;{ }^{13} \mathrm{C}$ NMR $\left(150 \mathrm{MHz}, \mathrm{CD}_{3} \mathrm{Cl}\right) \delta 167.0,146.6,123.8,78.3,76.6,74.2,33.8,30.4$, 27.5, 25.8, 24.4, 24.0, 20.7, 18.1, - 4.50, - 4.82; ESI HRMS Calcd for $\left[\mathrm{C}_{18} \mathrm{H}_{34} \mathrm{O}_{4} \mathrm{Si}+\mathrm{H}\right]^{+}$: 343.2299, Found: 343.2300.

(6R,12R,E)-6-(tert-butyldimethylsilyloxy)-12-methyloxacyclododec-3-ene-2,5-dione (III-3b)<smiles>CCCCCCC(O[SbH3])C(=O)/C=C/C(=O)OC1CCCCCC1</smiles> 
To a magnetically stirred solution of alcohol III-3a $(5 \mathrm{mg}, 0.015 \mathrm{mmol})$ in dry $\mathrm{CH}_{2} \mathrm{Cl}_{2}$ $(0.2 \mathrm{~mL})$ was added Dess-Martin periodinane $(16 \mathrm{mg}, 0.038 \mathrm{mmol})$ at room temperature. The reaction mixture was stirred for $1 \mathrm{~h} . \mathrm{CH}_{2} \mathrm{Cl}_{2}$ was added to dilute the reaction solution and then the reaction mixture was washed with $\mathrm{NaHCO}_{3}$, extracted with ether acetate, dried with $\mathrm{Na}_{2} \mathrm{SO}_{4}$ and evaporated under reduced pressure to leave the crude product which was then purified using silica gel flash chromatography to give ketone III-3b (4.6 $\mathrm{mg}, 0.014 \mathrm{mmol}, 95 \%)$ as a colorless oil; $R f\left(10 \% \mathrm{Et}_{2} \mathrm{O} / \mathrm{hexane}\right)=0.33 ;[\alpha]^{25}{ }_{\mathrm{D}}=-12$ $\left(\mathrm{CH}_{2} \mathrm{Cl}_{2}\right)$; IR (thin film, $\left.\mathrm{cm}^{-1}\right), 2931,2858,1721,1463,1251,1078,838,779 ;{ }^{1} \mathrm{H}$ NMR $\left(600 \mathrm{MHz}, \mathrm{CDCl}_{3}\right) \delta 7.21(\mathrm{~d}, J=16.8 \mathrm{~Hz}, 1 \mathrm{H}), 6.90(\mathrm{~d}, J=16.8 \mathrm{~Hz}, 1 \mathrm{H}), 4.89(\mathrm{dqd}, J=$ 7.8., $6.0,4.8 \mathrm{~Hz}, 1 \mathrm{H}), 4.41(\mathrm{dd}, J=6.0,4.2 \mathrm{~Hz}, 1 \mathrm{H}), 1.78-1.15(\mathrm{~m}, 10 \mathrm{H}), 1.34(\mathrm{~d}, J=6.0$ $\mathrm{Hz}, 3 \mathrm{H}), 0.91(\mathrm{~s}, 9 \mathrm{H}), 0.10(\mathrm{~s}, 3 \mathrm{H}), 0.07(\mathrm{~s}, 3 \mathrm{H}) ;{ }^{13} \mathrm{C} \mathrm{NMR}\left(150 \mathrm{MHz}, \mathrm{CD}_{3} \mathrm{Cl}\right) \delta 200.8$, $166.8,138.2,131.3,79.2,75.3,35.1,34.1,27.7,25.8,23.9,20.6,18.1,-4.79,-5.25$; ESI HRMS Calcd for $\left[\mathrm{C}_{18} \mathrm{H}_{32} \mathrm{O}_{4} \mathrm{Si}+\mathrm{Na}\right]^{+}: 363.1962$, Found: 363.1964 .

(6R,12R,E)-6-hydroxy-12-methyloxacyclododec-3-ene-2,5-dione (III-28)<smiles>O=C1C=CC(=O)C(O)CCCCCC1</smiles>

To a plastic flask were added III-3b (4 mg, $0.012 \mathrm{mmol})$ and 5\% $\mathrm{HF}$ in $\mathrm{CH}_{3} \mathrm{CN}(15 \mu \mathrm{L})$ at room temperature. The reaction mixture was stirred for $2 \mathrm{~h}$ and then ether acetate was added to dilute the reaction solution. The reaction mixture was washed with $\mathrm{NaHCO}_{3}$, 
extracted with ether acetate, dried with $\mathrm{Na}_{2} \mathrm{SO}_{4}$ and evaporated under reduced pressure to leave the crude product which was then purified using silica gel flash chromatography to give ketone III-28 $(2.3 \mathrm{mg}, 0.102 \mathrm{mmol}, 85 \%)$ as a colorless oil; $R f\left(50 \% \mathrm{Et}_{2} \mathrm{O} / \mathrm{hexane}\right)=$ $0.33 ;[\alpha]^{25}{ }_{\mathrm{D}}=-23\left(\mathrm{CH}_{2} \mathrm{Cl}_{2}\right)$; IR (thin film, $\left.\mathrm{cm}^{-1}\right), 3501,2931,1719,1249,1156 ;{ }^{1} \mathrm{H}$ NMR $\left(600 \mathrm{MHz}, \mathrm{CDCl}_{3}\right) \delta 7.37(\mathrm{~d}, J=16.2 \mathrm{~Hz}, 1 \mathrm{H}), 6.67(\mathrm{~d}, J=16.2 \mathrm{~Hz}, 1 \mathrm{H}), 4.94(\mathrm{dqd}, J=$ 9.6, 6.0, 3.0 Hz, 1H), $4.34(\mathrm{dd}, J=9.6,3.0 \mathrm{~Hz}, 1 \mathrm{H}), 2.01(\mathrm{~m}, 1 \mathrm{H}), 1.67-1.17(\mathrm{~m}, 9 \mathrm{H})$, $1.42(\mathrm{~d}, J=6.0 \mathrm{~Hz}, 3 \mathrm{H}) ;{ }^{13} \mathrm{C} \mathrm{NMR}\left(150 \mathrm{MHz}, \mathrm{CD}_{3} \mathrm{Cl}\right) \delta 201.5,165.6,137.5,131.2,77.3$, 75.2, 35.1, 33.5, 27.8, 24.5, 23.3, 20.8; ESI HRMS Calcd for $\left[\mathrm{C}_{12} \mathrm{H}_{18} \mathrm{O}_{4}+\mathrm{H}\right]^{+}: 227.1278$, Found: 227.1278.

(5S,6S,12R,E)-6-(tert-butyldimethylsilyloxy)-5-hydroxy-12-methyloxacyclododec-3en-2-one (III-18a)

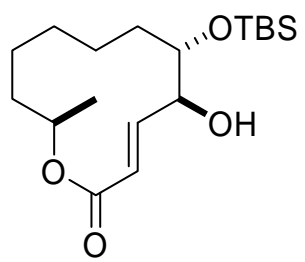

(5S,6S,12R,E)-5-(tert-butyldimethylsilyloxy)-6-hydroxy-12-methyloxacyclododec-3en-2-one (III-18a1)

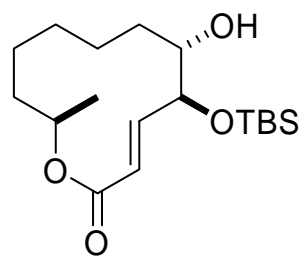


To a magnetically stirred solution of diol III-18 $(20 \mathrm{mg}, 0.088 \mathrm{mmol})$ and imidazole (15 $\mathrm{mg}, 0.22 \mathrm{mmol})$ in dry $\mathrm{CH}_{2} \mathrm{Cl}_{2}(1 \mathrm{~mL})$ was added dropwise $\mathrm{TBSCl}(38 \mathrm{mg}, 0.26 \mathrm{mmol})$ at $0{ }^{\circ} \mathrm{C}$. The reaction mixture was warmed to room temperature and stirred overnight. Water was added and then the reaction mixture was extracted with $\mathrm{Et}_{2} \mathrm{O}$, dried with $\mathrm{Na}_{2} \mathrm{SO}_{4}$ and evaporated under reduced pressure to leave the crude product which was then purified using silica gel flash chromatography to give alcohol III-18a (18 mg, $0.053 \mathrm{mmol}, 60 \%$ ) and alcohol III-18a1 (9 mg, $0.027 \mathrm{mmol}, 30 \%)$.

III-18a: colorless oil; $R f\left(10 \% \mathrm{Et}_{2} \mathrm{O} /\right.$ hexane $)=0.17 ;[\alpha]^{25}{ }_{\mathrm{D}}=+7\left(\mathrm{CH}_{2} \mathrm{Cl}_{2}\right)$; IR (thin film, $\left.\mathrm{cm}^{-1}\right), 3484,2931,2858,1716,1464,1252,1004,837,776 ;{ }^{1} \mathrm{H}$ NMR $\left(600 \mathrm{MHz}, \mathrm{CDCl}_{3}\right)$ $\delta 6.95(\mathrm{dd}, J=15.6,5.4 \mathrm{~Hz}, 1 \mathrm{H}), 6.10(\mathrm{dd}, J=15.6,1.8 \mathrm{~Hz}, 1 \mathrm{H}), 5.08(\mathrm{qdd}, J=6.0,6.0$, $2.4 \mathrm{~Hz}, 1 \mathrm{H}), 4.04(\mathrm{ddd}, J=7.2,5.4,1.8 \mathrm{~Hz}, 1 \mathrm{H}), 3.30(\mathrm{dd}, J=7.2,7.2, \mathrm{~Hz}, 1 \mathrm{H}), 1.60-$ $1.20(\mathrm{~m}, 10 \mathrm{H}), 1.28(\mathrm{~d}, J=6.0 \mathrm{~Hz}, 3 \mathrm{H}), 0.90(\mathrm{~s}, 9 \mathrm{H}), 0.10(\mathrm{~s}, 6 \mathrm{H}) ;{ }^{13} \mathrm{C}$ NMR $(150 \mathrm{MHz}$, $\left.\mathrm{CD}_{3} \mathrm{Cl}\right) \delta 166.9,146.8,121.9,78.9,77.3,72.6,33.7,32.4,28.0,25.8,24.5,22.9,19.0$, 18.0, - 4.20, - 4.19; ESI HRMS Calcd for $\left[\mathrm{C}_{18} \mathrm{H}_{34} \mathrm{O}_{4} \mathrm{Si}+\mathrm{Na}\right]^{+}: 365.2189$, Found: 365.2120 .

III-18a1: colorless oil; $R f\left(10 \% \mathrm{Et}_{2} \mathrm{O} /\right.$ hexane $)=0.20 ;[\alpha]_{\mathrm{D}}^{25}=+30\left(\mathrm{CH}_{2} \mathrm{Cl}_{2}\right)$; IR (thin film, $\left.\mathrm{cm}^{-1}\right), 3508,2932,2858,1716,1464,1251,1229,1004,837,778 ;{ }^{1} \mathrm{H}$ NMR $(600$ $\left.\mathrm{MHz}, \mathrm{CDCl}_{3}\right) \delta 6.94(\mathrm{dd}, J=16.2,6.0 \mathrm{~Hz}, 1 \mathrm{H}), 6.05(\mathrm{dd}, J=16.2,1.2 \mathrm{~Hz}, 1 \mathrm{H}), 5.11$ (dqd, $J=6.6,6.0,3.0 \mathrm{~Hz}, 1 \mathrm{H}), 4.03(\mathrm{ddd}, J=7.2,6.0,1.2 \mathrm{~Hz}, 1 \mathrm{H}), 3.29$ (ddd, $J=8.4$, 7.2, $1.2 \mathrm{~Hz}, 1 \mathrm{H}), 1.75-1.20(\mathrm{~m}, 10 \mathrm{H}), 1.28(\mathrm{~d}, J=6.0 \mathrm{~Hz}, 3 \mathrm{H}), 0.93(\mathrm{~s}, 9 \mathrm{H}), 0.10(\mathrm{~s}, 3 \mathrm{H})$, 0.07 (s, 3H); ${ }^{13} \mathrm{C}$ NMR (150 MHz, $\left.\mathrm{CD}_{3} \mathrm{Cl}\right)$ 8166.9, 147.9, 122.4, 78.7, 77.9, 72.7, 32.3, 
31.6, 28.0, 25.8, 24.8, 22.6, 18.8, 18.1, -5.35, -5.36; ESI HRMS Calcd for $\left[\mathrm{C}_{18} \mathrm{H}_{34} \mathrm{O}_{4} \mathrm{Si}\right.$ $+\mathrm{H}]^{+}: 343.2119$, Found: 343.2301 .

$(6 S, 12 R, E)-6$-(tert-butyldimethylsilyloxy)-12-methyloxacyclododec-3-ene-2,5-dione (III-18b)<smiles>CCCCC[C@H](O[SbH3])C(=O)/C=C/C(=O)OC1CCCCCC1</smiles>

To a magnetically stirred solution of alcohol III-18a $(10 \mathrm{mg}, 0.03 \mathrm{mmol})$ in dry $\mathrm{CH}_{2} \mathrm{Cl}_{2}$ $(0.4 \mathrm{~mL})$ was added Dess-Martin periodinane $(32 \mathrm{mg}, 0.076 \mathrm{mmol})$ at room temperature. The reaction mixture was stirred for $1 \mathrm{~h} . \mathrm{CH}_{2} \mathrm{Cl}_{2}$ was added to dilute the reaction solution and then the reaction mixture was washed with $\mathrm{NaHCO}_{3}$, extracted with ether acetate, dried with $\mathrm{Na}_{2} \mathrm{SO}_{4}$ and evaporated under reduced pressure to leave the crude product which was then purified using silica gel flash chromatography to give ketone III-18b $(9.2$ $\mathrm{mg}, 0.028 \mathrm{mmol}, 95 \%)$ as a colorless oil; $R f\left(10 \% \mathrm{Et}_{2} \mathrm{O} /\right.$ hexane $)=0.64 ;[\alpha]^{25}{ }_{\mathrm{D}}=+31$ $\left(\mathrm{CH}_{2} \mathrm{Cl}_{2}\right)$; IR (thin film, $\mathrm{cm}^{-1}$ ), 2932, 2858, 1723, 1464, 1254, 1095, 847, 779; ${ }^{1} \mathrm{H}$ NMR $\left(600 \mathrm{MHz}, \mathrm{CDCl}_{3}\right) \delta 7.41(\mathrm{~d}, J=16.8 \mathrm{~Hz}, 1 \mathrm{H}), 6.93(\mathrm{~d}, J=16.8 \mathrm{~Hz}, 1 \mathrm{H}), 4.63(\mathrm{qdd}, J=$ $6.0,6.0,6.0 \mathrm{~Hz}, 1 \mathrm{H}), 4.34(\mathrm{dd}, J=6.0,1.6 \mathrm{~Hz}, 1 \mathrm{H}), 1.90-1.06(\mathrm{~m}, 10 \mathrm{H}), 1.34(\mathrm{~d}, J=6.0$ $\mathrm{Hz}, 3 \mathrm{H}), 0.92$ (s, 9H), 0.08 (s, 3H), 0.07 (s, 3H); ${ }^{13} \mathrm{C}$ NMR $\left(150 \mathrm{MHz}, \mathrm{CD}_{3} \mathrm{Cl}\right) \delta$ 201.3, $167.5,134.2,132.0,77.2,74.9,34.8,34.2,27.9,25.7,24.1,21.4,20.6,18.1,-4.91$, 4.90; ESI HRMS Calcd for $\left[\mathrm{C}_{18} \mathrm{H}_{32} \mathrm{O}_{4} \mathrm{Si}+\mathrm{H}\right]^{+}: 341.2143$, Found: 341.2144 . 
(6S,12R,E)-6-hydroxy-12-methyloxacyclododec-3-ene-2,5-dione (III-29)<smiles>CC1CCCC[C@H](O)C(=O)/C=C/C(=O)O1</smiles>

To a plastic flask was added III-18b $(8 \mathrm{mg}, 0.024 \mathrm{mmol})$ and 5\% $\mathrm{HF}$ in $\mathrm{CH}_{3} \mathrm{CN}(30 \mu \mathrm{L})$ at room temperature. The reaction mixture was stirred for $2 \mathrm{hrs}$ and then ether acetate was added to dilute the reaction solution. The reaction mixture was washed with $\mathrm{NaHCO}_{3}$, extracted with ether acetate, dried with $\mathrm{Na}_{2} \mathrm{SO}_{4}$ and evaporated under reduced pressure to leave the crude product which was then purified using silica gel flash chromatography to give ketone III-29 (4.6 mg, $0.2 \mathrm{mmol}, 85 \%)$ as a colorless oil; $R f\left(50 \% \mathrm{Et}_{2} \mathrm{O} /\right.$ hexane $)=$ $0.56 ;[\alpha]_{D}^{25}=-10\left(\mathrm{CH}_{2} \mathrm{Cl}_{2}\right)$; IR (thin film, $\left.\mathrm{cm}^{-1}\right), 3508,2936,1714,1256,1194, \mathrm{~cm}-1 ;{ }^{1} \mathrm{H}$ NMR $\left(600 \mathrm{MHz}, \mathrm{CDCl}_{3}\right) \delta 7.12(\mathrm{~d}, J=16.2 \mathrm{~Hz}, 1 \mathrm{H}), 6.82(\mathrm{~d}, J=16.2 \mathrm{~Hz}, 1 \mathrm{H}), 4.92$ (qdd, $J=6.0,6.0,6.0 \mathrm{~Hz}, 1 \mathrm{H}), 4.52(\mathrm{dd}, J=8.4,4.2 \mathrm{~Hz}, 1 \mathrm{H}), 1.90(\mathrm{~m}, 1 \mathrm{H}), 1.72-1.18(\mathrm{~m}$, 9H), $1.36(\mathrm{~d}, J=6.0 \mathrm{~Hz}, 3 \mathrm{H}) ;{ }^{13} \mathrm{C} \mathrm{NMR}\left(150 \mathrm{MHz}, \mathrm{CD}_{3} \mathrm{Cl}\right) \delta 203.0,166.3,138.0,130.9$, 75.1, 74.6, 35.0, 34.8, 26.7, 24.0, 23.3, 20.7; ESI HRMS Calcd for $\left[\mathrm{C}_{12} \mathrm{H}_{18} \mathrm{O}_{4}+\mathrm{H}\right]^{+}$: 227.1278, Found: 227.1278. 
$(6 S, 12 R, Z)-6$-(tert-butyldimethylsilyloxy)-12-methyloxacyclododec-3-ene-2,5-dione (III-2a)<smiles>CO[C@@H](C)CCCCC[C@@H](O[SbH3])C(=O)/C=C\C(=O)O</smiles>

(A) To a magnetically stirred solution of Cladospolide B (5 mg, $0.022 \mathrm{mmol})$ and imidazole (4 mg, $0.05 \mathrm{mmol})$ in dry $\mathrm{CH}_{2} \mathrm{Cl}_{2}(0.2 \mathrm{~mL})$ was added dropwise $\mathrm{TBSCl}(9 \mathrm{mg}$, $0.06 \mathrm{mmol}$ ) at $0{ }^{\circ} \mathrm{C}$. The reaction mixture was warmed to room temperature and stirred overnight. Water was added and then the reaction mixture was extracted with $\mathrm{Et}_{2} \mathrm{O}$, dried with $\mathrm{Na}_{2} \mathrm{SO}_{4}$ and evaporated under reduced pressure to leave the inseparable 1:2 mixture of alcohol (6.8 mg, $0.02 \mathrm{mmol})$. To a magnetically stirred solution of mixture of $(2 \mathrm{mg}$, $0.006 \mathrm{mmol})$ in dry $\mathrm{CH}_{2} \mathrm{Cl}_{2}(0.06 \mathrm{~mL})$ was added Dess-Martin periodinane $(6 \mathrm{mg}, 0.015$ mmol) at room temperature. The reaction mixture was stirred for $1 \mathrm{~h} . \mathrm{CH}_{2} \mathrm{Cl}_{2}$ was added to dilute the reaction solution and then the reaction mixture was washed with $\mathrm{NaHCO}_{3}$, extracted with ether acetate, dried with $\mathrm{Na}_{2} \mathrm{SO}_{4}$ and evaporated under reduced pressure to leave the crude product which was then purified using silica gel flash chromatography to give ketone III-2a (0.6 mg, $0.0018 \mathrm{mmol}, 27 \%$ in 2 steps)

(B) To a plastic flask was added III-18b $(8 \mathrm{mg}, 0.024 \mathrm{mmol})$ and 5\% HF/Py. (2:1) in $\mathrm{CH}_{3} \mathrm{CN}(30 \mu \mathrm{L})$ at room temperature. The reaction mixture was stirred for $2 \mathrm{~h}$ and then ether acetate was added to dilute the reaction solution. The reaction mixture was washed with $\mathrm{NaHCO}_{3}$, extracted with ether acetate, dried with $\mathrm{Na}_{2} \mathrm{SO}_{4}$ and evaporated under reduced pressure to leave the crude product which was then purified using silica gel flash 
chromatography to give ketone III-2a $(7.4 \mathrm{mg}, 0.22 \mathrm{mmol}, 90 \%)$ as a colorless oil; $R f$ $\left(10 \% \mathrm{Et}_{2} \mathrm{O} /\right.$ hexane $)=0.37 ;[\alpha]^{25}{ }_{\mathrm{D}}=-80\left(\mathrm{CH}_{2} \mathrm{Cl}_{2}\right) ; \mathrm{IR}\left(\right.$ thin film, $\left.\mathrm{cm}^{-1}\right), 2931,2860,1726$, $1464,1251,1164,1119,839,778 ;{ }^{1} \mathrm{H}$ NMR $\left(600 \mathrm{MHz}, \mathrm{CDCl}_{3}\right) \delta 6.73(\mathrm{~d}, J=12.6 \mathrm{~Hz}$, $1 \mathrm{H}), 6.23(\mathrm{~d}, J=12.6 \mathrm{~Hz}, 1 \mathrm{H}), 4.91(\mathrm{dqd}, J=9.6,6.0,3.0 \mathrm{~Hz}, 1 \mathrm{H}), 4.32(\mathrm{dd}, J=7.8,4.2$ $\mathrm{Hz}, 1 \mathrm{H}), 1.93-1.26(\mathrm{~m}, 10 \mathrm{H}), 1.27(\mathrm{~d}, J=6.0 \mathrm{~Hz}, 3 \mathrm{H}), 0.93(\mathrm{~s}, 9 \mathrm{H}), 0.11(\mathrm{~s}, 3 \mathrm{H}), 0.09(\mathrm{~s}$, $3 \mathrm{H}) ;{ }^{13} \mathrm{C} \mathrm{NMR}\left(150 \mathrm{MHz}, \mathrm{CD}_{3} \mathrm{Cl}\right) \delta 203.2,166.3,133.6,130.6,76.4,73.9,33.3,330.5$, 26.3, 25.8, 22.1, 21.2, 20.0, 18.1, -4.91, -5.07; ESI HRMS Calcd for $\left[\mathrm{C}_{18} \mathrm{H}_{32} \mathrm{O}_{4} \mathrm{Si}+\mathrm{H}\right]^{+}$: 341.2143, Found: 341.2144

(6S,12R,Z)-6-hydroxy-12-methyloxacyclododec-3-ene-2,5-dione ((ent)-Cladospolide D III-31)<smiles>C[C@@H]1CCCCC[C@@H](O)C(=O)/C=C\C(=O)O1</smiles>

To a plastic flask was added III-2a (5 $\mathrm{mg}, 0.015 \mathrm{mmol})$ and $5 \% \mathrm{HF}$ in $\mathrm{CH}_{3} \mathrm{CN}(17 \mu \mathrm{L})$ at room temperature. The reaction mixture was stirred for $2 \mathrm{~h}$ and then ether acetate was added to dilute the reaction solution. The reaction mixture was washed with $\mathrm{NaHCO}_{3}$, extracted with ether acetate, dried with $\mathrm{Na}_{2} \mathrm{SO}_{4}$ and evaporated under reduced pressure to leave the crude product which was then purified using silica gel flash chromatography to give (ent)-Cladospolide D III-31 (3 mg, $0.013 \mathrm{mmol}, 87 \%)$ as a colorless oil; ${ }^{11} R f(30 \%$

\footnotetext{
${ }^{11}$ Zhang, H.; Tomoda, H.; Tabata, N.; Miura, H.; Namikoshi, M.; Yamaguchi, Y.; Masuma, R.; Omura, S. J. O. Anti. 54, 635-641
} 
$\mathrm{Et}_{2} \mathrm{O} /$ hexane $)=0.36 ;[\alpha]_{\mathrm{D}}^{25}=-28\left(\mathrm{CH}_{2} \mathrm{Cl}_{2}\right) ; \mathrm{IR}$ (thin film, $\left.\mathrm{cm}^{-1}\right), 3467,2936,1720$, 1224, 1167, 1083; ${ }^{1} \mathrm{H}$ NMR $\left(600 \mathrm{MHz}, \mathrm{CDCl}_{3}\right) \delta 6.40(\mathrm{~d}, J=13.2 \mathrm{~Hz}, 1 \mathrm{H}), 6.31(\mathrm{~d}, J=$ $13.2 \mathrm{~Hz}, 1 \mathrm{H}), 5.22(\mathrm{dqd}, J=9.0,6.0,6.0 \mathrm{~Hz}, 1 \mathrm{H}), 4.66(\mathrm{ddd}, J=8.4,6.0,5.4 \mathrm{~Hz}, 1 \mathrm{H})$, $3.16(\mathrm{~d}, J=6.0 \mathrm{~Hz}, 1 \mathrm{H}), 1.95(\mathrm{~m}, 1 \mathrm{H}), 1.72-1.18(\mathrm{~m}, 9 \mathrm{H}), 1.30(\mathrm{~d}, J=6.0 \mathrm{~Hz}, 3 \mathrm{H}) ;{ }^{13} \mathrm{C}$ NMR $\left(150 \mathrm{MHz}, \mathrm{CD}_{3} \mathrm{Cl}\right) \delta 203.5,165.4,133.3,130.9,73.5,71.5,33.2,31.1,23.0,21.6$, 21.5, 20.6; ESI HRMS Calcd for $\left[\mathrm{C}_{12} \mathrm{H}_{18} \mathrm{O}_{4}+\mathrm{H}\right]^{+}:$227.1278, Found: 227.1278.

(5R,6R,12R,Z)-6-(tert-butyldimethylsilyloxy)-5-hydroxy-12-methyloxacyclododec-3en-2-one (III-17a)<smiles>C[C@@H]1CCCCCC(O[Sb])/C=C\C(=O)O1</smiles>

(5R,6R,12R,Z)-5-(tert-butyldimethylsilyloxy)-6-hydroxy-12-methyloxacyclododec-3en-2-one (III-17a1)<smiles>C[C@H]1CCCCCC(O)C2C=CC(=O)O[Se]21</smiles>

To a magnetically stirred solution of diol III-17 (15 mg, $0.066 \mathrm{mmol})$ and imidazole (11.2 mg, $0.16 \mathrm{mmol})$ in dry $\mathrm{CH}_{2} \mathrm{Cl}_{2}(0.7 \mathrm{~mL})$ was added dropwise $\mathrm{TBSCl}$ (30 mg, 0.2 mmol) at $0{ }^{\circ} \mathrm{C}$. The reaction mixture was warmed to room temperature and stirred over night. Water was added and then the reaction mixture was extracted with $\mathrm{Et}_{2} \mathrm{O}$, dried with 
$\mathrm{Na}_{2} \mathrm{SO}_{4}$ and evaporated under reduced pressure to leave the crude product which was then purified using silica gel flash chromatography to give alcohol III-17a $(6.7 \mathrm{mg}, 0.02$ mmol, 30\%) and alcohol III-17a1 (13.4 mg, $0.04 \mathrm{mmol}, 60 \%)$.

III-17a: colorless oil; $R f\left(20 \% \mathrm{Et}_{2} \mathrm{O} /\right.$ hexane $)=0.39 ;[\alpha]^{25}=-56\left(\mathrm{CH}_{2} \mathrm{Cl}_{2}\right)$; IR (thin film, $\left.\mathrm{cm}^{-1}\right), 3482,2931,2858,1713,1463,1217,1075,835,778 ;{ }^{1} \mathrm{H}$ NMR (600 MHz, $\left.\mathrm{CDCl}_{3}\right)$ $\delta 6.14(\mathrm{dd}, J=12.0,7.2 \mathrm{~Hz}, 1 \mathrm{H}), 6.93(\mathrm{dd}, J=12.0,1.2 \mathrm{~Hz}, 1 \mathrm{H}), 5.14(\mathrm{qdd}, J=6.0,6.0$, $3.0 \mathrm{~Hz}, 1 \mathrm{H}), 4.94$ (dd, $J=7.2,1.2 \mathrm{~Hz}, 1 \mathrm{H}), 3.98$ (ddd, $J=10.2,3.0,1.2 \mathrm{~Hz}, 1 \mathrm{H}), 2.06-$ $1.26(\mathrm{~m}, 10 \mathrm{H}), 1.39(\mathrm{~d}, J=6.0 \mathrm{~Hz}, 3 \mathrm{H}), 0.90(\mathrm{~s}, 9 \mathrm{H}), 0.08(\mathrm{~s}, 3 \mathrm{H}), 0.05(\mathrm{~s}, 3 \mathrm{H}) ;{ }^{13} \mathrm{C} \mathrm{NMR}$ $\left(150 \mathrm{MHz}, \mathrm{CD}_{3} \mathrm{Cl}\right) \delta 165.8,148.7,121.0,74.3,72.5,67.4,31.9,29.3,25.8,24.4,21.5$, 21.2, 18.0, 16.8, - 4.70, - 5.09; ESI HRMS Calcd for $\left[\mathrm{C}_{18} \mathrm{H}_{34} \mathrm{O}_{4} \mathrm{Si}+\mathrm{H}\right]^{+}: 343.2299$, Found: 343.2300

III-17a1: colorless oil; $R f\left(20 \% \mathrm{Et}_{2} \mathrm{O} /\right.$ hexane $)=0.6 ;[\alpha]^{25}{ }_{\mathrm{D}}=+40\left(\mathrm{CH}_{2} \mathrm{Cl}_{2}\right)$; IR (thin film, $\left.\mathrm{cm}^{-1}\right), 3570,2931,2857,1713,1464,1199,1073,836,779 ;{ }^{1} \mathrm{H}$ NMR (600 MHz, $\left.\mathrm{CDCl}_{3}\right) \delta 6.14(\mathrm{~d}, J=12.0,8.4 \mathrm{~Hz}, 1 \mathrm{H}), 6.93(\mathrm{dd}, J=12.0,1.2 \mathrm{~Hz}, 1 \mathrm{H}), 5.13(\mathrm{qdd}, J=$ 6.0, 4.8, 3.6 Hz, 1H), 5.02 (ddd, $J=9.0,3.6,1.2 \mathrm{~Hz}, 1 \mathrm{H}), 3.75(\mathrm{ddd}, J=9.6,7.2,3.6, \mathrm{~Hz}$, $1 \mathrm{H}), 2.03-1.25(\mathrm{~m}, 10 \mathrm{H}), 1.27(\mathrm{~d}, J=6.0 \mathrm{~Hz}, 3 \mathrm{H}), 0.91(\mathrm{~s}, 9 \mathrm{H}), 0.11(\mathrm{~s}, 3 \mathrm{H}), 0.07(\mathrm{~s}, 3 \mathrm{H})$; ${ }^{13} \mathrm{C}$ NMR $\left(150 \mathrm{MHz}, \mathrm{CD}_{3} \mathrm{Cl}\right) \delta 166.1,146.9,121.6,73.6,72.6,69.0,31.5,29.9,26.0$, 24.9, 20.9, 20.7, 18.3, 17.3, - 4.66, - 4.68; ESI HRMS Calcd for $\left[\mathrm{C}_{18} \mathrm{H}_{34} \mathrm{O}_{4} \mathrm{Si}+\mathrm{H}\right]^{+}$: 343.2299, Found: 343.2301. 
(6R,12R,Z)-6-(tert-butyldimethylsilyloxy)-12-methyloxacyclododec-3-ene-2,5-dione (III-17b)

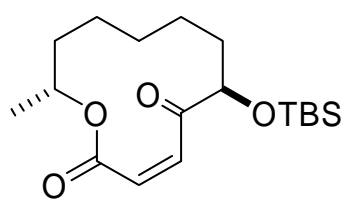

To a magnetically stirred solution of alcohol III-17a $(8 \mathrm{mg}, 0.023 \mathrm{mmol})$ in dry $\mathrm{CH}_{2} \mathrm{Cl}_{2}$ $(0.3 \mathrm{~mL})$ was added Dess-Martin periodinane $(24 \mathrm{mg}, 0.058 \mathrm{mmol})$ at room temperature. The reaction mixture was stirred for $1 \mathrm{~h} . \mathrm{CH}_{2} \mathrm{Cl}_{2}$ was added to dilute the reaction solution and then the reaction mixture was washed with $\mathrm{NaHCO}_{3}$, extracted with ether acetate, dried with $\mathrm{Na}_{2} \mathrm{SO}_{4}$ and evaporated under reduced pressure to leave the crude product which was then purified using silica gel flash chromatography to give ketone III-17b (7.4 $\mathrm{mg}, 0.022 \mathrm{mmol}, 95 \%)$ as a colorless oil; $R f\left(10 \% \mathrm{Et}_{2} \mathrm{O} /\right.$ hexane $)=0.50 ;[\alpha]^{25}{ }_{\mathrm{D}}=+18$ $\left(\mathrm{CH}_{2} \mathrm{Cl}_{2}\right)$; IR (thin film, $\mathrm{cm}^{-1}$ ), 2932, 2857, 1717, 1464, 1285, 1098, 838, 778; ${ }^{1} \mathrm{H}$ NMR $\left(600 \mathrm{MHz}, \mathrm{CDCl}_{3}\right) \delta 6.78(\mathrm{~d}, J=12.6 \mathrm{~Hz}, 1 \mathrm{H}), 5.95(\mathrm{~d}, J=12.6 \mathrm{~Hz}, 1 \mathrm{H}), 4.87(\mathrm{dqd}, J=$ 9.0, 6.0, 3.0 Hz, 1H), $4.14(\mathrm{dd}, J=9.6,3.0 \mathrm{~Hz}, 1 \mathrm{H}), 1.84-1.24(\mathrm{~m}, 10 \mathrm{H}), 1.25(\mathrm{~d}, J=6.0$ $\mathrm{Hz}, 3 \mathrm{H}), 0.91$ (s, 9H), 0.09 (s, 6H); ${ }^{13} \mathrm{C} \mathrm{NMR}\left(150 \mathrm{MHz}, \mathrm{CD}_{3} \mathrm{Cl}\right) \delta 204.3,164.5,140.4$, 125.9, 78.8, 73.9, 31.9, 31.3, 27.1, 25.7, 20.7, 19.5, 19.3, 18.0, - 4.80, - 5.13; ESI HRMS Calcd for $\left[\mathrm{C}_{18} \mathrm{H}_{32} \mathrm{O}_{4} \mathrm{Si}+\mathrm{Na}\right]^{+}: 363.1962$, Found: 363.1964 . 
(6R,12R,Z)-6-hydroxy-12-methyloxacyclododec-3-ene-2,5-dione (III-30)<smiles>C[C@@H]1CCCCC[C@@H](O)C(=O)/C=C\C(=O)O1</smiles>

To a plastic flask was added III-17b $(6 \mathrm{mg}, 0.018 \mathrm{mmol})$ and $5 \% \mathrm{HF}$ in $\mathrm{CH}_{3} \mathrm{CN}(20 \mu \mathrm{L})$ at room temperature. The reaction mixture was stirred for $2 \mathrm{~h}$ and then ether acetate was added to dilute the reaction solution. The reaction mixture was washed with $\mathrm{NaHCO}_{3}$, extracted with ether acetate, dried with $\mathrm{Na}_{2} \mathrm{SO}_{4}$ and evaporated under reduced pressure to leave the crude product which was then purified using silica gel flash chromatography to give alcohol III-30 (3.4 mg, $0.015 \mathrm{mmol}, 86 \%)$ as a colorless oil; $R f\left(30 \% \mathrm{Et}_{2} \mathrm{O} /\right.$ hexane $)$ $=0.32 ;[\alpha]^{25}=+4\left(\mathrm{CH}_{2} \mathrm{Cl}_{2}\right) ;$ IR (thin film, $\left.\mathrm{cm}^{-1}\right), 3453,2934,1712,1284,1083 ;{ }^{1} \mathrm{H}$ $\operatorname{NMR}\left(600 \mathrm{MHz}, \mathrm{CDCl}_{3}\right) \delta 6.56(\mathrm{~d}, J=13.2 \mathrm{~Hz}, 1 \mathrm{H}), 6.20(\mathrm{~d}, J=13.2 \mathrm{~Hz}, 1 \mathrm{H}), 4.96$ $(\mathrm{dqd}, J=9.6,6.0,3.6 \mathrm{~Hz}, 1 \mathrm{H}), 4.44(\mathrm{~m} \mathrm{1H}), 2.01(\mathrm{~m}, 1 \mathrm{H}), 1.82-1.18(\mathrm{~m}, 9 \mathrm{H}), 1.27(\mathrm{~d}, J=$ $6.0 \mathrm{~Hz}, 3 \mathrm{H}) ;{ }^{13} \mathrm{C} \mathrm{NMR}\left(150 \mathrm{MHz}, \mathrm{CD}_{3} \mathrm{Cl}\right) \delta 203.2,165.3,134.8,130.3,76.0,74.0,31.2$, 31.0, 27.3, 25.6, 21.5, 18.7; ESI HRMS Calcd for $\left[\mathrm{C}_{12} \mathrm{H}_{18} \mathrm{O}_{4}+\mathrm{H}\right]^{+}: 227.1278$, Found: 227.1278 .

(R,E)-12-methyloxacyclododec-3-ene-2,5,6-trione (III-26)<smiles>CC1CCCCCC(=O)C(=O)/C=C/C(=O)O1</smiles> 
A solution of diol III-3 (6 mg, $0.026 \mathrm{mmol})$ and trichloroisocyanuric acid $(6.1 \mathrm{mg}, 0.026$ mmol $)$ in ethyl ether $(0.3 \mathrm{ml})$ was stirred at $-30{ }^{\circ} \mathrm{C}$, then $2,2,6,6-$ tetramethylpeperidinooxy was added in one portion. The mixture was stirred for $60 \mathrm{~min}$ and then quenched with saturated sodium carbonate. The organic layer was separated and the aqueous layer was extracted with ether. Combined the organic layer, washed with brine, dried $\left(\mathrm{Na}_{2} \mathrm{SO}_{4}\right)$, filtered and concentrated. Chromatography on silica gel gave III26 (3.8 $\mathrm{mg}, 0.017 \mathrm{mmol}, 67 \%)$ as colorless oil.

A solution of diol III-18 (12 mg, $0.052 \mathrm{mmol})$ and trichloroisocyanuric acid (12.3 $\mathrm{mg}$, $0.053 \mathrm{mmol})$ in ethyl ether $(0.5 \mathrm{ml})$ was stirred at $-30{ }^{\circ} \mathrm{C}$, then $2,2,6,6-$ tetramethylpeperidinooxy was added in one portion. The mixture was stirred for $60 \mathrm{~min}$ and then quenched with saturated sodium carbonate. The organic layer was separated and the aqueous layer was extracted with ether. Combined the organic layer, washed with brine, dried $\left(\mathrm{Na}_{2} \mathrm{SO}_{4}\right)$, filtered and concentrated. Chromatography on silica gel gave III26 (7.6 mg, $0.034 \mathrm{mmol}, 65 \%)$ as colorless oil.

III-16: $R_{f}=0.67(7: 3(\mathrm{v} / \mathrm{v})$ hexane/EtOAc $) ;[\alpha]_{\mathrm{D}}^{20}=-2(c=0.1, \mathrm{MeOH}) ;$ IR (thin film, $\left.\mathrm{cm}^{-1}\right) \quad 3373,2934,1715,1134,1040,987,706 ;{ }^{1} \mathrm{H} \mathrm{NMR}\left(600 \mathrm{MHz}, \mathrm{CDCl}_{3}\right){ }^{1} \mathrm{H}$ spectra shows a mixture of diketone $\mathbf{1 6}$ and it's hydrate, peaks associated with the diketone: $\delta$ $6.87(\mathrm{~d}, J=15.6 \mathrm{~Hz}, 1 \mathrm{H}), 6.36(\mathrm{~d}, J=15.6 \mathrm{~Hz}, 1 \mathrm{H}), 5.05(\mathrm{~m}, 1 \mathrm{H}), 3.05(\mathrm{ddd}, J=13.2$, 7.8, $3.0 \mathrm{~Hz}, 1 \mathrm{H}), 2.36(\mathrm{ddd}, J=13.2,12.0,3.0 \mathrm{~Hz}, 1 \mathrm{H}), 1.41(\mathrm{~d}, J=7.8 \mathrm{~Hz}, 3 \mathrm{H})$, peaks associated with the hydride: $7.00(\mathrm{~s}, 2 \mathrm{H}), 4.60(\mathrm{~m}, 1 \mathrm{H}), 4.14(\mathrm{~s}, 1 \mathrm{H}), 3.73(\mathrm{~s}, 1 \mathrm{H}), 2.69$ 
$(\mathrm{m}, 1 \mathrm{H}), 2.54(\mathrm{~m}, 1 \mathrm{H}), 1.41(\mathrm{~d}, J=6.6 \mathrm{~Hz}, 3 \mathrm{H})$, peaks associated with the mixture: 1.67$1.54(\mathrm{~m}) ;{ }^{13} \mathrm{C} \mathrm{NMR}\left(150 \mathrm{MHz}, \mathrm{CD}_{3} \mathrm{Cl}\right)$ diketone 16: $\delta$ 204.2, 195.6, 164.9, 136.6, 136.0, 75.5, 39.5, 34.8, 24.8, 23.4, 21.8, 20.8; ESI HRMS Calcd for $\left[\mathrm{C}_{12} \mathrm{H}_{16} \mathrm{O}_{4}+\mathrm{H}\right]^{+}: 225.1121$, Found: 225.1122.

\section{(R,Z)-12-methyloxacyclododec-3-ene-2,5,6-trione (III-27)}

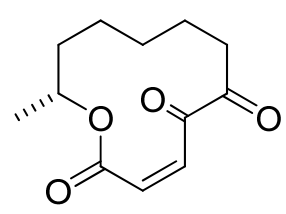

A solution of diol III-17 (6 mg, $0.026 \mathrm{mmol})$ and trichloroisocyanuric acid $(6.0 \mathrm{mg}$, $0.026 \mathrm{mmol})$ in ethyl ether $(0.3 \mathrm{ml})$ was stirred at $-30{ }^{\circ} \mathrm{C}$, then $2,2,6,6-$ tetramethylpeperidinooxy was added in one portion. The mixture was stirred for $40 \mathrm{~min}$ and then quenched with saturated sodium carbonate. The organic layer was separated and the aqueous layer was extracted with ether. Combined the organic layer, washed with brine, dried $\left(\mathrm{Na}_{2} \mathrm{SO}_{4}\right)$, filtered and concentrated. Chromatography on silica gel gave III27 (5.0 mg, $0.022 \mathrm{mmol}, 86 \%)$ as colorless oil.

A solution of diol III-2 (15 mg, $0.066 \mathrm{mmol})$ and trichloroisocyanuric acid (15.3 mg, $0.066 \mathrm{mmol})$ in ethyl ether $(0.6 \mathrm{ml})$ was stirred at $-30{ }^{\circ} \mathrm{C}$, then $2,2,6,6$ tetramethylpeperidinooxy was added in one portion. The mixture was stirred for $40 \mathrm{~min}$ and then quenched with saturated sodium carbonate. The organic layer was separated and the aqueous layer was extracted with ether. Combined the organic layer, washed with 
brine, dried $\left(\mathrm{Na}_{2} \mathrm{SO}_{4}\right)$, filtered and concentrated. Chromatography on silica gel gave III27 (12 mg, $0.056 \mathrm{mmol}, 85 \%)$ as colorless oil.

III-27: $R f=0.34\left(9: 1(\mathrm{v} / \mathrm{v})\right.$ hexane/EtOAc); $[\alpha]^{25}=+2\left(c=0.5, \mathrm{CH}_{2} \mathrm{Cl}_{2}\right)$; IR (thin film, $\left.\mathrm{cm}^{-1}\right), 2931,1710,1377,1284,995 ;{ }^{1} \mathrm{H}$ NMR $\left(600 \mathrm{MHz}, \mathrm{CDCl}_{3}\right) \delta 6.49(\mathrm{~d}, J=12.6 \mathrm{~Hz}$, $1 \mathrm{H}), 6.29(\mathrm{~d}, J=12.6 \mathrm{~Hz}, 1 \mathrm{H}), 5.02(\mathrm{dqd}, J=6.0,6.0,2.4 \mathrm{~Hz}, 1 \mathrm{H}), 3.13(\mathrm{ddd}, J=15.6$, 10.2, 2.4 Hz, 1H), 2.44 (ddd, $J=15.6,9.6,2.4 \mathrm{~Hz}, 1 \mathrm{H}), 1.98(\mathrm{~m}, 1 \mathrm{H}), 1.83(\mathrm{~m}, 1 \mathrm{H}), 1.59-$ $1.61(\mathrm{~m}, 6 \mathrm{H}), 1.18(\mathrm{~d}, J=6.0 \mathrm{~Hz}, 3 \mathrm{H}) ;{ }^{13} \mathrm{C} \mathrm{NMR}\left(150 \mathrm{MHz}, \mathrm{CD}_{3} \mathrm{Cl}\right) \delta 199.1,190.4$, 163.8, 135.7, 129.6, 73.2, 34.6, 31.6, 26.5, 21.4, 21.0, 19.0; ESI HRMS Calcd for $\left[\mathrm{C}_{12} \mathrm{H}_{16} \mathrm{O}_{4}+\mathrm{H}\right]^{+}:$225.1121, Found: 225.1122 .

(6S,12R,Z)-6-hydroxy-12-methyloxacyclododec-3-ene-2,5-dione (III-31)<smiles>C[C@@H]1CCCCC[C@@H](O)C(=O)/C=C\C(=O)O1</smiles>

To a solution of III-34 $(1.0 \mathrm{mg})$ in $3.0 \mathrm{ml} \mathrm{D}$-benzene, $0.1 \mathrm{mg} \mathrm{I}_{2}$ was added, the solution was irradiated with $300 \mathrm{~nm}$ UV light through a Pyrex filter for $20 \mathrm{~min}$, the chromatography gave double bond isomer III-35 $(0.95 \mathrm{mg}, 95 \%)$ was formed. To a plastic flask was added III-35 (5 mg, $0.015 \mathrm{mmol}) 0.1 \mathrm{~mL} \mathrm{CH}_{3} \mathrm{CN}$ and $5 \% \mathrm{HF}$ in $\mathrm{CH}_{3} \mathrm{CN}$ $(17 \mu \mathrm{L})$ at room temperature. The reaction mixture was stirred for $2 \mathrm{~h}$ and then ether acetate was added to dilute the reaction solution. The reaction mixture was washed with $\mathrm{NaHCO}_{3}$, extracted with EtOAc, dried with $\mathrm{Na}_{2} \mathrm{SO}_{4}$ and evaporated under reduced pressure to leave the crude product which was then purified using silica gel flash 
chromatography to give (ent)-cladospolide D III-31 (3 mg, $0.013 \mathrm{mmol}, 87 \%$ ) as a colorless oil;

III-31: $R f\left(30 \% \mathrm{Et}_{2} \mathrm{O} /\right.$ hexane $)=0.36 ;[\alpha]^{25}=-57\left(c=0.1, \mathrm{CH}_{2} \mathrm{Cl}_{2}\right) ;$ IR (thin film, $\mathrm{cm}^{-}$ $\left.{ }^{1}\right), 3467,2936,1720,1224,1167,1083 ;{ }^{1} \mathrm{H}$ NMR (600 MHz, $\left.\mathrm{CDCl}_{3}\right) \delta 6.40(\mathrm{~d}, J=13.2$ $\mathrm{Hz}, 1 \mathrm{H}), 6.31(\mathrm{~d}, J=13.2 \mathrm{~Hz}, 1 \mathrm{H}), 5.22(\mathrm{dqd}, J=9.0,6.0,6.0 \mathrm{~Hz}, 1 \mathrm{H}), 4.66(\mathrm{ddd}, J=$ 8.4, 6.0, $5.4 \mathrm{~Hz}, 1 \mathrm{H}), 3.16(\mathrm{~d}, J=6.0 \mathrm{~Hz}, 1 \mathrm{H}), 1.95(\mathrm{~m}, 1 \mathrm{H}), 1.72-1.18(\mathrm{~m}, 9 \mathrm{H}), 1.30(\mathrm{~d}, J$ $=6.0 \mathrm{~Hz}, 3 \mathrm{H}) ;{ }^{13} \mathrm{C} \mathrm{NMR}\left(150 \mathrm{MHz}, \mathrm{CD}_{3} \mathrm{Cl}\right) \delta 203.5,165.4,133.3,130.9,73.5,71.5$, 33.2, 31.1, 23.0, 21.6, 21.5, 20.6; ESI HRMS Calcd for $\left[\mathrm{C}_{12} \mathrm{H}_{18} \mathrm{O}_{4}+\mathrm{H}\right]^{+}: 227.1278$, Found: 227.1278

Photo equilibration of TBS-protected (E)-5-bis-cladospolide D (III-32) and TBSprotected (Z)-5-bis-cladospolide D (III-33)
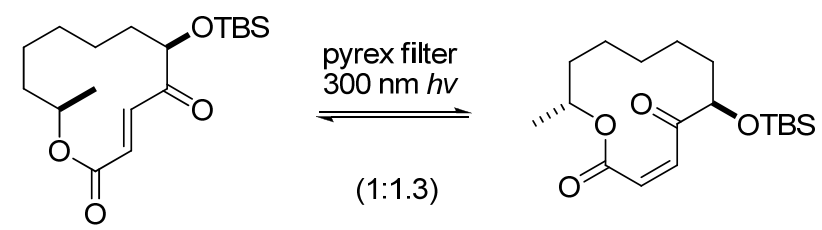

A solution of III-32 $(1.5 \mathrm{mg})$ in $3.5 \mathrm{ml} \mathrm{D}$-benzene was irradiated with $300 \mathrm{~nm}$ UV light through a Pyrex filter. After $24 \mathrm{~h}$, a photo equilibrium (1.3:1, III-33/ III-32) was established with the Z-isomer III-33 being slightly favored, III-32 and III-33 could be separated by chromatography. 
Pure III-33 (1.0 mg) was irradiated in $3.0 \mathrm{ml} \mathrm{D}_{6}$-benzene solution with $300 \mathrm{~nm} \mathrm{UV} \mathrm{light}$ through a Pyrex filter, the same photo equilibrium (1.3:1, III-33/ III-32) was established with the Z-isomer III-33 being slightly favored, III-32 and III-33 could be separated by chromatography.

III-32: $R f\left(10 \% \mathrm{Et}_{2} \mathrm{O} /\right.$ hexane $)=0.33 ;[\alpha]^{25}=-12\left(c=0.5, \mathrm{CH}_{2} \mathrm{Cl}_{2}\right)$; IR (thin film, $\mathrm{cm}^{-}$ $\left.{ }^{1}\right), 2931,2858,1721,1463,1251,1078,838,779 ;{ }^{1} \mathrm{H}$ NMR $\left(600 \mathrm{MHz}, \mathrm{CDCl}_{3}\right) \delta 7.21(\mathrm{~d}$, $J=16.8 \mathrm{~Hz}, 1 \mathrm{H}), 6.90(\mathrm{~d}, J=16.8 \mathrm{~Hz}, 1 \mathrm{H}), 4.89(\mathrm{dqd}, J=7.8 ., 6.0,4.8 \mathrm{~Hz}, 1 \mathrm{H}), 4.41$ $(\mathrm{dd}, J=6.0,4.2 \mathrm{~Hz}, 1 \mathrm{H}), 1.78-1.15(\mathrm{~m}, 10 \mathrm{H}), 1.34(\mathrm{~d}, J=6.0 \mathrm{~Hz}, 3 \mathrm{H}), 0.91(\mathrm{~s}, 9 \mathrm{H}), 0.10$ $(\mathrm{s}, 3 \mathrm{H}), 0.07(\mathrm{~s}, 3 \mathrm{H}) ;{ }^{13} \mathrm{C} \mathrm{NMR}\left(150 \mathrm{MHz}, \mathrm{CD}_{3} \mathrm{Cl}\right) \delta 200.8,166.8,138.2,131.3,79.2$, 75.3, 35.1, 34.1, 27.7, 25.8, 23.9, 20.6, 18.1, - 4.79, - 5.25; ESI HRMS Calcd for $\left[\mathrm{C}_{18} \mathrm{H}_{32} \mathrm{O}_{4} \mathrm{Si}+\mathrm{Na}\right]^{+}:$363.1962, Found: 363.1964 .

III-33: $R f\left(10 \% \mathrm{Et}_{2} \mathrm{O} /\right.$ hexane $)=0.50 ;[\alpha]^{25}=+18\left(c=0.4, \mathrm{CH}_{2} \mathrm{Cl}_{2}\right)$; IR (thin film, $\mathrm{cm}^{-}$ $\left.{ }^{1}\right), 2932,2857,1717,1464,1285,1098,838,778 ;{ }^{1} \mathrm{H}$ NMR $\left(600 \mathrm{MHz}, \mathrm{CDCl}_{3}\right) \delta 6.78(\mathrm{~d}$, $J=12.6 \mathrm{~Hz}, 1 \mathrm{H}), 5.95(\mathrm{~d}, J=12.6 \mathrm{~Hz}, 1 \mathrm{H}), 4.87(\mathrm{dqd}, J=9.0,6.0,3.0 \mathrm{~Hz}, 1 \mathrm{H}), 4.14(\mathrm{dd}$, $J=9.6,3.0 \mathrm{~Hz}, 1 \mathrm{H}), 1.84-1.24(\mathrm{~m}, 10 \mathrm{H}), 1.25(\mathrm{~d}, J=6.0 \mathrm{~Hz}, 3 \mathrm{H}), 0.91(\mathrm{~s}, 9 \mathrm{H}), 0.09(\mathrm{~s}$, $6 \mathrm{H}) ;{ }^{13} \mathrm{C} \mathrm{NMR}\left(150 \mathrm{MHz}, \mathrm{CD}_{3} \mathrm{Cl}\right) \delta 204.3,164.5,140.4,125.9,78.8,73.9,31.9,31.3$, 27.1, 25.7, 20.7, 19.5, 19.3, 18.0, - 4.80, - 5.13; ESI HRMS Calcd for $\left[\mathrm{C}_{18} \mathrm{H}_{32} \mathrm{O}_{4} \mathrm{Si}+\mathrm{Na}\right]^{+}$: 363.1962, Found: 363.1964. 
Iodine promoted photochemical equilibration of TBS-protected (E)-5-biscladospolide D (III-32) and TBS-protected (Z)-5-bis-cladospolide D (III-33)

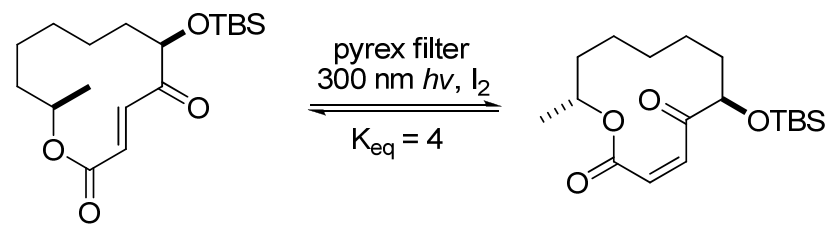

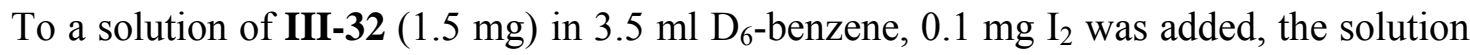
was irradiated with $300 \mathrm{~nm}$ UV light through a Pyrex filter. After $50 \mathrm{~min}$, a photochemical equilibrium $\left(\mathrm{K}_{\mathrm{eq}}=4\right.$, III-33/ III-32) was established with the $Z$-isomer III-33 being favored, III-32 and III-33 could be separated by chromatography.

To a solution of III-33 $(1.0 \mathrm{mg})$ in $3.0 \mathrm{ml} \mathrm{D}$-benzene, $0.1 \mathrm{mg} \mathrm{I} 2$ was added, the solution was irradiated with $300 \mathrm{~nm}$ UV light through a Pyrex filter. After $50 \mathrm{~min}$, the same photochemical equilibrium $\left(\mathrm{K}_{\mathrm{eq}}=4\right.$, III-33/ III-32) was established with the $Z$-isomer III-33 being favored, III-32 and III-33 could be separated by chromatography.

Photo equilibration of TBS-protected (E)-cladospolide D (III-34) and TBS-protected (Z)-cladospolide D (III-35)

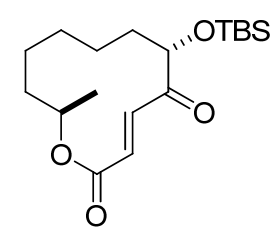

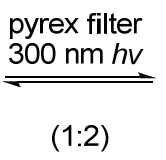<smiles>CC(C)O[C@H]1/C=C\C(=O)O[C@H](C)CCCCC1</smiles> 
A solution of III-34 $(1.0 \mathrm{mg})$ in $3.0 \mathrm{ml} \mathrm{D}$-benzene was irradiated with $300 \mathrm{~nm}$ UV light through a Pyrex filter. After $24 \mathrm{~h}$, a photo equilibrium $(1: 2$, III-34/III-35) was established with the Z-isomer III-35 being slightly favored, III-34 and III-35 could be separated by chromatography.

When pure III-35 $(1.0 \mathrm{mg})$ was irradiated in $3.0 \mathrm{ml} \mathrm{D}$-benzene solution with $300 \mathrm{~nm}$ UV light through a Pyrex filter, the same photo equilibrium (1:2, III-34/III-35) was established with the Z-isomer III-35 being slightly favored, III-34 and III-35 could be separated by chromatography.

III-34: $R f\left(10 \% \mathrm{Et}_{2} \mathrm{O} /\right.$ hexane $)=0.64 ;[\alpha]_{\mathrm{D}}^{25}=+31\left(c=0.4, \mathrm{CH}_{2} \mathrm{Cl}_{2}\right)$; IR (thin film, $\mathrm{cm}^{-}$ $\left.{ }^{1}\right), 2932,2858,1723,1464,1254,1095,847,779 ;{ }^{1} \mathrm{H} \mathrm{NMR}\left(600 \mathrm{MHz}, \mathrm{CDCl}_{3}\right) \delta 7.41(\mathrm{~d}$, $J=16.8 \mathrm{~Hz}, 1 \mathrm{H}), 6.93(\mathrm{~d}, J=16.8 \mathrm{~Hz}, 1 \mathrm{H}), 4.63(\mathrm{qdd}, J=6.0,6.0,6.0 \mathrm{~Hz}, 1 \mathrm{H}), 4.34(\mathrm{dd}$, $J=6.0,1.6 \mathrm{~Hz}, 1 \mathrm{H}), 1.90-1.06(\mathrm{~m}, 10 \mathrm{H}), 1.34(\mathrm{~d}, J=6.0 \mathrm{~Hz}, 3 \mathrm{H}), 0.92(\mathrm{~s}, 9 \mathrm{H}), 0.08(\mathrm{~s}$, 3H), 0.07 (s, 3H); ${ }^{13} \mathrm{C}$ NMR $\left(150 \mathrm{MHz}, \mathrm{CD}_{3} \mathrm{Cl}\right) \delta 201.3,167.5,134.2,132.0,77.2,74.9$, 34.8, 34.2, 27.9, 25.7, 24.1, 21.4, 20.6, 18.1, - 4.91, - 4.90; ESI HRMS Calcd for $\left[\mathrm{C}_{18} \mathrm{H}_{32} \mathrm{O}_{4} \mathrm{Si}+\mathrm{H}\right]^{+}:$341.2143, Found: 341.2144.

III-35: $R f\left(10 \% \mathrm{Et}_{2} \mathrm{O} /\right.$ hexane $)=0.37 ;[\alpha]_{\mathrm{D}}^{25}=-80\left(c=0.5, \mathrm{CH}_{2} \mathrm{Cl}_{2}\right)$; IR (thin film, $\mathrm{cm}^{-}$ $\left.{ }^{1}\right), 2931,2860,1726,1464,1251,1164,1119,839,778 ;{ }^{1} \mathrm{H}$ NMR $\left(600 \mathrm{MHz}, \mathrm{CDCl}_{3}\right) \delta$ $6.73(\mathrm{~d}, J=12.6 \mathrm{~Hz}, 1 \mathrm{H}), 6.23(\mathrm{~d}, J=12.6 \mathrm{~Hz}, 1 \mathrm{H}), 4.91(\mathrm{dqd}, J=9.6,6.0,3.0 \mathrm{~Hz}, 1 \mathrm{H})$, $4.32(\mathrm{dd}, J=7.8,4.2 \mathrm{~Hz}, 1 \mathrm{H}), 1.93-1.26(\mathrm{~m}, 10 \mathrm{H}), 1.27(\mathrm{~d}, J=6.0 \mathrm{~Hz}, 3 \mathrm{H}), 0.93(\mathrm{~s}, 9 \mathrm{H})$, $0.11(\mathrm{~s}, 3 \mathrm{H}), 0.09(\mathrm{~s}, 3 \mathrm{H}) ;{ }^{13} \mathrm{C}$ NMR $\left(150 \mathrm{MHz}, \mathrm{CD}_{3} \mathrm{Cl}\right) \delta$ 203.2, 166.3, 133.6, 130.6, 
76.4, 73.9, 33.3, 330.5, 26.3, 25.8, 22.1, 21.2, 20.0, 18.1, -4.91, -5.07; ESI HRMS Calcd for $\left[\mathrm{C}_{18} \mathrm{H}_{32} \mathrm{O}_{4} \mathrm{Si}+\mathrm{H}\right]^{+}:$341.2143, Found: 341.2144 .

Iodine promoted photochemical equilibration of TBS-protected $(E)$-cladospolide D (III-34) and TBS-protected (Z)-cladospolide D (III-35)

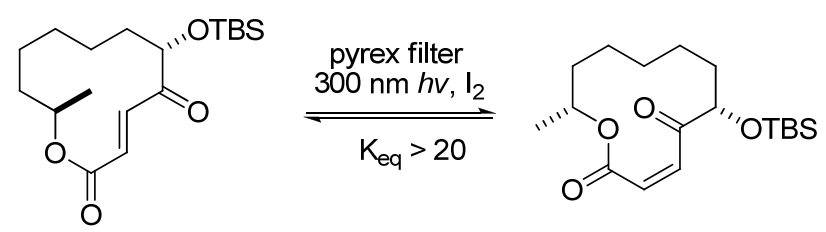

To a solution of III-34 $(1.0 \mathrm{mg})$ in $3.0 \mathrm{ml} \mathrm{D}$-benzene, $0.1 \mathrm{mg} \mathrm{I}_{2}$ was added, the solution was irradiated with $300 \mathrm{~nm}$ UV light through a Pyrex filter. After $20 \mathrm{~min}$, a photochemical equilibrium $\left(\mathrm{K}_{\mathrm{eq}}>20\right.$, III-35/III-34) was established with the $Z$-isomer III-35 being favored.

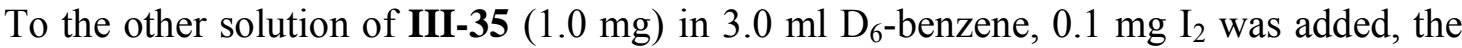
solution was irradiated with $300 \mathrm{~nm}$ UV light through a Pyrex filter. After $20 \mathrm{~min}$, the same photochemical equilibrium $\left(\mathrm{K}_{\mathrm{eq}}>20\right.$, III-35/III-34) was established with the $Z$ isomer III-35 being favored. 
Iodine promoted photochemical equilibration of cladospolide C (III-3) and 4,5-bisepi-cladospolide B (III-7)
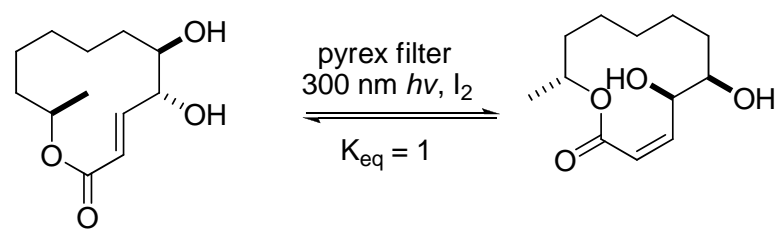

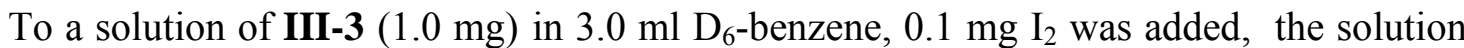
was irradiated with $300 \mathrm{~nm}$ UV light through a Pyrex filter. After $24 \mathrm{~h}$, a photochemical equilibrium $\left(\mathrm{K}_{\mathrm{eq}}=1\right.$, III-3/III-7) was established, III-3 and III-7 were separated by chromatography $\left(\mathrm{SiO}_{2}, 50 \% \mathrm{Et}_{2} \mathrm{O} /\right.$ hexane $)$.

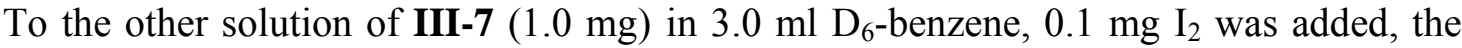
solution was irradiated with $300 \mathrm{~nm}$ UV light through a Pyrex filter. After $24 \mathrm{~h}$, the same photochemical equilibrium $\left(\mathrm{K}_{\mathrm{eq}}=1\right.$, III-3/ III-7) was established, III-3 and III-7 were separated by chromatography $\left(\mathrm{SiO}_{2}, 50 \% \mathrm{Et}_{2} \mathrm{O} /\right.$ hexane $)$.

III-3: $R f\left(50 \% \mathrm{Et}_{2} \mathrm{O} /\right.$ hexane $)=0.27 ; \mathrm{Mp} 91-92{ }^{\circ} \mathrm{C} ;[\alpha]_{\mathrm{D}}^{25}=+53 \quad(c=0.4, \mathrm{MeOH})$; IR (thin film, $\left.\mathrm{cm}^{-1}\right), 3415,2940,2864,1705,1650,1460,1258,1035 ;{ }^{1} \mathrm{H}$ NMR $(600 \mathrm{MHz}$, $\left.\mathrm{CDCl}_{3}\right) \delta 6.81(\mathrm{dd}, J=15.6,9.0 \mathrm{~Hz}, 1 \mathrm{H}), 6.05(\mathrm{~d}, J=15.6 \mathrm{~Hz}, 1 \mathrm{H}), 4.98(\mathrm{dqd}, J=8.4$, 6.0, $1.8 \mathrm{~Hz}, 1 \mathrm{H}), 3.98(\mathrm{dd}, J=8.4,7.8 \mathrm{~Hz}, 1 \mathrm{H}), 3.57(\mathrm{ddd}, J=7.8,7.8,1.8 \mathrm{~Hz}, 1 \mathrm{H}), 1.30$ $(\mathrm{d}, J=6.0 \mathrm{~Hz}, 3 \mathrm{H}) 1.69-1.11(\mathrm{~m}, 10 \mathrm{H}) ;{ }^{13} \mathrm{C} \mathrm{NMR}\left(150 \mathrm{MHz}, \mathrm{CD}_{3} \mathrm{Cl}\right) \delta 166.7,145.3$ 124.5, 77.5, 76.5, 74.3, 33.9, 32.1, 27.3, 24.5, 24.1, 20.8; ESI HRMS Calcd for $\left[\mathrm{C}_{12} \mathrm{H}_{20} \mathrm{O}_{4}+\mathrm{H}\right]^{+}:$229.1434, Found: 229.1435. 
III-7: $R f\left(50 \% \mathrm{Et}_{2} \mathrm{O} /\right.$ hexane $)=0.33 ;[\alpha]_{\mathrm{D}}^{25}=-1(\mathrm{c}=0.2, \mathrm{MeOH}) ;$ IR (thin film, $\left.\mathrm{cm}^{-1}\right)$, 3272, 2943, 2862, 1710, 1461, 1278, 1050; ${ }^{1} \mathrm{H}$ NMR $\left(600 \mathrm{MHz}, \mathrm{CDCl}_{3}\right) \delta 6.12(\mathrm{dd}, J=$ 12.0, $8.4 \mathrm{~Hz}, 1 \mathrm{H}), 6.05(\mathrm{dd}, J=12.0,1.2 \mathrm{~Hz}, 1 \mathrm{H}), 5.10$ (qdd, $J=6.0,5.4,3.0 \mathrm{~Hz}, 1 \mathrm{H})$, 4.97 (ddd, $J=8.4,4.8,1.2 \mathrm{~Hz}, 1 \mathrm{H}), 3.87(\mathrm{ddd}, J=9.0,4.8,3.0 \mathrm{~Hz}, 1 \mathrm{H}), 2.04-1.35(\mathrm{~m}$, $10 \mathrm{H}), 1.25(\mathrm{~d}, J=6.0 \mathrm{~Hz}, 3 \mathrm{H}) ;{ }^{13} \mathrm{C} \mathrm{NMR}\left(150 \mathrm{MHz}, \mathrm{CD}_{3} \mathrm{Cl}\right) \delta 166.7,145.3,124.5,77.5$, $76.5,74.3,33.9,32.1,27.3,24.5,24.1,20.8$; ESI HRMS Calcd for $\left[\mathrm{C}_{12} \mathrm{H}_{20} \mathrm{O}{ }_{4}+\mathrm{H}\right]^{+}$: 229.1434, Found: 229.1435.

\section{Isomerization of cladospolide B (III-2) and 4,5-bis-epi-cladospolide C (III-8)}<smiles>CC1CCCCCC(O)[C@H](O)C=CC(=O)O1</smiles>
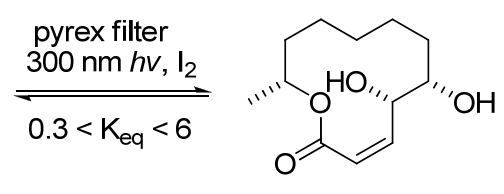

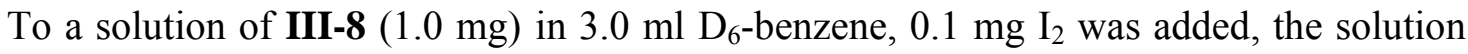
was irradiated with $300 \mathrm{~nm}$ UV light through a Pyrex filter. After $24 \mathrm{~h}$, a photochemical equilibrium $\left(\mathrm{K}_{\mathrm{eq}}=0.3\right.$, III-2/III-8) was established, III-8 and III-2 were separated by chromatography $\left(\mathrm{SiO}_{2}, 50 \% \mathrm{Et}_{2} \mathrm{O} /\right.$ hexane).

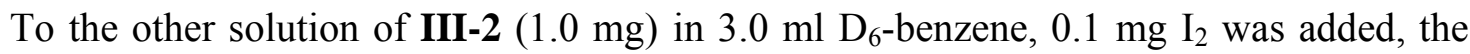
solution was irradiated with $300 \mathrm{~nm}$ UV light through a Pyrex filter. After $24 \mathrm{~h}$, a photochemical equilibrium $\left(\mathrm{K}_{\mathrm{eq}}=6\right.$, III-2/ III-8) was established, III-8 and III-2 were separated by chromatography $\left(\mathrm{SiO}_{2}, 50 \% \mathrm{Et}_{2} \mathrm{O} /\right.$ hexane $)$. 
III-8: $R f\left(50 \% \mathrm{Et}_{2} \mathrm{O} /\right.$ hexane $)=0.41 ;[\alpha]^{25}{ }_{\mathrm{D}}=-13(\mathrm{c}=0.1, \mathrm{MeOH}) ; \mathrm{IR}\left(\right.$ thin film, $\left.\mathrm{cm}^{-1}\right)$, 3396, 2937, 2870, 1711, 1464, 1247, 1028; ${ }^{1} \mathrm{H}$ NMR (600 MHz, $\left.\mathrm{CDCl}_{3}\right) \delta 6.94(\mathrm{dd}, J=$ 15.6, $6.0 \mathrm{~Hz}, 1 \mathrm{H}), 6.12(\mathrm{dd}, J=15.6,1.2 \mathrm{~Hz}, 1 \mathrm{H}), 4.96(\mathrm{dqd}, J=6.6,6.0,3.0 \mathrm{~Hz}, 1 \mathrm{H})$, $4.12(\mathrm{ddd}, J=7.2,6.0,1.2 \mathrm{~Hz}, 1 \mathrm{H}), 3.32(\mathrm{ddd}, J=9.6,7.8,1.8 \mathrm{~Hz}, 1 \mathrm{H}), 1.74-1.20(\mathrm{~m}$, $10 \mathrm{H}), 1.28(\mathrm{~d}, J=6.0 \mathrm{~Hz}, 3 \mathrm{H}) ;{ }^{13} \mathrm{C} \mathrm{NMR}\left(150 \mathrm{MHz}, \mathrm{CD}_{3} \mathrm{Cl}\right) \delta 167.2,146.9,122.5,78.0$, 77.8, 73.1, 33.2, 32.6, 28.3, 25.0, 22.8, 19.0; ESI HRMS Calcd for $\left[\mathrm{C}_{12} \mathrm{H}_{20} \mathrm{O}_{4}+\mathrm{Na}\right]^{+}$: 251.1254, Found: 251.1254.

III-2: $R f\left(50 \% \mathrm{Et}_{2} \mathrm{O} /\right.$ hexane $)=0.57 ; \mathrm{Mp} 108-109^{\circ} \mathrm{C} ;[\alpha]_{\mathrm{D}}^{25}=+24(\mathrm{c}=0.1, \mathrm{MeOH})$; IR (thin film, $\left.\mathrm{cm}^{-1}\right), 3345,2934,2865,1707,1461,1280,1046 ;{ }^{1} \mathrm{H}$ NMR (600 MHz, $\left.\mathrm{CDCl}_{3}\right) \delta 6.23(\mathrm{dd}, J=12.0,8.4 \mathrm{~Hz}, 1 \mathrm{H}), 5.77(\mathrm{dd}, J=12.0,1.2 \mathrm{~Hz}, 1 \mathrm{H}), 5.26(\mathrm{ddd}, J$ $=8.4,4.2,1.2 \mathrm{~Hz}, 1 \mathrm{H}), 4.88(\mathrm{dqd}, J=10.2,6.0,1.8 \mathrm{~Hz}, 1 \mathrm{H}), 3.77(\mathrm{ddd}, J=9.0,4.2,2.4$ $\mathrm{Hz}, 1 \mathrm{H}), 1.84-1.35(\mathrm{~m}, 10 \mathrm{H}), 1.28(\mathrm{~d}, J=6.0 \mathrm{~Hz}, 3 \mathrm{H}) ;{ }^{13} \mathrm{C} \mathrm{NMR}\left(150 \mathrm{MHz}, \mathrm{CD}_{3} \mathrm{Cl}\right)$ $\delta 165.8,148.5,121.9,74.4,73.9,67.5,32.0,30.6,25.7,24.1,21.3,19.7$; ESI HRMS Calcd for $\left[\mathrm{C}_{12} \mathrm{H}_{20} \mathrm{O}_{4}+\mathrm{H}\right]^{+}:$229.1434, Found: 229.1435 .

Methyl 3-(furan-2-yl)-3-oxopropanoate (IV-28)<smiles>COC(=O)CC(=O)c1ccco1</smiles> 
Sodium hydride $(5.71 \mathrm{~g}, 0.24 \mathrm{~mol})$ was placed in a flask and washed with dry hexane three times, dimethyl carbonate $(15.9 \mathrm{~g}, 0.177 \mathrm{~mol})$ and $60 \mathrm{~mL}$ THF was added to this flask. The mixture was heated to $50{ }^{\circ} \mathrm{C}$, a solution of 2-acetyl furan IV-30 $(6.54 \mathrm{~g}, 0.059$ mol) in $60 \mathrm{~mL}$ THF was added dropwise. The mixture was stirred for $1 \mathrm{~h}$ at $50{ }^{\circ} \mathrm{C}$ and then cooled to $0{ }^{\circ} \mathrm{C}$. After addition of glacial acetic acid followed by cold water, the mixture was extracted with ether acetate. The extracts were washed with cold water, dried $\left(\mathrm{Na}_{2} \mathrm{SO}_{4}\right)$ and concentrated under reduced pressure. The crude product was purified using silica gel flash chromatography to give furan ketone IV-28 (7.23 g, $0.043 \mathrm{~mol}$, $73 \%)$ as colorless oil: $R_{f}(10 \%$ EtOAc/hexane $)=0.15$; IR (thin film, $\left.\mathrm{cm}^{-1}\right) 3135,2956$,

1740, 1674, 1571, 1467, 1328, 1153, 1017, 883, 768; ${ }^{1} \mathrm{H}$ NMR $\left(600 \mathrm{MHz}, \mathrm{CDCl}_{3}\right) \delta 7.61$ (dd, $J=1.8,0.6 \mathrm{~Hz}, 1 \mathrm{H}), 7.27(\mathrm{dd}, J=3.6,0.6 \mathrm{~Hz}, 1 \mathrm{H}), 6.57(\mathrm{dd}, J=3.6,1.8 \mathrm{~Hz}, 1 \mathrm{H})$, $3.86(\mathrm{~s}, 2 \mathrm{H}), 3.75(\mathrm{~s}, 3 \mathrm{H}) ;{ }^{13} \mathrm{C}$ NMR $\left(150 \mathrm{MHz}, \mathrm{CDCl}_{3}\right) \delta 180.8,167.4,152.0,147.0$, $118.3,112.7,52.5,45.2$

(S)-Methyl 3-(furan-2-yl)-3-hydroxypropanoate (IV-28a)<smiles>COC(=O)CC(O)c1ccco1</smiles>

To a flask was added furan ketone IV-28 $(173 \mathrm{mg}, 1.03 \mathrm{mmol}), 2 \mathrm{M} \mathrm{NaCOOH}$ in water $(10 \mathrm{~mL})$, cetyl trimethylammonium bromide $(37.5 \mathrm{mg}, 0.10 \mathrm{mmol})$. The mixture was stirred at RT. for $5 \mathrm{~min}$, then Noyori catalyst $(S, S)(1.3 \mathrm{mg}, 0.002 \mathrm{mmol})$ was added. The reaction mixture was allowed to stir for $24 \mathrm{hr}$. Water was added to dilute and the mixture was extracted with $\mathrm{Et}_{2} \mathrm{O}$, dried $\left(\mathrm{Na}_{2} \mathrm{SO}_{4}\right)$ and concentrated under reduced pressure. The 
crude product was purified using silica gel flash chromatography to give $148 \mathrm{mg}(0.88$ mmol, $85 \%)$ of furan alcohol IV-28a as colorless oil: $R_{f}(30 \%$ EtOAc/hexane $)=0.18$; $[\alpha]^{25}{ }_{\mathrm{D}}=-24(c=1.0, \mathrm{MeOH}) ;$ IR (thin film, $\left.\mathrm{cm}^{-1}\right) 3450,2956,1733,1439,1362,1284$, 1163, 1011, 884, 805, 741; ${ }^{1} \mathrm{H}$ NMR (600 MHz, $\left.\mathrm{CDCl}_{3}\right) \delta 7.37(\mathrm{dd}, J=1.8,0.6 \mathrm{~Hz}, 1 \mathrm{H})$, $6.33(\mathrm{dd}, J=3.0,1.8 \mathrm{~Hz}, 1 \mathrm{H}), 6.277$ (ddd, $J=3.0,0.6,0.6 \mathrm{~Hz}, 1 \mathrm{H}), 5.14$ (ddd, $J=9.0$, $4.2,0.6 \mathrm{~Hz}, 1 \mathrm{H}), 3.75(\mathrm{~s}, 3 \mathrm{H}), \delta 2.91(\mathrm{dd}, J=16.8,9.0 \mathrm{~Hz}, 1 \mathrm{H}), 2.83(\mathrm{dd}, J=16.8,4.2$ $\mathrm{Hz}, 1 \mathrm{H}) ;{ }^{13} \mathrm{C} \mathrm{NMR}\left(150 \mathrm{MHz}, \mathrm{CDCl}_{3}\right) \delta 172.2,154.7,142.2,110.2,106.3,64.1,51.9$, 39.6

(S)-Methyl 2-(6-hydroxy-3-oxo-3,6-dihydro-2H-pyran-2-yl)acetate (IV-32)<smiles>COC(=O)C[C@H]1OC(O)C=CC1=O</smiles>

To a solution of furan alcohol IV-28a $(100 \mathrm{mg}, 0.59 \mathrm{mmol})$ in $4 \mathrm{~mL}$ THF- $\mathrm{H}_{2} \mathrm{O}(3: 1)$ was added $\mathrm{NaHCO}_{3}(98.8 \mathrm{mg}, 1.18 \mathrm{mmol}), \mathrm{NaOAc} .3 \mathrm{H}_{2} \mathrm{O}(80 \mathrm{mg}, 0.59 \mathrm{mmol})$ and NBS (105 $\mathrm{mg}, 0.59 \mathrm{mmol})$ at $0{ }^{\circ} \mathrm{C}$. The reaction mixture was kept stirring at this temperature for $1 \mathrm{~h}$, then saturated $\mathrm{NaHCO}_{3}$ was added to quench the reaction. The reaction mixture was extracted with EtOAc, dried $\left(\mathrm{Na}_{2} \mathrm{SO}_{4}\right)$ and concentrated under reduced pressure. The crude product was purified using silica gel flash chromatography to give $87 \mathrm{mg}(0.47$ mmol, $80 \%)$ of pyranone alcohol IV-32 as colorless oil: $R_{f}(30 \%$ EtOAc/hexane $)=0.14$ 


\section{Methyl-2-((2S,6S)-6-(tert-butoxycarbonyloxy)-3-0xo-3,6-dihydro-2H-pyran-2-}

yl)acetate (IV-33)<smiles>COC(=O)C[C@H]1OC(OC(C)=O)C=CC1=O</smiles>

To a solution of pyranone alcohol IV-32 (70 mg, $0.376 \mathrm{mmol})$ in $3 \mathrm{~mL} \mathrm{CH}_{2} \mathrm{Cl}_{2}$ was added DMAP (4.6 mg, $0.0376 \mathrm{mmol})$ at $-78{ }^{\circ} \mathrm{C}$, a pre-cooled solution of $(\mathrm{Boc})_{2} \mathrm{O}(164$ $\mathrm{mg}, 0.75 \mathrm{mmol}$ ) in $2 \mathrm{~mL} \mathrm{CH}_{2} \mathrm{Cl}_{2}$ was added dropwise. The reaction mixture was stirred at $-78{ }^{\circ} \mathrm{C}$ for $12 \mathrm{hr}$. The reaction mixture was diluted with EtOAc and quenched with saturated $\mathrm{NaHCO}_{3}$, extracted with EtOAc, washed $(\mathrm{NaCl})$, dried $\left(\mathrm{Na}_{2} \mathrm{SO}_{4}\right)$ and concentrated under reduced pressure. The crude product was purified using silica gel flash chromatography to give Boc-pyranone IV-33 $76 \mathrm{mg}(0.27 \mathrm{mmol}, 71 \%)$ as light yellow oil: $R_{f}(35 \%$ EtOAc/hexane $)=0.71 ;[\alpha]^{25}=+34.2(c=1.0, \mathrm{MeOH})$; IR (thin film, $\left.\mathrm{cm}^{-1}\right) 2919,1744,1702,1371,1275,1159,946,849 ;{ }^{1} \mathrm{H} \mathrm{NMR}\left(600 \mathrm{MHz}, \mathrm{CDCl}_{3}\right) \delta$ $6.87(\mathrm{dd}, J=10.2,3.6 \mathrm{~Hz}, 1 \mathrm{H}), 6.33(\mathrm{~d}, J=3.6 \mathrm{~Hz}, 1 \mathrm{H}), 6.25(\mathrm{~d}, J=10.2 \mathrm{~Hz}, 1 \mathrm{H}), 4.91$ $(\mathrm{dd}, J=6.0,4.2 \mathrm{~Hz}, 1 \mathrm{H}), 3.68(\mathrm{~s}, 3 \mathrm{H}), 2.95(\mathrm{dd}, J=16.8,4.8 \mathrm{~Hz}, 1 \mathrm{H}), 2.85(\mathrm{dd}, J=16.8$, $6.0 \mathrm{~Hz}, 1 \mathrm{H}), 1.52(\mathrm{~s}, J=9 \mathrm{H}) ;{ }^{13} \mathrm{C} \mathrm{NMR}\left(150 \mathrm{MHz}, \mathrm{CDCl}_{3}\right) \delta 193.8,170.3,151.7,140.8$, 128.5, 88.9, 83.7, 72.4, 51.9, 35.1, 27.6; CIHRMS: Calculated for $\left[\mathrm{C}_{13} \mathrm{H}_{18} \mathrm{O}_{7}+\mathrm{Na}^{+}\right]$: 309.0945, Found: 309.0946 


\section{Methyl-2-((2S,6R)-6-(tert-butoxycarbonyloxy)-3-oxo-3,6-dihydro-2H-pyran-2-}

yl)acetate (IV-34)<smiles>COC(=O)C[C@H]1O[C@@H](OC(C)=O)C=CC1=O</smiles>

To a solution of pyranone alcohol IV-32 (70 mg, $0.376 \mathrm{mmol})$ in $3 \mathrm{~mL} \mathrm{CH}_{2} \mathrm{Cl}_{2}$ was added DMAP (4.6 mg, $0.0376 \mathrm{mmol})$ at $-78{ }^{\circ} \mathrm{C}$, a pre-cooled solution of $(\mathrm{Boc})_{2} \mathrm{O}(164$ $\mathrm{mg}, 0.75 \mathrm{mmol}$ ) in $2 \mathrm{~mL} \mathrm{CH}_{2} \mathrm{Cl}_{2}$ was added dropwise. The reaction mixture was stirred at $-78{ }^{\circ} \mathrm{C}$ for $12 \mathrm{hr}$. The reaction mixture was diluted with EtOAc and quenched with saturated $\mathrm{NaHCO}_{3}$, extracted with EtOAc, washed $(\mathrm{NaCl})$, dried $\left(\mathrm{Na}_{2} \mathrm{SO}_{4}\right)$ and concentrated under reduced pressure. The crude product was purified using silica gel flash chromatography to give Boc-pyranone IV-34 $9 \mathrm{mg}(0.03 \mathrm{mmol}, 8 \%)$ as light yellow oil: $R_{f}(35 \%$ EtOAc/hexane $)=0.66 ;[\alpha]^{25}=-64.8(c=1.0, \mathrm{MeOH}) ; \mathrm{IR}\left(\right.$ thin film, $\left.\mathrm{cm}^{-1}\right)$ 2919, 1748, 1700, 1371, 1275, 1164, 1064, 945, 851; ${ }^{1} \mathrm{H}$ NMR $\left(600 \mathrm{MHz}, \mathrm{CDCl}_{3}\right) \delta 6.91$ $(\mathrm{dd}, J=10.2,2.4 \mathrm{~Hz}, 1 \mathrm{H}), 6.40(\mathrm{dd}, J=1.8,1.8 \mathrm{~Hz}, 1 \mathrm{H}), 6.26(\mathrm{dd}, J=10.2,1.2 \mathrm{~Hz}, 1 \mathrm{H})$, $4.73(\mathrm{dd}, J=7.8,5.4 \mathrm{~Hz}, 1 \mathrm{H}), 3.71(\mathrm{~s}, 3 \mathrm{H}), 2.91(\mathrm{dd}, J=16.8,4.8 \mathrm{~Hz}, 1 \mathrm{H}), 2.88(\mathrm{dd}, J=$ 16.8, 7.8 Hz, 1H), 1.52 (s, $J=9 \mathrm{H}) ;{ }^{13} \mathrm{C} \mathrm{NMR}\left(150 \mathrm{MHz}, \mathrm{CDCl}_{3}\right) \delta 193.6,170.1,151.4$, 143.5, 128.8, 90.0, 83.9, 75.8, 52.0, 37.4, 27.6; CIHRMS: Calculated for $\left[\mathrm{C}_{13} \mathrm{H}_{18} \mathrm{O}_{7}\right.$ $+\mathrm{Na}^{+}$]: 309.0945, Found: 309.0943 
Methyl-2-((2S,3R,6S)-6-(tert-butoxycarbonyloxy)-3-hydroxy-3,6-dihydro-2H-pyran2-yl)acetate (IV-48)<smiles>COC(=O)C[C@H]1OC(OC(C)(C)C)C=CC1O</smiles>

To a solution of pyranone IV-32 $(62 \mathrm{mg}, 0.22 \mathrm{mmol})$ in $0.5 \mathrm{~mL} \mathrm{CH}_{2} \mathrm{Cl}_{2}$ was added 0.5 $\mathrm{mL} \mathrm{CeCl} 3$ in $\mathrm{MeOH}(0.4 \mathrm{M})$ and $\mathrm{NaBH}_{4}(16 \mathrm{mg}, 0.43 \mathrm{mmol})$ at $-78{ }^{\circ} \mathrm{C}$. The reaction mixture was stirred at $-78{ }^{\circ} \mathrm{C}$ for $3 \mathrm{hr}$. The reaction mixture was diluted with EtOAc and quenched with saturated $\mathrm{NaHCO}_{3}$, extracted with EtOAc, washed $(\mathrm{NaCl})$, dried $\left(\mathrm{Na}_{2} \mathrm{SO}_{4}\right)$ and concentrated under reduced pressure. The crude product was purified using silica gel flash chromatography to give allylic alcohol IV-48 $57 \mathrm{mg}(0.20 \mathrm{mmol}, 90 \%)$ as colorless oil: $R_{f}(30 \%$ EtOAc/hexane $)=0.28 ;[\alpha]^{25}{ }_{\mathrm{D}}=-73.0\left(c=1.0, \mathrm{CH}_{2} \mathrm{Cl}_{2}\right) ;$ IR (thin film, $\left.\mathrm{cm}^{-1}\right)$ $3483,2981,1744,1438,1371,1275,1164,1057,938,847 ;{ }^{1} \mathrm{H}$ NMR $\left(600 \mathrm{MHz}, \mathrm{CDCl}_{3}\right)$ $\delta 6.07(\mathrm{ddd}, J=10.2,1.2,1.2 \mathrm{~Hz}, 1 \mathrm{H}), 6.03(\mathrm{ddd}, J=2.4,1.2,1.2 \mathrm{~Hz}, 1 \mathrm{H}), 5.78(\mathrm{ddd}, J=$ 10.2, 3.0, 2.4 Hz, 1H), 4.13-4.09 (m, 2H), 3.69 (s, 3H), 2.83 (dd, $J=16.2,4.2 \mathrm{~Hz}, 1 \mathrm{H})$, $2.63(\mathrm{dd}, J=16.2,7.2 \mathrm{~Hz}, 1 \mathrm{H}), 1.51(\mathrm{~s}, J=9 \mathrm{H}) ;{ }^{13} \mathrm{C} \mathrm{NMR}\left(150 \mathrm{MHz}, \mathrm{CDCl}_{3}\right) \delta 171.7$, 152.2, 134.8, 124.2, 90.3, 82.8, 70.3, 67.2, 51.9, 37.6, 27.7; CIHRMS: Calculated for $\left[\mathrm{C}_{13} \mathrm{H}_{20} \mathrm{O}_{7}+\mathrm{Na}^{+}\right]: 311.1101$, Found: 311.1092 
Methyl-2-((2S,3R,6S)-6-(tert-butoxycarbonyloxy)-3-(tert-butyldimethylsilyloxy)-3,6dihydro-2H-pyran-2-yl)acetate (IV-50)<smiles>[R5]OC1C=CC(OC(C)(C)C)[C@@H](CC(=O)OC)O1</smiles>

To a solution of alcohol IV-48 $(50 \mathrm{mg}, 0.17 \mathrm{mmol})$ in $1 \mathrm{~mL} \mathrm{CH}_{2} \mathrm{Cl}_{2}$ was added imidazol (24 mg, $0.35 \mathrm{mmol})$ and $\mathrm{TBSCl}(53 \mathrm{mg}, 0.35 \mathrm{mmol})$ at $0{ }^{\circ} \mathrm{C}$. The reaction mixture was stirred at RT overnight. The reaction mixture was diluted with EtOAc and quenched with saturated $\mathrm{NaHCO}_{3}$, extracted with EtOAc, washed $(\mathrm{NaCl})$, dried $\left(\mathrm{Na}_{2} \mathrm{SO}_{4}\right)$ and concentrated under reduced pressure. The crude product was purified using silica gel flash chromatography to give TBS-alcohol IV-50 $63 \mathrm{mg}(0.16 \mathrm{mmol}, 92 \%)$ as colorless oil: $R_{f}(10 \%$ EtOAc/hexane $)=0.5 ;[\alpha]^{25}=-83.5\left(c=1.3, \mathrm{CH}_{2} \mathrm{Cl}_{2}\right)$; IR (thin film, $\left.\mathrm{cm}^{-1}\right)$ 2960, 2932, 2859, 1748, 1370, 1254, 1139, 1096, 838; ${ }^{1} \mathrm{H}$ NMR $\left(600 \mathrm{MHz}, \mathrm{CDCl}_{3}\right) \delta$ $6.01(\mathrm{dd}, J=1.8,1.2 \mathrm{~Hz}, 1 \mathrm{H}), 5.96(\mathrm{ddd}, J=10.2,1.2,1.2 \mathrm{~Hz}, 1 \mathrm{H}), 5.70(\mathrm{ddd}, J=10.2$, $3.0,1.8 \mathrm{~Hz}, 1 \mathrm{H}), 4.18(\mathrm{ddd}, J=9.6,9.0,2.4 \mathrm{~Hz}, 1 \mathrm{H}), 4.08(\mathrm{ddd}, J=9.0,3.0,1.2 \mathrm{~Hz}, 1 \mathrm{H})$, $3.67(\mathrm{~s}, 3 \mathrm{H}), 2.77(\mathrm{dd}, J=15.6,2.4 \mathrm{~Hz}, 1 \mathrm{H}), 2.43(\mathrm{dd}, J=15.6,9.6 \mathrm{~Hz}, 1 \mathrm{H}), 1.51(\mathrm{~s}, J=$ $9 \mathrm{H}), 0.87(\mathrm{~s}, J=9 \mathrm{H}), 0.10(\mathrm{~s}, J=6 \mathrm{H}), 0.07(\mathrm{~s}, J=6 \mathrm{H}) ;{ }^{13} \mathrm{C} \mathrm{NMR}\left(150 \mathrm{MHz}, \mathrm{CDCl}_{3}\right)$ $\delta 171.0,152.3,135.9,123.4,90.5,82.6,70.4,67.0,51.6,36.6,27.7,25.6,17.8,-4.29,-$ 4.88; CIHRMS: Calculated for $\left[\mathrm{C}_{19} \mathrm{H}_{34} \mathrm{O}_{7} \mathrm{Si}+\mathrm{Na}^{+}\right]$: 425.1966, Found: 425.1970 
(2S,3S,6S)-6-(tert-butoxycarbonyloxy)-2-(2-methoxy-2-oxoethyl)-3,6-dihydro-2Hpyran-3-yl 4-nitrobenzoate (IV-48a)<smiles>COC(=O)C[C@H]1OC(OC(C)(C)C)C=C[C@@H]1OC(=O)OCc1ccccc1</smiles>

To a solution of alcohol IV-48 (100 mg, $0.35 \mathrm{mmol})$ in $5 \mathrm{~mL}$ THF was added $\mathrm{PPh}_{3}(182$ $\mathrm{mg}, 0.69 \mathrm{mmol}), p$-Nitrobenzoic acid $(116 \mathrm{mg}, 0.69 \mathrm{mmol})$ and DIAD (0.14 mL, 0.69 mmol) at $0{ }^{\circ} \mathrm{C}$. The reaction mixture was stirred at $\mathrm{RT}$ overnight. The reaction mixture was diluted with EtOAc and quenched with saturated $\mathrm{NaHCO}_{3}$, extracted with EtOAc, washed $(\mathrm{NaCl})$, dried $\left(\mathrm{Na}_{2} \mathrm{SO}_{4}\right)$ and concentrated under reduced pressure. The crude product was purified using silica gel flash chromatography to give IV-48a $125 \mathrm{mg}(0.29$ mmol, $82 \%)$ as colorless oil: $R_{f}(10 \%$ EtOAc/hexane $)=0.25 ;[\alpha]^{25}=148.4(c=1.0$, $\mathrm{CH}_{2} \mathrm{Cl}_{2}$ ); IR (thin film, $\mathrm{cm}^{-1}$ ) 2990, 2957, 1747, 1530, 1263, 1167, 942, 847; ${ }^{1} \mathrm{H}$ NMR $\left(600 \mathrm{MHz}, \mathrm{CDCl}_{3}\right) \delta 8.29(\mathrm{~d}, J=9.0,2 \mathrm{H}), \delta 8.21(\mathrm{~d}, J=9.0,2 \mathrm{H}), 6.36(\mathrm{ddd}, J=10.2$, 6.0, $1.2 \mathrm{~Hz}, 1 \mathrm{H}), 6.21(\mathrm{dd}, J=3.0,0.6 \mathrm{~Hz}, 1 \mathrm{H}), 6.13(\mathrm{ddd}, J=10.2,3.0,0.6 \mathrm{~Hz}, 1 \mathrm{H})$, $5.32(\mathrm{dd}, J=6.0,2.4 \mathrm{~Hz}, 1 \mathrm{H}), 4.75(\mathrm{ddd}, J=8.4,6.0,2.4 \mathrm{~Hz}, 1 \mathrm{H}), 3.66(\mathrm{~s}, 3 \mathrm{H}), 2.77(\mathrm{dd}$, $J=16.2,8.4 \mathrm{~Hz}, 1 \mathrm{H}), 2.66(\mathrm{dd}, J=16.2,6.0 \mathrm{~Hz}, 1 \mathrm{H}), 1.53(\mathrm{~s}, J=9 \mathrm{H}) ;{ }^{13} \mathrm{C} \mathrm{NMR}(150$ $\left.\mathrm{MHz}, \mathrm{CDCl}_{3}\right)$ 170.1, 164.0, 152.0, 150.8, 134.8, 130.9, 129.1, 126.4, 123.7, 90.0, 83.1, 67.4, 64.8, 51.9, 35.5, 27.7; CIHRMS: Calculated for $\left[\mathrm{C}_{20} \mathrm{H}_{23} \mathrm{NO}_{10}+\mathrm{Na}^{+}\right]$: 460.1214, Found: 460.1169 
Methyl-2-((2S,3S,6S)-6-(tert-butoxycarbonyloxy)-3-hydroxy-3,6-dihydro-2H-pyran2-yl)acetate (IV-52)<smiles>COC(=O)C[C@H]1OC(OC(=O)OC(C)(C)C)C=C[C@H]1O</smiles>

To a solution of nitrobenzoate IV-48a (34 mg, $0.08 \mathrm{mmol}$ ) in $0.8 \mathrm{~mL} \mathrm{MeOH}$ was added $\mathrm{K}_{2} \mathrm{CO}_{3}(1 \mathrm{mg}, 10 \% \mathrm{mmol})$ at $0{ }^{\circ} \mathrm{C}$. The reaction mixture was stirred at $\mathrm{RT}$ for $15 \mathrm{~min}$. The reaction mixture was diluted with EtOAc and quenched with $\mathrm{H}_{2} \mathrm{O}$, extracted with EtOAc, washed $(\mathrm{NaCl})$, dried $\left(\mathrm{Na}_{2} \mathrm{SO}_{4}\right)$ and concentrated under reduced pressure. The crude product was purified using silica gel flash chromatography to give allylic alcohol IV-52 $12 \mathrm{mg}(0.04 \mathrm{mmol}, 50 \%)$ as colorless oil: $R_{f}(30 \%$ EtOAc/hexane $)=0.26 ;[\alpha]^{25}=$ $2.4(c=1.0, \mathrm{MeOH})$; IR (thin film, $\left.\mathrm{cm}^{-1}\right)$ 3472, 2934, 1744, 1279, 1259, 1030, 938, 848; ${ }^{1} \mathrm{H}$ NMR $\left(600 \mathrm{MHz}, \mathrm{CDCl}_{3}\right) \delta 6.30(\mathrm{ddd}, J=9.6,6.0,0.6 \mathrm{~Hz}, 1 \mathrm{H}), 6.11(\mathrm{dd}, J=3.0,0.6$ $\mathrm{Hz}, 1 \mathrm{H}), 5.94(\mathrm{ddd}, J=9.6,3.0,1.2 \mathrm{~Hz}, 1 \mathrm{H}), 4.48(\mathrm{ddd}, J=7.2,6.0,1.2 \mathrm{~Hz}, 1 \mathrm{H}), 3.87$ $(\mathrm{m}, 1 \mathrm{H}), 3.70(\mathrm{~s}, 3 \mathrm{H}), 2.76(\mathrm{~d}, J=7.2,2 \mathrm{H}), 1.51(\mathrm{~s}, J=9 \mathrm{H}) ;{ }^{13} \mathrm{C} \mathrm{NMR}(150 \mathrm{MHz}$, $\left.\mathrm{CDCl}_{3}\right) \delta 171.1,152.1,131.0,126.2,90.4,82.9,69.4,63.0,51.8,35.3,27.7$; CIHRMS: Calculated for $\left[\mathrm{C}_{13} \mathrm{H}_{20} \mathrm{O}_{7}+\mathrm{Na}^{+}\right]: 311.1101$, Found: 311.1092 
Methyl-2-((2S,3S,6S)-6-(tert-butoxycarbonyloxy)-3-(tert-butyldimethylsilyloxy)-3,6dihydro-2H-pyran-2-yl)acetate (IV-54)<smiles>COC(=O)C[C@H]1OC(OC(=O)OC(C)(C)C)C=C[C@@H]1OC(C)(C)C</smiles>

To a solution of alcohol IV-52 (4 mg, $0.014 \mathrm{mmol})$ in $0.1 \mathrm{~mL} \mathrm{CH}_{2} \mathrm{Cl}_{2}$ was added imidazol (2 mg, $0.028 \mathrm{mmol})$ and $\mathrm{TBSCl}(4 \mathrm{mg}, 0.028 \mathrm{mmol})$ at $0{ }^{\circ} \mathrm{C}$. The reaction mixture was stirred at RT overnight. The reaction mixture was diluted with EtOAc and quenched with saturated $\mathrm{NaHCO}_{3}$, extracted with EtOAc, washed $(\mathrm{NaCl})$, dried $\left(\mathrm{Na}_{2} \mathrm{SO}_{4}\right)$ and concentrated under reduced pressure. The crude product was purified using silica gel flash chromatography to give TBS-alcohol IV-54 $5 \mathrm{mg}(0.013 \mathrm{mmol}, 90 \%)$ as colorless oil: $R_{f}(10 \%$ EtOAc/hexane $)=0.52 ;[\alpha]^{25}=12.4\left(c=0.8, \mathrm{CH}_{2} \mathrm{Cl}_{2}\right)$; IR (thin film, $\left.\mathrm{cm}^{-1}\right)$ 2960, 2932, 2859, 1748, 1368, 1256, 1139, 1096, 941, 838; ${ }^{1} \mathrm{H}$ NMR (600 MHz, $\mathrm{CDCl}_{3}$ ) $\delta 6.13(\mathrm{dd}, J=10.2,5.4 \mathrm{~Hz}, 1 \mathrm{H}), 6.12(\mathrm{~d}, J=3.0 \mathrm{~Hz}, 1 \mathrm{H}), 5.89(\mathrm{dd}, J=10.2,3.0, \mathrm{~Hz}$, 1H), 4.43 (ddd, $J=7.2,6.6,2.4 \mathrm{~Hz}, 1 \mathrm{H}), 3.96(\mathrm{dd}, J=5.4,2.4 \mathrm{~Hz}, 1 \mathrm{H}), 3.67$ (s, 3H), 2.71 $(\mathrm{dd}, J=16.8,7.2 \mathrm{~Hz}, 1 \mathrm{H}), 2.68(\mathrm{dd}, J=16.8,6.6 \mathrm{~Hz}, 1 \mathrm{H}), 1.51(\mathrm{~s}, J=9 \mathrm{H}), 0.88(\mathrm{~s}, J=$ 9H), $0.07(\mathrm{~s}, J=6 \mathrm{H}), 0.05(\mathrm{~s}, J=6 \mathrm{H}) ;{ }^{13} \mathrm{C} \mathrm{NMR}\left(150 \mathrm{MHz}, \mathrm{CDCl}_{3}\right) \delta 171.3,152.2$, 131.0, 125.4, 90.7, 82.6, 69.6, 62.3, 51.6, 35.4, 27.8, 25.7, 18.1, -4.27, -4.89; CIHRMS: Calculated for $\left[\mathrm{C}_{19} \mathrm{H}_{34} \mathrm{O}_{7} \mathrm{Si}+\mathrm{Na}^{+}\right]:$425.1966, Found: 425.1970 
(2S,6S)-6-(2-methoxy-2-oxoethyl)-5-oxo-5,6-dihydro-2H-pyran-2-yl pivalate (IV-43)<smiles>CCOC1C=CC(=O)[C@@H](CC(=O)OC)O1</smiles>

To a solution of pyranone alcohol (IV-32) $(55 \mathrm{mg}, 0.3 \mathrm{mmol})$ in $3 \mathrm{~mL} \mathrm{CH}_{2} \mathrm{Cl}_{2}$ was added PivCl $(0.07 \mathrm{~mL}, 0.6 \mathrm{mmol})$, pyridine $(0.07 \mathrm{~mL}, 0.9 \mathrm{mmol})$ at $-78{ }^{\circ} \mathrm{C}$. The reaction mixture was stirred at $-78{ }^{\circ} \mathrm{C}$ for $12 \mathrm{hr}$. The reaction mixture was diluted with EtOAc and quenched with saturated $\mathrm{NaHCO}_{3}$, extracted with EtOAc, washed $(\mathrm{NaCl})$, dried $\left(\mathrm{Na}_{2} \mathrm{SO}_{4}\right)$ and concentrated under reduced pressure. The crude product was purified using silica gel flash chromatography to give Piv-pyranone IV-43 $61 \mathrm{mg}(0.23 \mathrm{mmol}, 75 \%)$ as light yellow oil: $R_{f}(10 \%$ EtOAc/hexane $)=0.25 ;[\alpha]^{25}{ }_{\mathrm{D}}=+52.8\left(c=0.5, \mathrm{CH}_{2} \mathrm{Cl}_{2}\right)$; IR (thin film, $\left.\mathrm{cm}^{-1}\right) 2976,1742,1702,1370,1174,1127,1094,936 ;{ }^{1} \mathrm{H}$ NMR $\left(600 \mathrm{MHz}, \mathrm{CDCl}_{3}\right)$ $\delta 6.89(\mathrm{dd}, J=10.2,3.6 \mathrm{~Hz}, 1 \mathrm{H}), 6.48(\mathrm{~d}, J=3.6 \mathrm{~Hz}, 1 \mathrm{H}), 6.26(\mathrm{~d}, J=10.2 \mathrm{~Hz}, 1 \mathrm{H}), 4.89$ $(\mathrm{dd}, J=7.2,4.2 \mathrm{~Hz}, 1 \mathrm{H}), 3.69(\mathrm{~s}, 3 \mathrm{H}), 3.00(\mathrm{dd}, J=16.8,4.2 \mathrm{~Hz}, 1 \mathrm{H}), 2.89(\mathrm{dd}, J=16.8$, $7.2 \mathrm{~Hz}, 1 \mathrm{H}), 1.25(\mathrm{~s}, J=9 \mathrm{H}) ;{ }^{13} \mathrm{C} \mathrm{NMR}\left(150 \mathrm{MHz}, \mathrm{CDCl}_{3}\right) \delta 194.0,176.9,170.5,141.8$, 128.3, 86.6, 72.5, 52.0, 39.3, 35.0, 27.0; CIHRMS: Calculated for $\left[\mathrm{C}_{13} \mathrm{H}_{18} \mathrm{O}_{6}+\mathrm{Na}^{+}\right]$: 293.0996, Found: 309.0996 
(2R,6S)-6-(2-methoxy-2-oxoethyl)-5-oxo-5,6-dihydro-2H-pyran-2-yl pivalate (IV44) $)^{12}$<smiles>COC(=O)C[C@H]1O[C@@H](O[Na])C=CC1=O</smiles>

To a solution of pyranone alcohol (IV-32) $(55 \mathrm{mg}, 0.3 \mathrm{mmol})$ in $3 \mathrm{~mL} \mathrm{CH}_{2} \mathrm{Cl}_{2}$ was added PivCl $(0.07 \mathrm{~mL}, 0.6 \mathrm{mmol})$, pyridine $(0.07 \mathrm{~mL}, 0.9 \mathrm{mmol})$ at $-78{ }^{\circ} \mathrm{C}$. The reaction mixture was stirred at $-78{ }^{\circ} \mathrm{C}$ for $12 \mathrm{hr}$. The reaction mixture was diluted with EtOAc and quenched with saturated $\mathrm{NaHCO}_{3}$, extracted with EtOAc, washed $(\mathrm{NaCl})$, dried $\left(\mathrm{Na}_{2} \mathrm{SO}_{4}\right)$ and concentrated under reduced pressure. The crude product was purified using silica gel flash chromatography to give Piv-pyranone IV-44 $5 \mathrm{mg}(0.02 \mathrm{mmol}, 6 \%)$ as light yellow oil.

$(2 S, 5 R, 6 S)-5-h y d r o x y-6-(2-m e t h o x y-2-o x o e t h y l)-5,6-d i h y d r o-2 H-p y r a n-2-y l$ pivalate (IV-56)<smiles>COC(=O)C[C@@H]1OC(O[Na])C=CC1O</smiles>

To a solution of pyranone IV-43 $(56 \mathrm{mg}, 0.21 \mathrm{mmol})$ in $0.5 \mathrm{~mL} \mathrm{CH}_{2} \mathrm{Cl}_{2}$ was added 0.5 $\mathrm{mL} \mathrm{CeCl} 3$ in $\mathrm{MeOH}(0.4 \mathrm{M})$ and $\mathrm{NaBH}_{4}(24 \mathrm{mg}, 0.63 \mathrm{mmol})$ at $-78{ }^{\circ} \mathrm{C}$. The reaction mixture was stirred at $-78{ }^{\circ} \mathrm{C}$ for $3 \mathrm{hr}$. The reaction mixture was diluted with EtOAc and

\footnotetext{
12 This compound is not characterized.
} 
quenched with saturated $\mathrm{NaHCO}_{3}$, extracted with EtOAc, washed $(\mathrm{NaCl})$, dried $\left(\mathrm{Na}_{2} \mathrm{SO}_{4}\right)$ and concentrated under reduced pressure. The crude product was purified using silica gel flash chromatography to give allylic alcohol IV-56 $50 \mathrm{mg}(0.18 \mathrm{mmol}, 88 \%)$ as colorless oil: $R_{f}(30 \%$ EtOAc/hexane $)=0.25 ;[\alpha]^{25}{ }_{\mathrm{D}}=-45.5(c=0.29, \mathrm{MeOH}) ; \mathrm{IR}\left(\right.$ thin film, $\left.\mathrm{cm}^{-1}\right)$ 3480, 2974, 1741, 1439, 1280, 1122, 1035, 926; ${ }^{1} \mathrm{H}$ NMR $\left(600 \mathrm{MHz}, \mathrm{CDCl}_{3}\right) \delta 6.21(\mathrm{~d}, J$ $=2.4 \mathrm{~Hz}, 1 \mathrm{H}), 6.06(\mathrm{dd}, J=10.8,1.2 \mathrm{~Hz}, 1 \mathrm{H}), 5.78(\mathrm{ddd}, J=10.2,1.8,1.2 \mathrm{~Hz}, 1 \mathrm{H}), 4.09-$ $4.08(\mathrm{~m}, 2 \mathrm{H}), 3.70(\mathrm{~s}, 3 \mathrm{H}), 2.84(\mathrm{dd}, J=15.6,4.2 \mathrm{~Hz}, 1 \mathrm{H}), 2.60(\mathrm{dd}, J=15.6,7.8 \mathrm{~Hz}$ $1 \mathrm{H}), 1.23(\mathrm{~s}, J=9 \mathrm{H}) ;{ }^{13} \mathrm{C} \mathrm{NMR}\left(150 \mathrm{MHz}, \mathrm{CDCl}_{3}\right) \delta 177.3,171.7,134.2,124.9,89.3$, 87.7, 70.6, 67.2, 39.0, 37.6, 27.0; CIHRMS: Calculated for $\left[\mathrm{C}_{13} \mathrm{H}_{20} \mathrm{O}_{6}+\mathrm{Na}^{+}\right]$: 295.1152, Found: 295.1166

(2S,3S,6S)-2-(2-methoxy-2-oxoethyl)-6-(pivaloyloxy)-3,6-dihydro-2H-pyran-3-yl 4nitrobenzoate (IV-56a)<smiles>COC(=O)C[C@@H]1OC(O[Na])C=C[C@@H]1OC(C)(C)C</smiles>

To a solution of alcohol IV-56 (46 mg, $0.17 \mathrm{mmol})$ in $1 \mathrm{~mL}$ THF was added $\mathrm{PPh}_{3}(262$ mg, $0.25 \mathrm{mmol}), p$-Nitrobenzoic acid (167 mg, $0.25 \mathrm{mmol})$ and DIAD (0.05 mL, 0.25 mmol) at $0{ }^{\circ} \mathrm{C}$. The reaction mixture was stirred at $\mathrm{RT}$ overnight. The reaction mixture was diluted with EtOAc and quenched with saturated $\mathrm{NaHCO}_{3}$, extracted with EtOAc, washed $(\mathrm{NaCl})$, dried $\left(\mathrm{Na}_{2} \mathrm{SO}_{4}\right)$ and concentrated under reduced pressure. The crude product was purified using silica gel flash chromatography to give IV-56a $57 \mathrm{mg}(0.14$ 
mmol, $80 \%)$ as colorless oil: $R_{f}(10 \%$ EtOAc/hexane $)=0.29 ;[\alpha]^{25}{ }_{\mathrm{D}}=147.0(c=1.0$, $\mathrm{MeOH}$ ); IR (thin film, $\mathrm{cm}^{-1}$ ) 2981, 2879, 1728, 1530, 1270, 1113, 932, 718; ${ }^{1} \mathrm{H}$ NMR $\left(600 \mathrm{MHz}, \mathrm{CDCl}_{3}\right) \delta 8.29(\mathrm{~d}, J=9.0,2 \mathrm{H}), \delta 8.21(\mathrm{~d}, J=9.0,2 \mathrm{H}), 6.38(\mathrm{dd}, J=3.0,1.2$ $\mathrm{Hz}, 1 \mathrm{H}), 6.35$ (ddd, $J=10.2,6.0,1.2 \mathrm{~Hz}, 1 \mathrm{H}), 6.11(\mathrm{ddd}, J=10.2,3.0,0.6 \mathrm{~Hz}, 1 \mathrm{H}), 5.33$ $(\mathrm{dd}, J=6.0,2.4 \mathrm{~Hz}, 1 \mathrm{H}), 4.71(\mathrm{ddd}, J=8.4,4.8,2.4 \mathrm{~Hz}, 1 \mathrm{H}), 3.67(\mathrm{~s}, 3 \mathrm{H}), 2.75(\mathrm{dd}, J=$ 16.2, $9.0 \mathrm{~Hz}, 1 \mathrm{H}), 2.62(\mathrm{dd}, J=16.2,4.8 \mathrm{~Hz}, 1 \mathrm{H}), 1.25(\mathrm{~s}, J=9 \mathrm{H}) ;{ }^{13} \mathrm{C} \mathrm{NMR}(150 \mathrm{MHz}$, $\left.\mathrm{CDCl}_{3}\right) 177.1,170.3,164.1,150.8,134.8,130.9,129.7,125.9,123.7,87.5,67.7,65.1$, 52.0, 39.1, 35.7, 27.0; CIHRMS: Calculated for $\left[\mathrm{C}_{20} \mathrm{H}_{23} \mathrm{NO}_{9}+\mathrm{Na}^{+}\right]$: 444.1265, Found: 444.1248

(2S,5S,6S)-5-hydroxy-6-(2-methoxy-2-oxoethyl)-5,6-dihydro-2H-pyran-2-yl pivalate (IV-58)<smiles>COC(=O)C[C@@H]1OC(O[Na])C=C[C@H]1O</smiles>

To a solution of nitrobenzoate IV-56a $(18 \mathrm{mg}, 0.043 \mathrm{mmol})$ in $0.5 \mathrm{~mL} \mathrm{MeOH}$ was added $\mathrm{NEt}_{3}(3 \mathrm{~L})$ at $0{ }^{\circ} \mathrm{C}$. The reaction mixture was stirred at RT for $3 \mathrm{hr}$. The reaction mixture was diluted with EtOAc and quenched with $\mathrm{H}_{2} \mathrm{O}$, extracted with EtOAc, washed $(\mathrm{NaCl})$, dried $\left(\mathrm{Na}_{2} \mathrm{SO}_{4}\right)$ and concentrated under reduced pressure. The crude product was purified using silica gel flash chromatography to give allylic alcohol IV-58 $6 \mathrm{mg}(0.024 \mathrm{mmol}$, $60 \%)$ as colorless oil: $R_{f}(30 \%$ EtOAc/hexane $)=0.25 ;[\alpha]^{25}{ }_{\mathrm{D}}=37(c=1.0, \mathrm{MeOH})$; IR (thin film, $\mathrm{cm}^{-1}$ ) 3467, 2976, 2812, 1737, 1439, 1280, 1070, 919; ${ }^{1} \mathrm{H}$ NMR (600 MHz, 
$\left.\mathrm{CDCl}_{3}\right) \delta 6.30(\mathrm{ddd}, J=10.2,6.0,1.2 \mathrm{~Hz}, 1 \mathrm{H}), 6.28(\mathrm{~d}, J=3.6 \mathrm{~Hz}, 1 \mathrm{H}), 5.92(\mathrm{ddd}, J=$ 10.2, 3.6, 0.6 Hz, 1H), $4.46(\mathrm{ddd}, J=7.8,6.0,2.4 \mathrm{~Hz}, 1 \mathrm{H}), 3.85(\mathrm{~m}, 1 \mathrm{H}), 3.69(\mathrm{~s}, 3 \mathrm{H})$, $2.75(\mathrm{dd}, J=16.2,7.8 \mathrm{~Hz}, 1 \mathrm{H}), 2.71(\mathrm{dd}, J=16.2,6.0 \mathrm{~Hz}, 1 \mathrm{H}), 1.23(\mathrm{~s}, J=9 \mathrm{H}) ;{ }^{13} \mathrm{C}$ NMR (150 MHz, $\left.\mathrm{CDCl}_{3}\right)$ 177.2, 171.3, 130.7, 126.8, 87.9, 69.6, 62.3, 51.8, 39.0, 35.6, 27.0; CIHRMS: Calculated for $\left[\mathrm{C}_{13} \mathrm{H}_{20} \mathrm{O}_{6}+\mathrm{Na}^{+}\right]$: 295.1152, Found: 295.1143

\section{Hept-3-yn-2-ol (IV-31a)}

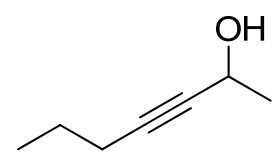

A solution of $n$-BuLi in hexane $(11.1 \mathrm{~mL}, 26.5 \mathrm{mmol})$ was added dropwise to the solution of 1-heptyn IV-31 (1.5 g, $22.1 \mathrm{mmol})$ in dry THF $(44 \mathrm{~mL})$ at $-78{ }^{\circ} \mathrm{C}$ under argon. After $30 \mathrm{~min}$, acetaldehyde $(1.2 \mathrm{~mL}, 22.1 \mathrm{mmol})$ was added. The reaction was stirred for 10 min at $0{ }^{\circ} \mathrm{C}$ and then the resulting solution was warmed to room temperature for $50 \mathrm{~min}$. Additional $\mathrm{CH}_{2} \mathrm{Cl}_{2}(10 \mathrm{~mL})$ was added to the reaction mixture followed by addition of saturated aqueous $\mathrm{NH}_{4} \mathrm{Cl}(20 \mathrm{~mL})$. The aqueous phase was separated and extracted with $\mathrm{CH}_{2} \mathrm{Cl}_{2}$. The combined organic layers were washed with brine, dried $\left(\mathrm{Na}_{2} \mathrm{SO}_{4}\right)$, filtered and concentrated. Chromatography on silica gel gave alcohol IV-31a $(2.03 \mathrm{~g}, 18.1 \mathrm{mmol}$, $82 \%)$ as a colorless oil; $R f(10 \%$ EtOAc/hexane $)=0.28$; IR (thin film, $\left.\mathrm{cm}^{-1}\right), 3340,2958$, $2869,2244,1458,1291,1155,1074,893 ;{ }^{1} \mathrm{H}$ NMR $\left(600 \mathrm{MHz}, \mathrm{CDCl}_{3}\right) \delta 4.45$ (qt, $J=$ 6.6, $1.8 \mathrm{~Hz}, 1 \mathrm{H}), 2.10(\mathrm{td}, J=6.6,1.8 \mathrm{~Hz}, 2 \mathrm{H}), 1.45(\mathrm{qt}, J=7.2,6.6 \mathrm{~Hz}, 2 \mathrm{H}), 1.35(\mathrm{~d}, J=$ 
$6.6 \mathrm{~Hz}, 3 \mathrm{H}), 0.91(\mathrm{t}, J=7.2 \mathrm{~Hz}, 3 \mathrm{H}) ;{ }^{13} \mathrm{C} \mathrm{NMR}\left(150 \mathrm{MHz}, \mathrm{CD}_{3} \mathrm{Cl}\right) \delta 84.1,82.4,58.2$, $24.5,21.9,20.5,13.2$

\section{Hept-3-yn-2-one (IV-35)}

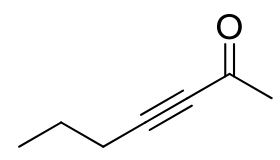

To a solution of alcohol (IV-31a) $(4.0 \mathrm{~g}, 35.7 \mathrm{mmol})$ in THF $(100 \mathrm{~mL})$ at room temperature was added activated $\mathrm{MnO}_{2}(20 \mathrm{~g})$. The reaction mixture was stirred for $24 \mathrm{~h}$ and then filtered through celite and concentrated under pressure to give ketone IV-35 (3.73 g, $33.9 \mathrm{mmol}, 95 \%)$ as a colorless oil. $R f(10 \%$ EtOAc/hexane $)=0.40$; IR (thin film, $\left.\mathrm{cm}^{-1}\right), 2960,2871,2210,1675,1358,1226 ;{ }^{1} \mathrm{H}$ NMR $\left(600 \mathrm{MHz}, \mathrm{CDCl}_{3}\right) \delta 2.30(\mathrm{t}, J$ $=7.2 \mathrm{~Hz}, 2 \mathrm{H}), 2.28(\mathrm{~s}, 3 \mathrm{H}), 1.57(\mathrm{qt}, J=7.2,7.2 \mathrm{~Hz}, 2 \mathrm{H}), 0.98(\mathrm{t}, J=7.2,3 \mathrm{H}) ;{ }^{13} \mathrm{C} \mathrm{NMR}$ $\left(150 \mathrm{MHz}, \mathrm{CD}_{3} \mathrm{Cl}\right) \delta 184.8,93.8,81.4,32.6,21.1,20.7,13.3$

\section{(S)-Hept-3-yn-2-ol (IV-36)}

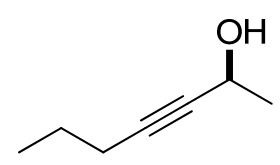

To a flask was added ketone IV-35 (10.8 g, $86.9 \mathrm{mmol}), 2 \mathrm{M} \mathrm{NaCOOH}$ in water (400 $\mathrm{mL})$, cetyl trimethylammonium bromide $(3.17 \mathrm{~g}, 8.69 \mathrm{mmol})$. The mixture was stirred at RT. for $5 \mathrm{~min}$, then Noyori catalyst (S, S) (105 mg, $0.174 \mathrm{mmol})$ was added. The reaction 
mixture was allowed to stir for $24 \mathrm{hr}$. Water was added to dilute and extracted with $\mathrm{Et}_{2} \mathrm{O}$, dried $\left(\mathrm{Na}_{2} \mathrm{SO}_{4}\right)$ and concentrated under reduced pressure. The crude product was purified using silica gel flash chromatography to give $9.5 \mathrm{~g}(84.8 \mathrm{mmol}, 97 \%)$ of alcohol IV-36 as colorless oil: $R f(10 \%$ EtOAc/hexane $)=0.28 ;[\alpha]^{25}=-10.3(c=0.29, \mathrm{MeOH})$; IR (thin film, $\left.\mathrm{cm}^{-1}\right), 3340,2958,2869,2244,1458,1291,1155,1074,893 ;{ }^{1} \mathrm{H}$ NMR $(600 \mathrm{MHz}$, $\left.\mathrm{CDCl}_{3}\right) \delta 4.45(\mathrm{qt}, J=6.6,1.8 \mathrm{~Hz}, 1 \mathrm{H}), 2.10(\mathrm{td}, J=6.6,1.8 \mathrm{~Hz}, 2 \mathrm{H}), 1.45(\mathrm{qt}, J=7.2$, $6.6 \mathrm{~Hz}, 2 \mathrm{H}), 1.35(\mathrm{~d}, J=6.6 \mathrm{~Hz}, 3 \mathrm{H}), 0.91(\mathrm{t}, J=7.2 \mathrm{~Hz}, 3 \mathrm{H}) ;{ }^{13} \mathrm{C}$ NMR $(150 \mathrm{MHz}$, $\left.\mathrm{CD}_{3} \mathrm{Cl}\right) \delta 84.1,82.4,58.2,24.5,21.9,20.5,13.2$

\section{(S)-Hept-6-yn-2-ol (IV-36a)}

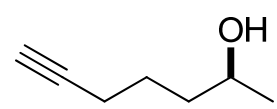

A $30 \%$ suspension of $\mathrm{KH}$ in mineral oil $(5.62 \mathrm{~g}, 140 \mathrm{mmol})$ was washed three times under Argon with dry ether and then the excess solvent was removed under vacuum, dry 1,3-diaminopropane $(28 \mathrm{~mL})$ was added with cooling $\left(15^{\circ} \mathrm{C}\right)$. After the mixture was stirred for $1 \mathrm{~h}$, the alkynol IV-36 $(3.15 \mathrm{~g}, 28.1 \mathrm{mmol})$ was added dropwise $(20 \mathrm{~min})$ at $\left(10-15{ }^{\circ} \mathrm{C}\right)$ to the mixture and the resulting slurry was stirred further under argon for 2 hrs. With cooling $\left(0^{\circ} \mathrm{C}\right)$, water was added dropwise and the mixture was extracted with ether, the combined extracts were washed with $1 \mathrm{~N} \mathrm{HCl}$, saturated aqueous sodium bicarbonate and brine, and then dried with anhydrous sodium sulfate. Flash chromatography gave alkynol IV-36a $(2.71 \mathrm{~g}, 24.2 \mathrm{mmol}, 85 \%)$ as a colorless oil: $[\alpha]^{25}$ $=8\left(c=1.0, \mathrm{CHCl}_{3}\right) ; \operatorname{Rf}(20 \% \mathrm{EtOAc} / \mathrm{Hexane})=0.28 ; \mathrm{IR}\left(\right.$ thin film, $\left.\mathrm{cm}^{-1}\right) 3309,2934$, 
2862, 2117, 1460, 1432, 1374, 1128, 1093, 937, 821; ${ }^{1} \mathrm{H}$ NMR $\left(600 \mathrm{MHz}, \mathrm{CDCl}_{3}\right) \delta 3.83$ (qt, $J=6.0,6.0 \mathrm{~Hz}, 1 \mathrm{H}), 2.23(\mathrm{td}, J=7.2,3.0 \mathrm{~Hz}, 2 \mathrm{H}), 1.95(\mathrm{t}, J=3.0 \mathrm{~Hz}, 1 \mathrm{H})$, $1.67 \sim 1.56(\mathrm{~m}, 4 \mathrm{H}), 1.20(\mathrm{~d}, J=6.0 \mathrm{~Hz}, 3 \mathrm{H}) ;{ }^{13} \mathrm{C} \mathrm{NMR}\left(150 \mathrm{MHz}, \mathrm{CDCl}_{3}\right) \delta 84.3,68.5$, $67.6,38.2,24.7,23.6,18.4$

\section{(S)-Hept-6-yn-2-yl pivalate (IV-29)}

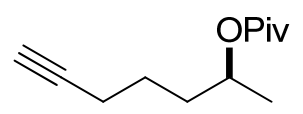

To a flask was added alkynol IV-36a (1.55 g, $13.8 \mathrm{mmol})$, PivCl (2.06 mL, $16.6 \mathrm{mmol})$, pyridine $(5.6 \mathrm{~mL}, 6.9 \mathrm{mmol})$ at $0{ }^{\circ} \mathrm{C}$. The mixture was then stirred at RT. for $3 \mathrm{hr}$. $\mathrm{NaHCO}_{3}$ was added and the mixture was extracted with $\mathrm{Et}_{2} \mathrm{O}$, dried $\left(\mathrm{Na}_{2} \mathrm{SO}_{4}\right)$ and concentrated under reduced pressure. The crude product was purified using silica gel flash chromatography to give $2.57 \mathrm{~g}(13.1 \mathrm{mmol}, 95 \%)$ of pivalate alcohol IV-29 as colorless oil: $R f(10 \%$ EtOAc/hexane $)=0.5 ;[\alpha]^{25}=27.8\left(c=1.0, \mathrm{CH}_{2} \mathrm{Cl}_{2}\right)$; IR (thin film, $\left.\mathrm{cm}^{-1}\right)$ 3300, 2976, 2874, 2244, 1725, 1288, 1166, 1088; ${ }^{1} \mathrm{H}$ NMR $(600 \mathrm{MHz}$, $\left.\mathrm{CDCl}_{3}\right) \delta 4.90(\mathrm{qt}, J=6.6,6.0 \mathrm{~Hz}, 1 \mathrm{H}), 2.20(\mathrm{td}, J=7.2,2.4 \mathrm{~Hz}, 2 \mathrm{H}), 1.95(\mathrm{t}, J=2.4 \mathrm{~Hz}$, 1H), $1.70 \sim 1.51(\mathrm{~m}, 4 \mathrm{H}), 1.20(\mathrm{~d}, J=6.6 \mathrm{~Hz}, 3 \mathrm{H}), 1.19(\mathrm{~s}, 9 \mathrm{H}) ;{ }^{13} \mathrm{C}$ NMR $(150 \mathrm{MHz}$, $\left.\mathrm{CD}_{3} \mathrm{Cl}\right) \delta 178.1,84.0,70.0,68.6,38.7,34.9,27.1,24.4,19.9,18.3$; CIHRMS: Calculated for $\left[\mathrm{C}_{12} \mathrm{H}_{20} \mathrm{O}_{2}+\mathrm{Na}^{+}\right]:$219.1356, Found: 219.1355 
(S)-6-Oxoheptan-2-yl pivalate (IV-37)

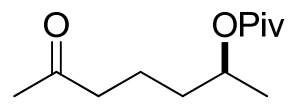

To a flask was added [(i-Pr)AuCl] $(21.9 \mathrm{mg}, 0.035 \mathrm{mmol})$ and small amount of $\mathrm{AgSbF}_{6}$ (covering the tip of a spatula) in $3 \mathrm{~mL} \mathrm{MeOH}$, the mixture was stirred at RT for $1 \mathrm{~min}$. Alkyne IV-29 (135 mg, $0.69 \mathrm{mmol})$ was added followed by addition of $1 \mathrm{~mL}$ water. The reaction mixture was heated at $65{ }^{\circ} \mathrm{C}$ for $12 \mathrm{hr}$. The solvent was removed by vacuum, the crude product was purified using silica gel flash chromatography to give ketone IV-37 $126 \mathrm{mg}(0.59 \mathrm{mmol}, 85 \%)$ as colorless oil: $R f(10 \%$ EtOAc/hexane $)=0.35 ;[\alpha]^{25}{ }_{\mathrm{D}}=34.7$ $\left(c=0.8, \mathrm{CH}_{2} \mathrm{Cl}_{2}\right) ; \mathrm{IR}$ (thin film, $\mathrm{cm}^{-1}$ ) $2976,1721,1481,1367,1284,1166,1088 ;{ }^{1} \mathrm{H}$ $\operatorname{NMR}\left(600 \mathrm{MHz}, \mathrm{CDCl}_{3}\right) \delta 4.90(\mathrm{qt}, J=6.0,6.0 \mathrm{~Hz}, 1 \mathrm{H}), 2.42(\mathrm{t}, J=7.2 \mathrm{~Hz}, 2 \mathrm{H}), 2.11$ (s, 3H), 1.65 1.48 (m, 4H), $1.18(\mathrm{~d}, J=6.0 \mathrm{~Hz}, 3 \mathrm{H}), 1.17(\mathrm{~s}, 9 \mathrm{H}) ;{ }^{13} \mathrm{C}$ NMR $(150 \mathrm{MHz}$, $\left.\mathrm{CD}_{3} \mathrm{Cl}\right) \delta 208.7,178.4,70.2,43.4,38.9,35.4,30.1,27.3,20.0,19.7$; CIHRMS: Calculated for $\left[\mathrm{C}_{12} \mathrm{H}_{22} \mathrm{O}_{3}+\mathrm{Na}^{+}\right]: 237.1461$, Found: 237.1460

(S)-7-Bromo-6-oxoheptan-2-yl pivalate (IV-38)<smiles>CC(CCCC(=O)CBr)O[Na]</smiles>

Bromine $(0.035 \mathrm{~mL}, 0.67 \mathrm{mmol})$ was added to a solution of ketone IV-37 (120 mg, 0.56 $\mathrm{mmol}$ ) in $3 \mathrm{~mL} \mathrm{MeOH}$ at $\mathrm{RT}$. for 10 min under $\mathrm{N}_{2}$. After the addition was completed, the reaction mixture was stirred for $2 \mathrm{hr}$. Then the $\mathrm{MeOH}$ was removed from the reaction 
mixture under reduced pressure. The residue was partitioned between ether and water. The $\mathrm{PH}$ of the resulting solution was adjusted to 8.0 by adding $\mathrm{NaHCO}_{3}$. The ether layer was washed with $\mathrm{NaHCO}_{3}$ and brine, dried over $\mathrm{Na}_{2} \mathrm{SO}_{4}$, filtered and concentrated to yield crude product, the crude product was purified using silica gel flash chromatography to give bromo-ketone IV-38 $114 \mathrm{mg}(0.39 \mathrm{mmol}, 70 \%)$ as colorless oil.

To a flask was added [(i-Pr)AuCl] $(50 \mathrm{mg})$ and small amount of $\mathrm{AgSbF}_{6}$ (covering the tip of a spatula) in $3 \mathrm{~mL}$ anhydrous $\mathrm{MeOH}$, the mixture was stirred at RT for $1 \mathrm{~min}$. Alkyne IV-29 (494 mg, $4.4 \mathrm{mmol}$ ) was added. The reaction mixture was heated at $65^{\circ} \mathrm{C}$ for $5 \mathrm{hr}$. NBS (801mg, $4.5 \mathrm{mmol}$ ) was added to the reaction solution. After another $5 \mathrm{hr}$, the solvent was removed by vacuum, the crude product was purified using silica gel flash chromatography to give ketone IV-38 $1.1 \mathrm{~g}(0.59 \mathrm{mmol}, 85 \%)$ as colorless oil.

IV-38: $R f(10 \% \mathrm{EtOAc} /$ hexane $)=0.53 ;[\alpha]_{\mathrm{D}}^{25}=0.60\left(c=1.0, \mathrm{CH}_{2} \mathrm{Cl}_{2}\right)$; IR (thin film, $\left.\mathrm{cm}^{-1}\right) 2974,1716,1460,1397,1284,1166,1127 ;{ }^{1} \mathrm{H}$ NMR $\left(600 \mathrm{MHz}, \mathrm{CDCl}_{3}\right) \delta 4.89$ (qt, $J=6.0,6.0 \mathrm{~Hz}, 1 \mathrm{H}), 3.86(\mathrm{~s}, 2 \mathrm{H}), 2.67(\mathrm{t}, J=7.2 \mathrm{~Hz}, 2 \mathrm{H}), 1.65 \sim 1.48(\mathrm{~m}, 4 \mathrm{H}), 1.19(\mathrm{~d}, J$ $=6.0 \mathrm{~Hz}, 3 \mathrm{H}), 1.19(\mathrm{~s}, 9 \mathrm{H}) ;{ }^{13} \mathrm{C} \mathrm{NMR}\left(150 \mathrm{MHz}, \mathrm{CD}_{3} \mathrm{Cl}\right) \delta 201.7,178.1,69.8,39.3$, 38.7, 35.0, 34.1, 27.2, 19.8, 19.6; CIHRMS: Calculated for $\left[\mathrm{C}_{12} \mathrm{H}_{21} \mathrm{BrO}_{3}+\mathrm{Na}^{+}\right]$: 317.0566, Found: 317.0544 
(S)-6-oxo-7-(1-phenyl-1H-tetrazol-5-ylthio)heptan-2-yl pivalate (IV-38a)

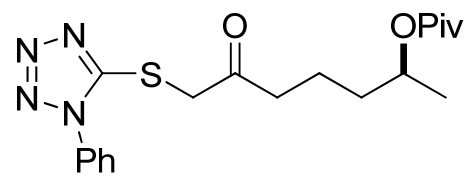

To the solution of ketone IV-38 (113 mg, $0.39 \mathrm{mmol})$ in MeOH (2 mL), PT-SH (68 mg, $0.39 \mathrm{mmol})$ and $\mathrm{KOH}(21 \mathrm{mg}, 0.39 \mathrm{mmol})$ was added at RT The mixture was then stirred at RT for $3 \mathrm{hr}$. $1 \mathrm{M} \mathrm{HCl}$ was added and extracted with $\mathrm{Et}_{2} \mathrm{O}$, dried $\left(\mathrm{Na}_{2} \mathrm{SO}_{4}\right)$ and concentrated under reduced pressure. The crude product was purified using silica gel flash chromatography to give $129 \mathrm{mg}(0.33 \mathrm{mmol}, 85 \%)$ of sulfide IV-38a as colorless oil: $R f(20 \%$ EtOAc/hexane $)=0.24 ;[\alpha]^{25}=42.3\left(c=0.65, \mathrm{CH}_{2} \mathrm{Cl}_{2}\right)$; IR (thin film, $\mathrm{cm}^{-}$ $\left.{ }^{1}\right) 2976,2939,2874,1718,1500,1169,698 ;{ }^{1} \mathrm{H} \mathrm{NMR}\left(600 \mathrm{MHz}, \mathrm{CDCl}_{3}\right) \delta 7.62 \sim 7.55$ $(\mathrm{m}, 5 \mathrm{H}), 4.89(\mathrm{qt}, J=6.0,6.0 \mathrm{~Hz}, 1 \mathrm{H}), 4.32(\mathrm{~s}, 2 \mathrm{H}), 2.69(\mathrm{t}, J=6.6 \mathrm{~Hz}, 2 \mathrm{H}), 1.65 \sim 1.48$ $(\mathrm{m}, 4 \mathrm{H}), 1.19(\mathrm{~d}, J=6.0 \mathrm{~Hz}, 3 \mathrm{H}), 1.19(\mathrm{~s}, 9 \mathrm{H}) ;{ }^{13} \mathrm{C} \mathrm{NMR}\left(150 \mathrm{MHz}, \mathrm{CD}_{3} \mathrm{Cl}\right) \delta 209.2$, $178.1,133.0,131.0,130.1,124.4,121.2,69.7,68.1,38.7,37.8,35.2,27.1,19.8,19.4$; CIHRMS: Calculated for $\left[\mathrm{C}_{19} \mathrm{H}_{26} \mathrm{~N}_{4} \mathrm{O}_{3} \mathrm{~S}+\mathrm{Na}^{+}\right]$: 413.1678, Found: 413.1618

(S)-6-oxo-7-(1-phenyl-1H-tetrazol-5-ylsulfonyl)heptan-2-yl pivalate (IV-27)<smiles>CC(CCCC(=O)CS(=O)(=O)c1nnnn1-c1ccccc1)O[Na]</smiles>

A solution of sulfide IV-38a $(66 \mathrm{mg}, 0.17 \mathrm{mmol})$ in ethanol $(1 \mathrm{~mL})$ at $0{ }^{\circ} \mathrm{C}$ was treated with ammonium heptamolybdate tetrahydrate $(31 \mathrm{mg}, 0.025 \mathrm{mmol})$ and $30 \%$ aqueous 
hydrogen peroxide solution $(0.17 \mathrm{ml}, 1.7 \mathrm{mmol})$, The reaction mixture was warmed to ambient temperature over $5 \mathrm{hr}$, stirred for additional $7 \mathrm{hr}$ and then brine was added. The mixture was extracted with $\mathrm{Et}_{2} \mathrm{O}$, dried $\left(\mathrm{Na}_{2} \mathrm{SO}_{4}\right)$ and concentrated under reduced pressure. The crude product was purified using silica gel flash chromatography to give sulfone IV-27 $50 \mathrm{mg}(0.12 \mathrm{mmol}, 70 \%)$ as colorless oil: $R f(30 \%$ EtOAc/hexane $)=0.54$; $[\alpha]^{25}=3.5\left(c=1.2, \mathrm{CH}_{2} \mathrm{Cl}_{2}\right)$; IR (thin film, $\left.\mathrm{cm}^{-1}\right)$ 2974, 2941, 2876, 1720, 1450, 1356, 1164, 764; ${ }^{1} \mathrm{H}$ NMR $\left(600 \mathrm{MHz}, \mathrm{CDCl}_{3}\right) \delta 7.67 \sim 7.58(\mathrm{~m}, 5 \mathrm{H}), 4.85$ (qt, $J=6.0,6.0 \mathrm{~Hz}$, 1H), $4.71(\mathrm{~s}, 2 \mathrm{H}), 2.67(\mathrm{t}, J=6.6 \mathrm{~Hz}, 2 \mathrm{H}), 1.65 \sim 1.48(\mathrm{~m}, 4 \mathrm{H}), 1.19(\mathrm{~d}, J=6.0 \mathrm{~Hz}, 3 \mathrm{H})$, $1.19(\mathrm{~s}, 9 \mathrm{H}) ;{ }^{13} \mathrm{C}$ NMR $\left(150 \mathrm{MHz}, \mathrm{CD}_{3} \mathrm{Cl}\right) \delta 196.0,178.2,153.4,132.9,131.6,129.6$, 125.5, 69.6, 64.8, 43.7, 38.7, 34.7, 27.1, 19.7, 19.7; CIHRMS: Calculated for $\left[\mathrm{C}_{19} \mathrm{H}_{26}\right.$ $\left.\mathrm{N}_{4} \mathrm{O}_{5} \mathrm{~S}+\mathrm{Na}^{+}\right]:$445.1516, Found: 445.1517

(2S)-5-((5S,7aS)-3-(sulfonyl)-5-(2-methoxy-2-oxoethyl)-6-0xo-5,6

7,7a-tetrahydro-3aH-furo[3,2-b]pyran-2-yl)pentan-2-yl-pivalate (IV-42)

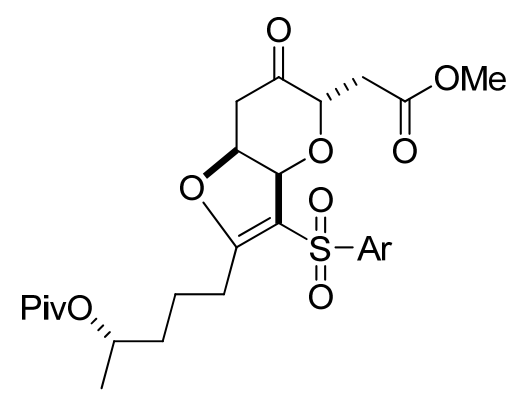

To a solution of Boc-pyranone IV-33 (10 mg, $0.024 \mathrm{mmol})$ and sulfone IV-27 (7 mg, $0.024 \mathrm{mmol})$ in dry $\mathrm{CH}_{2} \mathrm{Cl}_{2}(0.3 \mathrm{~mL})$, was added $\mathrm{Pd}_{2}(\mathrm{DBA})_{3} \cdot \mathrm{CHCl}_{3}(2.5 \mathrm{mg}, 10 \mathrm{~mol} \%)$ and $\mathrm{PPh}_{3}(2.5 \mathrm{mg}, 40 \mathrm{~mol} \%)$ at $0{ }^{\circ} \mathrm{C}$ under argon atmosphere. After stirring for $3 \mathrm{~h}$ at 0 
${ }^{\circ} \mathrm{C}$, the reaction mixture was quenched with saturated $\mathrm{NaHCO}_{3}$, extracted with $\mathrm{Et}_{2} \mathrm{O}$, dried $\left(\mathrm{Na}_{2} \mathrm{SO}_{4}\right)$, and concentrated under reduced pressure. The crude product was purified using silica gel flash chromatography to give $12 \mathrm{mg}$ IV-42 (0.02 mmol, 85\%) as colorless oil.

To a solution of Piv-pyranone IV-43 (11 mg, $0.026 \mathrm{mmol})$ and sulfone IV-27 (7 mg, $0.026 \mathrm{mmol})$ in dry $\mathrm{CH}_{2} \mathrm{Cl}_{2}(0.3 \mathrm{~mL})$, was added $\mathrm{Pd}_{2}(\mathrm{DBA})_{3} \cdot \mathrm{CHCl}_{3}(2.5 \mathrm{mg}, 10 \mathrm{~mol} \%)$ and $\mathrm{PPh}_{3}(2.5 \mathrm{mg}, 40 \mathrm{~mol} \%)$ at $0{ }^{\circ} \mathrm{C}$ under argon atmosphere. After stirring for $3 \mathrm{~h}$ at 0 ${ }^{\circ} \mathrm{C}$, the reaction mixture was quenched with saturated $\mathrm{NaHCO}_{3}$, extracted with $\mathrm{Et}_{2} \mathrm{O}$, dried $\left(\mathrm{Na}_{2} \mathrm{SO}_{4}\right)$, and concentrated under reduced pressure. The crude product was purified using silica gel flash chromatography to give $14 \mathrm{mg}$ IV-42 $(0.02 \mathrm{mmol}, 85 \%)$ as colorless oil.

IV-42: $R f(20 \%$ EtOAc/hexane $)=0.44 ;[\alpha]^{25}{ }_{\mathrm{D}}=-172.1\left(c=1.0, \mathrm{CH}_{2} \mathrm{Cl}_{2}\right)$; IR (thin film, $\left.\mathrm{cm}^{-1}\right) 2975,2957,2873,1739,1596,1369,1172,1083,764,632 ;{ }^{1} \mathrm{H}$ NMR $(600 \mathrm{MHz}$, $\left.\mathrm{CDCl}_{3}\right) \delta 7.71(\mathrm{~m}, 2 \mathrm{H}), 7.61(\mathrm{~m}, 3 \mathrm{H}), 5.57(\mathrm{~d}, J=7.2 \mathrm{~Hz}, 1 \mathrm{H}), 5.31(\mathrm{dt}, J=7.2,3.6 \mathrm{~Hz}$, $1 \mathrm{H}), 4.85(\mathrm{qt}, J=6.0,6.0 \mathrm{~Hz}, 1 \mathrm{H}), 4.29(\mathrm{t}, J=4.2 \mathrm{~Hz}, 1 \mathrm{H}), 3.61(\mathrm{~s}, 3 \mathrm{H}), 3.26(\mathrm{dd}, J=$ 16.2, $3.0 \mathrm{~Hz}, 1 \mathrm{H}), 3.04(\mathrm{dd}, J=16.2,3.0 \mathrm{~Hz}, 1 \mathrm{H}), 3.02(\mathrm{dd}, J=17.4,4.2 \mathrm{~Hz}, 1 \mathrm{H}), 2.78(\mathrm{t}$, $J=7.2 \mathrm{~Hz}, 2 \mathrm{H}), 2.54(\mathrm{dd}, J=17.4,4.2 \mathrm{~Hz}, 1 \mathrm{H}), 1.71 \sim 1.50(\mathrm{~m}, 4 \mathrm{H}), 1.17(\mathrm{~d}, J=6.0 \mathrm{~Hz}$, $3 \mathrm{H}), 1.16(\mathrm{~s}, 9 \mathrm{H}) ;{ }^{13} \mathrm{C} \mathrm{NMR}\left(150 \mathrm{MHz}, \mathrm{CD}_{3} \mathrm{Cl}\right) \delta 206.2,182.0,178.0,170.3,156.0$, $133.3,131.3,129.6,125.4,106.9,82.1,76.6,73.3,69.6,51.9,38.7,38.6,35.7,35.2,27.8$, 27.1, 22.6, 19.7; CIHRMS: Calculated for $\left[\mathrm{C}_{27} \mathrm{H}_{34} \mathrm{~N}_{4} \mathrm{O}_{9} \mathrm{~S}+\mathrm{Na}^{+}\right]$: 613.1939, Found: 613.1940 
$(S, E)-7-((2 S, 5 R, 6 S)-5-h y d r o x y-6-(2-m e t h o x y-2-o x o e t h y l)-5,6-d i h y d r o-2 H-p y r a n-2-$ yl)hept-6-en-2-yl pivalate (IV-41)

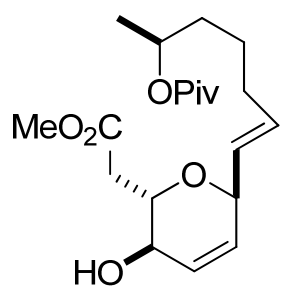

To a solution of allylic alcohol IV-48 $(15 \mathrm{mg}, 0.052 \mathrm{mmol})$ and sulfone IV-27 (22 $\mathrm{mg}$, $0.052 \mathrm{mmol})$ in dry $\mathrm{CH}_{2} \mathrm{Cl}_{2}(0.3 \mathrm{~mL})$, was added $\mathrm{Pd}_{2}(\mathrm{DBA})_{3} \cdot \mathrm{CHCl}_{3}(2.5 \mathrm{mg}, 5 \mathrm{~mol} \%)$ and $\mathrm{PPh}_{3}(2.5 \mathrm{mg}, 20 \mathrm{~mol} \%)$ at $0{ }^{\circ} \mathrm{C}$ under argon atmosphere. After stirring for $3 \mathrm{~h}$ at 0 ${ }^{\circ} \mathrm{C}$, the reaction mixture was cooled to $-78^{\circ} \mathrm{C}$ and $\mathrm{MeOH}$ was added to the solution. Then $\mathrm{NaBH}_{4}$ was added to the reaction solution, the reaction mixture was allowed to stir at -78 ${ }^{\circ} \mathrm{C}$ for $5 \mathrm{hr}$. The reaction was then quenched with saturated $\mathrm{NaHCO}_{3}$, extracted with $\mathrm{Et}_{2} \mathrm{O}$, dried $\left(\mathrm{Na}_{2} \mathrm{SO}_{4}\right)$, and concentrated under reduced pressure. The crude product was purified using silica gel flash chromatography to give $5 \mathrm{mg}$ IV-41 $(0.013 \mathrm{mmol}, 26 \%)$ as colorless oil: $R f(30 \%$ EtOAc/hexane $)=0.50 ;[\alpha]^{25}=57.0\left(c=0.75, \mathrm{CH}_{2} \mathrm{Cl}_{2}\right)$; IR (thin film, $\left.\mathrm{cm}^{-1}\right) 3485,2978,2936,1723,1456,1167,1070,1132 ;{ }^{1} \mathrm{H}$ NMR $(600 \mathrm{MHz}$, $\left.\mathrm{CDCl}_{3}\right) \delta 5.85(\mathrm{ddd}, J=10.8,1.8,1.8 \mathrm{~Hz}, 1 \mathrm{H}), 5.79(\mathrm{ddd}, J=10.8,3.0,1.8 \mathrm{~Hz}, 1 \mathrm{H}), 5.63$ $(\mathrm{dtd}, J=15.6,7.2,1.2 \mathrm{~Hz}, 1 \mathrm{H}), 5.53(\mathrm{dd}, J=15.6,5.4 \mathrm{~Hz}, 1 \mathrm{H}), 4.85(\mathrm{qt}, J=6.0,6.0 \mathrm{~Hz}$ 1H), 4.63 (dddd, $J=5.4,3.0,1.8,1.2 \mathrm{~Hz}, 1 \mathrm{H}), 3.94$ (ddd, $J=7.2,1.8,1.8 \mathrm{~Hz}, 1 \mathrm{H}), 3.89$ (ddd, $J=7.8,7.2,4.8 \mathrm{~Hz}, 1 \mathrm{H}), 3.71(\mathrm{~s}, 3 \mathrm{H}), 2.81(\mathrm{dd}, J=15.6,4.8 \mathrm{~Hz}, 1 \mathrm{H}), 2.57(\mathrm{dd}, J=$ 15.6, 7.8 Hz, 1H), $2.08(\mathrm{dt}, J=7.2,7.2 \mathrm{~Hz}, 2 \mathrm{H}), 1.64 \sim 1.38(\mathrm{~m}, 4 \mathrm{H}), 1.19(\mathrm{~d}, J=6.0 \mathrm{~Hz}$, $3 \mathrm{H}), 1.18(\mathrm{~s}, 9 \mathrm{H}) ;{ }^{13} \mathrm{C}$ NMR $\left(150 \mathrm{MHz}, \mathrm{CD}_{3} \mathrm{Cl}\right) \delta 178.3,172.1,133.9,129.5,128.6$, 
$127.9,72.5,70.5,67.2,57.0,51.8,38.7,37.9,35.0,32.1,27.1,24.5,19.7$; CIHRMS:

Calculated for $\left[\mathrm{C}_{20} \mathrm{H}_{32} \mathrm{O}_{6}+\mathrm{Na}^{+}\right]$: 391.2091, Found: 391.2079

$(S, Z)-7-((2 S, 5 R, 6 S)-5-h y d r o x y-6-(2-m e t h o x y-2-o x o e t h y l)-5,6-d i h y d r o-2 H-p y r a n-2-$

yl)hept-6-en-2-yl pivalate $(\mathbf{I V}-\mathbf{4 9})^{13}$

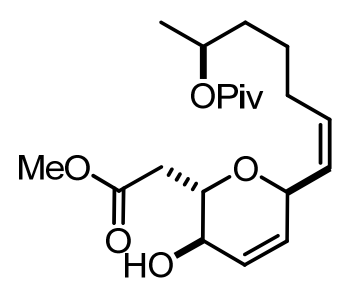

To a solution of allylic alcohol IV-48 $(15 \mathrm{mg}, 0.052 \mathrm{mmol})$ and sulfone IV-27 (22 $\mathrm{mg}$, $0.052 \mathrm{mmol})$ in dry $\mathrm{CH}_{2} \mathrm{Cl}_{2}(0.3 \mathrm{~mL})$, was added $\mathrm{Pd}_{2}(\mathrm{DBA})_{3} \cdot \mathrm{CHCl}_{3}(2.5 \mathrm{mg}, 5 \mathrm{~mol} \%)$ and $\mathrm{PPh}_{3}(2.5 \mathrm{mg}, 20 \mathrm{~mol} \%)$ at $0{ }^{\circ} \mathrm{C}$ under argon atmosphere. After stirring for $3 \mathrm{~h}$ at 0 ${ }^{\circ} \mathrm{C}$, the reaction mixture was cooled to $-78^{\circ} \mathrm{C}$ and $\mathrm{MeOH}$ was added to the solution. Then $\mathrm{NaBH}_{4}$ was added to the reaction solution, the reaction mixture was allowed to stir at -78 ${ }^{\circ} \mathrm{C}$ for $5 \mathrm{hr}$. The reaction was then quenched with saturated $\mathrm{NaHCO}_{3}$, extracted with $\mathrm{Et}_{2} \mathrm{O}$, dried $\left(\mathrm{Na}_{2} \mathrm{SO}_{4}\right)$, and concentrated under reduced pressure. The crude product was purified using silica gel flash chromatography to give $2 \mathrm{mg}$ IV-49 (0.006 mmol, 13\%) as colorless oil. ${ }^{1} \mathrm{H}$ NMR (600 MHz, $\left.\mathrm{CDCl}_{3}\right) \delta 5.84(\mathrm{ddd}, J=10.2,2.4,2.4 \mathrm{~Hz}, 1 \mathrm{H}), 5.71$ (ddd, $J=10.8,3.0,1.8 \mathrm{~Hz}, 1 \mathrm{H}), 5.55(\mathrm{~m}, 2 \mathrm{H}), 4.92(\mathrm{ddd}, J=4.8,2.4,1.8, \mathrm{~Hz}, 1 \mathrm{H}), 4.86$ (qt, $J=6.0,6.0 \mathrm{~Hz}, 1 \mathrm{H}), 3.97(\mathrm{ddd}, J=7.8,6.6,4.8 \mathrm{~Hz}, 1 \mathrm{H}), 3.92(\mathrm{ddd}, J=6.6,3.0,2.4$ $\mathrm{Hz}, 1 \mathrm{H}), 3.71(\mathrm{~s}, 3 \mathrm{H}), 2.77(\mathrm{dd}, J=15.6,4.8 \mathrm{~Hz}, 1 \mathrm{H}), 2.59(\mathrm{dd}, J=15.6,7.8 \mathrm{~Hz}, 1 \mathrm{H})$,

\footnotetext{
${ }^{13}$ This compound is not fully characterized.
} 
$2.33(\mathrm{ddd}, J=6.0,4.8,1.8 \mathrm{~Hz}, 2 \mathrm{H}), 1.64 \sim 1.38(\mathrm{~m}, 4 \mathrm{H}), 1.19(\mathrm{~d}, J=6.0 \mathrm{~Hz}, 3 \mathrm{H}), 1.18(\mathrm{~s}$, 9H);

(2R,7R)-7-(sulfonyl)-7-((2R,6S)-6-(2-methoxy-2-oxoethyl)-5-oxo-5,6-dihydro-2Hpyran-2-yl)-6-oxoheptan-2-yl pivalate (IV-25) ${ }^{14}$

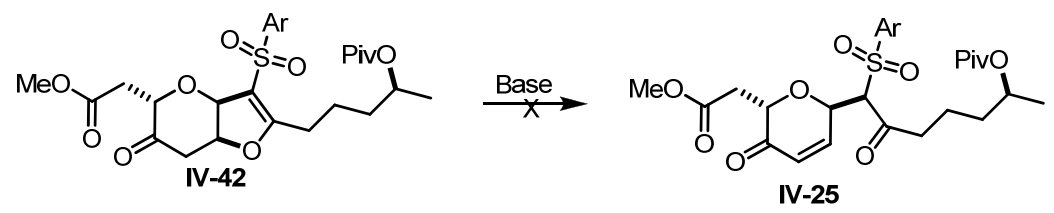

To three round bottle flasks, $\mathbf{I V - 4 2}$ in THF $(0.5 \mathrm{M})$ was added. $\mathrm{NaOMe}, \mathrm{KO} t-\mathrm{Bu}$ and $\mathrm{NaH}$ (catalytic to 5 equivalent) was added to the three bottles respectively. The reaction solutions were allowed to stir for $24 \mathrm{hr}$ at R.T. No desired product IV-25 can be isolated.

(2S)-5-((3S,3aR,5S,7aS)-3-(sulfonyl)-5-(2-methoxy-2-oxoethyl)-6-oxohexahydro-2Hfuro[3,2-b]pyran-2-yl)pentan-2-yl pivalate (IV-46) ${ }^{14}$

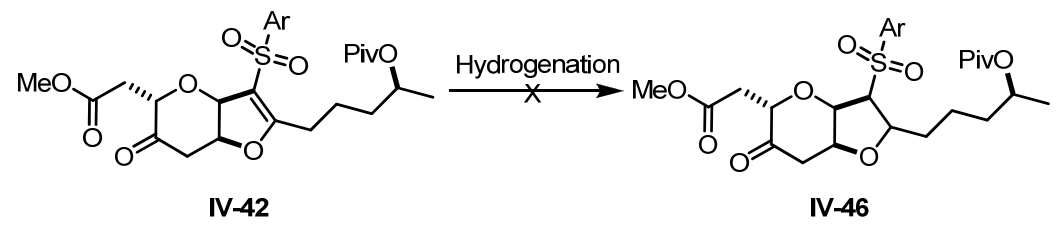

To a solution of IV-42 in dry $\mathrm{CH}_{2} \mathrm{Cl}_{2}$ was carefully added $\mathrm{Pd} / \mathrm{C}$ (3 equivalents) and the resulting suspension was stirred under $\mathrm{H}_{2}(1 \mathrm{~atm})$ for $24 \mathrm{hr}$ at room temperature. TLC

\footnotetext{
${ }^{14}$ This compound was not obtained.
} 
showed there was still starting materials. The reaction solution was allowed to stir under $\mathrm{H}_{2}$ (3 atm) for $24 \mathrm{hr}$, starting materials was fully recovered.

To a solution of IV-42 in dry $\mathrm{CH}_{2} \mathrm{Cl}_{2}$ was carefully added Wilkinson's catalyst (20 mol $\%)$ and the resulting suspension was stirred under $\mathrm{H}_{2}$ (1 atm) for $24 \mathrm{hr}$ at room temperature. TLC showed there was still starting materials. The reaction solution was allowed to stir under $\mathrm{H}_{2}$ (3 atm) for $24 \mathrm{hr}$, starting materials was fully recovered.

(2R,7R)-7-(sulfonyl)-7-((2R,5R,6S)-5-(tert-butyldimethylsilyloxy)-6-(2-methoxy-2oxoethyl)-5,6-dihydro-2H-pyran-2-yl)-6-oxoheptan-2-yl pivalate (IV-51) ${ }^{14}$

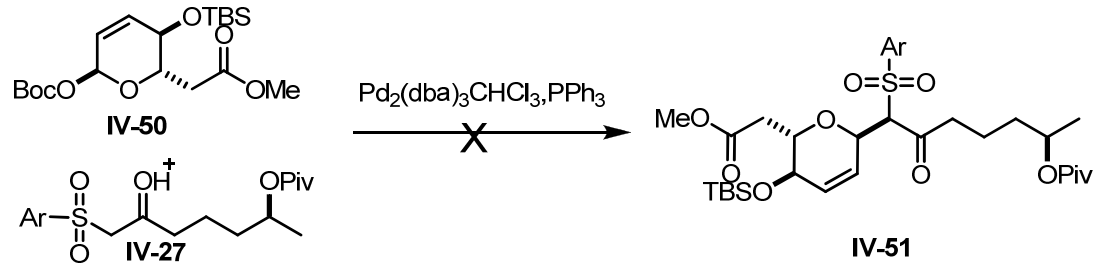

To a solution of IV-50 and sulfone IV-27 in dry $\mathrm{CH}_{2} \mathrm{Cl}_{2}(0.5 \mathrm{M})$, was added $\mathrm{Pd}_{2}(\mathrm{DBA})_{3} \cdot \mathrm{CHCl}_{3}(5 \mathrm{~mol} \%)$ and $\mathrm{PPh}_{3}(20 \mathrm{~mol} \%)$ at $0{ }^{\circ} \mathrm{C}$ under argon atmosphere. After stirring for $3 \mathrm{~h}$ at $0{ }^{\circ} \mathrm{C}$, the reaction mixture was warmed up to R.T. and allowed to stir for $5 \mathrm{hr}$, desired product IV-51 was not observed.

(2R,7R)-7-(sulfonyl)-7-((2R,5S,6S)-5-hydroxy-6-(2-methoxy-2-oxoethyl)-5,6-dihydro2H-pyran-2-yl)-6-oxoheptan-2-yl pivalate (IV-53) ${ }^{14}$ 


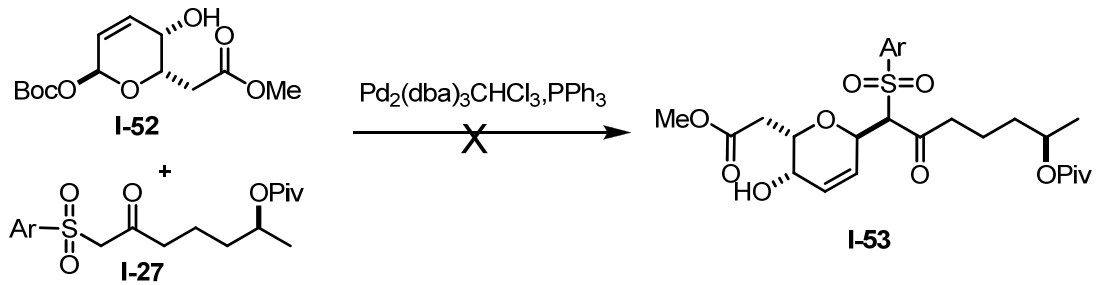

To a solution of IV-52 and sulfone IV-27 in dry $\mathrm{CH}_{2} \mathrm{Cl}_{2}(0.5 \mathrm{M})$, was added $\mathrm{Pd}_{2}(\mathrm{DBA})_{3} \cdot \mathrm{CHCl}_{3}(5 \mathrm{~mol} \%)$ and $\mathrm{PPh}_{3}(20 \mathrm{~mol} \%)$ at $0{ }^{\circ} \mathrm{C}$ under argon atmosphere. After stirring for $3 \mathrm{~h}$ at $0{ }^{\circ} \mathrm{C}$, the reaction mixture was warmed up to R.T. and allowed to stir for $5 \mathrm{hr}$, desired product IV-53 was not observed.

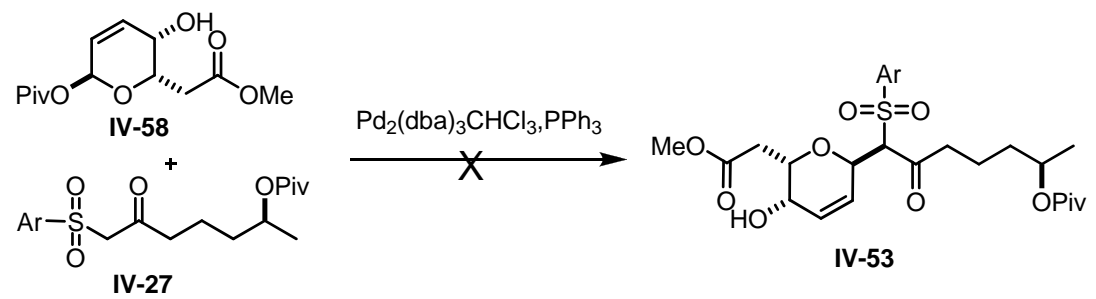

To a solution of IV-58 and sulfone IV-27 in dry $\mathrm{CH}_{2} \mathrm{Cl}_{2}(0.5 \mathrm{M})$, was added $\mathrm{Pd}_{2}(\mathrm{DBA})_{3} \cdot \mathrm{CHCl}_{3}(5 \mathrm{~mol} \%)$ and $\mathrm{PPh}_{3}(20 \mathrm{~mol} \%)$ at $0{ }^{\circ} \mathrm{C}$ under argon atmosphere. After stirring for $3 \mathrm{~h}$ at $0{ }^{\circ} \mathrm{C}$, the reaction mixture was warmed up to R.T. and allowed to stir for $5 \mathrm{hr}$, desired product IV-53 was not observed.

(2R,7R)-7-(sulfonyl)-7-((2R,5S,6S)-5-(tert-butyldimethylsilyloxy)-6-(2-methoxy-2oxoethyl)-5,6-dihydro-2H-pyran-2-yl)-6-oxoheptan-2-yl pivalate (IV-55) ${ }^{14}$ 


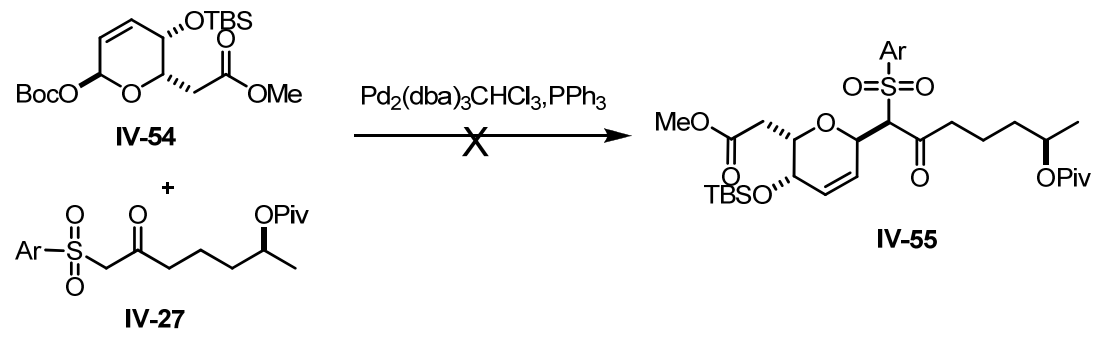

To a solution of IV-54 and sulfone IV-27 in dry $\mathrm{CH}_{2} \mathrm{Cl}_{2}(0.5 \mathrm{M})$, was added $\mathrm{Pd}_{2}(\mathrm{DBA})_{3} \cdot \mathrm{CHCl}_{3}(5 \mathrm{~mol} \%)$ and $\mathrm{PPh}_{3}(20 \mathrm{~mol} \%)$ at $0{ }^{\circ} \mathrm{C}$ under argon atmosphere. After stirring for $3 \mathrm{~h}$ at $0{ }^{\circ} \mathrm{C}$, the reaction mixture was warmed up to R.T. and allowed to stir for $5 \mathrm{hr}$, desired product IV-55 was not observed. 
Appendix 


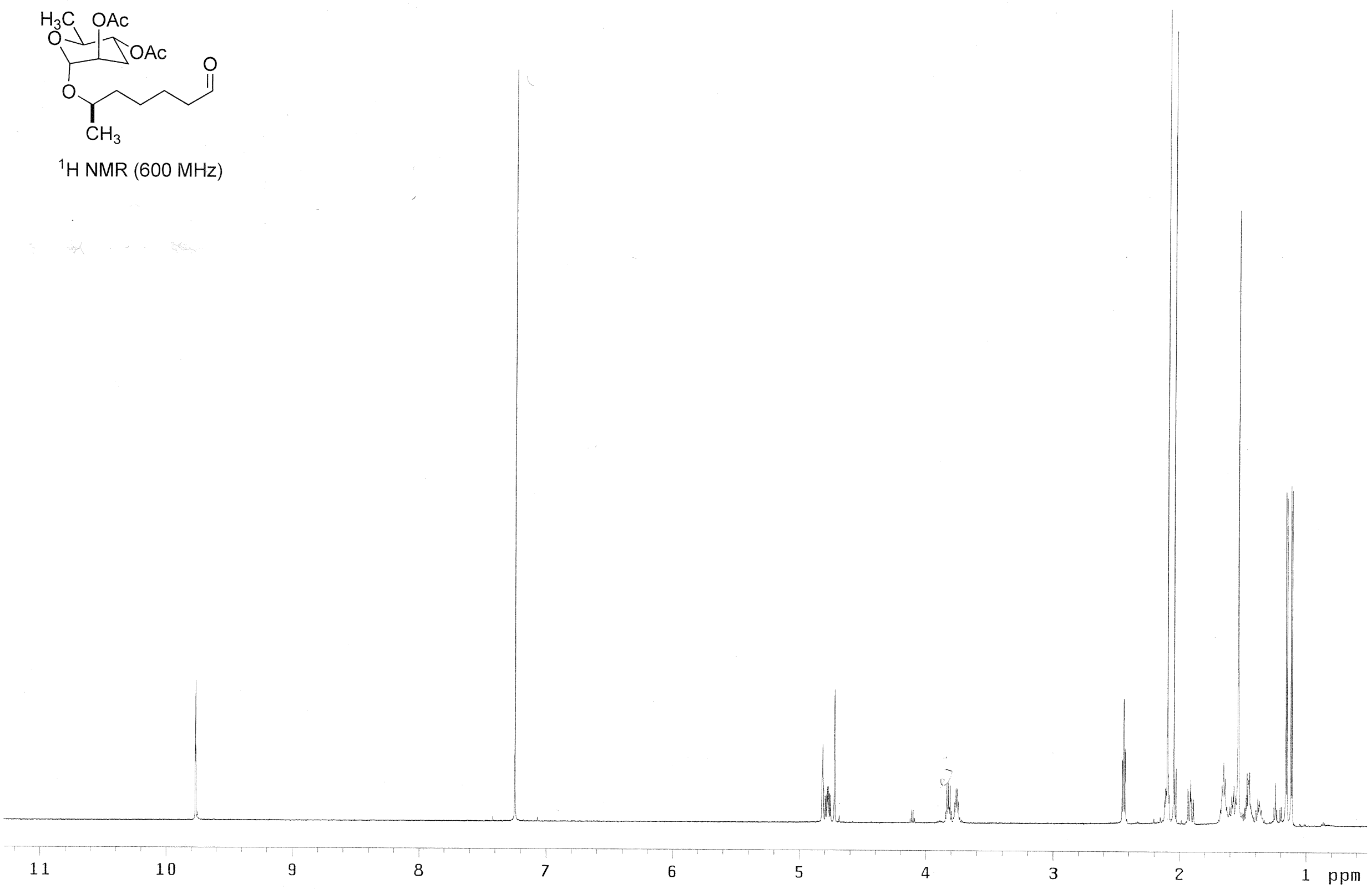




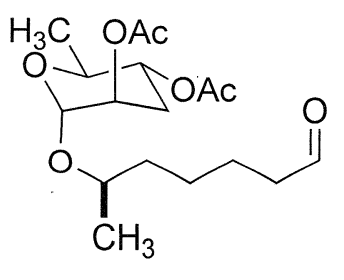

${ }^{13} \mathrm{C}$ NMR $(150 \mathrm{MHz})$

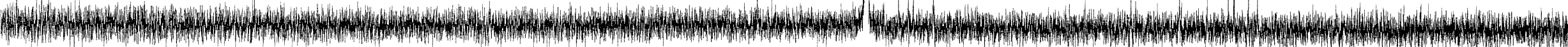




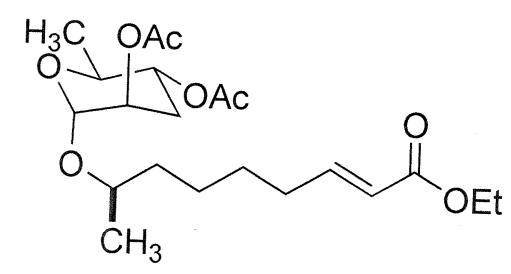

${ }^{1} \mathrm{H}$ NMR $(600 \mathrm{MHz})$

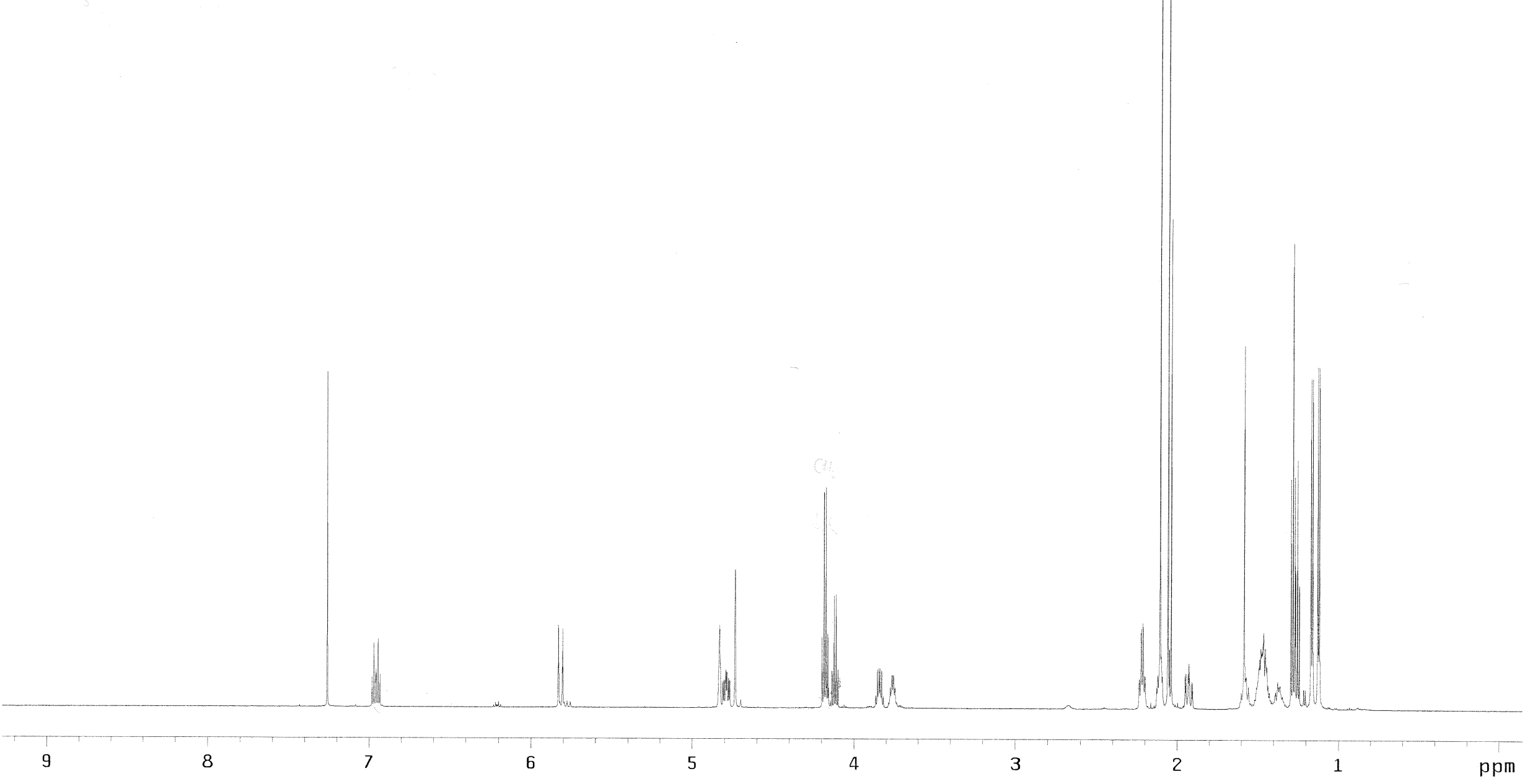




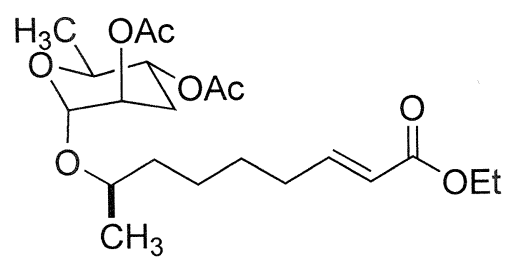

${ }^{13} \mathrm{C}$ NMR $(150 \mathrm{MHz})$

1

200

180

160

140

120

100

80

60

40

20

0

ppm

157 

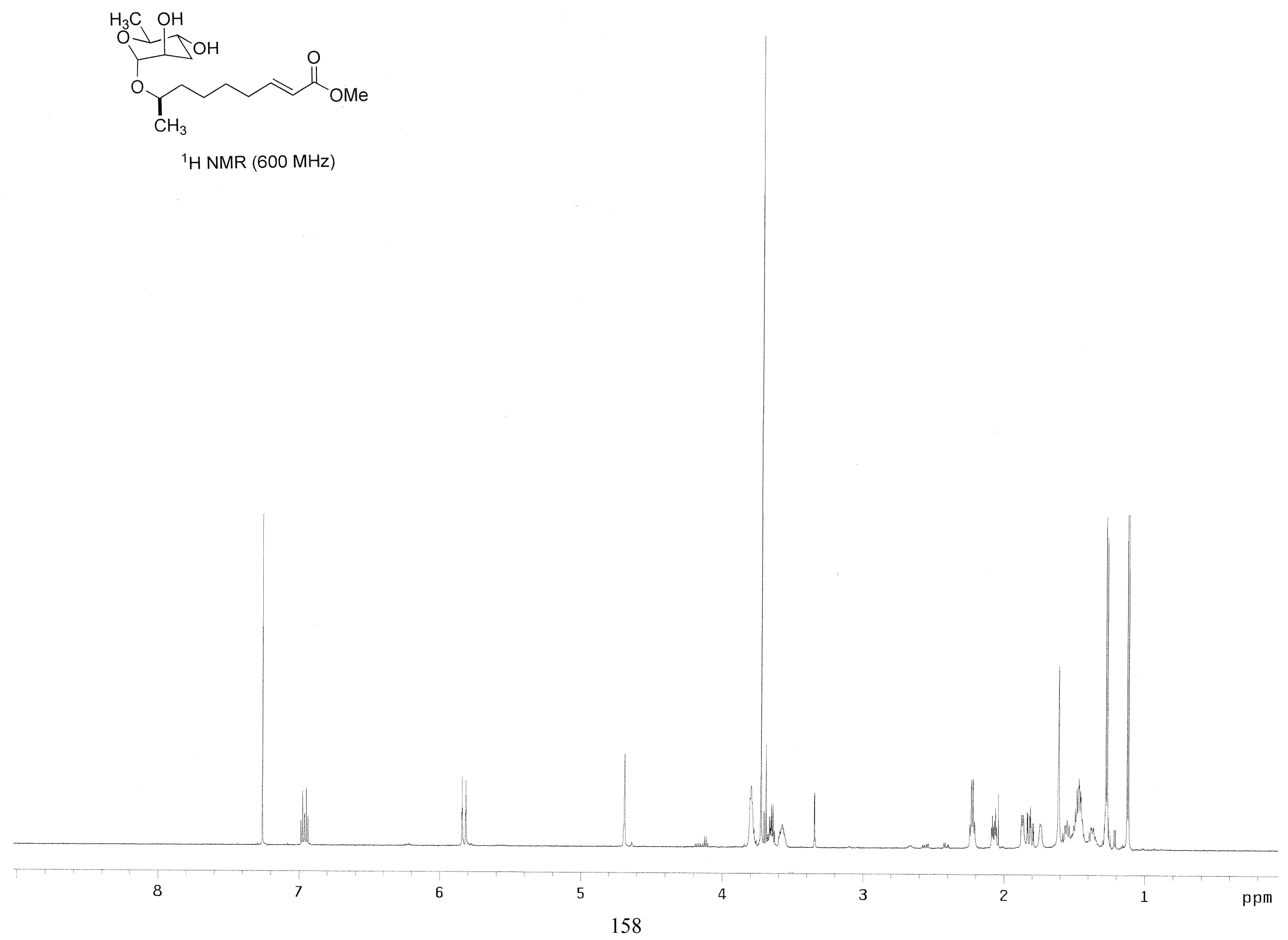


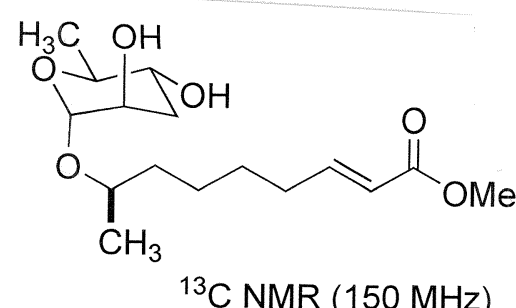

${ }^{13} \mathrm{C}$ NMR $(150 \mathrm{MHz})$

H. 
$\mathrm{H}_{3} \mathrm{C} \mathrm{OH}$

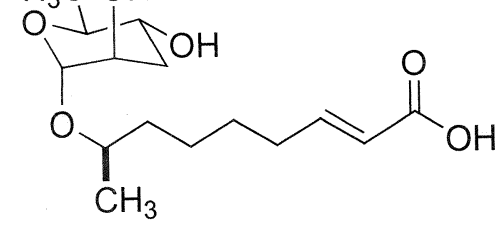

${ }^{1} \mathrm{H}$ NMR $(600 \mathrm{MHz})$

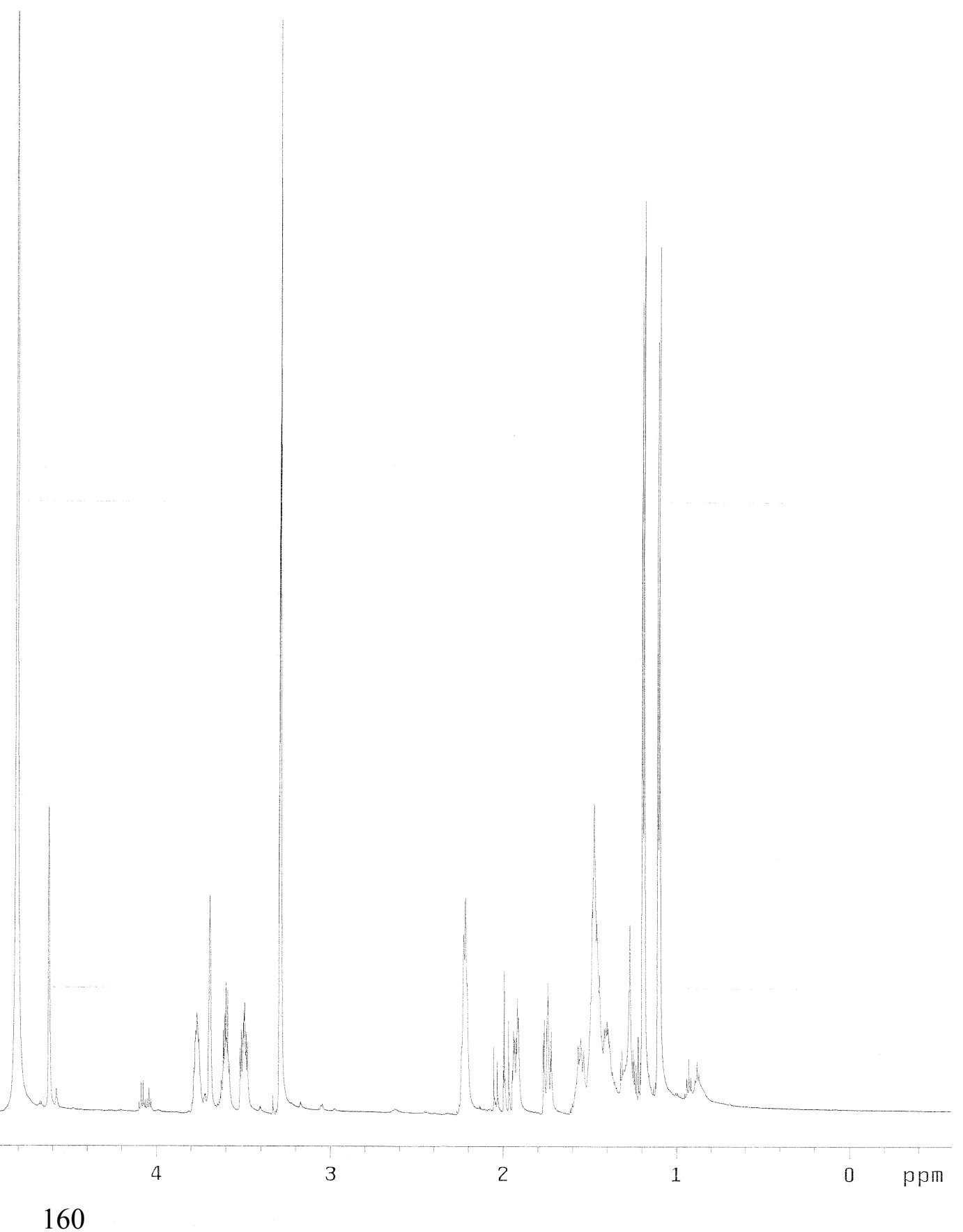




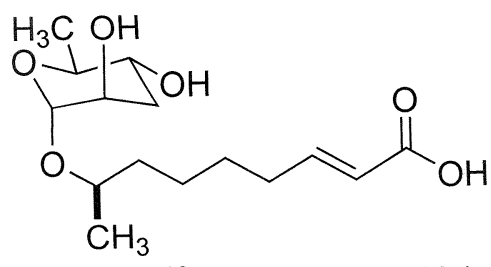

${ }^{13} \mathrm{C}$ NMR $(150 \mathrm{MHz})$

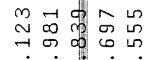

守标存

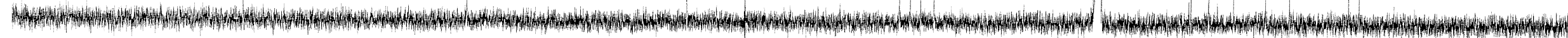




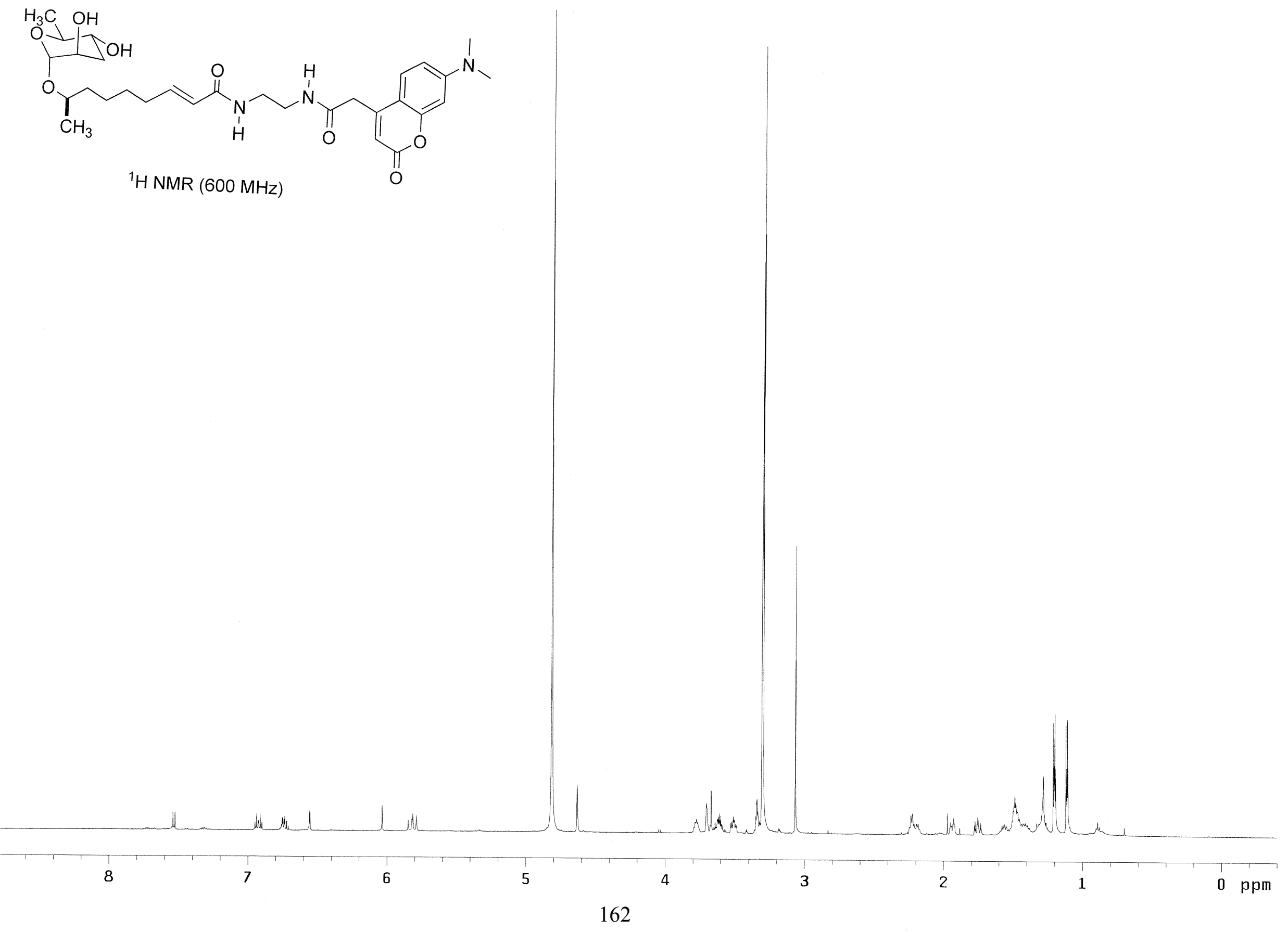



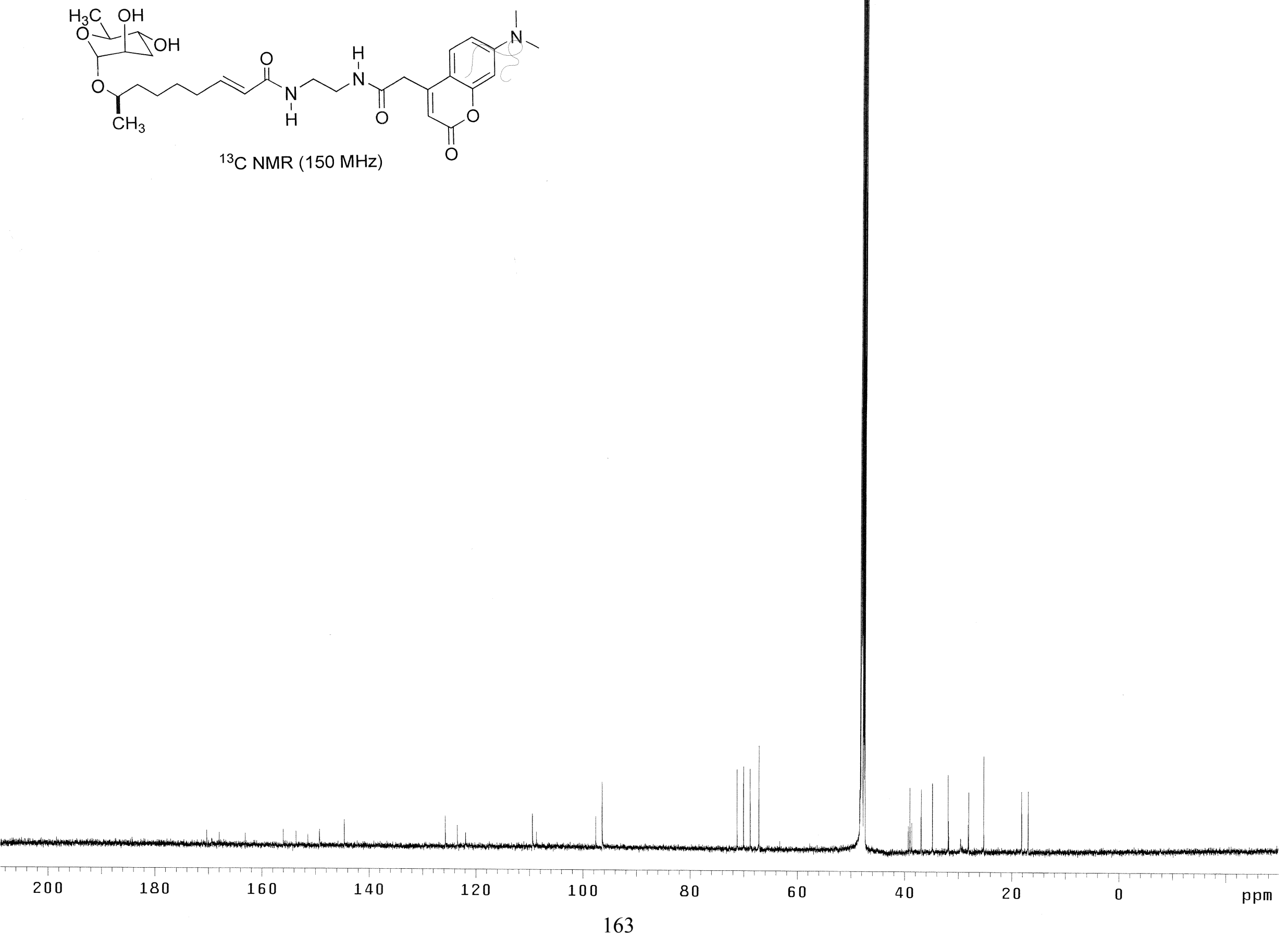
O:",<smiles>C[C@@H]1OC(O)CCC1=O</smiles>

${ }^{1} \mathrm{H}$ NMR $\left(270 \mathrm{MHz}, \mathrm{CDCl}_{3}\right)$

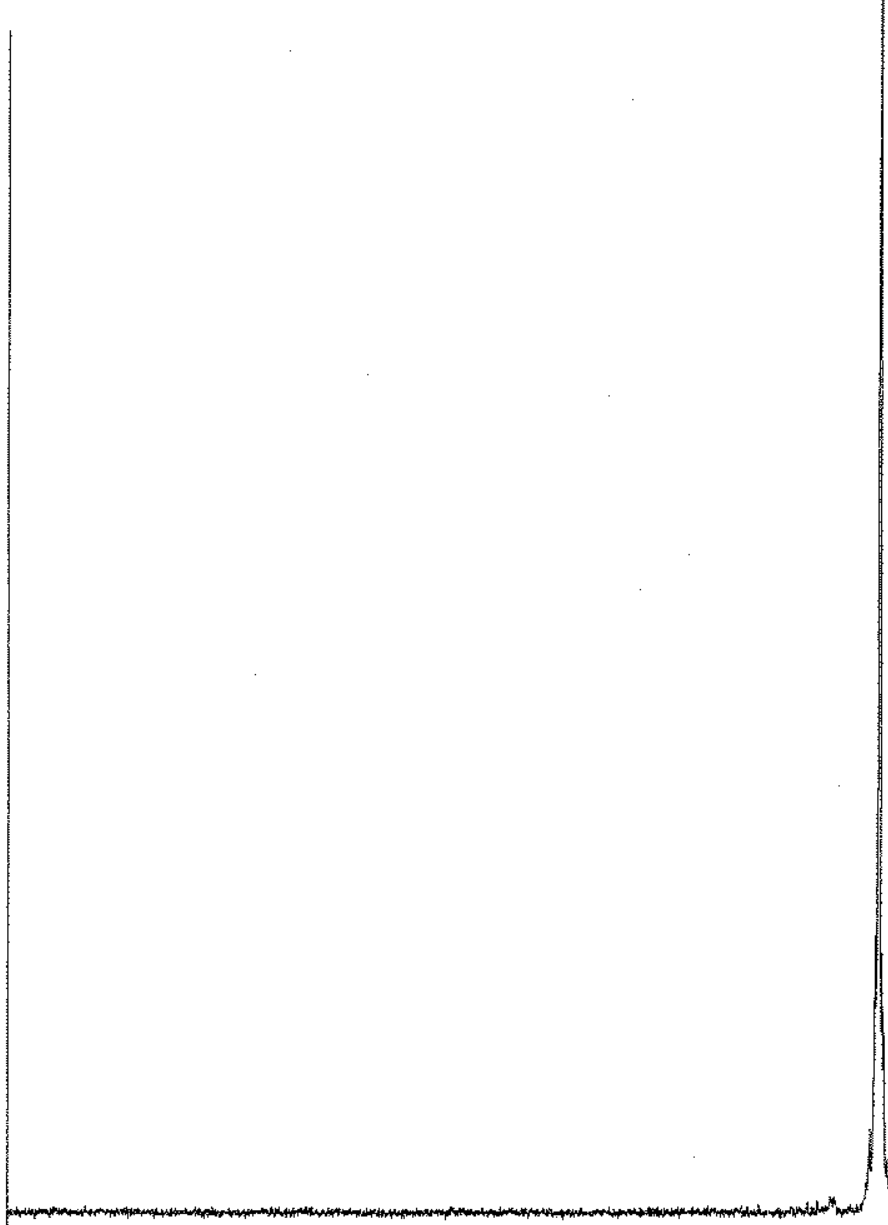

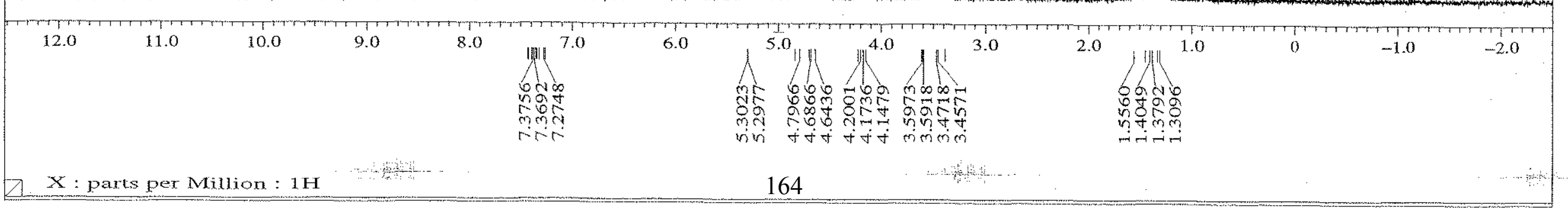




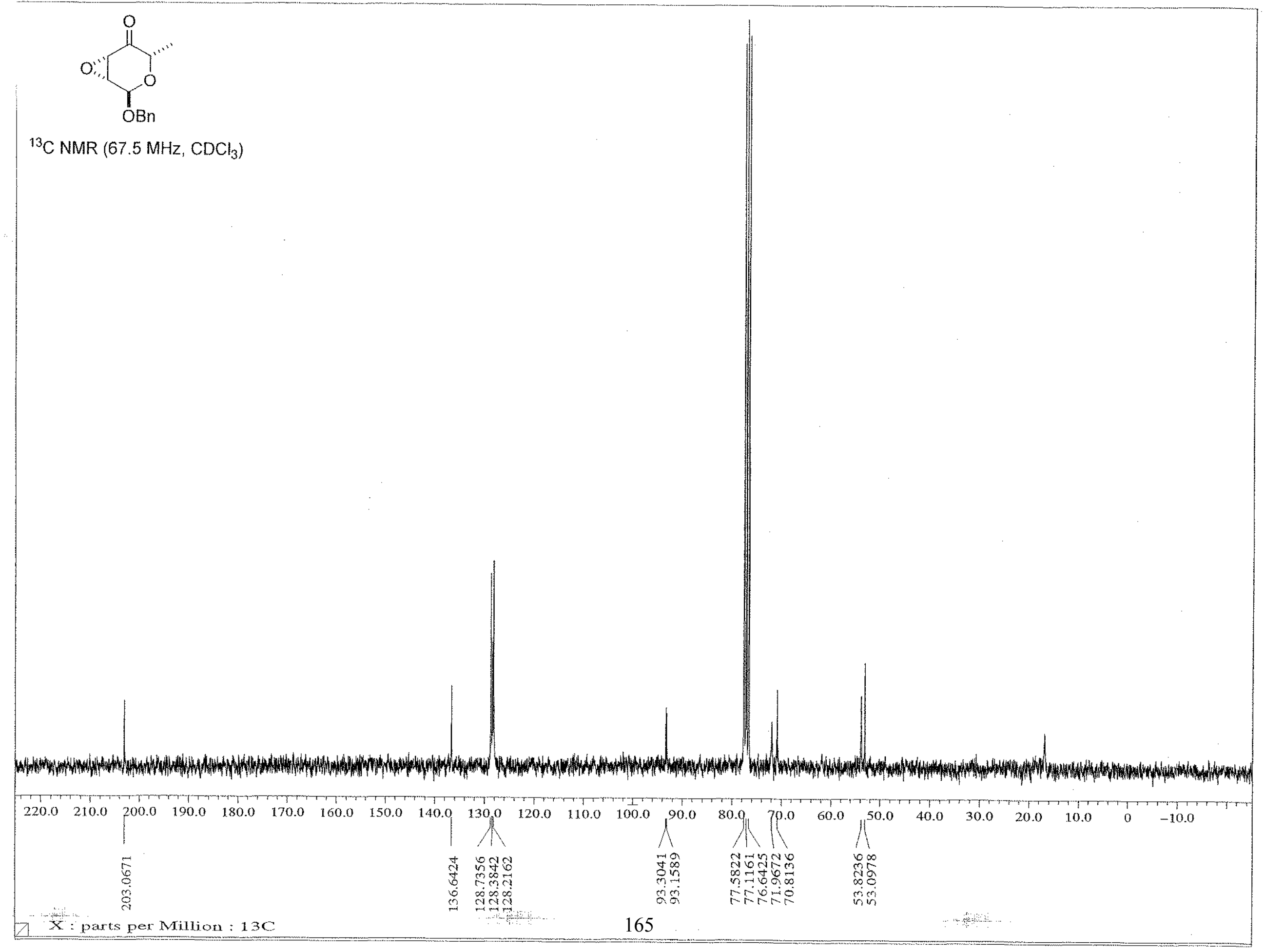




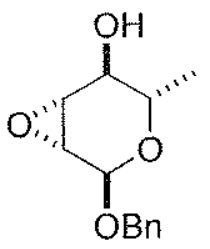

${ }^{1} \mathrm{H} \mathrm{NMR}\left(600 \mathrm{MHz}, \mathrm{CDCl}_{3}\right)$

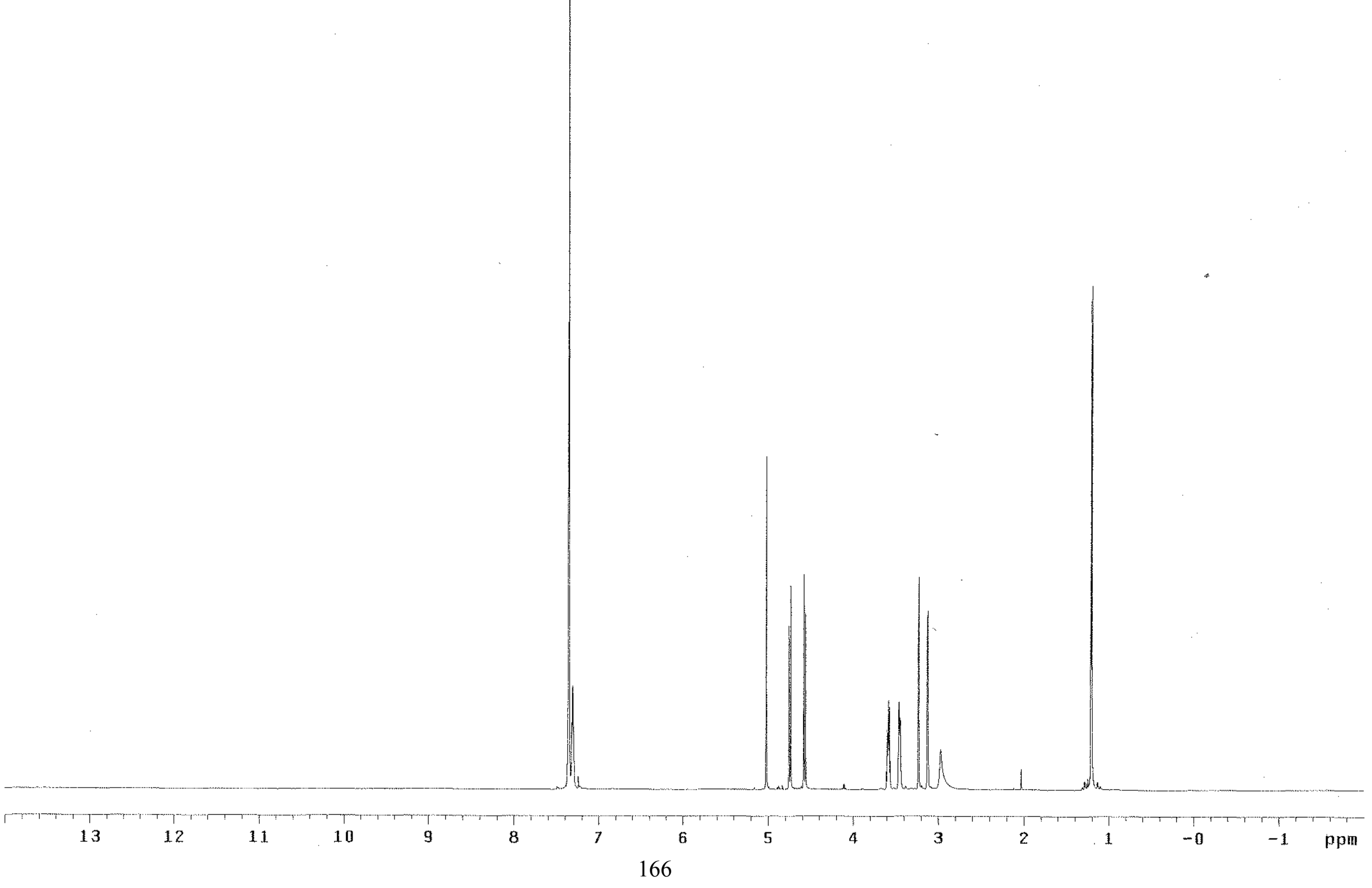




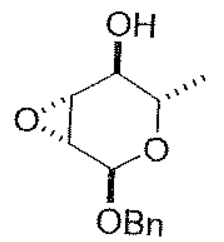

${ }^{13} \mathrm{C}$ NMR $\left(150 \mathrm{MHz}, \mathrm{CDCl}_{3}\right)$

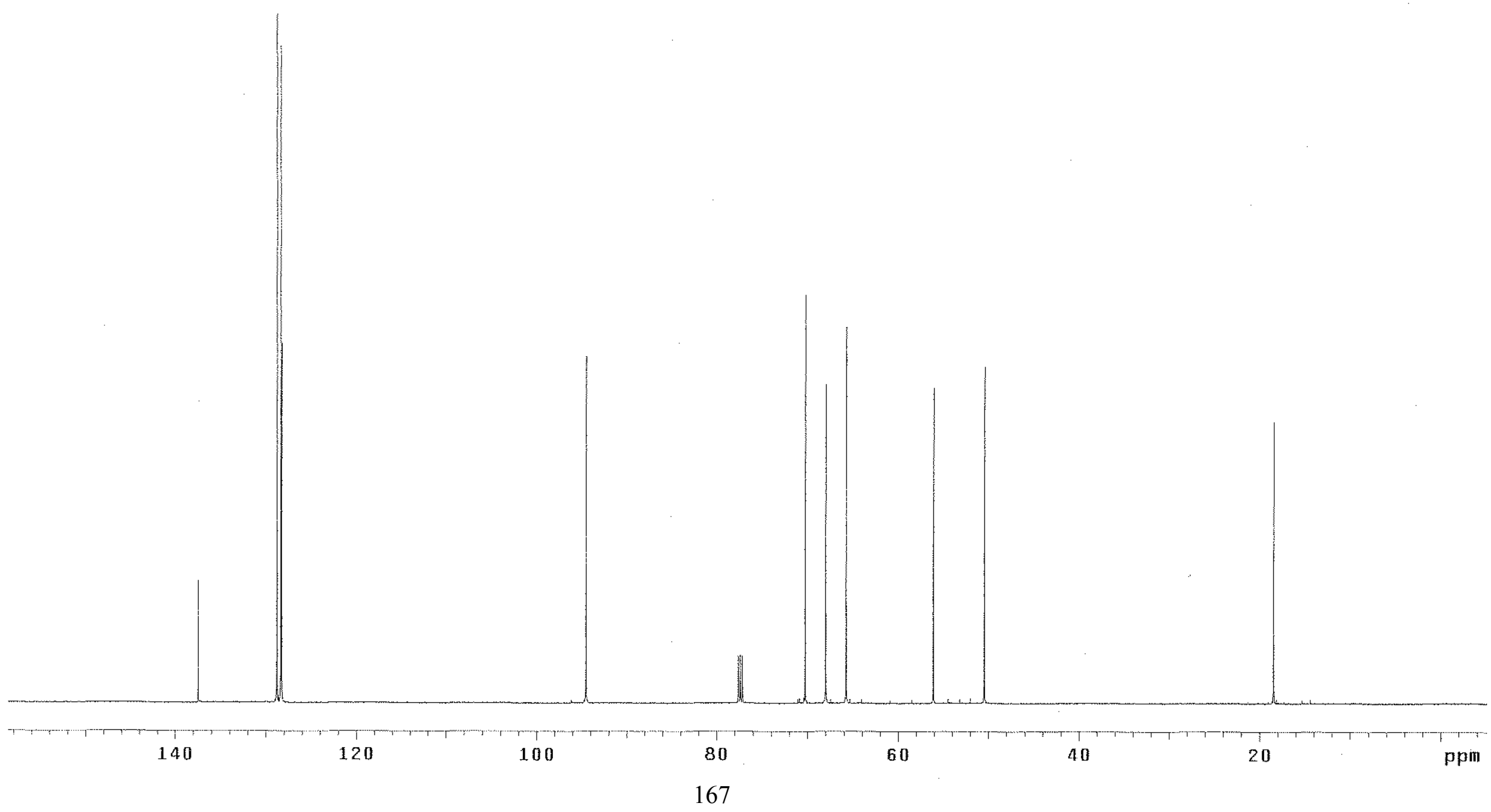


<smiles>C[C@@H]1O[C@H](O)[C@H](O)C(O)[C@H]1O</smiles>

${ }^{1} \mathrm{H} \mathrm{NMR}\left(600 \mathrm{MHz}, \mathrm{CDCl}_{3}\right)$

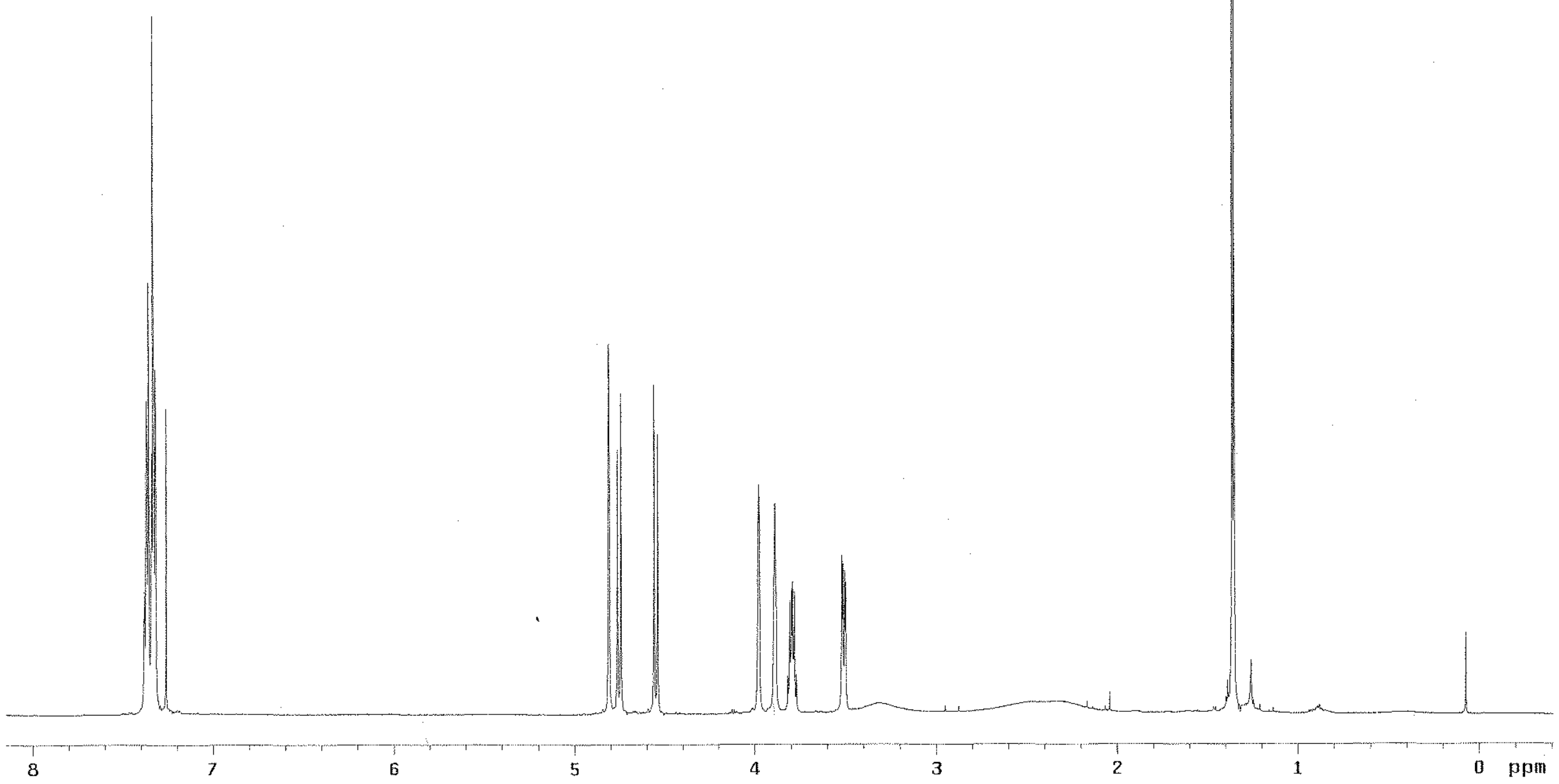

168 
<smiles>C[C@H]1OC(Cc2ccccc2)[C@H](O)C(O)C1O</smiles>

${ }^{13} \mathrm{C}$ NMR $\left(150 \mathrm{MHz} \mathrm{CDCl}_{3}\right)$

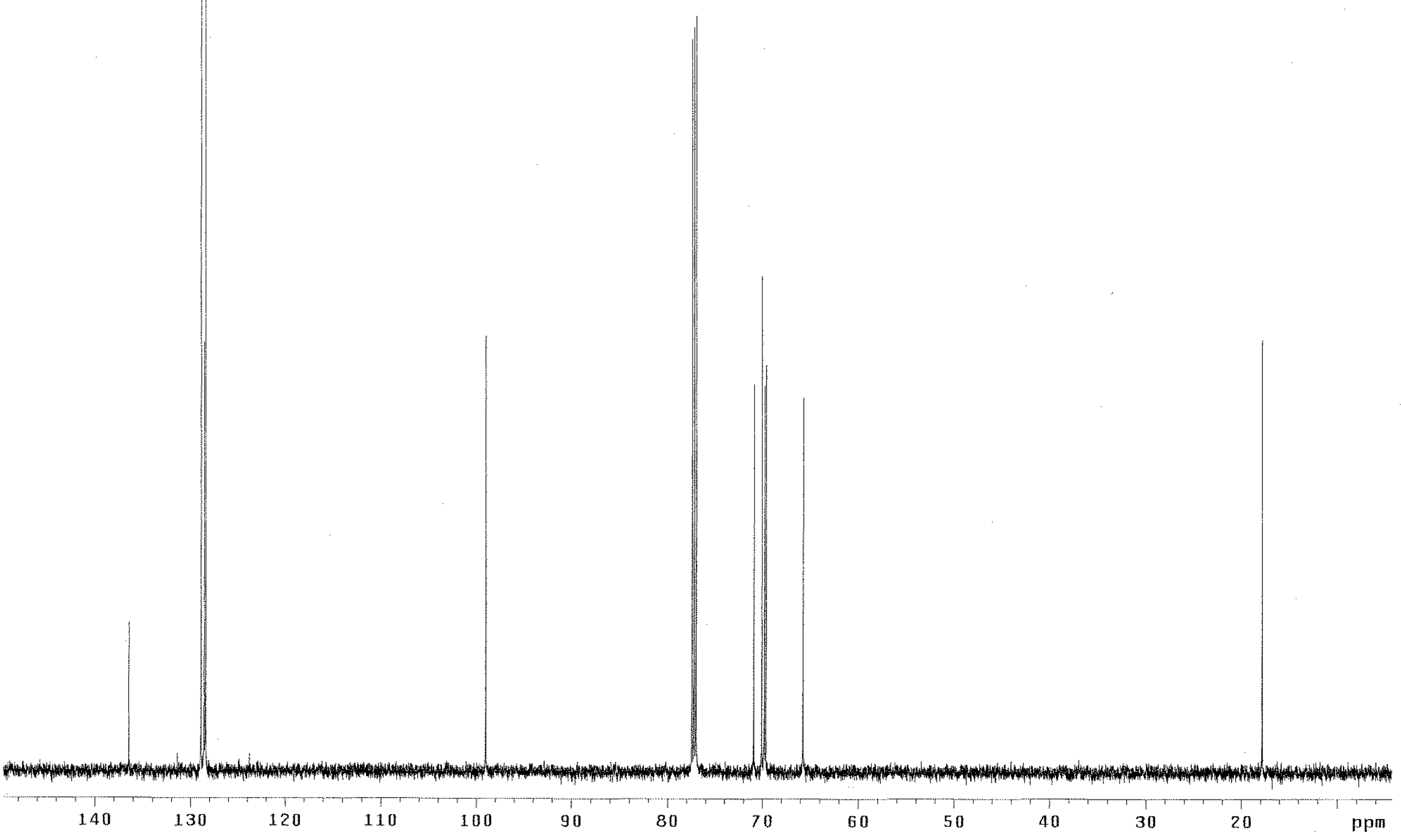

169 


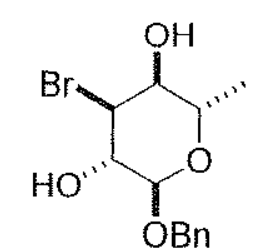

${ }^{1} \mathrm{H}$ NMR $\left(600 \mathrm{MHz}, \mathrm{CDCl}_{3}\right)$

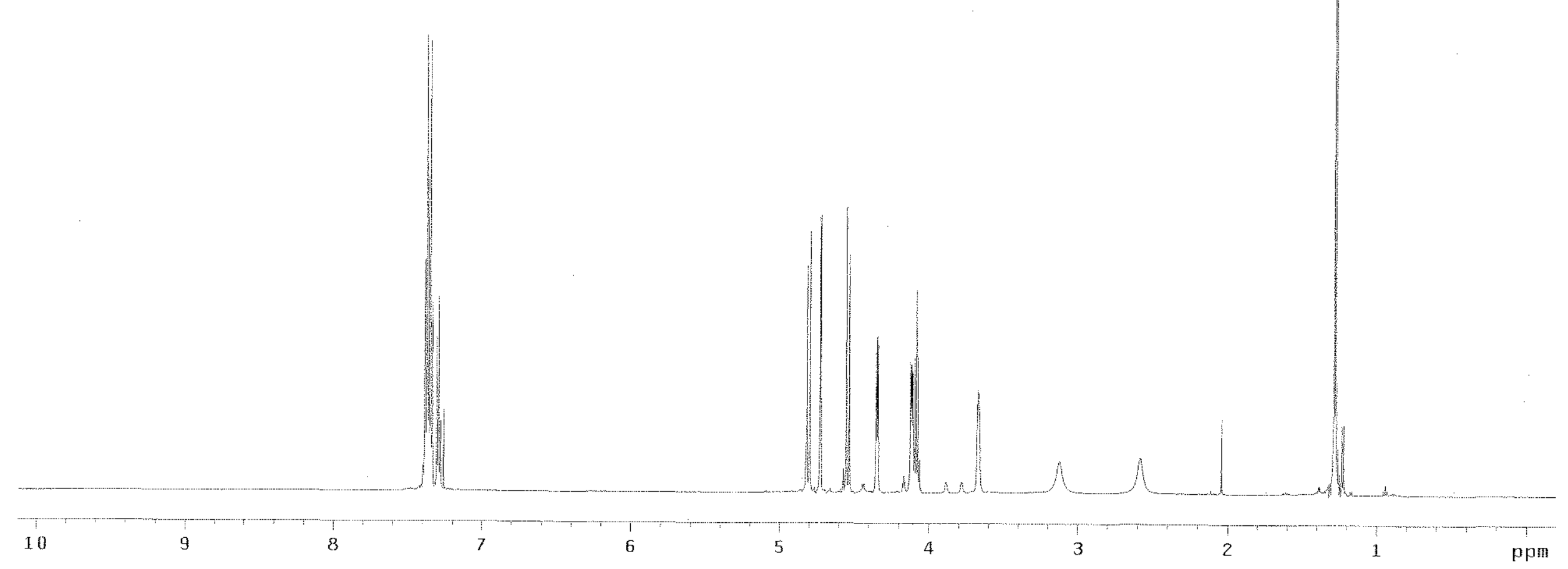




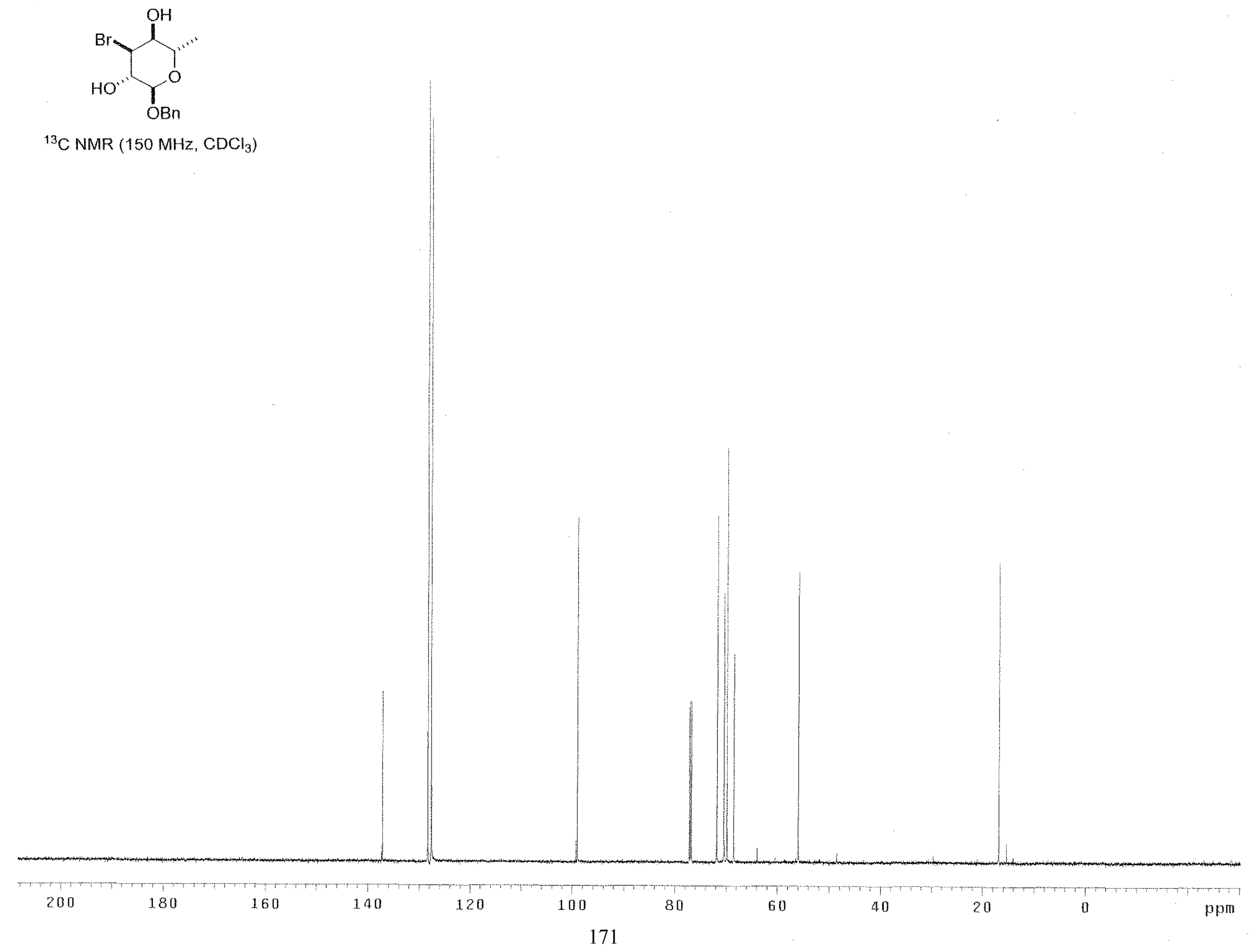




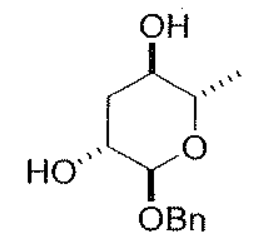

${ }^{1} \mathrm{HNMR}\left(600 \mathrm{MHz}, \mathrm{CDCl}_{3}\right)$

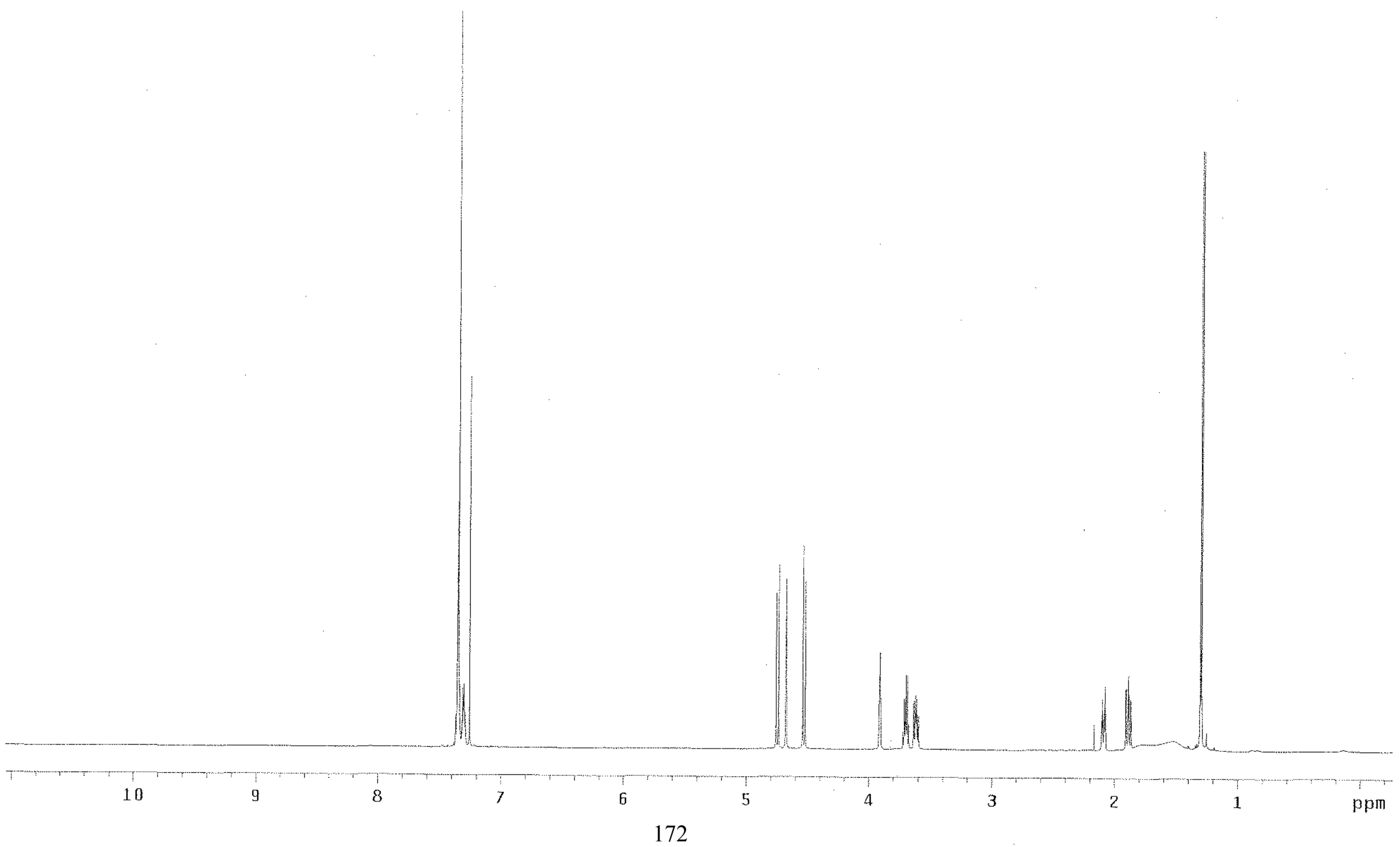




$$
\overbrace{\mathrm{OBn}}^{\mathrm{OH}}
$$

${ }^{13} \mathrm{C} \mathrm{NMR}\left(150 \mathrm{MHz}, \mathrm{CDCl}_{3}\right)$ 
$\overbrace{}^{\mathrm{OH}^{\mathrm{H}}}$

${ }^{1} \mathrm{H}$ NMR (600 MHz, CDCl3) 


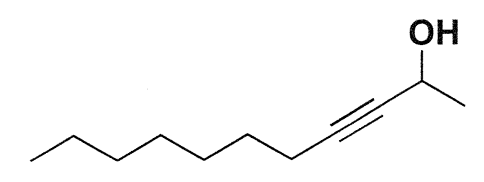

${ }^{13} \mathrm{C}$ NMR $(150 \mathrm{MHz}, \mathrm{CDCl} 3)$

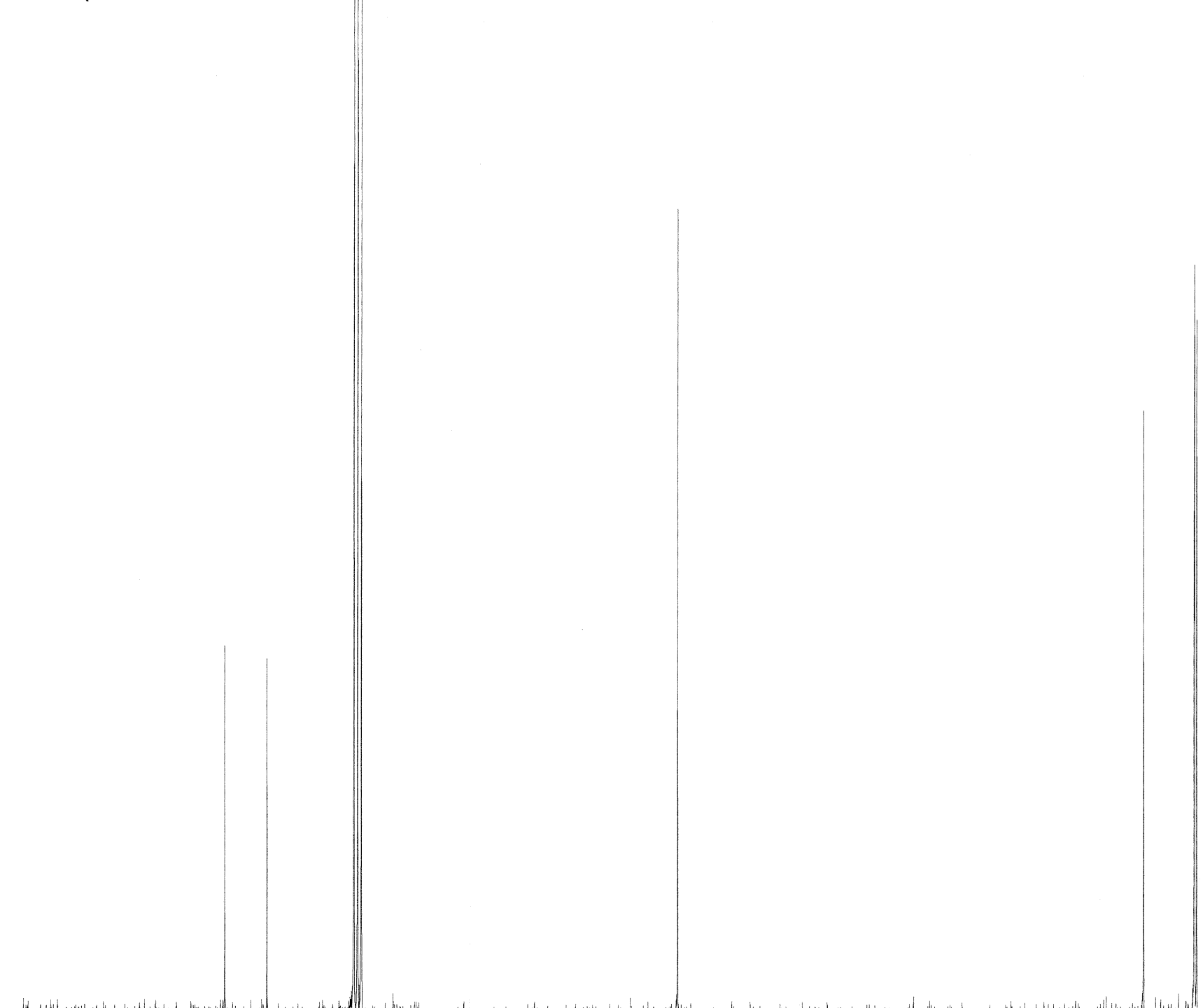

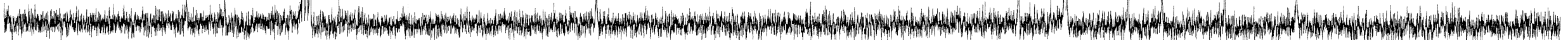




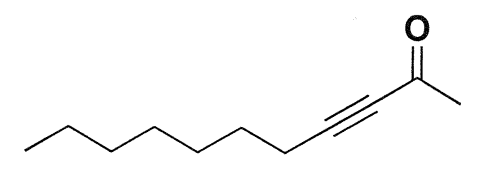

${ }^{1} \mathrm{H}$ NMR (600 MHz, CDCl3) 


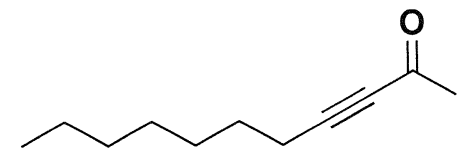

${ }^{13} \mathrm{C}$ NMR (150 MHz, CDCl3)

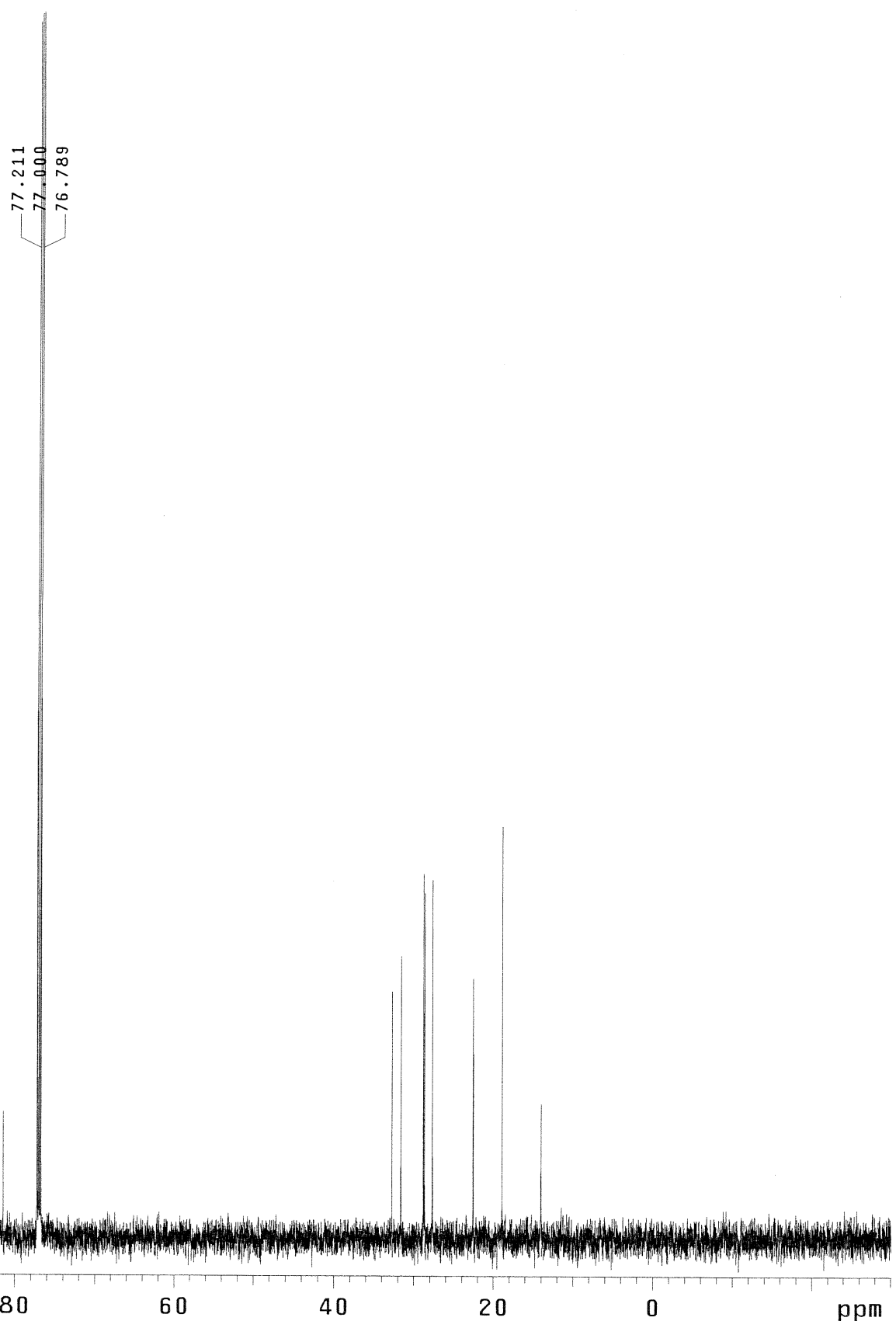


$\mathrm{OH}$

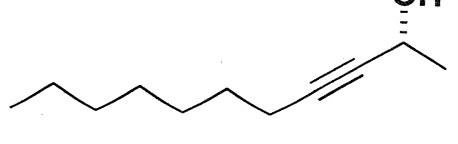

${ }^{1} \mathrm{H}$ NMR (600 MHz, CDCI3) 


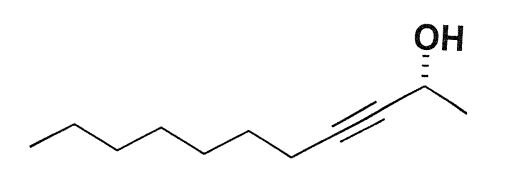

${ }^{13} \mathrm{C}$ NMR $(150 \mathrm{MHz}, \mathrm{CDCl} 3)$

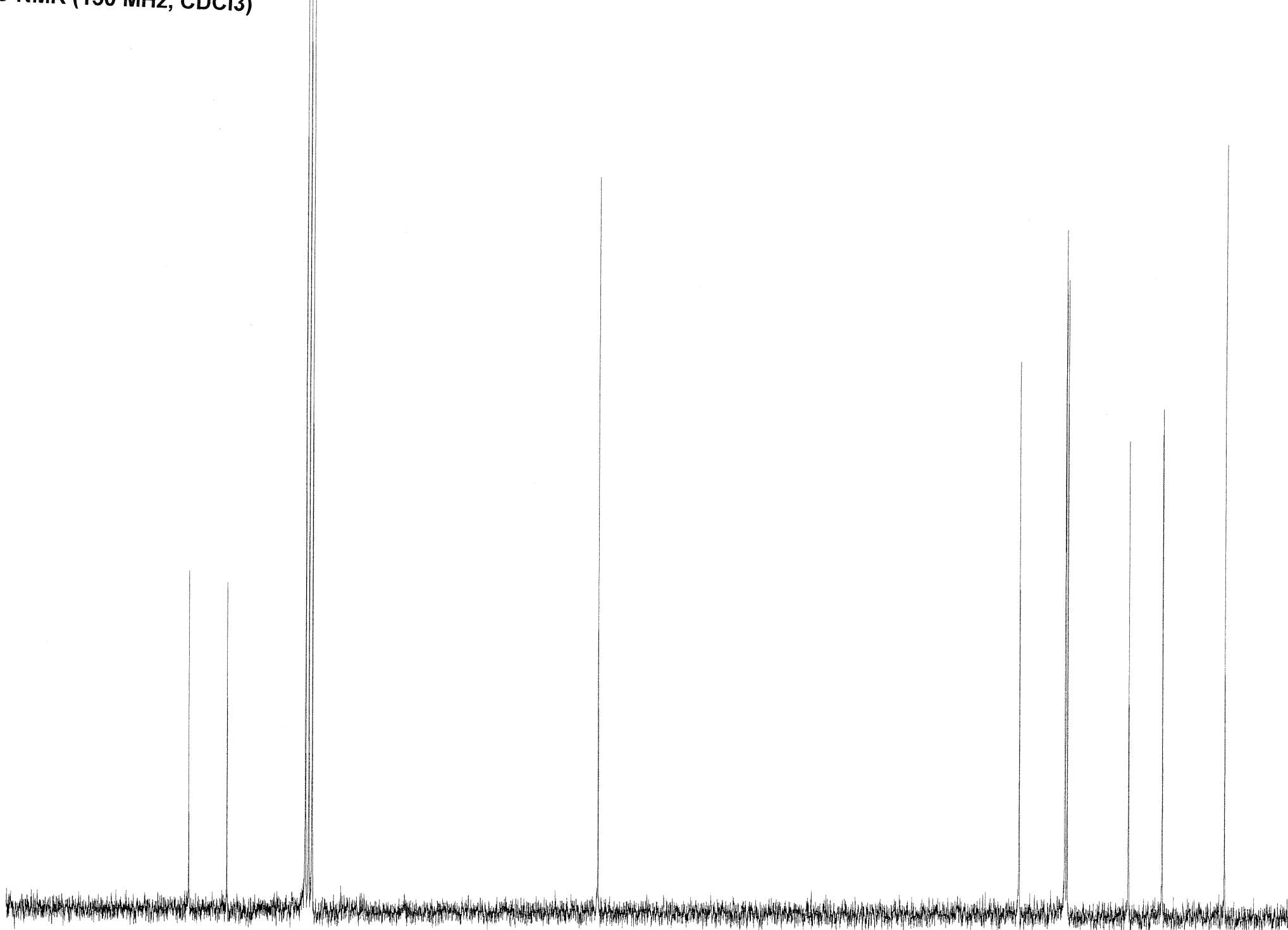

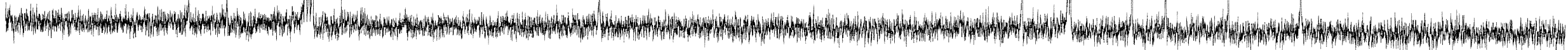




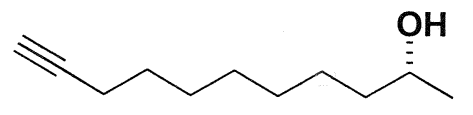

${ }^{1} \mathrm{H}$ NMR (600 MHz, CDCl3)

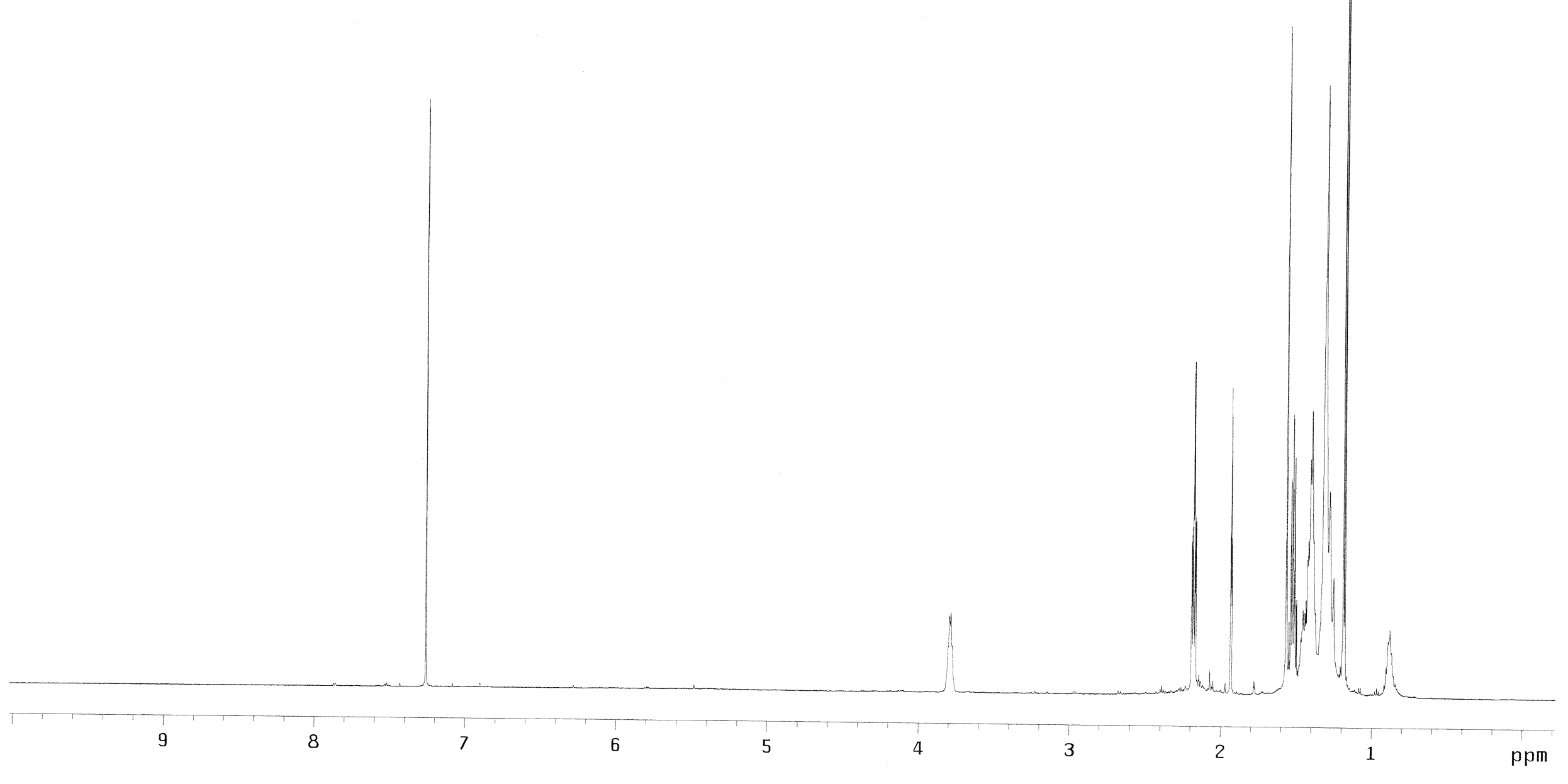




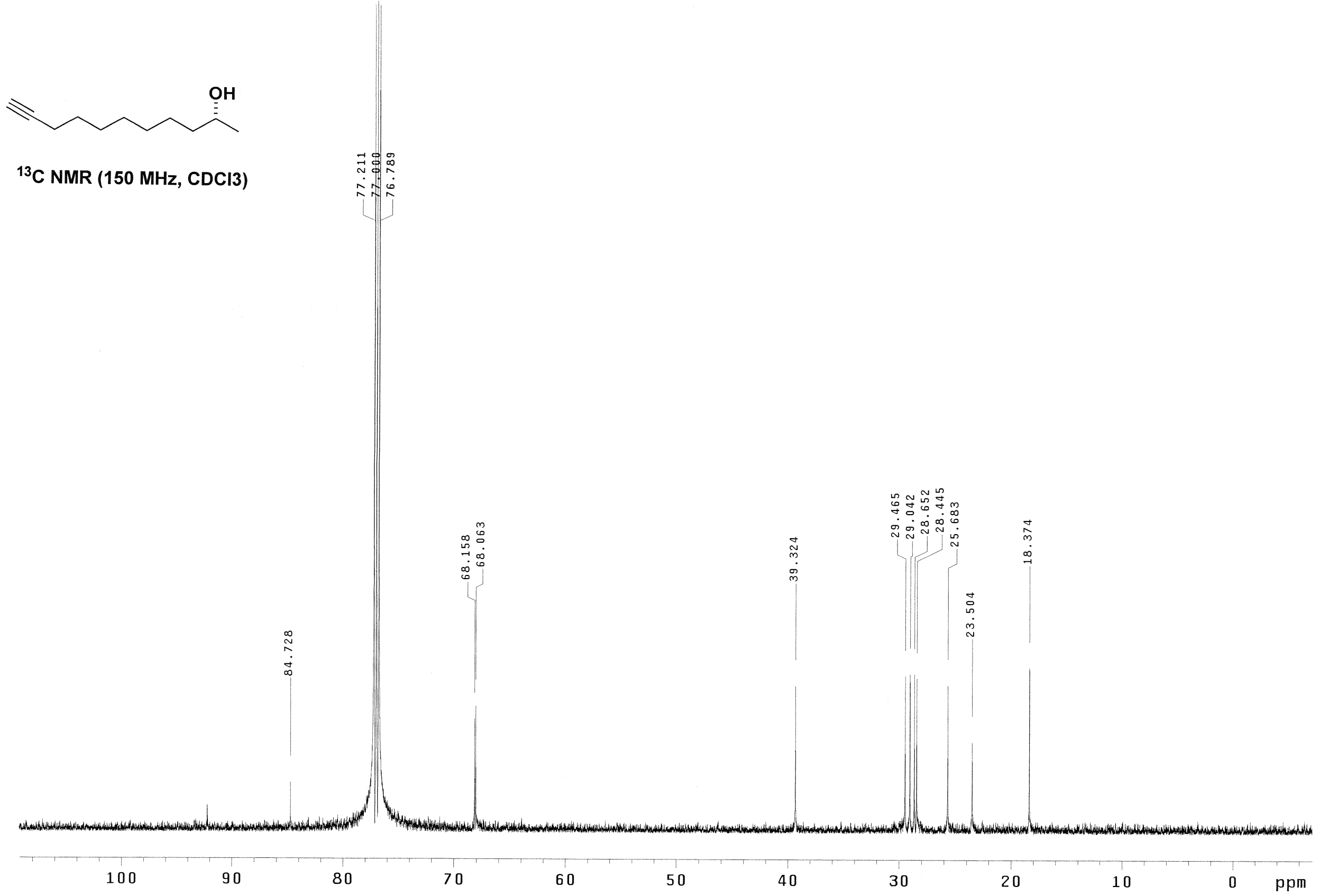




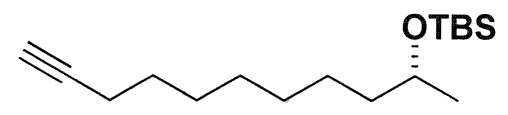

${ }^{1} \mathrm{H}$ NMR $(600 \mathrm{MHz}, \mathrm{CDCl} 3)$

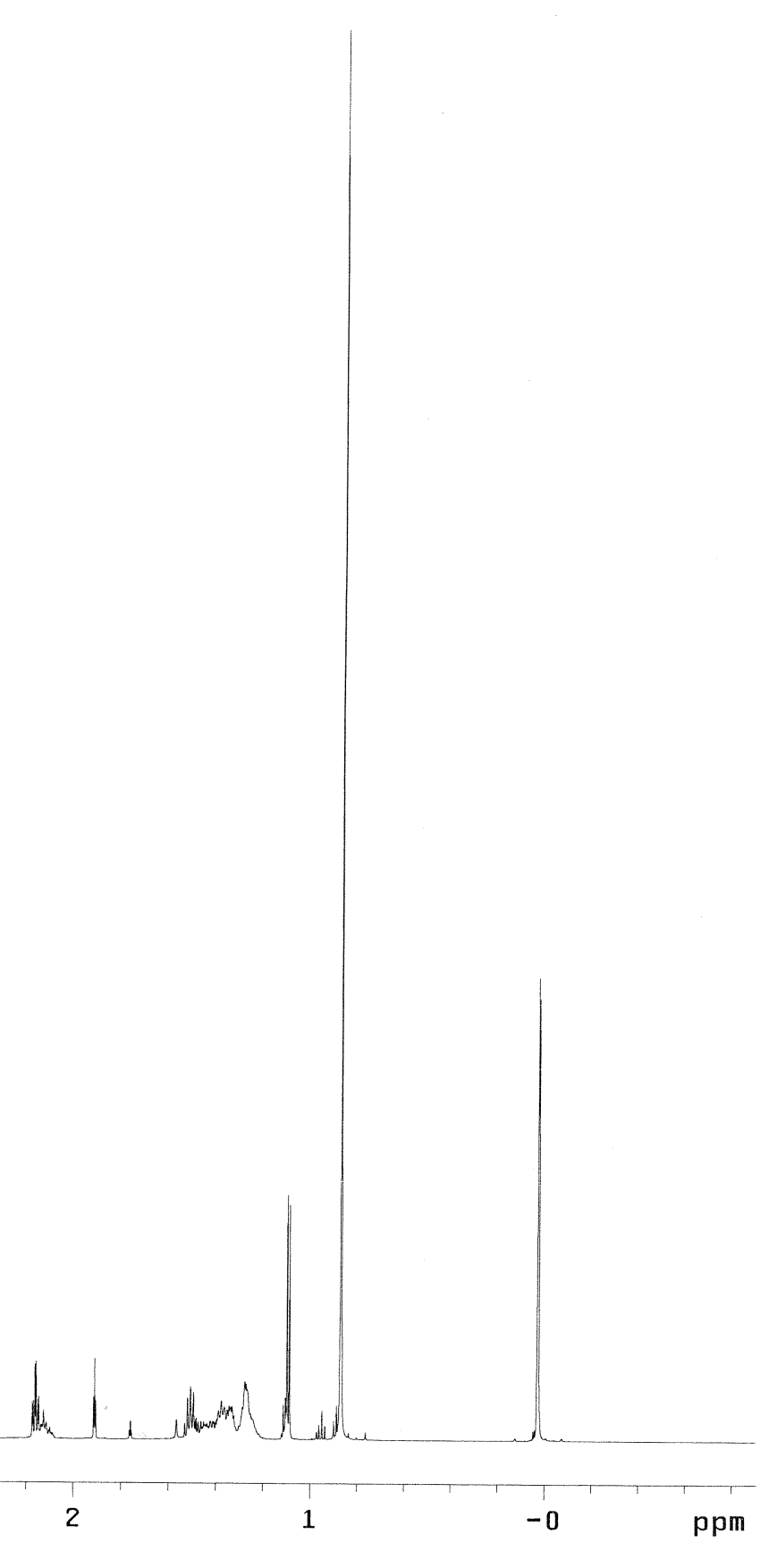




\section{OTBS}

${ }^{13} \mathrm{C}$ NMR (150 MHz, CDCI3)

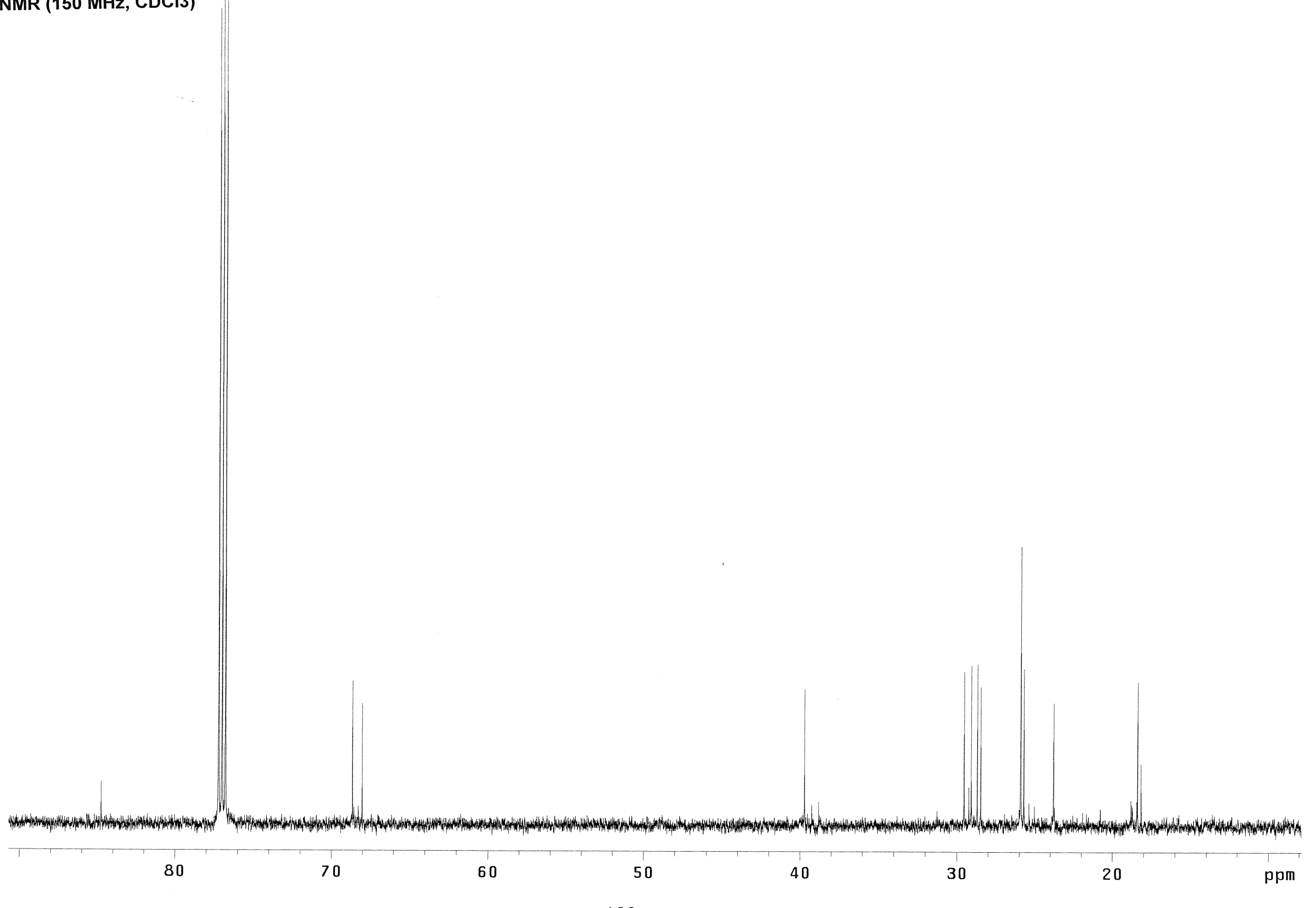




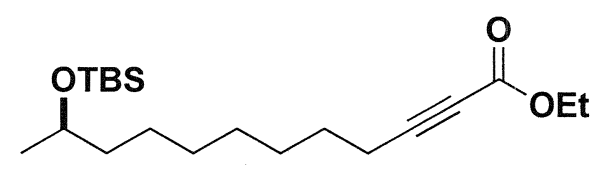

${ }^{1} \mathrm{H}$ NMR (600 MHz, CDCl3) 


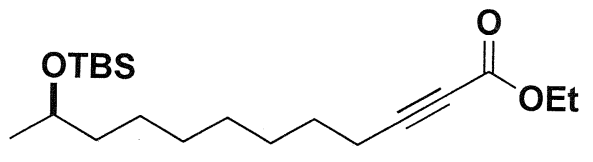

${ }^{13} \mathrm{C}$ NMR (150 MHz, CDCl3)

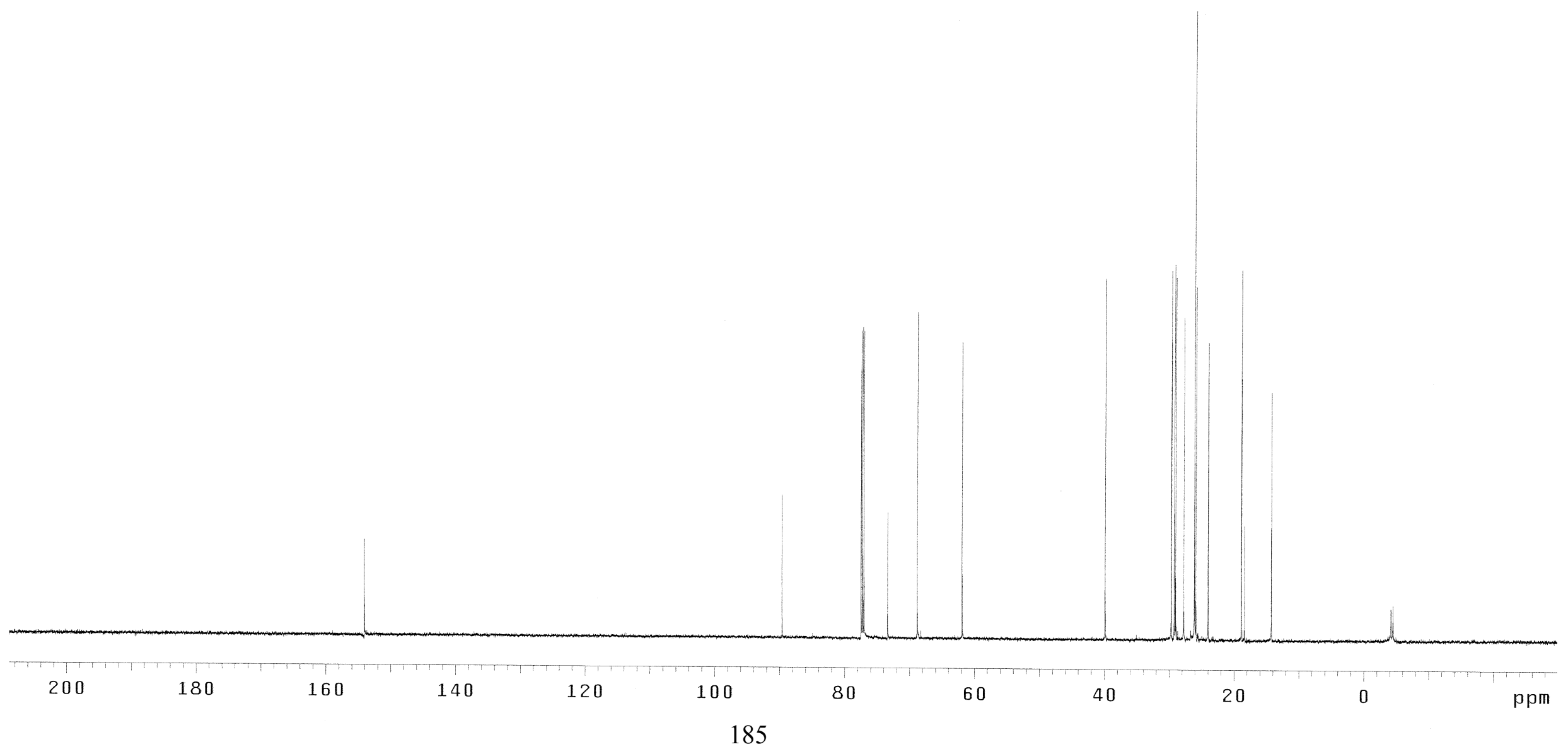




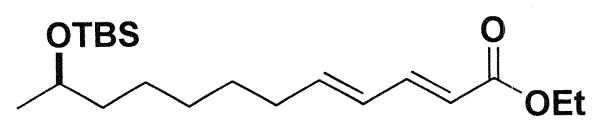

${ }^{1} \mathrm{H}$ NMR (600 MHz, CDCl3)

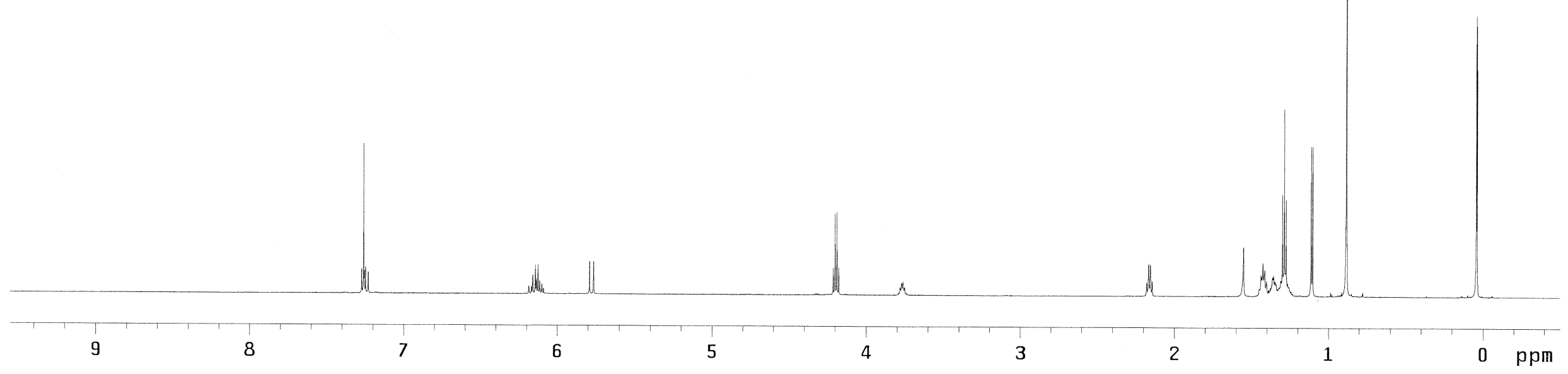




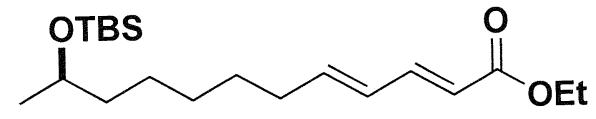

${ }^{13} \mathrm{C}$ NMR (150 MHz, CDCl3)

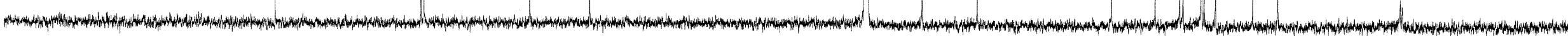

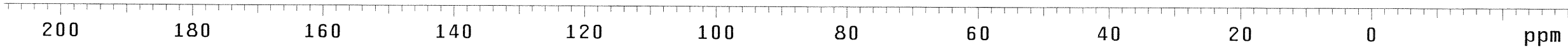




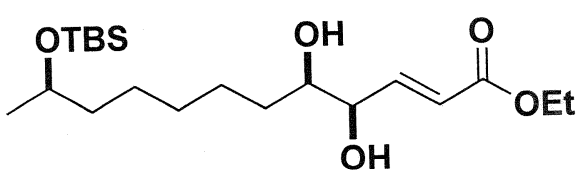

${ }^{1} \mathrm{H}$ NMR (600 MHz, CDCl3)

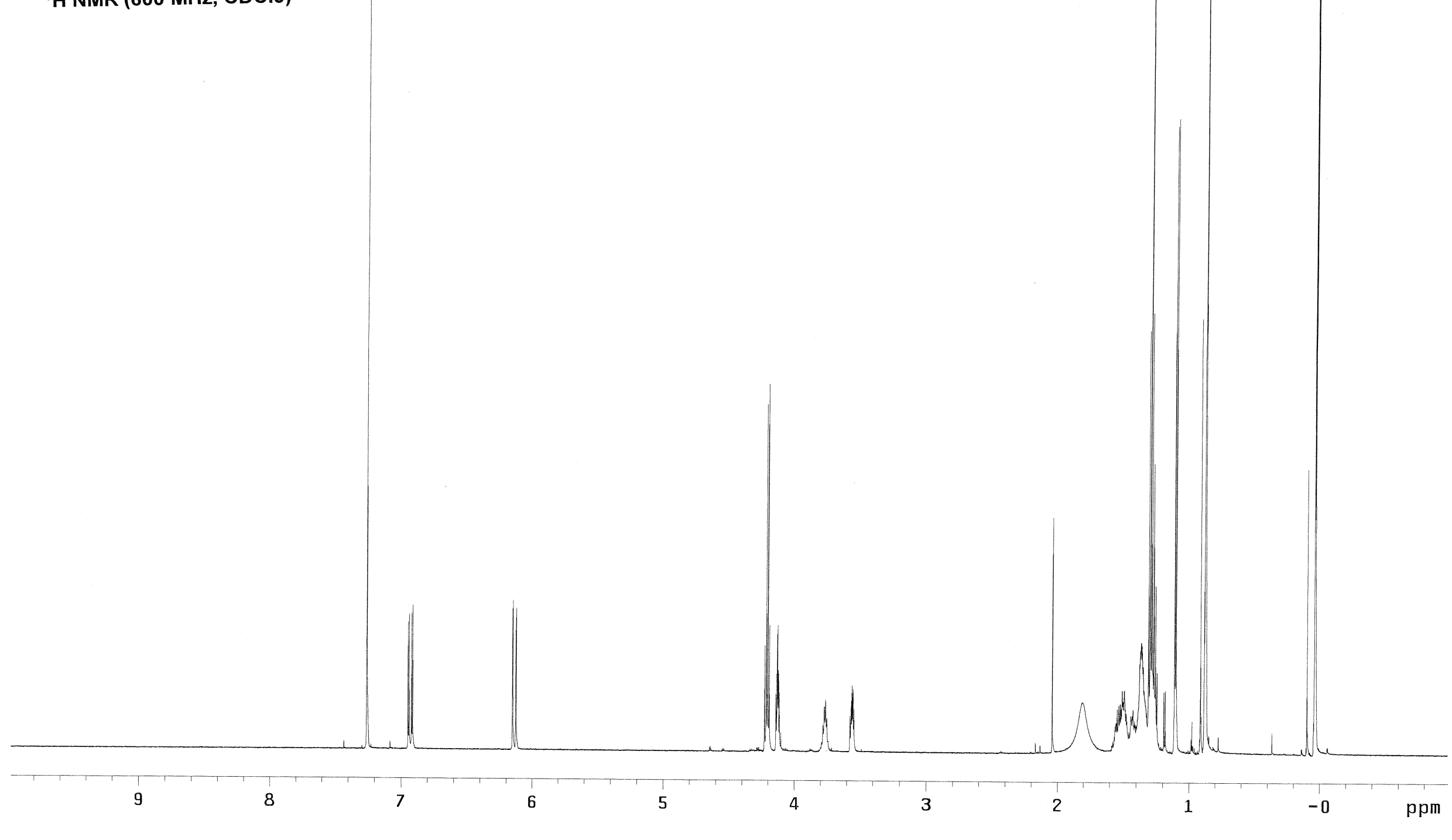




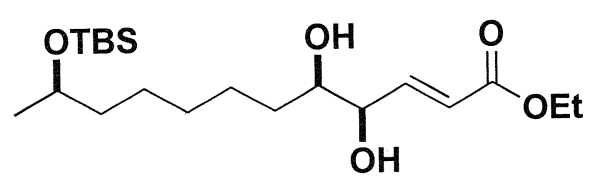

${ }^{13} \mathrm{C}$ NMR (150 MHz, CDCl3)

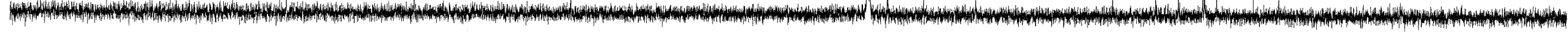

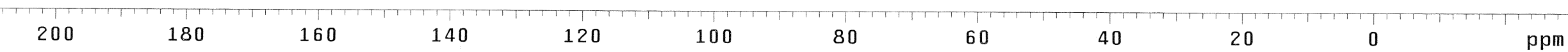




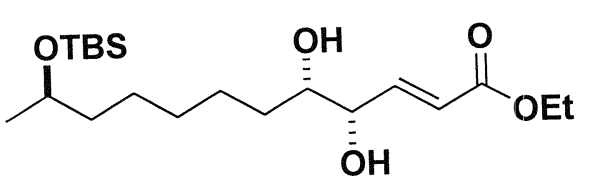

${ }^{1} \mathrm{H}$ NMR (600 MHz, CDCl3)

\section{-}




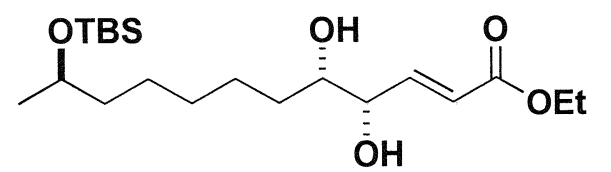

${ }^{13} \mathrm{C}$ NMR (150 MHz, CDCl3)

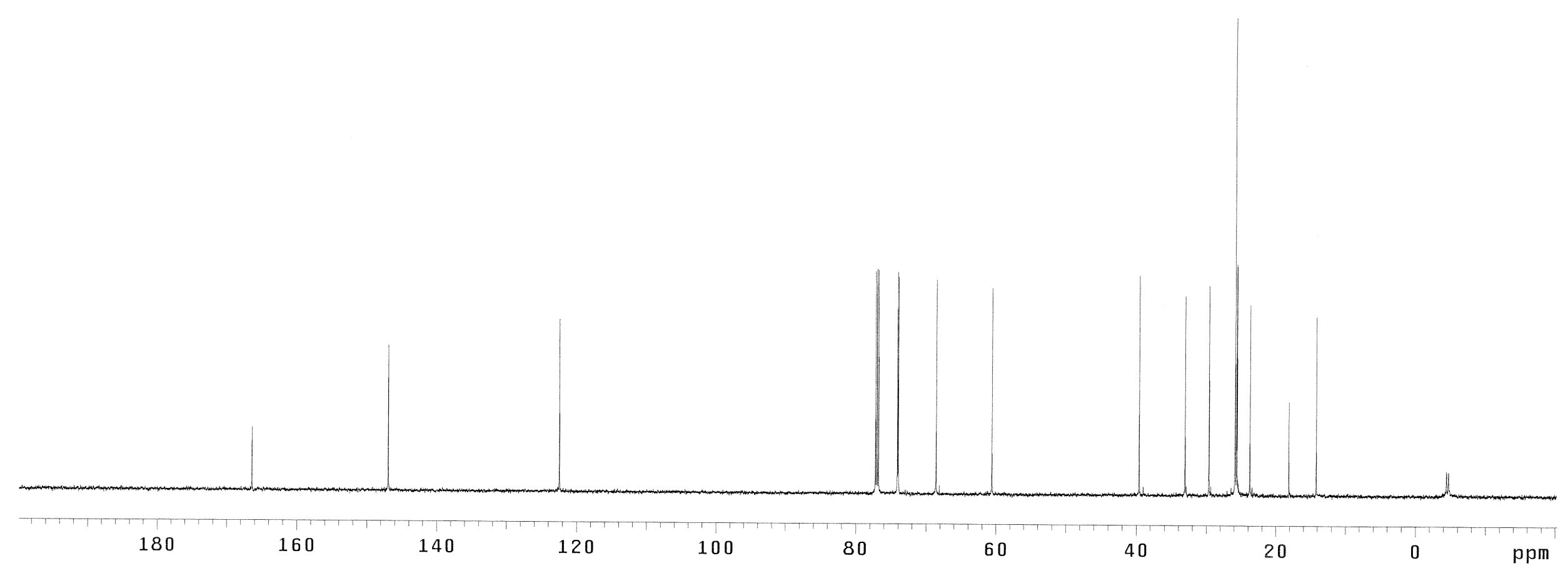




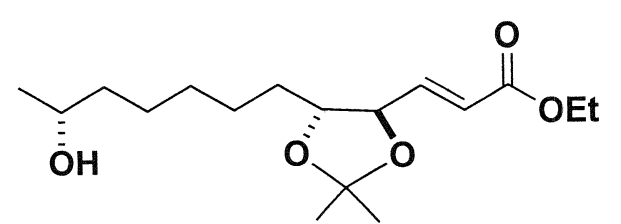

${ }^{1} \mathrm{H}$ NMR (600 MHz, CDCl3)

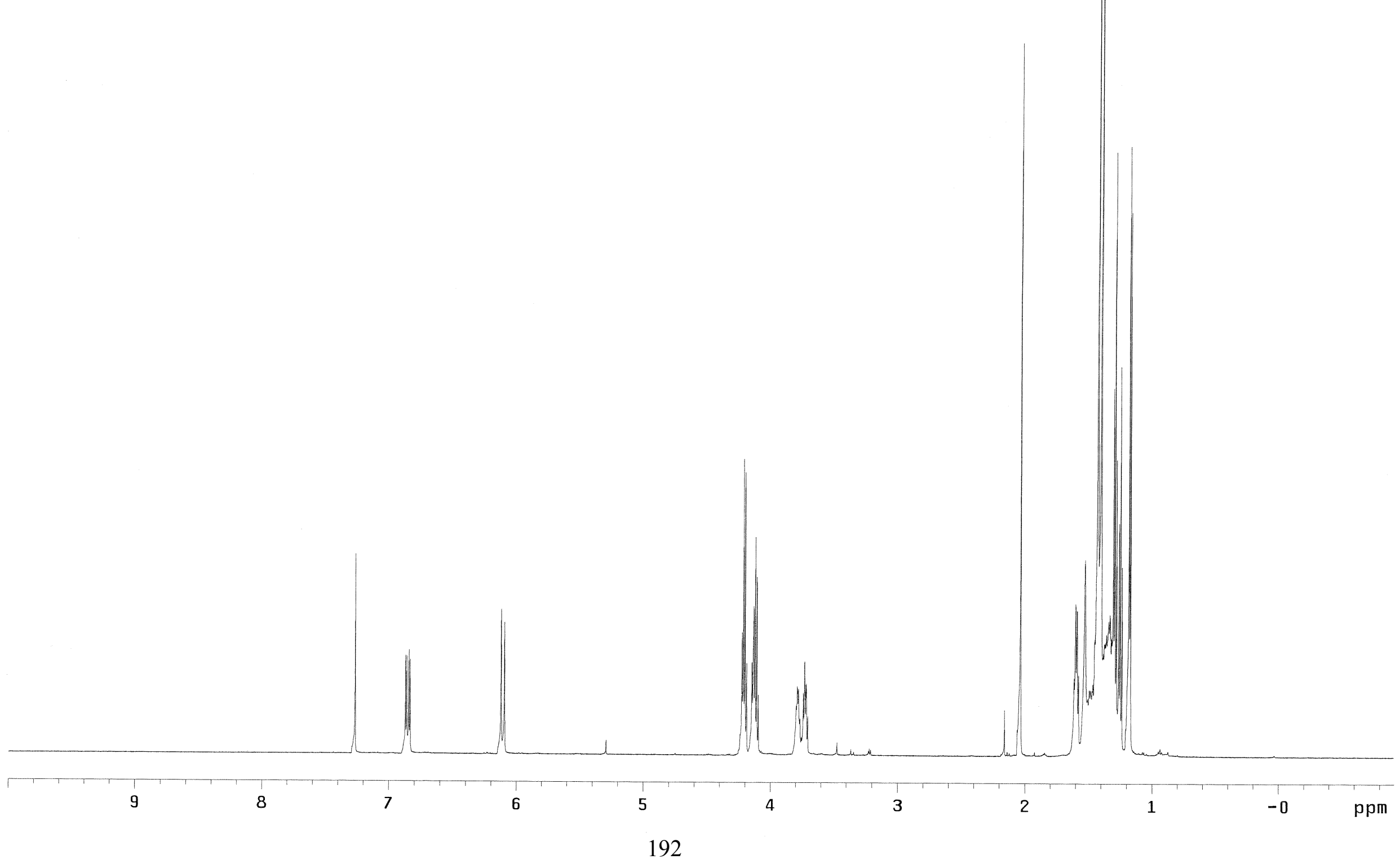




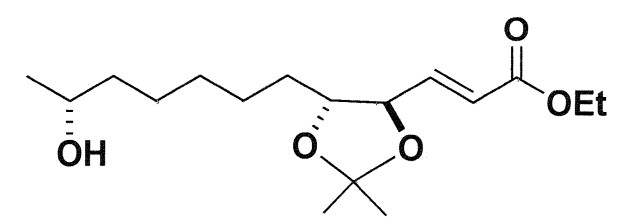

${ }^{13} \mathrm{C}$ NMR (150 MHz, CDCl3)

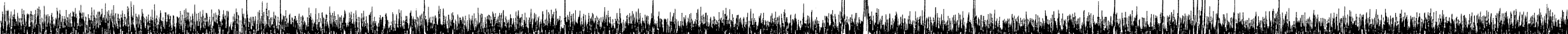
bow

160

140

120

100

80

60

40

20

0

ppm 


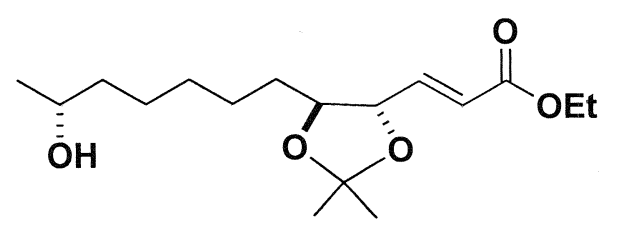

${ }^{1} \mathrm{H}$ NMR (600 MHz, CDCl3)

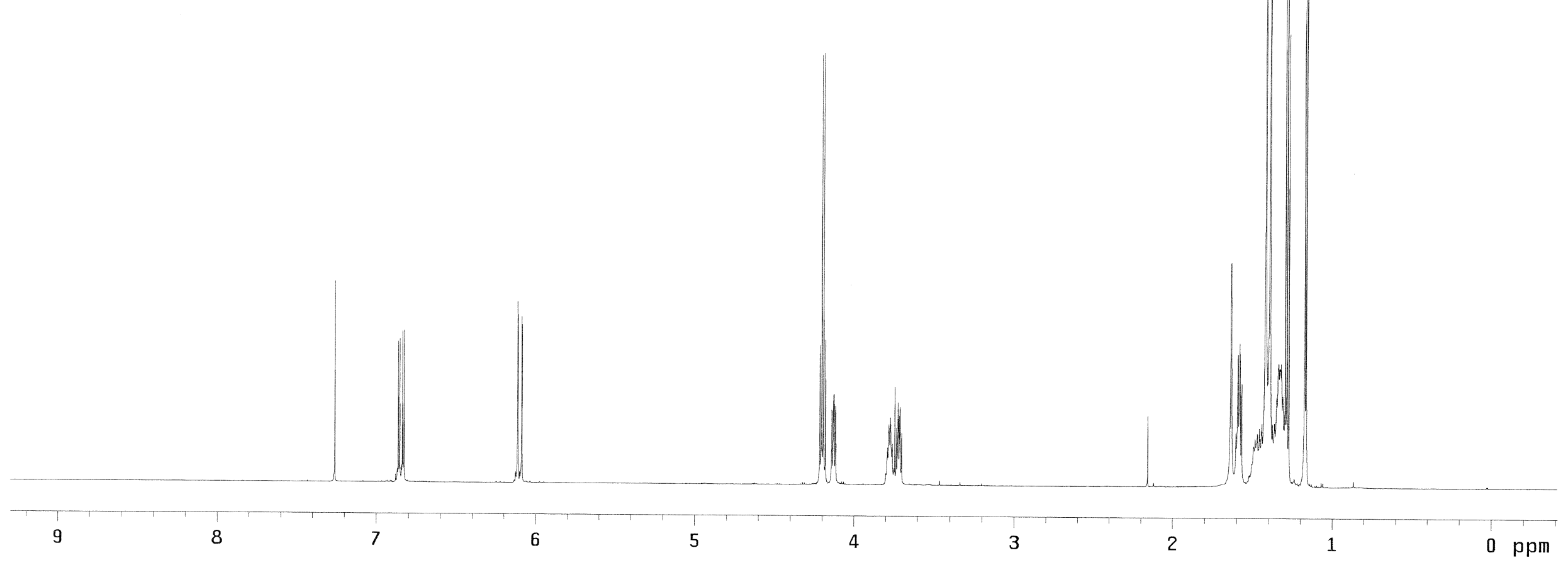




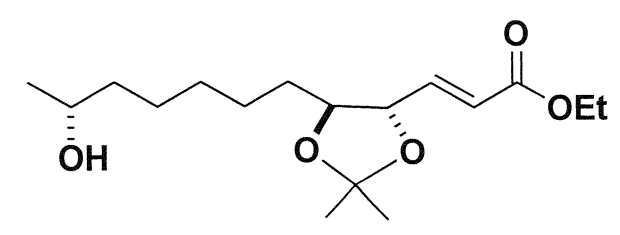

${ }^{13} \mathrm{C}$ NMR (150 MHz, CDCl3)

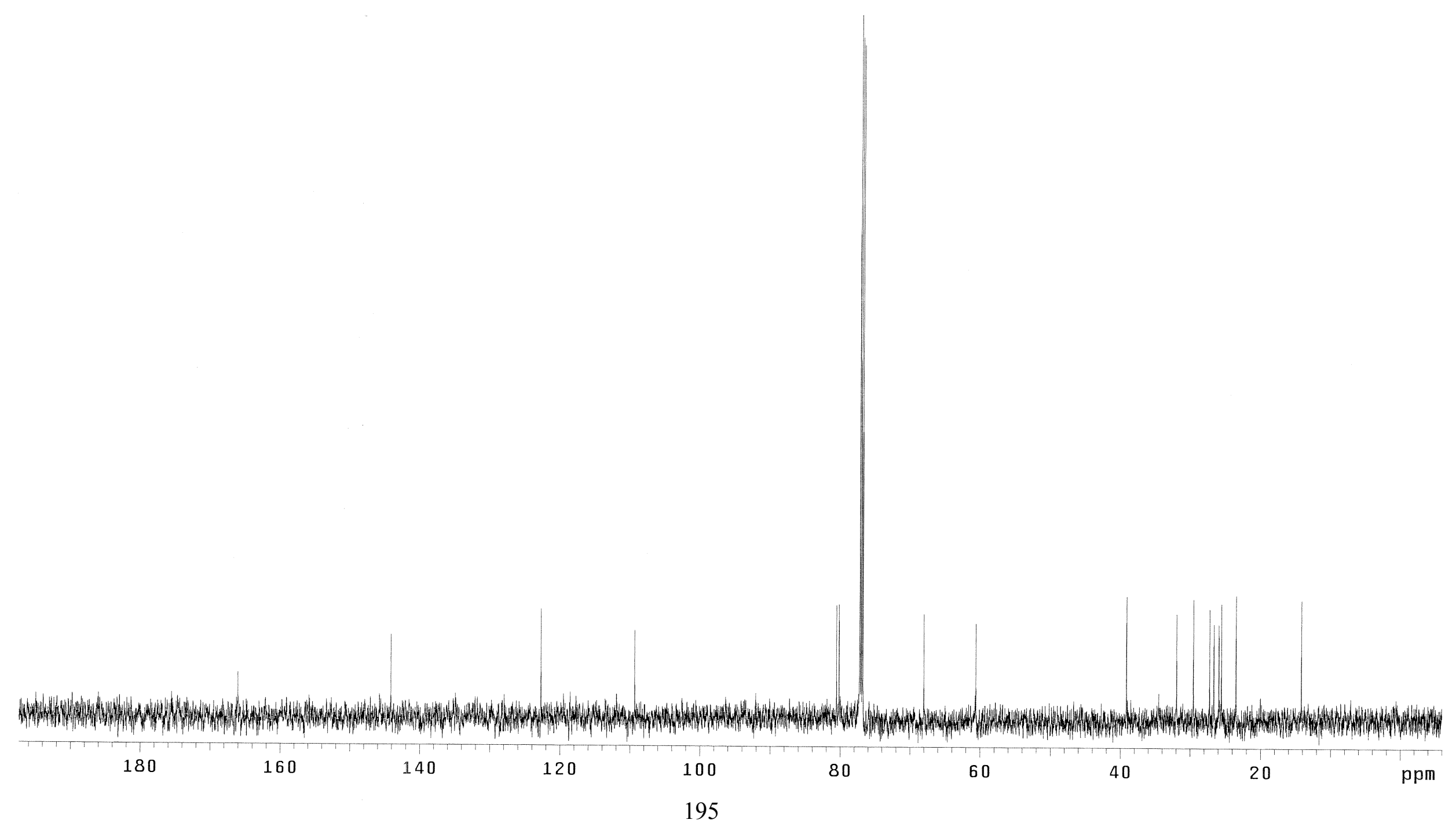




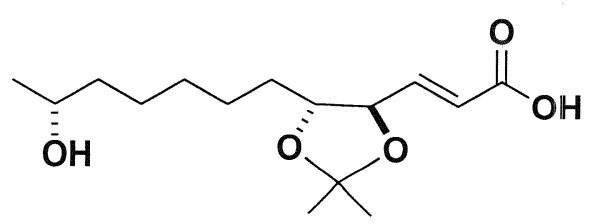

${ }^{1} \mathrm{H}$ NMR (600 MHz, CDCl3)

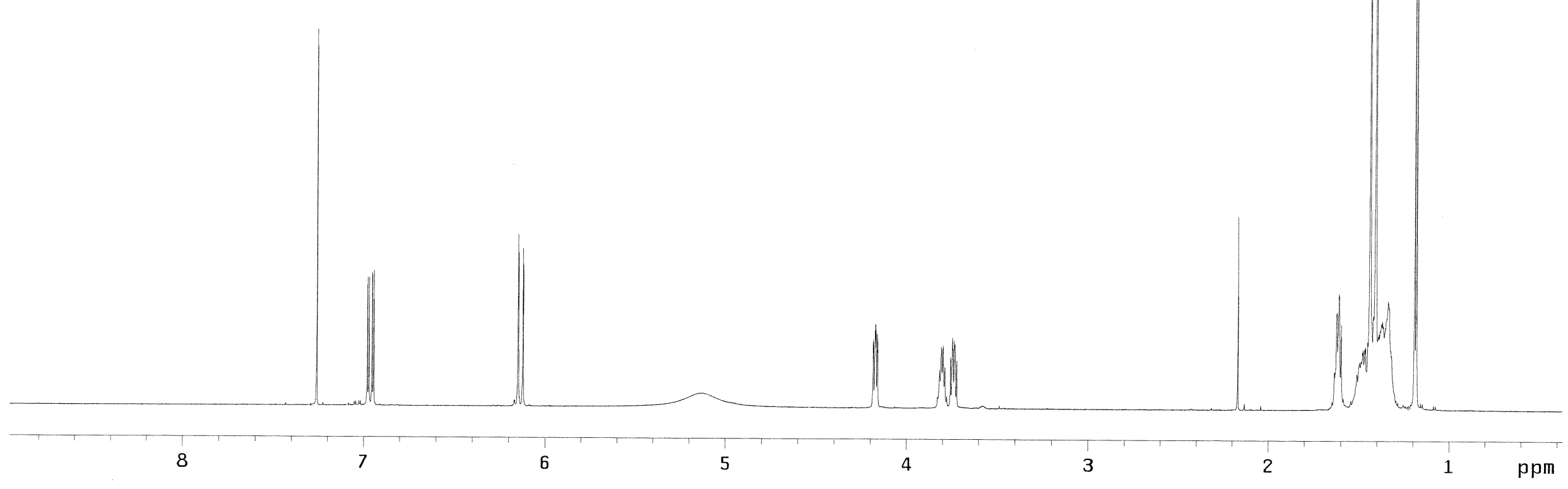




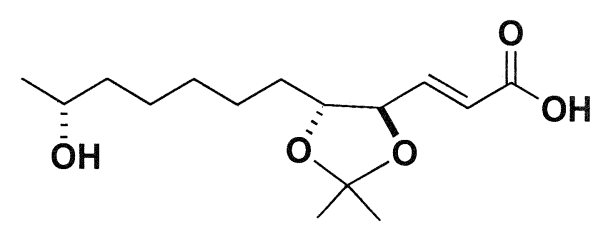

${ }^{13} \mathrm{C}$ NMR $(150 \mathrm{MHz}, \mathrm{CDCl})$

wow

\begin{tabular}{|c|c|c|c|c|c|c|c|c|c|c|}
\hline 200 & 180 & 160 & 140 & 120 & 100 & 80 & 60 & 40 & 20 & $\mathrm{ppm}$ \\
\hline
\end{tabular}




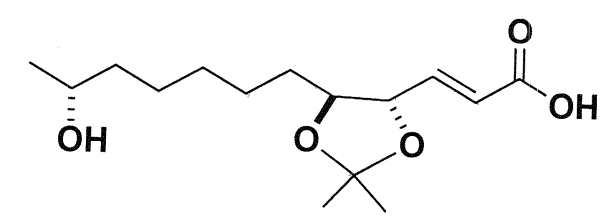

${ }^{1} \mathrm{H}$ NMR (600 MHz, CDCl3)

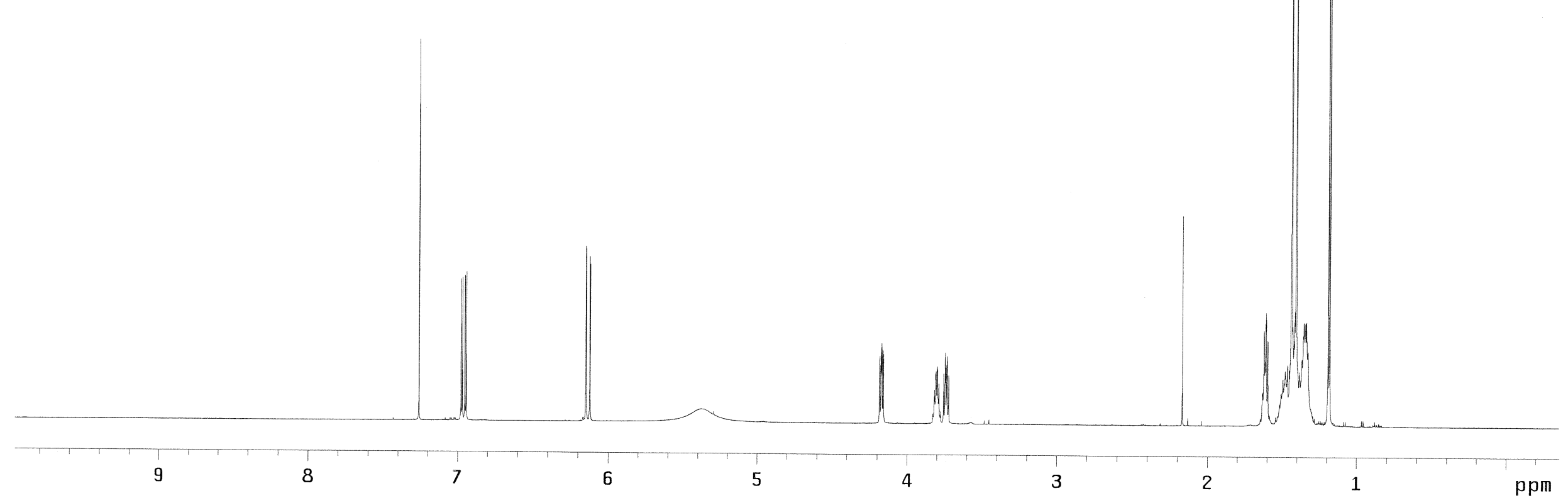




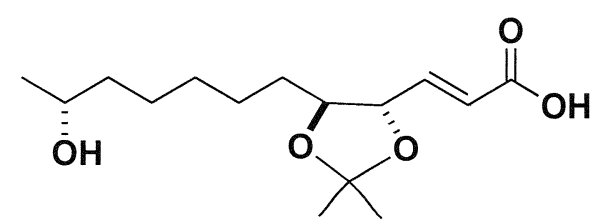

${ }^{13} \mathrm{C}$ NMR $(150 \mathrm{MHz}, \mathrm{CDCl} 3)$

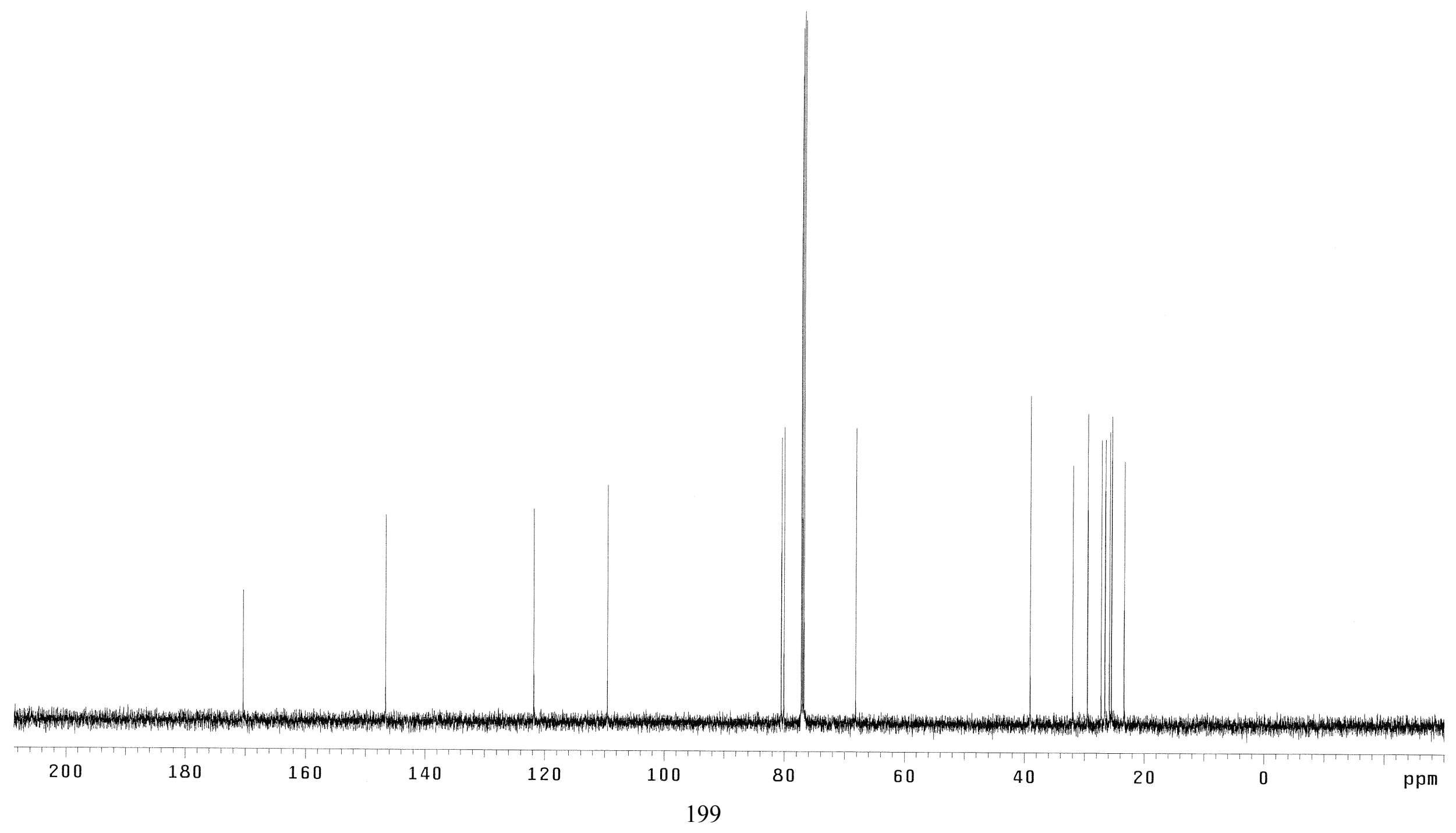




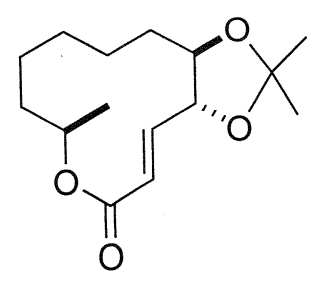

${ }^{1} \mathrm{H}$ NMR (600 MHz, CDCl3)

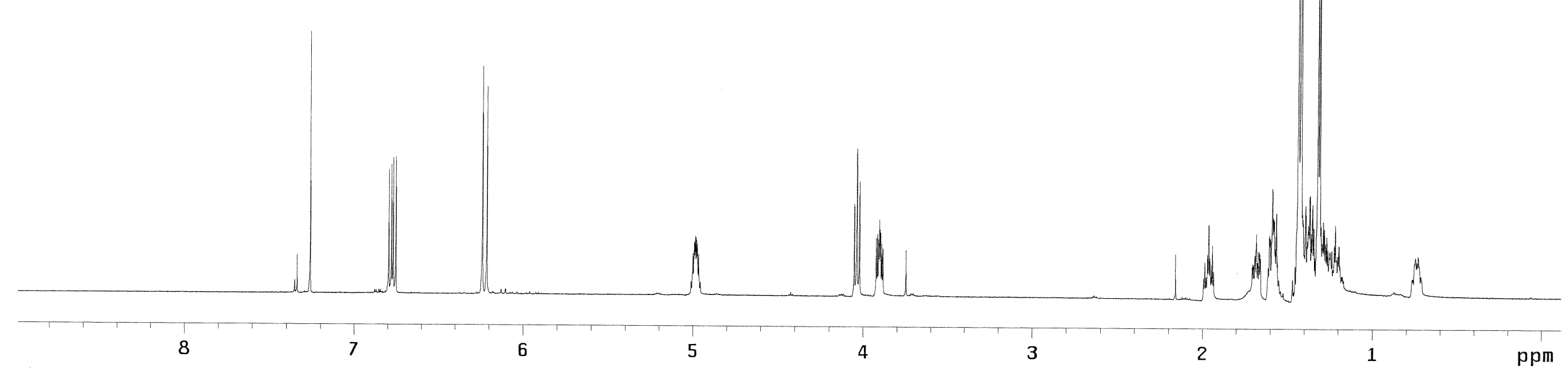




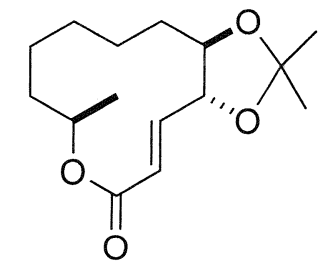

${ }^{13} \mathrm{C}$ NMR (150 MHz, CDCl3)

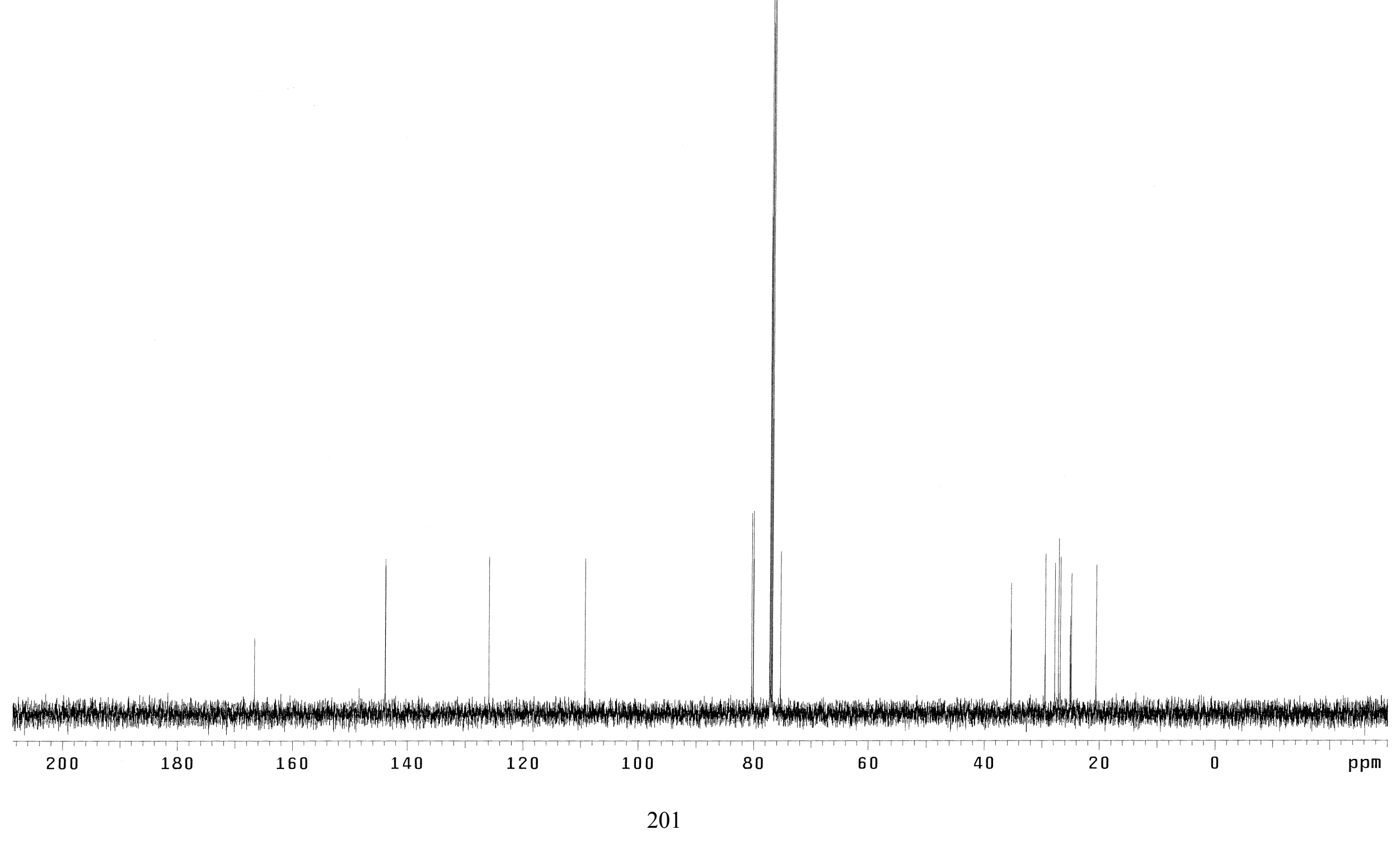




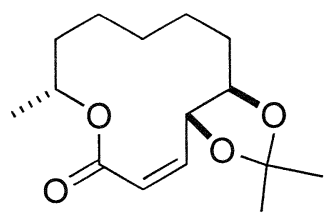

${ }^{1} \mathrm{H}$ NMR $(600 \mathrm{MHz}, \mathrm{CDCl} 3)$

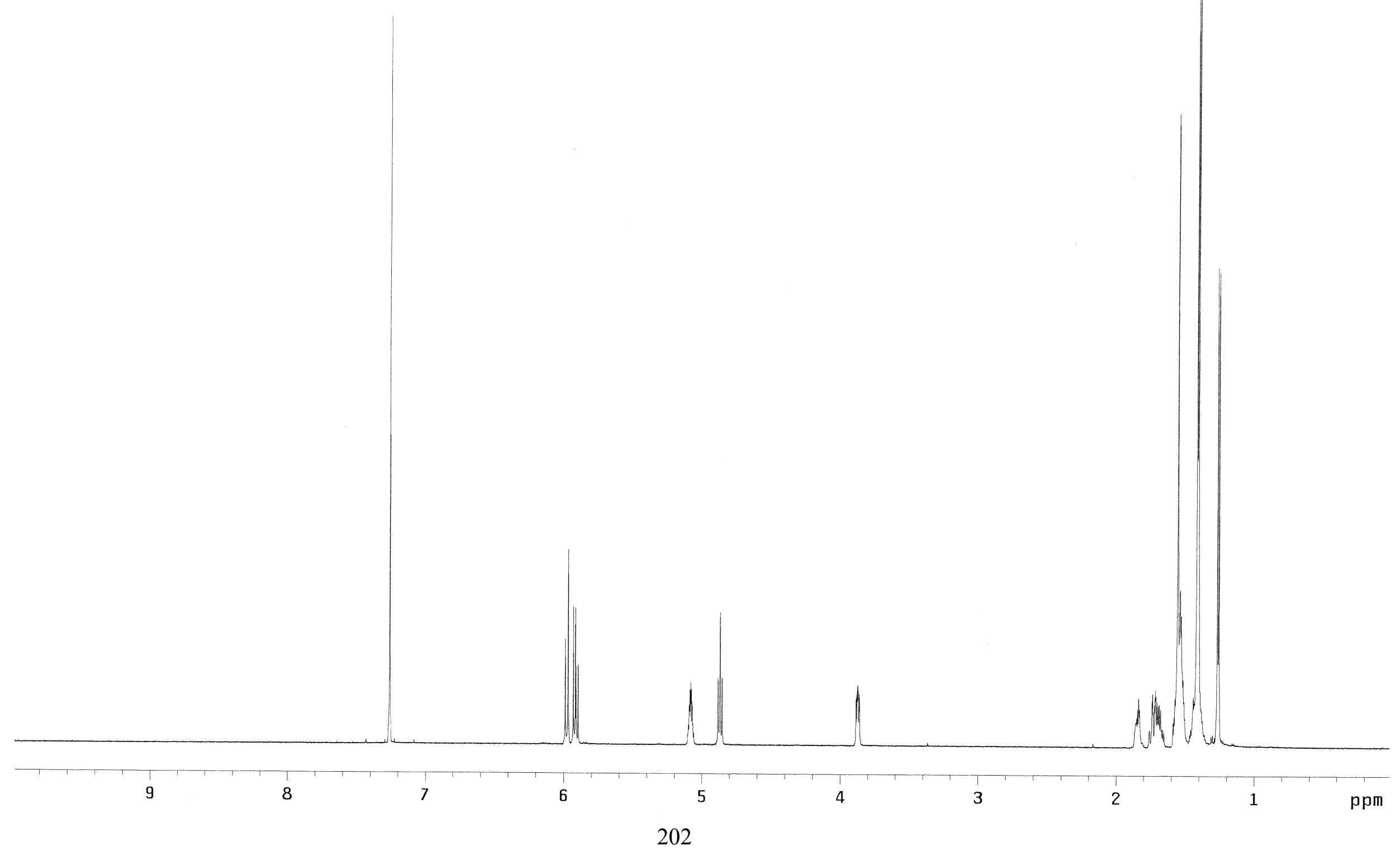




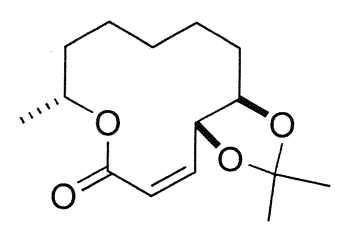

${ }^{13} \mathrm{C}$ NMR (150 MHz, CDCl3)

|

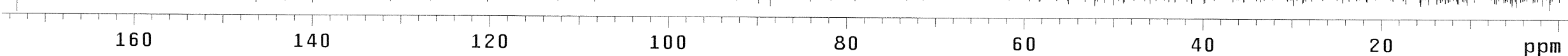




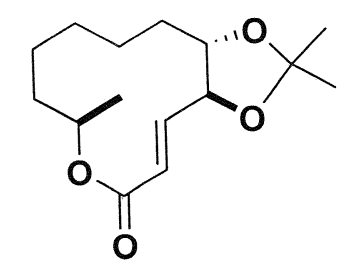

${ }^{1} \mathrm{H}$ NMR (600 MHz, CDCl3)

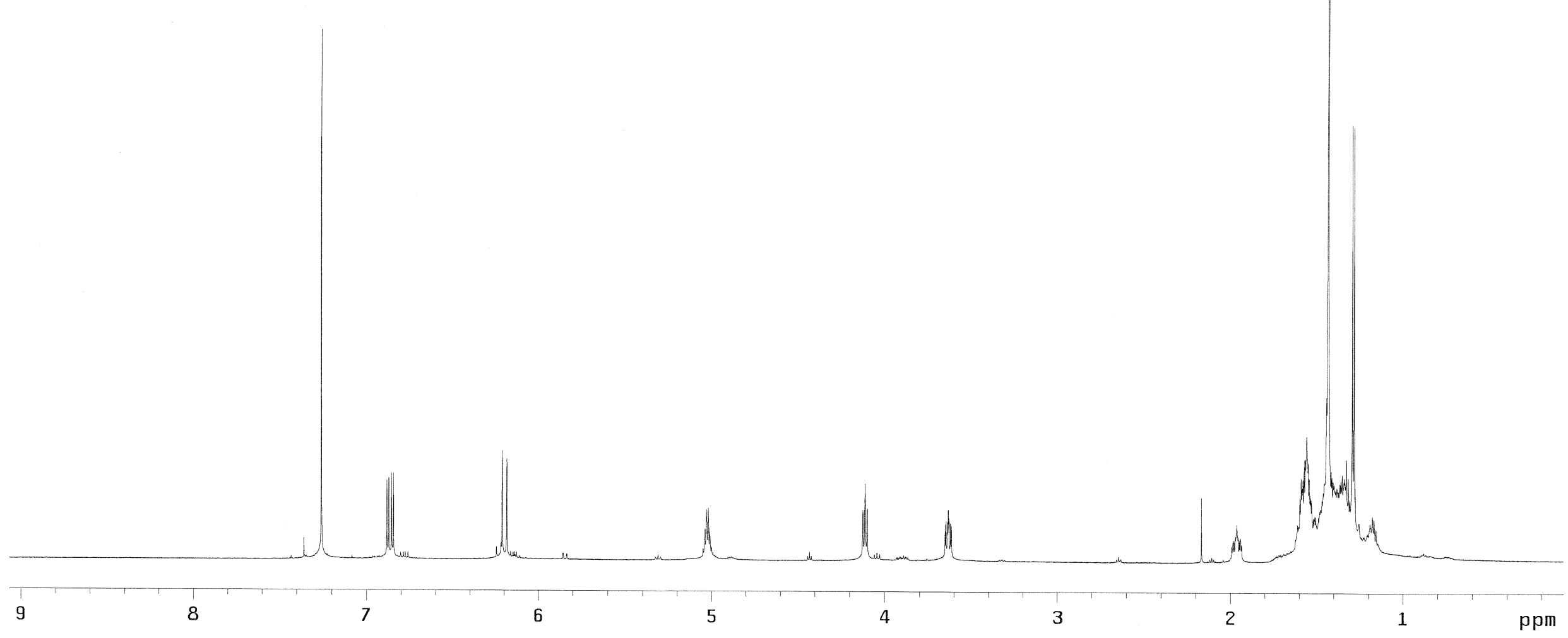




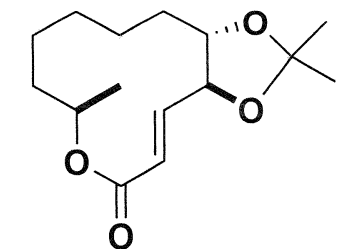

${ }^{13} \mathrm{C}$ NMR (150 MHz, CDCl3)

W 


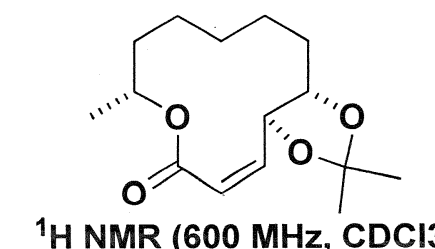

${ }^{1} \mathrm{H}$ NMR (600 MHz, CDCl3)

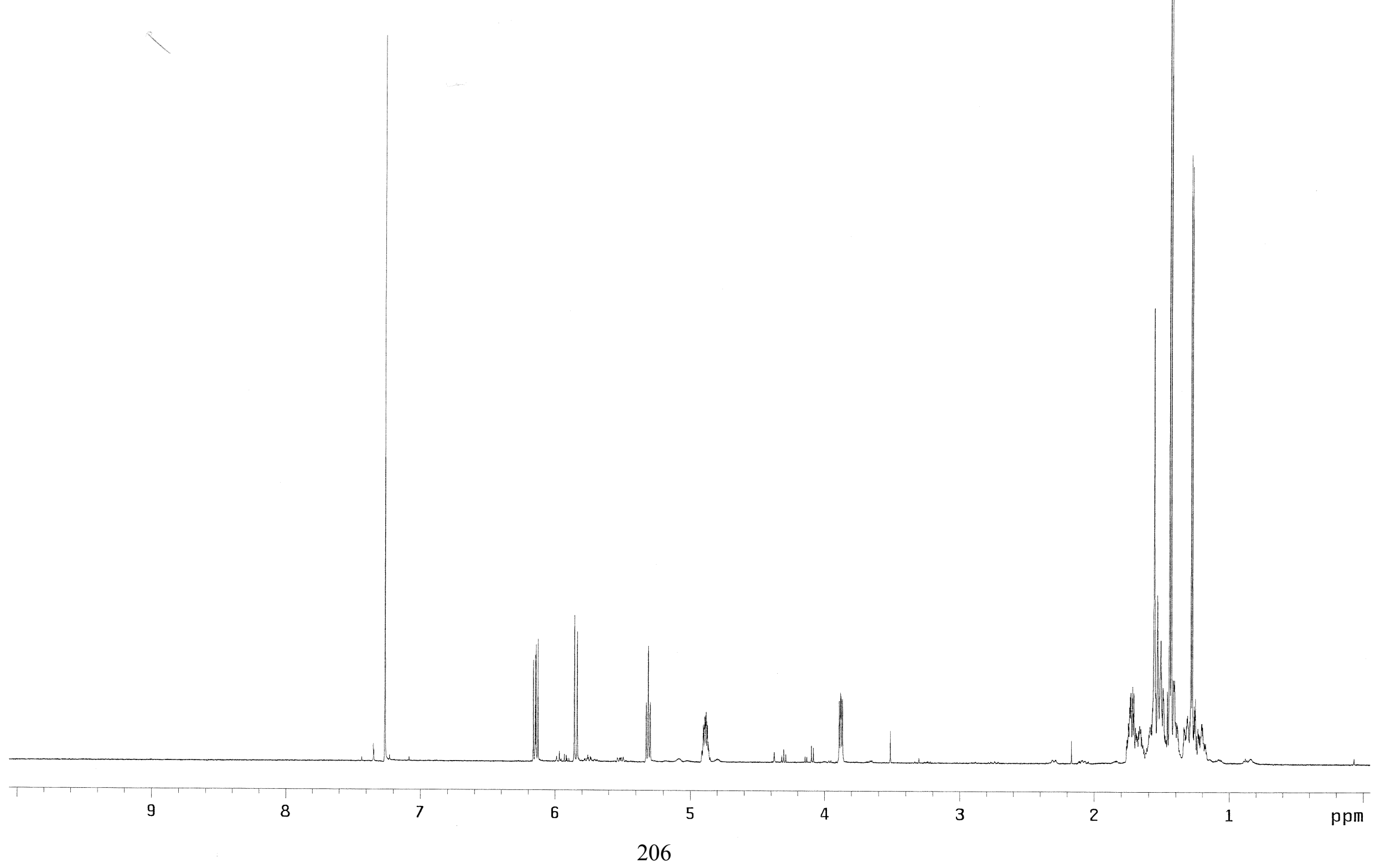




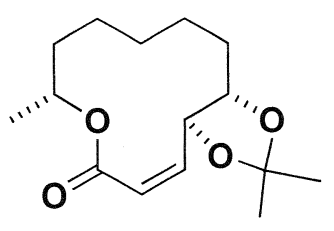

${ }^{13} \mathrm{C}$ NMR (150 MHz, CDCl3)

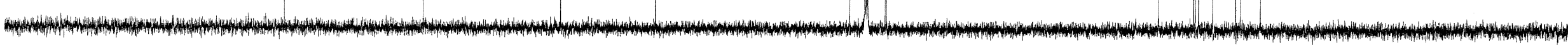

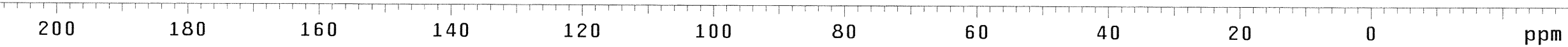




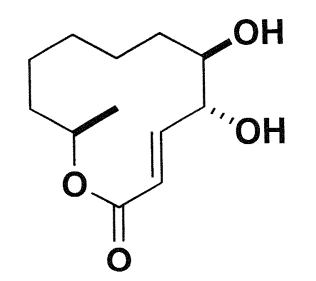

${ }^{1} \mathrm{H}$ NMR (600 MHz, CDCl3)

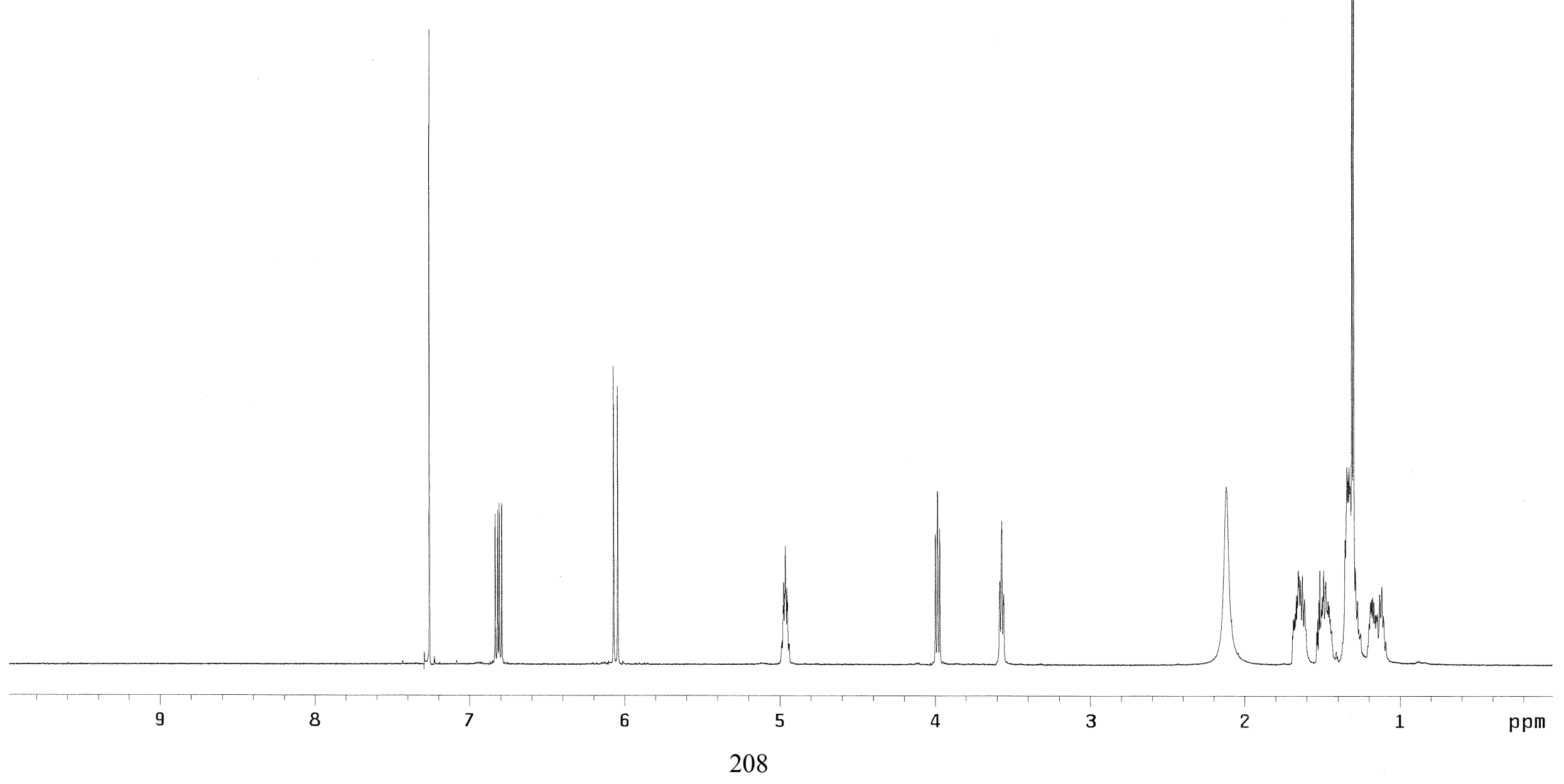




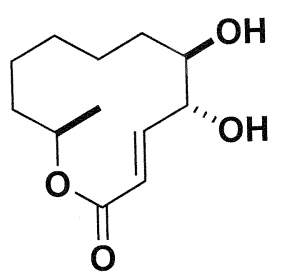

${ }^{13} \mathrm{C}$ NMR (150 MHz, CDCl3)

12tw

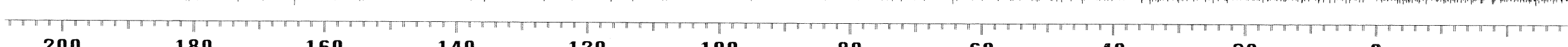




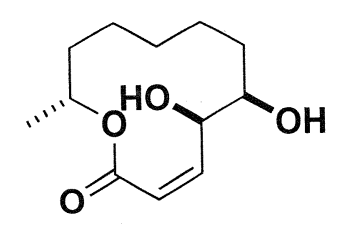

${ }^{1} \mathrm{H}$ NMR (600 MHz, CDCl3)

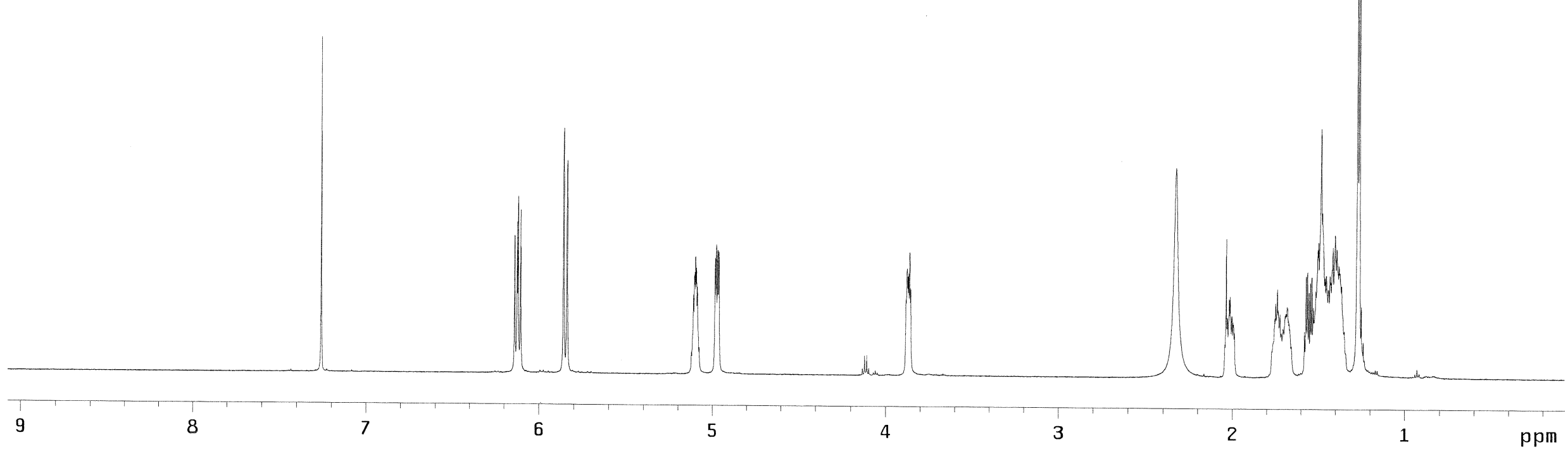




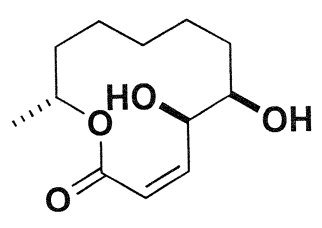

${ }^{13} \mathrm{C}$ NMR (150 MHz, CDCl3)

14tw 


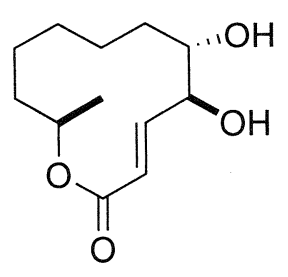

${ }^{1} \mathrm{H}$ NMR (600 MHz, CDCl3)

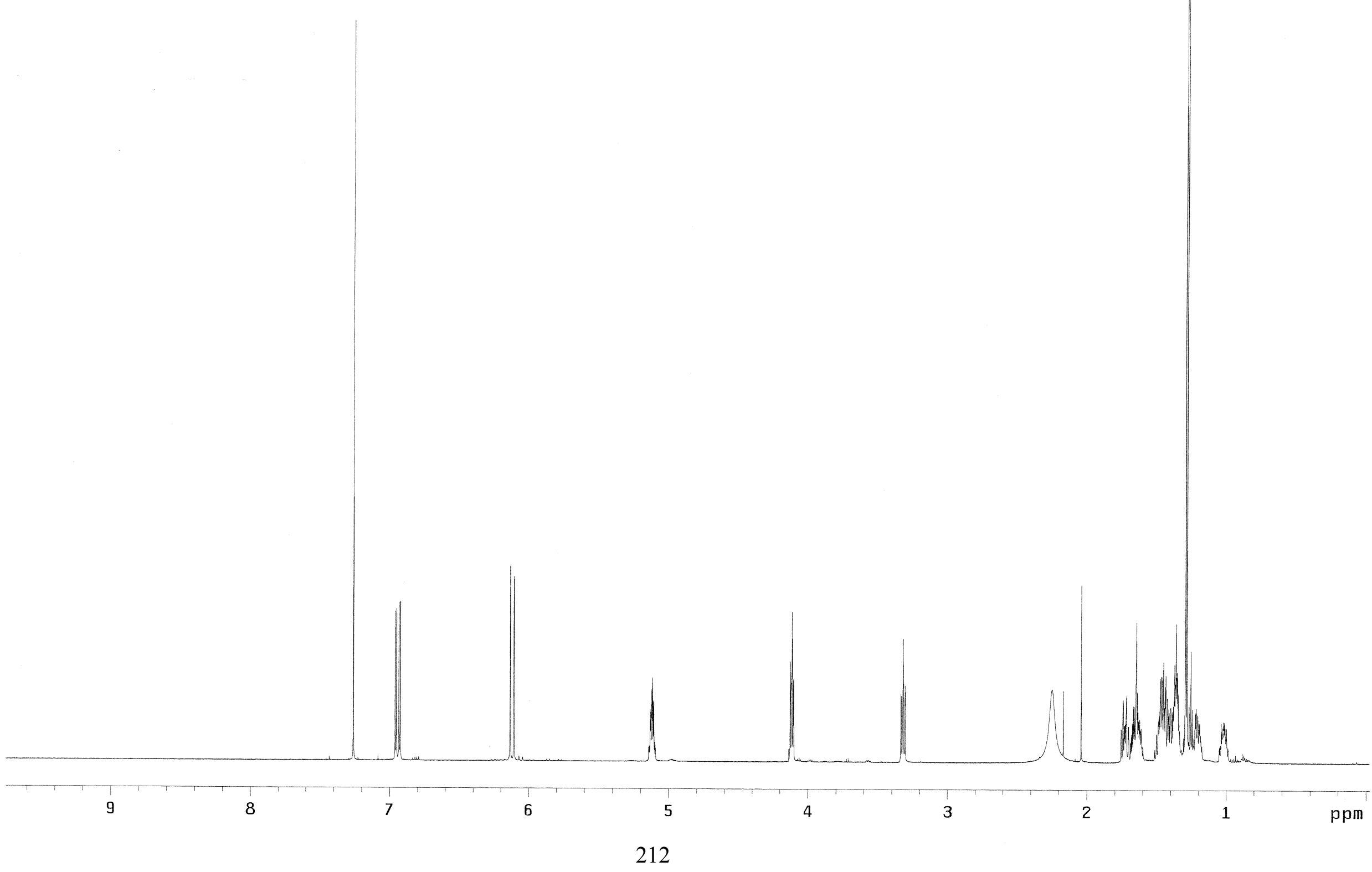




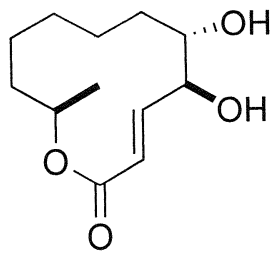

${ }^{13} \mathrm{C}$ NMR $(150 \mathrm{MHz}, \mathrm{CDCl} 3)$

W 


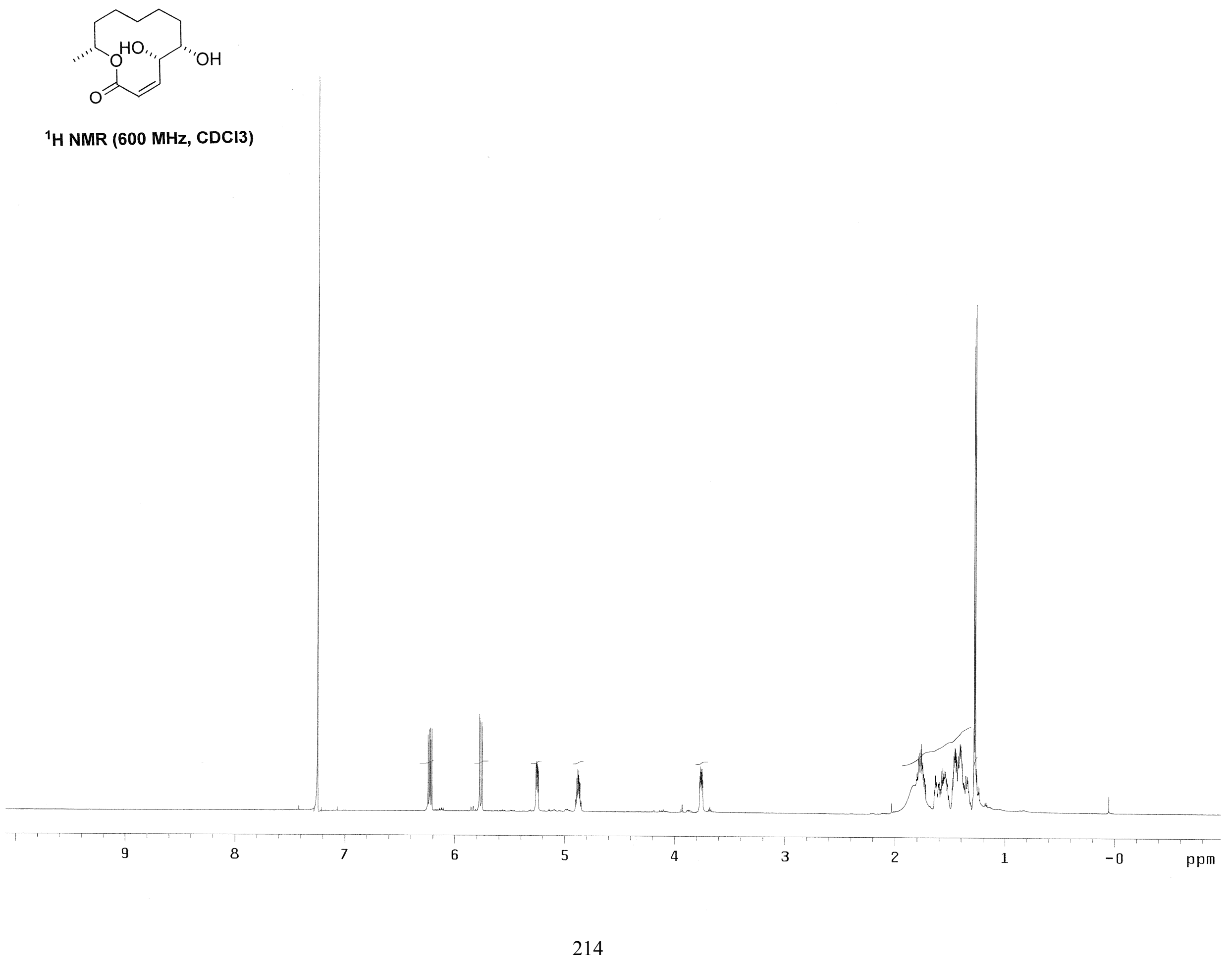




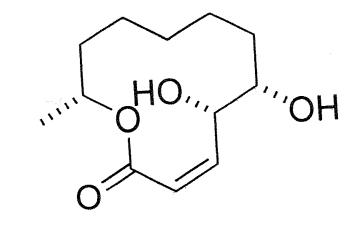

${ }^{13} \mathrm{C}$ NMR (150 MHz, CDCl3)

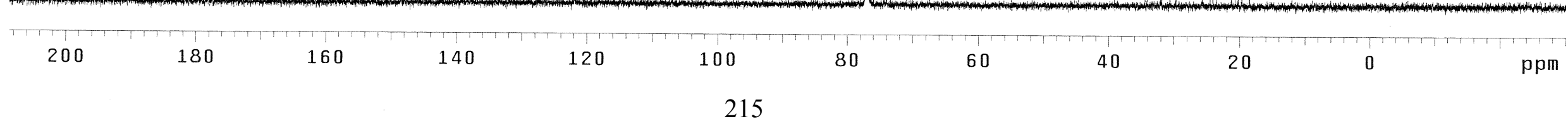




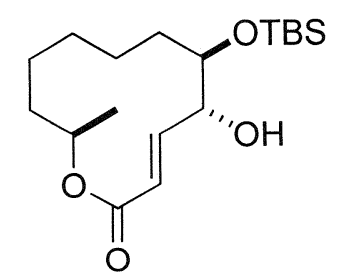

${ }^{1} \mathrm{H}$ NMR (600 MHz, CDCl3)

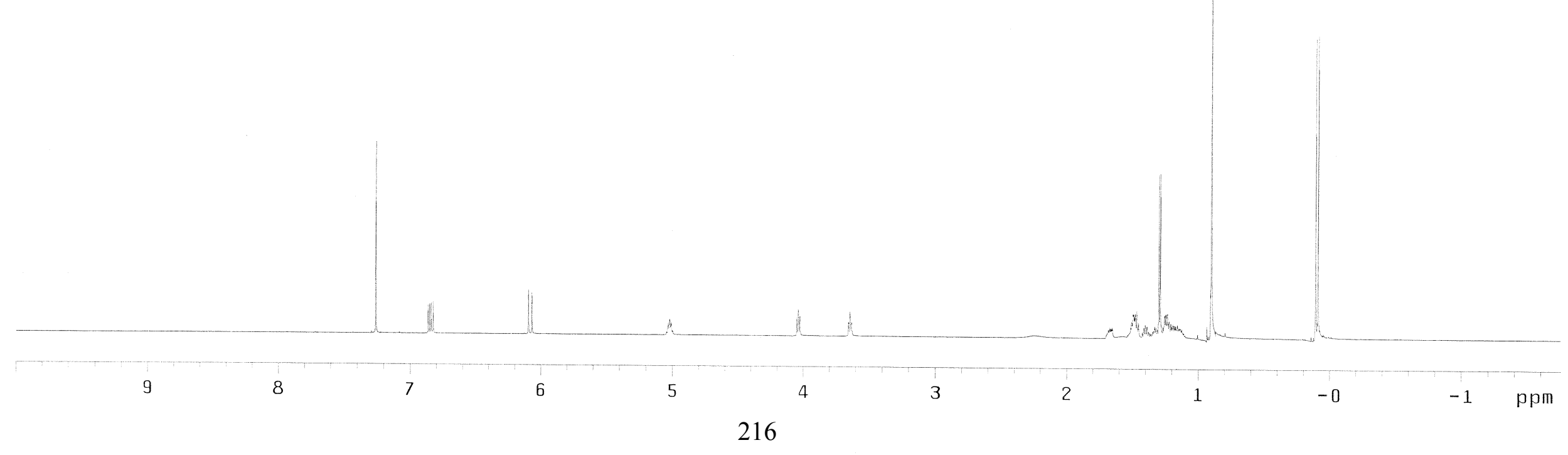




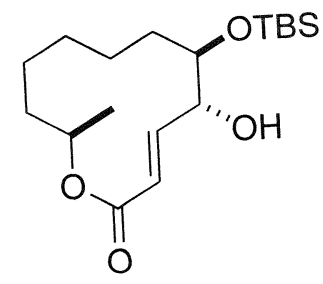

${ }^{13} \mathrm{C}$ NMR (150 MHz, CDCl3)

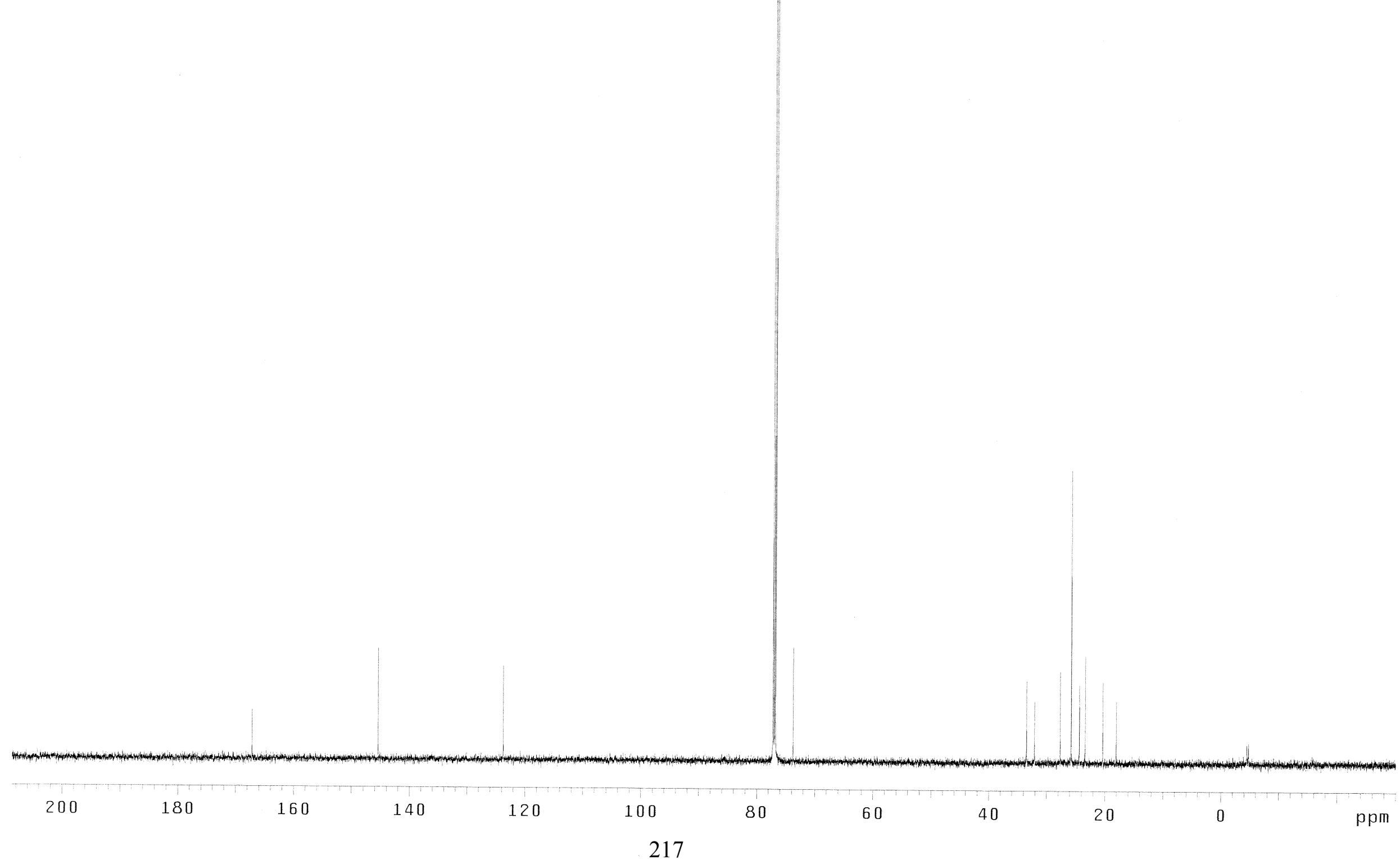




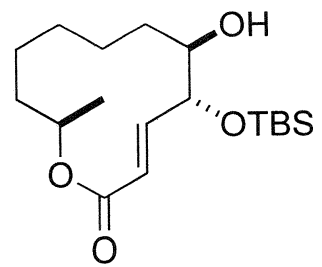

${ }^{1} \mathrm{H}$ NMR (600 MHz, CDCl3) 


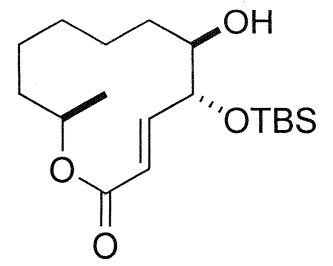

${ }^{13} \mathrm{C}$ NMR (150 MHz, CDCl3)

How

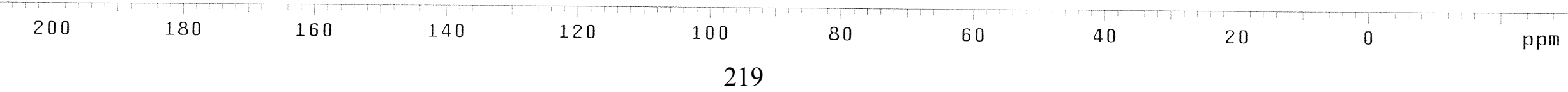




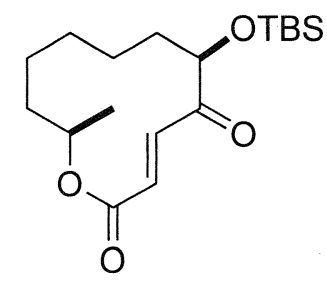

${ }^{1} \mathrm{H}$ NMR (600 MHz, CDCl3)

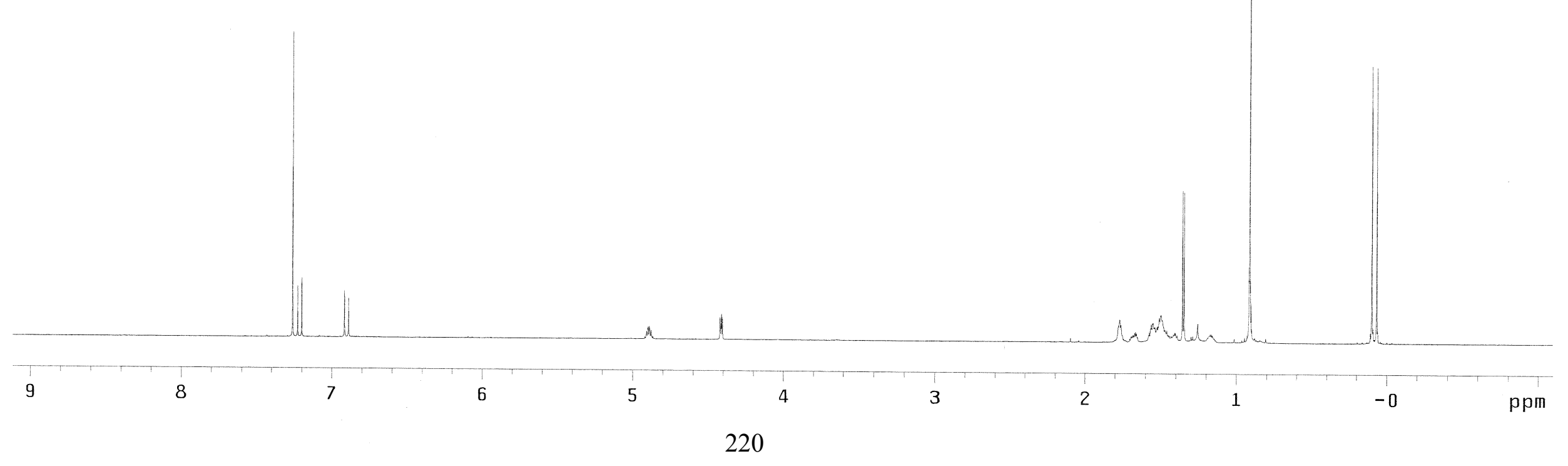




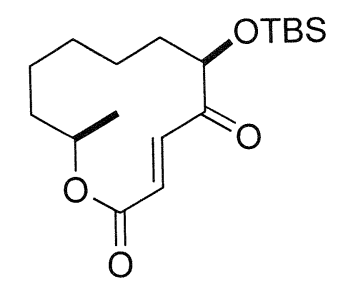

${ }^{13} \mathrm{C}$ NMR (150 MHz, CDCl3)

H.

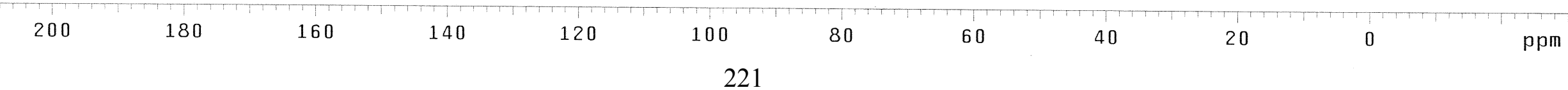




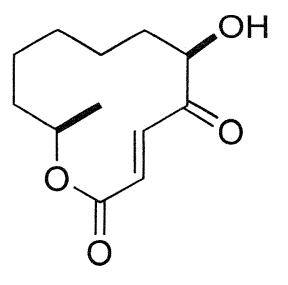

${ }^{1} \mathrm{H}$ NMR (600 MHz, CDCl3)

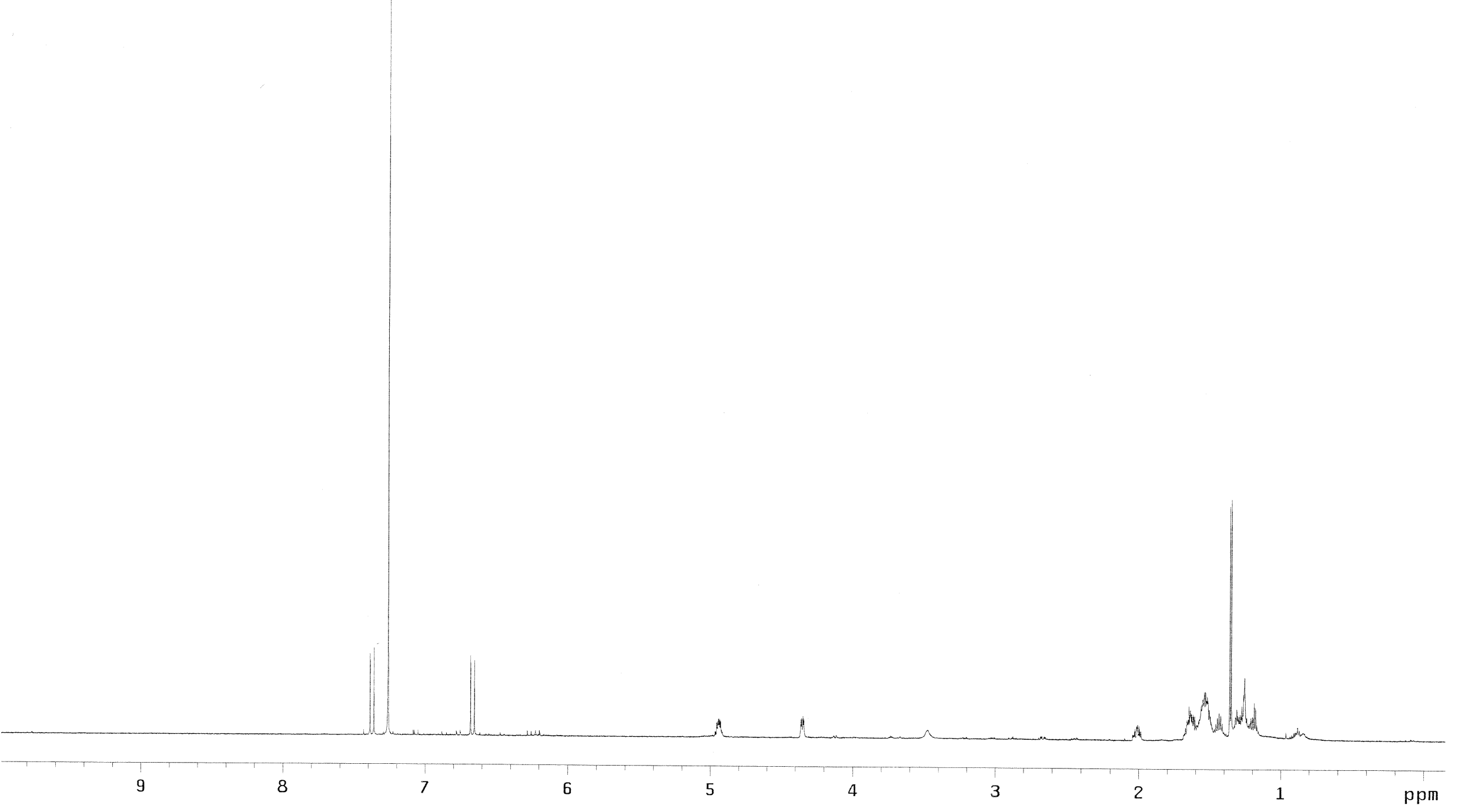




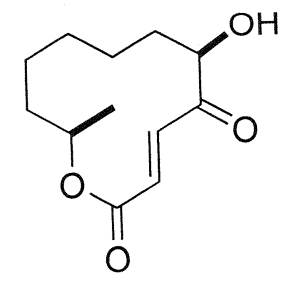

${ }^{13} \mathrm{C}$ NMR (150 MHz, CDCl3)

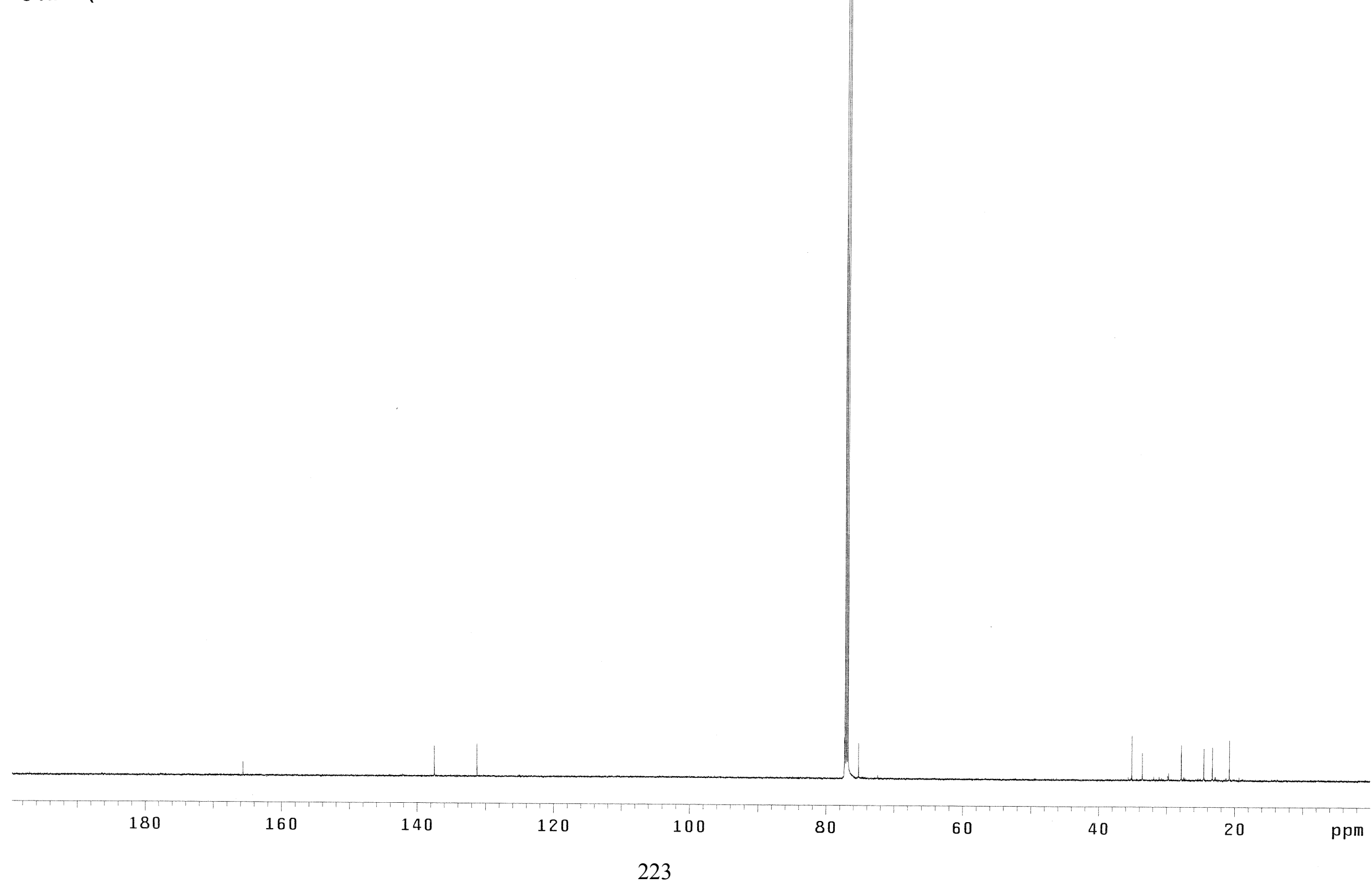




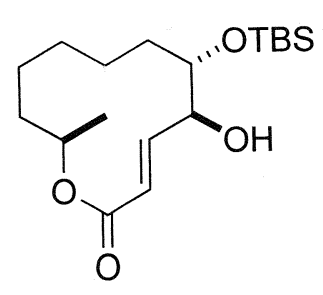

${ }^{1} \mathrm{H}$ NMR $(600 \mathrm{MHz}, \mathrm{CDCl} 3)$

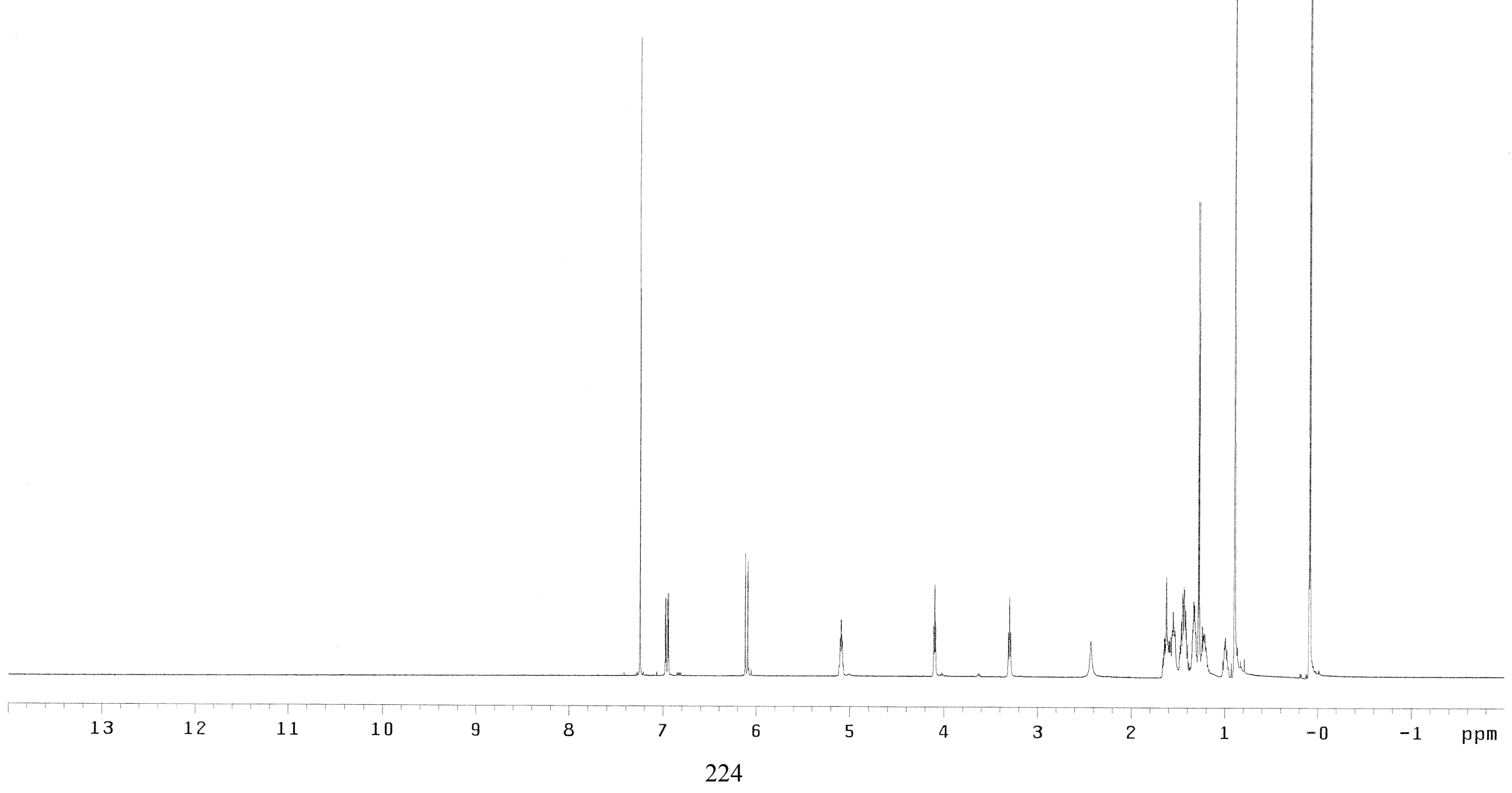




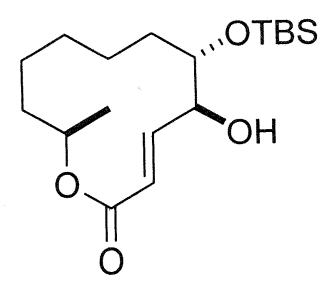

${ }^{13} \mathrm{C}$ NMR (150 MHz, CDCl3)

(w) 


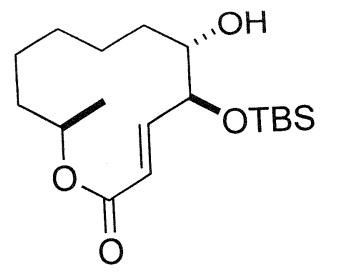

${ }^{1} \mathrm{H}$ NMR (600 MHz, CDCl3)

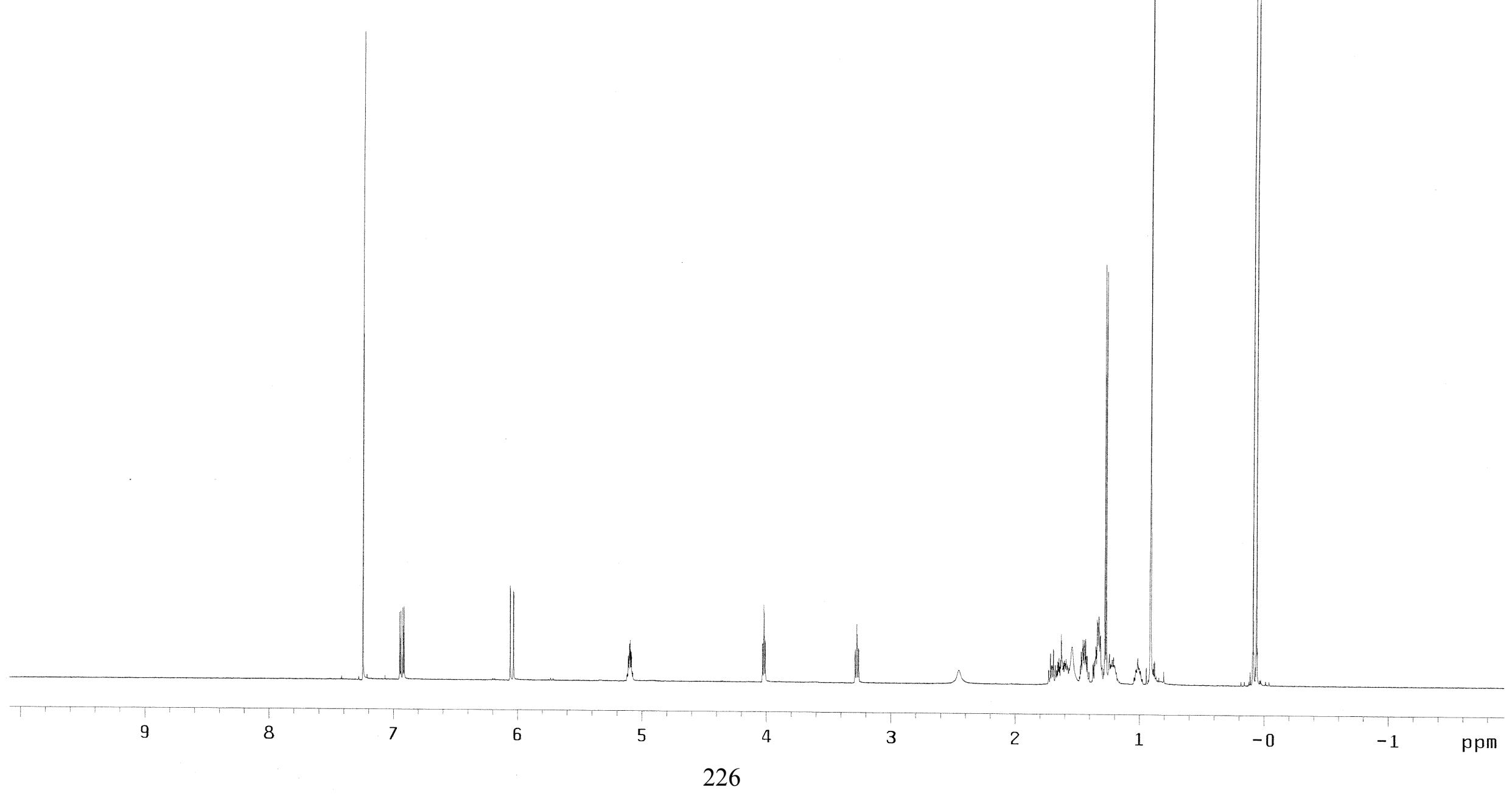




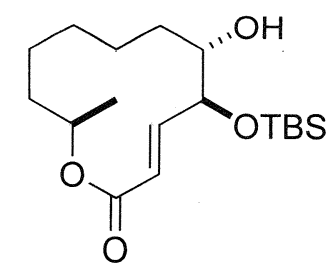

${ }^{13} \mathrm{C}$ NMR (150 MHz, CDCl3)

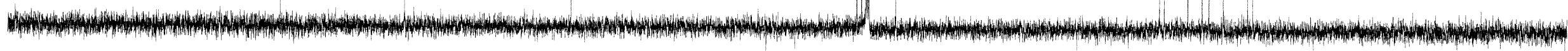




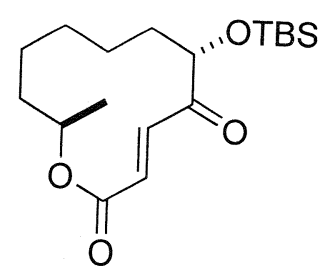

${ }^{1} \mathrm{H}$ NMR (600 MHz, CDCl3)

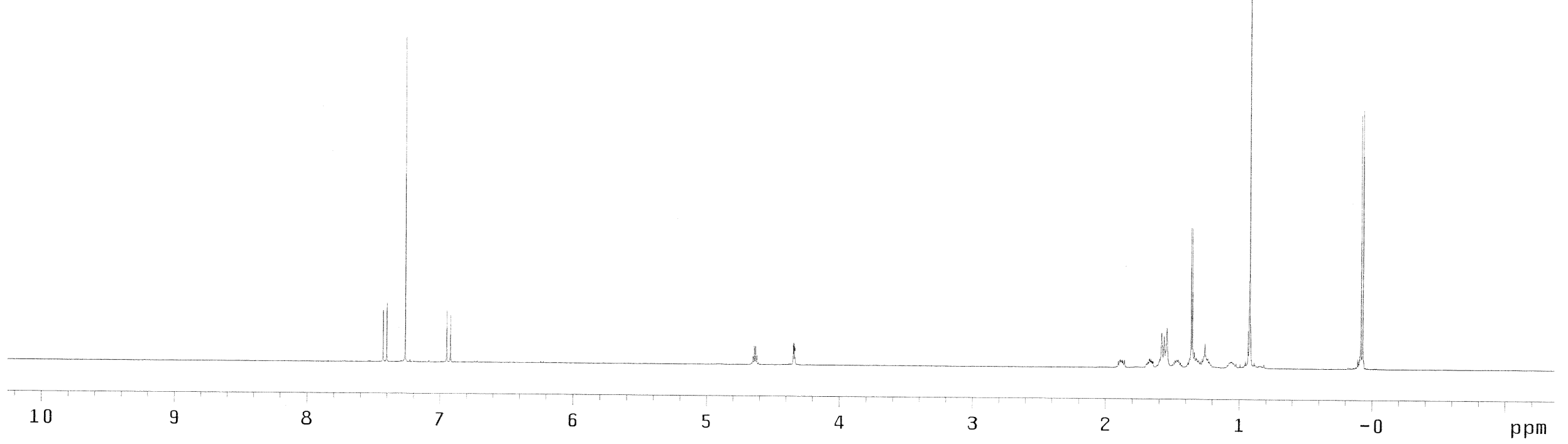




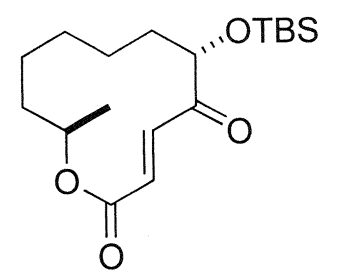

${ }^{13} \mathrm{C}$ NMR (150 MHz, CDCl3)

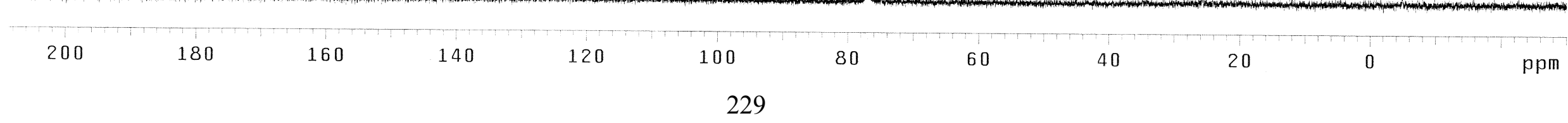




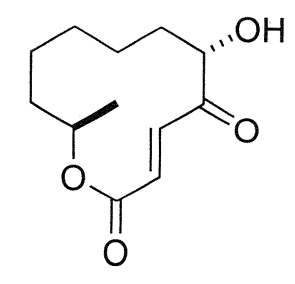

${ }^{1} \mathrm{H}$ NMR (600 MHz, CDCl3)

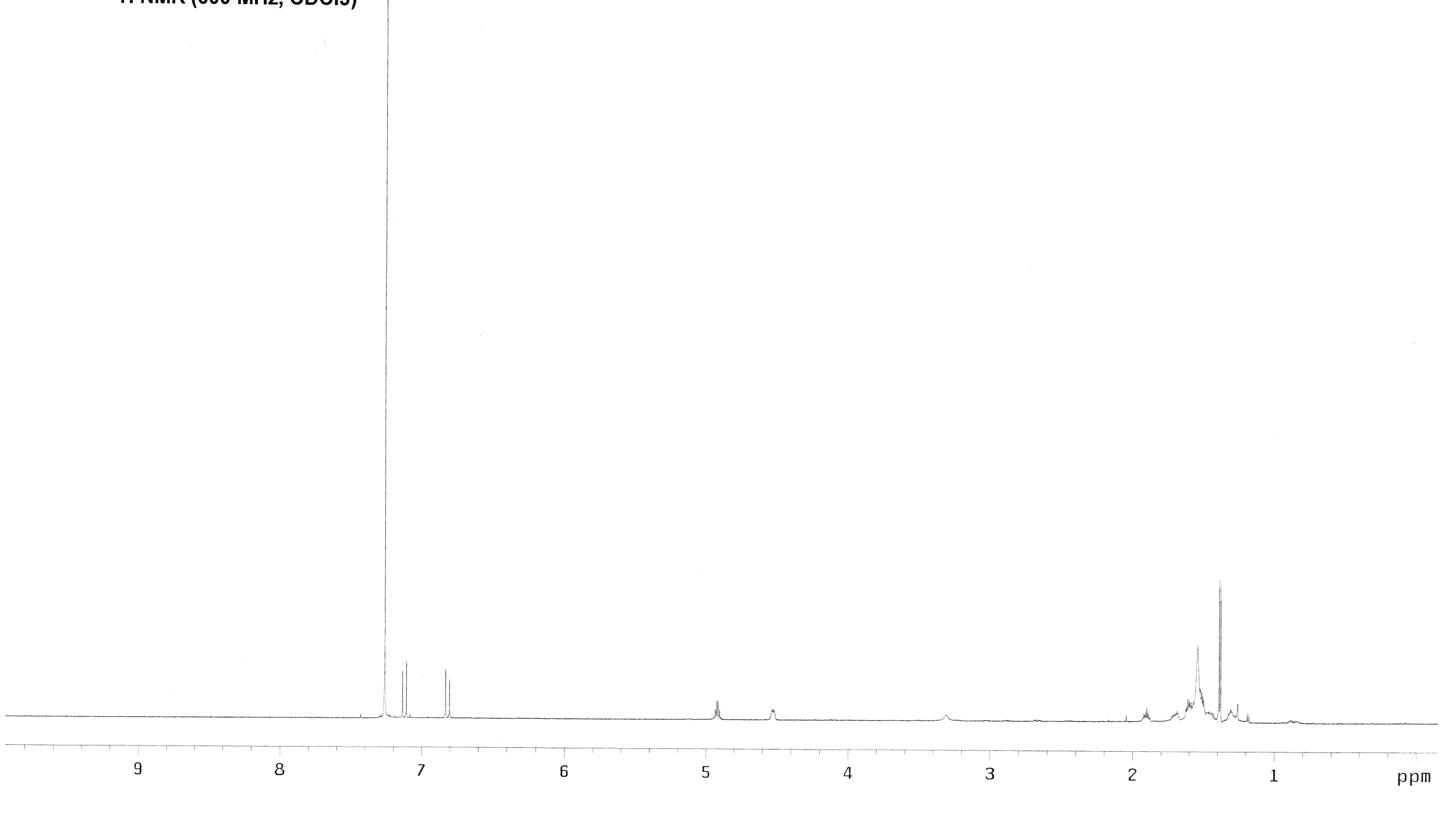




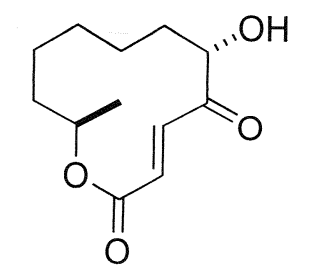

${ }^{13} \mathrm{C}$ NMR (150 MHz, CDCl3)

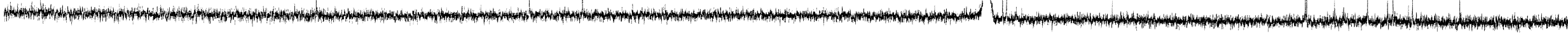




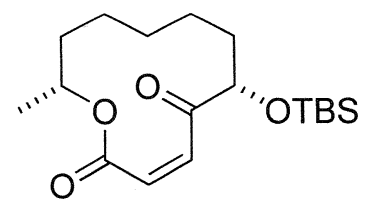

${ }^{1} \mathrm{H} \mathrm{NMR}(600 \mathrm{MHz}, \mathrm{CDCl} 3)$

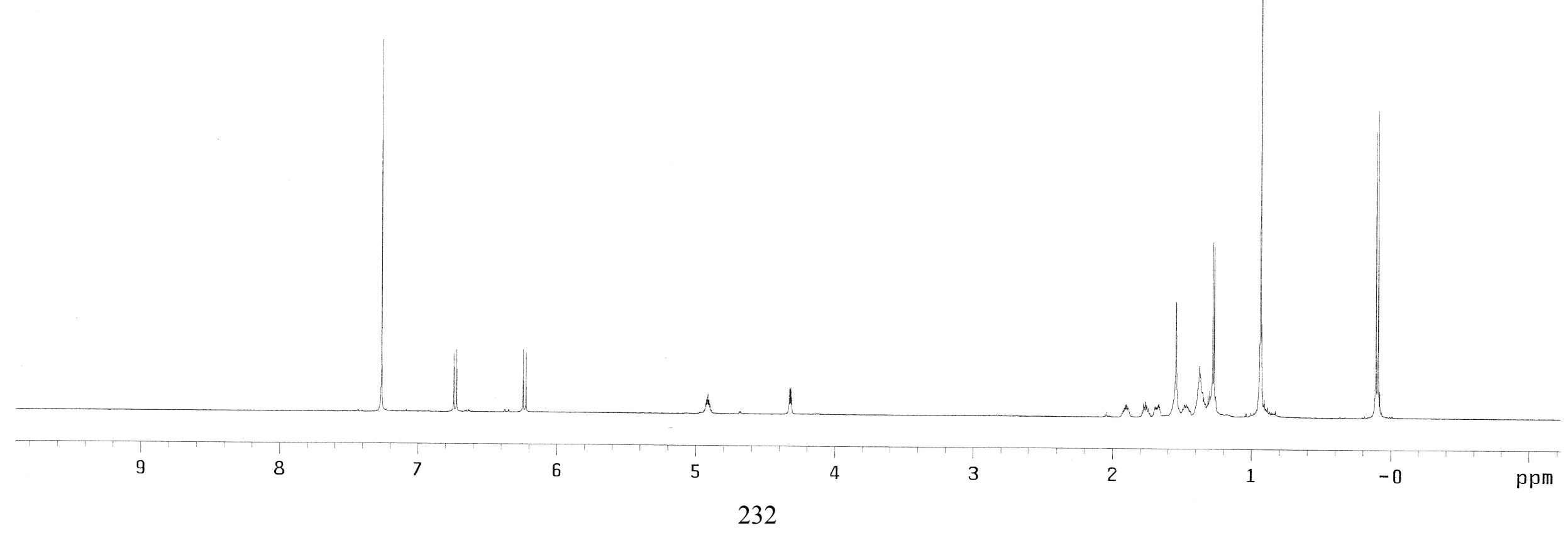




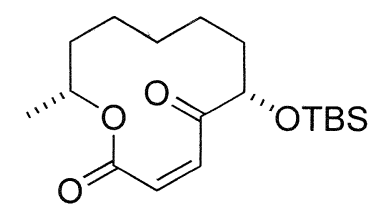

${ }^{13} \mathrm{C}$ NMR (150 MHz, CDCl3)

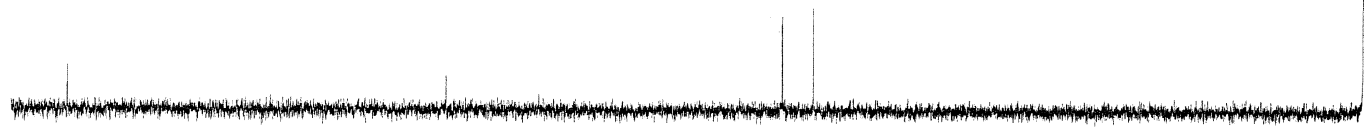

\begin{tabular}{|c|c|c|c|c|c|c|c|c|c|c|}
\hline 200 & 180 & 160 & 140 & 120 & 100 & 80 & 60 & 40 & 20 & 0 \\
\hline
\end{tabular}




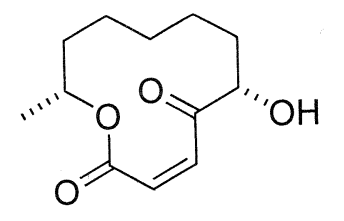

${ }^{1} \mathrm{H}$ NMR (600 MHz, CDCl3)

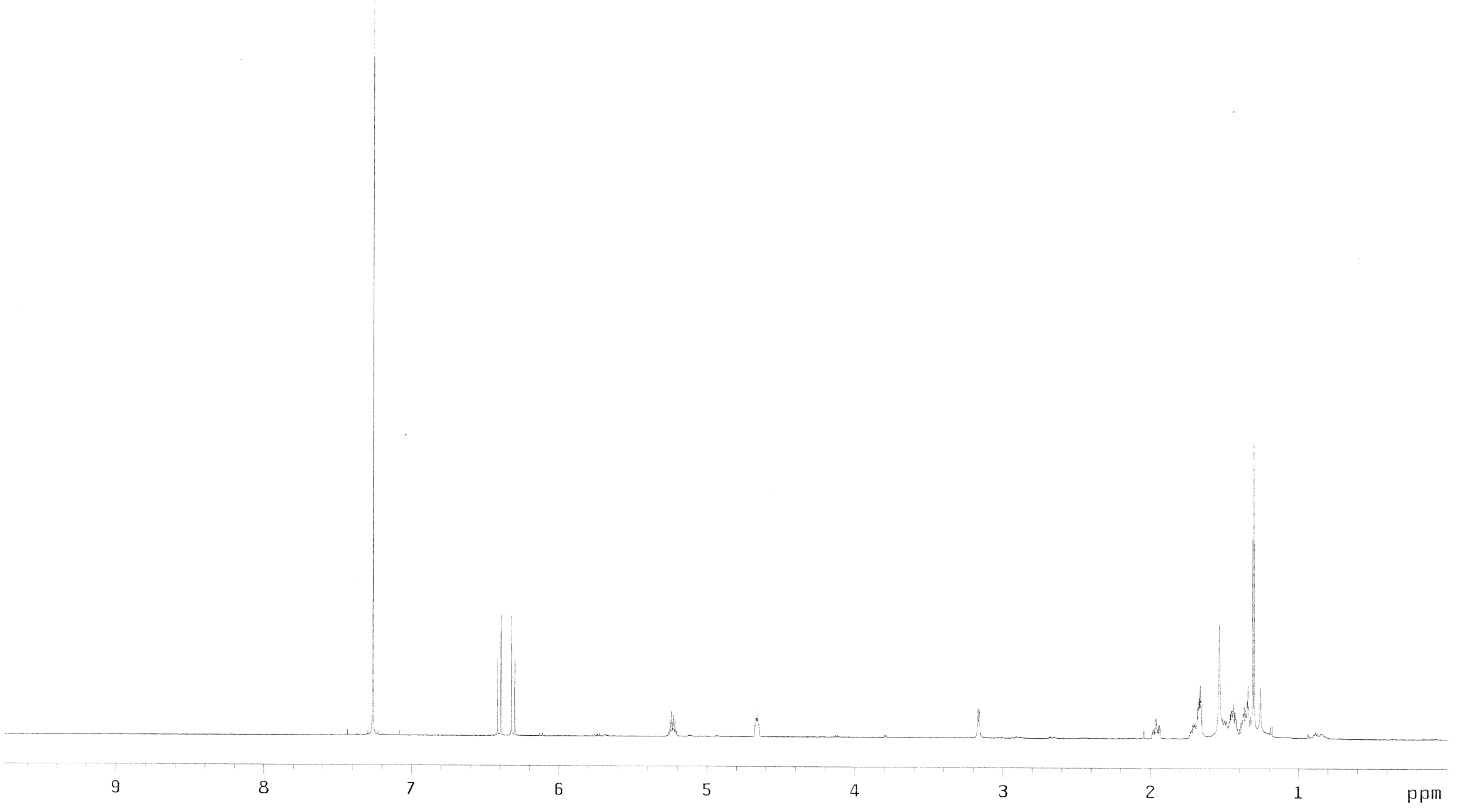




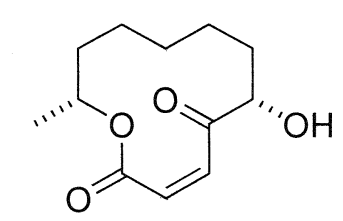

${ }^{13} \mathrm{C}$ NMR (150 MHz, CDCl3)

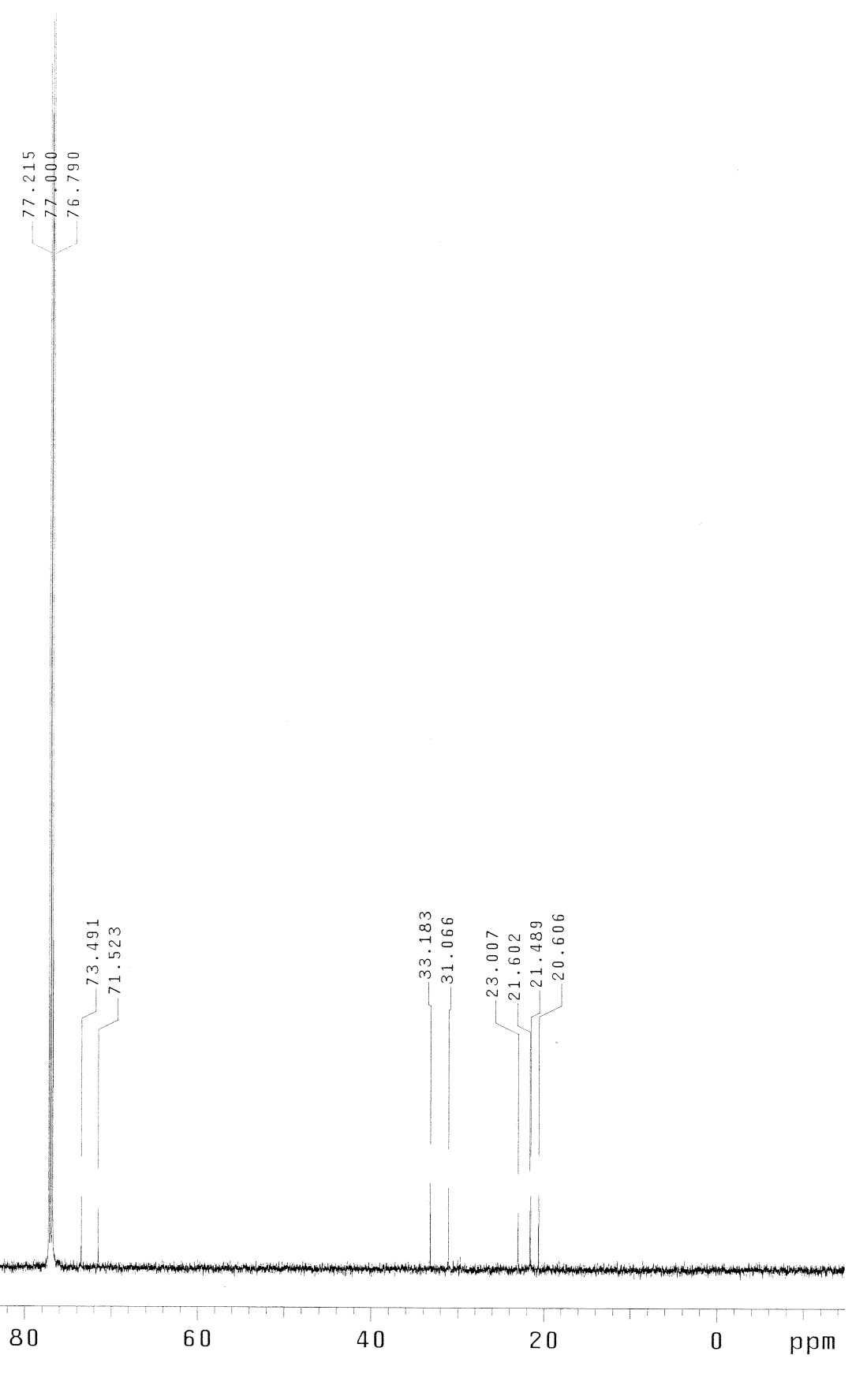




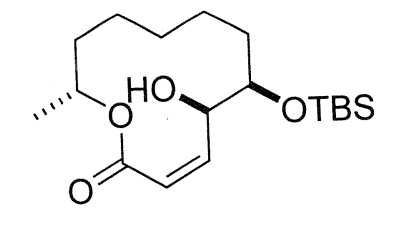

${ }^{1} \mathrm{H}$ NMR (600 MHz, CDCl3)

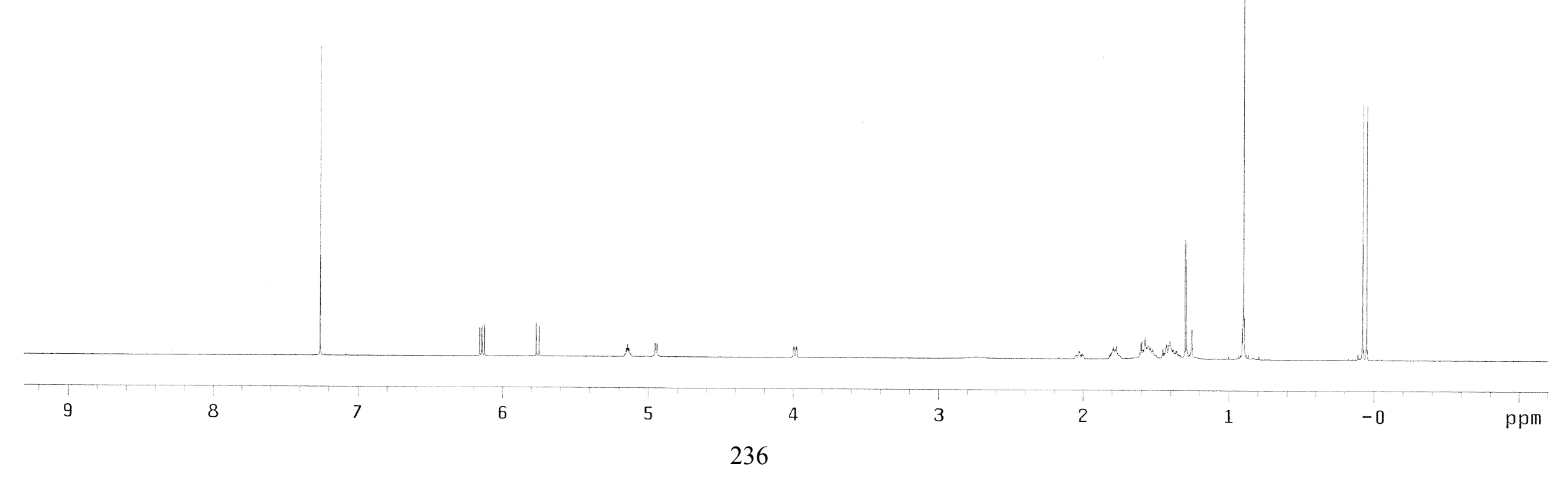




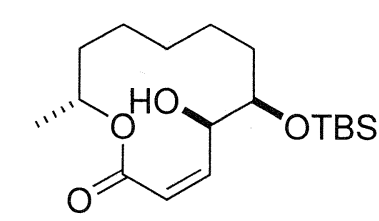

${ }^{13} \mathrm{C} \mathrm{NMR}(150 \mathrm{MHz}, \mathrm{CDCl} 3)$

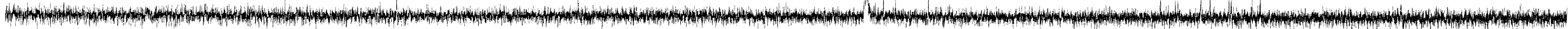




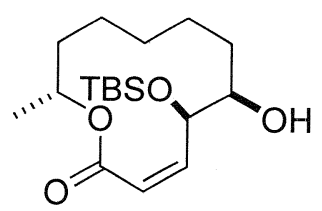

${ }^{1} \mathrm{H}$ NMR (600 MHz, CDCl3)

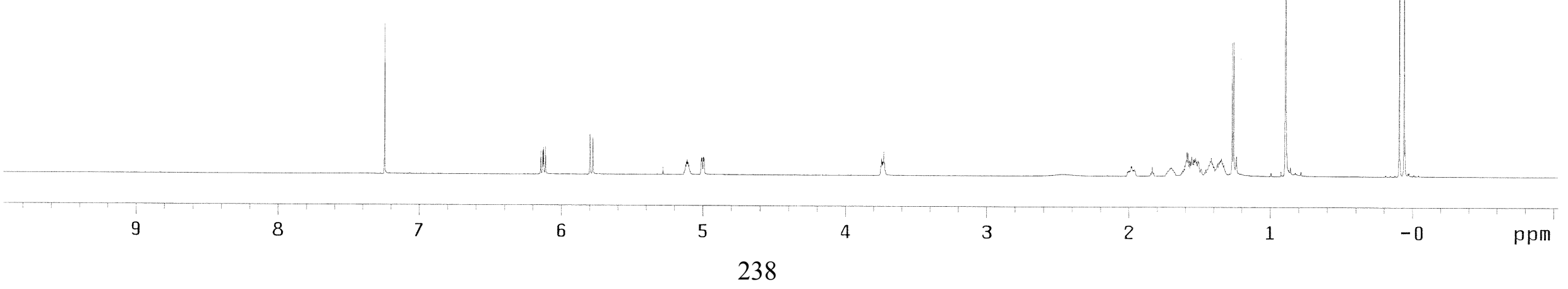




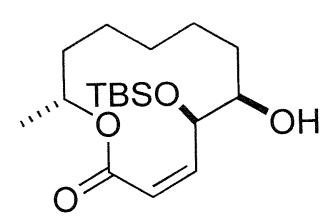

${ }^{13} \mathrm{C}$ NMR $(150 \mathrm{MHz}, \mathrm{CDCl} 3)$

16.

200

180

160

140

120

100

80

60

40

20

0

ppm 


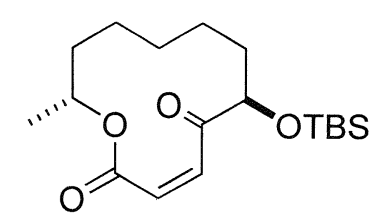

${ }^{1} \mathrm{H}$ NMR $(600 \mathrm{MHz}, \mathrm{CDCl} 3)$

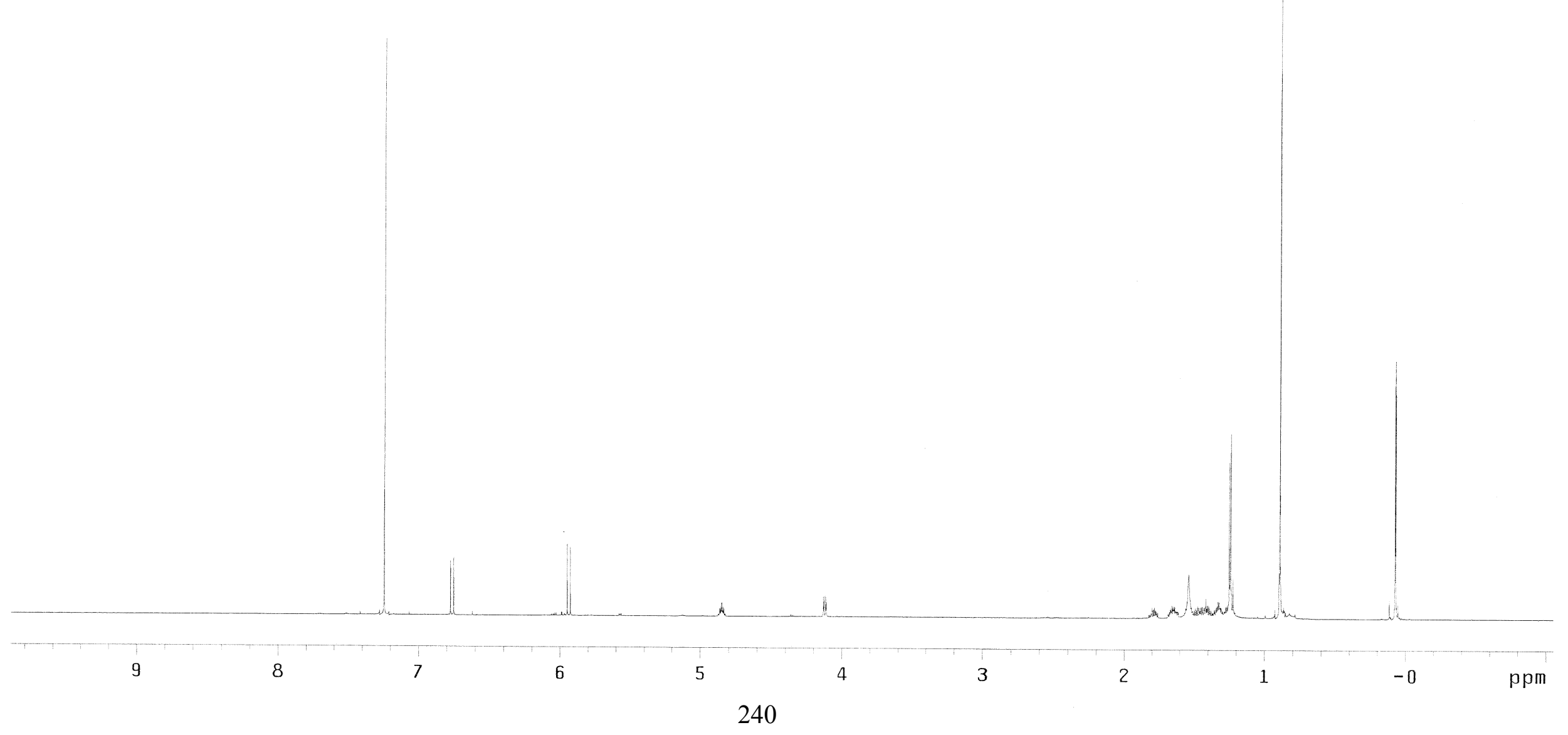




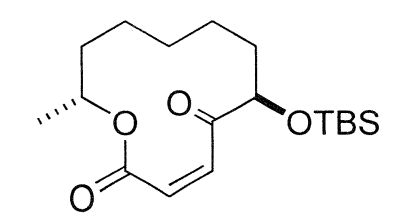

${ }^{13} \mathrm{C}$ NMR (150 MHz, CDCl3)

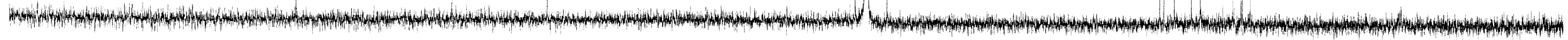

\begin{tabular}{|c|c|c|c|c|c|c|c|c|c|c|c|}
\hline 200 & 180 & 160 & 140 & 120 & 100 & 80 & 60 & 40 & 20 & 0 & ppm \\
\hline
\end{tabular}




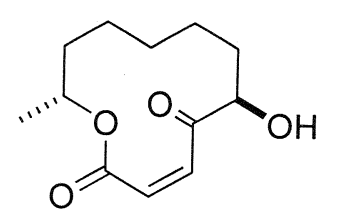

${ }^{1} \mathrm{H}$ NMR $(600 \mathrm{MHz}, \mathrm{CDCl})$

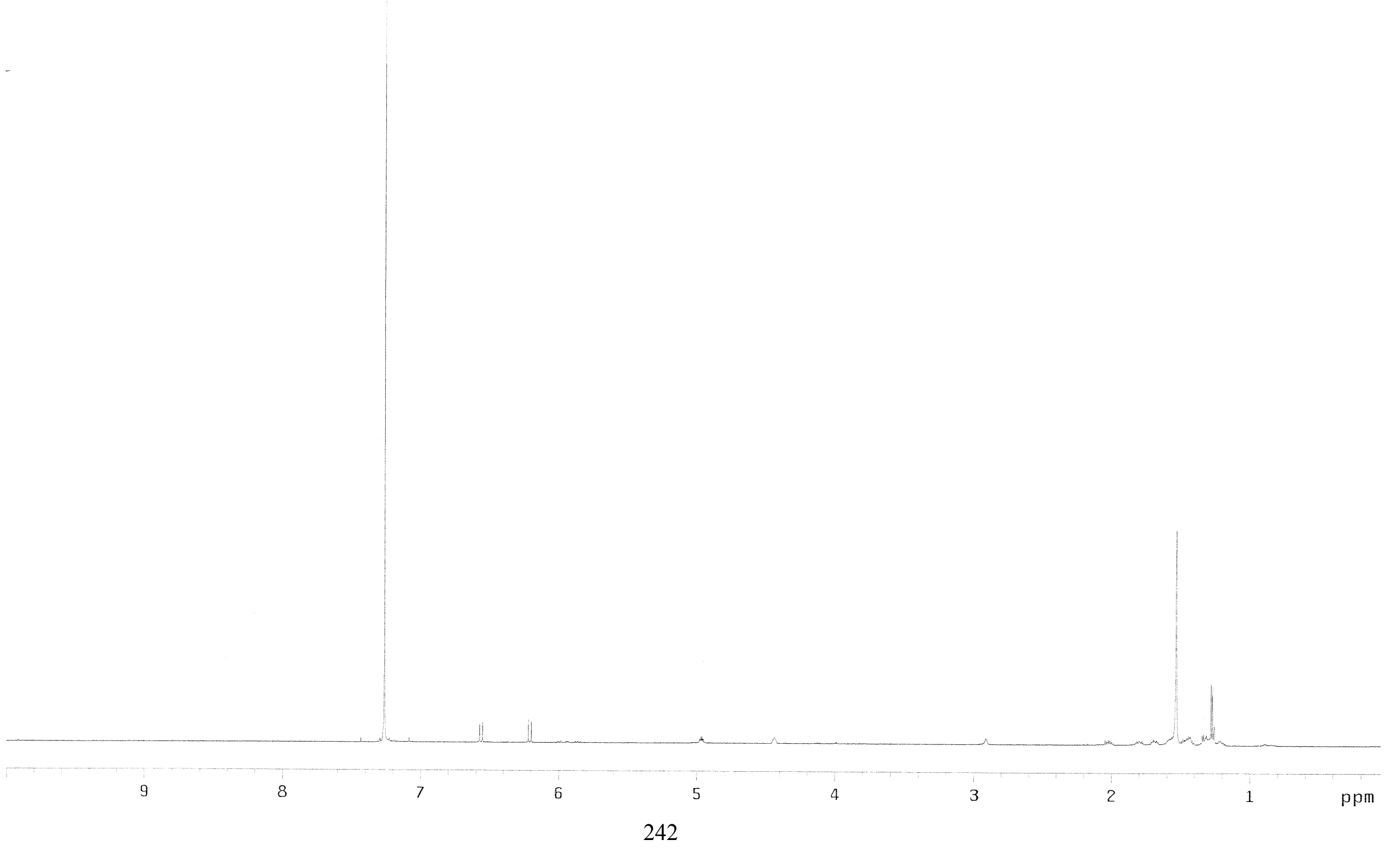




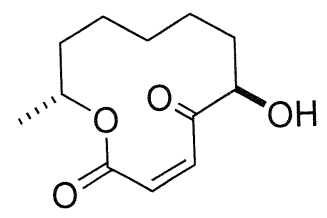

${ }^{13} \mathrm{C}$ NMR (150 MHz, CDCl3)

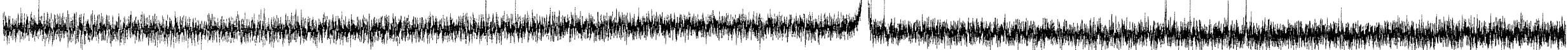




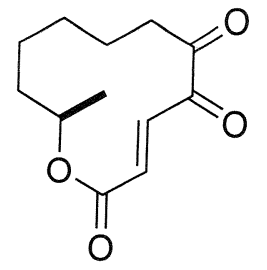

${ }^{1} \mathrm{H} \mathrm{NMR}\left(600 \mathrm{MHz}, \mathrm{CDCl}_{3}\right.$ )

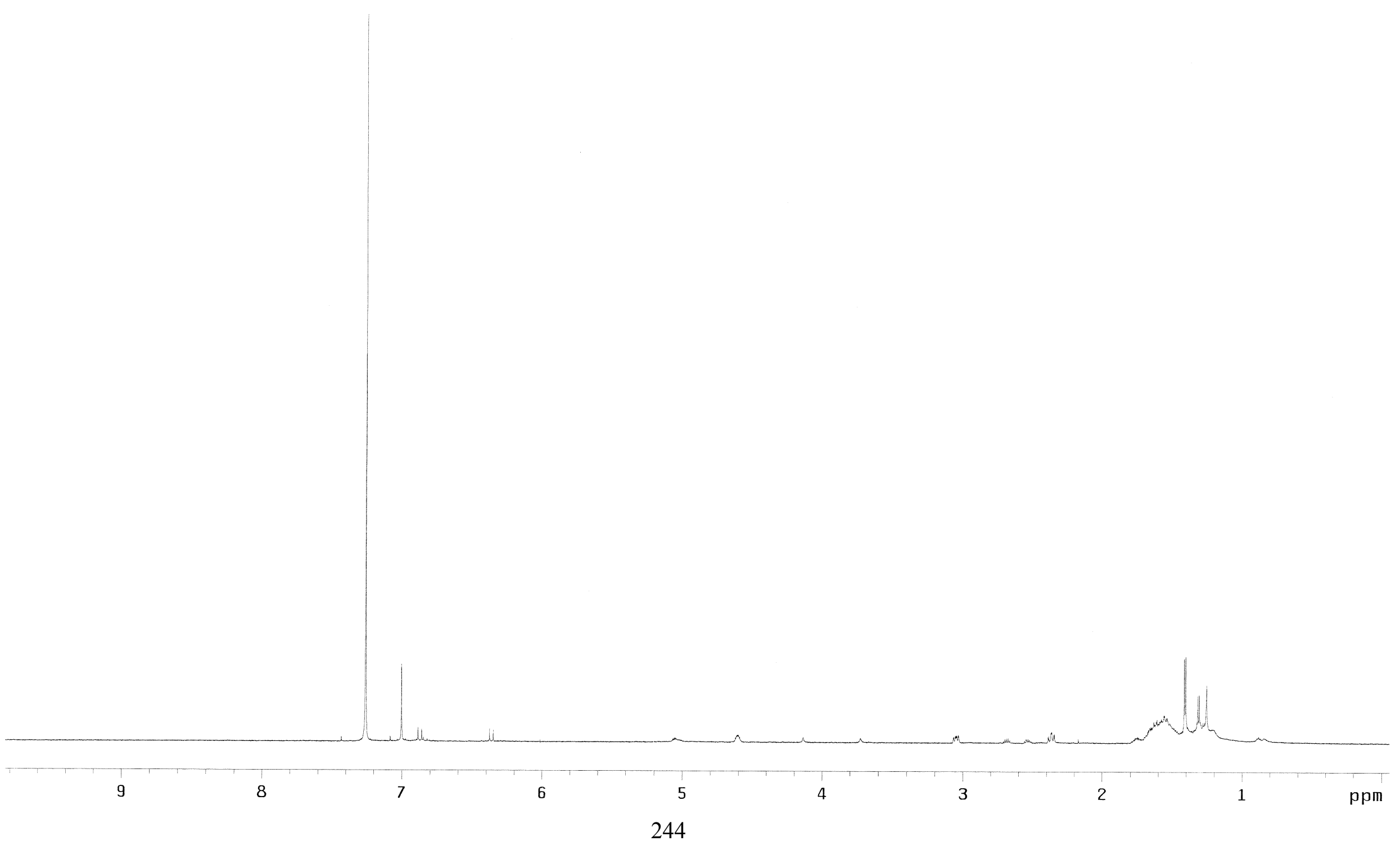




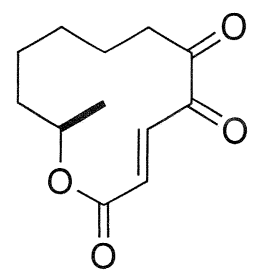

${ }^{13} \mathrm{C} \mathrm{NMR}\left(150 \mathrm{MHz}, \mathrm{CDCl}_{3}\right)$

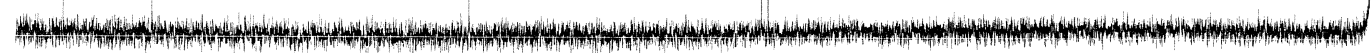

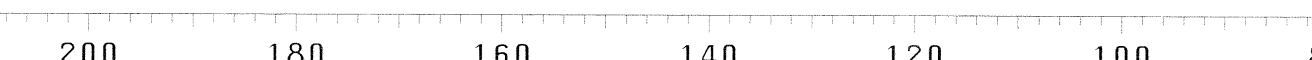

200

180

160

140

120

100

80

60

40

20

0

$\mathrm{ppm}$ 


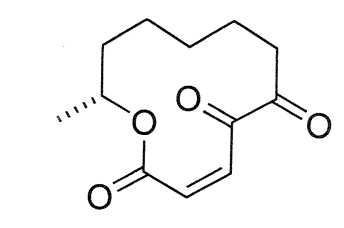

${ }^{1} \mathrm{H}$ NMR (600 MHz, $\left.\mathrm{CDCl}_{3}\right)$

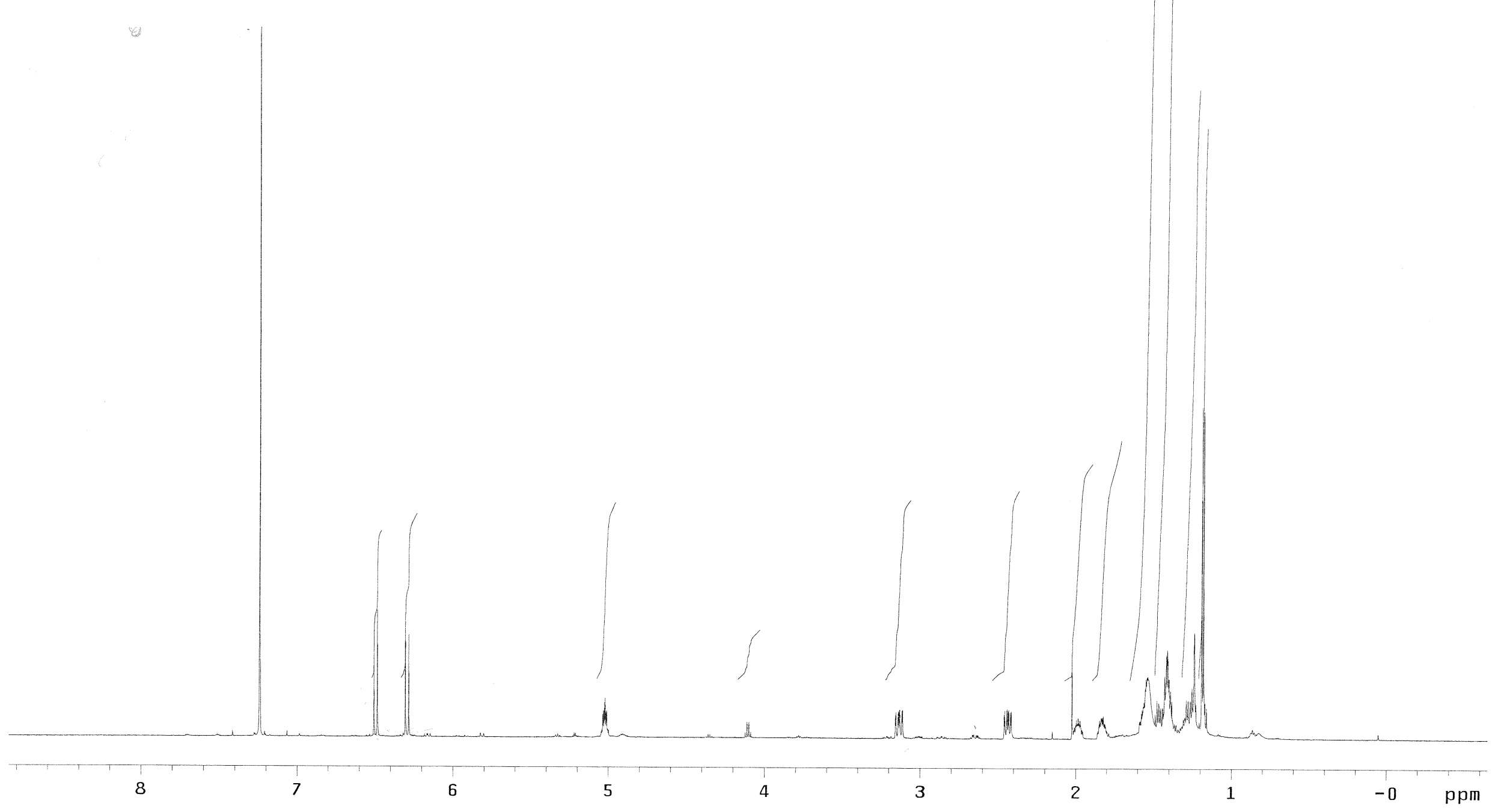




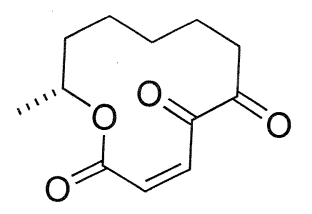

${ }^{13} \mathrm{C}$ NMR (150 MHz, $\mathrm{CDCl}_{3}$ )

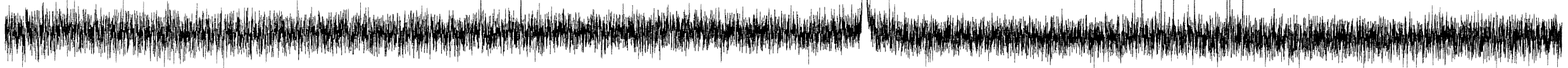




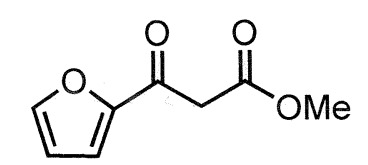

${ }^{1} \mathrm{H}$ NMR $(600 \mathrm{MHz})$

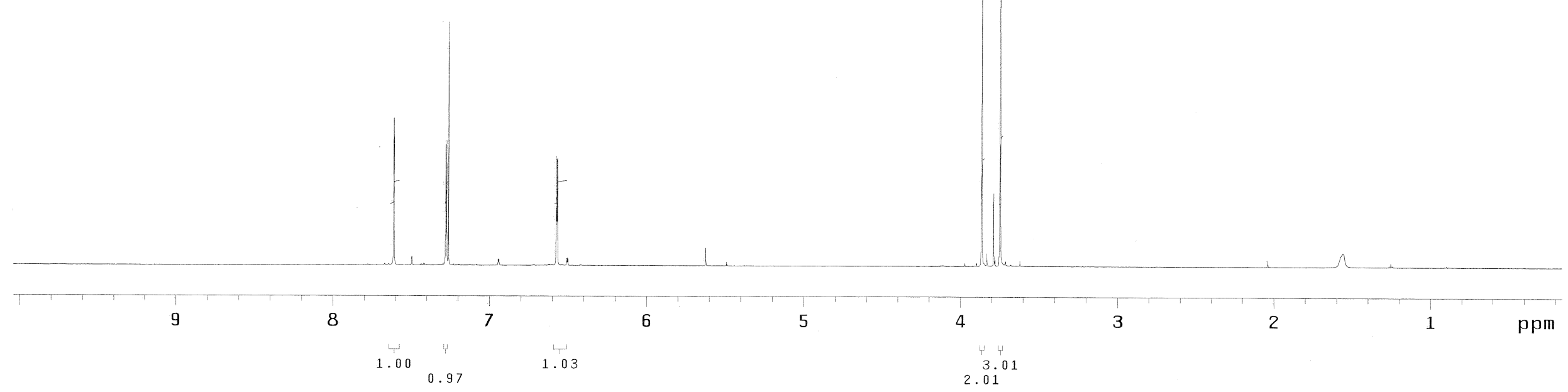




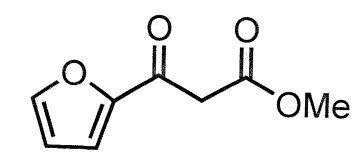

${ }^{13} \mathrm{C}$ NMR $(150 \mathrm{MHz})$ 


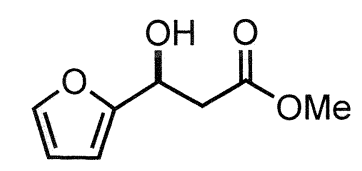

${ }^{1} \mathrm{H}$ NMR $(600 \mathrm{MHz})$

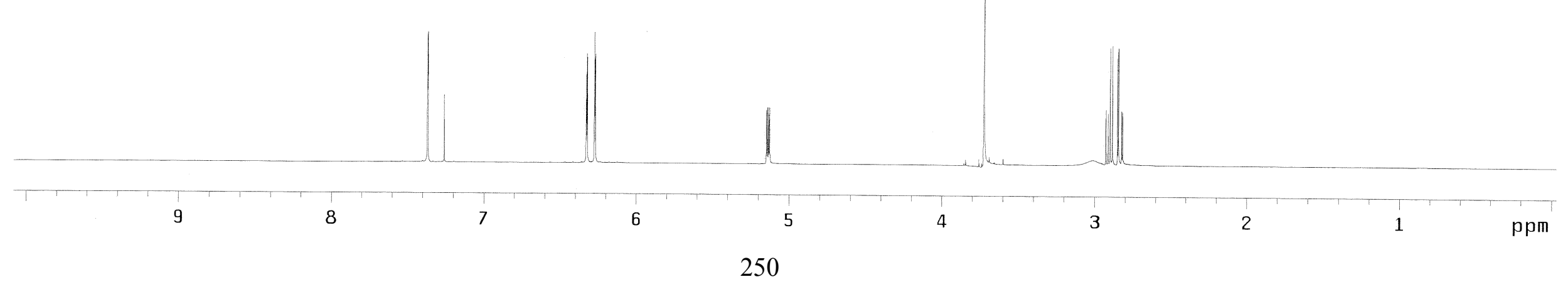




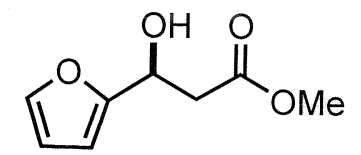

${ }^{13} \mathrm{C}$ NMR $(150 \mathrm{MHz})$

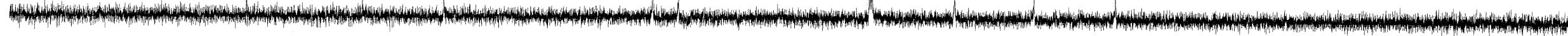

$\begin{array}{lllllllllll}200 & 180 & 160 & 140 & 120 & 100 & 80 & 60 & 40 & 20 & 0\end{array}$




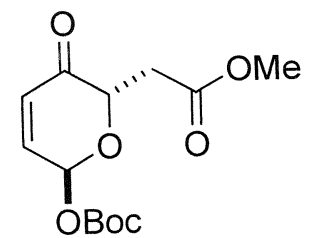

${ }^{1} \mathrm{H}$ NMR $(600 \mathrm{MHz})$

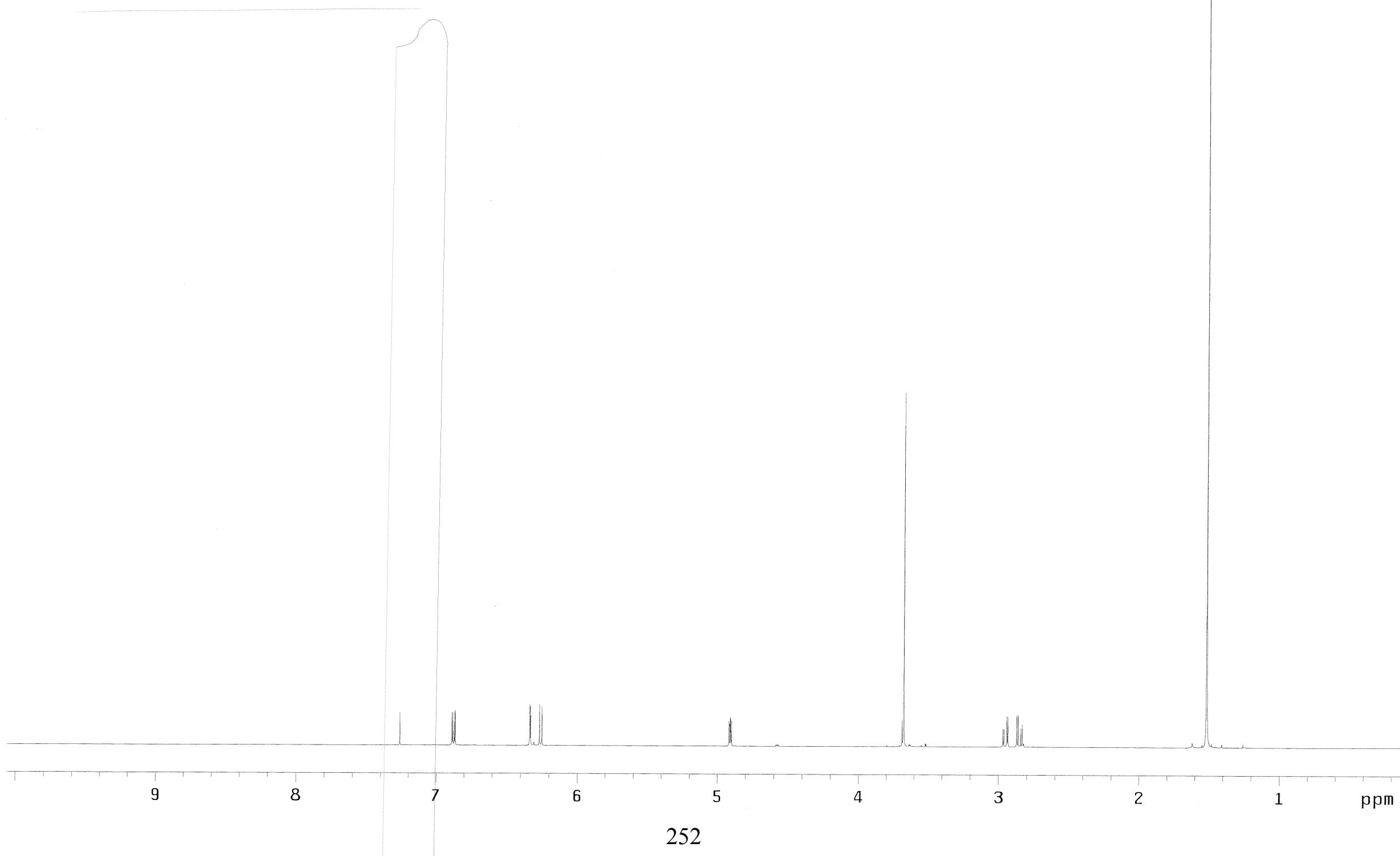




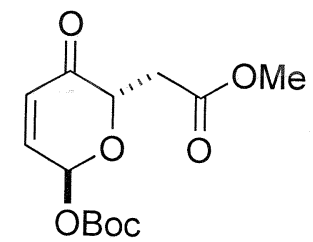

${ }^{13} \mathrm{C}$ NMR $(150 \mathrm{MHz})$

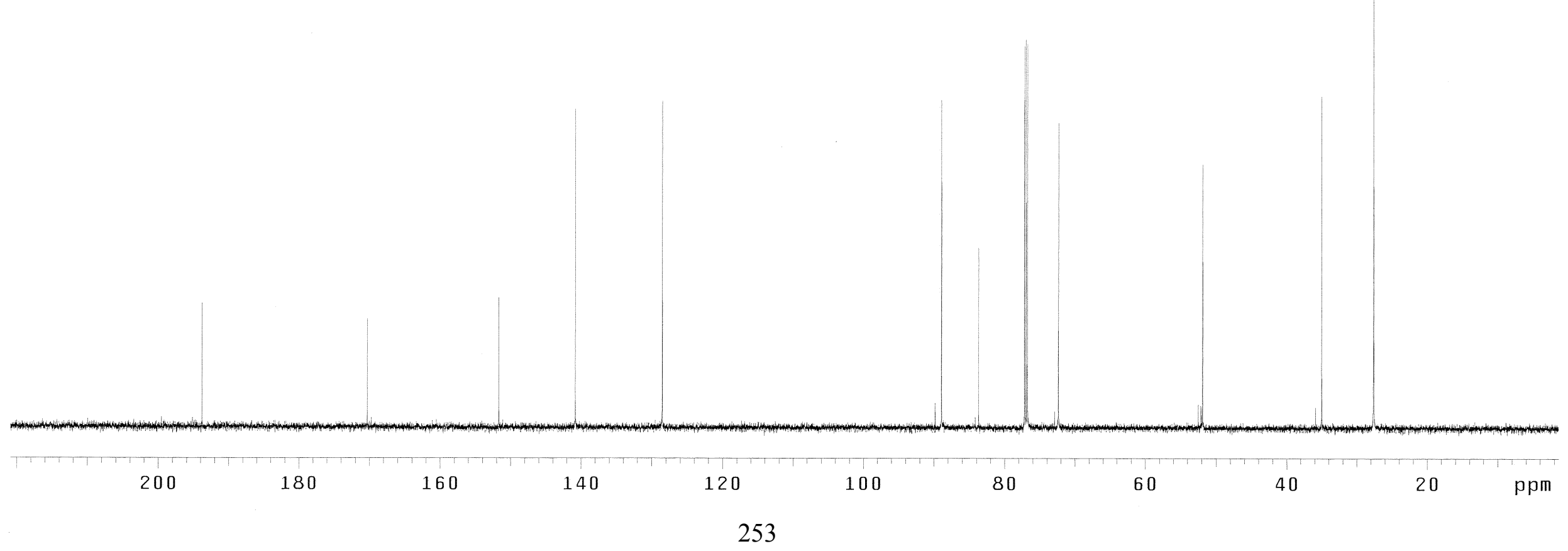




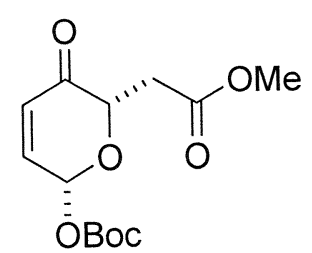

${ }^{1} \mathrm{H}$ NMR $(600 \mathrm{MHz})$

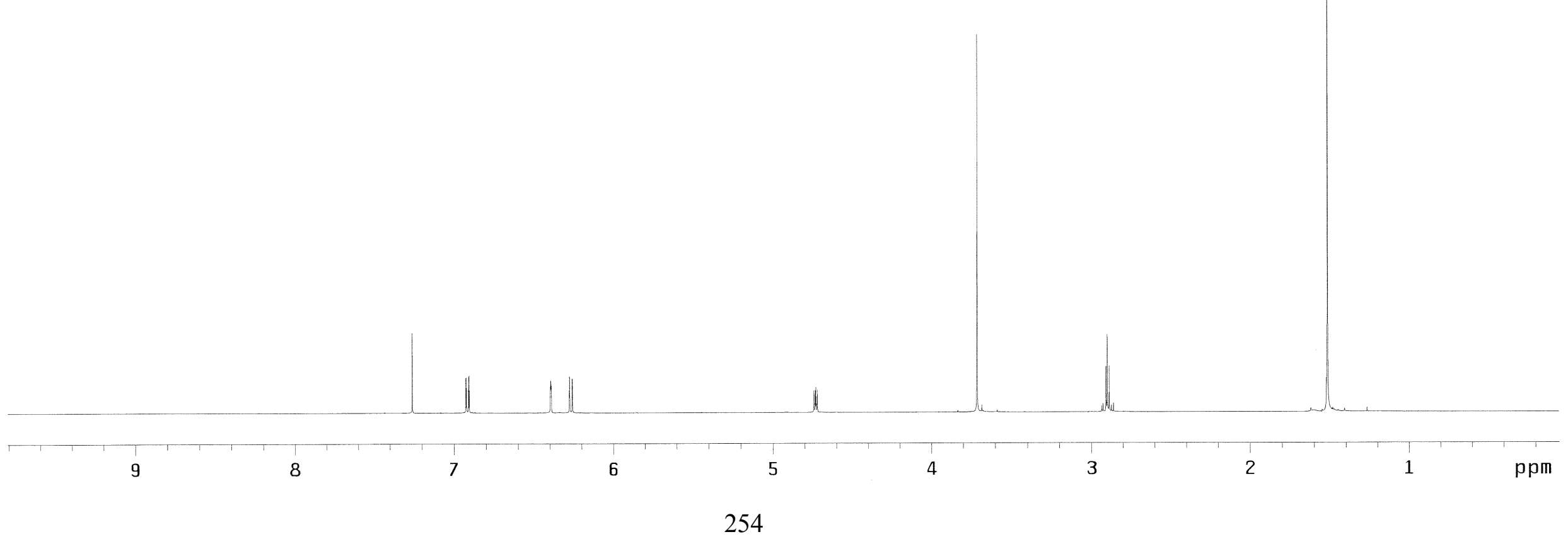




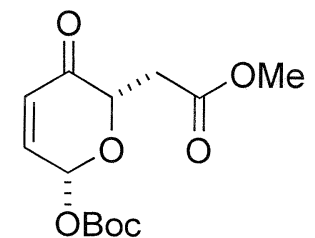

${ }^{13} \mathrm{C}$ NMR $(150 \mathrm{MHz})$

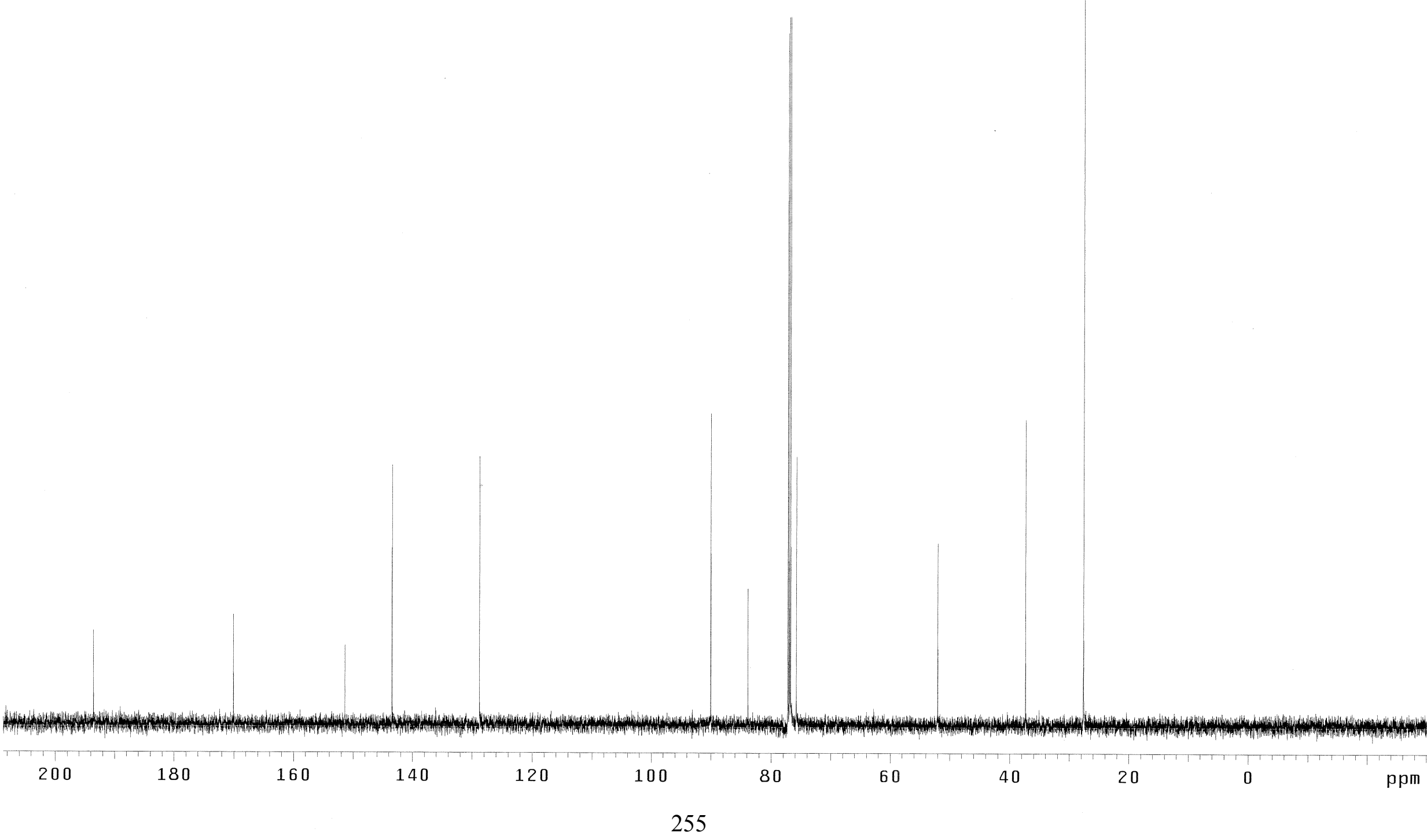




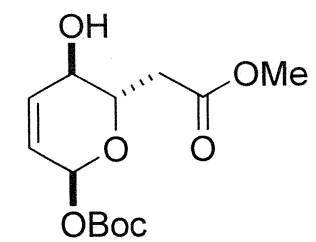

${ }^{1} \mathrm{H}$ NMR $(600 \mathrm{MHz})$

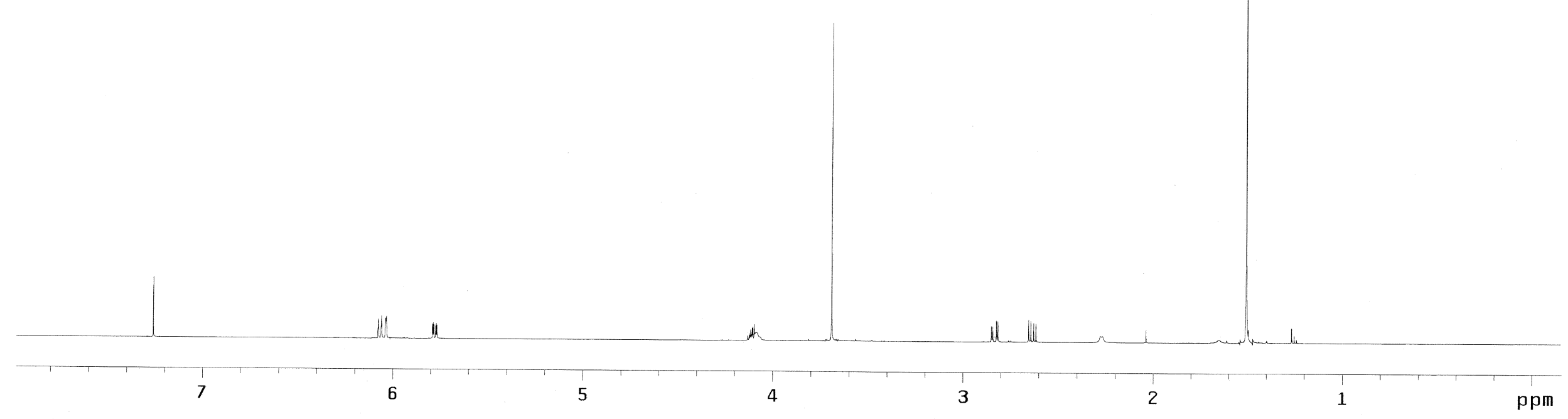


$\overbrace{\mathrm{OBOC}}^{\mathrm{OH}}$

${ }^{13} \mathrm{C}$ NMR (150 MHz)

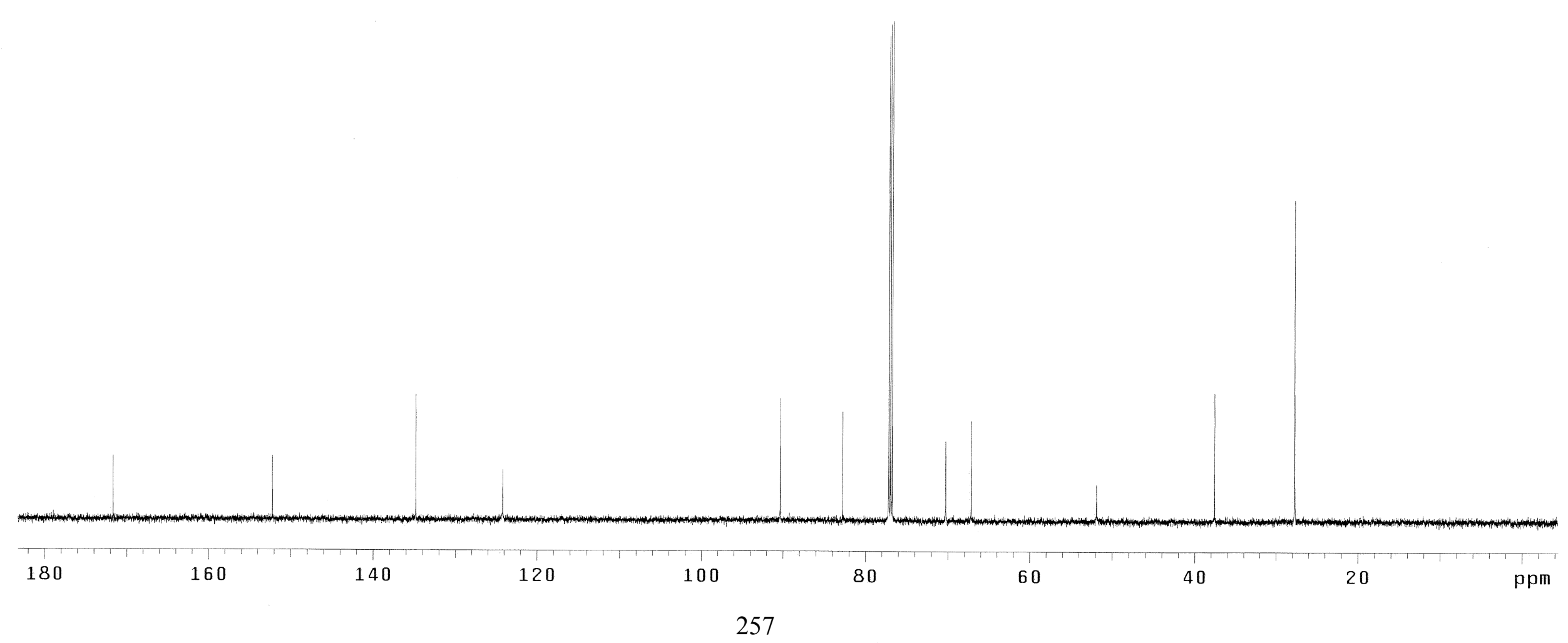


${ }_{\mathrm{OBOC}}^{\mathrm{O} \mathrm{H} \text { NMR }(600 \mathrm{MHz})}$

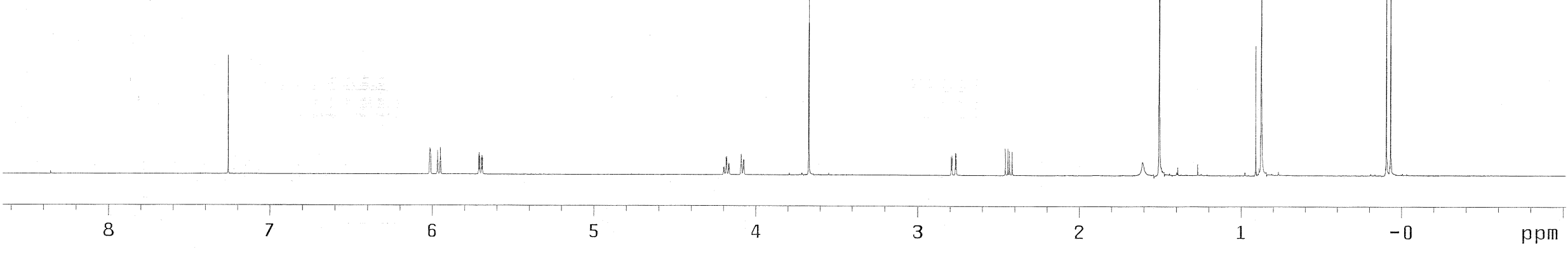


OTBS

$\left.\left.\right|_{O B O C} ^{O}\right|_{O} ^{O M e}$

${ }^{13} \mathrm{C}$ NMR $(150 \mathrm{MHz})$

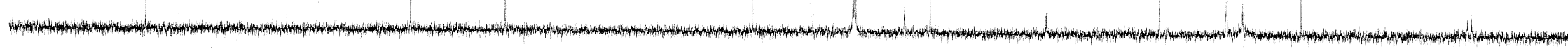


$\mathrm{OpNBz}$

$\overbrace{\mathrm{OBoc}}^{C_{O}^{\prime}} \prod_{O}^{\mathrm{OMe}}$

${ }^{1} \mathrm{H}$ NMR $(600 \mathrm{MHz})$

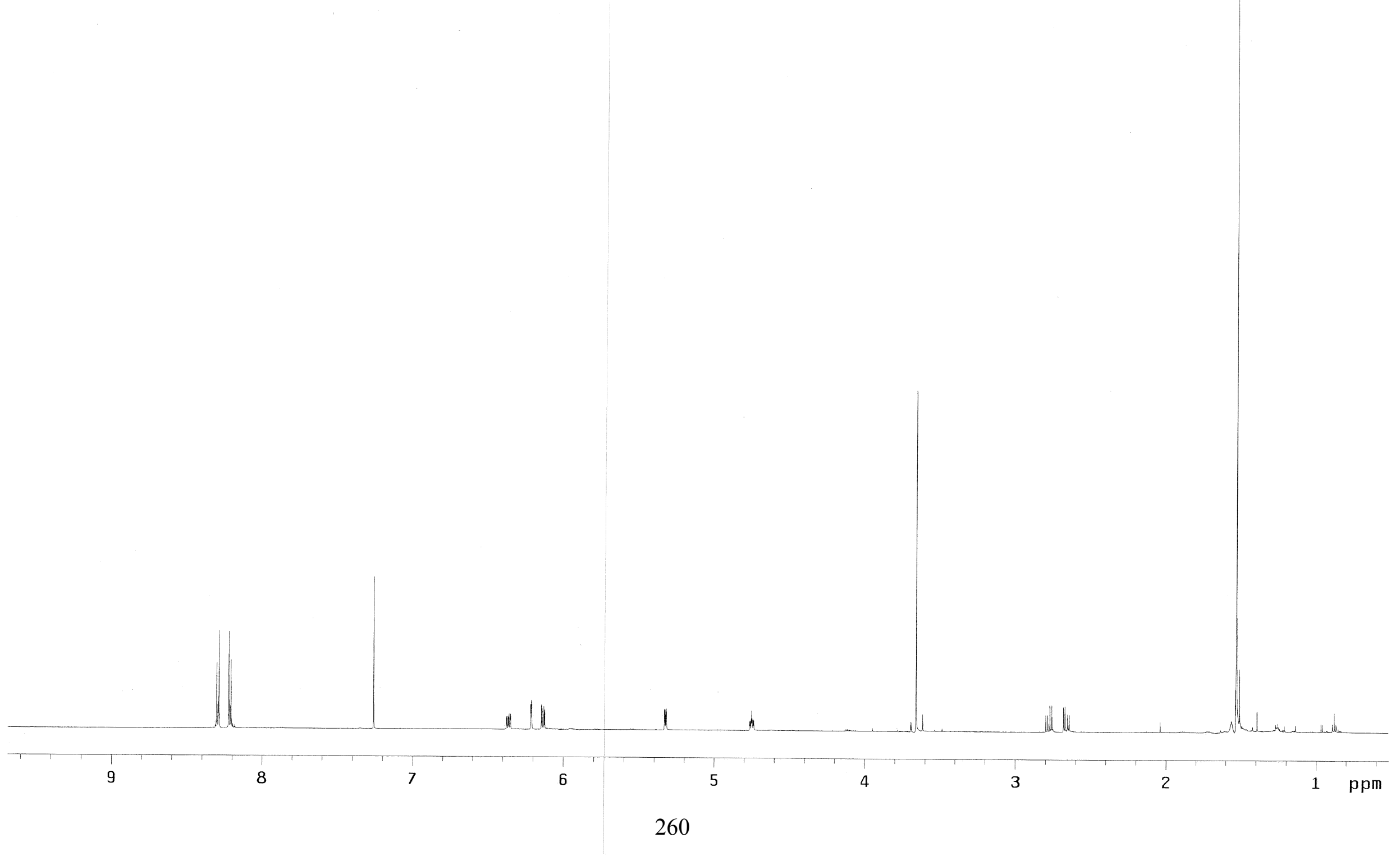


$\mathrm{OpNBz}$

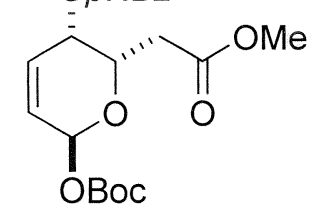

${ }^{13} \mathrm{C}$ NMR $(150 \mathrm{MHz})$ 
$\mathrm{OH}$

$\overbrace{\mathrm{OBOC}}^{\mathrm{O}} \prod_{\mathrm{O}}^{\mathrm{OMe}}$

${ }^{1} \mathrm{H}$ NMR $(600 \mathrm{MHz})$

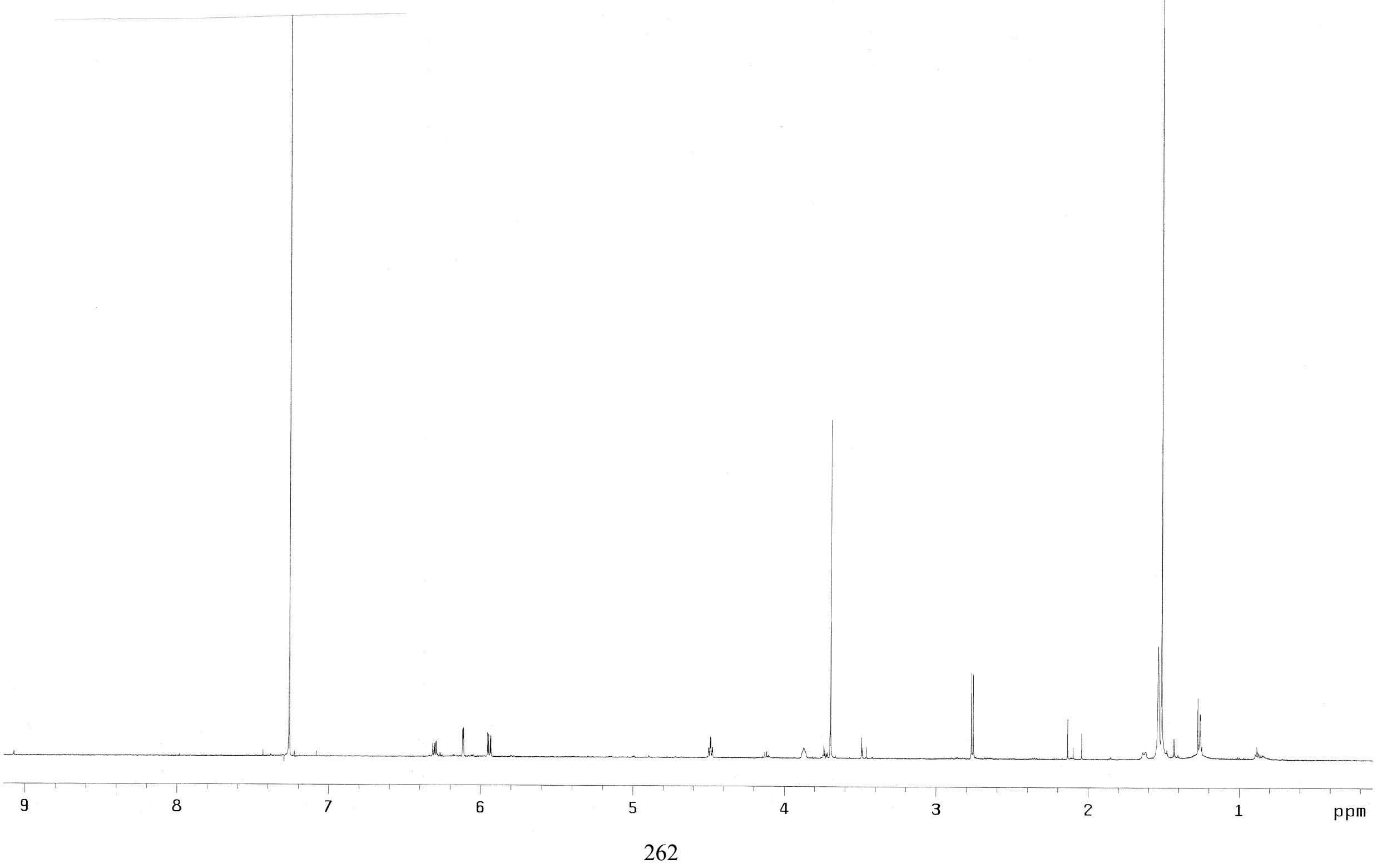




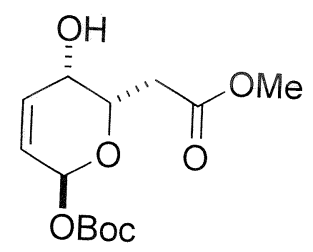

${ }^{13} \mathrm{C}$ NMR (150 MHz) 


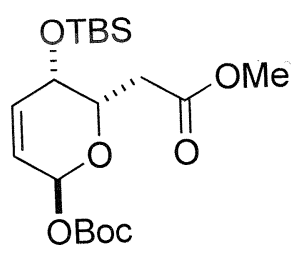

${ }^{1} \mathrm{H}$ NMR $(600 \mathrm{MHz})$

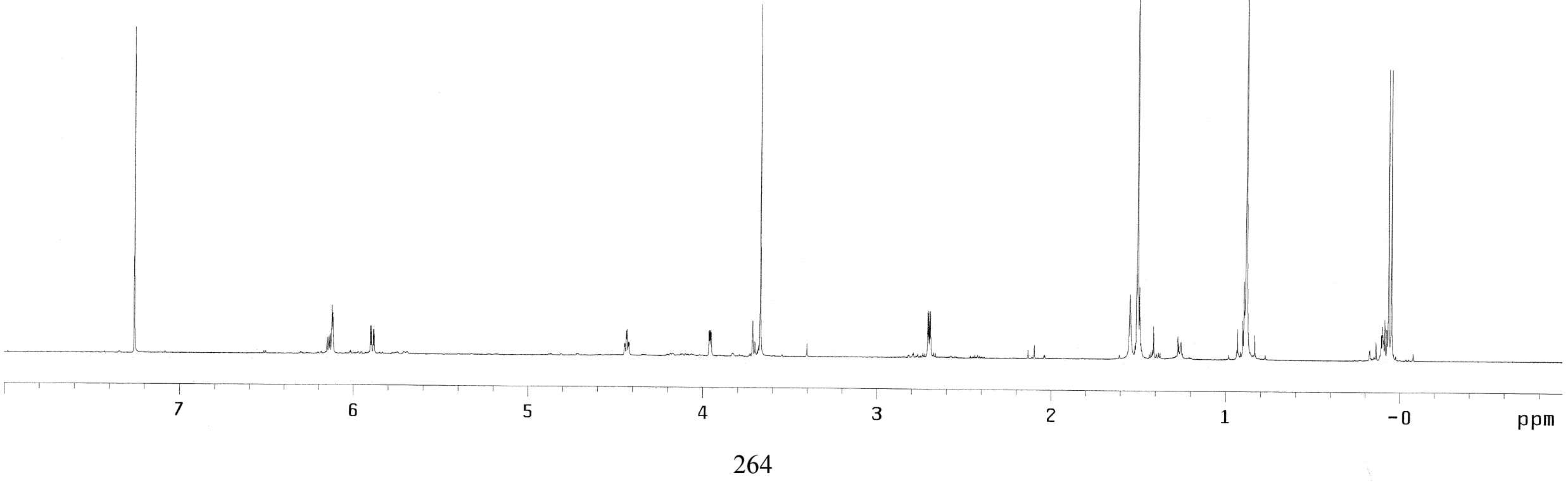




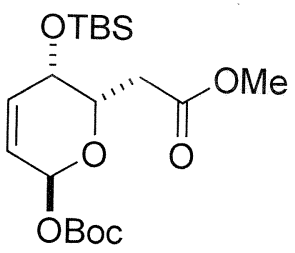

${ }^{13} \mathrm{C}$ NMR $(150 \mathrm{MHz})$

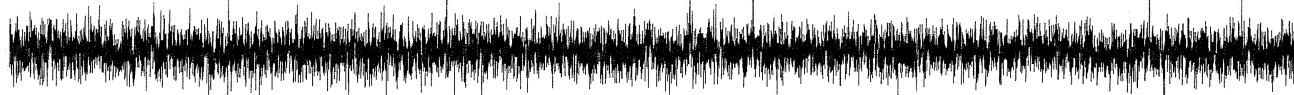




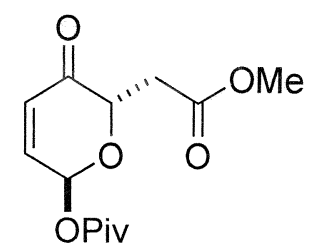

${ }^{1} \mathrm{H}$ NMR (600 MHz)

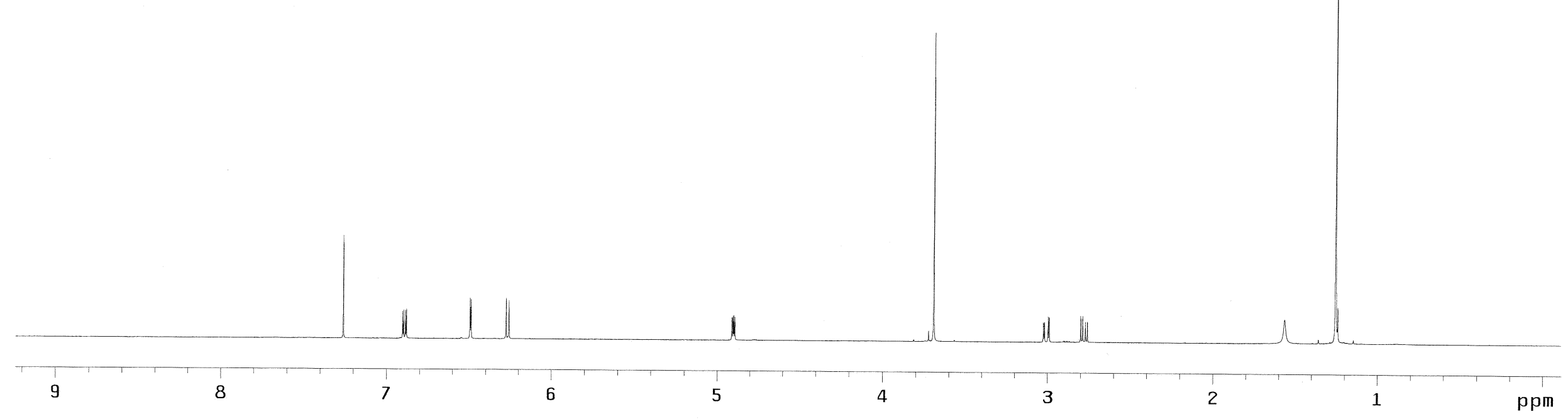




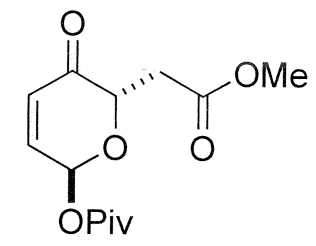

${ }^{13} \mathrm{C}$ NMR $(150 \mathrm{MHz})$

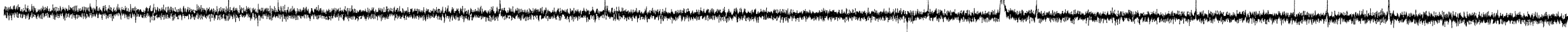

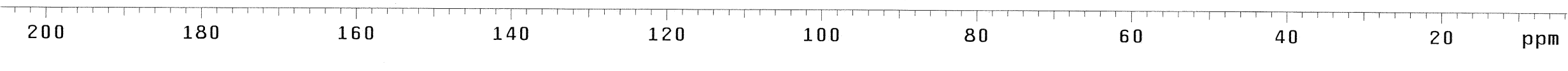


$\overbrace{O P i v}^{O H}$

${ }^{1} \mathrm{H}$ NMR $(600 \mathrm{MHz})$

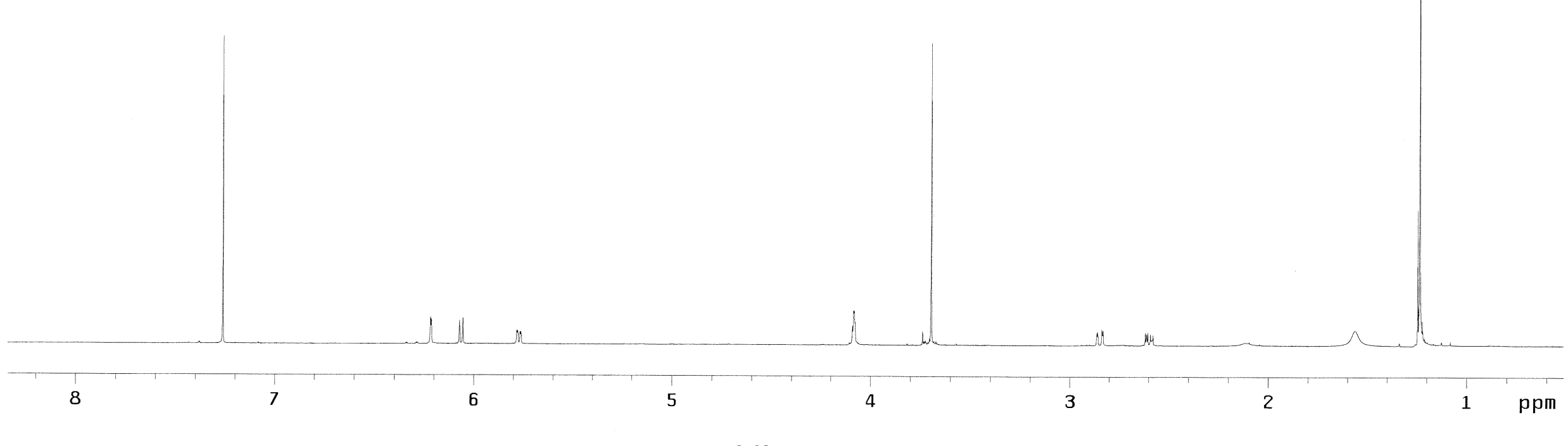



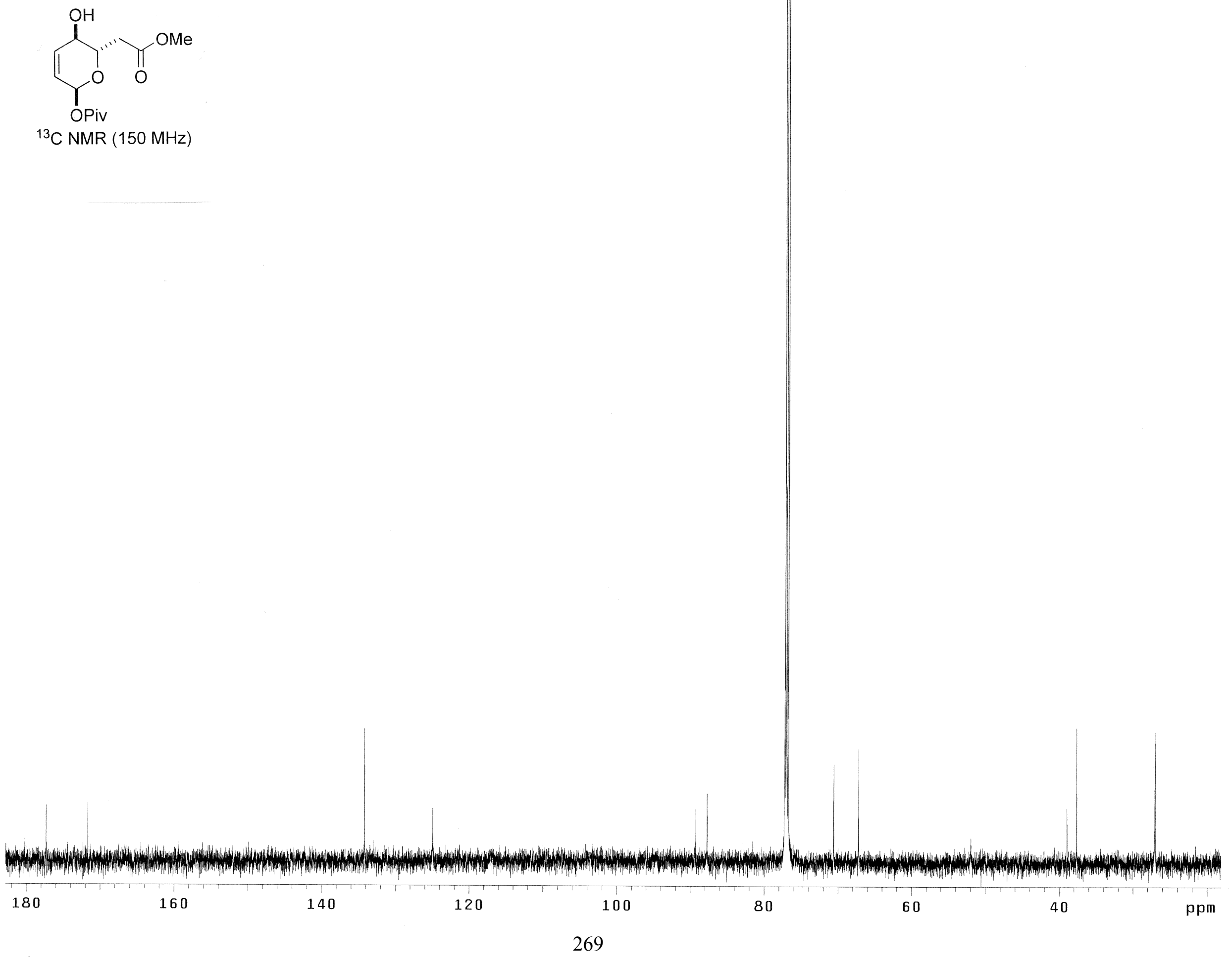


\section{$\mathrm{OpNBz}$}

$\overbrace{\text { OPiv }}^{C_{O}^{\prime \prime \prime \prime}} \prod_{O}^{O M e}$

${ }^{1} \mathrm{H}$ NMR $(600 \mathrm{MHz})$

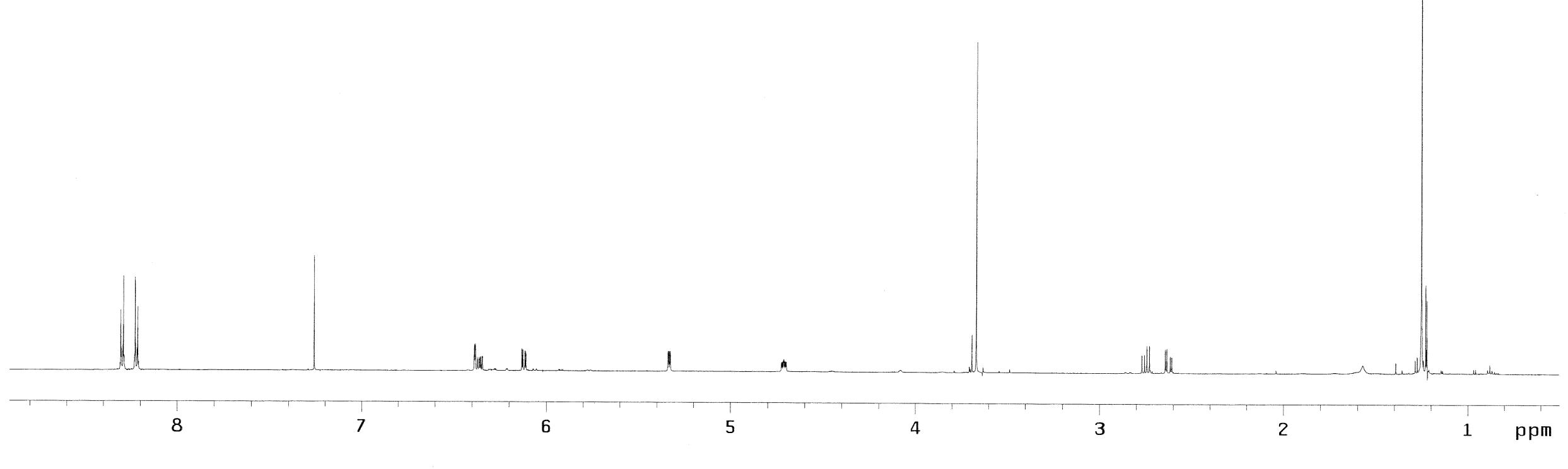


$\mathrm{OpNBz}$

$\overbrace{\text { OPiv }}^{\text {OP OMe }}$

${ }^{13} \mathrm{C}$ NMR $(150 \mathrm{MHz})$

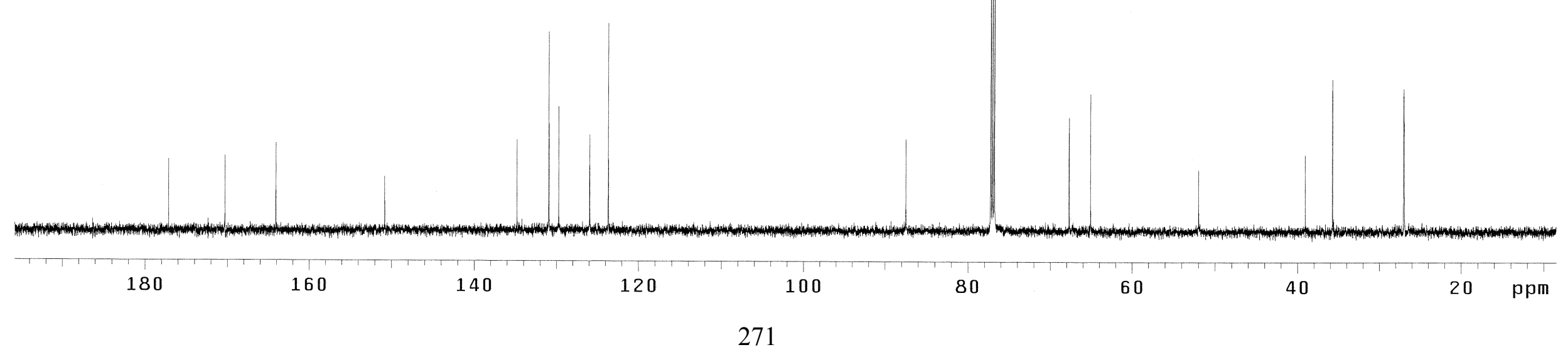


$\overbrace{\text { OPiv }}^{O H} \prod_{O}^{O H}$ OMe

${ }^{1} \mathrm{H}$ NMR $(600 \mathrm{MHz})$

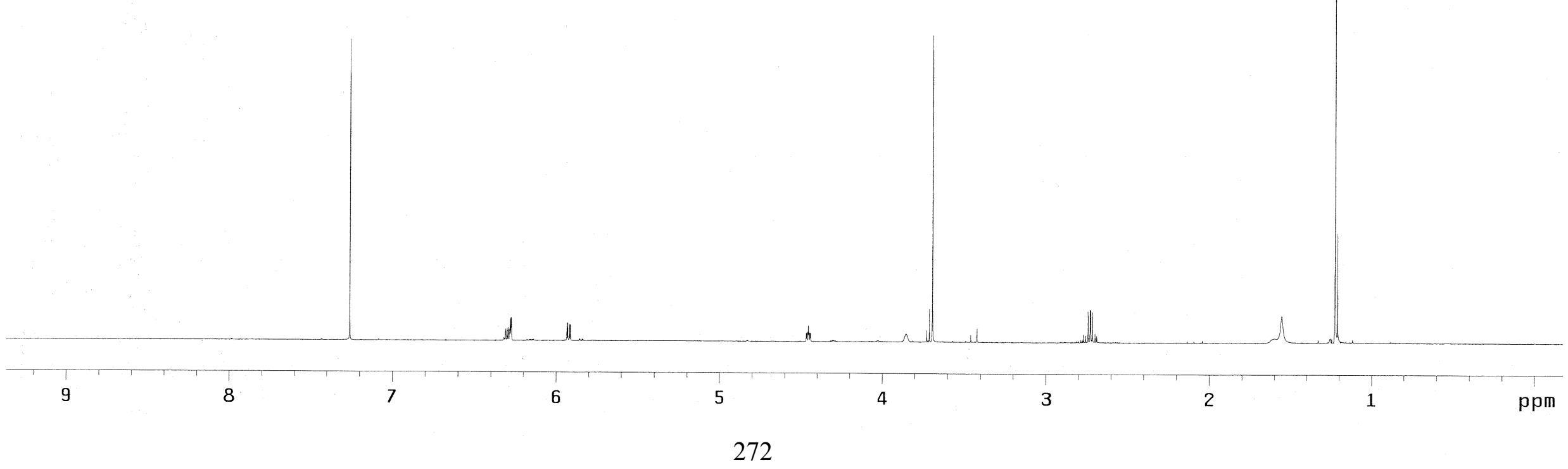




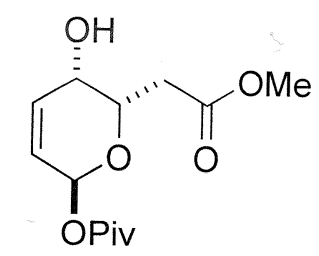

${ }^{13} \mathrm{C}$ NMR (150 MHz)

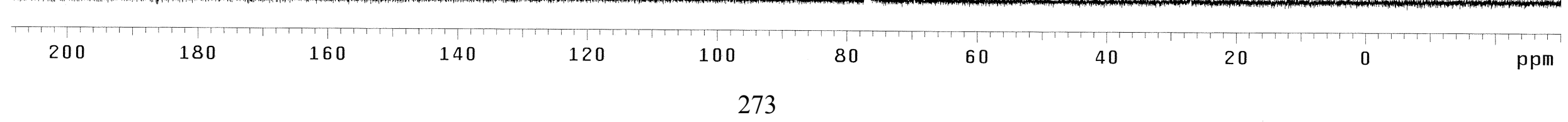



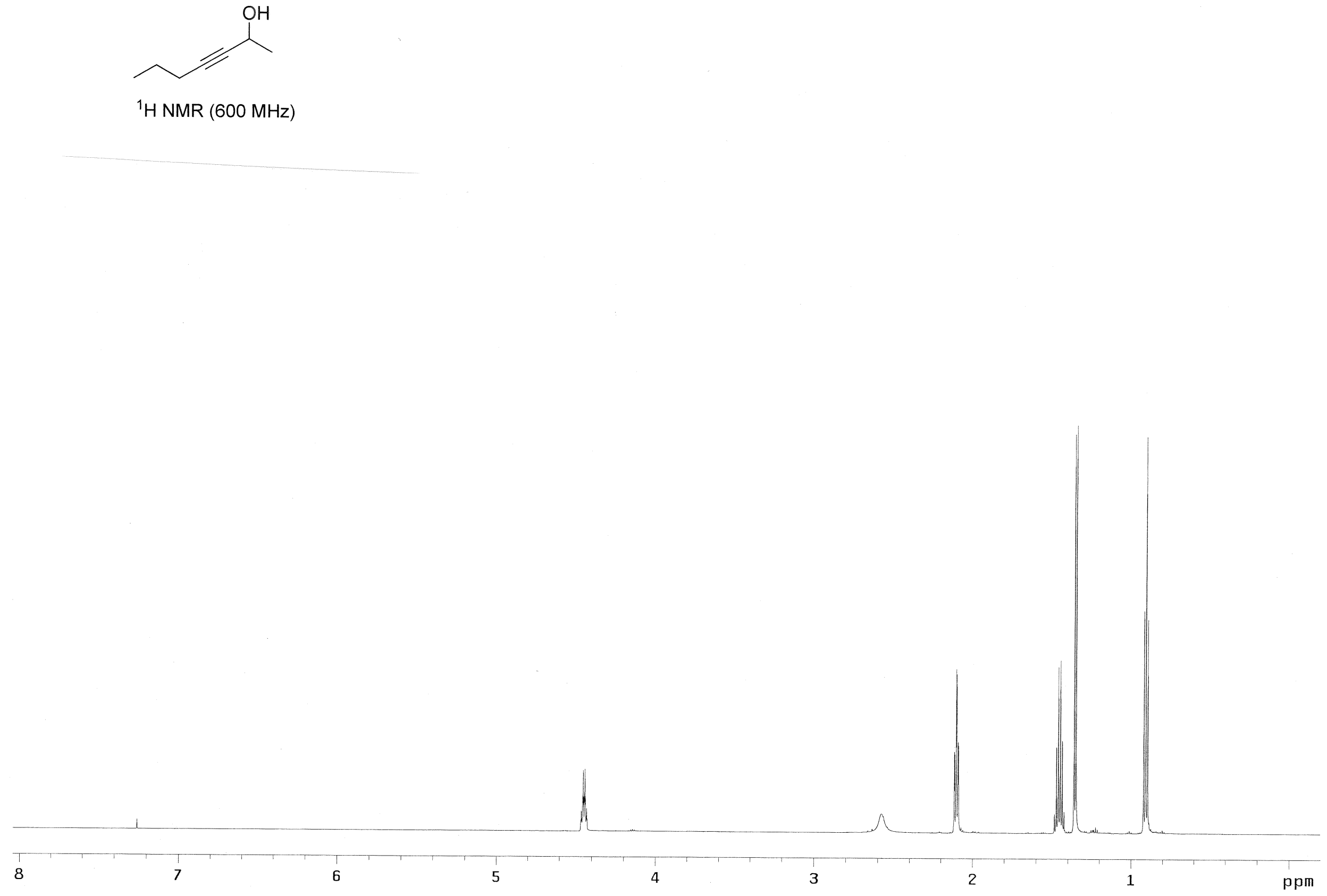


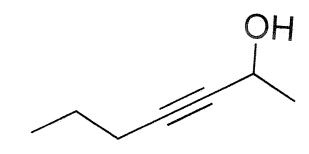

${ }^{13} \mathrm{C}$ NMR (150 MHz)

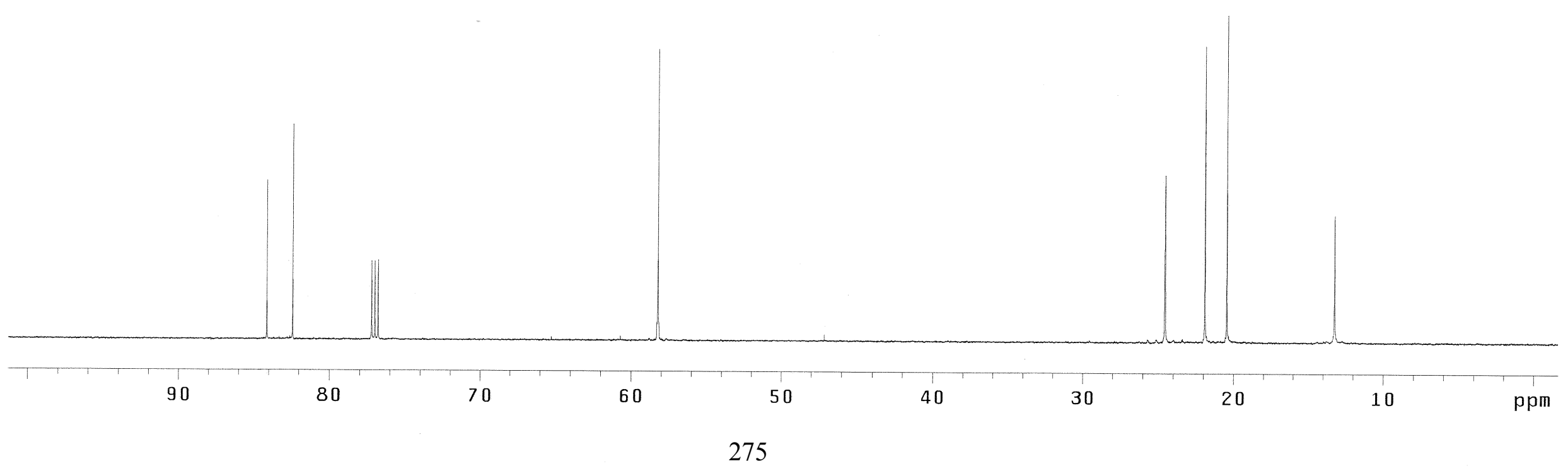




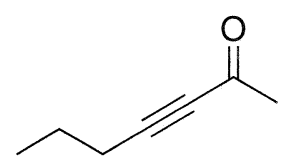

${ }^{1} \mathrm{H}$ NMR $(600 \mathrm{MHz})$

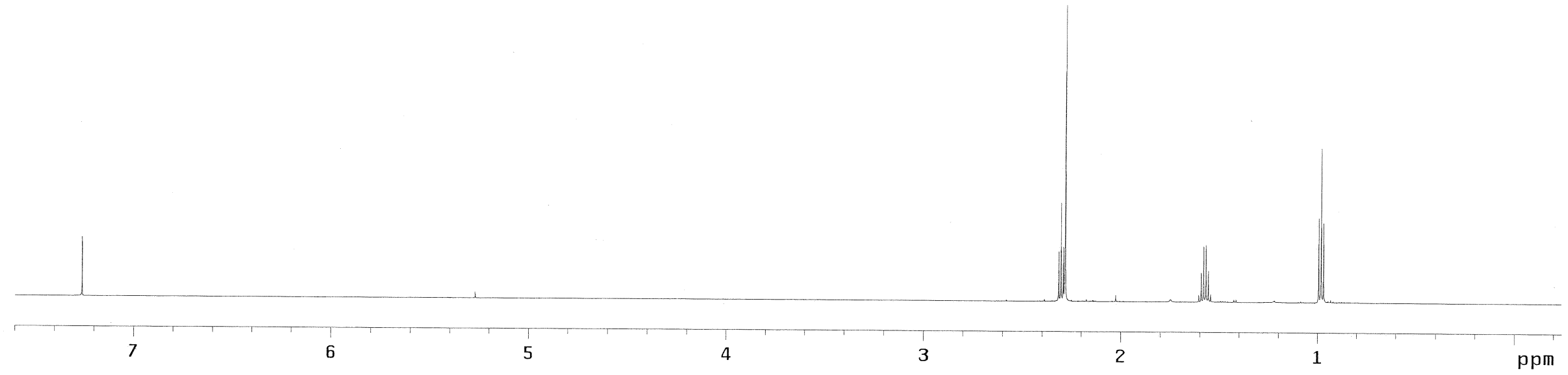




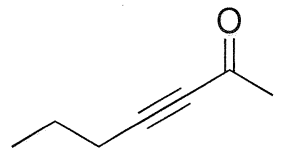

${ }^{13} \mathrm{C}$ NMR (150 MHz)

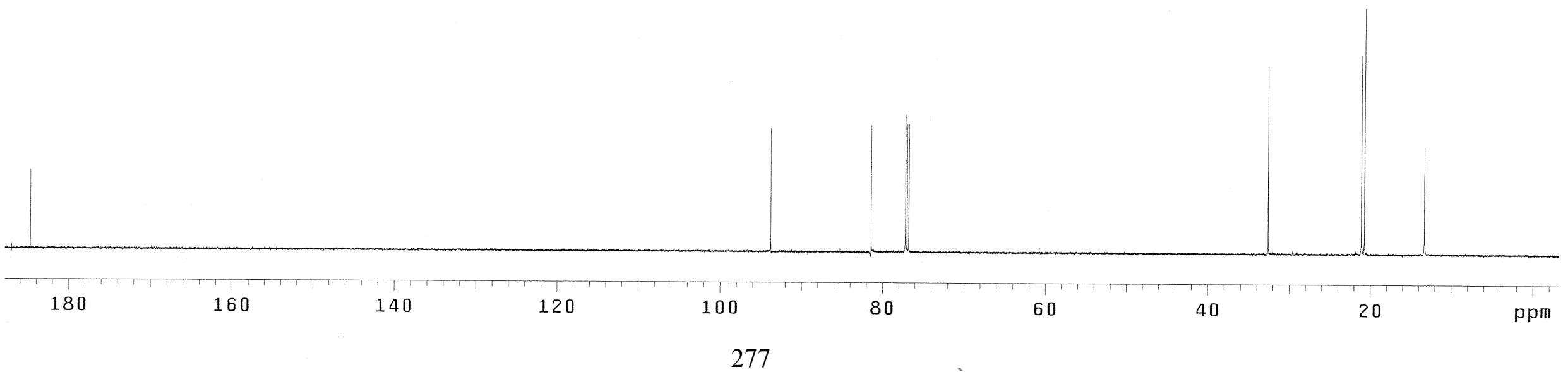




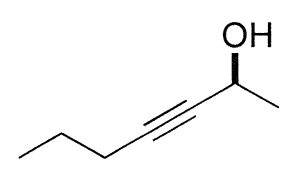

${ }^{1} \mathrm{H}$ NMR $(600 \mathrm{MHz})$

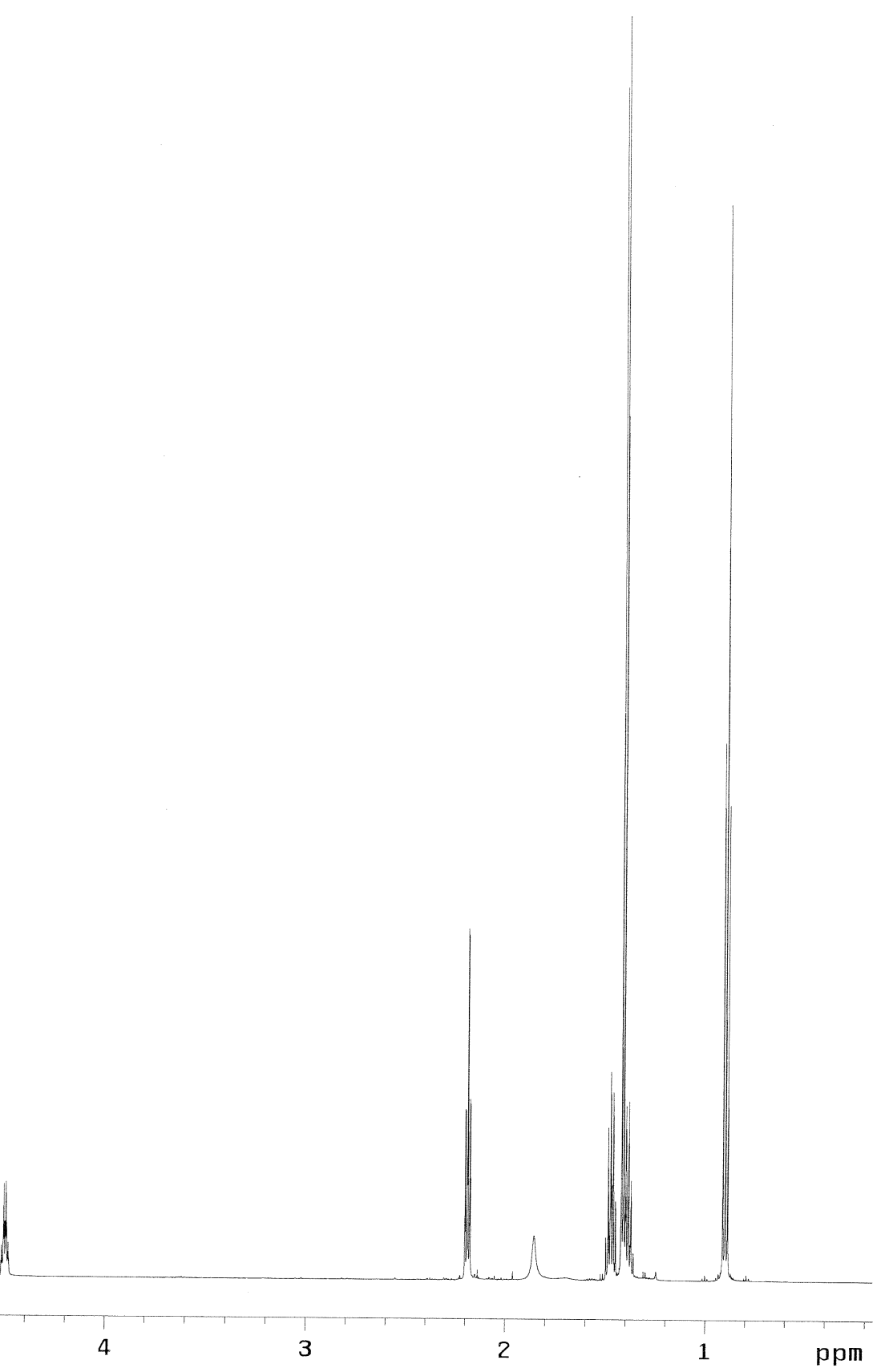




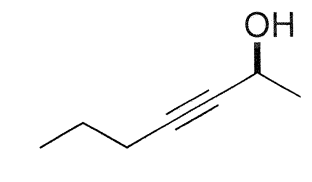

${ }^{13} \mathrm{C}$ NMR $(150 \mathrm{MHz})$

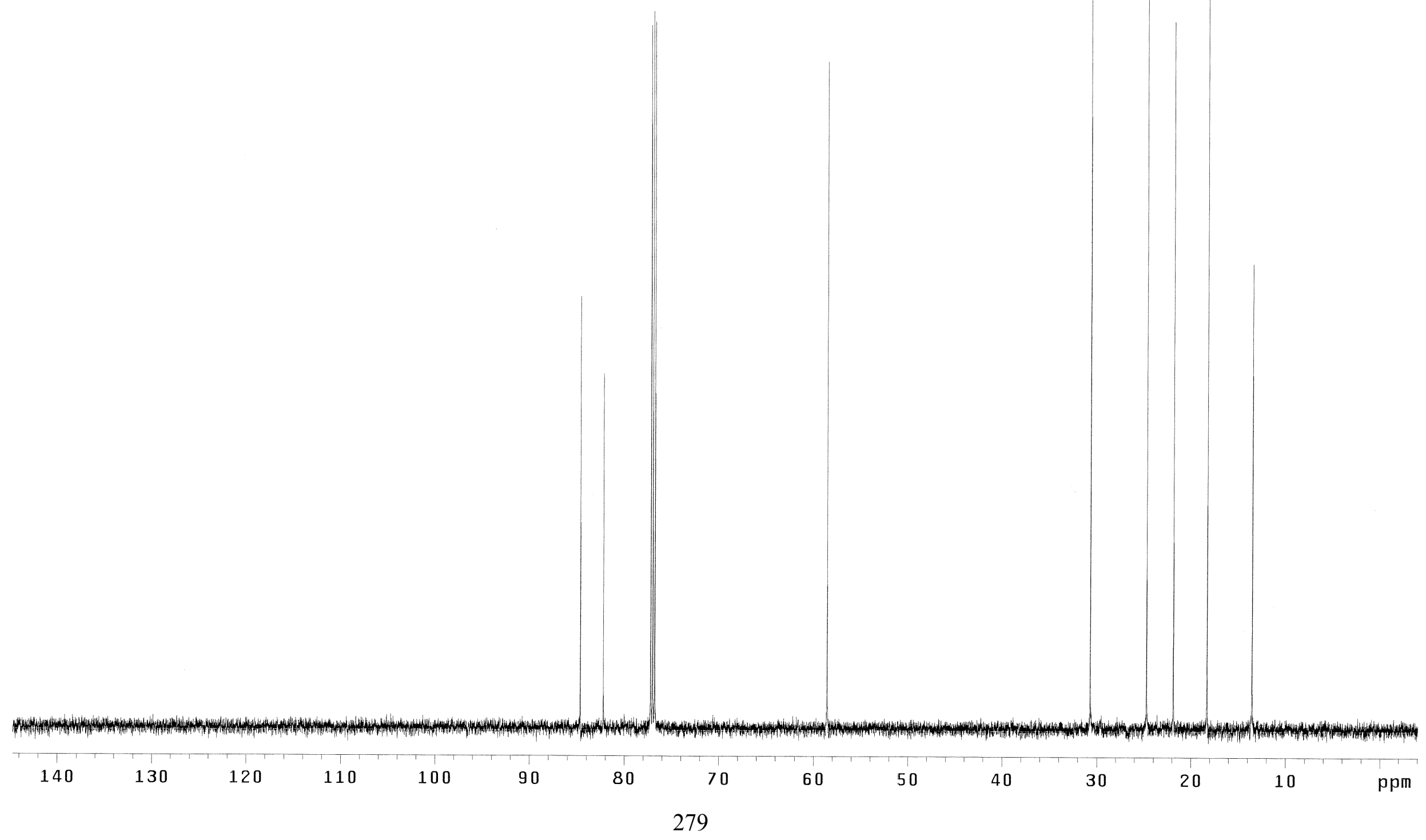




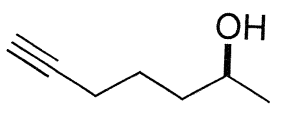

${ }^{1} \mathrm{H}$ NMR $(600 \mathrm{MHz})$

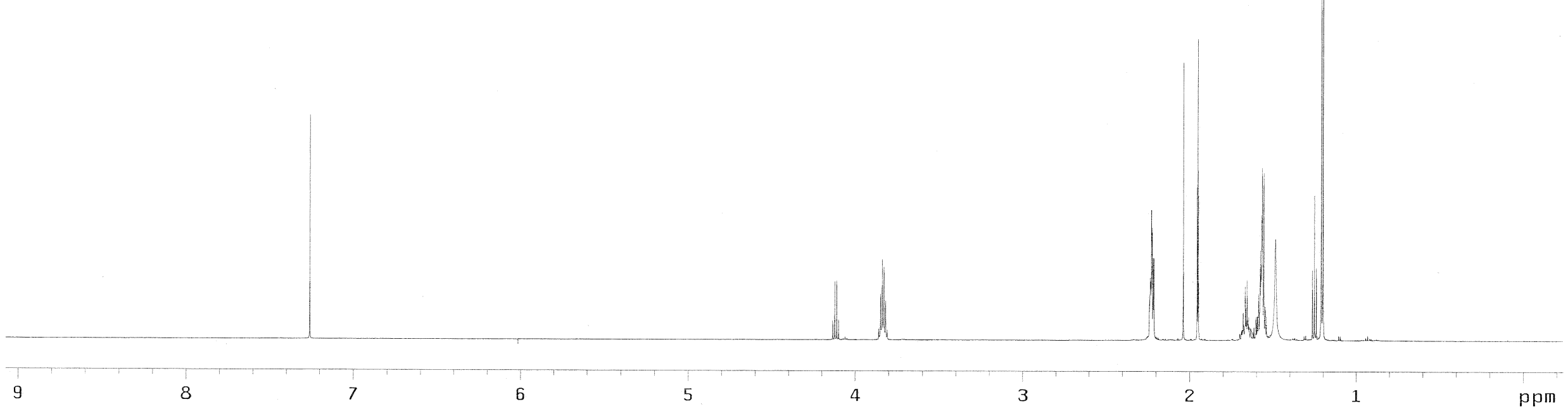




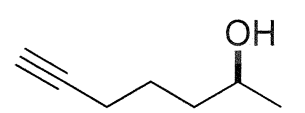

${ }^{13} \mathrm{C}$ NMR $(150 \mathrm{MHz})$

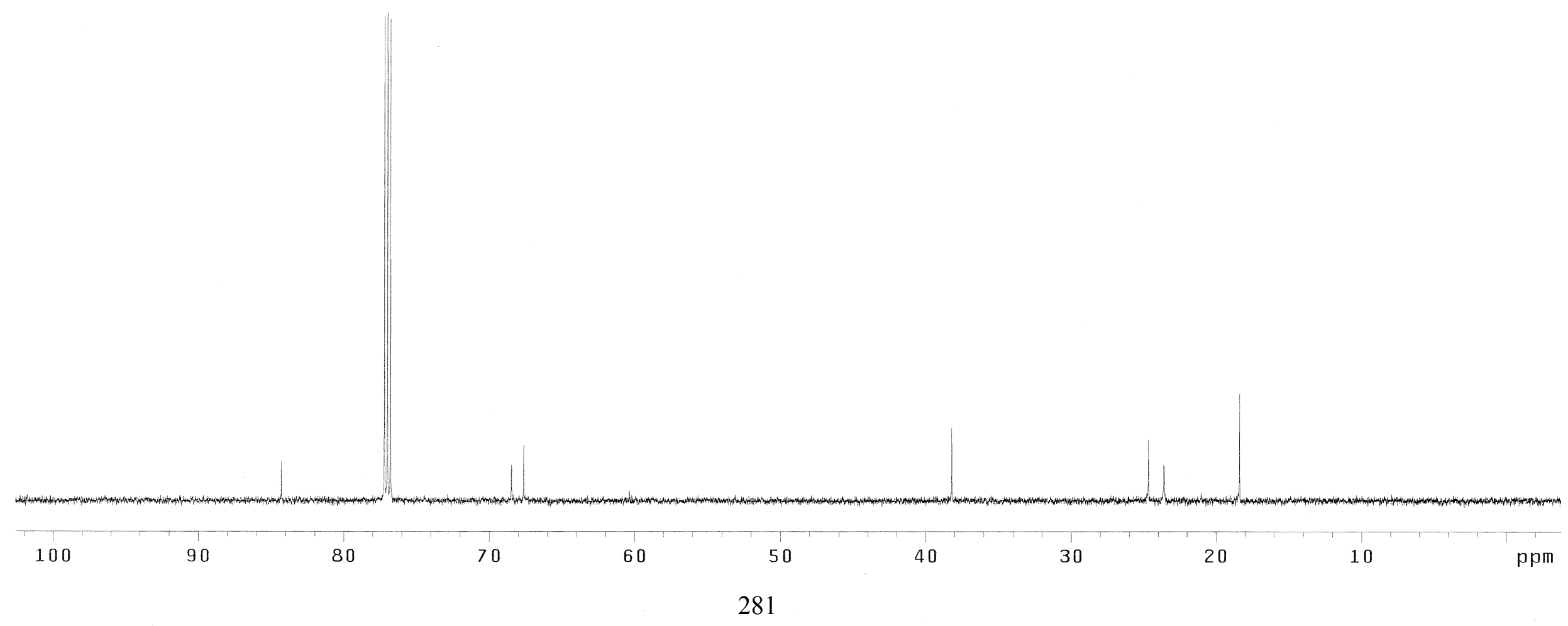




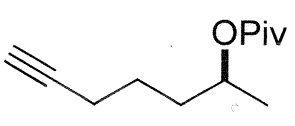

${ }^{1} \mathrm{H}$ NMR $(600 \mathrm{MHz})$

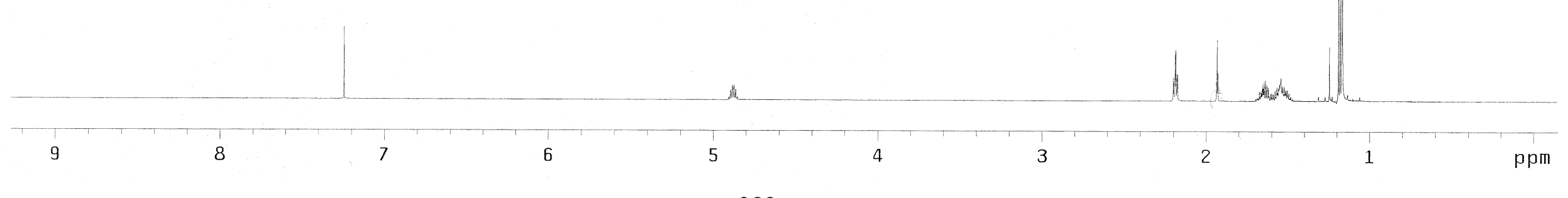




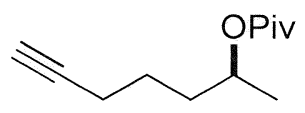

${ }^{13} \mathrm{C}$ NMR $(150 \mathrm{MHz})$

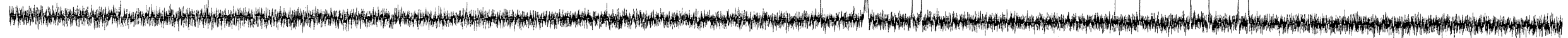

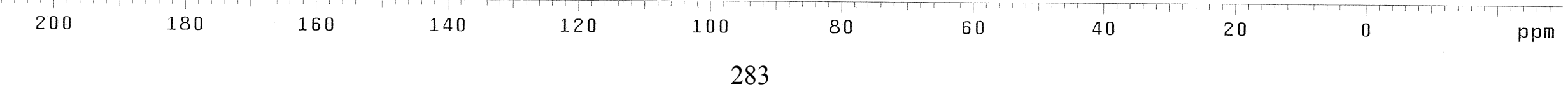




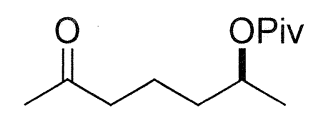

${ }^{1} \mathrm{H}$ NMR $(600 \mathrm{MHz})$

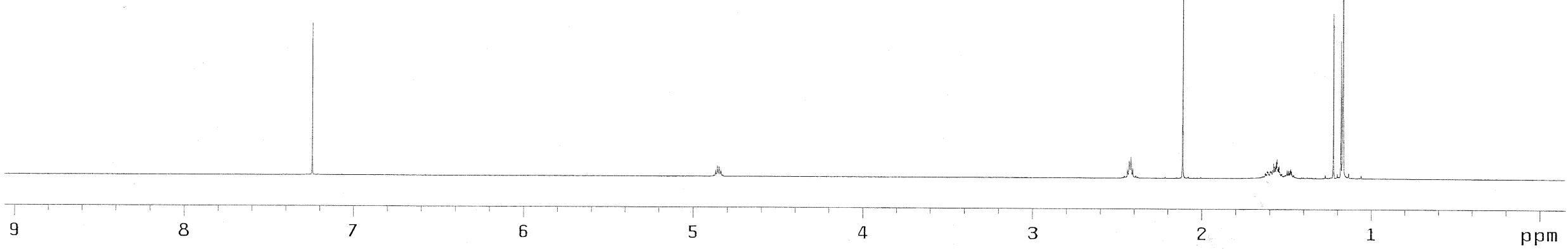




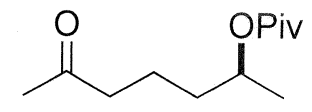

${ }^{13} \mathrm{C}$ NMR (150 MHz) 
OPiv

${ }^{1} \mathrm{H} \mathrm{NMR}(600 \mathrm{MHz})$

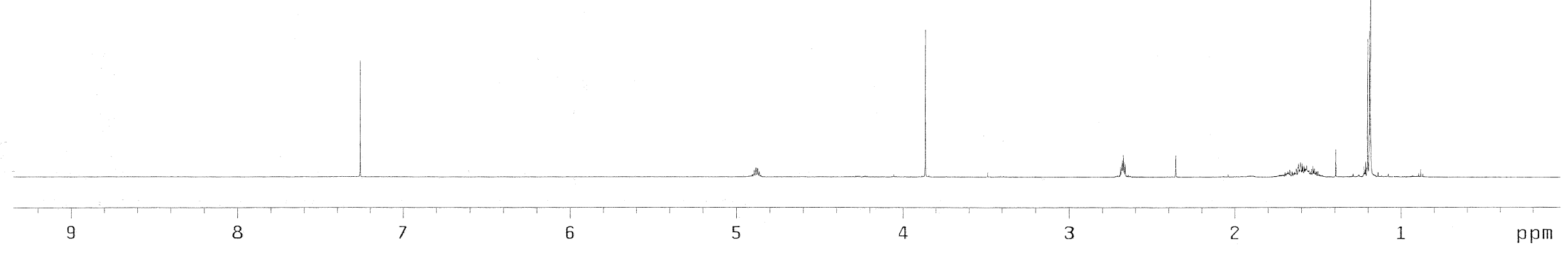


Br

${ }^{13} \mathrm{C}$ NMR $(150 \mathrm{MHz})$

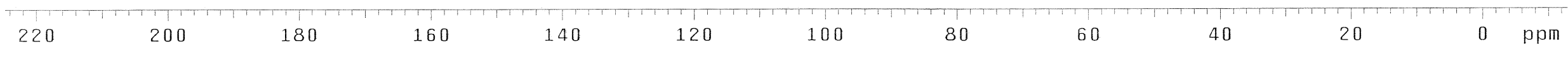


Priv

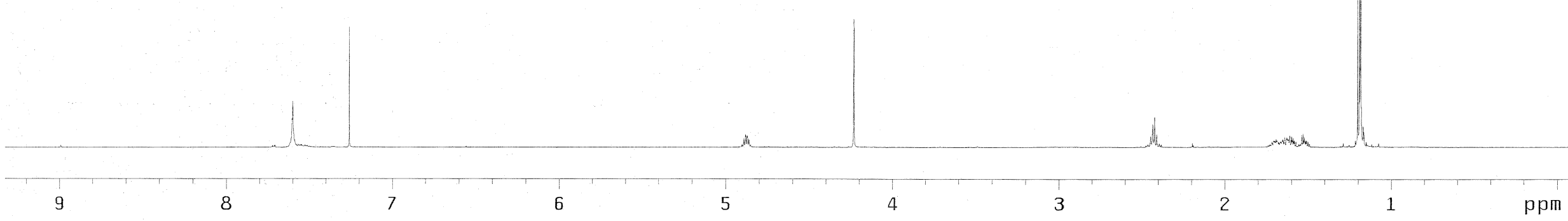




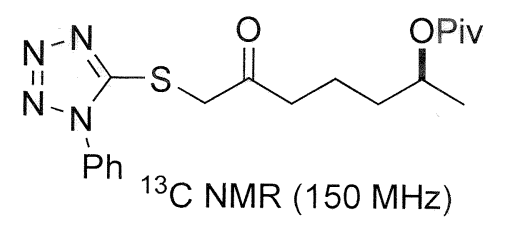



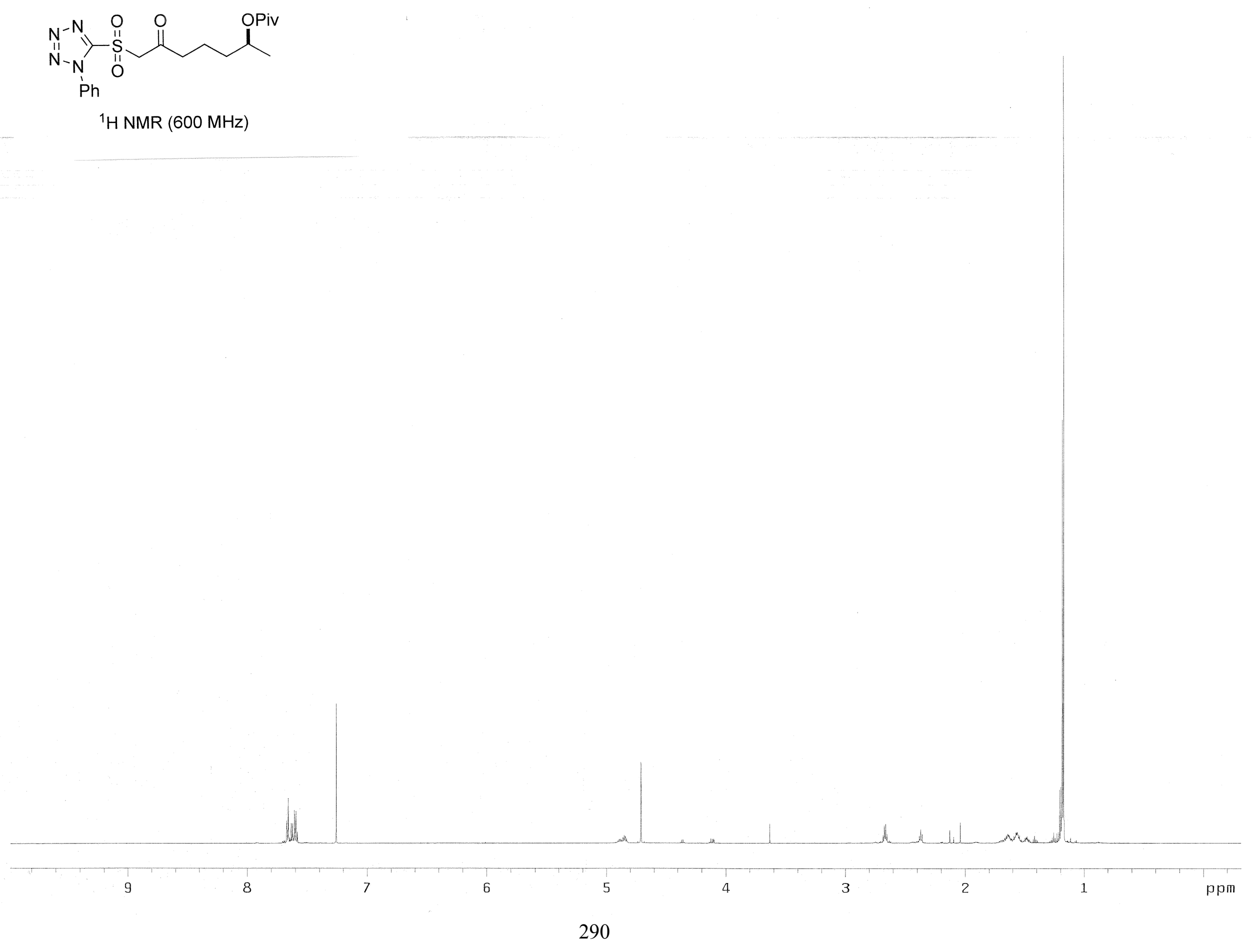


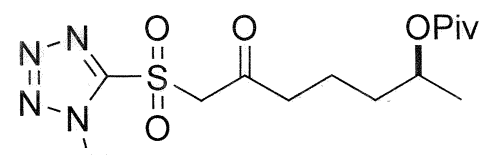

${ }^{13} \mathrm{C}$ NMR $(150 \mathrm{MHz})$

Whoth 


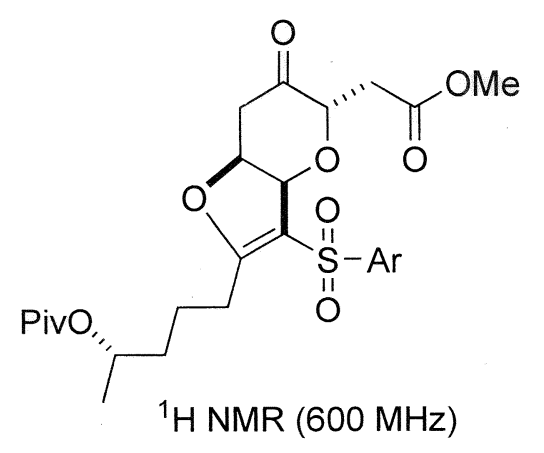




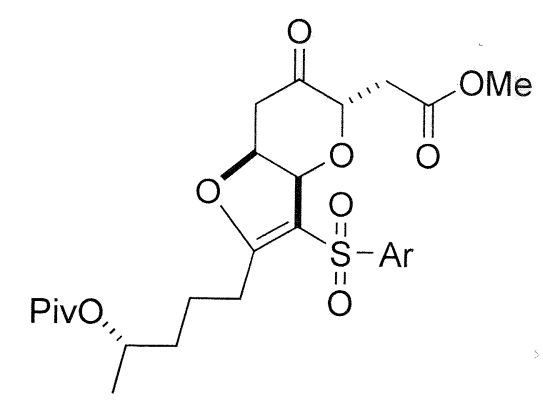

${ }^{13} \mathrm{C}$ NMR $(150 \mathrm{MHz})$

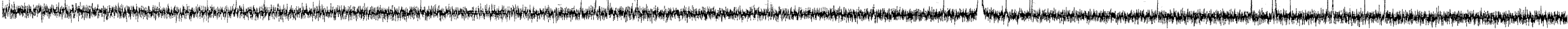

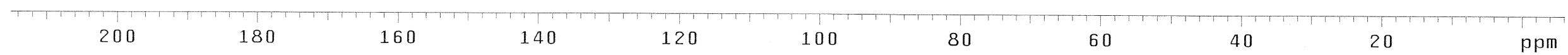




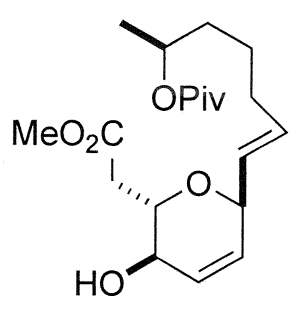

${ }^{1} \mathrm{H}$ NMR $(600 \mathrm{MHz})$ 


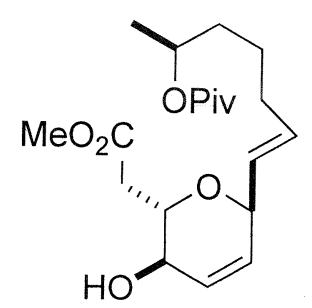

${ }^{13} \mathrm{C}$ NMR $(150 \mathrm{MHz})$ 

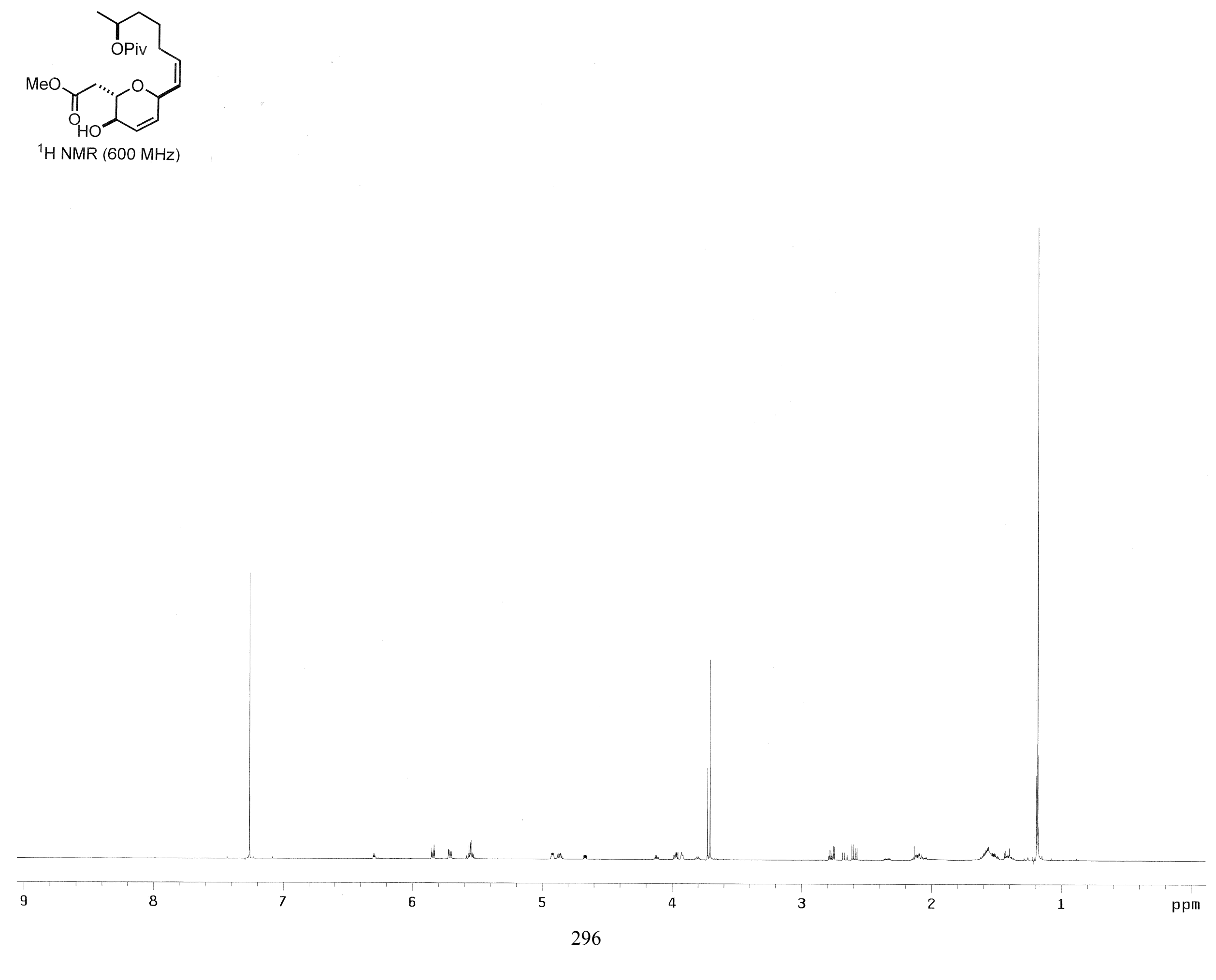